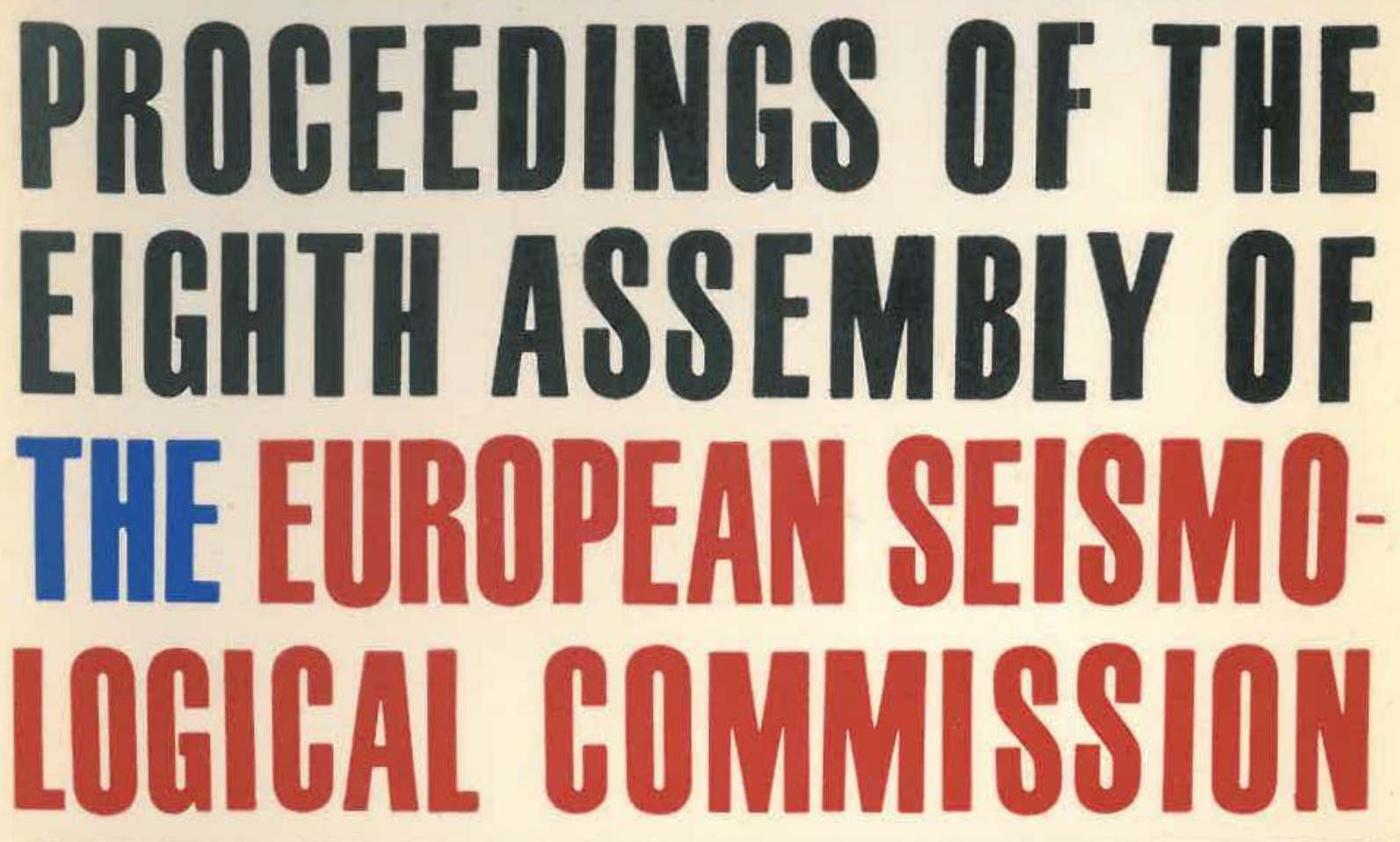


PROCEEDINGS OF THE EIGHTH ASSEMBLY OF THE EUROPEAN SEISMOLOGICAL COMMISSION 



\title{
PROCEEDINGS OF THE EIGHTH ASSEMBLY OF THE EUROPEAN SEISMOLOGICAL COMMISSION
}

\author{
EDITED BY \\ E. BISZTRICSÁNY
}

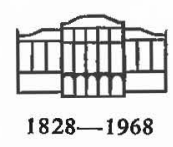

AKADÉMIAI KIADÓ, BUDAPEST 1968 
The Eighth Assembly of the European Seismologicai Commission under the auspices of the International Association of Seismology and Physics of the Earth's Interior, and the International Union of Geodesy and Geophysics was organized by the Hungarian Seismological Institute

\author{
Organizing Committee \\ LÁSZLÓ EGYED \\ EDE BISZTRICSÁNY \\ DEZSÕ CSOMOR \\ ZOLTÁN KISS
}

(C) Akadémiai Kiadó, Budapest 1969

\author{
Responsible for publication: Gy. Bernát
}

Director of the Publishing House of the Hungarian Academy of Sciences and the Academy Press

Responsible editor: L. Zombori Technical editor: A. Fülöp Cover and jacket: M. Kisfalusy

Printed in Hungary at the Academy Press, Budapest 


\section{CONTENTS}

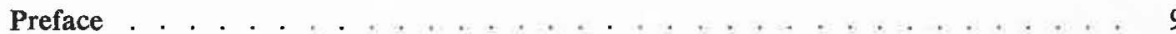

A. Zátopek

Presidential Address . . . . . . . . . . . . . . . . . . 11

A. Zátopek

Rapport d'activité $1962-1964$. . . . . . . . . . . . . . 13

\section{CRUSTAL AND UPPER MANTLE INVESTIGATIONS}

V. M. Arhangelskaya and I. M. Kuznetsova

Study of the earth's crust upper parts by surface waves dispersion . . . . . . 23

V. Babuška, L. Ruprechtová and O. Zenklová

Peculiarities in the energy propagation of the East-Alpine earthquakes . . . . 30

H. Burkhardt, O. Rosenbach und R. Vees

Der seismische Impuls bei Unterwassersprengungen in verschiedenen Registrier-

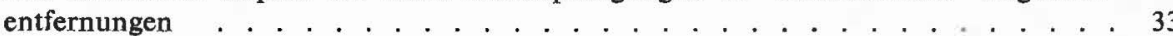

V. Cervený, A. M. Epinat'eva and I. P. Kosminskaya

Dynamic and kinematic properties of seismic waves in the region of critical angle 43

V. Červený and J. Jänský

The amplitudes of seismic body waves propagating in the earth's crust . . . . 44

N. Delibasis and A. G. Galanopoulos

Space and time variations of strain release in the Greek area . . . . . . . . 49

G. Dohr

Ergebnisse reflexionsseismischer Messungen zur Untersuchung des Baues der Erdkruste in der Bundesrepublik . . . . . . . . . . . . . . . . . . . 59

O. Förtsch

Ein Diskussionsbeitrag zur Ausbreitung der Longitudinalwelle . . . . . . . . . 82

$P$. Giese und $G$. de Visintini

Ergebnisse des Lago Lagorai-E-Profils . . . . . . . . . . . . . . . 95

E. Grigorova et S. Rijikova

Sur quelques particularités des ondes longitudinales enregistrées à Sofia . . . . 102 


\section{Güth}

Gruppengeschwindigkeitsdispersion kurzperiodischer Oberflächenwellen von eurasischen Erdbeben

\section{J. Hjelme}

Investigations of the crust below Denmark and the sea between Denmark and Norway 115

J. N. Jordan, R. A. Black and Ch. C. Bates

Patterns of maximum amplitudes of $\boldsymbol{P}_{n}$ and $\boldsymbol{P}$-waves over regional and continental

\section{N. K. Karapetian}

On determining the structure of the earth's crust of the Lesser Caucasus by seismic data

Z. Kiss

Untersuchung der durch Sprengung erzeugten Oberflächenwellen kurzer Periode

L. Knopoff

The International Upper Mantle Project

N. V. Kondorskaya and L. B. Slavina

Some results of investigation of regional peculiarities of travel-times of seismic waves by observation of seismic stations of the USSR . . . . . . . . . . . 146

I. Litvinenko

The crustal structure of the Baltic shield

A. A. Luck and I. L. Nersesov

The structure of the earth mantle's upper part according to the observations of the earthquakes with intermediate focal depth

E. Mituch and $K$. Posgay

Deep seismic sounding in Hungary

\section{L. Nersesov and T. G. Rautian}

The study of dynamics and kinematics of seismic waves at the profile Pamir-Baikal 174

N. Öcal

Fault-plane solutions for the Agadir, Morocco, earthquake of February 29, 1960 and the Skoplje, Yugoslavia, earthquake of July 26, 1963 . . . . . . . . . . 182

N. Öcal

Crustal structure in Anatolia

I. P. Passechnik

On determining frequency dependence of absorption coefficient of longitudinal seismic waves propagating in the earth's mantle . . . . . . . . . . . 183

K. Peغ et O. Novotný

La structure de l'écorce terrestre en Tchécoslovaquie à la base de la dispersion des ondes superficielles . . . . . . . . . . . . . . . . . . . . 189

I. V. Pomerantseva, A. N. Moszhenko, I. A. Sokolova, G. V. Egorkina and E. D. Taghay Structure of the earth's crust and upper mantle in the south-eastern part of the Russian platform .....................

I. I. Popov and S. A. Kapitanova

Group velocity dispersion of Rayleigh waves according to Simferopol observations 201 


\section{Prodehl}

Results of seismic refraction measurements in Southern Bavaria and along a profile through the Eastern Alps . . . . . . . . . . . . . . . . . . 207

Sh. S. Ragimov

The group character of observing surface seismic waves in a layered crust . . . 209

Sh. S. Ragimov

The phase velocities determination method and the earth's crust depth estimation in Azerbaijan by Rayleigh waves .... . . . . . . . . . . . . 212

V. Z. Ryaboy

Structure of the earth's crust and upper mantle in the central regions of Turkmenia according to deep seismic sounding data (DSS) . . . . . . . . . 216

E. F. Savarensky, V. B. Glasko and Ya. Sh. Granit

Rayleigh and Love waves dispersion curves for two- and three-layered earth's crust 222

E. F. Savarensky, O. E. Starovoit and S. A. Fedorov

Rayleigh long-period waves of Alaska earthquake on March 28, 1964 . . . . . 240

A. P. Sinitsyn

The influence of the obstacles on the parameters of seismic waves . . . . . . 247

V. B. Sollogub, N. I. Pavlenkova and A. V. Chekunov

Deep seismic research in Ukraine

J. Vaněk and C. Radu

Amplitude curves of seismic body waves at distances smaller than $12^{\circ}$. . . . 261

L. Waniek, J. Vaněk, Z. Pros and K. Klíma

Three-dimensional seismic models of the earth's upper mantle . . . . . . . . 264

P. S. Weizman

Characteristics of the crustal structure in some zones of transition from continent

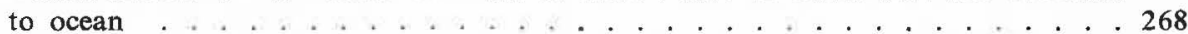

B. Wojtczak-Gadomska, A. Guterch and J. Uchman

Preliminary results of deep seismic sounding in Poland . . . . . . . . . . . 278

O. I. Yurkevich and B. I. Volosecky

Determination of the thickness of the earth's crust in the Carpathians from the dispersion of the surface waves from Chilean earthquakes in 1960 . . . . . . 285

\section{SEISMICITY AND MAGNITUDE}

L. Ahorner

Seismicity and quaternary structural activity in the Northern Rhine district . . 295

V. I. Bune

Seismological data on Europe seismotectonic map . . . . . . . . . . . 304

I. E. Gubin

Interrelations between earthquakes and geological structures . . . . . . 310

W. Sponheuer

Die Seismizität auf dem Gebiet der Deutschen Demokratischen Republik . . 313 


\section{A. Zátopek}

Seismicity of Macedonia

\section{A. Zátopek}

Tätigkeitsbericht der Subkommission für die seismische Untersuchung des Karpatenund Balkangebiets im Zeitraum 1962-1964

\section{INSTRUMENTS AND DEVICES}

E. Bisztricsány, D. Csomor, L. Egyed and Z. Kiss

New network of seismological stations in Hungary . . . . . . . . . . . . 333

G. N. Bozhko and O. E. Starovoit

Phase velocities of Rayleigh waves on the Russian platform

T. Iosif

Sur l'existence de la couche de faible vitesse dans la partie supérieure du manteau en Roumanie . . . . . . . . . . . . . . . . . . . . 344

R. Maaz

Theoretische Untersuchungen über einen eingebetteten Seismographen

R. Parks

Data acquisition and processing equipment for Edinburgh Royal Observatory . 356

Ch. Teupser

Frequenzabhängige Kopplung bei elektrodynamischen Seismographen . . . . 361

V. Tobyás

Electromagnetic velocity seismographs with non-negligible reaction of the galvanometer

A. Zátopek

Closing speech

List of Participants 
The Eighth Assembly of the European Seismological Commission was held in Budapest between the 7th and 13th of September, 1964. The ESC belongs to the International Association of Seismology and Physics of the Earth's Interior, and the International Union of Geodesy and Geophysics.

The ESC was invited by the Hungarian Academy of Sciences to hold the Eighth Assembly in Budapest. The Organizing Committee included the following members: Professor László Egyed, corresponding member of the Hungarian Academy of Sciences; Dr. Ede Bisztricsány, Hungarian Seismological Institute; Dezső Csomor, Hungarian Seismological Institute; and Zoltán Kiss, Hungarian Seismological Institute.

The manuscripts are generally published in their original form as presented to the Organizing Committee, apart from a few instances, where corrections were necessary to be carried out in order to unify the studies, which was for the benefit of the whole. 



\title{
PRESIDENTIAL ADDRESS
}

\author{
A. ZÁTOPEK
}

President of the ESC

(Prague, Czechoslovakia)

\section{Excellences, Mr. Chairman, Ladies and Gentlemen, Dear Colleagues}

At the foregoing seventh General Assembly of the European Seismological Commission, held in Jena in September 1962, it was decided to follow the invitation, kindly presented by the representative of the Hungarian People's Republic to organize the eighth Assembly of the European Seismological Commission in Budapest in 1964. In Berkeley, a year ago (1963), it was settled that it should be held in the beginning of September 1964.

Please allow me now to express, on behalf of the European Seismological Commission, our deeply felt gratitude for this invitation. I would also like to thank Szádeczky-Kardoss academician, the representative of the Hungarian Academy of Sciences, Mr. Gyalmos the Vice-President of the Municipal Council, representative of the President of the Municipal Council of Budapest, further Tárczy-Hornoch academician, the President of the National Committee of Geodesy and Geophysics, and Dr. Sebestyén the President of the Association of the Hungarian Geophysicists, for their kind and cordial words of welcome and for their good wishes addressed to our Assembly. I also have the honour to welcome at our Assembly Professor Rothé, Secretary General of the International Association of Seismology and Physics of the Earth's Interior, and Professor Knopoff, Secretary General of the IUGG Upper Mantle Committee.

It certainly will reflect the feelings of all of us, if I express our thanks to the Hungarian Organizing Committee, headed by Professor Egyed, member of the Hungarian Academy of Sciences, for their enthusiastic preparatory work which enabled us to start and ensure a rich programme at our sessions.

We desire that our work in Budapest be conformable to Hungarian scientific tradition in geophysics and seismology, as represented for many years by the names of Eötvös, Kövesligethy and Réthly, known all over the world, and is still living. Hungarian geophysicists, including seismologists, take care of its progressive development, practically in all lines of modern geophysics. But not only this; we know that Hungarian geophysical instruments of excellent quality, which have contributed to the fact that Hungary is one of the countries with a densest network of geophysical measurements, are exported into all parts of the world. 
Let us now have a look at the main tasks of our Assembly:

1. We shall review the work done by the ESC-Sub-Commissions and working groups during the past two years, as well as the research work accomplished in various $\mathrm{ESC}$-member-countries.

2. We have to discuss many original scientific contributions, presented to our Assembly, and, after having estimated their importance, we have to formulate some conclusions and recommendations in order to have fixed points facilitating the future seismological research in Europe.

3. We must try to find out in what way we should include the results of the Unesco Intergovernmental Meeting on Seismology and Earthquake Engineering. held in Paris in April, 1964, into our working programme. It should also be decided how we approach the implementation of the recommendation of the Upper Mantle Project Committee concerning the investigation of possibilities to carry out, in the framework of the European Seismological Commission, international long profile researches of the crust and upper mantle in Europe, the respective profiles going from the northern coast of Scandinavia to the southern coasts of the Mediterranean, and from Eastern Europe to the west towards the Atlantic Ocean.

4. Finally, we have to solve, in order to facilitate the implementation of the tasks just mentioned, a number of administrative questions.

All these subjects together represent a big working programme which is to be fulfilled in a fairly limited period of time. Therefore, measures must be taken requiring a high degree of discipline in order to spare as much time as possible on the most important topics of the programme.

I believe that in the traditional atmosphere of friendship and of deep interest in essential problems we take together a further step to the development of our science; the results of which may serve - as the present and future reconstruction programme of the city of Skopje, heavily destroyed by earthquake a year ago, demonstrates - the benefit of the whole of mankind.

I declare herewith the Eighth Assembly of the European Seismological Commission open and I wish full success to its work. 


\section{RAPPORT D'ACTIVITÉ \\ 1962-1964}

A. ZÁTOPEK

(Prague, Tchécoslovaquie)

\section{Introduction}

La Commission Séismologique Européenne dont nous avons ouvert la VIII ${ }^{\mathrm{e}}$ Assemblée Générale, a été créée dans le but de favoriser par tous les moyens possibles la solution des problèmes séismologiques concernant la zone nommée l'Europe qui est définie à l'Ouest par la crête médiane de l'Atlantique située au Nord du $30^{\mathrm{e}}$ parallèle, au Nord par l'Océan Arctique, à l'Est par l'Oural et les pays côtiers de la Mer Noire et de la Méditerranée (inclus) et au Sud par les pays côtiers de la Méditerranée (inclus).

C'était déjà à la première Assemblée Générale de Stuttgart en 1952, où on a fixé le sujet central: "La structure séismique du continent européen». Ce thème représente un complexe de problèmes qui sont étudiés à présent, (partiellement dans le cadre du Projet du Manteau Supérieur) dans les directions suivantes:

(1) L'établissement de nouvelles stations séismiques et le perfectionnement de l'appareillage (théorie et construction des séismomètres, homogénéisation des appareils et du réseau des stations).

(2) La séismicité (catalogues séismiques, cartes séismiques, localisation des foyers, classification énergétique des séismes et un nombre de problèmes associés).

(3) La séismotectonique (relations entre la séismicité et la tectonique du continent européen, carte séismotectonique de l'Europe).

(4) L'étude théorique et expérimentale des ondes séismiques, spécialement par rapport à la structure de l'Europe (propriétés cinématiques et dynamiques des ondes séismiques, le mécanisme des séismes, modèles séismiques etc.).

(5) L'étude des couches profondes de l'écorce terrestre et du manteau supérieur au moyen de diverses méthodes (grandes explosions - les explosions nucléaires y inclus, sondages séismiques profonds dans les Alpes, en Europe septentrionale, dans la zone carpathique, en Pologne, dans l'URSS etc.).

(6) L'étude des microséismes observés dans les stations européennes.

Naturellement, on prête attention aux problèmes méthodiques et organisatoires aussi bien qu'à l'application de nouvelles techniques.

\section{Sous-commission et groupes de travail}

Pour revoir le travail, accompli par les organes de la CSE et dans ses étatsmembres depuis l'Assemblée de Jena, nous examinerons comment on a rempli les huit résolutions adoptées à Jena. 
La Résolution No 1 qui recommandait qu'on devrait recueillir de nouvelles données dans les Alpes occidentales et procéder dans les régions encore non étudiées, a été remplie par la Sous-Commission des Explosions Alpines, sous la présidence du Professeur Closs, d'une manière excellente. A l'Assemblée générale de l'UGGI à Berkeley 1963, j'ai eu le privilège d'apprécier le remplissement du dernier point de la résolution, c'est à dire l'interprétation uniforme de l'analyse de l'ensemble des données.

Celle-ci a découvert la zone anomale d'Ivrée (caracterisée par des vitesses élevées et une anomalie gravimétrique) et a démontré l'existence des racines des Alpes occidentales. Les profondeurs maxima de la surface de Mohorovičić ont été trouvées environ à $60 \mathrm{~km}$ et diminuantes vers l'extérieur du massif alpin. Au Nord des Alpes les profondeurs du Moho sont de 26 à $30 \mathrm{~km}$.

Le livre sur ces recherches, publié en 1963 par le Centre National de la Recherche Scientifique à Paris, peut servir de modèle en ce qui concerne la présentation des résultats qui ont été atteints par la coopération étroite des savants allemands, français, anglais, suisses et italiens. Je félicite M. le Professeur Closs, Mme Labrouste et tous leurs collaborateurs et je les remercie. Je voudrais bien apprécier aussi la contribution et les suggestions que nos collègues américains ont apportées à la recherche de la structure de l'Europe occidentale.

La Sous-Commission a organisé plusieurs réunions et conférences de travail et a développé une coopération permanente avec la Deutsche Forschungsgemeinschaft et d'autres organisations. Ainsi, son radius d'activité s'est élargi même en dehors de la zone alpine et son travail futur exigera une réorganisation convenable suivant les recommendations qui seront présentées à notre Assemblée par le Président de la Sous-Commission.

La Résolution No 2 concerne la Sous-Commission de la Séismicité de l'Europe, présidée par le Dr. Kárník. Celui-ci a présenté, empêché de prendre part à nos séances, un rapport détaillé d'où on voit qu'il a continué à cataloguer les séismes européens; il a revu, au fond de commentaires de divers pays-membres, la première version des cartes des intensités maxima de l'Europe. En établissant la deuxième version de la carte des isoséistes maxima de l'Europe il a été, naturellement, lié par la présentation des cartes nationales. M. Kárník a procédé surtout dans l'analyse des relations fondamentales de classification des tremblements à l'aide de la magnitude et enfin a essayé de définir des régions naturelles caractérisées par les mêmes traits principaux de leur séismicité. Ce grand travail, accompli par M. Kárník, mérite une appréciation extraordinaire.

L'étude coordonnée du mécanisme au foyer des séismes européens n'a pas encore été commencée malgré le fait que le sujet a été traité théoriquement et expérimentalement et fut appliqué dans plusieurs régions de l'Europe.

Le problème de l'échelle macroséismique modifiée par MM. Medvedev, Sponheuer et Kárník a surpassé le cadre de notre Commission parce que, après la discussion devant l'AISPIT à Berkeley, l'Association a créé un groupe de travail, présidé par M. Sponheuer, pour la standardisation de l'échelle macroséismique. MM. Medvedev et Sponheuer ont apporté de nouvelles contributions à ce problème. 
A Jena, 1'Assemblée de la CSE a décidé de créer, dans le sein de la Sous-Commission de Séismicité, un groupe de travail pour l'étude du bouclier baltique. Les premiers commencements du travail ont été faits en Finlande et Norvège.

La Résolution No 3 concerne la Sous-Commission pour l'étude séismique des régions des Carpathes et des Balkans, présidée par le Professeur Zátopek.

Les appareils, possédant les caractéristiques uniformes, recommandés dans la résolution, ont été installés dans quelques stations en Tchécoslovaquie, Hongrie, Pologne et Bulgarie et sont planifiés en Yougoslavie et Roumanie. Néanmoins, le perfectionnement du réseau des stations dans cette région, surtout sur la Péninsule balcanique, laisse beaucoup à désirer quant à la densité des stations et à la modernisation de l'appareillage. L'obtention des données macroséismiques a été complétée par l'étude des archives dans des pays voisins (Tchécoslovaquie, Yougoslavie), analyse comparative etc. L'étude du tremblement destructif de Skopje le 26 juillet 1963 et du fort tremblement de Slavonski Brod du 13 avril 1964 ont donné l'occasion d'appliquer les directives de la CSE.

Le groupe de coordination des sondages profonds, créé à Jena au sein de cette Sous-Commission, est présidé par Mme Kosminskaya. Le groupe a abouti, après un échange initial de correspondance et après plusieurs conférences bilatérales, organisées en 1963 entre les séismologues de l'Allemagne Démocratique, de la Pologne, de la Hongrie et de la Tchécoslovaquie, en présence du Président de la Sous-Commission, à Moscou, en juin 1964, à un projet des observations coordonné pour les années 1964-1967, le long de 6 profiles traversant les frontières des dits pays. Les mesures seront organisées en collaboration avec des séismologues allemands, tchécoslovaques, polonais, hongrois, soviétiques, bulgaires et probablement aussi yougoslaves. En outre, on continuera les programmes nationaux établis dans cette région. Les résultats, disponibles de la région carpathique pour l'instant sur la profondeur du Moho, donnent $35 \mathrm{~km}$ environ dans la partie sud du Massif de Bohème, $25 \mathrm{~km}$ dans le terrain bas polonais et $29 \mathrm{~km}$ au nord des Carpathes occidentaux, tandis que, dans le Bassin Pannonien, on a reçu par les mesures de contrôle la même valeur de $24 \mathrm{~km}$ qu'auparavant. Considérant la valeur de $70 \mathrm{~km}$, trouvée en Roumanie par l'étude des tremblements, au-dessous du coude des Carpathes orientaux, on doit expecter dans la région carpathique des variations quelquefois très rapides de l'épaisseur de la croûte.

Les contacts avec les géodésiens et les géologues, nécessaires en vue d'améliorer les critères géologiques et tectoniques, ont été établis dans l'URSS, en Allemagne Démocratique (qui prend part aux sondages profonds de la région carpathique), en Tchécoslovaquie, en Pologne, en Hongrie et en Yougoslavie.

La Résolution No 4 concerne la carte séismotectonique de l'Europe. Les premiers tracés de cette carte, présentés à Jena par le Professeur Belousov et le Dr. Sorsky, ont été envoyés à tous les pays de l'Europe pour rassembler des commentaires sur le premier tracé. Ceux-ci ont été respectés et les auteurs les ont inclus, avec de nouvelles données séismiques du M. Kárník et des données sur les récents mouvements tectoniques et d'autres informations utilisables, dans la deuxième version de leur carte, «la Carte Séismotectonique Définitive de l'Europe». 
La Résolution No 5 concerne la collaboration des séismologues avec les groupes d'études chargés d'établir et de publier les cartes des mouvements récents de la croûte terrestre en Europe occidentale et orientale. Cette collaboration a été confiée au Professeur Meisser. La période écoulée est encore trop courte pour donner des résultats.

La Résolution No 6 concerne la création d'un groupe de travail pour le projet du manteau supérieur et son influence sur la croûte terrestre. Le groupe a été chargé d'établir, sous la présidence du Vice-Président Savarensky, le programme scientifique et de préparer un Symposium sur la structure du manteau supérieur et de la croûte terrestre pour la présente réunion. A cause de la maladie sérieuse du Professeur Savarensky le programme détaillé n'a pu être présenté, mais pour le Symposium qui sera dirigé par le Vice-Président Savarensky on a concentré plus de 50 contributions.

La Résolution No 7 concerne la création potentielle d'une Sous-Commission pour l'étude des raz de marée. La CSE a demandé le Professeur Galanopoulos d'organiser l'étude correspondante. Lui-même et le Professeur Ambraseys (Grande Bretagne) ont contribué à ce problème.

La Résolution No 8 concerne la publication du volume de l'Assemblée de Jena. La Sous-Commission pour les publications (dont le Président est le Professeur Meisser) a chargé le Dr. Sponheuer d'éditer un volume spécial sur l'Assemblée de Jena, étant assisté par le Dr. Vančk et le Dr. Van Gils. Le volume a été publié par les soins de l'Académie Allemande des Sciences de Berlin. Il est mon très agréable devoir de remercier particulièrement, au nom de la Commission Séismologique Européenne, l'Académie Allemande des Sciences pour l'édition de notre volume qui représente une carte de visite très jolie de l'Assemblée de Jena. Puis nous sommes très reconnaissants à $M$. le Professeur Meisser, initiateur et protecteur de cette action, et à MM. Sponheuer, Vaněk et Van Gils, et avec ceux-ci, de même à $M$. Maaz et Mme Resack pour leur travail dévoué et attentif concernant cet oeuvre.

\section{Travaux de recherches dans les pays-membres}

Permettez-moi maintenant d'attirer votre attention sur les principaux résultats scientifiques qui, dans le cadre de la CSE, ont été obtenus depuis 1'Assemblée de Jena. Partiellement, ils ont été mentionnés déjà l'année dernière à Berkeley. Je prie les Membres titulaires de la CSE de recevoir l'expression de ma profonde gratitude pour des informations sur l'état des recherches séismologiques dans leurs pays respectifs qu'ils ont offert à ma disposition. Je remercie, de même, autres informateurs dont les renseignements j'ai employé dans le présent rapport.

1. Le réseau des stations séismologiques en Europe a été considérablement amélioré par l'augmentation et modernisation de l'appareillage et l'établissement des nouvelles stations permanentes ou transportables. Un nombre de stations sont planifiées pour le plus proche futur. L'utilisation des systèmes homogènes et standardisés (parmi ceux-ci les complets USCGS) offre beaucoup d'avantages 
à i'étude des amplitudes et de l'énergie des séismes et de même à la recherche des microséismes. La théorie et construction des séismographs et des appareils spéciaux comme "strain-seismomètres», "strong-motion instruments» microbarographs etc. ont été développées surtout dans 1'URSS, mais aussi en Allemagne, en Finlande, en Hongrie, en Italie, en Pologne et en Tchécoslovaquie.

Il serait très désirable d'augmenter substantiellement le nombre de séismographes à longue période en Europe. Des recherches dans cette direction ont été organisées en Allemagne Démocratique, en Pologne et dans l'URSS. L'obtention d'un réseau fondamental, homogène et standardisé, possédant des instruments à courtes, médiocres et longues périodes, et complété d'un nombre suffisant de stations auxiliaires, surtout dans les régions actives de l'Europe, est devenue une des conditions inévitables en vue du progrès futur. On doit mentionner l'initiative de l'UNESCO qui, pendant la Réunion intergouvernementale de Séismologie tenue en avril 1964 à Paris, a souligné l'importance de ce point.

En ce qui concerne la mise en valeur des données séismiques et leurs présentation dans les bulletins, on a élaboré dans l'URSS les principes d'un bulletin opératif. Les principes de standardisation et unification proposés à la réunion mentionnée de l'UNESCO à Paris devraient être adoptés aussi par la CSE.

2. Des mémoires sur la séismicité ont été publiés presque dans tous les pays de l'Europe. En ce qui concerne les catalogues nationaux des séismes précédant l'année 1901, il est nécessaire de les compléter.

Les mémoires publiés sur la séismicité présentent d'une part des caractéristiques régionales, d'autre part, ils traitent des séismes individuels (p. e. ceux de Norvège, Roumanie, Tchécoslovaquie) ou s'occuppent de l'activité séismique d'une région étroitement limitée. Un cas que je voudrais bien introduire, est la catastrophe séismique de Skopje du 26 juillet 1963, 4h 17,2 m TMG, qui a exigé à peu près 1100 victimes. Sous le patronat des Nations Unies et de l'UNESCO on en a fait une étude complexe et a réussi à présenter une image détaillée du séisme et de ses répliques aussi bien que de la séismicité de la région de Skopje et de la Macédoine. Des méthodes analogues ont été appliquées plus tard dans le cas de Slavonski Brod, 1e 13 avril 1964.

Dans de nombreux pays, suivant l'exemple de la France, on a construit des isoséistes maxima. On en parlera pendant la réunion de la Sous-Commission de Séismicité.

L'étude de la séismicité se trouve en relation étroite avec le rayonnement séismique éxigé pour les buts du génie paraséismique. Les études de ce genre ont une tradition relativement longue dans l'URSS; en outre, au cours des dernières années, on y a beaucoup travaillé p. e. en Espagne (où on a même proposé une nouvelle échelle macroséismique), en Turquie, en Bulgarie, en Roumanie, en Yougoslavie etc.

3. A côté de la carte séismotectonique de l'Europe, des recherches séismotectoniques ont été poursuivies en Bulgarie, en Danemark, en Espagne, en Italie, dans l'URSS, en Tchécoslovaquie et en Yougoslavie.

4. On a étudié les propriétés cinématiques aussi bien que dynamiques des 
ondes préliminaires et superficielles, et, pour la plupart, on a fait des conclusions à la structure soit de la croûte, soit du manteau ou du noyau. Ces travaux, très nombreux, théoriques et expérimentaux, ont été élaborés en Allemagne, Bulgarie, Finlande, France, Italie, Norvège, Suède, Tchécoslovaquie, Turquie et dans l'URSS et se rapportent aussi à la crête médiane atlantique en Suède. L'épaisseur de la croûte en Eurasie a été déterminée au moyen de la dispersion des vitesses de groupe (Allemagne, Danemark, Espagne, Finlande, France, Hongrie, Norvège, Pologne, Roumanie, Suède, Tchécoslovaquie, Turquie et l'URSS); en Tchécoslovaquie aussi les vitesses de phase étaient usitées. Le résultat général est que la croûte sous le continent est plus épaisse que dans la zone méditerranéenne.

Pour compléter ce qu'il a été déjà dit sur les sondages séismiques profonds, il faut mentionner les mesures faites en Finlande (jusqu'à 1962, le Moho dans une profondeur de $33 \mathrm{~km}$ ) et les sondages continués en Norvège et Danemark le long d'un profile passant de la Norvège, du Nord jusqu'à la frontière DanemarkAllemagne. Ces mesures ont été réalisées par la collaboration des savants allemands, danois et norvégiens.

Il y a un nombre d'études théoriques et expérimentaux de modèles de l'écorce terrestre (p. e. dans 1'URSS, en Tchécoslovaquie, en Allemagne etc.).

Il faut mentionner encore, sans introduire des détails, des études faites sur les amplitudes de diverses phases, des recherches sur la magnitude et l'énergie des séismes (Allemagne Démocratique en collaboration avec des séismologues tchécoslovaques - pour un groupe des stations - en Bulgarie, en Hongrie, en Norvège et en Turquie).

En ce qui concerne la recherche sur les microséismes enregistrés dans les stations européennes, les travaux soviétiques sont les plus nombreux. La preuve de l'existence des microséismes au fond de la mer, semble-t-il, peut être considérée comme le résultat le plus important. En outre, on a étudié la localisation des sources des microséismes, leurs connections avec la situation météorologique. et leurs facteurs géologo-tectoniques (p. e. Allemagne, Danemark, Norvège, Suède). En Tchécoslovaquie l'étude s'est concentrée sur l'analyse statistique des périodes. On a étudié le bruit de fond aux environs des stations séismiques planifiées en Tchécoslovaquie, Roumanie etc.

6. J'introduirai encore qu'on a organisé en plus des recherches spéciales, telles que l'étude du régime des secousses extrêmement faibles dans des districts miniers, leur localisation etc. (Tchécoslovaquie, l'URSS, Allemagne Démocratique), leur rapports aux coups de toit, l'étude des pressions minières (collaboration des séismologues tchécoslovaques et soviétiques) in situ et en laboratoire, des mesures des pressions dans les blocs rocheux, etc. (1'URSS, la Tchécoslovaquie, la Suède).

Enfin, disons encore que quelques résultats progressifs ont été obtenus en applicant des calculateurs électroniques aux problèmes séismiques. On commence à travailler même en mécanisation et automatisation (URSS, Allemagne, Tchécoslovaquie, Norvège), mais les recherches de ce genre exigeraient une intensifcation substantielle. 
Deux sessions des membres de la CSE ont eu lieu depuis l'Asșemblée de Jena: une réunion officielle à Berkeley le 26 août 1963, sacrifiée à la préparation de notre Assemblée, et une rencontre inofficielle à Paris en avril 1964. Le Bureau s'est réuni à Prague du 3 au 5 sept. 1964. Le Professeur Rothé, Sécrétaire Général de l'AISPIT, et le Professeur Knopoff, Secrétaire Général du Projet du Manteau Supérieur, participaient à cette réunion.

J'espère que la partie scientifique de notre Assemblée présentera mon aperçu dans une forme plus concrète.

Et maintenant en peu de mots de ce qu'on a planifié: Il y a, en général, peu de substantiellement nouveau dans ces plans, excepté la recherche intensifiée de méthodes de mécanisation, automatisation et 1'usage des calculateurs électroniques, où on compte déjà avec une collaboration plus large avec le Centre Séismologique International d'Edinburgh.

Et quelles sont les conclusions et recommandations? Les voilà :

La recherche séismologique en Europe comprend pratiquement toutes les branches de la séismologie moderne; elle se développe assez spontanément et une coordination systématique devrait être introduite; les méthodes du travail profitent relativement peu de la technique et de l'organisation moderne du travail afin que la recherche devienne aussi économique.

A mon avis, on devrait recommander:

(1) Continuation coordonée des recherches déjà en cours et introduction de la coordination dans les recherches planifiées.

(2) Densification du réséau des stations séismologiques et leur équipage par des instruments convenables.

(3) Homogénéisation et standardisation des appareils fondamentaux des stations et des méthodes de la mise en valeur des données d'observation.

(4) Développement des procédés de mécanisation et automatisation du traitement de données et de résultats séismiques.

En concluant, je vous prie de me permettre quelques mots sur la situation.

La présente période est caractérisée par une évolution très rapide qui nous impose des tâches très complexes d'une part et très spécialisées d'autre part, p. e. les sondages profonds et, disons, l'automatisation des procédés de la mise en valeur des paramètres statistiques des microséismes, ou bien des problèmes reliés avec le projet du manteau supérieur. Malgré que la séismologie, étant une science internationale par excellence, a déjà quelques expériences, concernant la collaboration des groupes internationaux, il nous faudra, aussi dans la CSE, introduire une certaine tactique et stratégie qui serait capable de faire possible ou de faciliter la solution de tels problèmes du caractère international qui sont exigeants non seulement du point de vue scientifique et organisatoire, mais aussi du point de vue économique et politique. En outre, l'évolution a mis en contact, à la ligne de l'UNESCO, nous, séismologues, avec les ingénieurs du génie paraséismique pour réunir les forces pour le but de rechercher les moyens propres à réduire le plus possible les victimes et les dégâts causés par les tremblements de terre.

Je voudrais encore informer l"Assemblée d'une résolution que le Comité pour 
le Manteau Supérieur nous a adressée par l'intermédiaire de son Secrétaire Général, le Professeur Knopoff:

«Considering the complexity of the geological structure of Europe and the importance of detailed comparative studies of the crust and upper mantle in this region for the UMP, and acknowledging the work already done in the Alps by the European Seismological Commission as an example of effective international cooperation: the Upper Mantle Committee asks the European Seismological Commission to investigate the possibility of carrying out international profile researches of the crust and upper mantle on the territory from the northern coast of Scandinavia to the southern coasts of the Mediterranean, and to the West towards the Atlantic Ocean.»

Voici un problème pour la réalisation duquel la CSE devrait chercher et trouver la voie le plus tôt possible.

Il en suit:

(5) Formation d'un organe de la CSE pour la réalisation de la résolution du Comité du Manteau Supérieur sur l'étude de la croûte et du manteau supérieur le long d'un profil N-S, passant à travers tout le continent européen, et d'un profil analogue dans la direction $\mathrm{E}-\mathrm{W}$.

(6) Participation à l'activité de l'UNESCO concernant l'étude des tremblements de terre et la protection paraséismique en Europe.

En terminant, je voudrais encore remercier nos Vice-Présidents, les Professeurs Savarensky et Vesanen de leur travail dans le Bureau et spécialement je prie notre Sécrétaire perpétuel Monsieur Peterschmitt d'agréer l'expression de nos sentiments de profonde gratitude pour son activité infatigable et dévouée pendant toute la période. Je finis en remerciant tous les collègues qui ont contribué au développement prochain de notre belle science. 
CRUSTAL AND UPPER MANTLE INVESTIGATIONS 



\title{
STUDY OF THE EARTH'S CRUST UPPER PARTS BY SURFACE WAVES DISPERSION
}

\author{
V. M. Arhangelskaya and I. M. Kuznetsova
}

(Moscow, USSR)

During the past decades the surface waves are widely used at the study of the earth's crust and upper mantle. Until now mainly the surface waves records at distant earthquakes have been used in a wide range of periods from $15 \mathrm{sec}$ to many minutes. At near earthquakes $(800-1000 \mathrm{~km})$ rather intensive surface waves are observed. The investigation of these surface waves records gives a rich material for the study of sedimentary complexes and upper parts of the earth's crust. But unfortunately in this direction few investigations are carried out. For example the work of D. I. Sikharulidze, ${ }^{*}$ connected with determination of sedimentary complex by Love waves dispersion at near earthquakes.

At present we carried out the analysis of surface waves records at near earthquakes, recorded at Fergana, Prjevalsk, Alma-Ata stations and some other stations of Middle Asia.

About 150 earthquakes have been studied. Their epicentral distances varied from $30 \mathrm{~km}$ to $1000 \mathrm{~km}$.

As the records analysis have shown, at near earthquakes the surface wave has distinctly expressed normal dispersion.

The next seismogramm, Fig. 1 serves a good enough illustration to that. This record is from Alma-Ata station. The earthquake has occured in Tien-Shan ridge with coordinates: $\varphi=40^{\circ} 2 ; \lambda=76^{\circ} 98 ; \Delta=355 \mathrm{~km}, O=09-03-11$.

This earthquake has taken place to $S$ from Alma-Ata station.

Thus on $Z$ and $N S$ Rayleigh waves components are recorded, but on $E W$, Love waves are recorded. This division is distinctly marked on the records.

As it is seen from the seismogram the surface waves are recorded very distinctly. On the level of the strong transversal waves this arrival is marked by a considerable increase of period and more correct form of oscillations, and mainly by distinctly expressed dispersion.

It is possible to show the record of a near earthquake with $\Delta=130 \mathrm{~km}$ (Fig. 2), on which also it is possible to mark the surface waves with well expressed dispersion. This is the record of Fergana station. The earthquake has occured in the Pamirs.

* Sikharulidze, D. I.: The study of Love waves dispersion at near earthquakes. $I z v . A N$ $S S S R$, ser. geofiz., No. 4, 1959. 


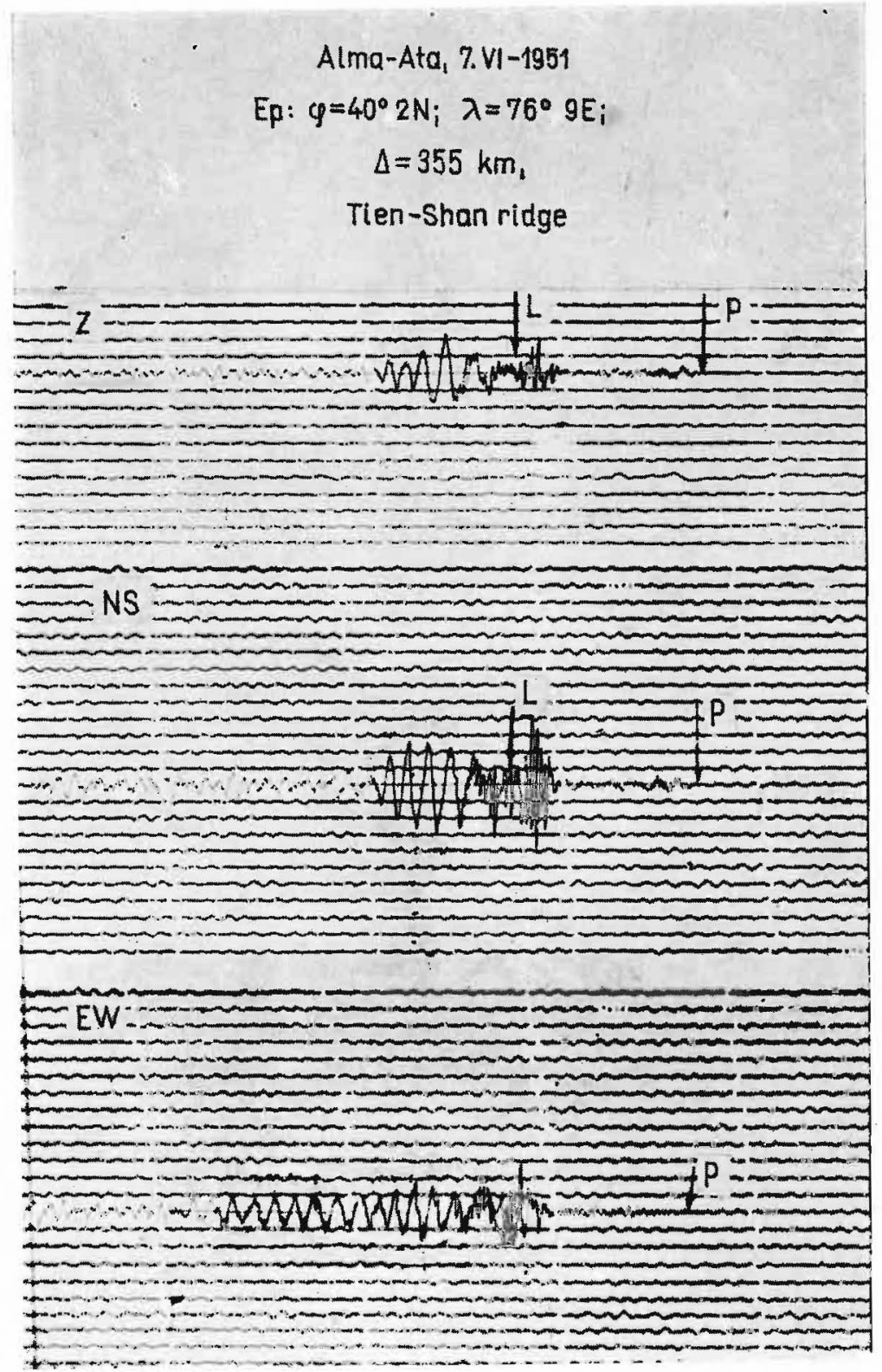

FIG. 1. The records of the surface waves of the earthquake 7. VI. 1951, Alma-Ata station $\Delta=355 \mathrm{~km}$ 


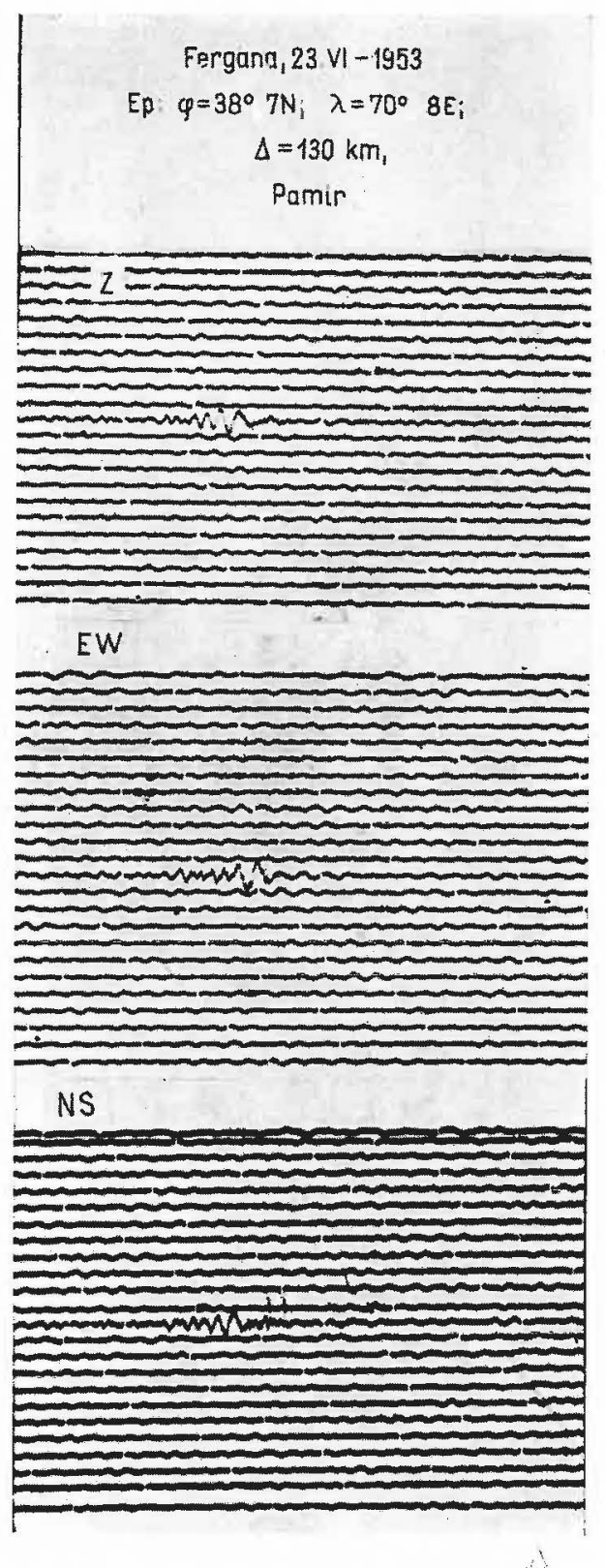

FIG. 2. The records of the surface waves of the earthquake 23. VI. 1953, Fergana station, $\Delta=130 \mathrm{~km}$ 
On the base of such records the experimental travel-time curves of maximum displacements in surface waves have been constructed. It is presented in Fig. 3. This travel-time curve makes it possible to determine approximate epicentral distances, especially in that cases, when we have not accurate arrivals of $S$ waves.

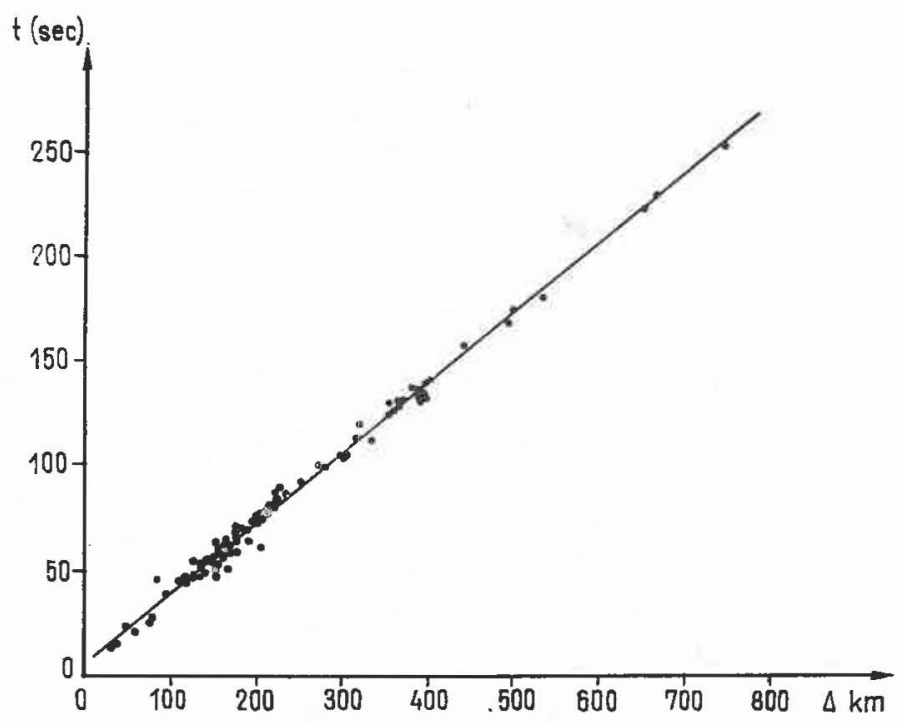

Fig. 3. The experimental travel time curve of the maximum displacements for surface waves

At near earthquakes decrease of the epicentral distance is compensated by the decrease of surface waves period. The observed periods of surface waves at near earthquakes vary in the range, mainly from 2 to $10-11 \mathrm{sec}$. Therefore, it is natural that these waves are connected with thinner layers of the earth crust, than to surface waves of the distant earthquakes.

The epicentres of earthquakes are plotted on a geological map, compiled by M. V. Gzovsky, N. N. Leonov and P. I. Nikolaev.

We see very clearly on the map that for Fergana station the epicentres of the selected earthquakes are placed mainly to the south from the station and to the north from it. The line of waves propagation is passing in very different geological conditions (Fig. 4).

On the north of Fergana the wave ways intersected the thick sedimentary stratas, where the thickness is of $\sim 10 \mathrm{~km}$. The southern sedimentary complexes are almost not found. As the result of such a difference in the structure on the line of the waves propagation, it is naturally to expect the great differences in observed dispersed data of these two groups of earthquakes.

Really, turning to Fig. 5 on which the observed group velocities depend on the wave period, we quite visually mark this difference in dispersion. The upper 
curve I corresponds to the data observed from earthquakes, placed to the south of Fergana. In this case oscillations possess relatively high velocities of propagation.

Curve II corresponds to the data of earthquakes, placed to the north in the upper group. The wave way is passing through thick sediments. In this case we

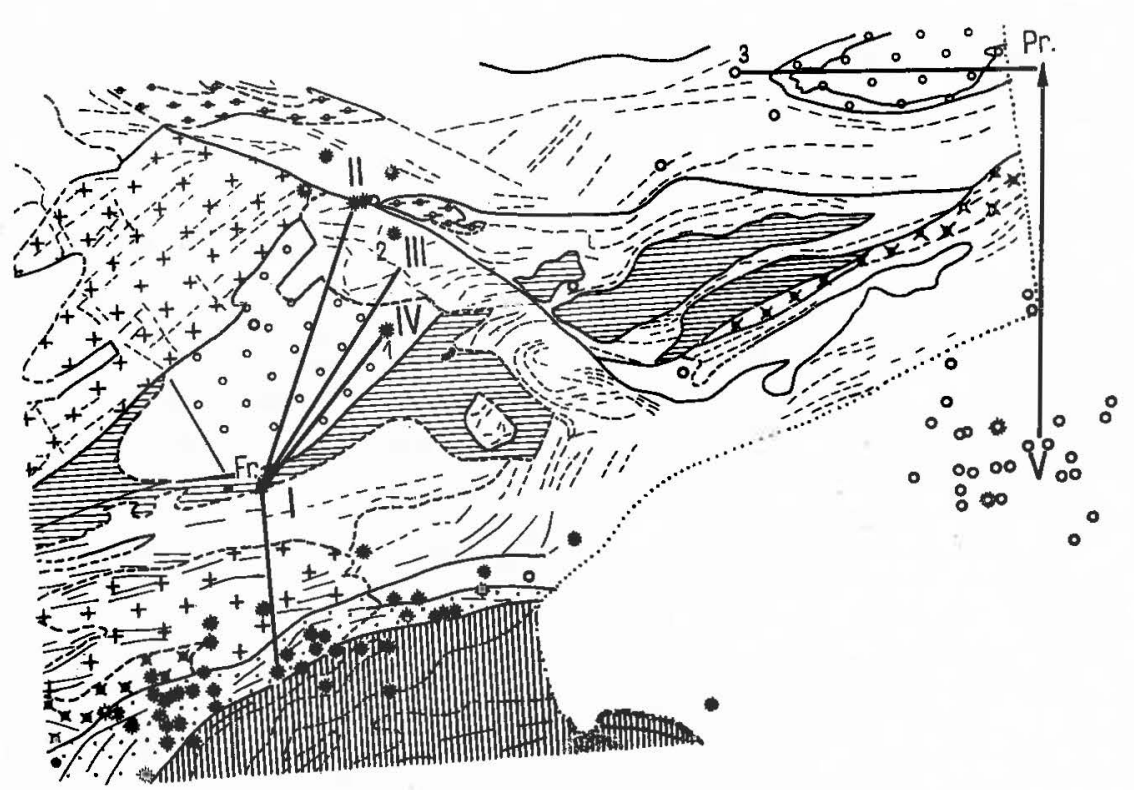

FIG. 4. The map of the epicentres of the investigated earthquakes

1. The thick sediment regions $(9-10 \mathrm{~km}$ ) and the average crust (from 35 to $55 \mathrm{~km}$ ).

2. The regions where a thin sedimental layer (up to $0.5 \mathrm{~km}$ ) lies on rocks of the $\gamma_{2}$ type.

3. The same as 2, except that the rocks are of the $\gamma_{1}$ type.

4. The regions where a thin (up to $0.5 \mathrm{~km}$ ) layer of sediments lies on that of the rocks of the $\gamma_{2}$ type (with thickness up to $3 \mathrm{~km}$ ). Under that layer are rocks of the $\gamma_{1}$ type.

5. Epicentre

$\gamma_{1}$ - the part of granitic layer with longitudinal waves velocities $6.0-7.0 \mathrm{~km} / \mathrm{sec}$

$\gamma_{2}$ - the part of granitic layer with longitudinal waves velocities $5.5-6.5 \mathrm{~km} / \mathrm{sec}$

Pr. - Prjevalsk

Fr. - Fergana

observe a considerable decrease of velocity. Thus the influence of thick sedimentary complex on velocities dispersion is visually seen. We have the variation of velocity $\sim 0.5 \mathrm{~km} / \mathrm{sec}$. As an example, let us pay attention to curves III and IV. Unfortunately, here we have the results of single observations, but they give visual quantative picture. Curve IV corresponds to the data of earthquake No. 1. 
In this case the waves from the focus to the station propagate only along the territory with sedimentary layer, and we observe still more considerable velocity decrease.

Curve III corresponds to the data of earthquake No. 2, i.e. an intermediate case of structure and correspondingly to an intermediate case by dispersed data within curves II and IV.

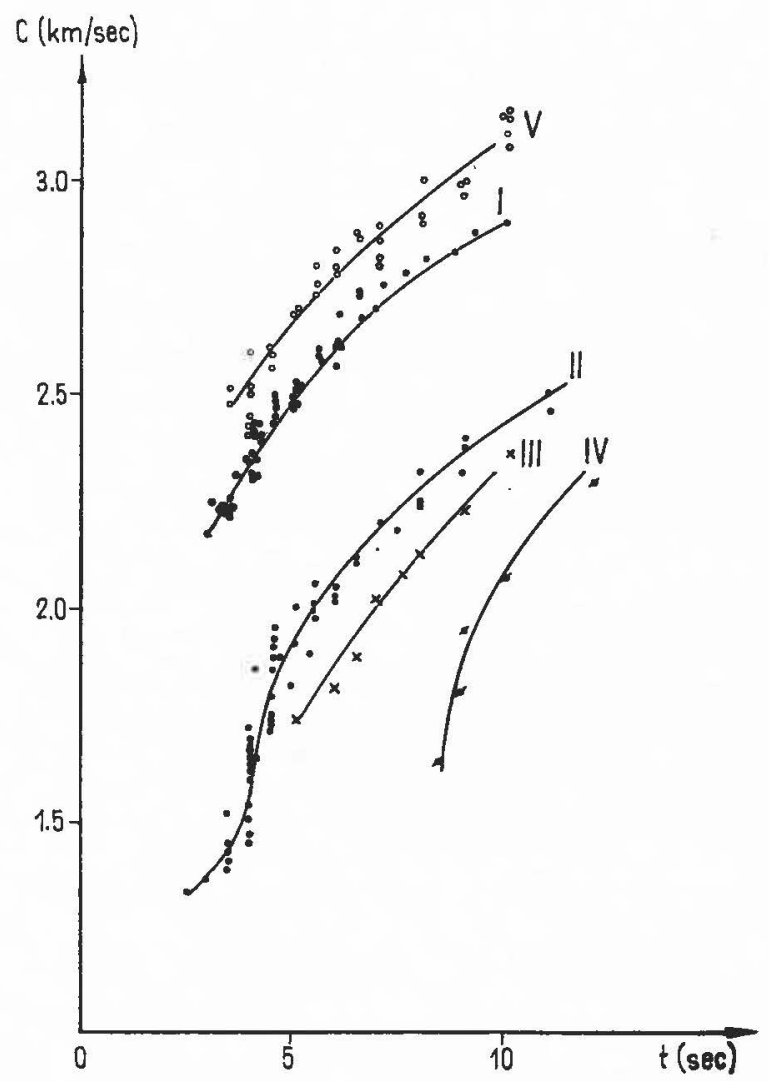

Fig. 5. Group velocities depend on the wave period of the surface waves for earthquakes

If we now turn to the observations of Prjevalsk station we see that almost all of the epicentres of earthquakes are placed to the south from the station. In this case the waves are passing the places, where sedimentary layers are absent, and on the way of these waves passing there are thick layers with consolidated metamorphosed rocks, more consolidated than on the line from Fergana to the south.

Now, if the obtained curve of dispersion $\mathrm{V}$ is compared with curve $\mathrm{I}$, it runs quite regularly above it (see Fig. 5), i.e. the waves are characterized by more high velocities, than the waves, propagating from the south to Fergana station. 
It is possible to continue the study of influence of the upper parts structure of the earth's crust on the dispersion of surface waves, if we turn to the earthquake No 3. In this case the line of the waves propagation is passing almost through the Issik-Kul Lake where the sedimentary layer is thick. Here the observed dispersion very well coincides with curve II, corresponding to the structure with a great sedimentary layer.

Thus, a good agreement of the observed dispersed data can be found with geology of the medium, consequently, we can use the surface waves dispersion at near earthquakes for the study of the upper parts of the earth's crust. At the same time it is necessary to account a very complex earth's crust structure of Middle Asia. In this connection it is desirable to use all the observations on less epicentral distances, i.e. expeditional observations. We have not carried out quantitative determinations up to now, but recently, the work in connection with selection and calculation of theoretical curves has been started accounting all the complexity of this regional structure. 


\section{PECULIARITIES IN THE ENERGY PROPAGATION OF THE EAST-ALPINE EARTHQUAKES

\author{
V. BABušKa, L. Ruprechtová and O. ZenkLová
}

(Prague, Czechoslovakia)

The East-Alpine earthquake of the 2nd December, 1963 the focus of which was south of Vienna $\left(\varphi=47.9^{\circ} \mathrm{N}, \lambda=16.4^{\circ} \mathrm{E}\right)$, was felt besides Austria also on the territory of Czechoslovakia, Hungary and Germany. The macroseismic material was investigated with the aim to find the relation between the shape of the macroseismic field and the geological structure of the quaked area.

The macroseismic field had approximately a rhomboidal form with the NW-SE axis. The focus is situated eccentrically on its SE border. According to the former investigations of A. Zátopek a similar anomalous form of the macroseismic field was observed also by other earthquakes from this epicentral zone. In our case, the earthquake manifestations were observed in the Bohemian Mass in distances reaching $400 \mathrm{~km}$ from the epicentre, as far as the surroundings of Dresden, whereas they were damped already in the distance of $150 \mathrm{~km}$ in the Carpathian system (Fig. 1).

The tectonic influence on the frequency and intensity of observations is more and more noticeable with the increasing epicentral distance. On the Austrian territory the macroseismic field maintains, on the whole, its classic form; a striking increase of intensity takes place only at the southern boundary of the Bohemian Mass, along the contact with the Alpine foredeep.

As to the character of the macroseismic field on the Czechoslovak territory, we can distinguish two categories of the distribution of the macroseismic phenomena. First, the observations are arranged mainly in a linear way, reflecting the discontinuities dipping at a high angle, in the first place boundaries of geologic units with the different physical properties, mostly of different age and petrographical character. The second category, the observations are distributed approximately homogeneously on the quaked area; they occur chiefly in the Moldanubian block, this is the oldest and most denudated part of the Bohemian Mass and it represents, at the same time, the greatest elevation of its underground structure, that means the most elevated uparching of the crustal granite layer. Moldanubikum is also the most consolidated tectonic unit, a relatively homogeneous mass, where we can hardly presume the existence of discontinuities of subhorizontal orientation expressive enough to prevent the seismic energy from propagating upward to the surface. Such discontinuities are far more probable in other younger units of the Bohemian Mass where the seismic waves travel from the depth mainly along the tectonic lines of the first order, according to our first category. This presumption 
corresponds with the conception of A. Zátopek that the energy of East-Alpine earthquakes propagates along a hypothetical subhorizontal discontinuity which ascends out of the base of the Alps to the surface in the Bohemian Mass.

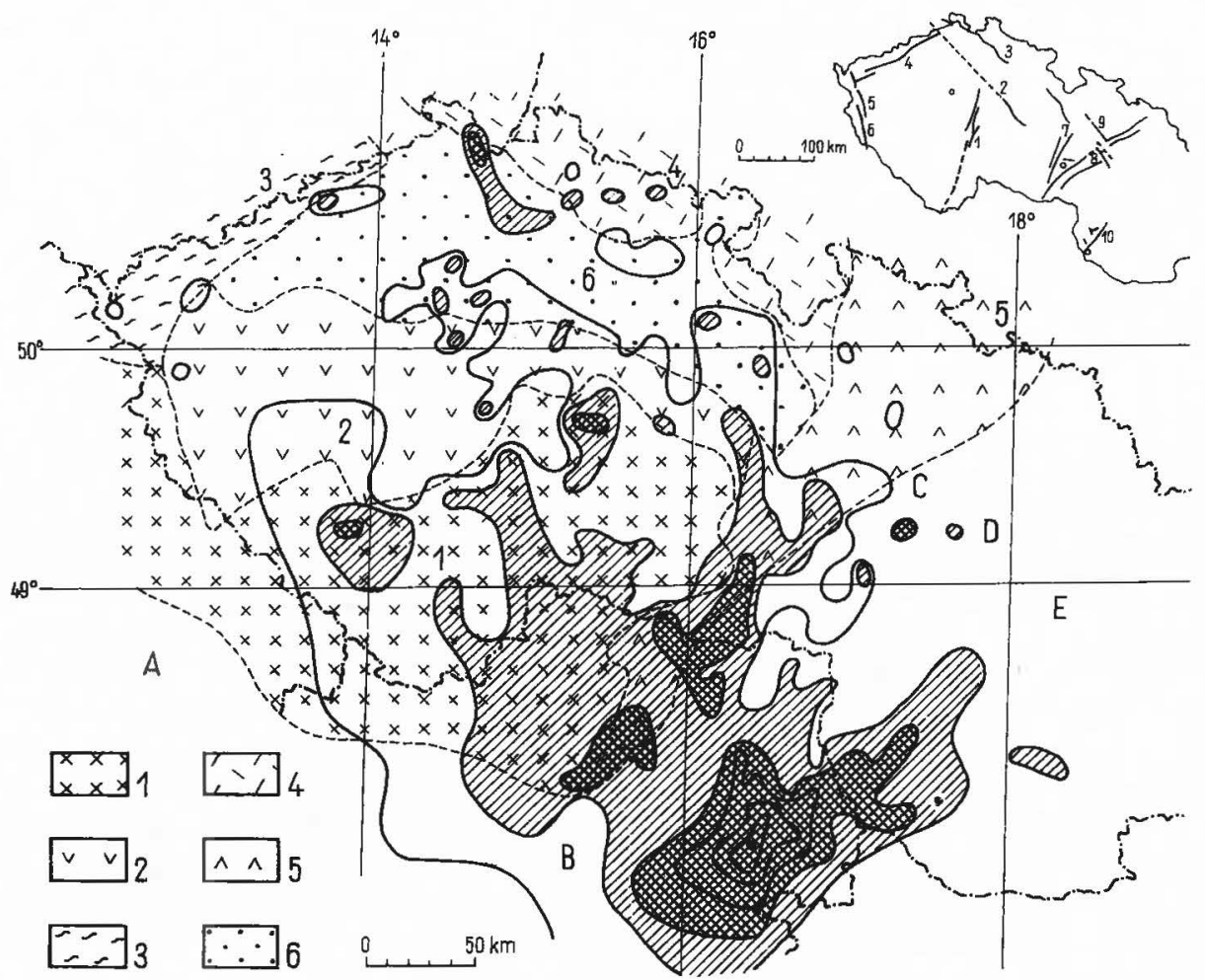

FIG. 1

Isoseismal map with the schematic outline of the main tectonic units of the Bohemian Mass $(1$ - Moidanubian block of the Bohemian Mass, 2 - Assyntian block of the Bohemian Mass, 3 - Thüringen region, 4 - WestSudeten region, 5 - Moravo-Silesian region, 6 - North-Bohemian Cretaceous Platform) and the surrounding units (A - Alpine foredeep, B - The Alps, C - Carpathian foredeep, D - The Outer Carpathians, E The Inner Carpathians). The inset shows the scheme of the main tectonic lines in the macroseismic area $(1-$ Blanice Graben, 2 - Labeline, 3 - Lužice-fault, 4 - Krušné Hory-fault, 5 - Marienbad-fault, 6 - Czech great quartz lode, 7 - Boskovice Graben, 8 - Border faults of the Carpathian foredeep, 9 - Haná-line, 10 Border faults of Malé Karpaty)

The increasing macroseismic activity was observed on the contact of the Bohemian Mass with the Carpathian system (especially in north-east Austria and south Moravia), on the eastern tectonic limitation of Malé Karpaty and in the southern part of the Boskovice Graben.

The macroseismic observations from the greatest epicentral distances are also connected with the important tectonic lines: fault at the foot of Krušné Hory (Erzgebirge) and the Lužice-fault. 
A rather expressive decrease of the number of macroseismic observations occurs in the Assyntian block of the Bohemian Mass and in the central part of the North Bohemian Cretaceous. The density of observations decreases there to $0.29 / 100 \mathrm{~km}^{2}$ in the other parts of the Bohemian Mass. The screen for the seismic waves propagation is caused very likely by the complicated tectonics with a quantity of discontinuities dipping at a low angle, as well as by the considerable thickness of sediments.

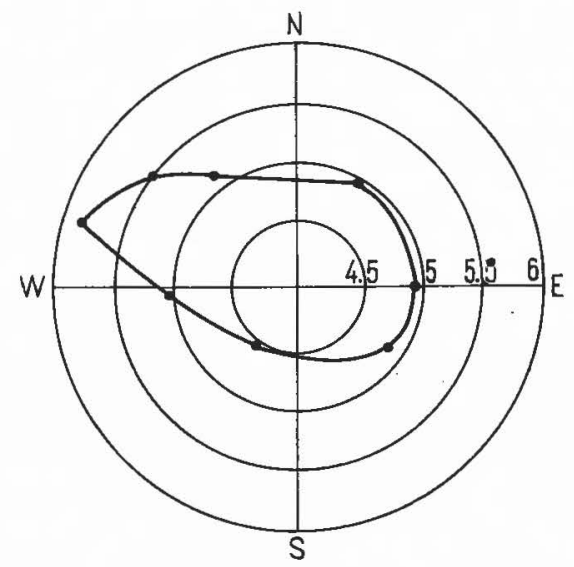

FIG. 2. The dependance of the magnitude $M_{\mathrm{LH}}$ on the azimuth

The propagation of the seismic energy was investigated also on the basis of the microseismic data. Our earthquake was recorded by more than 50 European stations. The magnitude from the surface waves $\left(M_{\mathrm{LH}}\right)$ was determined for 36 stations; the mean value is 5.1 . The values of magnitude for different stations show, however, a considerable dispersion; the influence of the epicentral distance being eliminated by the procedure of the magnitude determination, the dependence of the magnitude on the azimuth was investigated (Fig. 2). A decrease of the magnitude was found in the 2nd and 3rd quadrant, in comparison with a noticeable increase in the 4th quadrant, that meant in the west to the northwest from the focus. The propagation of the surface waves energy shows then a similar asymmetry as the macroseismic phenomena. 


\title{
DER SEISMISCHE IMPULS BEI UNTERWASSER- SPRENGUNGEN IN VERSCHIEDENEN REGISTRIER- ENTFERNUNGEN
}

\author{
H. Burkhardt, O. Rosenbach und R. Vees
}

(Mainz, BRD)

\section{Zusammenfassung}

Für die Auswertung refraktionsseismischer Messungen ist die Kenntnis der Gestalt des angeregten seismischen Signals von großer Bedeutung.

Während der Lago-Lagorai-Experimente 1962 wurden daher an mehreren Feststationen in verschiedenen Entfernungen vom Schußpunkt ( $80 \mathrm{~m}$ bis $70 \mathrm{~km}$ ) quantitative seismische Messungen durchgeführt.

Spektralanalysen der Aufzeichnungen einer schußpunktnahen Station zeigen eine systematische Änderung der Spektren bei Variation der Ladungsmenge und der Schußtiefe im See. Ein Vergleich dieser Spektren mit den Ergebnissen der Analyse bei entfernteren Stationen ermöglicht Aussagen über die Ausbreitungsbedingungen eines Wellentyps auf einem bestimmten Laufweg.

Amplituden-Ladungsgesetze für die Amplitude des Ersteinsatzes ergaben einen Exponenten $n \approx 2 / 3$. Dieser Wert stimmt recht gut mit denen überein, die bei Messungen in anderen Seen unterschiedlicher Größe gefunden wurden und scheint daher innerhalb eines gewissen Ladungsbereichs für Unterwasserexplosionen charakteristisch zu sein. Bei den Amplitudenuntersuchungen zeigte sich auch ein Zusammenhang zwischen Anregungsbedingungen und der Entstehung von Kompressions- und Scherungswellen.

Die bei Unterwasserexplosionen auftretende Mehrfachanregung wird bezüglich ihrer seismischen Wirkung ebenfalls untersucht. Dabei zeigt sich der große Nutzen schußpunktnaher Feststationen für die Identifikation späterer Anregungen in den Seismogrammen entfernterer Stationen.

\section{A) Einleitung}

Bei der Untersuchung der Erdkruste benutzt man zur Erzeugung des seismischen Signals neben Steinbruchsprengungen häufig Unterwassersprengungen, weil man dabei erfahrungsgemäß einen höheren seismischen Wirkungsgrad erreicht.

Neben den refraktions- und reflexionsseismischen Beobachtungen haben wir bei einer Reihe solcher Unterwassersprengungen systematische Untersuchungen des abgestrahlten Impulses in verschiedenen Registrierentfernungen durchgeführt. Im folgenden soll über einige dabei auftretende charakteristische Erscheinungen berichtet werden. Diese können für die Auswertung der Refraktionsund Reflexionsmessungen von großer Bedeutung sein.

Das Charakteristische solcher Explosionen wird am Beispiel der Lago-LagoraiExperimente erläutert. Zum Vergleich werden Ergebnisse von einem weiteren großen See, dem Lago Bianco, und von vier verschiedenen kleinen Seen herangezogen. 


\section{B) Registrierungen Lago Lagorai 1962}

Abb. 1 zeigt die Anordnung der Feststationen bei den Lago-Lagorai-Experimenten. Der Schußpunkt lag normalerweise auf einer vertikalen Achse an der tiefsten Stelle des Sees.

\section{VERSUCHE 1962}

1) Lad serie in $29 \mathrm{m:} 0,1-5480 \mathrm{~kg}$

2) Lad. serie in $8 \mathrm{~m}: 0,1-320 \mathrm{~kg}$

3) Tiefenserie mit $160 \mathrm{~kg}$

4) Verschiedene Schußstellen

5) Reproduzierbarkeit

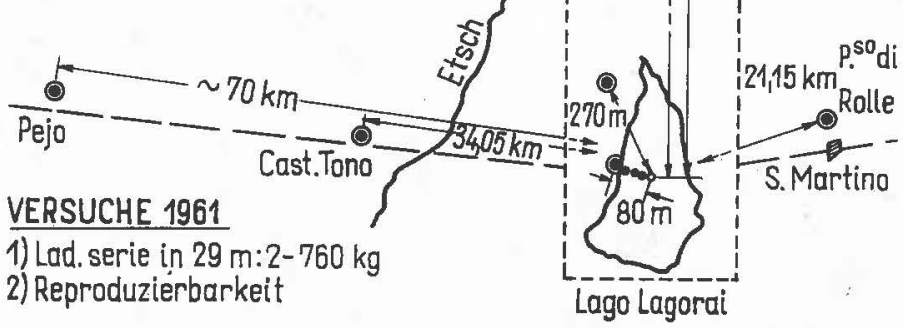

Aвв. 1. Lago Lagorai 1962 - Skizze der festen Meßuunkte

$\Delta[\mathrm{m}] \quad V\left[\mathrm{~mm}\right.$ Film $\left./ \mathrm{cm}^{-4} \mathrm{~s}^{-1}\right]$

80

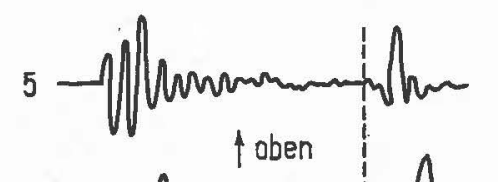

270

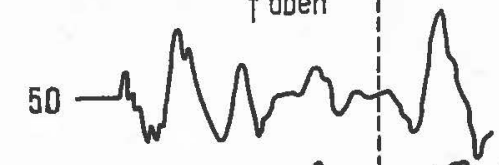

1100

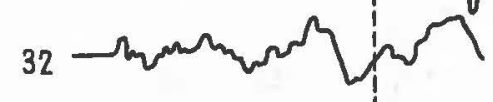

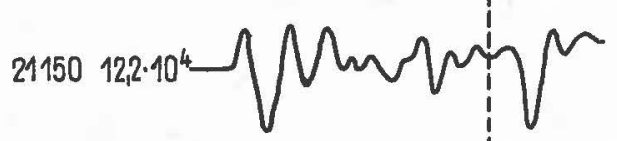

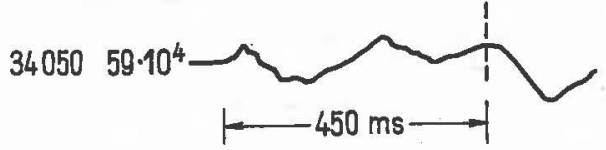

\section{$80 \mathrm{~kg}$ TNT in $29 \mathrm{~m}$ Tiefe $\$ 10 \mathrm{~mm}$ Film}

AвB. 2. Lago Lagorai 1962 - Der seismische Impuls in verschiedenen Entfernungen

In Abb. 2 ist eine Seismogramm-Montage der vertikalen Bodengeschwindigkeit für eine Ladung von $80 \mathrm{~kg}$ TNT, in $29 \mathrm{~m}$ Tiefe auf Grund gezündet, für Ent- 
fernungen von $80 \mathrm{~m}$ bis $34 \mathrm{~km}$ dargestellt. Während man in $80 \mathrm{~m}$ Entfernung mit einem Zeitintervall von weniger als $1 / 2 \mathrm{sec}$ einen vollständigen Anregungsvorgang erfaßt, stellt das gleiche Intervall in größerer Entfernung naturgemäß nur einen Bruchteil des gesamten Vorgangs dar.

In Schußnähe erkennt man deutlich einen zweiten Anregungsvorgang, da bei Unterwasserexplosionen neben der primären, eigentlichen Detonations-Stoßwelle weitere Druckwellen erzeugt werden, welche die aus den heißen Explosionsgasen bestehende Blase infolge ihrer Pulsationen nach außen abstrahlt. Der zeitliche Abstand derartiger aufeinanderfolgender Pulsationsdruckwellen ist abhängig vom hydrostatischen Druck und von der Energie des Gases, die bei jeder erneuten Abstrahlung abnimmt.

In größerer Entfernung ist dieser zweite Anregungsvorgang nur noch in Ausnahmefällen aus den Seismogrammen eindeutig zu identifizieren. Außerdem läßt sich aus dieser Darstellung gut die allgemein bekannte Tiefpaßwirkung des übertragenden Mediums erkennen.

Um die Form des seismischen Impulses auf seinem Laufweg verfolgen zu können, empfiehlt es sich daher, wegen der Frequenzabhängigkeit der Übertragungseigenschaften der Gesteine, mit Spektralanalysen zu arbeiten.

Im folgenden werden u. a. harmonische Analysen von Seismogrammausschnitten dargestellt. In Schußnähe wurde dabei im allgemeinen ein Zeitintervall von $250 \mathrm{~ms}$ verwendet; damit erfaßt man im wesentlichen den gesamten ersten Anregungsvorgang. In größerer Entfernung haben wir die Länge und die Lage der Intervalle variiert. In den folgenden Beispielen, die aus $21 \mathrm{~km}$ Entfernung stammen, wird der Seismogrammanfang bis $200 \mathrm{~ms}$ benutzt. Mit diesem Intervall erfaßt man den am Kristallin refraktierten P-Einsatz; die direkt durch den Bozener Quarzporphyr gelaufene P-Welle ist bis dahin noch nicht angekommen.

In Abb. 3 werden Spektren aus zwei verschiedenen Entfernungen $(80 \mathrm{~m}$ und $21 \mathrm{~km}$ ) miteinander verglichen; es handelt sich dabei um Beispiele aus einer Ladungsserie in $8 \mathrm{~m}$ Schußtiefe. Die Amplituden der einzelnen Spektren sind jeweils auf die maximalen spektralen Amplituden normiert.

Bei konstanter Entfernung von $80 \mathrm{~m}$ zeigt sich eine Variation des Spektrums mit der Ladung: Während sich die Maxima bei höheren Frequenzen mit zunehmender Ladung abbauen, bauen sich gleichzeitig bei tieferen Frequenzen neue Maxima auf. Der Anteil tieferer Freuqenzen nimmt also mit wachsender Ladung zu.

Ähnliches zeigt sich auch bei den Spektren in $21 \mathrm{~km}$ Entfernung. Der Hauptteil des Spektrums liegt jetzt zwar bei tieferen Frequenzen. Verfolgt man jedoch zwei benachbarte Maxima, z. B. bei ca. $26 \mathrm{~Hz}$ und bei ca. $16 \mathrm{~Hz}$, so sieht man auch hier: Das höherfrequente Maximum baut sich ab, das tieferfrequente auf. Diese Beziehung gilt nicht für das Maximum bei ca. $5 \mathrm{~Hz}$.

Es sei noch bemerkt, daß man aus dem Verhältnis von je zwei gleichfrequenten spektralen Amplituden in verschiedenen Entfernungen in Abhängigkeit von der Frequenz Durchlaßkurven für einen bestimmten Laufweg erhält, sofern es sich bei den analysierten Seismogrammteilen um Anregungsvorgänge gleicher 


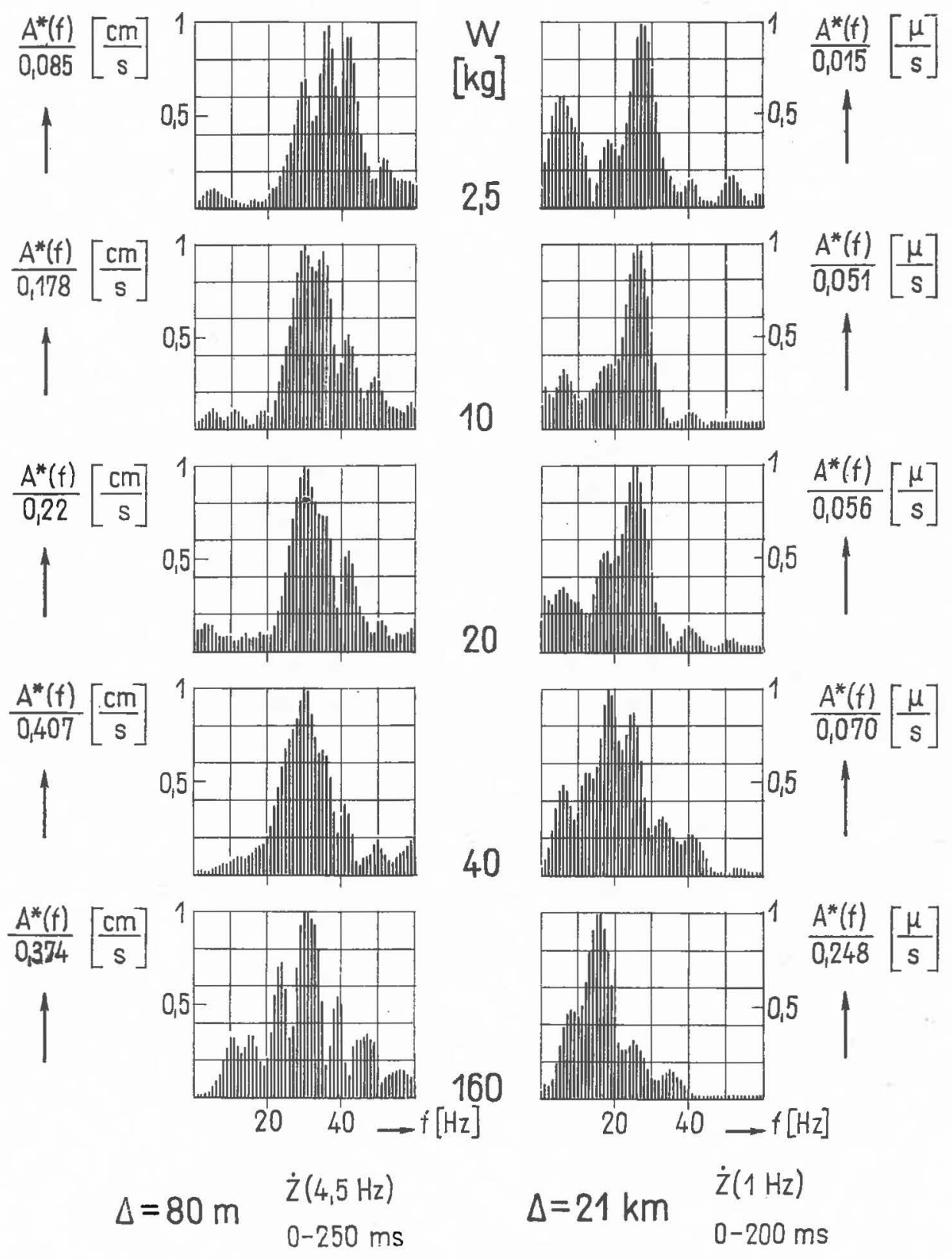

AвB. 3. Lago Lagorai 1962 - Ladungsserie in $8 \mathrm{~m}$ Schußtiefe 

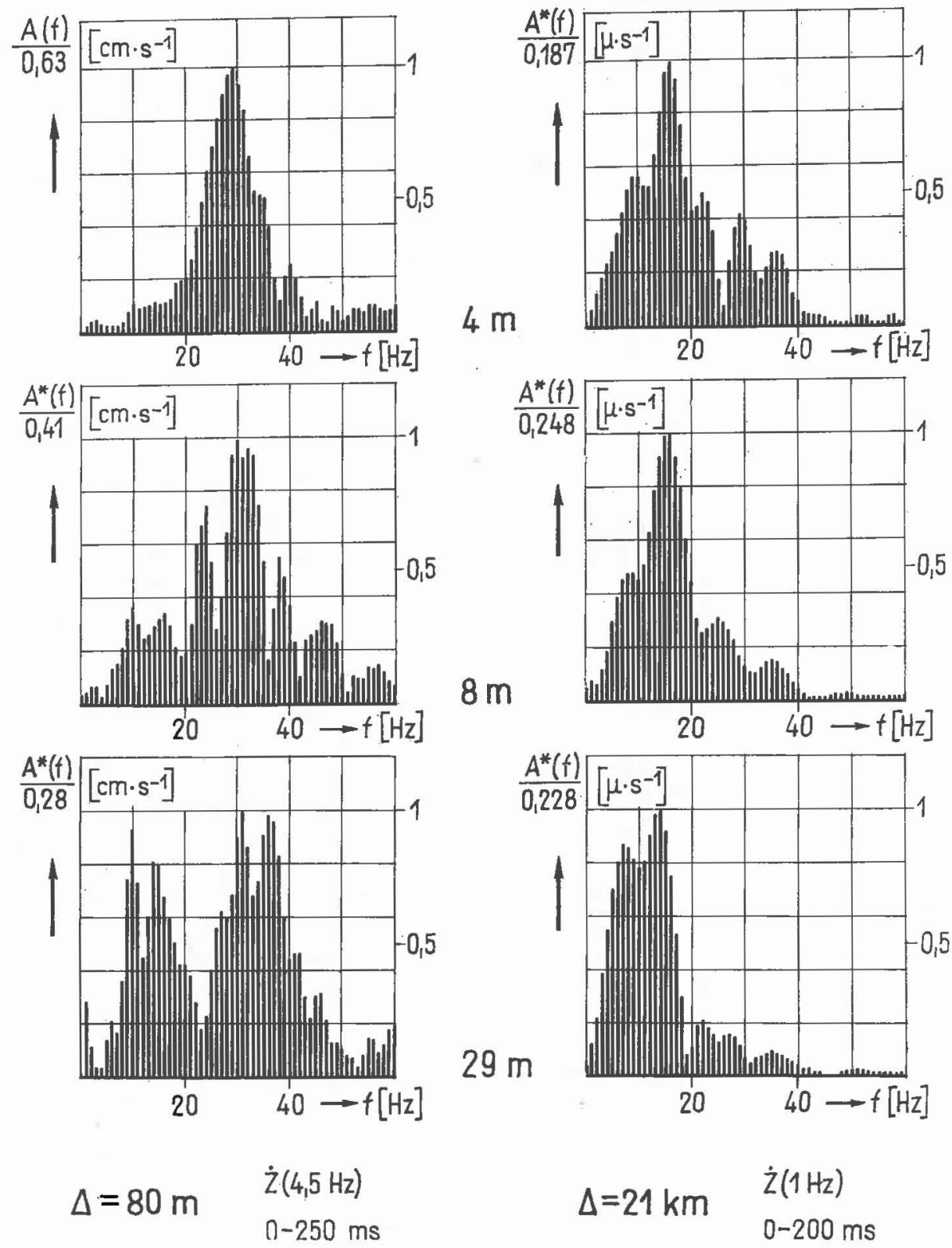

$$
\Delta=21 \mathrm{~km} \quad \begin{aligned}
& \dot{z}(1 \mathrm{~Hz}) \\
& 0-200 \mathrm{~ms}
\end{aligned}
$$

AвB. 4. Lago Lagorai 1962 - Tiefenserie mit $160 \mathrm{~kg}$ 
Wellentypen handelt. Die Kurven zeigen im Groben die Tiefpaßwirkung des übertragenden Mediums, haben aber daneben eine Feinstruktur mit einzelnen stärker absorbierenden Frequenzbändern.

Abb. 4 zeigt eine ähnliche Darstellung für eine Tiefenserie mit konstanter Ladung von $160 \mathrm{~kg}$. Die Spektren sind wieder aus Seismogrammen in Entfernungen von $80 \mathrm{~m}$ und $21 \mathrm{~km}$ berechnet. In $80 \mathrm{~m}$ Entfernung zeigt sich bei einer Schußtiefe von $29 \mathrm{~m}$ eine breite Verteilung der spektralen Amplituden. Mit abnehmender Schußtiefe bleibt im wesentlichen nur noch ein Maximum bei ca. $30 \mathrm{~Hz}$ bestehen. Insgesamt nimmt mit wachsender Schußtiefe der Anteil höherer Frequenzen ab. Das tieffrequente Maximum bei ca. $10 \mathrm{~Hz}$ könnte unmittelbar mit der Erzeugung von Scherungswellen zusammenhängen, da in ähnlicher Weise wie hier dieses Maximum auch der Anteil der erzeugten Scherungswellen mit zunehmender Schußtiefe größer wird (siehe auch Abb. 7b).

In $21 \mathrm{~km}$ Entfernung sieht man - ähnlich wie bei zunehmender Ladungsmenge in konstanter Tiefe - eine Betonung tieferer Frequenzen mit zunehmender Schußtiefe.

\section{C) Amplituden-Ladungs-Gesetze}

Abb. 5 zeigt eine Zusammenstellung von Amplituden-Ladungs-Gesetzen aus Sprengungen in Seen unterschiedlicher Größe. Die Registrierungen stammen aus verschiedenen Entfernungen.

Als Amplitude ist jeweils die Hälfte der ersten Doppelamplitude im Seismogramm in Abhängigkeit von der Ladung doppellogarithmisch aufgetragen.

Allgemein läßt sich feststellen, daß man über einen sehr weiten Ladungsbereich (ca. $1 \mathrm{~g}$ bis ca. 10 to) für alle benutzten Registrierentfernungen ähnliche Potenzgesetze erhält.

Verfolgt man bei den Lago-Lagorai-Versuchen bei einer Schußtiefe von $29 \mathrm{~m}$ die einzelnen Amplituden-Ladungs-Gesetze mit wachsender Entfernung, so findet man jeweils ähnliche Steigungen.

Dagegen ist z. B. die Steigung der Geraden bei der $8 \mathrm{~m}$-Landungsserie von der Entfernung abhängig, da sich bei dieser Serie das abgestrahlte Spektrum sehr viel stärker ändert, als bei einer Serie in $29 \mathrm{~m}$ Tiefe (in Abb. 5 ist für diese Serie nur die Kurve in 1,5 km Entfernung eingezeichnet). Für verschiedene Entfernungen kommen daher verschiedene Teile des Spektrums als Hauptbestandteile des Ersteinsatzes in Frage.

Das Amplituden-Ladungs-Gesetz der im Jahre 1964 durchgeführten LagoBianco-Sprengungen ist ebenfalls in Abb. 5 eingezeichnet. Zu kleineren Ladungen hin setzt sich diese Gerade in ähnlicher Weise aus den 1963 durchgeführten Vorversuchen bis zu Ladungen von $3 \mathrm{~kg}$ fort.

Im Lago Bianco wurden drei verschiedene Sprengstoffarten verwendet, außerdem mußten wegen der geringen Seetiefe die Ladungen geteilt werden. Die eingezeichneten Punkte sind direkte Meßwerte. Es wurde bis jetzt noch kein Versuch unternommen, die Amplituden auf einheitlichen Sprengstoff und auf eine 
einteilige Ladung zu reduzieren. Daraus erklärt sich die hier wesentlich größere Streuung der Meßwerte.

Auch bei kleineren Seen findet man ähnliche Steigungen. Beim kleinsten See mit einem Volumen von ca. $200 \mathrm{~m}^{3}$ ergibt sich ein Abknicken der Geraden mit wachsenden Ladungen, und man stellt das Amplituden-Ladungs-Gesetz am besten

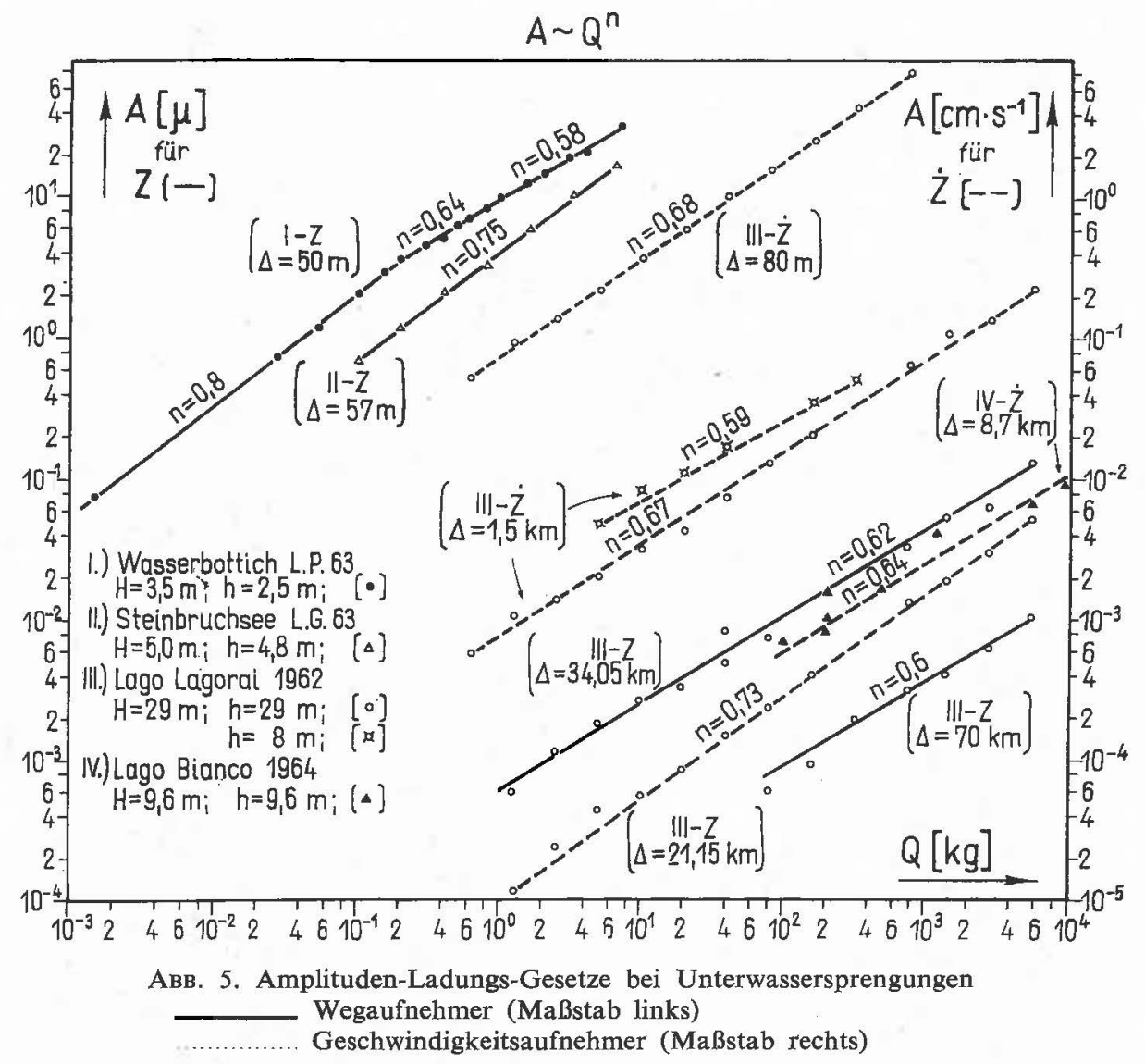

durch drei einzelne Potenzgesetze dar. Die Abnahme des Exponenten mit wachsender Ladung hängt mit dem Verhältnis von Ladungsmenge zu Seegröße zusammen und bedeutet im wesentlichen eine Abnahme des seismischen Wirkungsgrads der Sprengung.

Die Amplituden-Ladungs-Gesetze der Abb. 5 sind in Abb. 6 dreidimensional gezeichnet, um auch die Amplituden-Entfernungs-Abhängigkeit anschaulich zu zeigen. Die verschiedenen Achsen bezeichnen die Amplitude, die Ladung und die Entfernung; es sind dabei nur Geschwindigkeitsamplituden verwendet worden. 
Der zusammenhängende Block gehört zu den Ergebnissen der Lago-LagoraiMessungen. Die dicken Striche stellen die Amplituden-Ladungs-Gesetze dar, die nach hinten verlaufenden Flächen die Amplituden-Entfernungs-Gesetze. Die entsprechenden Werte von Sprengungen in einem künstlich angelegten Wasserbottich (I), in einem Steinbruchsee (II) und im Lago Bianco (IV) sind ebenfalls eingezeichnet.

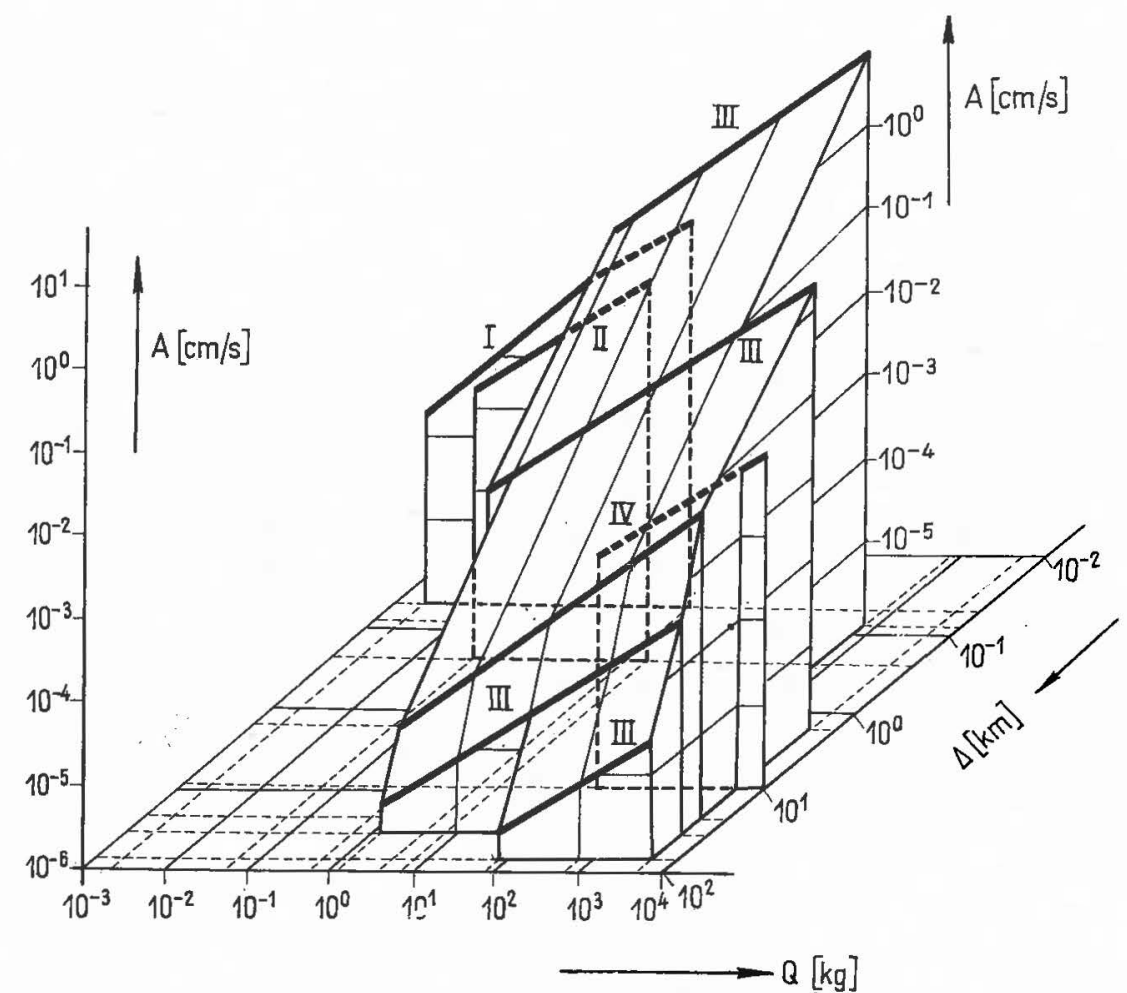

Авв. 6. Amplituden-Ladungs-Gesetze für Unterwassersprengungen in verschiedenen Registrierentfernungen:

I: Wasserbottich L.P. 63

II: Steinbruchsee L.G. 63

III: Lago Lagorai 1962

IV: Lago Bianco 1964

D) Anregung von Kompressions- und Scherungswellen bei verschiedenen Schußtiefen

In Abb. 7 ist der Einfluß der Schußtiefe auf die Entstehung von Kompressionsund Scherungswellen dargestellt. Die Amplituden der Kompressionswellen nehmen nach einem Maximum bei ca. $8 \mathrm{~m}$ mit der Schußtiefe wieder ab. Die Amplituden der Scherungswellen dagegen nehmen mit wachsender Schußtiefe kontinuierlich 
zu. Dieser Sachverhalt ist nicht verwunderlich, da die Scherungswelle bei Unterwassersprengungen mit frei im Wasser schwebender Ladung erst beim Auftreffen der Kompressionswellen auf die Grenzfläche flüssig-fest entsteht, während die Zündung einer Ladung, die auf dem Seegrund aufliegt, auch die Entstehung direkter Scherungswellen bewirkt. Der Zusammenhang zwischen Amplitude der
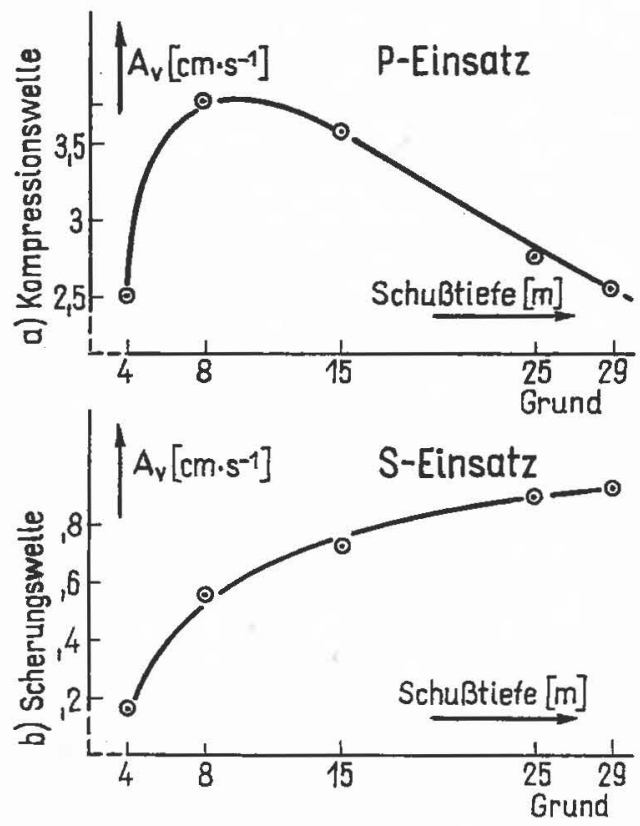

Aвв. 7. Lago Lagorai 1962 - Einfluß der Schußtiefe auf die Entstehung von Kompressions und Scherungswellen bei konstanter Lađung $160 \mathrm{~kg}$

Kompressionswellen und der Schußtiefe wird sowohl von der Ladung als auch von der Gestalt des Sees abhängen. Die Zunahme der Scherungswellen-Amplituden bei Annäherung der Ladung an den Seeboden wird dagegen bei allen Unterwassersprengungen ähnlich sein.

\section{E) Mehrfachanregungen durch pulsierende Gasblasen}

In Abb. 8 ist eine Seismogramm-Montage für einen Geschwindigkeitsaufnehmer in $80 \mathrm{~m}$ Entfernung bei einer Ladungsserie in $8 \mathrm{~m}$ Schußtiefe dargestellt. Neben den Hauptimpulsen erkennt man eine Reihe von Folgeimpulsen, die sogenannten »bubble-pulses». Die Impulsfolgezeiten liegen dabei auf einer Geraden, da die Seismogrammspuren im Abstand $W^{1 / 3}$ montiert sind.

Die Folgeimpulse haben eine ähnliche Form wie die Hauptimpulse; dies zeigt auch eine Frequenzanalyse. Ihre Energie dagegen nimmt bei der $8-\mathrm{m}-\mathrm{Serie}$ schon bis zu $20 \mathrm{~kg}$ Ladungsmenge merklich ab. Demgegenüber hat man bei der La- 
dungsserie auf Grund, z. B. noch bei $160 \mathrm{~kg}$, einen ersten Folgeimpuls, der etwa dieselbe Energie wie der erste Impuls hat.

Aus der Kenntnis der Impulsfolgezeiten und dem spektralen Aufbau der Folgeimpulse ergibt sich die Möglichkeit, auch Seismogrammspuren in größerer Entfernung, wo die Sekundäranregungen oft nicht mehr einwandfrei zu erkennen

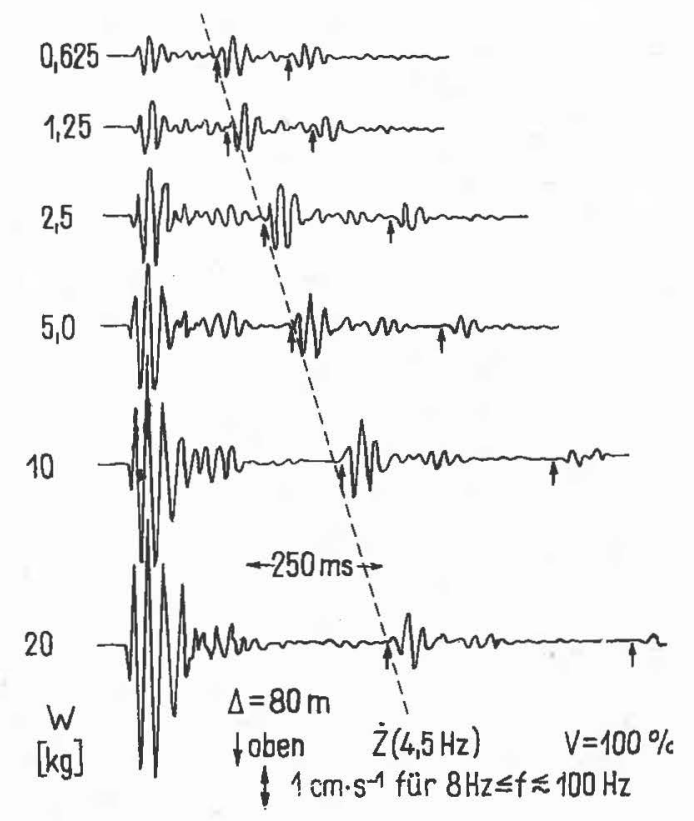

Авв. 8. Lago Lagorai — Mehrfachanregungen

$$
\begin{aligned}
& \text { Für die Periode der Pulsationen gilt die Formel von Willis } \\
& \qquad \begin{array}{c}
T=C \cdot \frac{W^{1 / 3}}{P^{5 / 6}} \\
C=\text { Sprengstoffkonstante } \\
W=\text { Ladungsmenge } \\
P=\text { hydrostatischer Druck }
\end{array}
\end{aligned}
$$

sind, zu reduzieren. Sinnvoll ist dies jeweils bis zur doppelten Periode der Impulsfolge. Während unkorrigierte Seismogrammspuren von unterschiedlichen Schüssen bei gleichem Laufweg nach den ersten Schwingungen oft stark voneinander abweichen, ergeben sich bei korrigierten Spuren wieder sehr ähnliche Schwingungsbilder.

Aber auch hinsichtlich der Interpretation späterer Seismogrammstücke, wo Mehrfachanregungen reelle Einsätze vortäuschen können, ist die Kenntnis der Eigenschaften dieser Mehrfachanregungen durch schußpunktnahe Messungen ein wertvolles Hilfsmittel für die Auswertung. 


\title{
DYNAMIC AND KINEMATIC PROPERTIES OF SEISMIC WAVES IN THE REGION OF CRITICAL ANGLE
}

\author{
V. Červení, A. M. Epinat'eva and I. P. Kosminskaya
}

(Prague, Czechoslovakia)

\begin{abstract}
Summary
The results of theoretical and experimental investigations of the region of critical angle $\left(i_{1}^{*}=\sin ^{-1} v_{p 1} / v_{p 2}\right)$ are given. At the critical point itself no kinematic or dynamic peculiarities appear. But there are some peculiarities in a certain distance beyond the critical point. In this distance lies the maximum of amplitude curve of the interference reflected-head wave, there occur changes in spectrum of this wave. In the region beyond the critical point the travel-time curves of phases differ from those of first arrivals. While the travel-time curve of first arrivals of head waves is tangent to the travel-time curve of reflected waves in the critical point, the travel-time curves of first maxima of these waves intersect each other beyond the critical point. The apparent velocity of the interference reflected head wave differs from the geometrically determined velocity of the reflected wave. All these peculiarities depend on frequency. The region of the second critical angle $\left(i_{2}^{*}=\sin ^{-1} v_{p 1} / v_{s 2}\right)$ was also investigated. Theoretical calculations are compared with field data.

The position of the critical point may be of use to determine the depth of a refracting or reflecting boundary as well as the average velocity in the overlying medium provided the boundary velocity is known. It may help also to clear up the nature of waves on great distance from the source, and especially to identify the overcritical reflexions and refractions.
\end{abstract}




\title{
THE AMPLITUDES OF SEISMIC BODY WAVES PROPAGATING IN THE EARTH'S CRUST
}

\author{
V. ČERVENÝ and J. JÁNSKÝ \\ (Prague, Czechoslovakia)
}

The present communication is concerned with the amplitude curves of a series of waves which originate on an interface, the parameters of which are close to the parameters of the Mohorovičic discontinuity. The source is assumed to be symmetric, harmonic, with a frequency 6.4 cycles and to be situated in the proximity of the earth's surface. We suppose that the earth's crust of $H=30 \mathrm{~km}$ thickness is homogeneous, with the velocity of compressional waves $v_{p 1}=6.4 \mathrm{~km} / \mathrm{sec}$, the velocity of compressional waves below Moho $v_{p 2}=8 \mathrm{~km} / \mathrm{sec}$; the ratio of compressional to shear waves is $\sqrt{3}$ inside the crust and below it. Since the amplitude curves are not noticeably influenced by the ratio of densities, we shall assume $\rho_{1} / \rho_{2}=1$. As we are not interested in the details of the amplitude curves, it is possible to use the methods of geometric ray theory for the calculations.

In the case of an incidence of a $\mathrm{P}$ wave 4 waves are produced: PP and PS reflected waves together with PPP and PPS head waves (see Fig. 1). The critical point of PPP wave lies at $80 \mathrm{~km}$, the critical point of PPS wave at $56 \mathrm{~km}$. In the regions of the critical points, where the formulae of geometric ray theory lack precision, the amplitudes are given by dashed lines. Besides, the reflected wave and corresponding head wave interfere after the critical point. The length of the interference zone has been calculated for a pulse of the length of one period. At the point where the dashed line changes into a continuous one, there is an end of the interference zone. In Fig. 1 we can see that the dominant wave is the PP reflected wave. Only at distances between 50 and $70 \mathrm{~km}$ the PS reflected wave reaches higher amplitudes, while at the majority of the distances the PS wave is noticeably weaker. Both the head waves are even weaker than the PS wave at the ends of the interference zone. They are, in both cases, approximately one order weaker than the corresponding reflected waves. The PPS wave is about 5 to 10 times weaker than a PPP wave. It is, consequently, improbable that it could be found in the records.

In the case of an incidence of S wave 5 waves are produced: SS and SP reflected waves, together with SSS, SPS and SPP head waves. The dominant wave over the whole range of distances is the reflected SS wave, with the exception of short distances from the source, where it is commensurate with the SP reflected wave. The head waves are again very weak. There is very little probability that the SPP and SPS waves might exceed the noise level. 
Now again to the amplitudes of the PP and PPP waves. Of considerable importance is the determination of the amplitude ratio of both waves directly at the point where both waves divide, i.e. at the end of their interference zone. At the

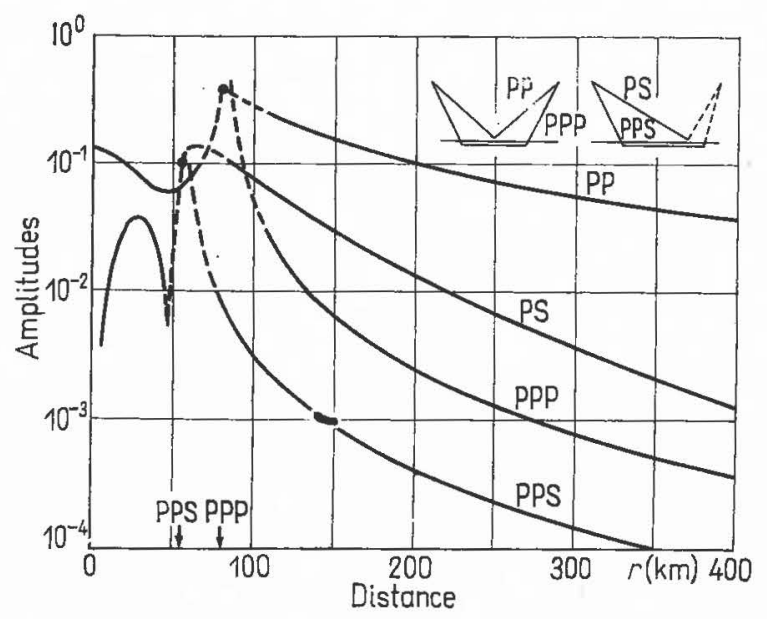

Fig. 1

end of the interference zone we obtain a very simple formula for the amplitude ratio of both waves:

$$
\mathrm{A}_{\mathrm{PPP}} / \mathrm{A}_{\mathrm{PP}}=F(n)(H / \lambda)^{-1 / 4}
$$

The values of $F(n)$ are given in Fig. 2 (for $v_{p 1} / v_{s 1}=v_{p 2} / v_{s 2}=\sqrt{3} ; \rho_{1} / \rho_{2}=1$ ). From Fig. 2 it can be seen that $F(n)$ depends only to a slight extent on the refraction index $n$. So we obtain the following approximate relation:

$$
\mathrm{A}_{\mathrm{PPP}} / \mathrm{A}_{\mathrm{PP}} \sim 0.22(H / \lambda)^{-1 / 4^{4}}
$$

In Table 1 the amplitude ratio $\mathrm{A}_{\mathrm{PPP}} / \mathrm{A}_{\mathrm{PP}}$ is given for certain $H / \lambda$.

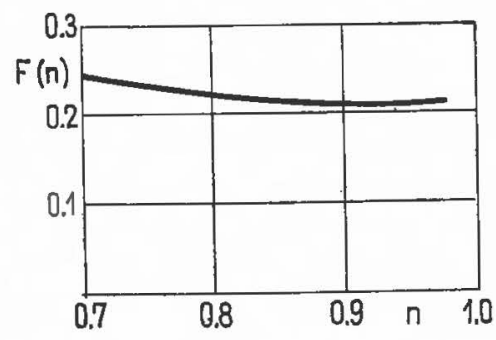

Fig. 2 
It can be seen that the said amplitude ratio depends only very slightly on $H / \lambda$ and thus also on the frequency. The general result is that the amplitudes of PPP waves are at the end of the interference zone at least about one order weaker than the amplitudes of reflected PP waves. It would, therefore, be extremely difficult to register the pure head wave at all.

TABLE 1

\begin{tabular}{c|c|c|c|c|c|c|c|c|c|c}
\hline $\mathrm{H} / \lambda$ & 10 & 20 & 30 & 40 & 50 & 60 & 70 & 80 & 90 & 100 \\
\hline $\mathrm{A}_{\mathrm{PPP}} / \mathrm{A}_{\mathrm{PP}}$ & 0.12 & 0.10 & 0.09 & 0.09 & 0.08 & 0.08 & 0.08 & 0.07 & 0.07 & 0.07
\end{tabular}

The amplitude ratio of head and reflected waves determined experimentally is, however, as a rule larger than a ratio determined theoretically. This discrepancy can be accounted for by different reasons. The absorption may influence the situation only to a slight extent. Much more important are other factors. It is well known that the geometric ray theory in its approximation of zero order

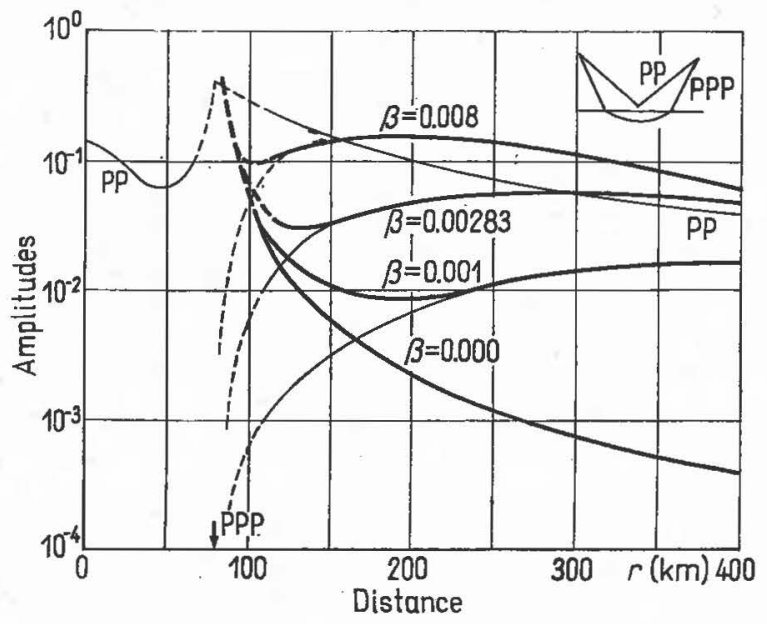

Fig. 3

gives for reflected wave non-zero amplitudes, but zero amplitudes for head waves. The head wave is only an effect of the first order. This is due to the fact that the head wave is propagating through the second medium in a horizontal direction along the interface. However, as soon as the ray of a head wave has arrived at 1 or 2 wavelengths from the interface, a wave of the first order is converted into a wave of a zero order, its amplitudes being thereby noticeably increased. No changes in the kinematics of the PPP wave can meanwhile be observed (Fig. 3). 
There may be a number of reasons which account for the deviation of a ray from the interface. If, for example, there is a positive velocity gradient below Moho, the ray does not propagate in a direction parallel to that of the interface, but immerses below it (see Fig. 4). We shall call this wave immerged wave. In Fig. 4 there are given the amplitude curves of immerged waves for three

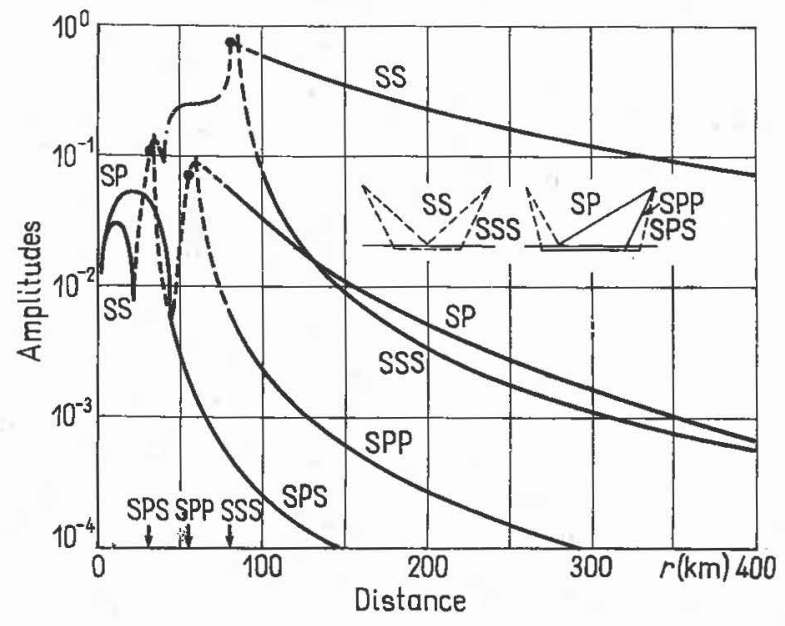

Fig. 4

gradients $\beta: \beta=0.001 \mathrm{~km}^{-1} ; \beta=0.00283 \mathrm{~km}^{-1}$ and $\beta=0.008 \mathrm{~km}^{-1}$. Figure 4 further gives the amplitude curves of a reflected PP wave and of a PPP head wave originating in the same medium without a gradient $\left(\beta=0.000 \mathrm{~km}^{-1}\right)$. From Fig. 4 it is seen that at larger distances from the critical point the amplitudes of immerged waves are about 1 or 2 orders stronger than the amplitudes of head waves. In this distances it is possible to use for the amplitudes of immerged waves the formulas of zero order aproximation - see thin lines in Fig. 4. On the contrary, for short distances from the critical point it is possible to use for the amplitudes of immerged waves the formulae for head waves. In the transition zone, where neither of the above formulae can be used, the formulae of Čekin (Institute of the Physics of the Earth, Moscow, - private communication), were used for the connection of both curves.

So even for very little pronounced gradients about $10 \mathrm{~m} / \mathrm{sec} / \mathrm{km}$ the amplitudes of immerged waves are by 1 or 2 orders stronger than the amplitudes of head waves at distances of $200 \mathrm{~km}$ or more from the source. It is sufficient when the ray immerses into the other medium about 3 wavelengths, that is, in our case about $4 \mathrm{~km}$, to make the amplitude increase by one order.

A similar effect can be exerted also by the Moho curvature due to the earth's sphericity or to other factors. Already at distances of about $400 \mathrm{~km}$ from the source the curvature of the earth will noticeably influence the amplitudes of the PPP waves, as the ray of a PPP wave penetrates about $3 \mathrm{~km}$ below Moho. It 
is further possible to obtain much stronger amplitudes of the PPP waves if under the basic interface there is situated another interface, even a weak one, at not too great a distance. In this case a wave reflected on the second interface is much stronger than the head wave produced on the first interface, while in the kinematics these waves differ only very little.

So we think that the pure head wave exists only very rarely in real media. The waves interpreted as head waves are in most cases the waves of other type, with kinematic characteristics very near to those of head waves, but with quite different dynamic characteristics.

In a similar way, there exist mechanisms which, conversely, attenuate the amplitudes of reflected PP waves. The amplitudes of PP waves are attenuated beyond the critical point in the case of a positive velocity gradient inside the earth's crust, as well as through a presence of intermediate interfaces between earth's surface and Moho. 


\title{
SPACE AND TIME VARIATIONS OF STRAIN RELEASE IN THE GREEK AREA
}

\author{
N. Delibasis and A. G. Galanopoulos \\ (Athens, Greece)
}

\begin{abstract}
Summary
Six cross-sections show the latitude and longitude variations of the total strain released by all earthquakes of magnitude $>43 / 4$ occurred in the Greek area bounded by the parallels of $34^{\circ} \mathrm{N}$ and $42^{\circ} \mathrm{N}$ and the meridians of $19^{\circ} \mathrm{E}$ and $29^{\circ} \mathrm{E}$ during the period 1841-1959, and the cumulative magnitude corresponding to the total strain released in the eastern and western section, bounded by the $24^{\circ} \mathrm{E}$ meridian, over the 120-year interval 1841-1960 in dependence of time. It was verified by the presence of an oscillation pattern with two migration cycles of the maximum of strain release between the two sections. The oscillation period was found to be 52 years and the oscillation amplitude almost constant, i.e. about 8 to $81 / 4$ cumulative earthquake magnitude.
\end{abstract}

\section{Introduction}

In a previous investigation (Galanopoulos 1963) two maps of earthquake efficiency were constructed showing the number of shocks of magnitude 6.8 per square degree per hundred years over the periods 1710-1959 and 1841-1959. Both maps reveal that the main part of the earthquake energy released in the Greek area comes from two well developed centres of higher seismic activity, located along the large fault zone bordering the western and southern coasts of Greece.

In the centre, situated along the western coast of Greece, the earthquake activity rises rapidly towards the middle region of the centre, and culminates between the islands of Cephalonia and Zante and the entrance to the Gulf of Patras. The middle region of the centre situated in the south-eastern Aegean Sea covers the area between Rhodes, Karpathos and Kos.

In the second map of earthquake efficiency derived from earthquake data over the period 1841-1959 for which the available data were proved to be fairly consistent - the earthquake activity rises towards the middle regions of the two centres of higher seismicity to the same rate of 12 shocks of magnitude 6.8 per square degree per hundred years. In both maps the two centres mentioned above are connected by a bridge of minor earthquake activity confined nearly along the $24^{\circ}$ meridian.

In the present investigation an attempt is made to stress the details of the spatial course of the strain release by drawing cross-sections showing the distribution of earthquake foci, the cumulative and the average cumulative magnitude against 
latitude and longitude, alternately. Considering the loose connection of the two centres of higher seismic activity along the $24^{\circ}$ meridian, two additional crosssections were made showing the distribution of earthquake foci, the cumulative and the average cumulative magnitude against latitude in the eastern and western section of the Greek area. These two additional cross-sections show clearly the different structure of the earth's crust in the eastern and western section of the Greek area, evidenced also by the failure of D. Taner (1962) to find a unique solution for the structure of the whole area.

Taking into consideration that the two centres of higher seismic activity are located on the same fault zone, an attempt was made to find out if there is any pattern of migration of the maximum strain release between the two ends of the elongated earthquake belt bordering the western and southern coasts of Greece. By plotting the cumulative earthquake magnitude observed in the eastern and western section during the period 1841-1960 against time, we have verified an oscillation pattern with two migration cycles. The oscillation period was found to be 52 years and the oscillation amplitude almost constant, i.e. about 8 to $81 / 4$ cumulative earthquake magnitude. It should be mentioned that the occurrence of the strain release at the ends of elongated fault zones in the oscillatory manner was previously asserted for a number of aftershock sequences in the Circum-Pacific Belt (Duda 1961, 1962; Duda and Båth 1963).

\section{Method Applied}

In the present investigation the observational data, taken from Table I of a previous paper (Galanopoulos 1963), cover only the 119-year interval 1841-1959, for which period the available data were proved to be fairly consistent.

At the first step we have calculated the sum of the earthquake energy released by all surface shocks having occurred at the same latitude or the same longitude. From the cumulative earthquake energy we have determined the corresponding "cumulative earthquake magnitude", i.e. the magnitude of the earthquake which could have released the energy released by all normal shocks having occurred at the same latitude or the same longitude during the period 1841-1959.

All surface shocks were considered to have occurred at the same focal depth; the corresponding cumulative magnitudes, divided in four classes, were plotted at the depth of $15 \mathrm{~km}$. The four classes, 4.8-5.7, 5.8-6.7, 6.8-7.7 and 7.8, were marked by open circles of four different diameters. The cumulative earthquake magnitudes corresponding to the sum of the energy released by all shocks considered to have occurred at "intermediate depth" were plotted at a depth of 100 $\mathrm{km}$. When the focal depth was known, the cumulative magnitude corresponding to the sum of the energy released by all shocks having the same focal depth were plotted at the given depth.

The curve in the middle of the cross-sections refers to the cumulative earthquake magnitude corresponding to the sum of the energy liberated by all shocks 
at the indicated latitude or longitude, irrespective of their depths. Considering that the coordinates of the epicentres are given with an accuracy of a tenth of a degree to quarter or half degrees, we plotted another curve showing the "average cumulative magnitude" corresponding to the middle value of the cumulative energies assigned to a full degree of latitude or longitude. The average cumulative magnitude corresponding to the middle value, i.e. to the sum of the cumulative energies devided by ten, was plotted in the middle of the degree. The next average value was calculated using ten data points starting with the second and ending with the eleventh. The same method of running averages was used by Berg and associates (1964). The successive average values were calculated by a continuous overlapping of the cumulative energies corresponding to a full degree, i.e. each successive value was calculated "by chopping one data point from the beginning and adding one to the end of the preceding set of data points". It is believed that, after this smoothing process, any exaggerations in the spatial course of the cumulative magnitudes, produced from uncertainties in the location of earthquakes, should be negligible.

Suspecting the existence of an oscillation of the maximum strain release between the two centres of higher seismic activity previously mentioned, we have determined the cumulative magnitude corresponding to the sum of the strain released in the eastern and western section bounded by the $24^{\circ}$ meridian for every year since 1841, respectively. The cumulative magnitudes corresponding to the western and eastern section were plotted above and below the time axis, respectively. After the plotting the time axis was tentatively divided into parts, such that for every part could be established, a predominance of the strain released in one of the two sections. For everyone of the tentatively selected time intervals we have marked by a star the cumulative magnitude corresponding to the difference of the strains released above and below the time axis. The stars were placed in the middle of the corresponding time intervals.

After many attempts we have found a clear oscillation of the strain release between the two centres of higher seismicity located at the ends of the earthquake belt bordering the western and southern coasts of Greece. The oscillation period, being twice the finally selected time interval, was found to be 52 years. Thus, the oscillation pattern shows two cycles of migration of the strain release during the 120-year interval 1841-1960. In both cycles the amplitudes of the oscillation are equal to 8-8 1/4 earthquake magnitude, but one being 8.9.

\section{Space Variations of the Strain Release}

It was previously asserted that the earthquake activity in the Greek area is concentrated along the large fault zone bordering the western and southern coasts of Greece, mostly in the region of the Ionian Islands and in the southeastern Aegean Set, in the region of the Dodecanese (Fig. 1). 


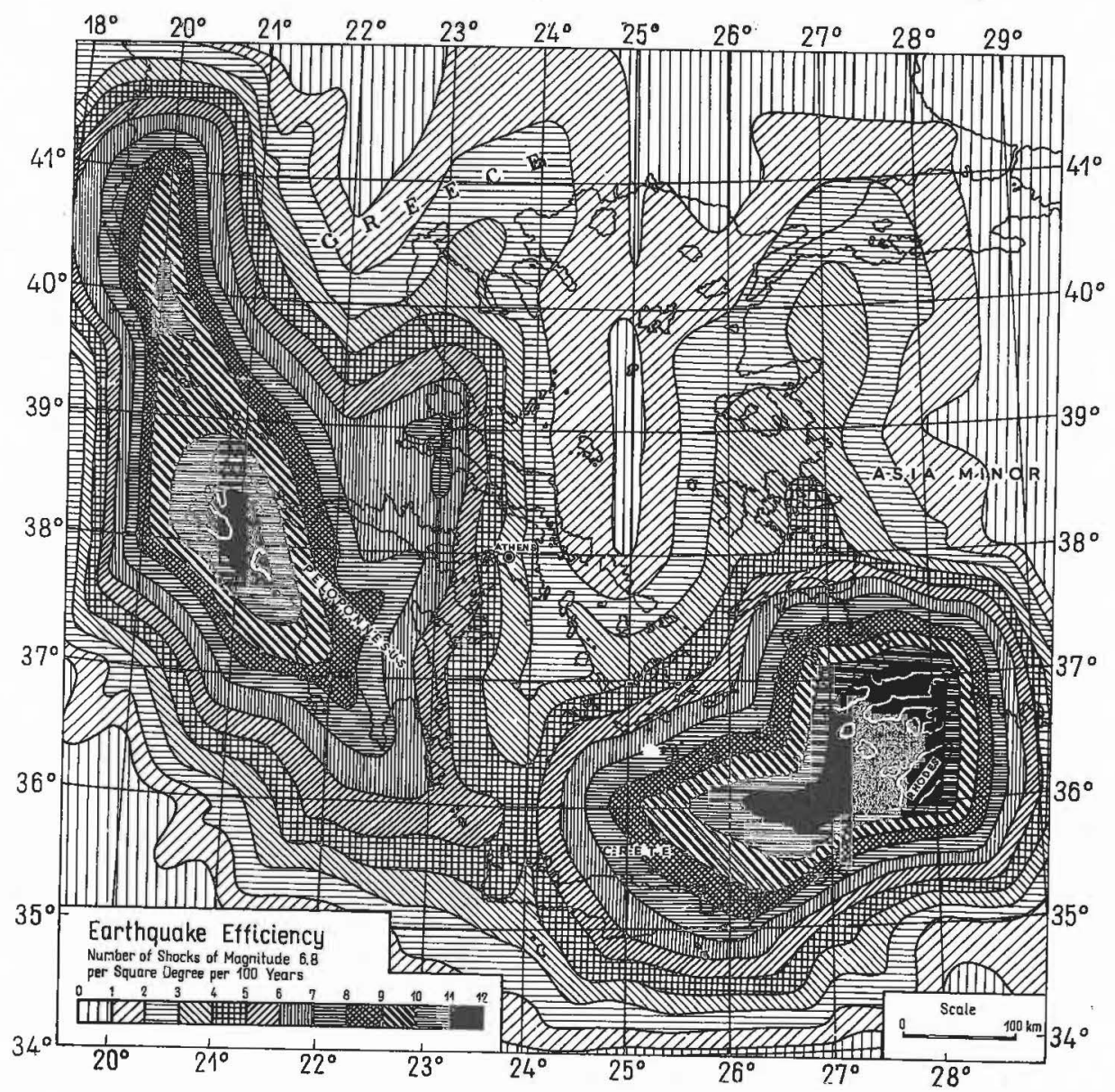

FIG. 1. Earthquake efficiency in Greece derived from earthquake data over the period $1841-1959$

Figure 2 shows that there is a continuous occurrence of foci of normal shocks from the $19^{\circ} 2 \mathrm{E}$ meridian to the $28^{\circ} \mathrm{OE}$ meridian. For the present, the intermediate shocks appear to occur between the meridians of $20^{\circ} \mathrm{OE}$ and $28^{\circ} \mathrm{OE}$ with a clear increase of their focal depth from West to East. The distribution of the foci of the intermediate shocks suggests the existence of a boundary surface dipping between the meridians $20^{\circ} 2 \mathrm{E}$ and $28^{\circ} \mathrm{OE}$ from a depth of $100 \mathrm{~km}$ to a depth of $200 \mathrm{~km}$.

Over the 119-year interval 1841-1959 the earthquake activity increases in the east of the $19^{\circ} 4 \mathrm{E}$ meridian rather abruptly and builds up a high with an average cumulative magnitude $71 / 2$ to 8 between the meridians $19^{\circ} 5 \mathrm{E}$ and $21^{\circ} 2 \mathrm{E}$. From $21^{\circ} 3 \mathrm{E}$ to $21^{\circ} 9 \mathrm{E}$ the strain release remains close to the level of an earthquake 
magnitude $71 / 2$, but beyond that increases again and builds up a second maximum between the meridians $22^{\circ} 5 \mathrm{E}$ and $23^{\circ} 4 \mathrm{E}$. From $23^{\circ} 5 \mathrm{E}$ to $25^{\circ} 4 \mathrm{E}$ the earthquake activity stands below $71 / 2$ and forms a minimum at the level of 7 between the meridians $23^{\circ} 8 \mathrm{E}$ to $24^{\circ} 4 \mathrm{E}$. Between $25^{\circ} 5 \mathrm{E}$ and $26^{\circ} 4 \mathrm{E}, 27^{\circ} \mathrm{OE}$ and $27^{\circ} 9 \mathrm{E}$, the

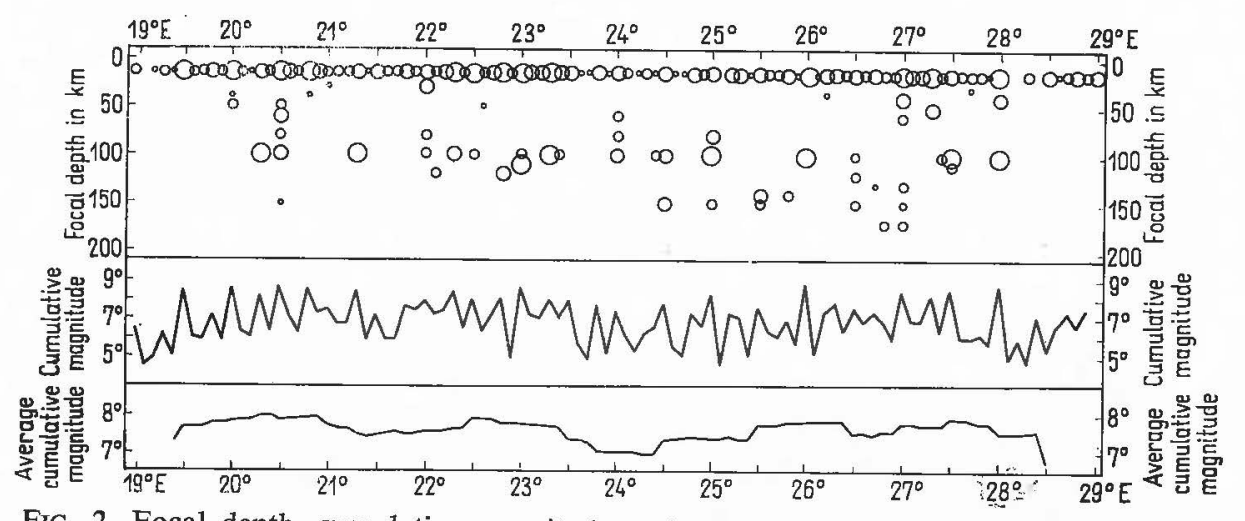

Fig. 2. Focal depth, cumulative magnitude and average cumulative magnitude of earthquakes of magnitude $>43 / 4$, occurred in the Greek area bounded by the parallels of $34^{\circ} \mathrm{E}$ and $42^{\circ} \mathrm{N}$ during the period 1841-1959, plotted against longitude

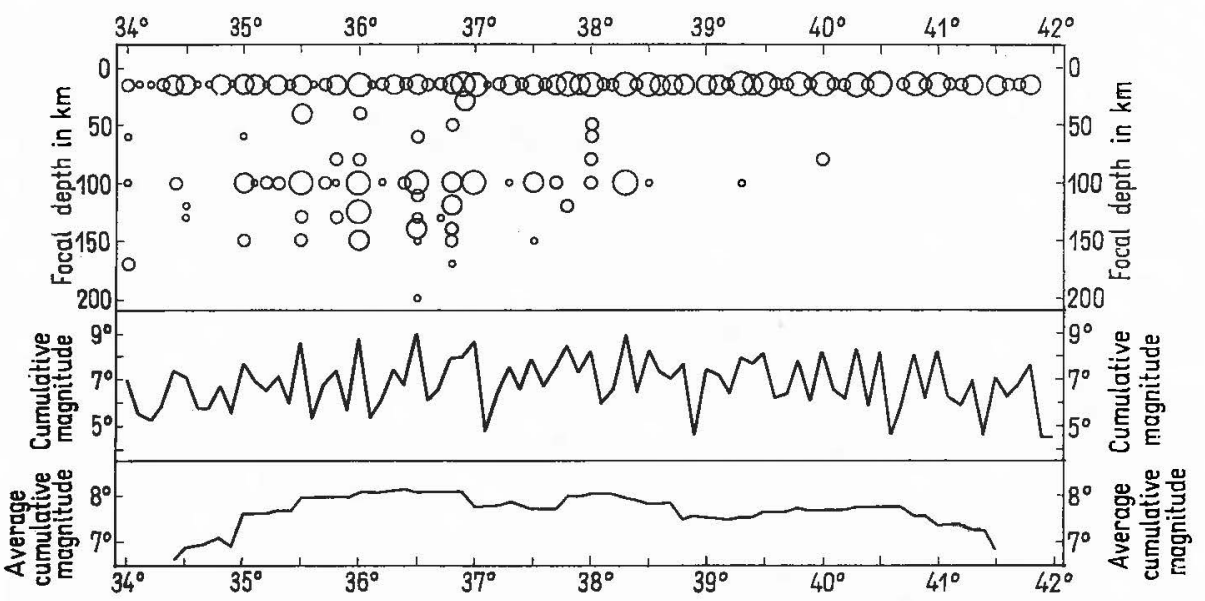

FIG. 3. Focal depth, cumulative magnitude and average cumulative magnitude of earthquakes of magnitude $>43 / 4$, occurred in the Greek area bounded by the meridians of $19^{\circ} \mathrm{E}$ and $29^{\circ} \mathrm{E}$ during the period $1841-1959$, plotted against latitude

strain release forms two highs of the same width, separated by a bridge at which the earthquake activity remains close to $71 / 2$. From $28^{\circ} \mathrm{OE}$ to $28^{\circ} 4 \mathrm{E}$ the strain release stands constantly at the level of $71 / 2$ and beyond that drops below 7 .

Figure 3 shows a continuous occurrence of surface shocks between the parallels $34^{\circ} \mathrm{ON}$ and $41^{\circ} 8 \mathrm{~N}$ with three gaps at the latitudes $38^{\circ} 9 \mathrm{~N}, 40^{\circ} 6 \mathrm{~N}$ and $41^{\circ} 4 \mathrm{~N}$. 
The foci of the intermediate shocks appear to occur between the parallels $34^{\circ} \mathrm{ON}$ and $40^{\circ} \mathrm{ON}$, but their majority is expressedly confined between the parallels $34^{\circ} \mathrm{ON}$ and $38^{\circ} \mathrm{ON}$. If we disregard the focal depth of $170 \mathrm{~km}$, appeared at the latitude $34^{\circ} \mathrm{ON}$, the distribution of the intermediate foci marks a wedge plunging $200 \mathrm{~km}$ deep at the $36^{\circ} 5 \mathrm{~N}$ latitude. The two surfaces of the wedge dipping from a depth of $130 \mathrm{~km}$ at the latitude $34^{\circ} 5 \mathrm{~N}$, and $80 \mathrm{~km}$ at the latitude $40^{\circ} \mathrm{ON}$, towards

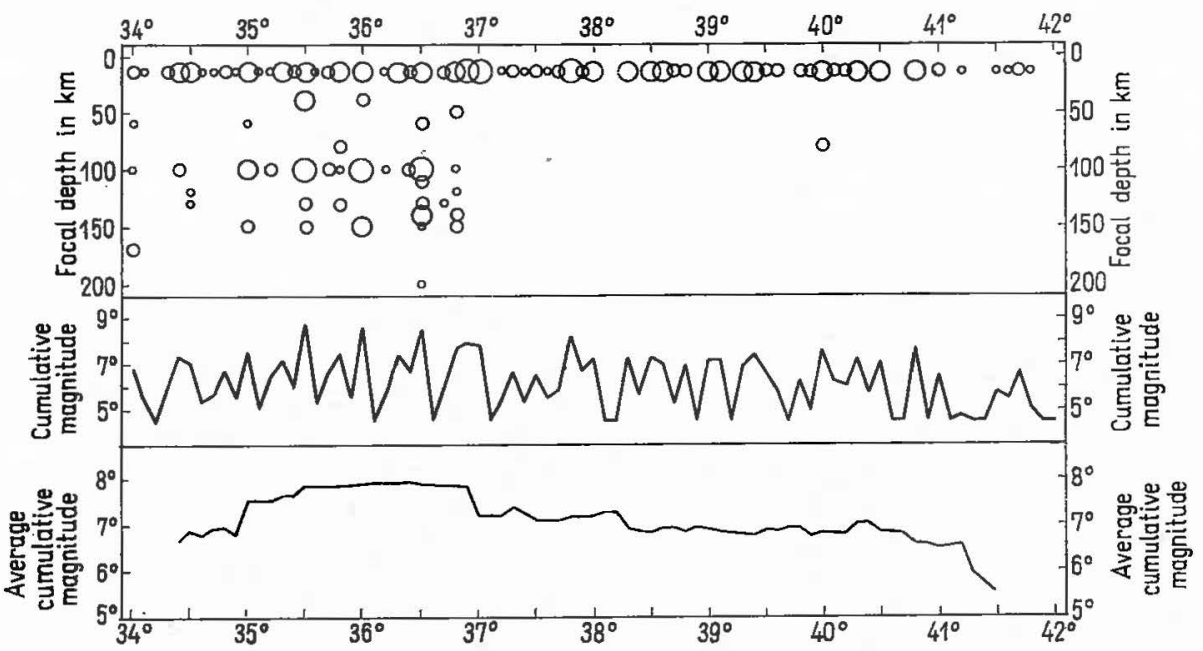

FTG. 4. Focal depth, cumulative magnitude and average cumulative magnitude of earthquakes of magnitude $>43 / 4$, occurred in the western section of the Greek area during the period 1841-1959, plotted against latitude

the depth of $200 \mathrm{~km}$ at the latitude $36^{\circ} 5 \mathrm{~N}$, show the same inclination. It is interesting to note that the cusp of the wedge is at the $36^{\circ} 5 \mathrm{~N}$ parallel close to which the active volcanoes of Santorin and Nisyros are situated.

The earthquake activity increases abruptly at the $35^{\circ} \mathrm{ON}$ parallel and remains above the level of an average magnitude $71 / 4$ up to the parallel $41^{\circ} 4 \mathrm{~N}$, beyond which it drops abruptly. Two highs with an average magnitude $73 / 4$ to 8 appear between the parallels $35^{\circ} 5 \mathrm{~N}$ and $36^{\circ} 9 \mathrm{~N}, 37^{\circ} 8 \mathrm{~N}$ and $38^{\circ} 7 \mathrm{~N}$, separated by a bridge at which the earthquake activity remains close to the level of $73 / 4$. The two highs correspond to the Dodecanese centre and to the Ionian centre of higher earthquake activity evidenced in the previous paper (Fig. 1).

Figures 4 and 5 show the latitude variations of the total strain released in the western and eastern section, respectively. In the western section (Fig. 4) the earthquake activity rises almost one unit at the $35^{\circ} 5 \mathrm{~N}$ and remains above 7 up to the $41^{\circ} 4 \mathrm{~N}$ parallel. The silhouette of the Ionian centre of higher strain release is fairly defined between the parallels $37^{\circ} 8 \mathrm{~N}$ and $38^{\circ} 7 \mathrm{~N}$. The foci of the intermediate shocks are nearly $2 / 3$ of those appeared in the eastern section. All the intermediate foci but one are located between the parallels $35^{\circ} 5 \mathrm{~N}$ and $38^{\circ} 5 \mathrm{~N}$. 


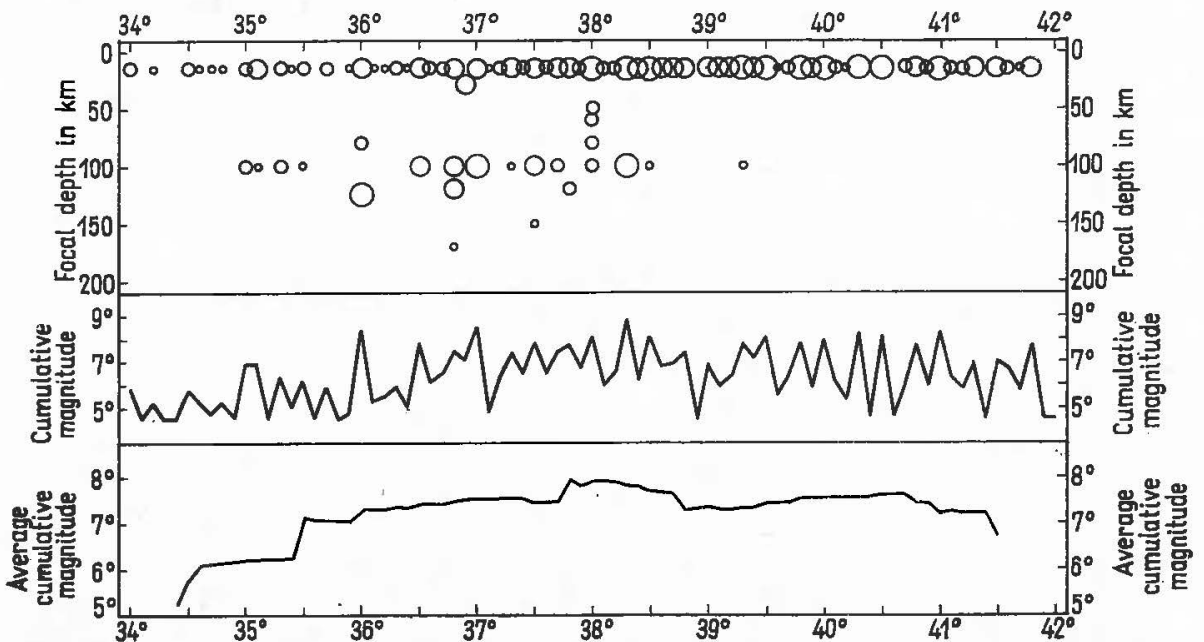

FIG. 5. Focal depth, cumulative magnitude and average cumulative magnitude of earthquakes of magnitude $>43 / 4$, occurred in the eastern section of the Greek area during the period 1841-1959, plotted against latitude

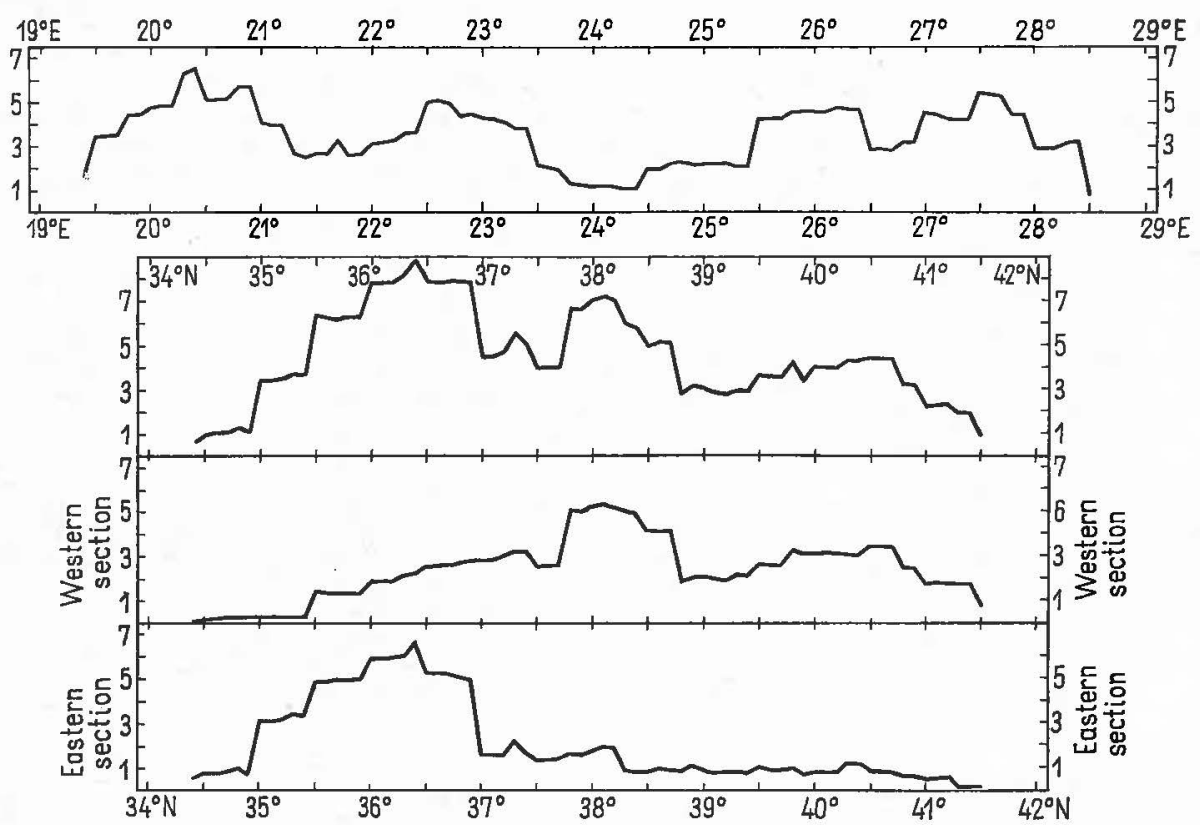

FIG. 6. Number of shocks of magnitude 6.8 per hundred years corresponding to the average cumulative strain released in the Greek area, bounded by the parallels of $34^{\circ} \mathrm{N}$ and $42^{\circ} \mathrm{N}$ and the meridians of $19^{\circ} \mathrm{E}$ and $29^{\circ} \mathrm{E}$ during the period $1841-1959$, plotted against longitude and latitude 
During the period under investigation all the intermediate earthquakes having occurred in the east of the $24^{\circ} \mathrm{OE}$ meridian (Fig. 5) are limited between the parallels $34^{\circ} \mathrm{ON}$ and $36^{\circ} 8 \mathrm{~N}$, except one. The deepest earthquake focus $(200 \mathrm{~km})$ known in the Greek area has been observed in the eastern section. In the section shown in Fig. 5 the strain release remains above an average cumulative magnitude $63 / 4$ over the region bounded by the parallels $34^{\circ} 5 \mathrm{~N}$ and $40^{\circ} 7 \mathrm{~N}$ and forms a high well-expressed between $35^{\circ} \mathrm{ON}$ and $36^{\circ} 9 \mathrm{~N}$.

The spatial variations of the earthquake activity are strongly marked in Fig. 6 showing the average number of earthquakes of magnitude 6.8 per hundred years, calculated from the average cumulative energy at the corresponding longitude or latitude. Figure 6 has the additional advantage of showing directly the cumulative strain energy release expressed in number of shocks occurring in the Greek area at a given longitude or latitude over a 100-year interval.

\section{Time Variations of the Strain Release}

It was previously asserted (Galanopoulos 1955a, b) that the strain relief in the Greek area occurs approximately at the same rate on both sides of the Aegean intermediate mass. However, the lack of a measure of the strain release before the use of the concept of the earthquake magnitude handicapped the disclosure of the time relation existing in the shift of the earthquake activity between the eastern and western section of the Greek area (Galanopoulos 1941).

It is true that weak earthquakes occur in the eastern and western section of the Greek area not rarely at the same day or even at the same hour, but severe earthquakes have never happened on both sides of the Aegean crystalline mass within the time interval of one day. The shift of the earthquake activity from one section to another takes several days, usually months or years.

It was found that there is a tendency of a regular migration of the earthquake foci from one side of the Aegean intermediate mass to the other predetermined by the fault structure of the Greek area (Galanopoulos 1955b), but we did not succeed to find a clear oscillation of the maximum of strain release from one section to the other within a time increment shorter than that shown in Fig. 7. There are, of course, some kinds of shorter oscillation during the 120 -year interval

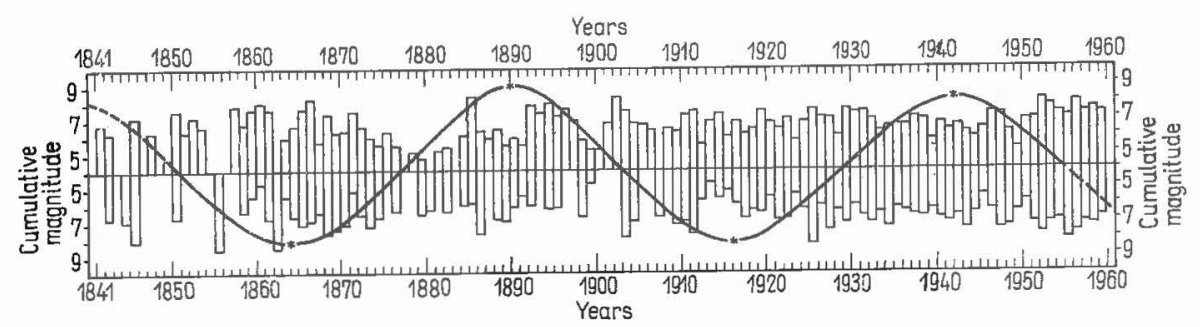

FIG. 7. Oscillation pattern of the maximum of the annual strain-expressed in earthquake magnitudes - released in the western (above time axis) and the eastern section of the Greek area (below time axis) during the period 1841-1960 
$1841-1960$, but it is hard to attach any importance to a discontinuous occurrence of an alternative predominance of strain release of different period and different intensity in the eastern and western section. Whether the time increment of 26 years, in the present paper, has any special meaning is beyond our judgement.

\section{Azimuthal Variation of the Residuals of Arrival Times of $\mathbf{P}$ Waves}

It was previously stated that there is evidence of a marked difference in the structure of the earth's crust in the eastern and western section of the Greek area. Residuals of arrival times of $\mathrm{P}$ waves in Athens $\left(P_{g}, P^{*}, P_{n}\right)$ radiated from

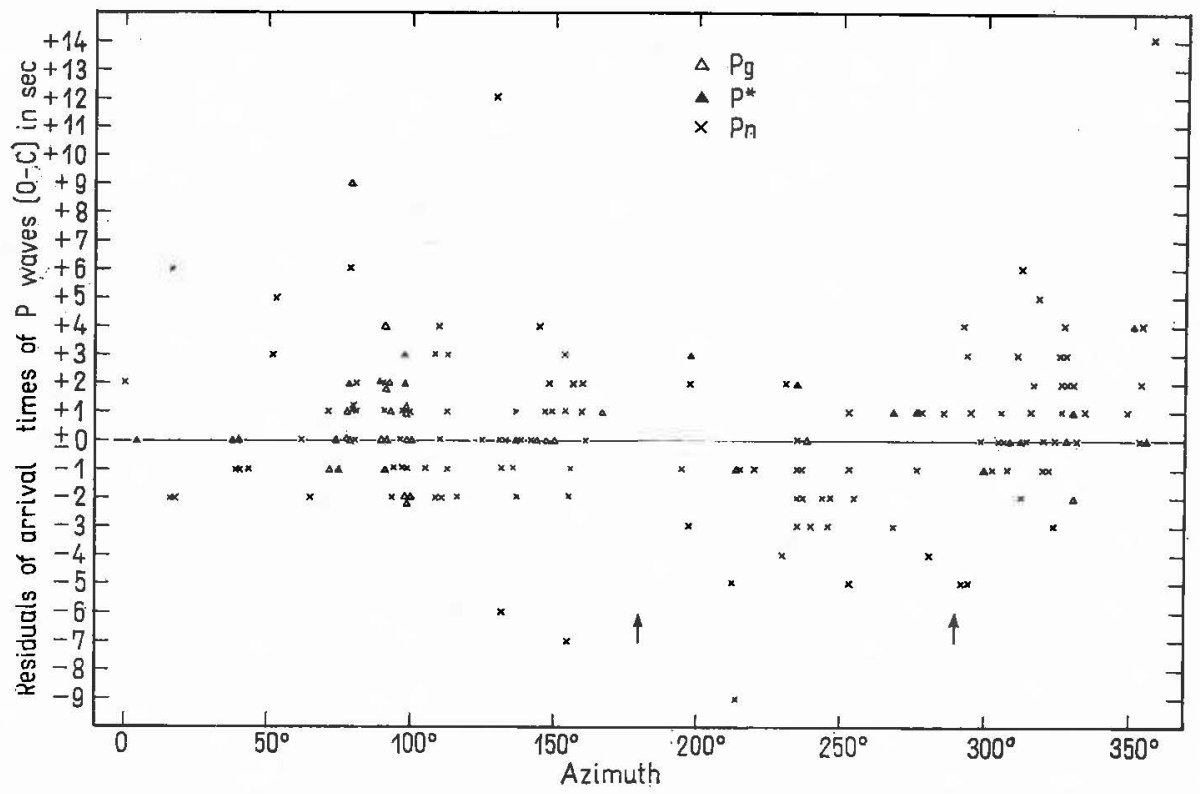

FIG. 8. Residuals of arrival times of $P$ waves, as given in the I.S.S. for Athens over the years 1950 to 1957

earthquake foci within the Greek area reveal an azimuthal variation (Fig. 8), which points to a different crustal and upper mantle structure in the eastern section, and particularly in the Northern Aegean Sea. The arrival times of $P_{n}$ waves, coming from earthquake foci situated between the azimuths of $190^{\circ}$ and $290^{\circ}$, are systematically too early, with a mean residual of -1.59 seconds, while the mean residual of $P_{n}$ arrivals from the Southern Aegean Sea $\left(290^{\circ}-360^{\circ}\right)$ is +0.86 seconds. The mean residual of $P_{n}$ arrivals from the western section $\left(0^{\circ}-180^{\circ}\right)$ is +0.42 seconds, almost half of the mean difference found for the $290^{\circ}-360^{\circ}$ section. All the residuals of arrival times of $P_{n}$ waves, as given in the International Seismological Summary for Athens over the years 1950-1957 were used, but 4 out of 124 - showing a scattering over 6 seconds - were not considered in the 
calculation of the mean difference. A systematic investigation of the azimuthal variation of the residuals of $P$ arrivals over a longer period (1950-1964) is now in progress. No variation of the residuals of arrival times of $P$ waves in dependence of distance was revealed for the range $0^{\circ} 5$ to $4^{\circ} 5$.

In view of the few score of reliable data available for the time being, we shall confine ourselves to saying that there is a tendency of a positive distribution of the residuals corresponding to the western section, indicative of an average continental structure associated with the Alpine folding. The early arrival of $\boldsymbol{P}_{n}$ waves may be due to a smaller depth of the M-discontinuity in the section between the azimuths of $190^{\circ}$ and $290^{\circ}$. The relatively lower $P_{n}$ velocity in the $290^{\circ}-360^{\circ}$ section indicated by the explicitly positive mean residual might be attributed to a greater thickness of the crust and to the presence of magma chambers in the Southern Aegean Sea. It is worth noting that the volcano of Nisyros is situated in the transitional zone between the azimuths of $290^{\circ}$ and $295^{\circ}$. If we drop the residuals corresponding to the $290^{\circ}-295^{\circ}$ section, where a sudden change in the $P_{n}$ velocity should presumably occur, the mean residual of $P_{n}$ arrivals from the Southern Aegean Sea $\left(300^{\circ}-360^{\circ}\right)$ is +1.06 seconds.

\section{Acknowledgments}

The research reported in this document has been sponsored by the Air Force Office of Scientific Research under Contract AF 61 (052)-803 through the European Office of Aerospace Research (OAR), United States Air Force, as part of the Advanced Research Projects Agency's Project VELA UNIFORM.

The authors would like to express their sincere thanks to Dr. B. Papazachos for reading critically the manuscript of this paper.

\section{REFERENCES}

[1] Berg, W. J. JR., Gaskell, R. and RINehart, V.: Earthquake Energy Release and Isostasy, Bull. Seism. Soc. Am. 542 777-784 (1964)

[2] DudA, S. J.: Phänomenologische Untersuchung einer Nachbebenserie aus dem Gebiet der Alëuten Inseln. Zeitschr. Geophys. 27 207-213 (1961); und Freiberger Forschungsh, C 132 Geophys. 1-90 (1962)

[3] DudA, S. J.: Strain release in the Circum-Pacific Belt, Chile: 1960, J. Geophys. Res. $68195531-5544$ (1962)

[4] DudA, S. J. and B\&TH, M.: Strain release in the Circum-Pacific Belt: Kern Country 1952, Desert Hot Springs 1948, San Francisco 1957, Geophys. J. R. A. S. 75 554570 (1963)

[5] Galanopoulos, A.: Gleichzeitige Erdbebentätigkeit im Jonischen und Ägäischen Gebiet, Gerl. Beitr. z. Geoph. 572 117-131 (1941)

[6] Galanopoulos, A.: The earthquake activity in the Greek area from 1950 to 1953 , Prakt. Acad. Athenes 30 38-49 (1955a)

[7] Galanopoulos, A.: Strain relief at the same rate on both sides of the Aegean Mass, Prakt. Acad. Athènes 30 49-57 (1955b)

[8] Galanopoulos, A.: On mapping of seismic activity in Greece, Ann. di Geof. 16 137100 (1963)

[9] TANER, D.: Sur la structure de la croûte terrestre en Grèce, en Mer Egée et auprès des côtes occidentales de la Turquie, Ann. Geophys. 291-293 (1962) 
ERGEBNISSE REFLEXIONSSEISMISCHER MESSUNGEN ZUR UNTERSUCHUNG DES BAUES

DER ERDKRUSTE IN DER BUNDESREPUBLIK

G. DOHR

(Hannover, BRD)

Unsere Kenntnisse über den Bau der Erdkruste sind - verglichen etwa mit den Fortschritten, welche beispielsweise die Erforschung des Weltraumes in den letzten Jahrzehnten gemacht hat — noch recht dürftig. Zwar wissen wir in großen Zügen einiges über den grundsätzlichen Aufbau der oberen Erdschichten; bei der Frage nach Details müssen wir uns aber leider vorerst noch sehr bescheiden.

Die Erforschung des tieferen Untergrundes, zumindest, soweit wir ihn unter dem Begriff Erdkruste zusammenfassen, geht zum großen Teil auf die Verdienste der Göttinger Schule zurück, die die Ausnutzung künstlicher Energie, wie sie bei Steinbruchsprengungen erzeugt wird, zur seismischen Erforschung des Untergrundes in die Beobachtungspraxis einführte. Solche Untersuchungen haben, neben den bedeutsamen Arbeiten von Conrad, Mohorovičić, der Gutenbergschen Schule und anderen, welche sich auf das in der Erdbebenseismik anfallende Material stützten, zu einer ersten Vorstellung über den Bau der Erdkruste, die Erkenntnisse über die Existenz der Conrad- und Mohorovičić-Diskontinuität und ihre mögliche geologische und petrographische Bedeutung geführt. In Deutschland sind dann die Beobachtungena nläßlich großer Sprengungen nach dem letzten Kriege von besonderer Bedeutung für die Erweiterung unserer Kenntnis über den Verlauf der tiefen Unstetigkeitsflächen in Mitteleuropa gewesen. Es ist unbestreitbar das Verdienst dieser Forschungen - hier seien Namen wie Reich, Schulze, Förtsch, Rothe, Peterschmitt u. a. genannt - die Existenz einer durchgehenden Conrad-Diskontinuität und einer überall erkennbaren MohorovičićDiskontinuität in Mitteleuropa aufgezeigt zu haben.

Die Fortsetzung dieser refraktionsseismischen Messungen bildet das systematisch durchgeführte Meßprogramm, welches im Rahmen des Schwerpunktprogramms der Deutschen Forschungsgemeinschaft in den lezten Jahren durchgeführt wurde und über welches an anderer Stelle zu berichten ist.

Neben diesen Arbeiten hat sich in den letzten Jahren in der Bundesrepublik ein anderes Verfahren der seismischen Untersuchung durchgesetzt, welches sich der reflexionsseismischen Methode bedient. Die Existenz und vor allem der praktische Nachweis verwertbarer reeller Reflexionswellen von den tieferen Grenzflächen ist längere Zeit sehr skeptisch betrachtet und in Zweifel gezogen worden. Reich hat anläßlich einer Sprengung bei Blaubeuren Reflexionen von tiefen Grenzflächen mit einer Laufzeit von etwa 9 Sekunden nachweisen können.

Es zeigte sich jedoch später, daß zur Registrierung solch tiefer Reflexionen 
auch wesentlich geringere Energiemengen auf der Sendeseite ausreichten. Von A. Junger ist in Kanada auf die Beobachtung reflektierter Wellen von tiefen Grenzflächen bei routinemäßigen reflexionsseismischen Untersuchungen berichtet worden. In der Bundesrepublik ist kurze Zeit später von Schulz, Holste und Dohr aus den Gebieten der Pfalz, des Rheintalgrabens und des Alpenvorlandes ebenfalls über tiefe Reflexionen, welche mit großer Wahrscheinlichkeit dem Bereich der Conrad- und Mohorovičić-Diskontinuität zugeordnet werden können, berichtet worden. Diese ersten Arbeiten und Erfolge waren Anlaß, die Registrierung von Tiefenreflexionen in der Bundesrepublik ständig bei einer Anzahl seismischer Meßtrupps durchzuführen. Bei der großen Zahl ständig laufender seismischer Untersuchungsprogramme konnten jeweils 4-5 Meßtrupps ausgewählt werden, welche bei allen Schüssen Seismogramme bis zu einer Laufzeit von etwa 12 Sekunden aufnahmen.

In der ersten Abbildung ist ein Überblick über die Arbeitsgebiete gegeben, in welchen in den letzten Jahren sichere Tiefenreflexionen beobachtet wurden. Man erkennt, daß sich die Beobachtungen auf zwei Gebiete konzentrieren: einmal auf den Raum des Alpenvorlandes und zum anderen auf den Südteil des nordwestdeutschen Beckens. Diese Häufung ist nicht zufällig. Einmal handelt es sich in Süddeutschland um einen Raum mit besonders starker seismischer Aktivität und durchweg recht guten Tiefenreflexionen, zum anderen hat sich der Nordteil des nordwestdeutschen Beckens als besonders schwierig für die Bearbeitung von Tiefenreflexionen erwiesen. Hier stört vor allem die sehr starke Bedeckung mit Tertiär, die Salzstöcke und -Kissen im Keuper und Zechstein und überdies das Auftreten zahlreicher und starker Mehrfachreflexionen, die Registrierung von Reflexionen mit langen Laufzeiten.

Es zeigte sich sehr bald, da $ß$ in der Mehrzahl der Untersuchungsgebiete Reflexionen bei sehr langen Laufzeiten beobachtet werden konnten. Es war jedoch nur in wenigen Fällen möglich, die einzelnen Reflexe über lange Strecken zu korrelieren. In der Mehrzahl der Fälle ließen sich zwar ganze Gruppen und Bänder von Reflexionen über lange Strecken und viele Seismogramme verfolgen, die einzelnen Phasen hielten aber gewöhnlich nur über höchstens 6-8 Seismogramme durch. Zudem traten die Reflexe bei langen Laufzeiten in manchen Gebieten nur sporadisch auf. Aus diesem Grunde wurde ein Auswerteverfahren gewählt, welches einerseits einen raschen Ubberblick über die Ergebnisse in den einzelnen Arbeitsgebieten gestattete, andererseits aber auch eindeutige und verwertbare Aussagen über die Existenz und Tiefenlage tiefer Grenzflächen gestattete.

Die Aufstellung von sogenannten Häufigkeitsverteilungskurven in den einzelnen Meßgebieten erwies sich als brauchbarste Methode. Die in den Einzelseismogrammen beobachteten Reflexionen oder Reflexionsandeutungen wurden aufsummiert und ihre absolute Anzahl in einer jeweils für ein Teilgebiet gezeichneten Kurve als Funktion der Reflexionszeit aufgetragen. Als Intervallbreite für die Aufsummierung der Reflexe wurde 0,2 oder 0,1 Sekunden gewählt. Dort, wo innerhalb des Meßgebietes tiefe Grenzflächen reflektieren, zeichnen sich in den Kurven der Reflexionshäufigkeit mehr oder minder scharfe Maxima ab. 
$\phi 10 \mathrm{~F}$-Diskontinuität

Q17 C-

$\times 31 \mathrm{M}-$

- 23 Zwischen-Diskontinuität

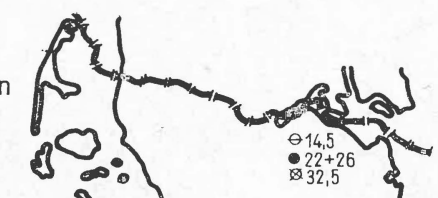

in $\mathrm{km}$ unter $\mathrm{NN}$
(3,

- 3
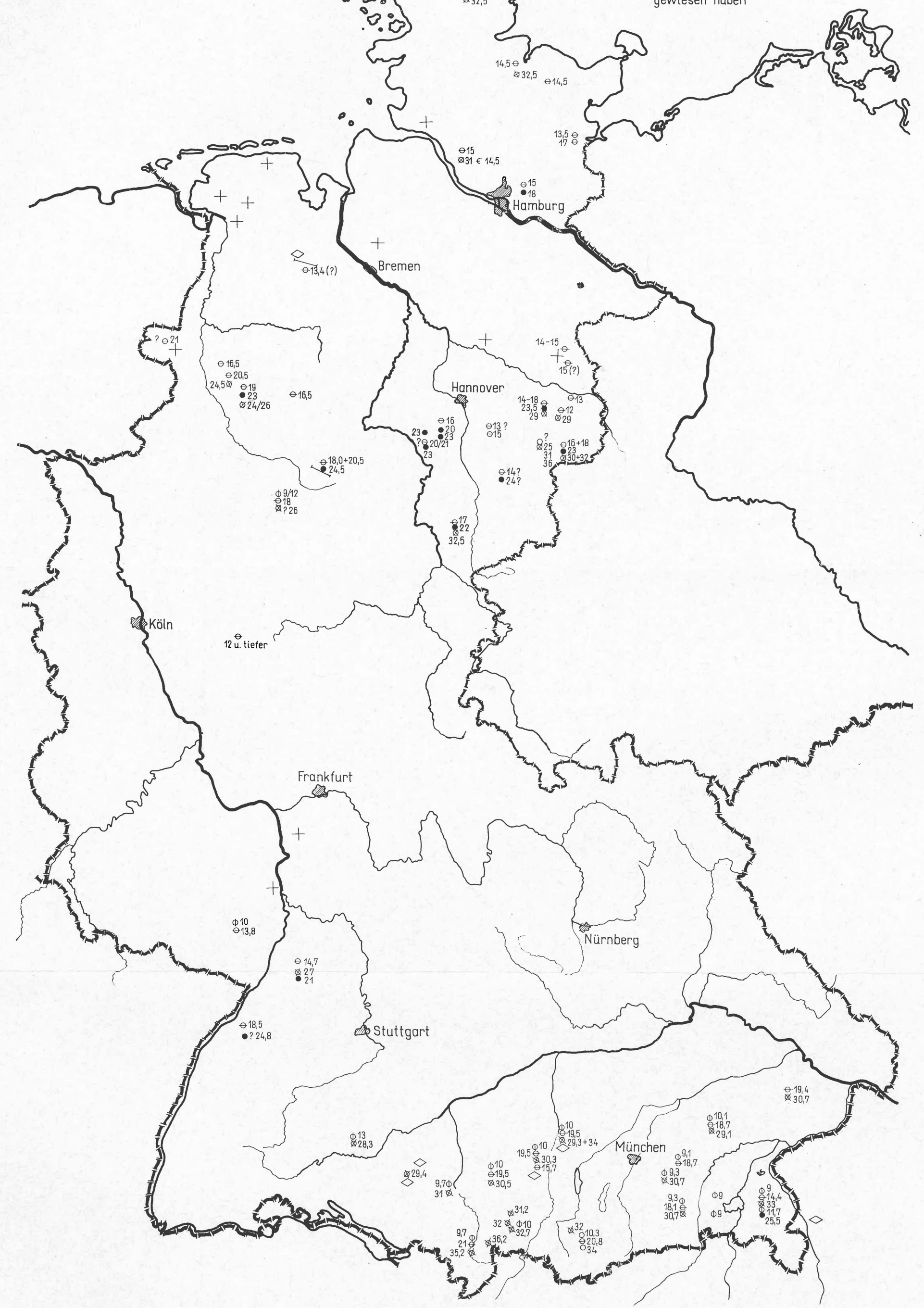
Die Frage, ob es sich bei diesen tiefen Reflexionen tatsächlich um reelle Reflexe und nicht etwa um Mehrfachreflexionen oder apparative Reflexe handelt, ist lange diskutiert worden. An dieser Stelle soll im einzelnen nicht auf diese Untersuchungen eingegangen werden. Im Laufe der Zeit hat sich die Realität der tiefen Reflexionen aber immer mehr bestätigt, und sie haben sich sowohl in der Bearbeitung durch verschiedene Auswerter als auch bei Wiederholung der Messungen in gleichen oder eng benachbarten Gebieten als reproduzierbar erwiesen. Die statistische Methode erscheint uns auch zur weiteren Untersuchung gut geeignet.

Der süddeutsche Raum ist in einer kürzlich publizierten Arbeit von Liebscher behandelt worden. Die Ausarbeitungen sind hier am weitesten fortgeschritten und haben bereits zu recht abgerundeten und geschlossenen Ergebnissen gefübrt. Als Beispiel für die Beobachtungsergebnisse und die oben diskutierte Darstellung der Ergebnisse in Form von Häufigkeitsverteilungskurven sei hier einmal ein

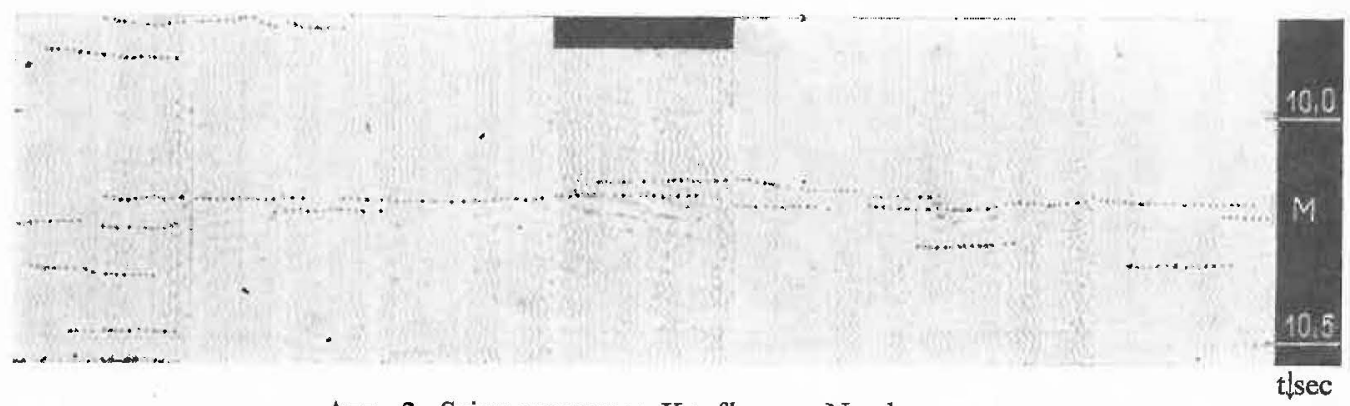

AвB. 2. Seismogramme: Kaufbeuren-Nord

Seismogrammausschnitt mit Reflexionen aus dem Bereich der Conrad-Diskontinuität (Abb. 2) und zum anderen eine Zusammenstellung von Häufigkeitsverteilungskurven aus dem Gebiet der Vorland- und der gefalteten Molasse gezeigt (Abb. 3). Man erkennt sehr gut, daß sich die Maxima in den einzelnen Verteilungskurven überraschend gut entsprechen. Die Buchstaben $F, C$ und $M$ sollen darauf hinweisen, daß sich die hier erkennbaren Reflexionsanhäufungen sehr wahrscheinlich dem Bereich der Förtsch-, Conrad- und Moho-Unstetigkeitszone zuordnen lassen.

Von Liebscher sind zur weiteren Interpretation und zur Ergänzung des seismischen Materials die vorliegenden Gravimetermessungen der Reichsaufnahme hinzugezogen worden. Durch Einhängung eines gravimetrischen Modells in die aus der neuen Reflexionsseismik gewonnenen Fixpunkte ergibt sich bei Einsetzung plausibler Dichten im Alpenvorland ein vollständiger Tiefenlinienplan für die Conrad- und Moho-Diskontinuität. In den nachfolgenden Abbildungen (Abb. 4 und 5) sind diese - wohl zumeist schon bekannten - Pläne dargestellt.

Die Ausarbeitungen im süddeutschen Raum haben darüber hinaus weitere Besonderheiten aufgezeigt, auf die noch kurz hingewiesen sei. 


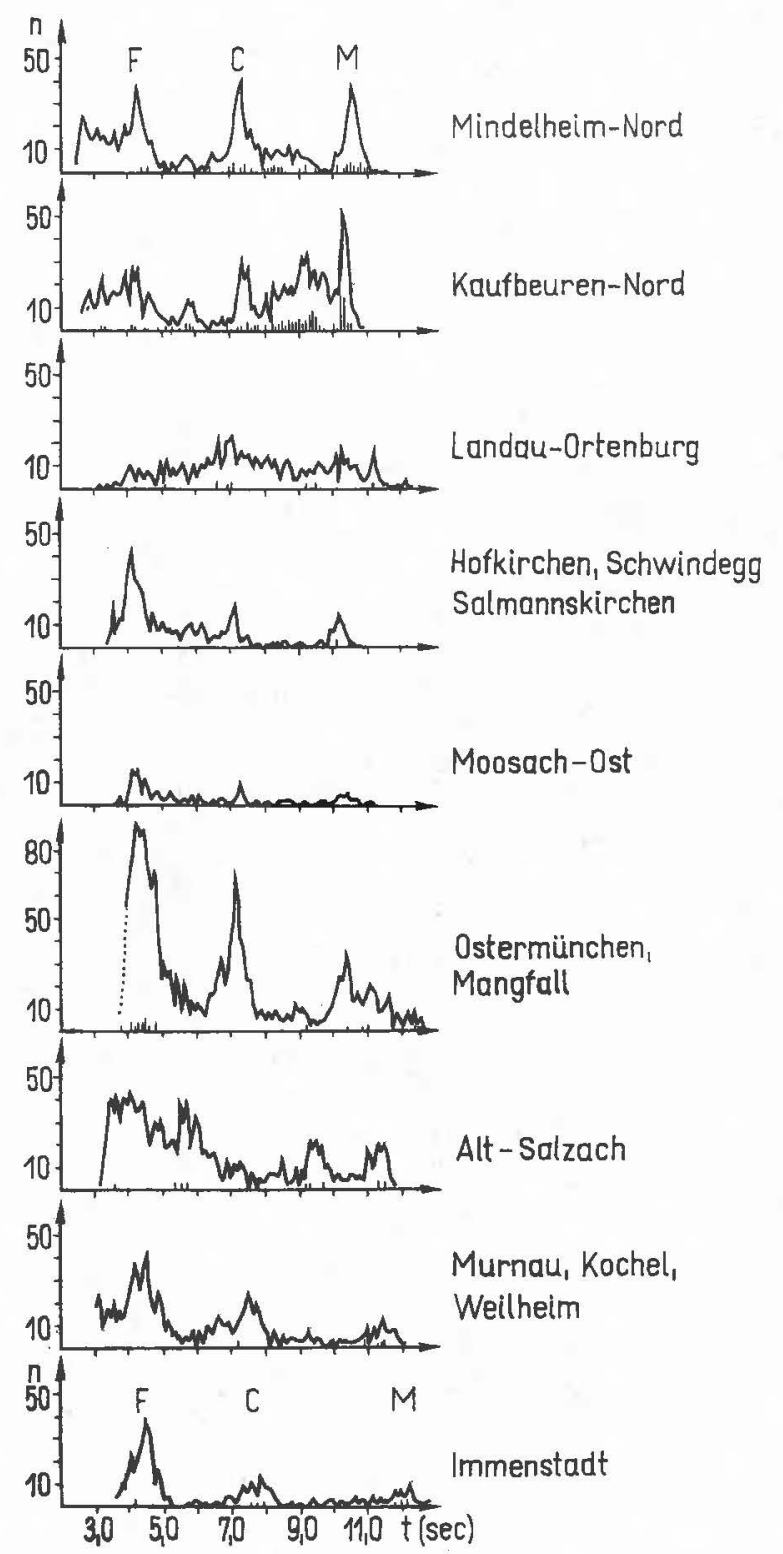

Aвв. 3. Absolute Häufigkeit tiefer Reflexionen Intervallbreite $0,1 \mathrm{sec}$ 


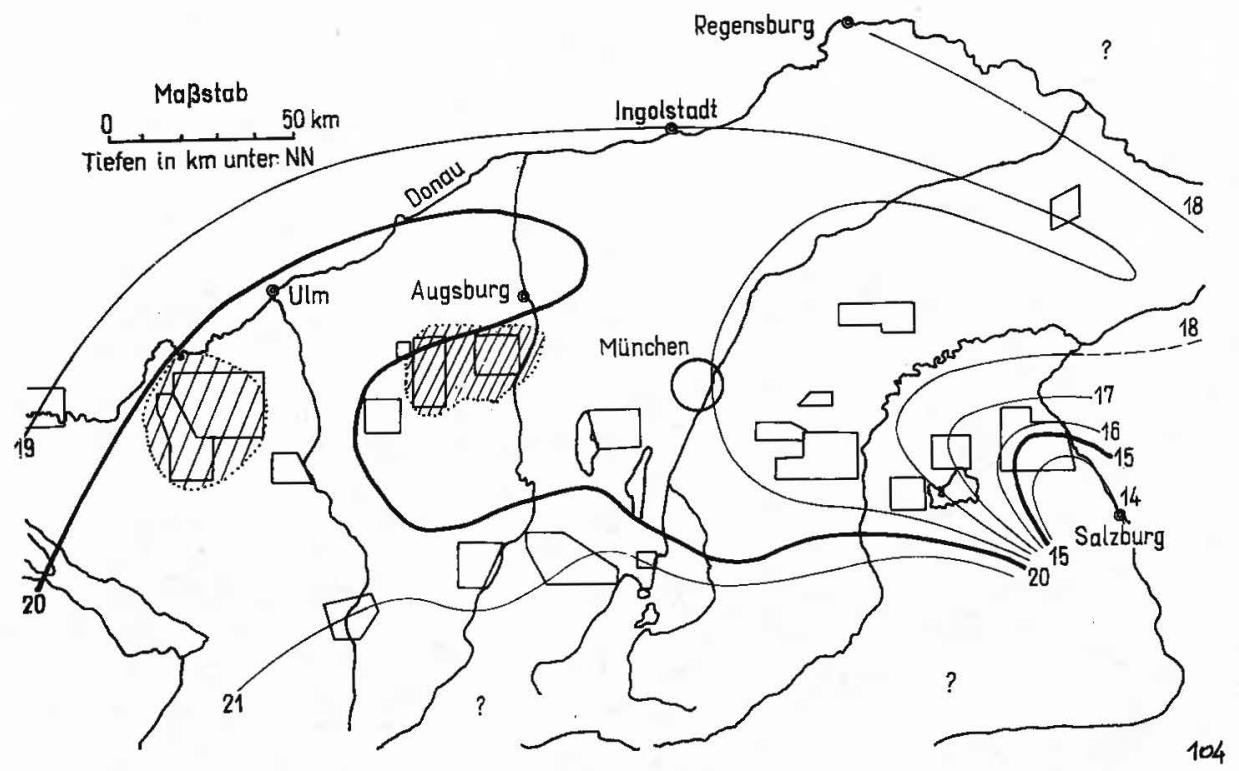

ABB. 4. Tiefenlinienplan für die C-Diskontinuität mit Tiefenkörper

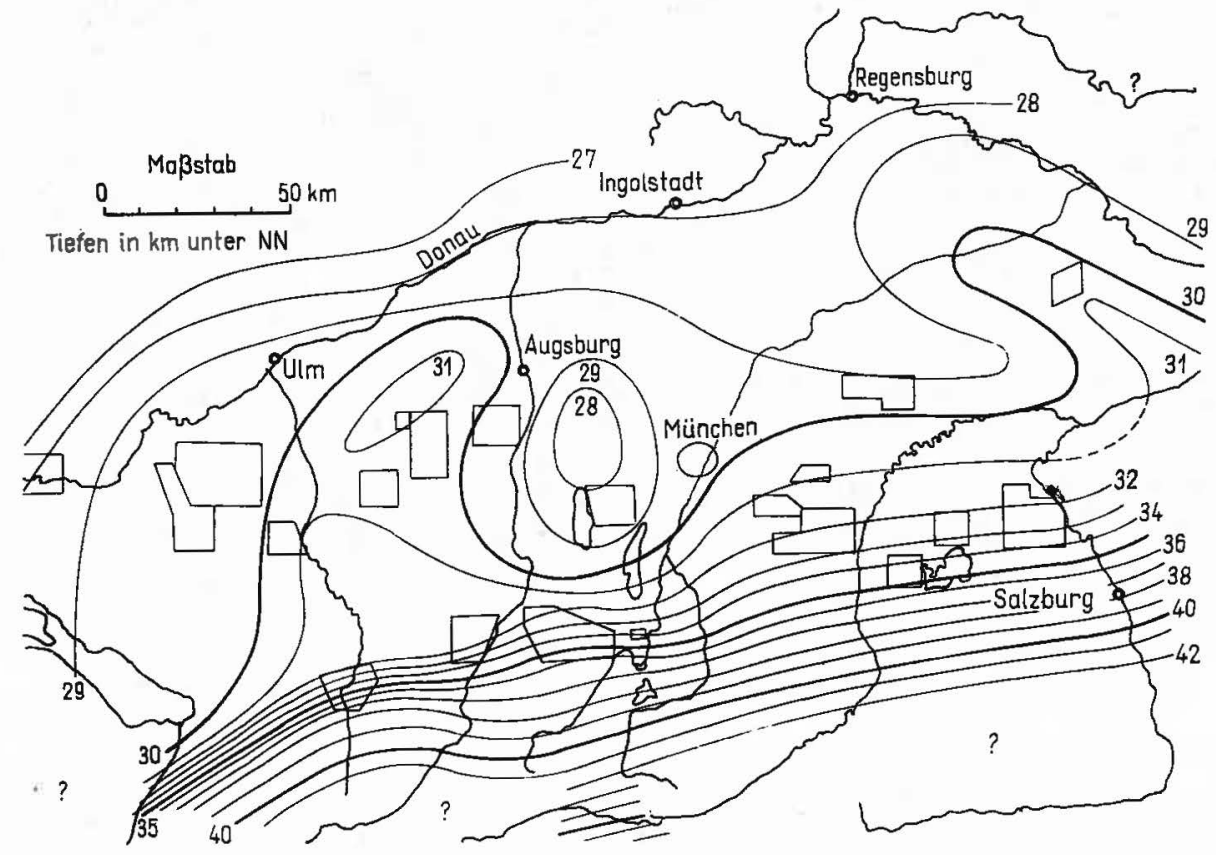

ABв. 5. Tiefenlinienplan für die M-Diskontinuität 

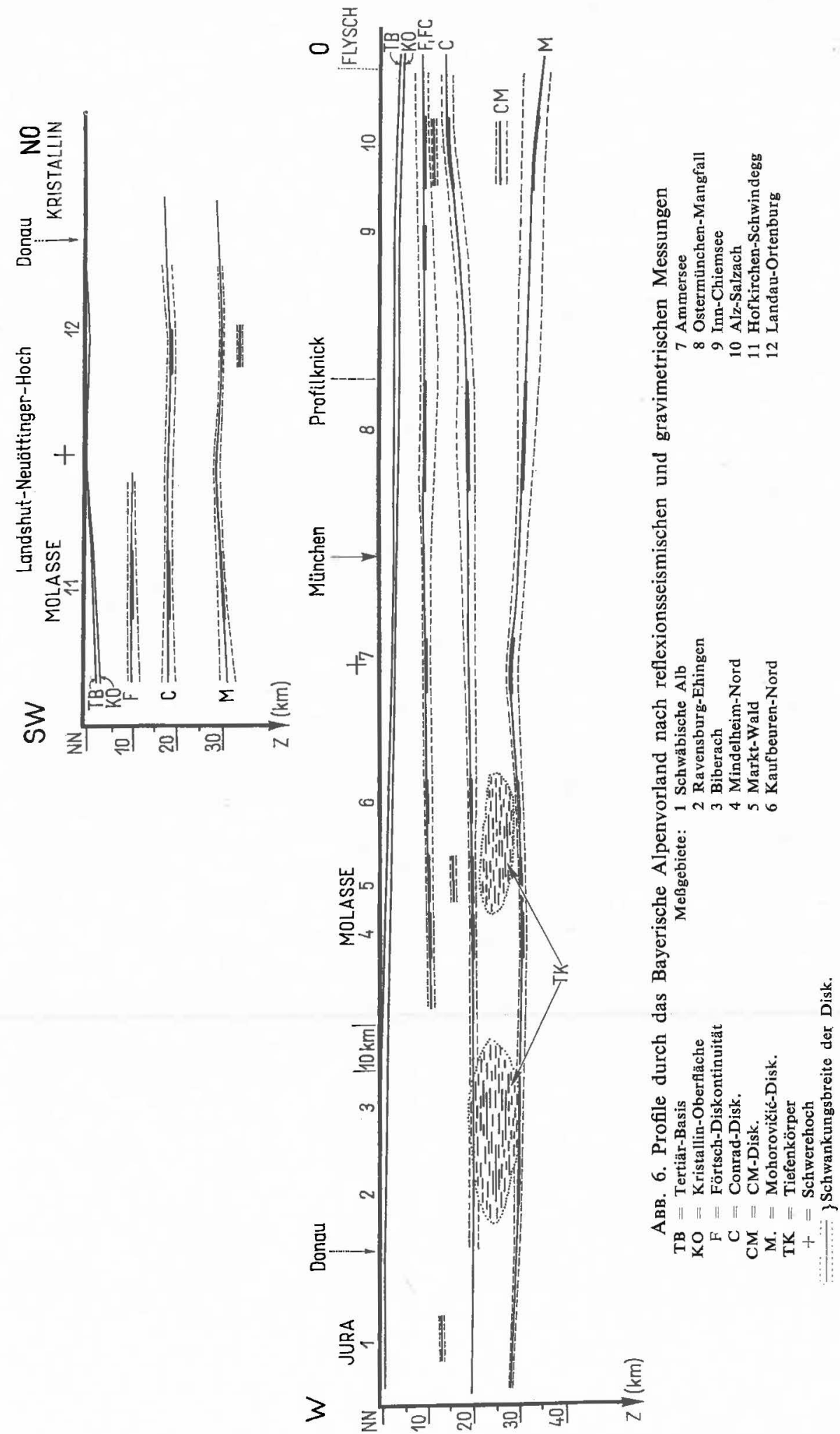
Die Hochachse im Tiefenlinienplan der Conrad-Diskontinuität weist mit ihrer höchsten Lokation auf ein Gebiet hin, in welchem ein ausgeprägtes magnetisches Hochgebiet liegt, nämlich den Raum Berchtesgaden-Salzburg. Die Möglichkeit, daß hier ein ursächlicher Zusammenhang zwischen der magnetischen Anomalie und einem Strukturbereich der Conrad-Diskontinuität vorliegt, ist nicht von der Hand zu weisen und auch von Liebscher diskutiert. Ähnliche Überlegungen werden uns auch im norddeutschen Raum wieder begegnen, und es scheint eine der Aufgaben der nahen Zukunft zu sein, das Beobachtungsmaterial zu diesem Fragenkomplex zu erweitern und Untersuchungen über die Deutung der großen magnetischen Anomalien, wie sie z. B. in Norddeutschland mehrfach seit langem bekannt sind, unter Zuhilfenahme der Seismik anzustellen.

Auf eine andere Besonderheit weist Liebscher in der Westmolasse hin. An einigen Stellen deuten die Anhäufungen von Reflexionen - zumeist im Bereich zwischen der Conrad- und der Moho-Diskontinuität - auf die Möglichkeit hin, hier sogenannte Tiefenkörper anzunehmen. Auch ein Zusammenhang z. B. mit den Vulkanvorkommen des Hegau und des Uracher Raumes erscheint denkbar. Der Wert solcher Hinweise liegt weniger in seinem zunächst noch problematischen wissenschaftlichen Gehalt als vielmehr in seiner Forderung für weitere zukünftige Untersuchungen. So kann man überhaupt sagen, daß die reflexionsseismischen Ergebnisse im Alpenvorland, welche wir derzeit als am besten durchgearbeitet ansehen können, neben den sehr schönen und weitreichenden Ergebnissen, wie der Kartierung der Conrad- und Moho-Diskontinuität, auch eine ganze Reihe von Fragen aufgeworfen haben, die weitere Untersuchungen nach sich ziehen werden, Arbeiten, die nicht zuletzt zu einer Kombination verschiedener Verfahren führen werden.

Im Raum östlich und südöstlich der Stadt Augsburg ist das sogenannte Augsburger Schwerehoch - eine positive Schwereanomalie von etwa 11 Milligal - seit langem Gegenstand der Diskussionen. Versuche, diese Anomalie mit einer Aufwölbung des varistischen Grundgebirges unter der ungefalteten Molasse zu deuten, lassen sich nach den seismischen Ergebnissen nicht halten. Ebenso führt die Annahme, das Schwerehoch durch den Ausfall der im ostbayrischen Molassebecken vorhandenen Kreide einerseits und das Einschalten leichterer mesozoischer Schichten in der Westmolasse andererseits zu deuten, nicht auf den tatsächlichen Betrag der Anomalie. Die Deutung Liebschers verbindet die Deutung des Augsburger Schwerehochs mit einer lokalen Aufwölbung der Moho-Diskontinuität. Sie kann sich dabei auf einige seismische Fixpunkte stützen, in welche sich die Berechnung des gravimetrischen Modells zwanglos einfügt.

Es wurde bereits erwähnt, daß die Conrad-Diskontinuität sich durch eine lange, aus dem Berchtesgadener-Salzburger Raum nach WNW vorspringende Hochachse auszeichnet. Betrachten wir die Moho-Diskontinuität, so fällt neben dem eben erwähnten Hoch bei Augsburg der kräftige Abfall unmittelbar am Alpenrand auf. Die Ergebnisse Liebschers stützen sich gerade hier im Gebiet der gefalteten Molasse auf qualitativ besonders gute Reflexionen. Diese Aussage erscheint bedeutsam, zumal sie vielen bisherigen Annahmen, die von einer kräfti- 
gen Einsenkung der Conrad-, nicht der Moho-Diskontinuität, am Alpenrand ausgingen, widerspricht.

In der Bearbeitung des Materials aus dem Alpenvorland fällt auf, daß in zahlreichen Gebieten zwischen den bereits genannten Unstetigkeitsflächen zum Teil recht deutliche Hinweise auf dazwischenliegende Diskontinuitäten existieren. Vor allem im Bereich zwischen der Conrad- und Moho-Diskontinuität zeigen die Häufigkeitsverteilungskurven mancher Arbeitsgebiete ein deutliches Zwischenmaximum, welches einer Inter-Diskontinuität zugeordnet werden muß. Eine ähnliche Erscheinung wird uns im norddeutschen Raum wieder begegnen. Überhaupt scheint sich der Bau des tieferen Untergrundes zwischen der Conrad- und MohoDiskontinuität im Alpenvorland von Westen nach Osten zu ändern. Je weiter wir nach Osten kommen, desto größer wird der Abstand zwischen den beiden Zonen, und die Ausprägung der Inter-Diskontinuitäten wird immer charakteristischer (Abb. 6). Zweifellos stehen wir hier vor einer Erscheinung, die Interesse und weitere Bearbeitung verdient, zumal wir auch in Norddeutschland ähnliche Beobachtungen gemacht haben. Die Frage, wann und wo Interdiskontinuitäten auftreten, sollte uns weiterhin beschäftigen - nicht zuletzt im Hinblick auf die von Bederke geäußerten Gedankengänge.

Als weiteres Arbeitsgebiet bot sich der Raum des Oberrheintalgrabens an, in welchem schon in den Jahren 1955 und 1956 tiefe Reflexionen beobachtet worden waren. Im Gegensatz zum Alpenvorland ist hier die geophysikalische Prospektionstätigkeit nahezu zum Erliegen gekommen, so daß sich die Ausarbeitungen auf frühere Registrierungen stützen mußten. Von Demnati sind aus Arbeitsgebieten im mittleren Oberrheintalgraben, etwa im Gebiet zwischen Durmersheim (nördlich Rastatt) und Kehl annähernd 700 Seismogramme neu bearbeitet worden. Die Auswertung bestätigte die bereits früher in einem Teil dieses Materials beobachteten Anhäufungen von z. T. guten Reflexionen bei etwa 6,5 bis $9 \mathrm{sec}$ Reflexionslaufzeit. Die statistische Bearbeitung und die Aufstellung von Häufigkeitsverteilungskurven zeigt recht ausgeprägt eine Reflexionsgruppe bei etwa 6,5-7 sec und eine spätere, schwächere Gruppe bei ca. $9 \mathrm{sec}$ Reflexionszeit. In der nachfolgenden Abbildung (Abb. 7) sind die Verteilungskurven aus 5 Teilgebieten dargestellt, welche in dem genannten Teil des Rheintalgrabens von Nord nach Süd angeordnet sind. Hier ist besonders bemerkenswert, daß sich das recht scharf ausgeprägte Maximum in den Kurven von Nord nach Süd systematisch zu kürzeren Laufzeiten verlagert, um in der letzten Kurve wieder eine längere Reflexionszeit aufzuzeigen. Wir erkennen also aus dieser Darstellung bereits ein Kurzzeitgebiet für den Bereich dieser Reflexionsgruppen in dem vorletzten Teilgebiet. Betrachten wir dazu nun die Karte der Bouguerschen Schwerestörungen (Abb. 8), so erkennt man genau an dieser Stelle auch ein Schwerehoch von ca. 4 Milligal. Diese Übereinstimmung ist sehr bemerkenswert, und es fragt sich nun, ob die Ursache in einer regionalen Hochlage des tiefen Untergrundes oder in einer wechselnden Sedimentationsmächtigkeit in der Grabenzone selbst liegt. Wir wissen aus den Ergebnissen der Prospektionsseismik, daß die Tertiärfüllung des Rheintalgrabens von Norden her sehr schnell abnimmt und daß wir etwa im Raum von Kork- 

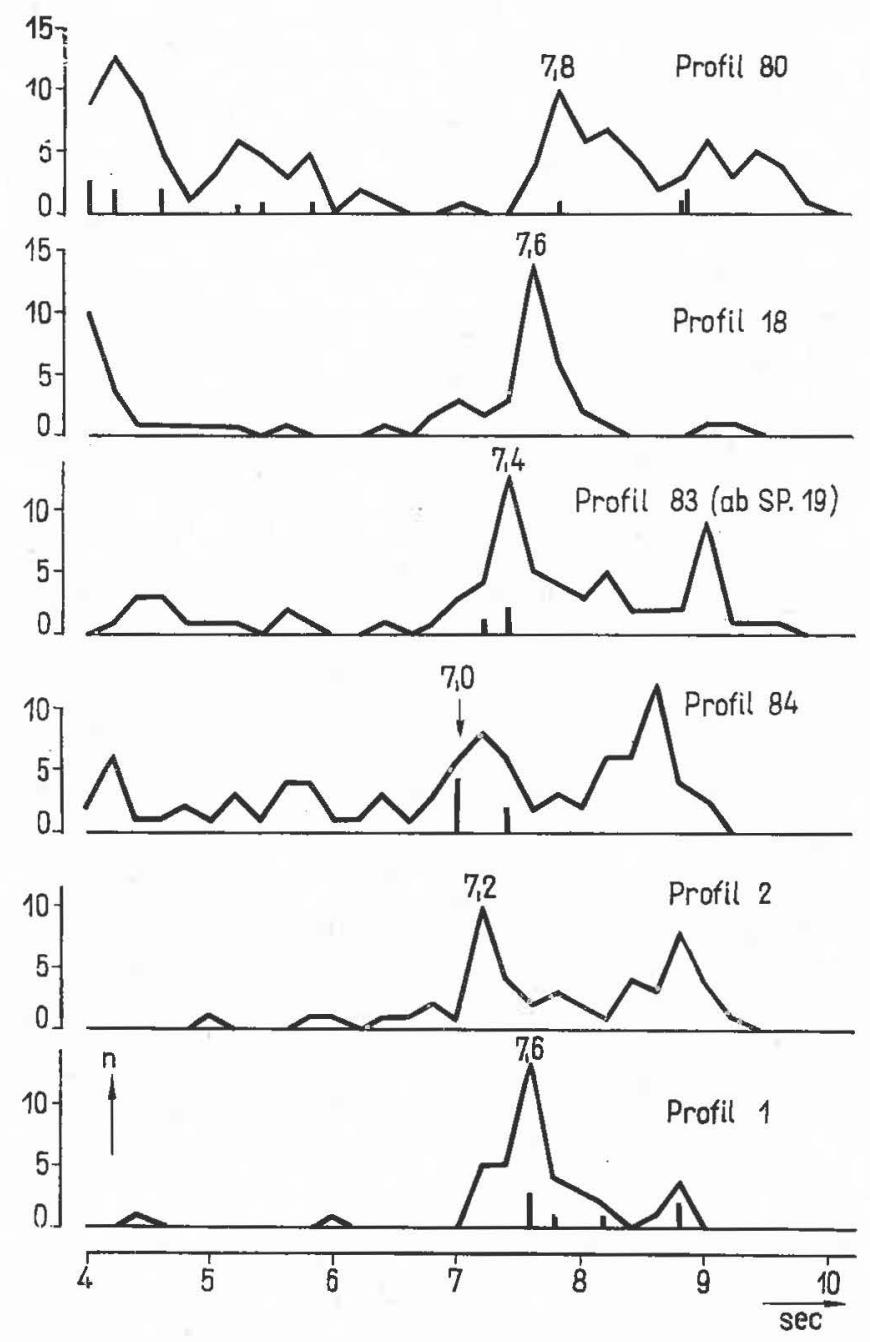

AвB. 7

Kehl mit einer relativ geringen Teufenlage des Mesozoikums und des paläozoischen Untergrundes zu rechnen haben. Somit könnte das Kurzzeitgebiet und die Schwereanomalie in diesem Falle ihre Ursache in der wechselnden Sedimentfüllung des Grabens selbst haben. Andererseits führt eine Teufenberechnung für die Reflexionen der Conrad-Diskontinuität unter Berücksichtigung dieser wechselnden Sedimentfüllung und Anlehnung an die Geschwindigkeitsmessungen in den Tiefbohrungen Weitenung und Kork tatsächlich zu einer Hochlage der Conrad-Diskontinuität in diesem Gebiet. Bereits früher war auf Grund der Beobachtungen von Reflexionsgruppen auf West-Ost verlaufenden Profilen nahe des Grabenrandes 


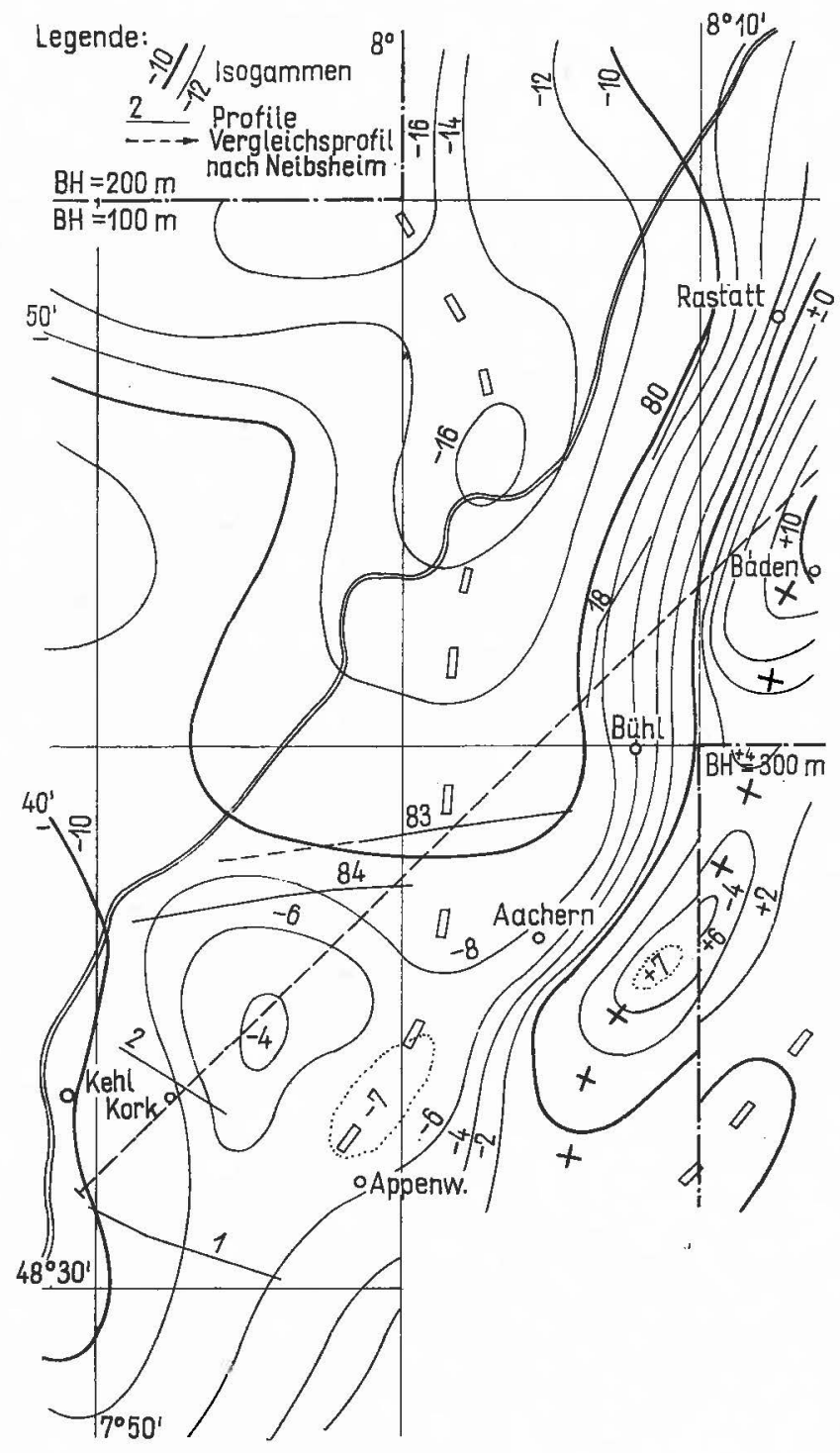

Aвв. 8. Lageplan mit Bouguer-Anomalie (Schleusener u. Closs) Maßstab $1: 200000$

und im Vergleich zu den Ergebnissen von Schulz in der Pfalz die Frage aufgeworfen, ob der Oberrheintalgraben auch einer Einsenkung etwa im Bereich der Conrad-Diskontinuität korrespondiert. Da mit Ausnahme der Beobachtungen von Schulz im Gebiet der Nahe-Senke seismische Messungen außerhalb der 
eigentlichen Grabenzone fehlen und auch in absehbarer Zeit mit derartigen Arbeiten nicht zu rechnen ist, wurde im Oktober des Jahres $1962 \mathrm{im}$ Gebiet des Kraichgaues, etwa nördlich der Stadt Bretten, ein kurzes seismisches Meßprogramm durchgeführt. Es war das erste Mal, daß seitens der deutschen Forschungsgemeinschaft Gelder für gezielte, zusätzliche reflexionsseismische Messungen bereitgestellt wurden. Zum Einsatz kamen Bohrgeräte der Firma W. Thiele, Celle und der Seismos GmbH., Hannover. Registriert wurde mit der SIE-Apparatur des Geophysikalischen Institutes der Bergakademie Clausthal. In Anbetracht der nur begrenzten finanziellen Mittel und der schwierigen Bohrverhältnisse konnten nur 9 Schußpunkte abgetan werden, welche sich auf zwei sich kreuzenden Profilstücken anordnen.

Zweifellos ist der Kraichgau als eine morphologische und geologische Senke nicht ideal für ein derartiges Versuchsprogramm, er bot sich jedoch zunächst wegen der leichten Gangbarkeit im Gegensatz zum Schwarzwald oder Odenwald an.

Auf 8 der neun Schußpunkte konnten Tiefenreflexionen mit Laufzeiten 5,2-5,4 sec sowie bei 7 und $9 \mathrm{sec}$ registriert werden. Soweit sich aus den relativ kurzen Profilstücken eine solche Aussage mit hinreichender Sicherheit machen läßt, zeigen alle Reflexionsgruppen einen leichten Einfall nach SW, d. h. etwa in Richtung auf den Oberrheintalgraben.

Es liegt nun nahe, auch einen Teufenvergleich der bildmäßig vermutlich zusammengehörenden Reflexionsgruppen anzustellen. Für die Reflexionen der Grabenzone, die mit einer Laufzeit von 7-8 sec die oben erwähnten charakteristischen Maxima der Verteilungskurven erzeugen, kommt Demnati zu einer Teufe von 18,5-20,5 km, für die Gruppen bei ca. 9 sec ergibt sich eine wahre Teufe von etwa $23-25 \mathrm{~km}$.

Nimmt man nun an - und das Bild der Reflexionen legt diese Deutung nahe-, daß die Reflexionen von $7 \mathrm{sec}$ im Rheintal denen bei 5,2-5,4 sec im Kraichgau entsprechen, so liegen letztere unter plausiblen Geschwindigkeitsannahmen (T. B. Neibsheim) bei etwa $14,8 \mathrm{~km}$ Teufe. Diese Zuordnung würde bedeuten, daß die Conrad-Diskontinuität, welcher die genannten Refiexionsgruppen sehr wahrscheinlich zugeordnet werden müssen, außerhalb des Rheintalgrabens im Gebiet des Kraichgaues, etwa 4-5 km höher liegt. Diese Vorstellung und Zuordnung der fraglichen Reflexionen würde dem angedeuteten Einfallen durchaus entsprechen. Demnati hat darüber hinaus versucht, diese Deutungen mit dem gravimetrischen Bild einer schiefen Stufe zu vergleichen. Er findet unter Annahme plausibler Dichten und Verwendung der aus der Reflexionsseismik gewonnenen Teufenangaben eine gute Übereinstimmung der theoretischen und beobachteten Werte, die lediglich innerhalb der Grabenzone etwas differieren. Dies dürfte angesichts der nicht genau bekannten und in ihren Dichtewerten ebenfalls nicht sehr sicheren sedimentären Grabenfülllung begründet sein.

Zieht man abschließend in die Betrachtungen noch die Ergebnisse von Schulz aus dem Gebiet der Nahe-Senke ein, so ergibt sich im Großen das Bild einer schwach durchgewölbten Conrad-Diskontinuität unter dem Rheintalgraben (Abb. 
9). Diese Vorstellung ist in der nachfolgenden Abbildung dargestellt. Wenngleich alle Einzelbeobachtungen vorerst noch mit einer gewissen Vorsicht - soweit es die Interpretation der Meßergebnisse anbelangt - betrachtet werden müssen, so ist doch auffällig, daß sich alle Deutungen zwanglos in das hier gegebene Bild einer Depression der Conrad-Diskontinuität unter der Zone des Oberrheintalgrabens einfügen.

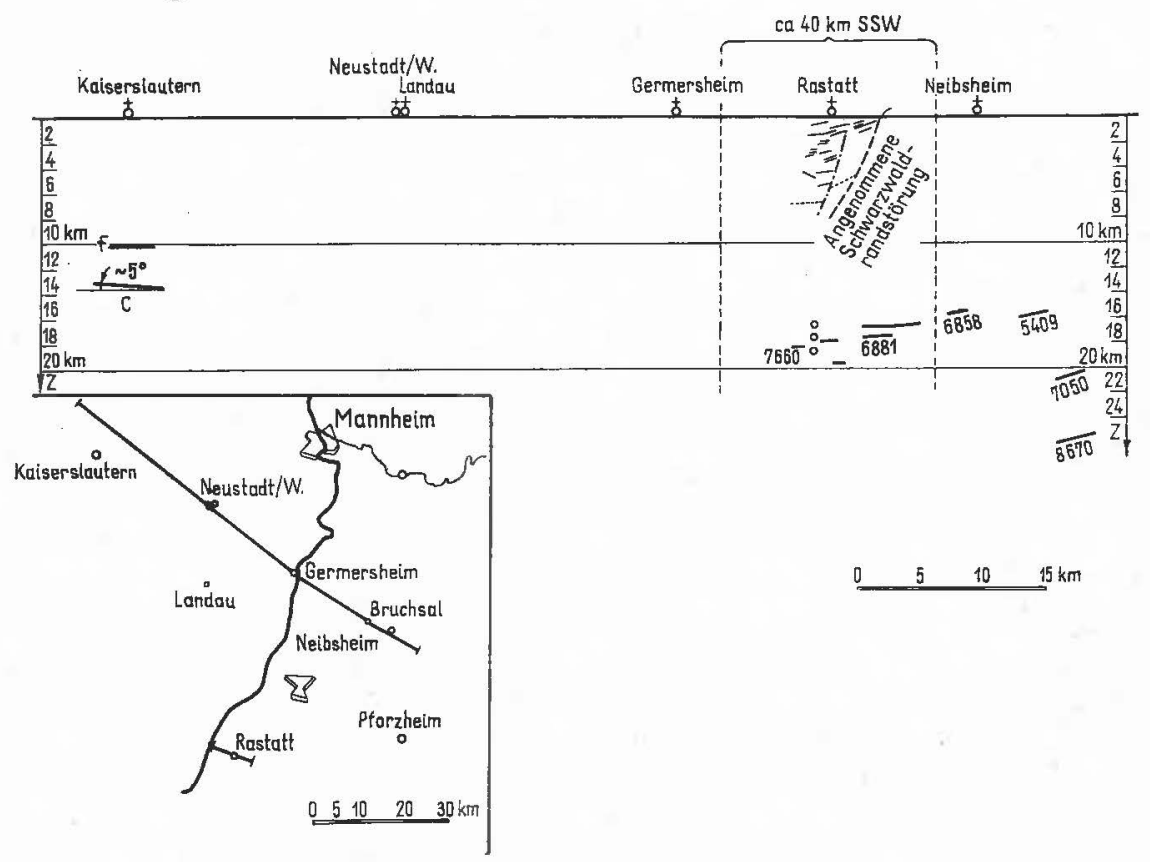

Авв. 9. Tiefenseismische Ergebnisse im Bereich des Oberrheintalgrabens

In Norddeutschland sind die Bearbeitungen der Tiefenreflexionen noch im Gange. Dies liegt einmal begründet in der Größe des Gebietes, zum anderen aber in den besonderen Problemen, welche sich hier der Erlangung schneller zusammenfassender Resultate entgegenstellen. Die Aufnahmen von Tiefenreflexionen konzentrierten sich im wesentlichen auf den Südrand des nordwestdeutschen Beckens unter Einschluß des Münsterlandes sowie auf den Raum von Osthannover und Schleswig-Holstein. Es handelt sich hier fast durchweg um Randzonen des Beckens, nämlich Gebiete, in denen eine nicht allzu mächtige Sedimentfüllung zunächst energetisch bessere Voraussetzungen bot als in jenen Gebieten, in denen wir bereits die Zechsteinformation in fast $5000 \mathrm{~m}$ Teufe zu suchen haben. Wesentlicher aber hat sich das Auftreten von multiplen Reflexionen in diesen Gebieten erwiesen. Die Seismogramme zeigen bis zu langen Laufzeiten zahlreiche Reflexionen, die in der Mehrzahl als Mehrfachreflexionen nachzuweisen sind und die reellen - wahrscheinlich ebenfalls vorhandenen Reflexe - völlig verdecken. Dennoch ist es zu früh, diese Meßunterlagen als unbrauchbar zu bezeichnen. Eine detaillier- 


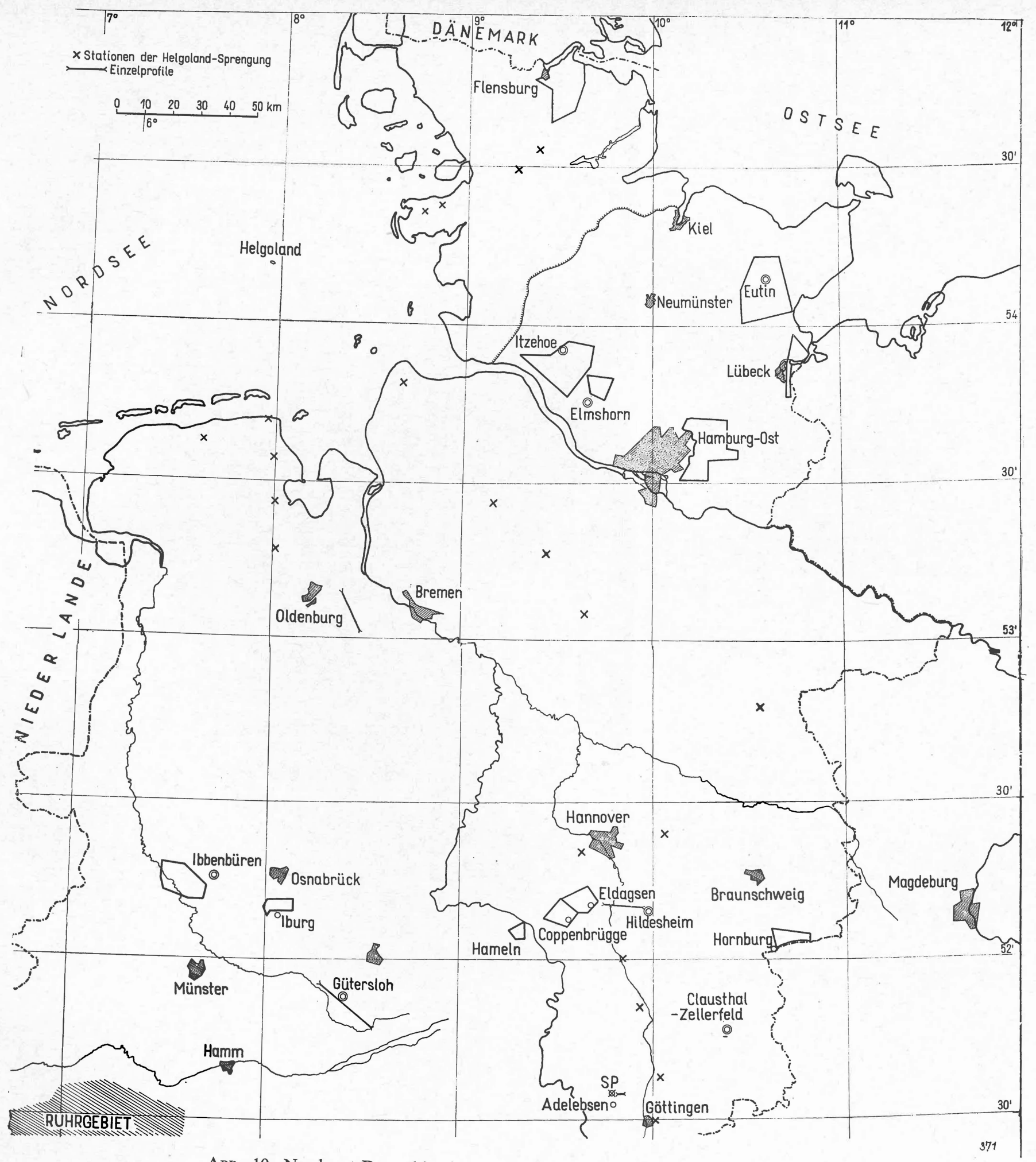

Авв. 10. Nordwest-Deutschland. Gebiete mit beobachteten Tiefenreflexionen 
tere Betrachtung könnte m. E. auch hier noch zusätzliche wertvolle Aussagen bringen.

Die erste umfassende Sichtung des Materials - mehr als 3000 Seismogramme ist von Hehn im Institut für Geophysik der T. H. Clausthal durchgeführt worden. Die Arbeiten werden z. Zt. von Herrn Hadjebi im Institut für Physik des festen

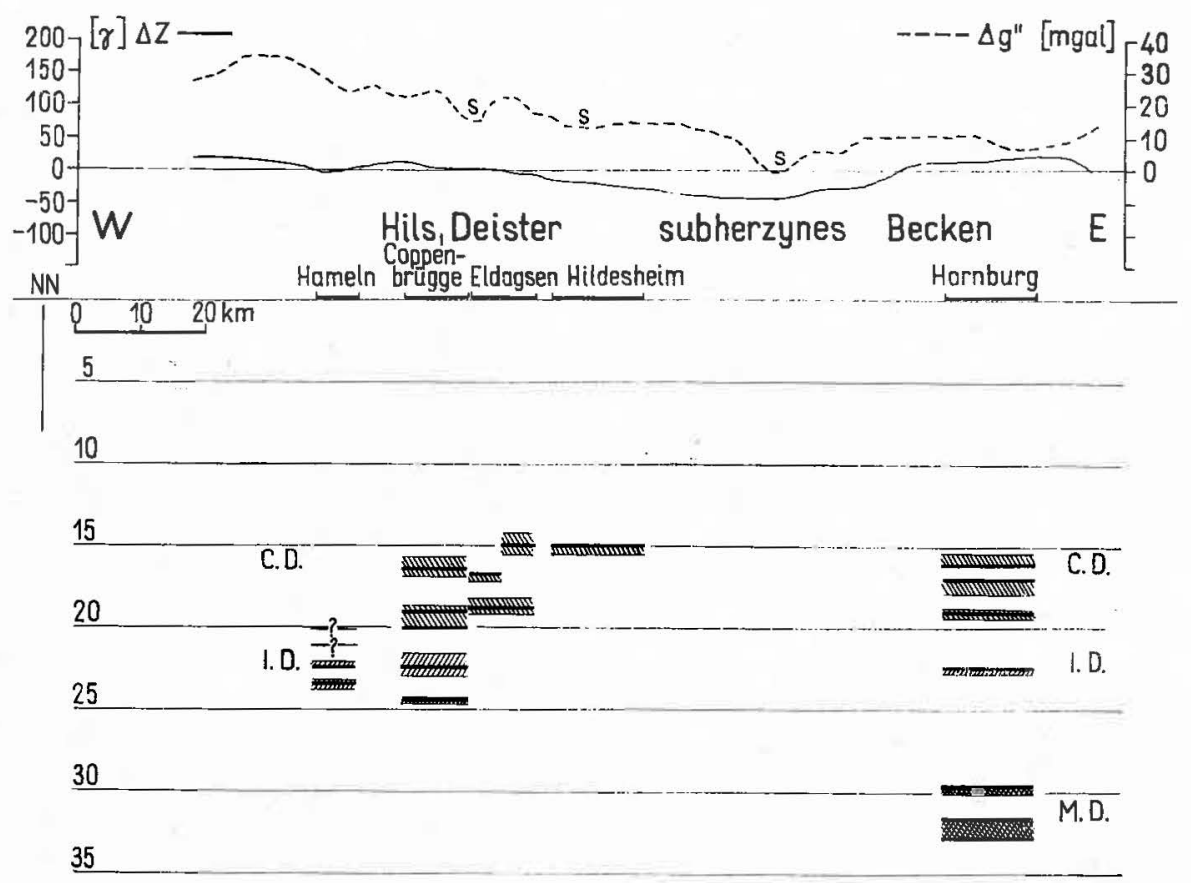

Aвb. 11. Profil A

Erdkörpers der Universität Hamburg unter Konzentration auf den Südrand des nordwestdeutschen Beckens fortgesetzt.

Tiefenreflexionen wurden in folgenden Arbeitsgebieten registriert und ausgewertet:

Flensburg, Eutin, Lübeck, Itzehoe, Elmshorn, Hamburg-Ost, Oldenburg, Ibbenbüren, Iburg, Gütersloh, Hameln, Eldagsen, Coppenbrügge, Hildesheim, Hornburg.

In der Abb. 10 ist die Lage dieser Untersuchungsgebiete dargestellt.

Bei der Auswertung kam wieder das Prinzip der Darstellungen in Häufigkeitsverteilungen zur Ausführung. Daneben sind die Ergebnisse nach Umrechnung in Teufen in Form von Profilschnitten über jeweils 3 bis 4 Arbeitsgebiete dargestellt. Die Abb. 11-14 zeigen diese aus den Häufigkeitsverteilungskurven abgeleiteten Profilschnitte. Wie man aus den Darstellungen erkennt, ist in fast allen Arbeitsgebieten der Bereich der vermutlichen Conrad-Diskontinuität sowie die Moho-Diskontinuität erfaßt worden. Dabei ist bemerkenswert, daß in vielen 

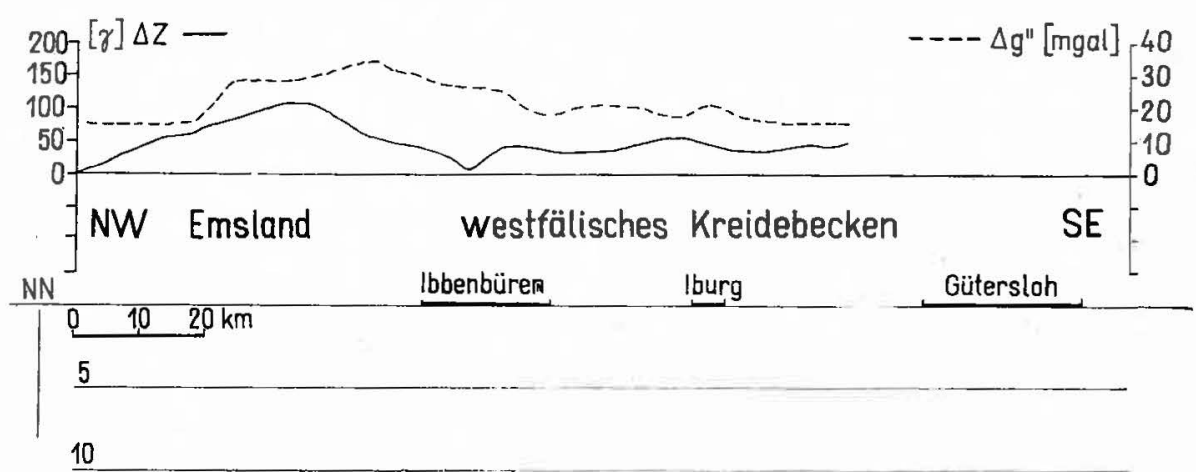

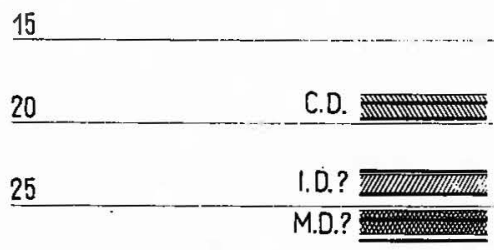

30

$\underline{35}$

AвB. 12. Profil B

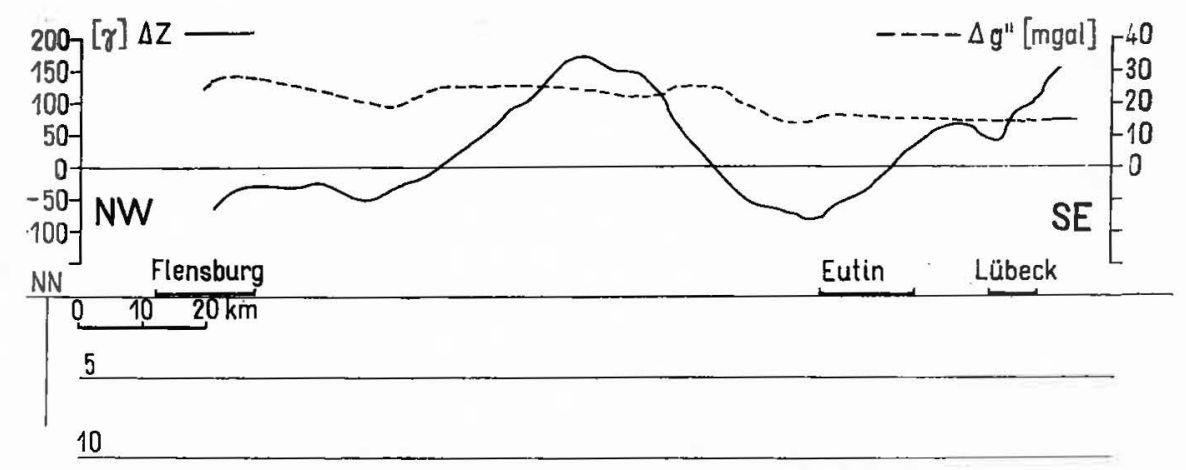

15 C.D.

C.D.

20

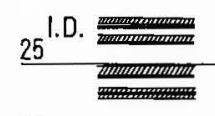

$\underline{30}$

$\underline{35}$ M.D.

Авв. 13. Profil C 
Fällen nicht etwa ein, sondern mehrere Maxima im Bereich der Diskontinuitäten — vor allem aber der Conrad-Diskontinuität - beobachtet wurden. Dies kommt auch in den profilmäßigen Darstellungen zum Ausdruck, welche vielfach einen beträchtlichen Teufenbereich für die Diskontinuitäten aufzeigen.
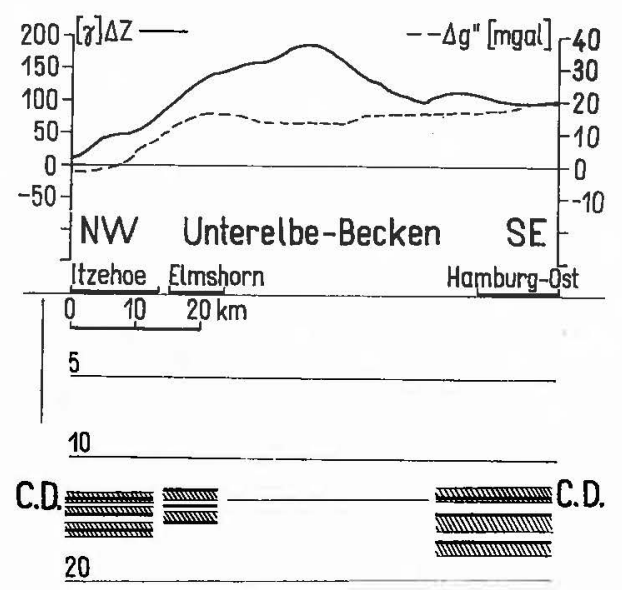

$\underline{25}$

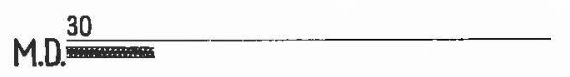

35

Aвв. 14. Profil D

Trotz relativ guter Ergebnisse gerade am Südrand des norddeutschen Beckens treten bei der Korrelation der einzelnen Maxima der Verteilungskurven vielfach besondere Probleme auf. So ist beispielsweise in den Gebieten Springe/Eldagsen und Coppenbrügge eine recht deutliche Ablösung der Maxima aus dem C-Bereich zu erkennen dergestalt, daß diese Maxima der Häufigkeitsverteilungen von einer bestimmten Linie ab plötzlich um ca. 1/2 sec nach längeren Laufzeiten zu verschoben erscheinen. Ob hier ein tatsächlicher Versatz in den tiefen Horizontgruppen vorliegt oder ob eine verschiedenartige fazielle Ausbildung einzelner Schichtpakete innerhalb einer breiteren Übergangszone eine Veränderung der Reflexionsbilder bewirkt, kann vorläufig nicht entschieden werden. Die zweite Deutung erscheint jedoch zunächst plausibler.

Ebenso ist eine endgültige Deutung der scharfen Maxima in den Verteilungskurven des Gebietes Ibbenbüren (Abb. 15) noch nicht sicher. Es könnte sich bei dem späteren Maximum um eine Hochlage im Bereich der Moho handeln, oder aber wir müßten auch dieses Reflexionsband einer Zwischendiskontinuität zuordnen. Es ist zu hoffen, daß solche Fragen bei einer Verdichtung der Messungen geklärt werden können. Das vordere Maximum der Ibbenbürener Kurve läßt 


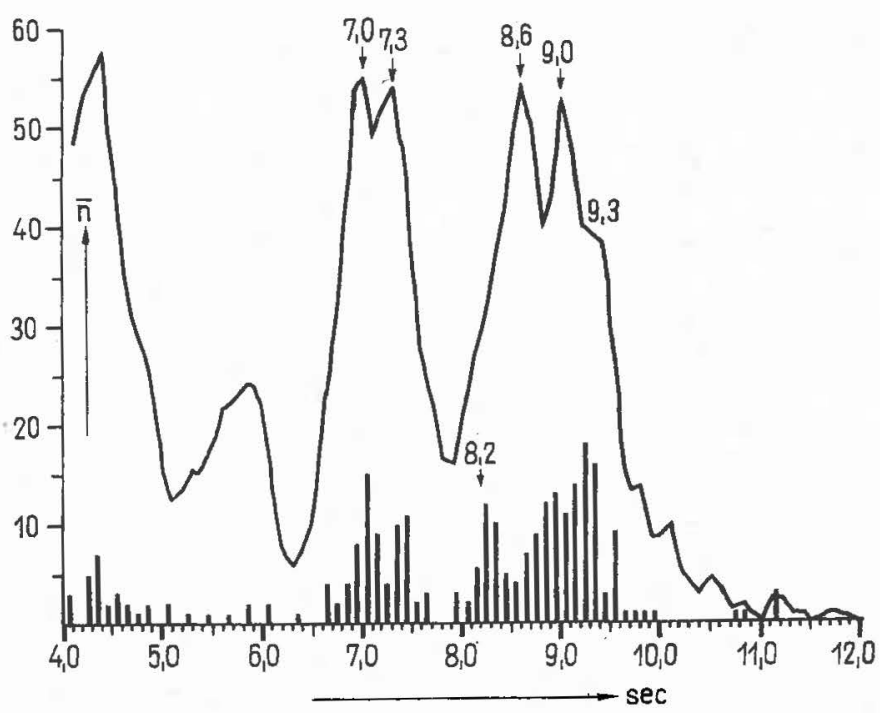

AвB. 15. Ibbenbüren

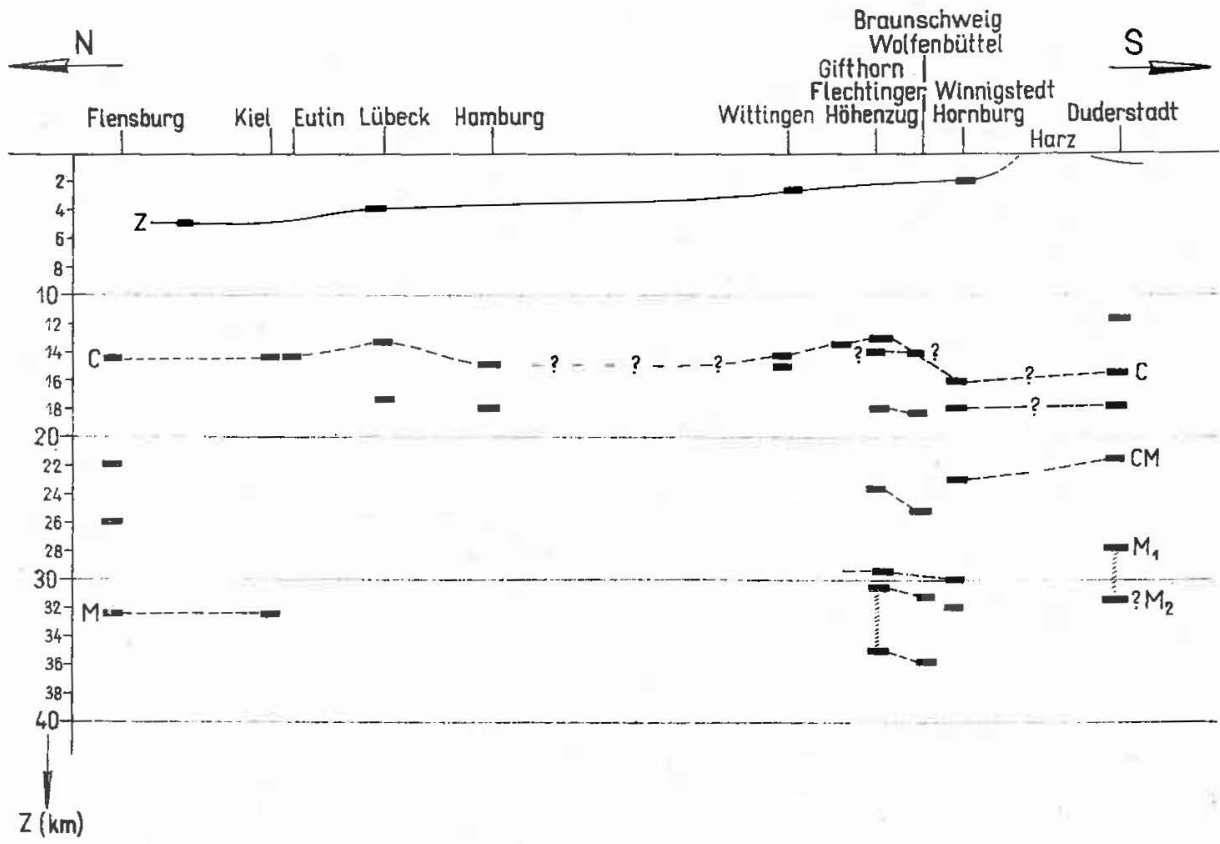

Авв. 16. Profilschnitt Flensburg-Harz nach Beobachtungen von Tiefenreflexionen Maßstab: Horizontal 1:1000 000

Maßstab: Vertikal 1:200 000

(5-fach überhöht) 
sich zwanglos dem Bereich der sogenannten Conrad-Diskontinuität zuordnen und fügt sich relativ gut in die aus den Gebieten Iburg und Gütersloh ermittelten Teufen ein. Aus diesen 3 Arbeitsgebieten läßt sich ein flaches Einfallen der ConradDiskontinuität nach Südwesten ableiten.

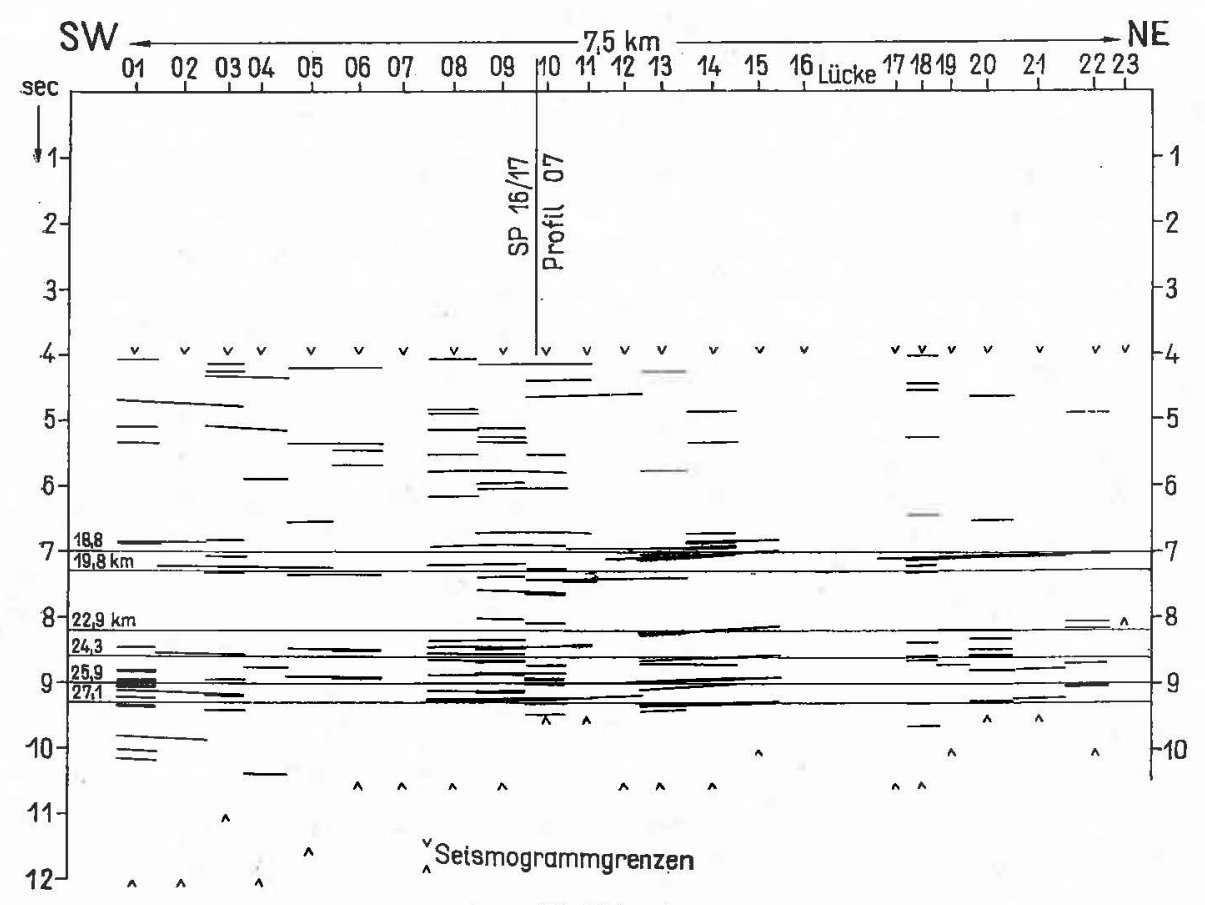

Авв. 17. Ibbenbüren Lotzeit-Profil

05

Obgleich das Netz der Beobachtungsgebiete noch relativ weitmaschig ist, lassen sich doch schon gewisse Aussagen über den Verlauf der tieferen Grenzflächen in Norddeutschland machen. So gibt Abb. 16 einen Profilschnitt aus dem Raum Flensburg bis in das Gebiet von Göttingen-Duderstadt wieder, in welchem bei 5-facher Überhöhung die Anhäufungen von Tiefenreflexionen durch schwarze Striche markiert sind. Auffällig ist hierbei eine mögliche Bewegung (Hochlage) im Bereich der Conrad-Diskontinuität in der Nähe des Flechtinger Höhenzuges. Dieses Ergebnis ist jedoch zunächst mit großer Vorsicht aufzufassen, und erst weitere Messungen in unmittelbarer Nähe werden eine weitere Diskussion dieser hier aufgeworfenen Möglichkeit erlauben.

Im Herbst des Jahres 1963 ist von der Erdölindustrie im Raum Osthannover ein großräumiges Refraktionsprogramm zur Untersuchung präpermischer Schichten durchgeführt worden. Die starken Schüsse dieser Messungen sind auf einer Profillinie, welche etwa aus dem Raum von Wittingen bis in die Gegend von 
Seesen verläuft, von der Reflexionsapparatur des Institutes für Geophysik der Bergakademie Clausthal zwecks Aufnahme von Tiefenreflexionen mitregistriert worden. Eine Ausarbeitung der Ergebnisse - es wurden durchweg zufriedenstellende bis gute Tiefenreflexionen registriert - ist im Institut in Vorbereitung. Gerade diese Messungen dürften von besonderem Interesse sein, da das Profil die oben erwähnte Linie des Flechtinger Höhenzuges überquert und möglicherweise durch diese Ergebnisse die Frage einer lokalen Aufwölbung oder Bewegung im Bereich der Conrad-Diskontinuität geklärt werden kann.

$\mathrm{Zu}$ bemerken ist, daß in vielen Arbeitsgebieten zwischen den bekannten Diskontinuitäten Reflexionsflächen mit zum Teil recht guten Reflexionen beobachtet wurden. Solche Zwischendiskontinuitäten sind bekanntlich auch von Liebscher im Alpenvorland und hier vornehmlich im ostbayerischen Raum beobachtet worden. Es wäre zweifellos für die Zukunft von Interesse, die räumliche Verteilung solcher Zwischendiskontinuitäten zu verfolgen und in Profilschnitten den Übergang von Gebieten ohne Zwischendiskontinuitäten in solche mit Zwischendiskontinuitäten $\mathrm{zu}$ untersuchen.

Ưber die von Hehn in seiner Arbeit dargestellten Meßgebiete hinaus ist in einer Anzahl weiterer Arbeitsräume die Registrierung von Langseismogrammen durchgeführt worden. In einem Teil dieser Messungen sind die Filme bis zu langen Laufzeiten derartig stark von Mehrfachreflexionen überlagert, daß die statistische Auslese von reellen Reflexionsgruppen außerordentlich erschwert, wenn nicht gar unmöglich gemacht wird. Dies trifft vor allem auf die Meßgebiete in Ostfriesland, Westholstein und den Raum von Hamburg zu.

Wie in Süddeutschland, so sind auch im norddeutschen Raum profilmäßige Darstellungen der Meßergebnisse versucht worden. Als Beispiel für die Darstellung von Laufzeitprofilen ist ein Profil mit guten Tiefenreflexionen aus dem Raum Ibbenbüren wiedergegeben (Abb. 17). Bereits im vergangenen Jahre war ein längeres Profil mit Tiefenreflexionen - vermutlich aus dem Bereich der Conrad-Diskontinuität - aus den Messungen im Gebiet Gütersloh gezeigt worden (Abb.18).

Diese Beispiele zeigen, daß es durchaus zweckmäßig und erfolgversprechend ist, auf längeren reflexionsseismischen Profilen in konventioneller Form Reflexionen aus großen Tiefen zu registrieren. In fast allen Gebieten, in denen die Registrierung von Langseismogrammen durchgeführt wurde, sind auch Reflexionen aus großen Tiefen beobachtet worden. Wenn in einzelnen Fällen die Versuche nicht erfolgreich verliefen, so lag dies zumeist an anderen Begleitumständen, wie z. B. an der Überlagerung durch multiple Reflexionen in Norddeutschland oder an topographischen Verhältnissen, wie z. B. Gebieten mit extrem ungünstigen Oberflächenbedingungen (etwa bestimmte Moränengebiete Nord- oder Süddeutschlands). Wenn auch die Erprobung neuer Registrier- und Aufnahmeverfahren - wie z. B. die Beobachtung von Weitwinkelreflexionen oder das Verfahren der Mehrfachüberdeckung - für die Weiterentwicklung der Tiefenseismik unbedingt notwendig ist, so scheint mir zum gegenwärtigen Zeitpunkt das konventionelle Verfahren bei hinreichender Planung und Sorgfallt in der Ausführung durchaus geeignet, die Untersuchung der tieferen Stockwerke mit reflexionsseismischen 


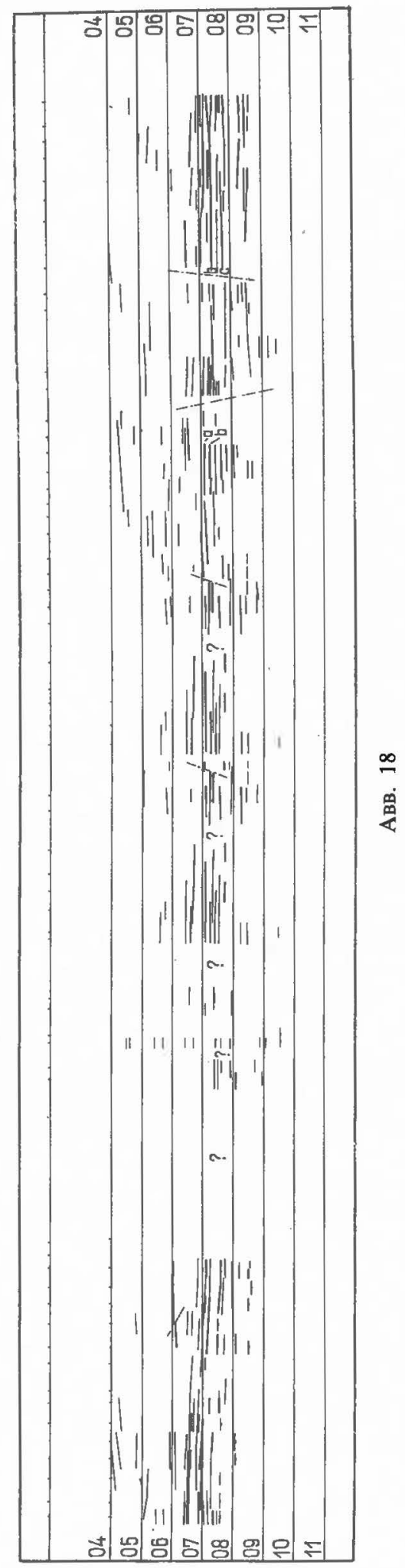


Methoden weiterzuführen und spezielle Probleme anzugehen. Es ist jedoch einleuchtend, daß die Aussagekraft reflexionsseismischer Profile für tiefe Grenzflächen mit der Länge der Meßlinien wächst. Es dürfte wenig Zweck haben, etwa ein reflexionsseismisches Meßprogramm mit Profillängen von 20 oder $30 \mathrm{~km}$ Länge zu planen. Unseres Erachtens ist nur die Anlage von Profillinien von wenigstens $100 \mathrm{~km}$ Länge sinnvoll.

Vergleicht man die Teufen der Reflexionsgruppen, welche im norddeutschen Raum der Conrad-Diskontinuität zugeordnet wurden, mit den Ergebnissen und Deutungen der Helgolandsprengung, so ergibt sich hier ein merklicher Widerspruch. Die von Hehn und anderen Bearbeitern für die vermutliche ConradDiskontinuität in Norddeutschland angegebenen Werte liegen zwischen etwa 14 und $19 \mathrm{~km}$. Diese Teufenwerte sind durchweg $3-4 \mathrm{~km}$ größer als die Ergebnisse der Auswertung der Helgolandsprengung. Nach den Beobachtungen in Süddeutschland - man siehe hierzu die Ausführungen von Liebscher und Giese - liegen erfahrungsgemäß die Teufenangaben aus reflexionsseismischen Beobachtungen um 1,5-3 km höher als die Werte aus refraktionsseismischen Messungen. Ein Ergebnis, das in gutem Einklang mit der z. B. von Giese dargelegten Vorstellung ist, daß im oberen Kristallin (und entsprechend möglicherweise im Bereich der großen Diskontinuitäten) ein Geschwindigkeitsgradient vorhanden ist, der die Refraktionswelle mehr oder weniger tief in das Medium mit der höheren Geschwindigkeit eintauchen läßt. Wenn man diese Teufenvariation zwischen beiden Meßprinzipien zuläßt, so steht die für die Teufen der Conrad-Diskontinuität in Norddeutschland angegebene Differenz gerade im umgekehrten Verhältnis, und der Widerspruch zwischen beiden Werten wird noch größer. Es ist zur Zeit noch nicht möglich, eine Erklärung für diesen Widerspruch zu geben. Wenig wahrscheinlich ist, daß die für die Teufenbestimmung der Reflexionen verwendeten Werte einen solch großen Fehler aufweisen. Die größte Fehlerquelle - nämlich der Einfluß der sedimentären Schichten bis zum Zechstein - wurde durch Verwendung der aus der Prospektionsseismik bekannten Durchschnittsgeschwindigkeiten bis zum tiefen Zechstein ausgeschaltet. Darunter wurde mit den Bestwerten der aus verschiedenen Refraktionsmessungen bekannten Intervallgeschwindigkeiten gerechnet. Rechnet $\mathrm{x}$ an dabei mit einem maximalen Fehler von $\pm 5 \%$, so bedeutet dies eine Unsicherheit in der Teufenabschätzung von höchstens $\pm 1 \mathrm{~km}$, ein Wert, der wesentlich unterhalb der genannten Differenz liegt.

Es muß bei der Diskussion dieser Fragen betont werden, daß die angegebenen Werte aus Reflexionsmessungen nicht in Gebieten gewonnen wurden, welche genau auf der Profillinie der Beobachtungsstationen der Helgolandsprengung liegen. Hier könnte eine natürliche Differenz begründet sein. Wesentlicher scheint jedoch die Frage, ob bei beiden Auswertungen wirklich die gleichen Unstetigkeitsflächen erfaßt wurden. Mit anderen Worten: Wir wissen noch nicht, ob wir in Norddeutschland die Zuordnung der Reflexions- bzw. der Refraktionseinsätze zu der Conrad-Diskontinuität als richtig ansehen dürfen. Es muß allerdings vermerkt werden, daß bei der Auswertung der Helgolandsprengung 
keine Gegenschußbeobachtungen zur Verfügung standen. Dies scheint vor allem im Hinblick auf die vielfach beobachteten Inter-Diskontinuitäten für die Ausdeutung gefährlich.

Die für die Moho-Diskontinuität bestimmten Teufenwerte fügen sich allgemein in die bisherige Vorstellung gut ein. Nach den bisherigen Ergebnissen sind mit Sicherheit noch keine größeren Bewegungen innerhalb der C- und M-Diskontinuität nachzuweisen. Dies besagt nichts über die tatsächlichen Verhältnisse. Die Beobachtungen in verschiedenen Gebieten, wie Ibbenbüren, Iburg, Gütersloh und Osthannover legen sehr wohl den Gedanken an großräumige Bewegungen in den tiefen Grenzflächen nahe. Es ist jedoch eine Aufgabe der Zukunft, durch weitere Beobachtungen und Verdichtung des Beobachtungsnetzes diese Fragen weiter zu untersuchen. Es wäre sehr wünschenswert, wenn zur detaillierteren Untersuchung lange Reflexionsprofile geschossen werden könnten, dort, wo besonders wesentliche Bewegungen in den tieferen Diskontinuitäten vermutet werden.

Angesichts der Größe des norddeutschen Raumes und der komplizierten Tektonik muß die Bearbeitung von Tiefenreflexionen noch als im Anfangsstadium stehend angesehen werden. Die umfangreiche und sorgfältige Bearbeitung von Hehn ist ein erster Schritt zur Sichtung des Materials, welche bereits jetzt eine Reihe von Fragen und Problemen aufgeworfen hat.

Die Arbeiten im norddeutschen Raum werden gegenwärtig von Herrn Hadjebi im Institut für Physik des festen Erdkörpers der Universität Hamburg fortgeführt. Die Ausarbeitungen konzentrieren sich dabei vor allem auf die Arbeitsgebiete am Südrand des nordwestdeutschen Beckens.

Im September dieses Jahres ist durch die Unterstützung der Deutschen Forschungsgemeinschaft in Süddeutschland erstmalig ein größeres reflexionsseismisches Meßprogramm zur Registrierung von Tiefenreflexionen durchgeführt worden. Bei diesen Arbeiten sollten insbesondere Erfahrungen mit Weitwinkeleinsätzen aus dem Bereich der Conrad- und Mohorovičić-Diskontinuität gesammelt werden. $\mathrm{Zu}$ diesem $\mathrm{Zwecke}$ wurde ein Reflektorstück aus verschiedenen Entfernungen angeschossen dergestalt, daß Schußpunkte und Registrieraufstellungen im gleichen Maße nach beiden Seiten wanderten. Nach den Ergebnissen der Reflexionsseismik wurde ein Reflexionselement östlich der Stadt Augsburg ausgewählt. Die Schußpunkte reichten im Süden bis etwa an den Südrand der ungefalteten Molasse im Raum von Weilheim und im Norden über den Fränkischen Jura hinweg bis in den Raum von Weißenburg. Registriert wurde mit den institutseigenen Apparaturen der Geophysikalischen Institute der Universitäten Hamburg, Kiel, Münster und des Niedersächsischen Landesamtes für Bodenforschung. Außerdem wurden gleichzeitig Refraktionsaufnahmen durch das Institut für Angewandte Geophysik der Universität München im Gebiet der Fränkischen Alb bei Sollenhofen durchgeführt. Die Registrierung der Reflexionen erfolgte mit drei Apparaturen auf der vorgenannten Profillinie, wobei an jedem Tag ein Schußpunkt aufgenommen wurde. Die Aufnahmen schlossen kontinuierlich auf dem Nord-Süd-Profil aneinander an. Die 4. Apparatur registrierte die Senkrechtreflexionen direkt am Schußpunkt. Zusätzlich war noch eine 4-spurige Magnet- 
bandapparatur des Institutes für Meteorologie und Geophysik der Universität Frankfurt eingesetzt, welche der Untersuchung spezieller Fragen - wie Frequenzanalysen, Amplitudenmessungen usw. - dienen sollte.

Die Qualität der aufgenommenen Seismogramme schwankte zwischen sehr mäßigen Reflexionsandeutungen im Bereich zwischen etwa 10 und $30 \mathrm{~km}$ Entfernung vom Schußpunkt bis zu außerordentlich kräftigen Reflexionsbändern bei rund $100 \mathrm{~km}$. Es zeigte sich, daß bereits ab etwa $45 \mathrm{~km}$ Entfernung SPRegistrieraufstellung deutliche Einsätze auftraten, welche sich bis zu einer Entfernung über $100 \mathrm{~km}$ in steigender Qualität zu einem sehr markanten Reflexionsband aufbauten. Es scheint, als ob diese Reflexionsgruppen dem Bereich der MohoDiskontinuität zugeordnet werden können. Eine genaue Auswertung des vorliegenden Materials wird noch einige Zeit in Anspruch nehmen und soll im Institut für Meteorologie und Geophysik der Universität Frankfurt durchgeführt werden.

Über das genannte Programm ist zusammenfassend zu sagen, daß sowohl die Durchführung der Feldarbeiten im Zusammenspiel der verschiedenen Institutsapparaturen mit dem von der Firma Göttker, Wathlingen, gestellten Bohrbetrieb und dem von der Firma Prakla GmbH., Hannover, gestellten Aufsichtspersonal einwandfrei war und die seismischen Ergebnisse, soweit bislang zu übersehen, durchaus ermutigend und für künftige Arbeiten sehr wertvoll sein dürften.

Erstmalig in Deutschland sind kürzlich erste Versuche mit dem Verfahren des "weight dropping" zur Registrierung von Tiefenreflexionen in der Nähe von Hannover durchgeführt worden. Die Ergebnisse sind durchaus positiv, und bei den ersten Versuchen konnte ein Reflexionsband bei 5 bis $6 \mathrm{sec}$ Laufzeit beobachtet werden. Bei diesen Arbeiten wurde auf der Sendeseite mit 15 bis 120 "drops" gearbeitet. Weitere etwas umfangreichere Versuche zur Erprobung dieses Verfahrens sind in Vorbereitung.

Bereits in früheren Reflexionsseismogrammen sind verschiedentlich Reflexionsandeutungen unter dem Bereich der Moho-Diskontinuität beobachtet worden.

Bei den Registrierungen der starken Refraktionsschüsse im osthannoverschen Raum wurden auf mehreren Reflexionsseismogrammen sehr markante und zum Teil korrelierende Reflexionen bei ungewöhnlich großen Laufzeiten beobachtet. Die Abbildung 19 zeigt zum Beispiel einen Seismogrammausschnitt mit Tiefenreflexionen bei etwa $15 \mathrm{sec}$ (die Moho liegt in diesem Gebiet bei ca. $10 \mathrm{sec}$ Reflexionszeit). Die Herkunft dieser Reflexionen mit Reflexionszeiten, welche größer sind als die der Reflexionen aus dem Bereich der Moho ist noch nicht eindeutig klar. Es erscheint aber gerade in dem vorliegenden Fall sehr schwierig, diese Reflexionen als Mehrfachreflexionen zu interpretieren. Wahrscheinlicher ist, daß es sich hierbei tatsächlich um Reflexe handelt, welche aus dem Bereich unterhalb der Moho kommen.

Mit dieser Fragestellung greift die Reflexionsseismik aber bereits in das Gebiet des oberen Erdmantels, und es ist eine der Zukunftsaufgaben, die Möglichkeit von Reflexionen aus dem oberen Erdmantel im Rahmen des UppermantleProjektes zu untersuchen. $\mathrm{Zu}$ diesem $\mathrm{Zwecke}$ werden bereits verschiedentlich die 
Reflexionsseismogramme bis zu etwa 18 oder $20 \mathrm{sec}$ aufgenommen und sollten auch alle Registrierungen von Kammersprengungen zumindest bis $\mathrm{zu}$ diesen Laufzeiten durchgeführt werden.

Wir stehen heute in der seismischen Erforschung des tieferen Untergrundes von Mitteleuropa trotz der in den letzten Jahren erzielten Fortschritte noch am

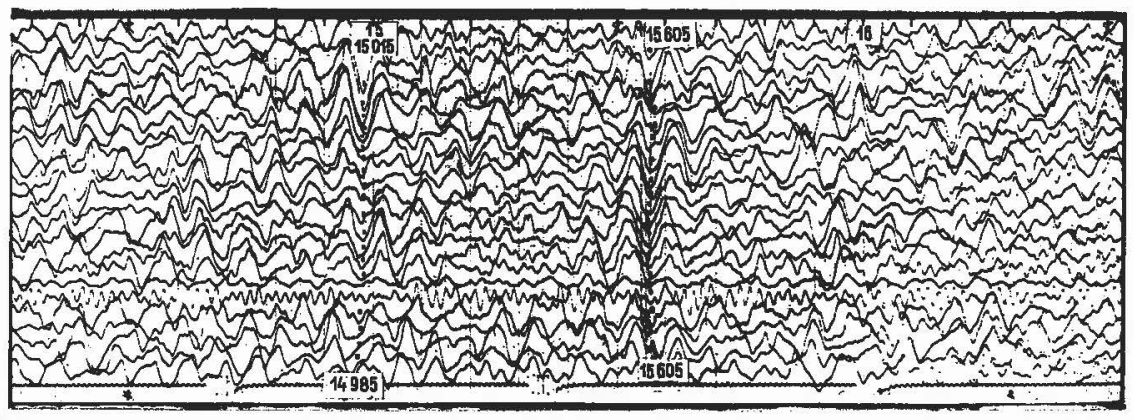

Aвв. 19

Anfang. Anstelle einzelner Refraktionslinien, welche ihren Ausgangspunkt an verschiedenen Steinbrüchen finden, und reflexionsseismischer Untersuchungen mit Hilfe des "Abfallmaterials» der Prospektionsseismik sollte ein gezieltes, großräumiges seismisches Meßprogramm treten, welches sowohl Reflexions- als auch Refraktionsmessungen einschließt. Vor allem hinsichtlich der Reflexionsmessungen wären mehrere langdurchlaufende Profile durch die Bundesrepublik außerordentlich wünschenswert.

Nach all unseren bisherigen Erfahrungen spricht vieles dafür, daß wir es im Bereich der tieferen Erdkruste keineswegs überall mit einer ruhigen und flachen Lagerung zu tun haben. Wir dürfen vermuten, daß sich ein Teil der geologischen Großformen, die wir kennen, auf großräumige Bewegungen im Bereich der tieferen Diskontinuitäten zurückführen läßt. Es erscheint daher meines Erachtens als eine der wesentlichsten Zukunftsaufgaben, diese Beziehungen aufzuzeigen und $\mathrm{zu}$ untersuchen und damit $\mathrm{zu}$ einer neuen Vorstellung geologischer Vorgänge zu gelangen.

Letztlich ist heute eine eventuelle wirtschaftliche Bedeutung der Erkenntnisse über strukturelle Zusammenhänge im tieferen Untergrund noch nicht abzusehen. Es kann aus diesem Grunde heute lediglich die Frage aufgeworfen werden, ob bei der Abschließung nutzbarer Lagerstätten und der Suche nach ergiebigen Energiequellen Großstrukturen im tieferen Untergrund nicht in absehbarer Zeit eine maßgebliche Rolle spielen werden. Aus dieser Sicht sollte meines Erachtens zum gegenwärtigen Zeitpunkt eine großzügige und großräumige Untersuchung des tieferen Untergrundes in der Bundesrepublik geplant werden. Die in dem vorliegenden Referat dargelegten Ergebnisse - ebenso wie die Resultate der refraktionsseismischen Messungen - könnten die Voraussetzungen bilden, auf welchen ein umfassendes Arbeitsprogramm aufgebaut werden kann. 


\title{
EIN DISKUSSIONSBEITRAG ZUR AUSBREITUNG
}

DER LONGITUDINALWELLE

\author{
O. FöRTSCH
}

(München, BRD)

Seit O. v. Schmidt (1939) die ersten schlierenoptischen Aufnahmen vom Ausbreitungsvorgang elastischer Wellen veröffentlicht hat, sind eine Reihe von Arbeiten erschienen, welche die sehr mannigfaltige und unerwartet komplizierte Art und Weise der Ausbreitung eines elastischen Impulses in einem Stab anschaulich zeigen. Es sei hier nur auf die sehr instruktiven Aufnahmen von David, Schall und Schardin (1964) hingewiesen. In den Publikationen wird immer wieder zum Ausdruck gebracht, wie schwierig es ist, die beachteten Phänomene theoretisch zu deuten. So erhebt sich die Frage, wie kommt es, daß, obwohl nur ein Impuls angeregt wird, in den Aufnahmen stets eine große Anzahl von Wellenfronten zu sehen ist, welche in einem bestimmten Abstand aufeinander folgen. Von den Details, die außerdem noch deutlich zu erkennen sind, soll gar nicht gesprochen werden. Es gibt eine Theorie von Pochhammer (1878) und Chree (1889), welche die Ausbreitung von Longitudinalwellen in Stäben beschreibt. Die Handhabung dieser Theorie ist aber so umfangreich und schwierig, daß bis heute noch keine Lösung durchgerechnet worden ist. Die Theorie ist für die Praxis bis heute noch nicht zum Tragen gekommen. Als ganz grobe Näherung ist der bekannte, einfache Ansatz zu betrachten, welcher nur elastische Verschiebungen parallel zur Stabachse vorsieht. Dabei wird noch angenommen, daß die Verschiebungen innerhalb eines Querschnittes gleich groß sein sollen. Dieser sehr vereinfachende Ansatz liefert nur den richtigen Wert für die Ausbreitungsgeschwindigkeit der Longitudinalwelle, nämlich $a=\sqrt{E / \rho}$, die anderen Phänomene vermag er aber nicht mehr zu deuten. Weitere, bessere Näherungen sind von Mindlin und Hermann (1952) sowie von Hunter und Johnson (1952) angegeben worden. Aber auch diese vermögen die Beobachtungen bei weitem nicht befriedigend $\mathrm{zu}$ deuten. Es erscheint daher mehr wie angebracht, daß noch weitere Anstrengungen unternommen werden, welche zu einer Erklärung der Ausbreitung longitudinaler Wellen in Stäben und darüber hinaus in elastischen Körpern allgemein beitragen. Dabei muß man sich im voraus im klaren sein, daß man mit den bisher verwendeten Überlegungen nicht weiter kommen kann, man muß vielmehr in sie neue Gedankengänge einbauen. Die vorliegende Arbeit soll einen Diskussionsbeitrag in dieser Richtung liefern. 


\section{A) Einführende Bemerkungen}

Bei unseren Betrachtungen wollen wir uns auf die Wellenausbreitung in einem stabförmigen Körper von rechteckigem Querschnitt und auf diejenige im elastischen Vollraum beschränken. Der Fall der dünnen Platte, der zwischen den beiden genannten liegt, bringt nichts Neues. Seine Behandlung ist dann nur eine rein formale Angelegenheit. Bei der Betrachtung der Ausbreitung einer Longitudinalwelle im Vollraum werden wir uns diesen in viele Stäbe unterteilt denken und so diesen Fall auch wieder auf die Wellenausbreitung in einem Stab zurückführen.

Um die theoretischen Ableitungen einfacher und vor allem anschaulicher gestalten zu können, wollen wir ein rechtwinkliges Koordinatensystem so wählen, daß die Richtung der $x$-Achse mit derjenigen der Längsachse der Stäbe zusammenfällt (s. Fig. 1). Die $y$-bzw. $z$-Achse soll parallel zu einer Seite der Stabquer-

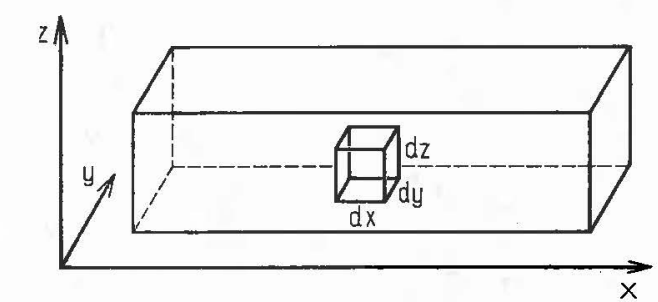

Fig. 1. Lage des Stabes im Koordinatensystem

schnitte verlaufen. Wie üblich denken wir uns aus dem Stab ein kleines Parallelepiped mit den Kantenlängen $d x, d y$ und $d z$ herausgeschnitten. Auf dieses Volumenelement sollen die Normalspannungen $\tau_{x x}, \tau_{y y}$ und $\tau_{z z}$ wirken. Sie erzeugen die elastischen Verschiebungen $u, v$ und $w$, welche parallel zur $x$-, $y$-, bzw. $z$-Achse gerichtet sein sollen, sowie die Deformationen $\frac{\partial u}{\partial x}, \frac{\partial v}{\partial y}$ und $\frac{\partial w}{\partial z}$. In der üblichen Weise sollen die elastischen Konstanten durch folgende Buchstaben dargestellt werden:

$$
\begin{aligned}
& E=\text { Elastizitätsmodul, kurz E-Modul genannt, } \\
& \sigma=\text { Poissonsche Zahl, } \\
& \mu=\frac{E}{2(1+\sigma)}=\text { Scher- oder Schubmodul, } \\
& k=\frac{E}{3(1-2 \sigma)}=\text { Kompressibilitätsmodul, } \\
& \lambda=\frac{\sigma E}{(1+\sigma)(1-2 \sigma)}=\text { Lamésche Konstante. }
\end{aligned}
$$

Durch die Normalspannungen werden Deformationen erzeugt, die man in eine reine, allseitig gleiche Volumenänderung und in eine reine Scherung zerlegen kann. Auch die Normalspannungen lassen sich in Anteile, die eine reine Volumen- 
änderung und in solche, welche eine reine Scherung verursachen, aufteilen. Die reine Volumenänderung ist bestimmt durch die Terme:

$$
p=\left(\tau_{x x}+\tau_{y y}+\tau_{z z}\right) / 3
$$

bei den Spannungen und durch

$$
\varepsilon=\left(\frac{\partial u}{\partial x}+\frac{\partial v}{\partial y}+\frac{\partial w}{\partial z}\right) / 3
$$

bei den Deformationen. Die entsprechenden Ausdrücke für die reine Scherung lauten:

$$
\begin{aligned}
& q_{x x}=\left(2 \tau_{x x}-\tau_{y y}-\tau_{z z}\right) / 3=\tau_{x x}-p, \\
& q_{y y}=\left(2 \tau_{y y}-\tau_{z z}-\tau_{x x}\right) / 3=\tau_{y y}-p, \\
& q_{z z}=\left(2 \tau_{z z}-\tau_{x x}-\tau_{y y}\right) / 3=\tau_{z z}-p,
\end{aligned}
$$

bei den Spannungen und

$$
\begin{aligned}
& \vartheta_{x x}=\left(2 \frac{\partial u}{\partial x}-\frac{\partial v}{\partial y}-\frac{\partial w}{\partial z}\right) / 3=\frac{\partial u}{\partial x}-\varepsilon, \\
& \vartheta_{y y}=\left(2 \frac{\partial v}{\partial y}-\frac{\partial w}{\partial z}-\frac{\partial u}{\partial x}\right) / 3=\frac{\partial v}{\partial y}-\varepsilon, \\
& \vartheta_{z z}=\left(2 \frac{\partial w}{\partial z}-\frac{\partial u}{\partial x}-\frac{\partial v}{\partial y}\right) / 3=\frac{\partial w}{\partial z}-\varepsilon
\end{aligned}
$$

bei den Deformationen. Die Beziehungen zwischen den Spannungs- und den entsprechenden Deformationsgrößen lauten:

$$
\begin{aligned}
& \frac{\partial u}{\partial x}=\frac{1}{E}\left(\tau_{x x}-\sigma \tau_{y y}-\sigma \tau_{z z}\right), \\
& \frac{\partial v}{\partial y}=\frac{1}{E}\left(\tau_{y y}-\sigma \tau_{z z}-\sigma \tau_{x x}\right), \\
& \frac{\partial w}{\partial z}=\frac{1}{E}\left(\tau_{z z}-\sigma \tau_{x x}-\sigma \tau_{y y}\right) .
\end{aligned}
$$

Löst man diese Gleichungen nach den Normalspannungen auf und verwendet die bekannte Abkürzung $\Theta=3 \varepsilon$, so erhält man:

$$
\begin{gathered}
\tau_{x x}=2 \mu \frac{\partial u}{\partial x}+\lambda \Theta, \\
\tau_{y y}=2 \mu \frac{\partial v}{\partial y}+\lambda \Theta, \\
\tau_{z z}=2 \mu \frac{\partial w}{\partial z}+\lambda \Theta .
\end{gathered}
$$




\section{B) Die Longitudinalwelle im freien Stab}

In diesem Abschnitt der Abhandlung wollen wir uns bewußt auf die eingangs erwähnte erste, grobe Näherung beschränken. Sie gibt den richtigen Wert der Ausbreitungsgeschwindigkeit, worauf es hier in der Hauptsache ankommt. Es sei dahingestellt, ob der Ansatz noch weit von den tatsächlichen Verhältnissen abweicht.

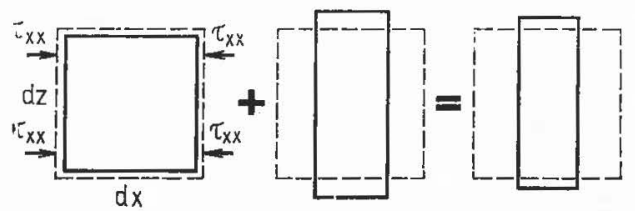

FIG. 2. Deformation eines freien Stabes durch die Normalspannung $\tau_{x x}$

Wir betrachten wie üblich das kleine Volumenelement $d x d y d z$, das in Fig. 1 gesondert dargestellt ist. In Fig. 2 ist die Vorderseite des Elementes mit den Seiten $d x$ und $d z$ vergrößert in Form des gestrichelt gezeichneten Quadrates wiedergegeben. Ohne Beschränkung der Allgemeinheit, nur der Anschaulichkeit wegen, sind die Seiten $d x$ und $d z$ gleich lang gewählt worden. Auf die beiden Flächen $d y d z$, welche auf der Richtung der $x$-Achse senkrecht stehen, wirke die Normalspannung $\tau_{x x}$. Die beiden anderen Normalspannungen $\tau_{y y}$ und $\tau_{z z}$ seien nicht vorhanden. Das Element erfährt durch $\tau_{x x}$ folgende Deformation, welche man den Gln. (3) sofort entnehmen kann:

$$
\begin{aligned}
& \frac{\partial u}{\partial x}=\frac{1}{E} \tau_{x x}=\frac{1-2 \sigma}{E} \frac{1}{3} \tau_{x x}+\frac{1+\sigma}{E} \frac{2}{3} \tau_{x x}=\frac{p}{3 k}+\frac{1}{2 \mu} q_{x x} ; \\
& \frac{\partial v}{\partial y}=-\frac{\sigma}{E} \tau_{x x}=\frac{1-2 \sigma}{E} \frac{1}{3} \tau_{x x}-\frac{1+\sigma}{E} \frac{1}{3} \tau_{x x}=\frac{p}{3 k}+\frac{1}{2 \mu} q_{y y} \\
& \frac{\partial w}{\partial z}=-\frac{\sigma}{E} \tau_{x x}=\frac{1-2 \sigma}{E} \frac{1}{3} \tau_{x x}-\frac{1+\sigma}{E} \frac{1}{3} \tau_{x x}=\frac{p}{3 k}+\frac{1}{2 \mu} q_{z z} .
\end{aligned}
$$

Im hinteren Teil der Gleichungen ist die Deformation in die allseitige Kompression und in die reine Scherung aufgespalten worden. Diese Aufspaltung wird in Fig. 2 veranschaulicht. Die Darstellung gilt für die Poissonsche Zahl $\sigma=0,25$. Im linken Teil der Figur ist die reine Volumenänderung veranschaulicht. Durch die allseitige Kompression wird das gestrichelt gezeichnete, ursprüngliche Quadrat in das kleinere, ausgezogen gezeichnete übergeführt. Der mittlere Teil zeigt uns in der gleichen Weise den Betrag der Scherung. Der Betrag der Dehnung in der $y$ - und $z$-Richtung ist halb so groß wie der der Kontraktion in der $x$-Richtung. Schließlich sind im rechten Teil der Figur beide Grunddeformationen addiert worden. Durch die Normalspannung $\tau_{x x}$ wird das ursprüngliche Quadrat in das 
ausgezogen gezeichnete Rechteck übergeführt. Man erkennt, daß der überwiegende Anteil an der Deformation von der reinen Scherung herrührt.

Durch die Normalspannung $\tau_{x x}$ wird im Stab Energie gespeichert. Ihren Betrag pro Volumeneinheit $W_{1}$ können wir sofort angeben:

$$
W_{1}=\frac{1}{2} \tau_{x x} \frac{\partial u}{\partial x}=\frac{E}{2} \cdot\left(\frac{\partial u}{\partial x}\right)^{2} .
$$

Die Ableitung der Differentialgleichung für die Longitudinalwelle im freien Stab ist, wenn wir uns auf die grobe Näherung beschränken, sehr einfach. Sie lautet bekanntlich:

$$
\rho \frac{\partial^{2} u}{\partial t^{2}}=\frac{\partial \tau_{x x}}{\partial x}
$$

Da $\tau_{x x}=E \frac{\partial u}{\partial x}$, ergibt sich

$$
\frac{\partial^{2} u}{\partial t^{2}}=\frac{E}{\rho} \frac{\partial^{2} u}{\partial x^{2}} ; \quad \text { oder } \frac{\rho}{E} \frac{\partial^{2} \tau_{x x}}{\partial t^{2}}=\frac{\partial^{2} \tau_{x x}}{\partial x^{2}} .
$$

Für die Ausbreitungsgeschwindigkeit dieser Longitudinalwelle erhalten wir den Ausdruck:

$$
a_{1}=\sqrt{\frac{E}{\rho}}=\sqrt{\frac{1}{\rho} \frac{\tau_{x x}}{\partial u / \partial x}} .
$$

Der Index 1 von $a$ dient nur zur Unterscheidung von ähnlichen Ausdrücken, welche in den folgenden Abschnitten vorkommen. Um die Hinweise weiterhin zu erleichtern, soll die Longitudinalwelle im freien Stab als Fall 1 bezeichnet werden. Die Longitudinalwellen, die in den beiden folgenden Abschnitten behandelt werden, sollen dann kurz entsprechend mit Fall 2 bzw. 3 bezeichnet werden.

\section{C) Die Longitudinalwelle in einem Stab, der speziellen Bedingungen unterliegt}

Wir wenden uns nun dem elastischen Vollraum zu. In ihm soll sich eine Longitudinalwelle in Richtung der $x$-Achse ausbreiten. Die $y-z$-Ebene ist dann Wellenfront. In ihr sind alle Phasen und Zustände überall gleich. Wir denken uns nun den Vollraum, wie eingangs schon erwähnt worden ist, in Stäbe unterteilt, deren Achsen wiederum wie im vorstehenden Abschnitt parallel zur $x$-Achse orientiert sein sollen. Die Unterteilung soll so erfolgen, daß die Querschnitte der Stäbe rechteckig werden. Wirkt auf alle Stäbe einheitlich eine zeitlich konstante Normalspannung $\tau_{x x}$, so tritt wie im Fall 1 eine Querdehnung bzw. -kontraktion auf. Die Stäbe werden dadurch in der $y$ - und $z$ - Richtung gegeneinander verschoben. 
Bei den elastischen Wellen und Schwingungen handelt es sich um oszillatorische Bewegungen um die Ruhelage. Würde hierbei wechselseitig im Rhythmus der Frequenz der Welle eine Querdehnung und Querkontraktion auftreten, so müßten alle Stäbe Querbewegungen von nicht geringem Betrage ausführen. Die damit verbundenen Trägheitskräfte, die wegen der zu bewegenden großen Masse gleich sehr beträchtliche Ausmaße annehmen müßten, verbieten aber von vorneherein solche Bewegungen. Eine Querkontraktion oder -dilatation ist also nicht möglich. Es ist zu fordern, $\mathrm{da} B \frac{\partial v}{\partial y}=\frac{\partial w}{\partial z}=0$ ist. Auch bei der Behandlung dieses Problems können wir wieder von den allgemeinen Gleichungen (3) ausgehen und erhalten:

$$
\begin{aligned}
& \frac{\partial u}{\partial x}=\frac{1}{E}\left(\tau_{x x}-\sigma \tau_{y y}-\sigma \tau_{z z}\right) ; \\
& \frac{\partial v}{\partial y}=0=\frac{1}{E}\left(\tau_{y y}-\sigma \tau_{z z}-\sigma \tau_{x x}\right) ; \\
& \frac{\partial w}{\partial z}=0=\frac{1}{E}\left(\tau_{z z}-\sigma \tau_{x x}-\sigma \tau_{y y}\right) .
\end{aligned}
$$

Während beim freien Stab $\tau_{y y}=\tau_{z z}=0$ gesetzt werden konnte und $\frac{\partial v}{\partial y}=\frac{\partial w}{\partial z} \neq 0$ sind, ist es hier umgekehrt. Die beiden letzten Gleichungen von (10) fordern, daß neben $\tau_{x x}$ auch noch $\tau_{y y}$ und $\tau_{z z}$ existieren und daß diese in einem bestimmten Verhältnis zu $\tau_{x x}$ stehen müssen. Es muß sein:

$$
\tau_{y y}=\tau_{z z}=\frac{\sigma}{1-\sigma} \cdot \tau_{x x}
$$

Weiterhin ergibt sich daraus:

$$
\begin{array}{rlrl}
p & =\frac{1+\sigma}{1-\sigma} \frac{1}{3} \tau_{x x} ; & q_{x x}=\frac{1-2 \sigma}{1-\sigma} \frac{2}{3} \tau_{x x} ; \\
q_{x x}=2 \frac{1-2 \sigma}{1+\sigma} p=2 \frac{2 \mu}{3 k} p ; & q_{y y}=q_{z z}=-\frac{1}{2} q_{x x} .
\end{array}
$$

Im vorliegenden Fall 2 findet nur in Richtung der $x$-Achse eine Deformation statt. Die Volumenänderung $\Theta$ ist dem Betrage nach identisch mit $\frac{\partial u}{\partial x}$. Hierfür erhalten wir:

$$
\frac{\partial u}{\partial x}=\Theta=\frac{3 p}{3 k}=\frac{p}{3 k}+\frac{q_{x x}}{2 \mu}=\frac{(1+\sigma)(1-2 \sigma)}{(1-\sigma) E} \tau_{x x}=\frac{\tau_{x x}}{\lambda+2 \mu}=\frac{\tau_{x x}+\tau_{y y}+\tau_{z z}}{3 k} .
$$


Das zweite Gleichheitszeichen ergibt sich aus der allgemeinen Beziehung $p=k \Theta$. Das dritte Gleichheitszeichen resultiert aus der Aufspaltung der Deformation in die reine Kompression und die reine Scherung. Das vierte Gleichheitszeichen ist eine Folgerung aus der ersten der drei obigen Gleichungen (10). Auf den Ausdruck hinter dem letzten Gleichheitszeichen werden wir später zurückkommen.

Für die Energie, welche bei einer solchen Deformation in der Volumeneinheit gespeichert wird, erhalten wir:

$$
W_{2}=\frac{1}{2} \tau_{x x} \frac{\partial u}{\partial x}=\frac{E}{2} \frac{1-\sigma}{(1+\sigma)(1-2 \sigma)}\left(\frac{\partial u}{\partial x}\right)^{2} .
$$

Die beiden anderen Normalspannungen $\tau_{y y}$ und $\tau_{z z}$ leisten wegen $\frac{\partial v}{\partial y}=\frac{\partial w}{\partial z}=0$ keine Arbeit.

Für die Longitudinalwelle in einem Stab, der den obigen Bedingungen unterworfen ist, erhalten wir die Differentialgleichung (7):

$$
\rho \frac{\partial^{2} u}{\partial t^{2}}=\frac{\partial \tau_{x x}}{\sigma x}
$$

Unter Verwendung von Gl. (13) erhalten wir aus Gl. (7):

$\frac{\partial^{2} u}{\partial t^{2}}=\frac{E}{\rho} \frac{1-\sigma}{(1+\sigma)(1-2 \sigma)} \frac{\partial^{2} u}{\partial x^{2}} ; \quad \frac{\rho}{E} \frac{(1+\sigma)(1-2 \sigma)}{1-\sigma} \frac{\partial^{2} \tau_{x x}}{\partial t^{2}}=\frac{\partial^{2} \tau_{x x}}{\partial x^{2}}$.

Die Ausbreitungsgeschwindigkeit $a_{2}$ dieser Longitudinalwelle ergibt sich daraus zu:

$$
a_{2}=\sqrt{\frac{E}{\rho} \frac{1-\sigma}{(1+\sigma)(1-2 \sigma)}}=\sqrt{\frac{\lambda+2 \mu}{\rho}}=\sqrt{\frac{1}{\rho} \frac{\tau_{x x}}{\partial u / \partial x}} .
$$

Die obige Ableitung der Ausbreitungsgeschwindigkeit $a_{2}$ ist im Prinzip identisch mit derjenigen, nach der in der Elastodynamik die Differentialgleichungen der elastischen Wellen gewonnen werden. Für unser spezielles Koordinatensystem ist sie nur etwas einfacher und anschaulicher. Es ist daher nicht verwunderlich, daß für die Ausbreitungsgeschwindigkeit der Longitudinalwelle der gleiche Ausdruck erhalten wird. Das spezielle Koordinatensystem hat aber gezeigt, daß neben $\tau_{x x}$ auch noch $\tau_{y y}$ und $\tau_{z z}$ in einem bestimmten Verhältnis zu $\tau_{x x}$ vorhanden sein müssen, was bisher übersehen worden ist. Diese Forderung meldet aber erhebliche Bedenken gegenüber der Existenzmöglichkeit einer solchen Welle an.

In Fig. 3 soll in Anlehnung an Fig. 2 die Deformation des Stabes ohne Querdehnung veranschaulicht werden. Zunächst wird wieder die allseitige Kompression, dann die reine Scherung und schließlich die Summe der beiden dargestellt. Man erkennt, daß im Vergleich mit den Verhältnissen beim freien Stab der Kompressionsanteil der Deformation größer und der Anteil der Scherung wesentlich kleiner geworden ist. Entscheidend ist hier, daß in Richtung der $y$-bzw. $z$-Achse 
die Kompression und die Scherung dem Betrage nach gleich groß sind, sie unterscheiden sich nur im Vorzeichen. In Richtung der $x$-Achse ist der Betrag der Scherung doppelt so groß wie derjenige der Kompression. Die Gesamtdeformation ist somit in dieser Richtung dreimal größer als die Kompression.

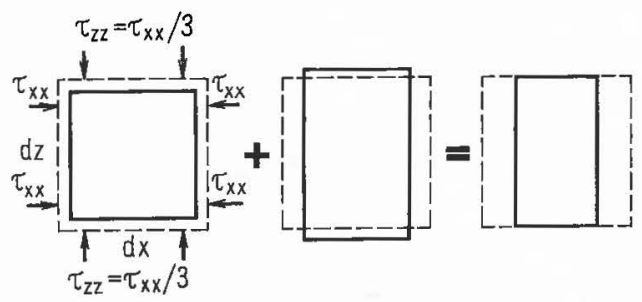

FrG. 3. Deformation eines Stabes, der der Bedingung $\partial v / \partial y=\partial w / \partial z=0$ unterliegt

Die oben geäußerten Bedenken gegenüber der Existenzmöglichkeit der Longitudinalwelle in einem Stab, der den oben geforderten Bedingungen unterliegt, und damit auch gegenüber derjenigen Longitudinalwelle, die sich nach der Theorie im Vollraum ausbreiten soll, gründen sich in erster Linie auf die Forderung, daß die beiden Normalspannungen $\tau_{y y}$ und $\tau_{z z}$ mit der Welle mitlaufen und sich im gleichen Rhythmus ändern müssen, ebenso wie es bei der Verschiebung $u$ oder der relativen Volumenänderung $\Theta$ oder der Normalspannung $\tau_{x x}$ der Fall ist. Während die zuletzt erwähnten Größen im Wellenmechanismus verankert sind, d. h. für sie läßt sich eine Wellendifferentialgleichung aufstellen, ist dies für die Normalspannungen $\tau_{y y}$ und $\tau_{z z}$ nicht möglich. Wie sollen diese Spannungen bei einer geschlossenen Wellenfront im Vollraum auch erzeugt werden? Es bereitet daher ernste Schwierigkeiten, ihre Existenz und ihr Mitwandern mit der Welle physikalisch zu erklären. Im folgenden Abschnitt soll daher eine andere Möglichkeit für den Mechanismus der Wellenausbreitung im Stab und im Vollraum zur Diskussion gestellt werden, welche die erwähnten Schwierigkeiten umgeht.

\section{D) Die Longitudinalwelle im Stab unter Zwang}

Die Ausgangsposition im Fall des Stabes unter Zwang soll die gleiche sein wie im vorstehenden Fall. Es wird auch hier die Forderung erhoben, daß $\frac{\partial v}{\partial y}=\frac{\partial w}{\partial z}=0$ ist. Darüber hinaus soll auch noch die gleiche Bedingung wie beim freien Stab erfüllt werden, nämlich $\tau_{y y}=\tau_{z z}=0$. Es ist klar, daß sich angesichts der vier Bedingungen keine zwanglose Lösung des Problems finden läßt. Bei der Behandlung desselben wollen wir ebenso vorgehen wie in den Figuren 2 und 3. Wir betrachten der Reihe nach die beiden Grunddeformationen. Zunächst erzeugt $\tau_{x x}$ eine allseitige Kompression, die ebenso groß ist wie diejenige beim freien Stab (s. Fig. 2). Dafür wird die Spannung $p=\tau_{x x} / 3$ benötigt, welche in 
allen drei Achsenrichtungen wirkt. Es verbleiben die Spannungen für die Scherung, nämlich $q_{x x}=2 \tau_{x x} / 3, q_{y y}=-\tau_{x x} / 3$ und $q_{z z}=-\tau_{x x} / 3$. Diese Spannungen können nun aber nicht wie beim freien Stab den vollen Betrag der Scherung erzeugen. Wegen der Konstanz der Querschnittsflächen der Stäbe kann vielmehr der Betrag der Scherung in Richtung der $y$ - und $z$-Achse nur ebenso groß werden, wie in diesen Richtungen von der Kompression vorher Platz geschaffen worden ist. Für diesen geringeren Betrag der Scherung gegenüber demjenigen beim freien Stab wird nur ein Teil der zur Verfügung stehenden Scherspannung benötigt. Es verbleibt ein Rest, den wir folgerichtig als Restspannung bezeichnen wollen. Diese Restspannung versucht, das den Stab umgebende Material nach außen zu verdrängen, was ihr aber angesichts der großen Masse und der damit verbundenen großen Trägheit nicht gelingt. Die Restspannung rührt von der Normalspannung $\tau_{x x}$ her, sie wird daher von ihr gesteuert und läuft somit automatisch mit der Welle mit. In der Sprache der Mathematik läßt sich das Gesagte folgendermaßen ausdrücken, wobei sich die mit dem Index $k$ versehenen Terme auf die reine Kompression, die mit dem Index $s$ versehenen auf die reine Scherung und die mit dem Index $r$ versehenen auf die Restspannung beziehen:

$$
p=\tau_{x x} / 3 ; \quad\left(\frac{\partial u}{\partial x}\right)_{k}=\frac{1-2 \sigma}{E} \frac{1}{3} \tau_{x x} \quad \text { (s. freier Stab). }
$$

Wegen

und wegen

$$
\frac{\partial v}{\partial y}=\frac{\partial w}{\partial z}=0
$$

muß sein:

$$
\left(\frac{\partial v}{\partial y}\right)_{k}=\left(\frac{\partial w}{\partial z}\right)_{k}=\left(\frac{\partial u}{\partial x}\right)_{k}
$$

$$
\begin{gathered}
\left(\frac{\partial v}{\partial y}\right)_{s}=\left(\frac{\partial w}{\partial z}\right)_{s}=-\left(\frac{\partial u}{\partial x}\right)_{k}=-\frac{1-2 \sigma}{E} \frac{1}{3} \tau_{x x} ; \\
\left(\frac{\partial u}{\partial x}\right)_{s}=2\left(\frac{\partial u}{\partial x}\right)_{k}=\frac{1-2 \sigma}{E} \frac{2}{3} \tau_{x x}
\end{gathered}
$$

Die für die Deformation $\left(\frac{\partial u}{\partial x}\right)_{s}$ benötigte Scherspannung $\left(\tau_{x x}\right)_{s}$ errechnet sich aus:

zu:

$$
\left(\frac{\partial u}{\partial x}\right)_{s}=\frac{1+\sigma}{E}\left(\tau_{x x}\right)_{s}
$$

$$
\left(\tau_{x x}\right)_{s}=\frac{1-2 \sigma}{1+\sigma} \frac{2}{3} \tau_{x x} ;
$$

weiterhin ist: 


$$
\left(\tau_{y y}\right)_{s}=\left(\tau_{z z}\right)_{s}=-\left(\tau_{x x}\right)_{s} / 2 .
$$

Für die Restspannung ergibt sich schließlich:

$$
\begin{gathered}
\left(\tau_{x x}\right)_{r}=\frac{2}{3} \tau_{x x}-\left(\tau_{x x}\right)_{s}=\frac{2 \sigma}{1+\sigma} \tau_{x x} ; \\
\left(\tau_{y y}\right)_{r}=\left(\tau_{z z}\right)_{r}=-\left(\tau_{x x}\right)_{r} / 2 .
\end{gathered}
$$

Für die Deformation in Richtung der $x$-Achse erhalten wir in diesem Fall:

$$
\frac{\partial u}{\partial x}=\Theta=\frac{3 p}{3 k}=\frac{p}{3 k}+\frac{q_{x x}}{2 \mu}=3\left(\frac{\partial u}{\partial x}\right)_{k}=\frac{1-2 \sigma}{E} \tau_{x x}=\frac{\tau_{x x}}{3 k}=\frac{\tau_{x x}-\left(\tau_{x x}\right)_{r}}{\lambda+2 \mu} .
$$

In Fig. 4 sind die Deformationsverhältnisse für diesen Lösungsversuch dargestellt. Sie unterscheiden sich von denen in Fig. 3 nur durch die Beträge. Im Fall des Stabes unter Zwang sind für $\sigma=0,25$ die Deformationen im Verhältnis $3: 5$

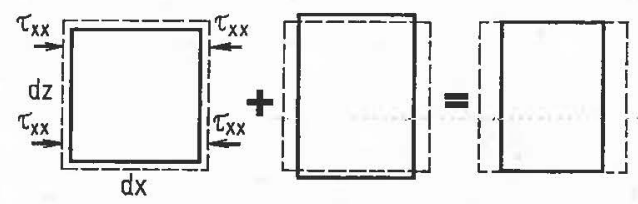

FIG. 4. Deformation eines Stabes unter Zwang

kleiner als diejenigen im Falle des Stabes unter den speziellen Bedingungen. Hierbei ist vorausgesetzt, daß die Normalspannung $\tau_{x x}$ jeweils den gleichen Wert besitzt. Würde man die Deformationen auf die Summe der Normalspannungen, also auf $3 p$, beziehen, so würde sich in beiden Fällen der gleiche Wert ergeben.

Wir haben oben gesagt, da $ß$ die Restspannung versucht, das den Stab umgebende Material wegzuschieben, wobei sie es beschleunigen muß. Die dabei auftretenden Trägheitskräfte halten der Restspannung das Gleichgewicht. Wegen der großen Masse, die im Vollraum vorhanden ist, erreichen die Beschleunigungen und damit auch die Verschiebungen nur Beträge, die vernachlässigt werden können. Lassen wir aber das den Stab umgebende Material weniger und weniger werden, so wachsen die Querverschiebungen mehr und mehr an und erreichen merkbare Beträge. Dabei treten im benachbarten Material Trägheits- und Scherkräfte auf. Weiterhin wird aber nun auch eine zusätzliche Scherung des betrachteten Stabelementes ermöglicht und zwar in einem solchen Ausmaß, als dies die Querverschiebungen des benachbarten Materials erlauben. Die Restspannung, die all dies bewirken muß, wird aufgespalten in einen Anteil, der den Spannungen und Kräften im Nachbarmaterial das Gleichgewicht hält, und in einen Anteil, der für die zusätzliche Scherung erforderlich ist. Das Verhältnis des ersten Anteils zum zweiten ist für den Vollraum unendlich groß. Nimmt das umgebende Material 
mehr und mehr ab, so wird das Verhältnis immer kleiner. Verschwindet schließlich das umgebende Material ganz, d. h. es ist ein dünner, schlanker Stab gegeben, so geht das Verhältnis gegen Null. Wir sind dann beim freien Stab des Abschnittes $B$ angelangt. Der Lösungsvorschlag 3 mit den Restspannungen ermöglicht es uns, den Übergang von der Vollraumgeschwindigkeit $\mathrm{zu}$ der Longitudinalgeschwindigkeit im freien Stab einfach und zwanglos zu erklären. Auch enthält er Ansätze zur Deutung der schon erwähnten Mannigfaltigkeit der Beobachtungen. Eine eingehende Behandlung dieses Problems soll einer weiteren Abhandlung vorbehalten bleiben.

Die Energiedichte errechnet sich im Falle des Stabes unter Zwang in der gleichen Weise wie in den beiden vorher behandelten Fällen zu:

$$
W_{3}=\frac{1}{2} \tau_{x x} \frac{\partial u}{\partial x}=\frac{1}{2} \frac{E}{1-2 \sigma}\left(\frac{\partial u}{\partial x}\right)^{2} .
$$

Für die Longitudinalgeschwindigkeit im Stab unter Zwang und damit auch für diejenige im Vollraum erhalten wir zunächst wieder (7):

$$
\rho \frac{\partial^{2} u}{\partial t^{2}}=\frac{\partial \tau_{x x}}{\partial x}
$$

Ersetzen wir hier in der üblichen Weise $\tau_{x x}$ durch die Deformation $\frac{\partial u}{\partial x}$ bzw. umgekehrt $\frac{\partial u}{\partial x}$ durch $\tau_{x x}$, so ergibt sich:

$$
\frac{\partial^{2} u}{\partial t^{2}}=\frac{E}{\rho} \frac{1}{1-2 \sigma} \frac{\partial^{2} u}{\partial x^{2}} ; \quad \frac{\rho}{E}(1-2 \sigma) \frac{\partial^{2} \tau_{x x}}{\partial t^{2}}=\frac{\partial^{2} \tau_{x x}}{\partial x^{2}} .
$$

Die Geschwindigkeit $a_{3}$, mit der sich diese Longitudinalwelle ausbreitet, wird bestimmt durch:

$$
a_{3}=\sqrt{\frac{E}{\rho} \frac{1}{1-2 \sigma}}=\sqrt{\frac{3 k}{\rho}}=\sqrt{\frac{1}{\rho} \frac{\tau_{x x}}{\partial u / \partial x}} .
$$

\section{E) Diskussion über die Geschwindigkeitswerte $a_{2}$ und $a_{3}$}

Im Fall 3 erhalten wir einen größeren Wert für die Longitudinalgeschwindigkeit als den herkömmlichen im Fall 2. Der Wert $a_{2}$ würde sich im Fall 3 ergeben, wenn wir nicht die gesamte Spannung $\tau_{x x}$ in Gl. (25) einsetzen, sondern nur denjenigen Teil von ihr, der für die Deformation gebraucht wird, nämlich $\left[\tau_{x x}-\left(\tau_{x x}\right)_{r}\right]$. Umgekehrt würden wir im Fall 2 den Geschwindigkeitswert $a_{3}$ erhalten, wenn wir in Gl. (16) statt $\tau_{x x}$ die Summe aller Normalspannungen $\left(\tau_{x x}+\tau_{y y}+\tau_{z z}\right)$ einsetzen. 
Es ist nun zur Diskussion zu stellen, ob für die Geschwindigkeit der Longitudinalwelle die Summe aller vorhandenen Spannungen oder nur derjenige Teil, welcher für die Deformationen benötigt wird, von Bedeutung ist. Die Geschwindigkeit $a_{2}$ erhalten wir, wenn wir schreiben:

$$
\begin{aligned}
\text { im Falle 2: } & a_{2}=\sqrt{\frac{1}{\rho} \frac{\tau_{x x}}{\partial u / \partial x}} ; \\
\text { im Falle 3: } & a_{2}=\sqrt{\frac{1}{\rho} \frac{\tau_{x x}-\left(\tau_{x x}\right)_{r}}{\partial u / \partial x} .}
\end{aligned}
$$

Die Geschwindigkeit $a_{3}$ erhalten wir, wenn wir schreiben:

$$
\begin{aligned}
\text { im Falle 2: } & a_{3}=\sqrt{\frac{1}{\rho} \frac{\tau_{x x}+\tau_{y y}+\tau_{z z}}{\partial u / \partial x}} ; \\
\text { im Falle 3: } & a_{3}=\sqrt{\frac{1}{\rho} \frac{\tau_{x x}}{\partial u / \partial x}} .
\end{aligned}
$$

Welche von den aufgeführten Möglichkeiten die physikalisch richtige ist, läßt sich nur an Hand von Argumenten entscheiden. Zur Anregung einer Diskussion seien einige erwähnt. Zunächst sei nochmals auf die Schwierigkeiten hingewiesen, die das Vorhandensein der Normalspannungen $\tau_{y y}$ und $\tau_{z z}$ im Falle 2 verursachen.

Ein wichtiges Argument liefern uns Energiebetrachtungen. Die Energiedichte ist schließlich die wichtigste Größe einer Welle. Solange der Querschnitt der Stäbe sich nicht verändert, leisten nur diejenigen Normalspannungen Arbeit, welche parallel zur Richtung der Wellenausbreitung wirken. Daraus ergibt sich, daß im Fall 2 nur $a_{2}$ und im Fall 3 nur $a_{3}$ die möglichen Geschwindigkeitswerte sind. Zusammen mit dem zuerst genannten Argument sprechen die Energiebetrachtungen für den Geschwindigkeitswert $a_{3}$.

Weiterhin sei an das erinnert, was im Abschnitt $D$ angedeutet worden ist, daß nämlich in der Theorie des Falles 3 Ansätze enthalten sind, welche das sehr komplizierte Bild der Ausbreitung eines Impulses in einem Stab, seine Aufspaltung in eine Gruppe von aufeinanderfolgenden Wellenfronten, zu deuten vermögen.

Schließlich sei in diesem Zusammenhang auf eine andere, bekannte Tatsache hingewiesen. Die $E$-Module, welche aus seismischen Messungen unter Verwendung der Formel für $a_{2}$ erhalten werden, sind im allgemeinen größer als diejenigen, die am gleichen Gestein mittels statischer Methoden ermittelt werden. Die dynamisch bestimmten Werte liegen im Mittel um $10 \%$ über den statisch ermittelten. Um etwa denselben Betrag werden die dynamisch bestimmten Werte kleiner, wenn sie unter Verwendung der Formel für $a_{3}$ berechnet werden. Gewiß kann man sagen, der Unterschied von $10 \% 1$ läßt sich auch leicht mit dem nicht vollkommen elastischen Verhalten der Gesteine erklären. Diese zeigen Klüftung, Schiefe- 
rung, besitzen kleinere und größere Risse und sind nicht als völlig homogen anzusehen. Bevor man aber zu dieser Erklärungsmöglichkeit greift, die mehr als Lückenbüßer anzusehen ist, sollte man die Möglichkeiten ausschöpfen, die sich von der Theorie her ergeben.

\section{LITERATUR}

[1] Chree, C.: Trans. Camb. Phil. Soc. 14250 (1889)

[2] Davio, E., Schall, R. und Schardin, H.: Visualisation of Wave Propagation in PulseLoaded Bars. Artikel aus dem Buch: Kolsky, H. and Prager, W. Stress-Waves in Anelastic Solids. Springer Verlag 1964.

[3] Hunter, S. C. und Johnson, I. A.: Proceedings of the First U.S. National Congress of Applied Mechanics, New York. Amer. Soc. Mech. Engineers. S. 140 (1952)

[4] Mindlin, R. D. und Herrmann, G.: Proceedings of the First U.S. National Congress of Applied Mechanics, New York. Amer. Soc. Mech. Engineers. 187 (1952)

[5] Pochнammer, L.: J. reine angew. Math. 81324 (1878)

SCHмid, O. v.: Über Kopfwellen in der Seismik. Ztschrift Geophysik, 15 S. 141 (1939) 


\title{
ERGEBNISSE DES LAGO LAGORAI-E-PROFILS
}

\author{
P. Giese und G. De Visintini \\ (München, BRD; Triest, Italien)
}

\section{Einleitung}

Das Lago Lagorai-E-Profil ist das längste der von diesem Schußpunkt ausgehenden Profile. Bis zum Dreiländereck Italien-Österreich-Jugoslawien verläuft die Profillinie durch die Südalpen (SE-Dolomiten, Venezianische Alpen, westliche Julische Alpen). Hier wird bei Tarvisio die Periadriatische Linie (Grenze Zentralalpen - Südalpen bzw. Dinariden) geschnitten. Die folgenden Stationen der zweiten Profilhälften liegen auf der Nordseite der Karawanken und auf der südlichen Koralpe. Die letzte brauchbare Registrierung wurde in $310 \mathrm{~km}$ Entfernung bei Leibnitz/Mur gewonnen (Abb. 1).

Im Jahre 1961 beobachteten die geophysikalischen Institute aus Clausthal, Mainz, München und Triest und das Amt für Bodenforschung in Hannover auf diesem Profil. Die Sprengungen im folgenden Jahr wurden mit Refraktionsstationen nur von den Instituten aus München und Triest registriert. Die Ergebnisse einer vorläufigen Auswertung der Messungen aus dem Jahre 1961 sind bereits für alle Lago Lagorai-Profile in einer Arbeit veröffentlicht worden (Behnke, Giese, Prodehl u. de Visintini, 1962). Das Ziel der vorliegenden Arbeit ist es, an Hand der Meßergebnisse des E-Profils einige neue Gedankengänge bezüglich der Auswertung zu erläutern.

Der Auswertung liegt eine normierte Seismogramm-Montage zugrunde (Normiergeschwindigkeit $2 \mathrm{~cm} / \mathrm{s}$ ). Die Registrierungen aus dem Jahre 1961 wurden in Straßburg mit Hilfe eines Affinographen normiert (Peterschmitt, 1961), die aus dem Jahre 1962 auf photographischem Wege in München.

\section{Ergebnisse}

\subsection{Grundlagen der Auswertung}

Da auf dem Lago Lagorai-E-Profil keine Beobachtung in Gegenrichtung vorliegt, muß jede Tiefenberechnung, gleich auf welche Art sie durchgeführt wird, von der Annahme ausgehen, daß sich in horizontaler Richtung die Geschwindigkeit nicht ändert. Abb. 2 zeigt ein reduziertes Laufzeitdiagramm zwischen 0 und $170 \mathrm{~km}$ Entfernung. Es sind die ersten und markante zweite Einsätze (bis 0,5 s nach dem Ersteinsatz) eingetragen. Diese besondere Darstellungsweise zeigt deutlich, daß sich die Punkte nicht auf einer, sondern auf wenigstens 4 getrennten Laufzeitkurven mit allmählich zunehmender Durchschnittsneigung anordnen lassen. 


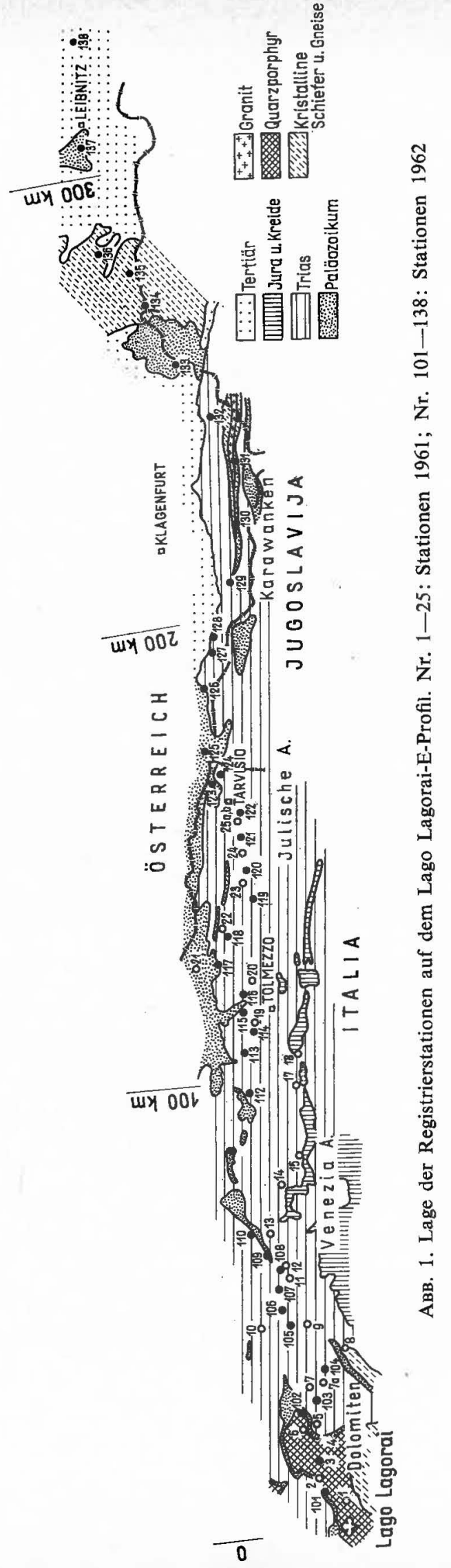


Wendet man die in der Refraktionsseismik üblichen Formeln für die Tiefenberechnung an, so ist das Ergebnis sehr unsicher, da die Geschwindigkeitsunterschiede nur sehr gering sind (eine geringe Änderung des Geschwindigkeitsverhältnisses bewirkt in diesem Falle eine starke Änderung von cos $i$ ). Da es außerdem recht wahrscheinlich ist, daß innerhalb einer Schicht die Geschwindigkeit mit der Tiefe (Druck-Temperatur-Bedingungen und zunehmender Anteil der basischen Bestandteile) zunimmt, wurde hier zur Bestimmung des Geschwindigkeitsprofils das Verfahren von Herglotz-Wiechert angewendet.

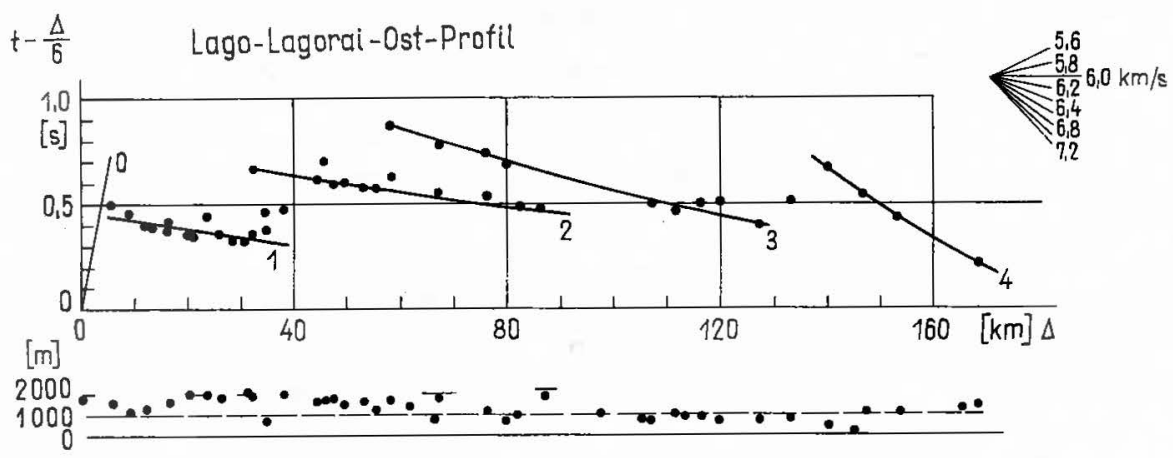

Aвв. 2. Reduzierter Laufzeitplan der ersten Profilhälfte

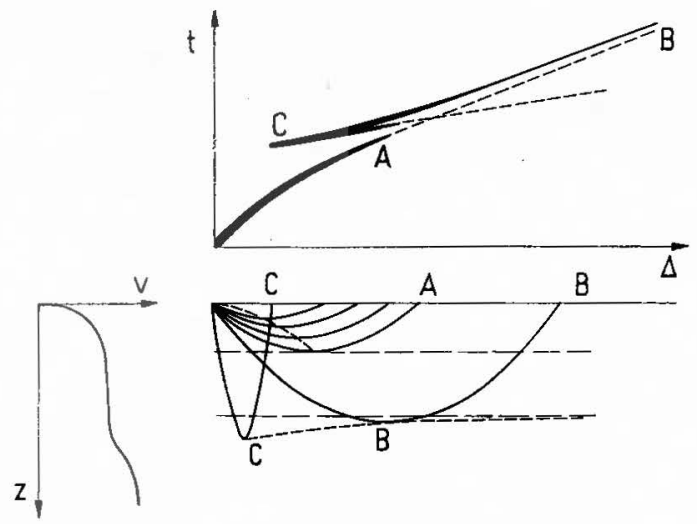

Aв8. 3. Schematische Darstellung des Strahlenverlaufs und der Amplitudenverteilung für die im linken Diagramm gezeichnete Geschwindigkeitsfunktion. Die Strichstärke der Laufzeitkurven soll ein qualitatives $\mathrm{Maß}$ für die Energieverteilung sein

Die in Abb. 2 eingetragenen Laufzeitkurven 1-4 schneiden sich nicht. Die größten Amplituden treten am Beginn jeder Kurve auf. Geht man von der Vorsstellung aus, daß sich innerhalb der Erdkruste Bereiche mit schwächerem und ștärkerem Gradienten ablösen, so folgt aus einer Intensitätsbetrachtung, ‘daß die 
beobachteten Laufzeitkurven als rückläufig zu deuten sind (Abb. 3). Daher sind durch die einzelnen Punktfolgen konkav gekrümmte Laufzeitkurven gelegt worden (Abb. 2).

\subsection{Geschwindigkeitsverteilung in der Kruste}

Die im Bozener Quarzporphyr gelaufene Welle (die eingetragene Laufzeitkurve 0 ist dem Nordprofil entnommen) wird nach etwa $4 \mathrm{~km}$ Entfernung von einer Welle 1 (gemittelte Geschwindigkeit $6,1 \mathrm{~km} / \mathrm{s}$ ) überholt. Für die Tiefenlage der Grenzfläche ergibt sich ein Wert von $1500 \mathrm{~m}$, der etwa der Mächtigkeit des Quarzporphyrs entspricht.

Bemerkenswert bei der Wellengruppe 1, die den oberen Bereich des Grundgebirges durchlaufen hat, ist das Verschwinden der Einsätze ab $30 \mathrm{~km}$ Entfernung. In Süddeutschland ist die Laufzeitkurve der Welle, die den Gneis--Granit-Bereich
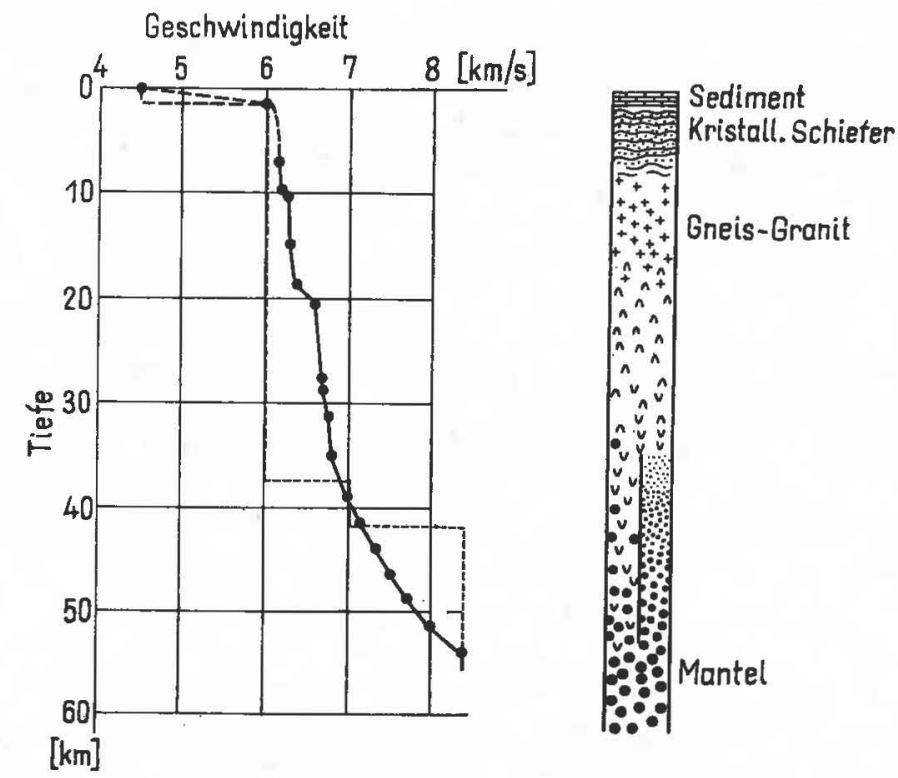

AвB. 4. Ausschnitt aus der Seismogramm-Montage zwischen $\Delta=130$ und $170 \mathrm{~km}$. Infolge der photographischen Normierung sind die Amplituden untereinander quantitativ nicht mehr vergleichbar

passiert, wenigstens $50-60 \mathrm{~km}$ lang. Da die geologischen Verhältnisse in Profilrichtung annähernd konstant bleiben, muß das Abbrechen der Laufzeitkurve 1 auf Geschwindigkeitsverhältnisse zurückgeführt werden, die sich von denen für Gnei:--Granit unterscheiden. Wie geologische Aufnahmen zeigen, bilden Quarzphyllite die Unterlage des Quarzporphyrs. Aus dem Laufzeitdiagramm ergibt sich, daß der Bereich der Phyllite etwa $8 \mathrm{~km}$ mächtig ist. - Mit 0,3 s Abstand folgt die Wellengruppe 2, die sich, da in diesem Abschnitt die Registriergeräte größten- 
teils homogen waren, phasenrichtig bis $90 \mathrm{~km}$ Entfernung verfolgen läßt (mittlere Geschwindigkeit 6,2 km/s). Die eigentliche Gneis-Granit-Zone mit einer Mächtigkeit von $8-9 \mathrm{~km}$ ist von dieser Wellengruppe durchlaufen worden.

Die Einsätze der Laufzeitkurve 3 und 4 entstammen Wellen, deren Scheiteltiefen zwischen 20 und $35 \mathrm{~km}$ liegen. In diesem Bereich steigt die Geschwindigkeit mit fast konstantem Gradienten von 6,5 auf 6,8, $\mathrm{km} / \mathrm{s}$ an (Abb. 4). Welchen Gesteinstypen diese Geschwindigkeitswerte zuzuordnen sind, muß offenbleiben. Eine scharfe Trennung zwischen einem sauren und einem basischen Gestein (z. B. Granit-Gabbro) muß auf Grund der stetigen Zunahme der Geschwindigkeit abgelehnt werden.

\subsection{Die Grenze Kruste-Mantel}

Neben der gut belegten Laufzeitkurve der im oberen Mantel (MohorovičićDiskontinuität) gelaufenen Welle (Giese u. de Visintini, 1963), sind die späteren großen Einsätze zwischen 120 und $170 \mathrm{~km}$ Entfernung (Abb. 5) von besonderem

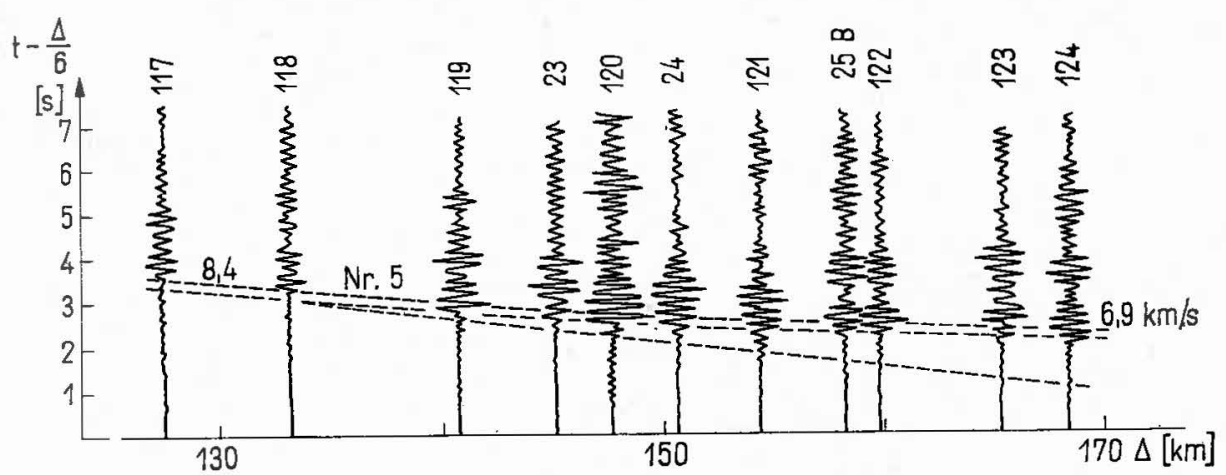

Aв8. 5. Deutungsmöglichkeiten für die in Abb. 4 dargestellte Laufzeitkurve

Interesse. Man könnte diese Laufzeitkurve mit Hilfe einer an der Conrad-Diskontinuität geführten Welle deuten. Da aber diese Kurve schwach konkav gekrümmt ist und mit der Laufzeitkurve der im oberen Mantel gelaufenen Welle bei $130 \mathrm{~km}$ Entfernung zusammen läuft, bieten sich zwei andere Deutungsmöglichkeiten an, die in Abb. 6 dargestellt sind:

1. Der nächstliegende Gedanke ist, diese Wellengruppe als Weitwinkel-Reflexion der Grenze Kruste-Mantel aufzufassen. Die großen Amplituden jenseits des kritischen Winkels ließen sich mit Hilfe der Theorie von Červený (Červený, 1962 u. 1963) erklären. Da die Geschwindigkeitsverteilung in der Erdkruste (Abschnitt 2.2.) bekannt ist, kann der Verlauf von $d \Delta / d t$ berechnet und mit dem beobachteten verglichen werden (Abb. 6).

2. $\mathrm{Da}$ keine Übereinstimmung vorliegt, wird auch diese Laufzeitkurve als rückläufig gedeutet, d. h. zwischen Kruste und Mantel nimmt in einem Bereich 
von etwa $15 \mathrm{~km}$ die Geschwindigkeit kontinuierlich von 6,8 auf $8,3 \mathrm{~km} / \mathrm{s} \mathrm{zu}$ (Abb. 4). Auf Grund dieser Deutung muß die "scharfe« Mohorovičić-Diskontinuität durch eine breite Übergangszone ersetzt werden. Diese Wellengruppe ist auf

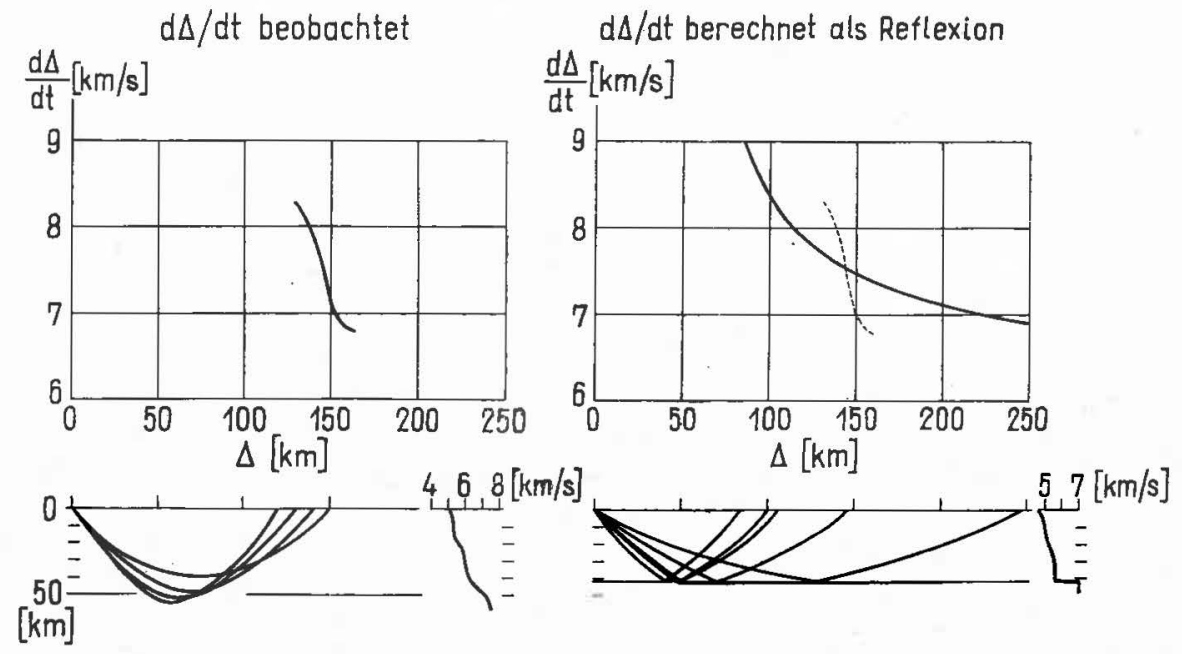

ABB. 6. Tiefen-Geschwindigkeitsfunktion für das Lago Lagorai-E-Profil, abgeleitet aus den Laufzeitkurven

sehr vielen Refraktionsprofilen auch aus außeralpinen Bereichen zu erkennen. Da sie in vielen Fällen die Grundlage für die Ableitung der Conrad-Diskontinuität gibt, bedarf dieser Begriff einer Klärung. - Der Übergangsbereich zwischen Kruste und Mantel muß entweder aus einer Mischung zwischen Krusten- und Mantelmaterial (linke Säulenhälfte, Abb. 4) bestehen, oder das Mantelmaterial unterliegt einer allmählichen Phasentransformation (rechte Profilhälfte). In jedem Falle würde diese Deutung der Laufzeitkurve eine schärfere Definition der Grenze Kruste-Mantel verlangen. Auf Grund der bisherigen Auffassung liegt die Mohorovičić-Diskontinuität in der Tiefe, in der der Geschwindigkeitswert von $8,3 \mathrm{~km} / \mathrm{s}$ erreicht wird. Wie oben aber gezeigt wurde, ist damit zu rechnen, daß auch am Aufbau der unteren Kruste noch Mantelmaterial beteiligt ist.

\subsection{Krustenmächtigkeit und geologische Entwicklung in den Südalpen}

Zum Abschluß soll auf eine Besonderheit in der Entwicklung der Erdkruste in den Südalpen hingewiesen werden. Nach der variskischen Ära regenerierte sich am Ende des Paläozoikums der Bereich, aus dem die heutigen Alpen entstanden sind. Im Gegensatz zu den Zentralalpen waren jedoch in den Südalpen die tektonische Beanspruchung und die Einengung nur sehr gering. Daher ist es überraschend, daß zwischen Zentral- und Südalpen kaum ein Unterschied 
in der Krustenmächtigkeit besteht (Schneider, 1964). In beiden Bereichen muß etwa mit einem Wert von 50-55 km gerechnet werden (Prodehl, 1964). Die Absenkung der Erdoberfläche in den Südalpen betrug während des Mesozoikums etwa $2 \mathrm{~km}$. In derselben Größenordnung liegt die Mächtigkeit der mesozoischen Sedimente auf der süddeutschen Großscholle. Doch hier liegt die Grenze KrusteMantel in einer Tiefe von nur $30 \mathrm{~km}$ (The German Research Group for Explosion Seismology, 1964). Es muß daher vermutet werden, daß sich diese Grenze zwischen Kruste und Mantel in vertikaler Richtung verschieben kann, ohne daß dabei an der Erdoberfläche größere Vertikalbewegungen und intensive tektonische Prozesse stattfinden. $\mathrm{Ob}$ der Kruste bei diesem möglichen Prozeß Material von unten oder von der Seite her zugeführt oder entzogen wird, muß vorerst offen bleiben. Im Falle Süddeutschland-Südalpen ist der vertikale Prozeß wahrscheinlicher, da Deformationen größeren Ausmaßes an der Erdoberfläche fehlen.

\section{LITERATUR}

[1] Behnke, Cl., Giese, P., Prodehl, Cl. und de Visintint, G. : Seismic Refraction Investigation in the Dolomites for the Exploration Earth's Crust in the Eastern Alpine Area 1961. Boll. di Geofisica Teor. ed Appl., 4 110-132 (1962)

[2] ČERvenÝ, V.: The amplitude curves of reflected harmonic waves around the critical point. Studia Geogr. et geophys. 5 Prag

[3] Črrvený, V.: On the position of the maximum of the amplitude curves of reflected waves. Studia Geogr. et geophys. Prag 6 (1962)

[4] Cervenx́, V.: On the length of the interference zone. Studia Geogr. et geophys. Prag 6 (1963)

[5] GIese, P. und De Visintini, G.: Vorläufige Auswertung des Lago Lagorai-Ostprofils. 2. Stuttgarter DFG-Kolloquium, 23166 (1963)

[6] Peterschmitt, E.: Flächenhafte Darstellung von Laufzeitkurven mittels normierter Seismogramme: Anwendung bei den Registrierungen der Alpensprengungen 1958. Frankfurter DFG-Kolloquium 4/1-23 (1961)

[7] ProdeHL, Cl.: Vorläufiger Tiefenlinienplan der Mohorovičić-Diskontinuität im Bayerischen Alpenvorland und in den Ostalpen zwischen München und Padua. Bad Kreuznacher DFG-Kolloquium, 974 (1964)

[8] SCHNEIDER, H.-J.: Diskussionsbeitrag. Bad Kreuznacher DFG-Kolloquium (1964)

[9] The German Research Group for Explosion Seismology: Crustal Structure in Western Germany. Ztschrift Geophys. 30 209-234 (1964) 


\title{
SUR QUELQUES PARTICULARITÉS DES ONDES LONGITUDIN ALES ENREGISTRÉES A SOFIA
}

\author{
E. Grigorova et S. Rijikova
}

(Sofia, Bulgarie)

Cette étude comporte les observations faites sur les phases d'ondes longitudinales enregistrées sur la base de séismes arrivés des distances de 130 à $830 \mathrm{~km}$ pour la période 1947-1957.

On n'a utilisé que des enregistrements d'une seule station, celle de Sofia, obtenus à l'aide du séismographe "Wiechert" à deux composantes horizontales avec des constantes moyennes $V=200, T_{0}=9 \sec \varepsilon: 1=4$.

On a observé uniquement des séismes peu profonds dont la plus grande partie avait des azimuts de $100^{\circ}$ à $260^{\circ}$, c'est-à-dire des tremblements de terre en Asie Mineure, et ceux dans la mer Egée et en Grèce. La figure 1 représente la carte avec les épicentres des séismes étudiés.

Dans tous les séismes observés après le temps d'arrivée des ondes longitudinales $P_{n}, P_{b}$ et $P_{g}$ avant que la première phase transversale $S$ soit observée, on remarque trois phases brusquement émergeantes des ondes longitudinales marquées par $P_{1}, P_{2}$ et $P_{3}$ (Fig. 2). Le plus souvent l'onde $P_{1}$ se trouve aussi dans les tremblements de terre à tout intervalle observé des distances épicentrales. L'onde $\boldsymbol{P}_{2}$ se trouve à une distance de $\Delta>240 \mathrm{~km}$. L'onde $P_{3}$ est observée à une distance qui ne dépasse pas $560 \mathrm{~km}$.

L'intensité de toutes les trois phases $P_{1}, P_{2}$ et $P_{3}$ est supérieure à celle de l'onde $P_{g}$, ainsi le rapport moyen de leurs amplitudes maxima est:

$$
\frac{A_{P_{1}}}{A_{P_{g}}}=1.45 ; \quad \frac{A_{P_{2}}}{A_{P_{g}}}=1.51 ; \quad \frac{A_{P_{3}}}{A_{P_{g}}}=1.56 .
$$

Les hodographes des ondes $P_{1}, P_{2}$ et $P_{3}$, ainsi que celles de $P_{n}, P_{b}$ et $P_{g}$ sont calculés au moyen de la formule

$$
\tau=a+\frac{\Delta}{v}
$$

où $\tau$ est le parcours de chaque onde étudiée, $v$ sa vitesse et le coefficient $a$ le temps de parcours des ondes pour $\Delta=0$.

A cet effet sont utilisées les données de Bulletin International de Strasbourg, International Seismological Summary, ainsi que les données littéraires des coordonnées des épicentres, le temps dans le foyer, la distance épicentrale et la profondeur du foyer. 
La méthode de Gutenberg étant appliquée, les déviations du parcours moyen ont été trouvées dans l'intervalle choisi pour $d \Delta$ (dans ce cas on a choisi $20 \mathrm{~km}$ comme intervalle commensurable avec les déviations dans la détermination de $\Delta$ ).

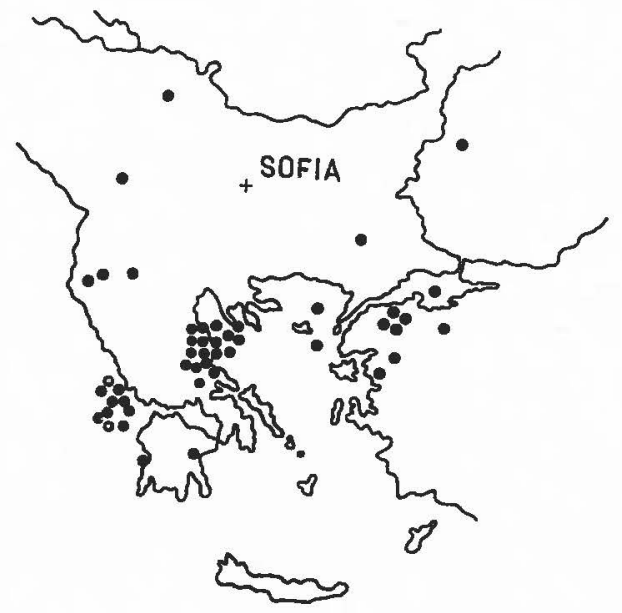

FIG. 1

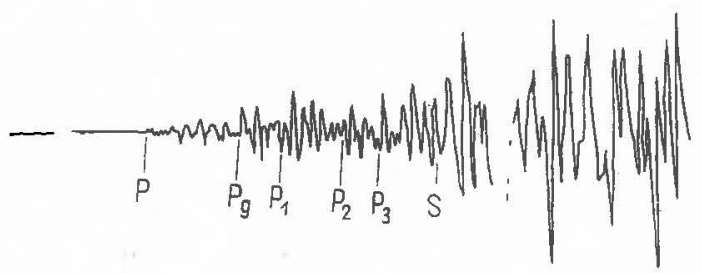

FIG. 2. Enregistrement de séismogramme $(E-W)$ du tremblement de terre en Grèce le 3. I. $19550107 \quad 03$

Au moyen de la méthode des moindres carrés, on a déterminé les inconnus $r$ et $a$ pour toutes les six ondes. Les coefficients obtenus et les vitesses figurent dans le tableau 1.

Tableau 1

\begin{tabular}{c|c|c|c|c|c|c} 
& $P$ & $P_{b}$ & $P_{\mathrm{g}}$ & $P_{1}$ & $P_{2}$ & $P_{3}$ \\
\hline$a$ & 12,5 & 4,9 & 0,2 & 4,0 & 1,7 & 1,2 \\
\hline 0 & 8,28 & 6,62 & 5,56 & 5,21 & 4,70 & 4,37
\end{tabular}




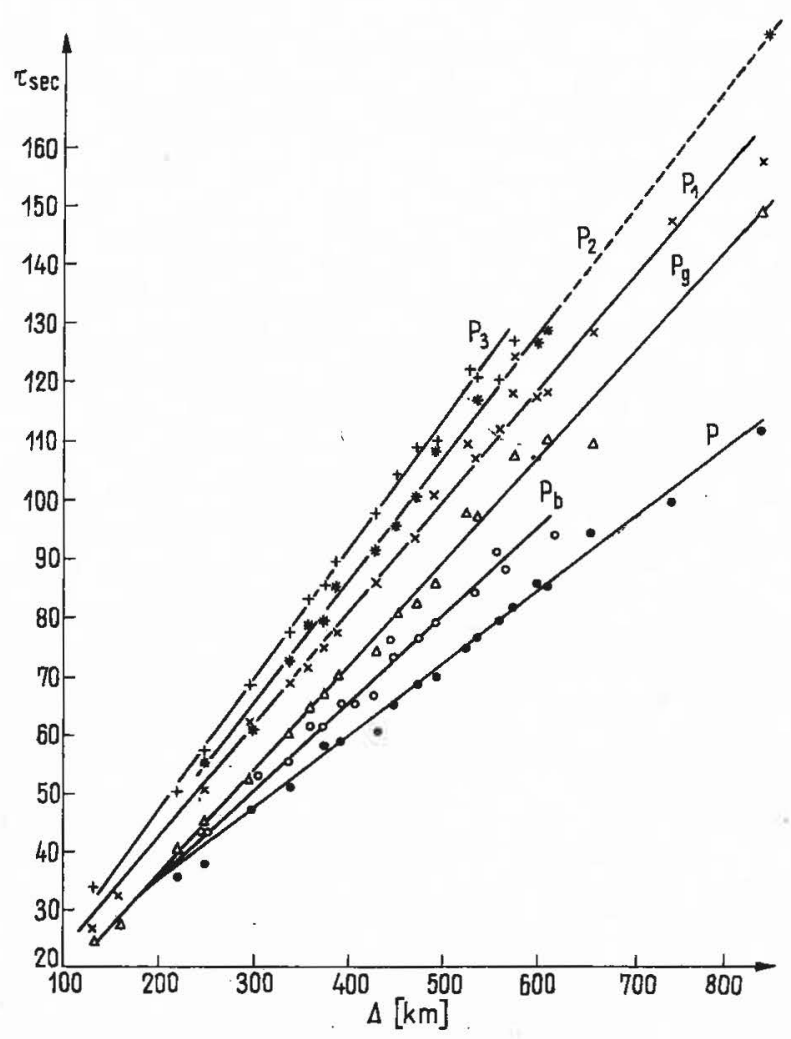

Fig. 3. Hodographes des ondes longitudinales pour Sofia. Les points désignent les parcours moyens pour tous les $20 \mathrm{~km}$ déterminés par des observations. Les hodographes calculés théoriquement sont désignés par de gros traits

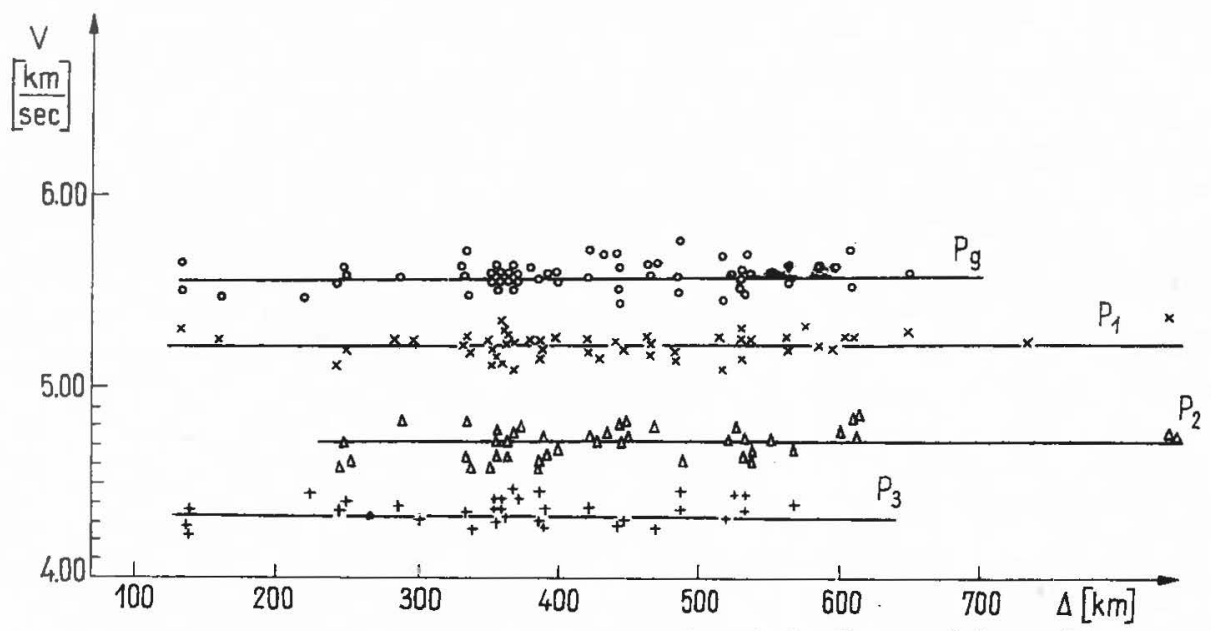

FIG. 4. Répartition de la vitesse en fonction de la distance épicentrale 
La figure 3 représente les hodographes obtenus ainsi et montre qu'ils coïncident avec les parcours moyens, pour tous les $20 \mathrm{~km}$, déterminés grâce aux observations.

On a déterminé empiriquement la dépendance $v=v(\Delta)$ (Fig. 4) autant des ondes $P_{1}, P_{2}$ et $P_{3}$ que de $P_{g}$ à titre de comparaison. Il est évident que la vitesse de ces ondes ne change pas en raison de la distance, il en est de même pour $\boldsymbol{P}_{g}$, tout au moins pendant l'intervalle étudié de la distance épicentrale. Cela démontre que les ondes étudiées se propagent dans le même milieu.

On a essayé de déterminer approximativement la profondeur de la croûte terrestre, ainsi que l'épaisseur de la couche de granit sur la base des hodographes construits en ce qui concerne $P_{n}, P_{b}$ et $P_{g}$.

On a utilisé la dépendance de Gutenberg

$$
t_{1}-t_{2}=\frac{\sqrt{h^{2}+\Delta^{2}}}{v_{1}}-\frac{\Delta}{v_{2}}-(2 d-h) \sqrt{\frac{1}{V_{1}^{2}}-\frac{1}{V_{2}^{2}}},
$$

où $t_{1}$ et $t_{2}$ représentent le temps d'arrivée des ondes longitudinales dans les deux couches, $v_{1}$ et $v_{2}$ les vitesses respectives, $d$ la puissance cherchée.

$\mathrm{Si}$ on partage les épicentres étudiés en quelques groupes d'après leurs azimuts (Fig. 1) pour l'épaisseur moyenne de la croûte dans les directions différentes, les résultats suivants sont obtenus:

$$
\begin{array}{rrrrrr}
\text { Pour } A_{z} & \text { de } & 100^{\circ} & \text { à } & 160^{\circ} & d_{1}=43 \pm 5 \mathrm{~km} \\
A_{z} & \text { de } & 160^{\circ} & \text { à } & 195^{\circ} & d_{1}=47 \pm 4 \mathrm{~km} \\
A_{z} & \text { de } & 195^{\circ} & \text { à } & 215^{\circ} & d_{1}=48 \pm 5 \mathrm{~km} \\
A_{z} & \text { de } & 215^{\circ} & \text { à } & 310^{\circ} & d_{1}=35 \pm 5 \mathrm{~km} .
\end{array}
$$

Pour les groupes des azimuts mentionnés on obtient les valeurs numériques respectives de l'épaisseur du granit:

$$
\begin{array}{llll}
A_{z}=100^{\circ} & \text { à } & 160^{\circ} & d_{2}=20 \pm 4 \mathrm{~km} \\
A_{z}=160^{\circ} & \text { à } & 195^{\circ} & d_{2}=17 \pm 3 \mathrm{~km} \\
A_{z}=195^{\circ} & \text { à } & 215^{\circ} & d_{2}=18 \pm 3 \mathrm{~km} \\
A_{z}=215^{\circ} & \text { à } & 310^{\circ} & d_{2}=15 \mathrm{~km} .
\end{array}
$$

\section{RÉFÉRENCES}

[1] International Seismological Summary, Kew Observatory, for 1945, 1946, 1947, 1948, 1949 , $1950,1951,1952,1953,1954,1955$

[2] Bulletin Séismique International de Strasbourg, Bureau Central International de Séismologie 1955, 1956, 1957

[3] Gutenberg, B.: Travel time curves at small distances and wave velocities in S. California, Gerl. Beitr. zur Geoph. 35 (1932)

[4] Rozova, E. A.: Stroenie zemnoj kori v Srednej Azii. Trudi Seismitcheskovo Instituta, $A N$ SSSR, 94 (1939)

[5] Gutenberg, B.: Handbuch der Geophysik, 4, p. 67 


\section{GRUPPENGESCHWINDIGKEITSDISPERSION KURZPERIODISCHER OBERFLÄCHENWELLEN VON EURASISCHEN ERDBEBEN}

D. GüTH

(Jena, DDR)

Die Frage nach dem Aufbau der Erdkruste und des Erdmantels besitzt gegenwärtig in der Fachwelt besonderes Interesse. Da der Verlauf der Dispersionskurven von den Dicken der Schichten und ihren elastischen Eigenschaften abhängt, stellt die Untersuchung der Dispersion von Erdbeben-Oberflächenwellen eine Methode zur Ermittlung der Schichtstruktur dar. In vorliegender Arbeit wird aus Registrierungen an den Stationen Jena, Moxa, Potsdam und Stuttgart die Dispersion von Oberflächenwellen im Periodenbereich 5 bis $30 \mathrm{~s}$ für Erdbeben aus Asien und Südeuropa mittels der Gruppengeschwindigkeitsmethode untersucht. Da zur Bestimmung der Erdkrustendicke aus der Grundmode der untersuchte Periodenbereich nicht ausreicht, wurden auch die höheren Moden ausgewertet, soweit sie registriert worden sind. Die Epizentren der verwendeten asiatischen Beben liegen in der Provinz Sinkiang in China und den benachbarten Gebieten. Die Wellenwege nach den Stationen gehen größtenteils durch Tiefebene. Aus Südeuropa wurden einige griechische Beben gewählt. Auf ihrem Weg durchlaufen die Wellen größtenteils Gebirge.

\section{Vergleichsmodelle}

Zum Vergleich mit den empirischen Werten wurden Dispersionskurven für die in folgender Tabelle aufgeführten Schichtmodelle von Dorman [10], [8] verwendet.

TABELLE 1

\begin{tabular}{|c|c|c|c|c|c|}
\hline Schicht & $\alpha[\mathrm{km} / \mathrm{s}]$ & $\beta[\mathrm{km} / \mathrm{s}]$ & $\rho\left[\mathrm{g} / \mathrm{cm}^{3}\right]$ & $h[\mathrm{~km}]$ & $\begin{array}{c}\text { Modell } \\
\text { Dorman }\end{array}$ \\
\hline $\begin{array}{l}\text { Sedimente } \\
\text { Granit } \\
\text { Gabbro } \\
\text { Peridotit }\end{array}$ & $\begin{array}{l}3,98 \\
6,15 \\
6,58 \\
8,14\end{array}$ & $\begin{array}{l}2,30 \\
3,55 \\
3,80 \\
4,70\end{array}$ & $\begin{array}{l}2,340 \\
2,817 \\
2,922 \\
3,300\end{array}$ & $\left.\begin{array}{r}2,105 \\
17,895 \\
20,000 \\
\infty\end{array}\right\}$ & 8043 \\
\hline $\begin{array}{l}\text { Granit } \\
\text { Gabbro } \\
\text { Peridotit }\end{array}$ & $\begin{array}{l}6,15 \\
6,58 \\
8,14\end{array}$ & $\begin{array}{l}3,55 \\
3,80 \\
4,70\end{array}$ & $\begin{array}{l}2,817 \\
2,922 \\
3,300\end{array}$ & $\left.\begin{array}{c}20,000 \\
20,000 \\
\infty\end{array}\right\}$ & 8012 \\
\hline $\begin{array}{l}\text { Granit } \\
\text { Gabbro } \\
\text { Peridotit }\end{array}$ & $\begin{array}{l}6,15 \\
6,58 \\
8,14\end{array}$ & $\begin{array}{l}3,55 \\
3,80 \\
4,70\end{array}$ & $\begin{array}{l}2,740 \\
3,000 \\
3,300\end{array}$ & $\begin{array}{c}20,000 \\
20,000 \\
\infty\end{array}$ & 8007 \\
\hline
\end{tabular}


Die Modelle 8043 [10] und 8012 [10] wurden zum Vergleich mit den empirischen Rayleighwellenwerten verwendet. Sie unterscheiden sich voneinander nur darin, daß im Modell 8043 eine Sedimentschicht von ca. $2 \mathrm{~km}$ Mächtigkeit vorhanden ist und dafür die Granitschicht um diesen Betrag dünner ist. Für die Lovewellen wurde das Modell 8007 [8] verwendet. Die Geschwindigkeiten und Schichtdicken sind die gleichen wie bei dem Modell 8012 . Nur die Dichtewerte sind andere. Mit dimensionslosen Tabellen von Dorman für Modelle mit zwei Schichten über einem Halbraum [3] wurden die ersten beiden Moden berechnet.

\section{Die asiatischen Erdbeben}

Die Epizentren der untersuchten Beben sind auf Bild 1 eingezeichnet und in Tabelle 2 zusamengestellt. Die Wellen laufen nach den Stationen Jena und Stutt-

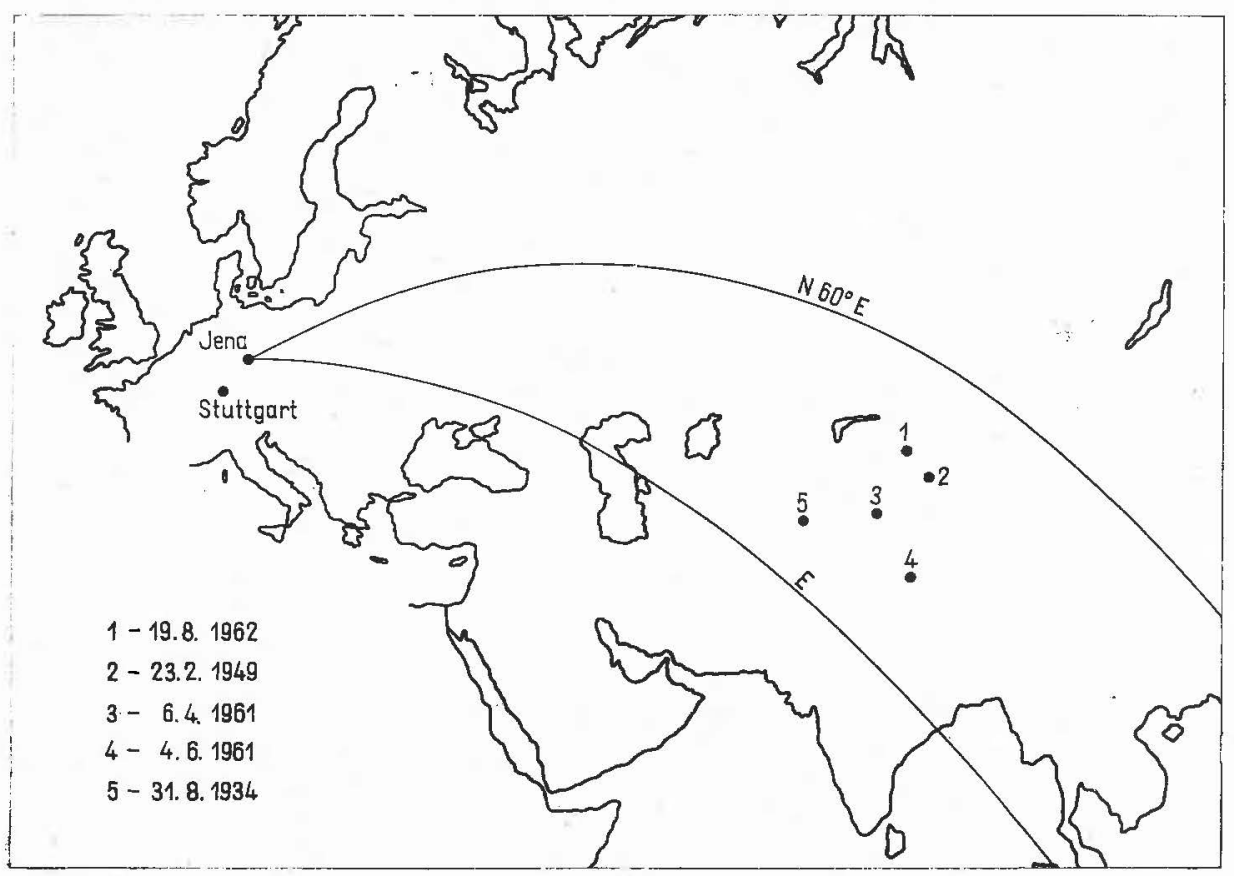

Авв. 1. Epizentren der untersuchten asiatischen Erdbeben

gart durch den südlichen Teil der Russischen Tafel. Sehr groß sind die Unterschiede der Wellenwege nach den beiden Stationen nicht. Die Epizentralentfernungen betragen 5000 bis $5800 \mathrm{~km}$. Da die Wellenwege höchstens um $20^{\circ}$ von der Ostrichtung abweichen, kann man die Lovewellen von der NS-Komponente und die Rayleighwellen von der EW-Komponente und natürlich auch von der Z-Komponente ablesen. 
TABELLE 2

\begin{tabular}{l|r|c|c|c|c}
\hline Nr. & Datum & Herdzeit & \multicolumn{2}{|c|}{ Koordinaten } & Herdgebiet \\
\hline 1 & 19.8 .1962 & $18^{\mathrm{h}} 26^{\mathrm{m}} 39^{\mathrm{s}}$ & $44,6^{\circ} \mathrm{N}$ & $81,7^{\circ} \mathrm{E}$ & Prov. Sinkiang \\
2 & 23.2 .1949 & $16^{\mathrm{h}} 08^{\mathrm{m}} 07^{\mathrm{s}}$ & $42,2^{\circ} \mathrm{N}$ & $84,1^{\circ} \mathrm{E}$ & $"$ \\
3 & 6.4 .1961 & $01^{\mathrm{h}} 33^{\mathrm{m}} 47^{\mathrm{s}}$ & $39,6^{\circ} \mathrm{N}$ & $77,8^{\circ} \mathrm{E}$ & $"$ \\
4 & 4.6 .1961 & $07^{\mathrm{h}} 33^{\mathrm{m}} 06$ & $34,1^{\circ} \mathrm{N}$ & $82,0^{\circ} \mathrm{E}$ & Tibet \\
5 & 31.8 .1934 & $14^{\mathrm{h}} 57^{\mathrm{m}} 51^{\mathrm{s}}$ & $38,7^{\circ} \mathrm{N}$ & $70,5^{\circ} \mathrm{E}$ & Alai-Gebirge \\
& & & & &
\end{tabular}

Die Herdzeit und -koordinaten sind für die Beben 2 und 5 aus [20] und für die Beben 1, 3 und 4 aus [18] entnommen.

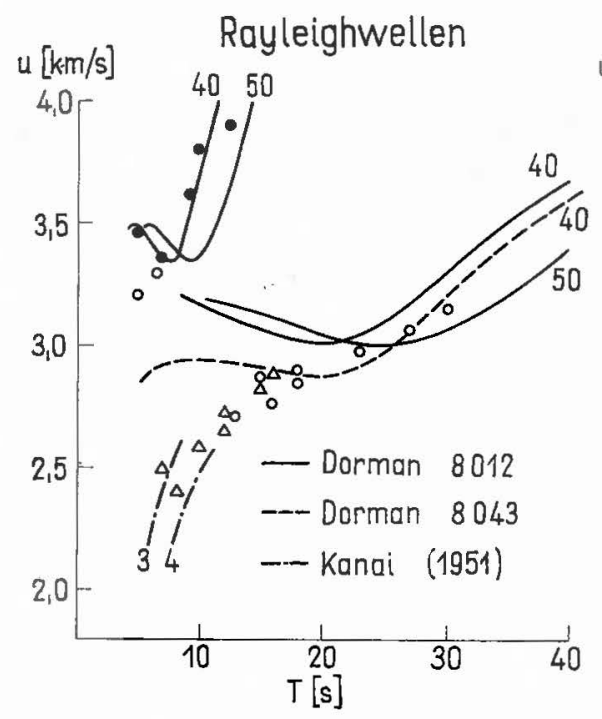

19.8.1962 Prav. Sinkiang-Jena •

6.4.1961 Prov. Sinkiang-Jena 。

4.6.1961 Tibet - Jena $\Delta$
Lovewellen

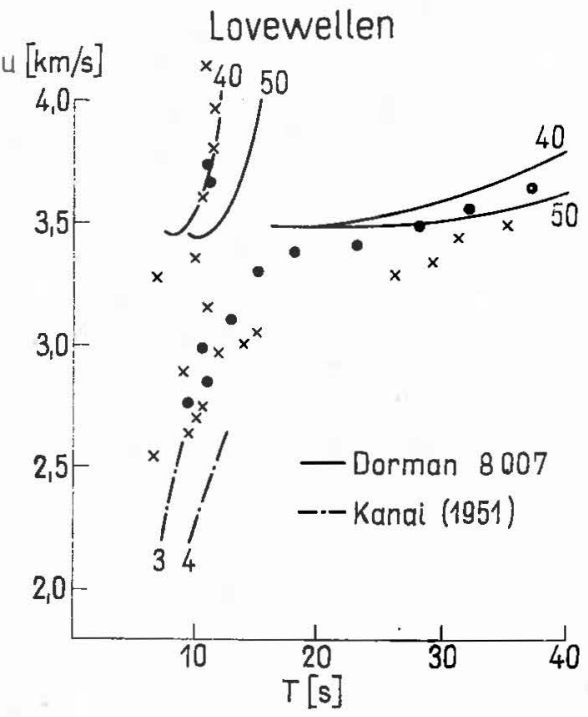

23.2.1949 Prov. Sinkiang-Jena

31.8.1934 Alai-Gebirge-Suttgart $\times$

Авв. 2. Dispersion der Oberflächenwellen von fünf Erdbeben in Asien

Die Dispersion der Rayleigh- und Lovewellen ist im Bild 2 dargestellt. Die ausgezogenen Vergleichskurven für die Rayleighwellen gehören zum Modell 8012 für 40 und $50 \mathrm{~km}$ Krustendicke. Gestrichelt ist die Rayleight-Grundmode für das Modell 8043 für $H=40 \mathrm{~km}$ eingezeichnet. Die höheren Moden der beiden Modelle unterscheiden sich im gezeichneten Periodenbereich praktisch gar nicht. Die Sedimentschicht macht sich erst bei kleineren Perioden bemerkbar.

Von einem Sinkiangbeben ist die erste Schermode ausgewertet worden. Sie ist ein regulär dispergierter Wellenzug, der ungefähr auf der Kurve für $H=40 \mathrm{~km}$ 
liegt. Er setzt sich jedoch unterhalb des Minimums noch bis zu einer Gruppengeschwindigkeit $u=3,2 \mathrm{~km} / \mathrm{s}$ fort. Dasselbe ist bei der zweiten Lovemode zu beobachten. Für zwei Beben ist die Grundmode ausgewertet. Die drei Rayleighwellenwerte mit Perioden über 20 s lassen nach der Kurve 8012 auf eine Krustendicke von ungefähr $45 \mathrm{~km}$ schließen, während nach der Kurve 8043 die Mohorovičić-

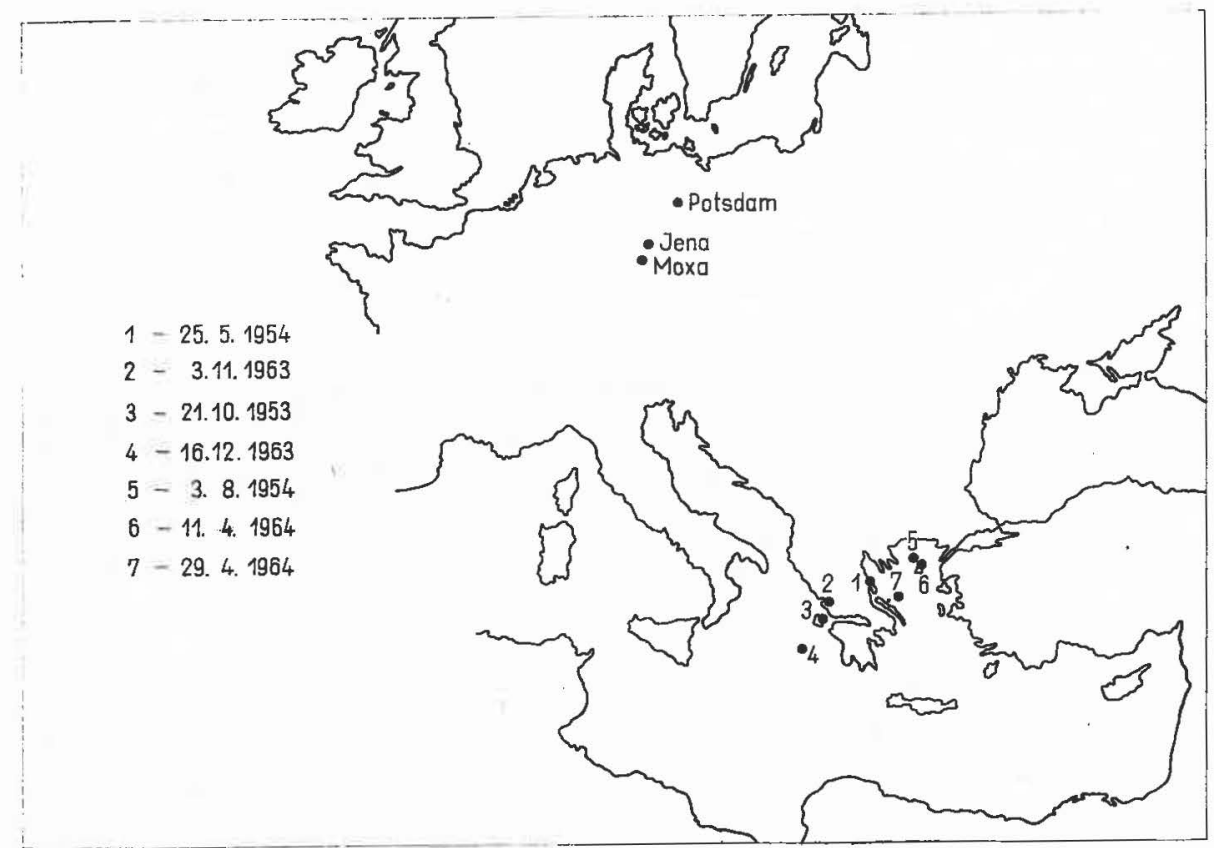

Авв. 3. Epizentren der untersuchten griechischen Erdbeben

Schicht etwa $40 \mathrm{~km}$ tief liegt, also eine Übereinstimmung mit der ersten Schermode besteht. Bei den Lovewellen liefern die Grundmode und die erste höhere Mode ebenfalls verschiedene Ergebnisse. Durch Hinzufügen einer geeigneten Sedimentschicht könnte vielleicht die Diskrepanz beseitigt werden. Diese Schicht müßte allerdings für das Sinkiangbeben und das Alaibeben verschieden sein, denn die Ergebnisse der beiden Beben stimmen nur in der zweiten Mode überein. In der Grundmode liegen die Werte des Alaibebens tiefer.

Auffällig ist sowohl bei den Rayleigh- als auch bei den Lovewellen die starke Geschwindigkeitsabnahme bei Verkleinerung der Perioden in der Grundmode. Diese Erscheinung ist auf das Vorhandensein der Sedimente zurückzuführen. Das Modell 8043 enthält eine Sedimentschicht. Die Grundmode ist von Dorman [10] bis $u=2,84 \mathrm{~km} / \mathrm{s}$ berechnet worden.

In der vorliegenden Arbeit sind zum Vergleich Kurven von Kanai [7] für ein Modell mit einer Sedimentschicht über einem Granithalbraum verwendet worden, welche von Schneider [15] zur Ermittlung der Sedimentmächtigkeit in Süddeutsch- 
land benutzt worden sind. Die Lovewellenkurven sind mit Hilfe der Nomogramme von Satô [12], [13] konstruiert worden. Für die Sedimentschicht gelten die Geschwindigkeiten $\alpha=3,5 \mathrm{~km} / \mathrm{s}, \beta=2,0 \mathrm{~km} / \mathrm{s}$, für die Granitschicht $\alpha=6,0$ $\mathrm{km} / \mathrm{s}$ und $\beta=3,5 \mathrm{~km} / \mathrm{s}$. Nach diesen Kurven beträgt die durchschnittliche Sedimentdicke für die Rayleighwellen 3 bis $4 \mathrm{~km}$ (nach Modell 8043 etwas mehr) und für die Lovewellen ungefähr $3 \mathrm{~km}$.

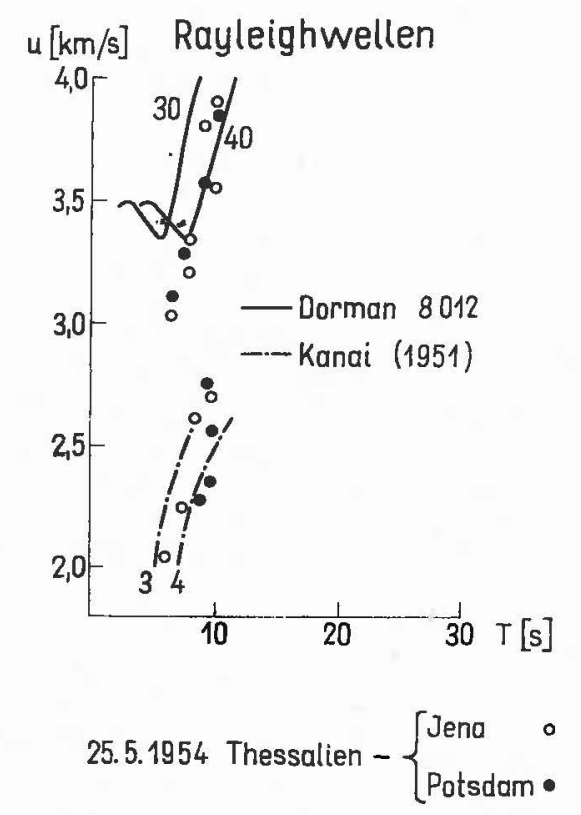

ABB. 4. Dispersion der Rayleighwellen eines Erdbebens in Griechenland

Die Kurven von Kanai sind gegenüber den Kurven 8043 von Dorman für die gleiche Sedimentdicke nach größeren Perioden verschoben. Diese Verschiebung kann (wenigstens zum Teil) mit der geringeren Sedimentgeschwindigkeit erklärt werden.

Für die Tiefe der Conrad- und Mohorovičić-Schicht in der Russichen Tafel liegen von einigen Autoren Ergebnisse vor. Für die Erdkrustendicke im zentralen und westlichen Teil werden von Šečkov [16] Tiefen zwischen 35 und $45 \mathrm{~km}$ berichtet. In der Ostukraine haben Demidenko u. a. [2] ein Absinken der MohorovičićSchicht auf 45 bis $53 \mathrm{~km}$ beobachtet. Auch nach Savarenskij u. a. [14] nimmt die Erdkrustendicke vom Zentralteil der Russischen Tafel nach Süden hin zu und erreicht im Kaukasus eine Mächtigkeit von 45 bis $55 \mathrm{~km}$. Für den gesamten Wellenweg von Sinkiang nach Stuttgart bzw. Uppsala haben Müller [9] und Kovach [8] aus Dispersionsuntersuchungen eine mittlere Krustendicke von $45 \mathrm{~km}$ erhalten. Šečkov [16] gibt für Mitteleuropa 35-40 km Tiefe für die MohorovičićDiskontinuität an. Die Tiefe der Conrad-Schicht in der Russischen Tafel ist 
von Rezanov [11] zu 10 bis $12 \mathrm{~km}$ bestimmt worden. Egorkin [4] hat für den Südwestteil der Russischen Tafel 21,5 km erhalten.

Die nach Modell 8043 erhaltene Krustendicke von $40 \mathrm{~km}$ steht $\mathrm{zu}$ diesen Daten nicht im Widerspruch. Die Conrad-Schicht liegt nach diesem Modell $20 \mathrm{~km}$ tief. Demidenko u. a. [2] geben für die Granit- und Gabbroschicht ungefähr dieselben Geschwindigkeiten wie die der verwendeten Modelle an.

Die in der Literatur angegebenen Geschwindigkeiten und Mächtigkeiten für die Sedimentschicht sind sehr unterschiedlich, so daß sich kein Mittelwert abschätzen läßt. Die Sedimentgeschwindigkeit im Modell von Kanai scheint etwas zu gering zu sein. Demnach müßte die mittlere Sedimentdicke für den untersuchten Wellenweg größer als 3 bis $4 \mathrm{~km}$ sein.

\section{Die griechischen Erdbeben}

Die untersuchten Beben sind auf der Karte (Bild 3) eingezeichnet und in Tabelle 3 zusammengestellt

TABELLE 3

\begin{tabular}{|c|c|c|c|c|c|}
\hline Nr. & Datum & Herdzeit & \multicolumn{2}{|c|}{ Koordinaten } & Herdgebiet \\
\hline 1 & 25. 5. 1954 & $22^{\mathrm{h}} 03^{\mathrm{m}} 32^{\mathrm{s}}$ & $39,3^{\circ} \mathrm{N}$ & $22,2^{\circ} \mathrm{E}$ & Thessalien \\
\hline 2 & 3.11. 1963 & 143601 & $38,9^{\circ} \mathrm{N}$ & $21,2^{\circ} \mathrm{E}$ & Ionische Inseln \\
\hline 3 & 21.10. 1953 & $\begin{array}{lll}18 & 19 & 52\end{array}$ & $38,3^{\circ} \mathrm{N}$ & $20,8^{\circ} \mathrm{E}$ & , \\
\hline 4 & 16.12. 1963 & $13 \quad 47 \quad 59$ & $37,3^{\circ} \mathrm{N}$ & $19,9^{\circ} \mathrm{E}$ & \\
\hline 5 & 3. 8. 1954 & $\begin{array}{lll}18 & 18 & 13\end{array}$ & $40,1^{\circ} \mathrm{N}$ & $24,5^{\circ} \mathrm{E}$ & Ägäisches Meer \\
\hline 6 & 11. 4. 1964 & $1600 \quad 39$ & $40,0^{\circ} \mathrm{N}$ & $25,3^{\circ} \mathrm{E}$ & , $\quad$, \\
\hline 7 & 29. 4. 1964 & 042104 & $39,0^{\circ} \mathrm{N}$ & $24,3^{\circ} \mathrm{E}$ & , \\
\hline
\end{tabular}

Für die Beben 1, 3 und 5 sind die Herdzeiten und -koordinaten aus [20] und für die übrigen Beben aus [19] entnommen.

Die Wellenwege von den Epizentren nach den Stationen sind etwas unterschiedlich, aber alle verlaufen größtenteils durch Gebirge. Den geringsten Gebirgsanteil haben die Wege vom Ägäischen Meer aus. Sie durchqueren den südwestlichen Teil der Ungarischen Tiefebene. Die Epizentralentfernungen betragen 1500 bis 1800 $\mathrm{km}$.

Bei den Thessalienbeben (Bild 4) sind nur Perioden bis $10 \mathrm{~s}$ gemessen worden. Die erste Schermode für $H=40 \mathrm{~km}$ ist dicht mit Punkten belegt. Der regulär dispergierte Wellenzug setzt sich unterhalb des Minimums von der Vergleichskurve noch fort. Diese Erscheinung ist bei den Balkanbeben noch deutlicher als bei den asiatischen. Die Ursache kann nicht die Sedimentschicht sein, da sie sich erst bei noch kürzeren Perioden auswirkt. Wahrscheinlich sind die Zwischenschichten hierfür verantwortlich. Die sedimentär beeinflußten Grundmodewerte liegen zwischen den Vergleichskurven von Kanai für 3 und $4 \mathrm{~km}$ Sedimentdicke. 
Die Lovewellen konnten nicht ausgewertet werden, weil das Azimut zu stark von der Süd- oder Ostrichtung abweicht.

Die Ergebnisse der Beben von den Ionischen Inseln (Bild 5) unterscheiden sich nicht sehr von denen des Thessalienbebens. Die erste Schermode war erst ab
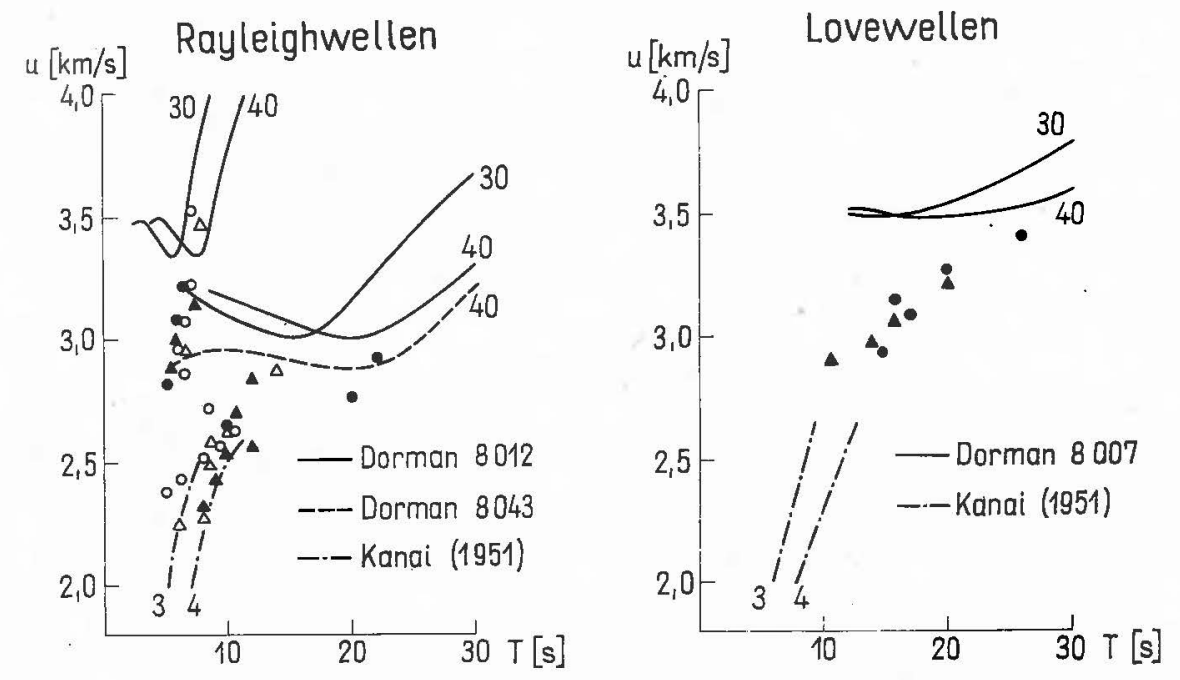

$$
\begin{aligned}
& \text { 21. 10.1953 lon. Inseln - } \begin{cases}\text { Jena } & \circ \\
\text { Potsdam } & \Delta\end{cases} \\
& \text { 3.11.1963 lon. Inseln - Moxa }
\end{aligned}
$$

Авв. 5. Dispersion der Oberflächenwellen dreier griechischer Erdbeben

$u=3,5 \mathrm{~km} / \mathrm{s}$ zu erkennen. Der Wellenzug läßt sich verfolgen' bis $u=2,8 \mathrm{~km} / \mathrm{s}$. Der sedimentär beeinflußte Teil der Grundmode entspricht im Mittel ungefähr dem des Thessalienbebens. Die beiden Punkte mit längeren Perioden genügen nicht, um die Krustendicke zu bestimmen. Aber auf keinen Fall besteht für Modell 8012 eine Übereinstimmung der Grund- und höheren Moden.

Ein deutlicher Lovewellenzug konnte auf den Horizontalkomponenten der Station Moxa identifiziert werden. In diesem Periodenbereich überlagern sich die Love- und Rayleighgrundmoden noch nicht. Irgendwelche Aussagen über die Tiefe der Mohorovičić-Schicht oder Dicke der Sedimentschicht können aber aus diesem Stück nicht gemacht werden.

Die Dispersion von drei Beben im Ägäischen Meer ist im Bild 6 dargestellt. Die höheren Moden weisen auf eine Krustendicke von $35 \mathrm{~km}$ oder etwas weniger hin. Die Fortsetzung des Wellenzuges unterhalb des Minimums der Vergleichskurve ist nicht so deutlich wie bei den Beben von Thessalien und den Ionischen Inseln. Die Grundmode gibt für Modell 8012 etwa dieselbe Tiefe für die Mohoro- 
vičić-Diskontinuität an wie die höheren Moden. Der sedimentär beeinflußte Teil der Grundmode liegt zwischen den Kurven für $h=2$ und $3 \mathrm{~km}$.

Die empirischen Lovewellenwerte lassen auf eine andere Krustendicke, nämlich $H=40 \mathrm{~km}$ schließen. Aus den kurzperiodischen Love-Grundmodewerten ist ein sedimentärer Einfluß zu erkennen. Weiter ließ sich der Wellenzug wegen der Überlagerung mit den Rayleighwellen nicht auswerten.
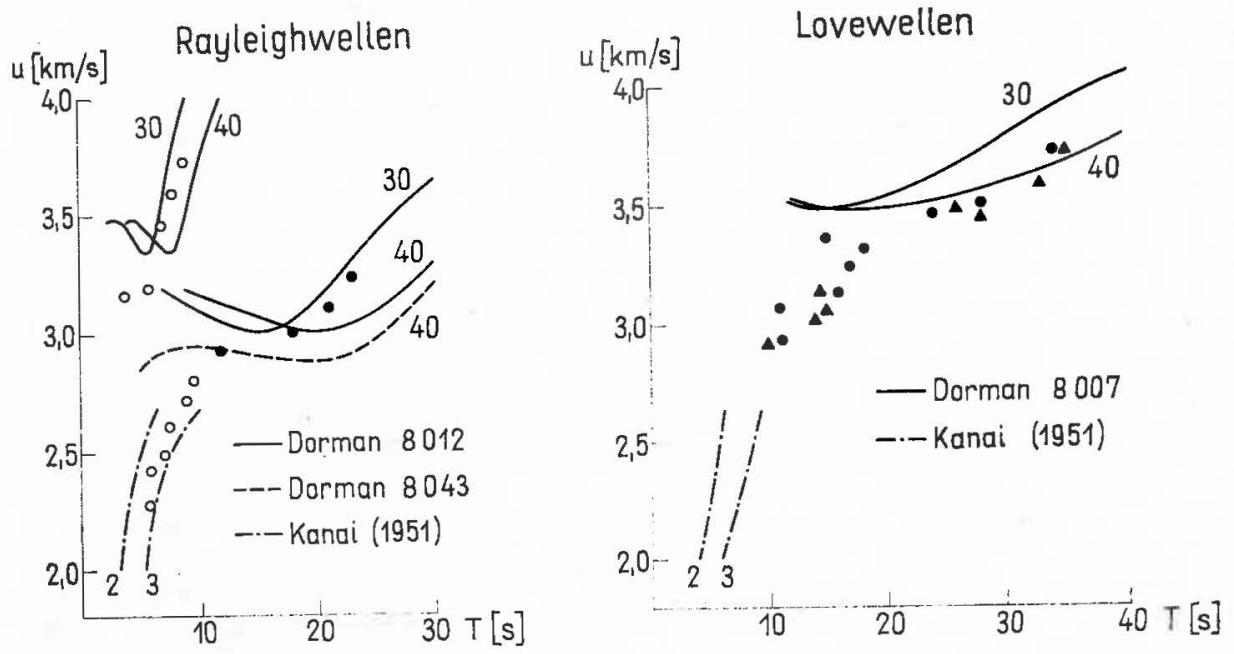

\subsubsection{4 Ägäisches, Meer - Jena 。 \\ 11. 4.1964 Ägäisches Meer-Moxa • \\ 29.4. 1964 Ägäisches Meer-Moxa \}

Авв. 6. Dispersion der Oberflächenwellen von drei griechischen Erdbeben

Die unterschiedlichen Ergebnisse aus Love- und Rayleighwellen beweisen, daß die Eigenschaften der Vergleichsmodelle nicht den mittleren Eigenschaften des Wellenweges entsprechen. Die theoretischen Kurven müßten variiert werden, bis eine Übereinstimmung der Rayleigh- und Lovewellen-Ergebnisse zugleich mit einer Übereinstimmung der Resultate aus den höheren Moden erreicht ist.

Aber bereits jetzt ist zu erkennen, daß die Krustendicke längs des Wellenweges vom Ägäischen Meer nach Moxa und Jena im Durchschnitt geringer ist als von Thessalien und den Ionischen Inseln nach diesen Stationen. Vom Ägäischen Meer aus durchqueren die Wellen den südwestlichen Teil der Ungarischen Tiefebene. Für die Ungarische Tiefebene sind Erdkrustenmächtigkeiten zwischen 20 und $40 \mathrm{~km}$ ermittelt worden [1], [5], [6], [17]. Csomor und Gálfi [1] haben festgestellt, daß die Tiefe der Mohorovičić-Diskontinuität nach Südwesten größer und nach Norden geringer wird. Von Gálfi und Stegena [6], [17] sind für die Sedimente Schergeschwindigkeiten zwischen 2,2 und $2,4 \mathrm{~km} / \mathrm{s}$ gemessen worden, also etwas größere als bei dem Modell von Kanai. 


\section{LITERATUR}

[1] Csomor, D. und Gálfi, J.: A földkéreg felépítése a Magyar Medencében az 1951. II. 20-i Nógrádi földrengés adatai szerint (The Structure of the Earths Crust in the Hungarian Basin According to the Data of the Nógrád-Earthquake on the 20 February 1951). Geofizikai Közlemények Budapest 12 49-56 (1963)

[2] ДЕмиденко,Ю. Б.; МАнютА, М. Г., ЛЫСенко, В. А. и СПихинА, Л. М.: Результаты сейсмических исследований глубинногостроения земной коры в предедах восточной Украины. Инст. Геофиз. АН УССР, Киев 57 107-114(1963) (Геофиз. Сборник)

[3] Dorman, J.: Numerical solutions for Love wave dispersion on a half-space with double surface layer. Geophysics 24 12-29 (1959)

[4] Егоркин, А. В.; Строение земной коры на юго-востоке Русской платформы. Глубинное сейсмическое зондирование земной коры в СССР, Ленинград, 145-165 (1962)

[5] Gálfi, J. und Stegena, L.: Általánosított módszer a földkéreg vastagságának megállapítására $P_{p}$ és $P_{s}$ típusú váltóhullámokkal (A Generalized Method for the Determination of Crustal Thickness by Means of $P_{s}$ and $P_{p}$ Waves). Geofizikai Közlemények Budapest 12 57-64 (1963)

[6] Gálfi, J. und Stegena, L.: Mélységi reflexiók és a földkéreg szerkezete a Magyar medencében (Deep Reflections and the Structure of the Earth's Crust in the Hungarian Plain). Geofizikai Közlemények Budapest 8 189-195 (1959)

[7] KanaI, K.: On the group velocity of dispersive surface waves. Bull. Earthqu. Res. Inst. Tokyo 29 49-60 (1951)

[8] Kovach, R.: Surface wave dispersion for an Asio-African and a Eurasian path. Jour. Geophys. Res. Richmond 64 805-813 (1959)

[9] MÜller, ST.: Untersuchungen an kontinentalen Rayleighwellen in Eurasien. Veröff. Inst. Bodendyn. Erdbebenforsch. Jena 77 165-170 (1964)

[10] Oliver, J., DoRman, J. und Sutton, G.: The second shear mode of continental Rayleigh waves. Bull. Seism. Soc. Amer. Berkeley 49 379-389 (1959)

[11] Rezanov, J. A.: Über die Beschaffenheit und die Entstehung der Basaltschicht der Erdkruste. Veröff. Inst. Bodendyn. Erdbebenforsch. Jena 77 109-112 (1964)

[12] SATô, Y.: Study on surface waves VIII. Nomogram for the phase velocity of Love waves. Bull. Earthqu. Res. Inst. Tokyo 31 81-87 (1953)

[13] Satô, Y.: Study on surface waves IX. Nomogram for the group velocity of Love waves. Bull. Earthqu. Res. Inst. Tokyo 31 255-260 (1953)

[14] Savarenskis, E. F., Popov, I. I. und Lazareva, A. P.: The long waves of the Chile earthquake of 1960 and the estimation of the thickness of the crust by phase velocities. Veröff. Inst. Bodendyn. Erdbebenforsch. Jena 77 185-192 (1964)

[15] Schneider, G.: Einfluß der Sedimentmächtigkeit auf die Dispersion von Rayleighwellen. Hermann-Aldinger-Festschrift Stuttgart 159-162 (1962)

[16] ШЕчков, Б. Н.: Дисперсии поверхностных сейсмических волн и строение земной коры Евразик. Изв. АН СССР, сер. геоф. Москва 3 313-321 (1964)

[17] Stegena, L.: Seismische Untersuchungen der Tiefenstruktur der Erdkruste in Ungarn. Studia geophys. et geodät. Praha 2 177-180 (1958)

[18] Bureau Central International de Séismologie: Bulletin mensuel, Strasbourg (1961), (1962)

[19] Bureau Central International de Séismologie: Determinations préliminaires, Strasbourg (1963), (1964)

[20] International Seismological Summary, Richmond (1934), (1949), (1953), (1954) 


\title{
INVESTIGATIONS OF THE CRUST BELOW DENMARK AND THE SEA BETWEEN DENMARK AND NORWAY
}

\author{
J. HJELME
}

(Copenhagen, Denmark)

\begin{abstract}
Summary
Since 1962 the Committee of Geophysical and Geological Investigation of the Skagerrak and the North Sea has carried out a seismic investigation of the crust below Denmark and Norway.

The investigation has been made along a line from Grimstad in Norway, crossing the Skagerrak and Jutland, and ending on Funen. In the Skagerrak, in 1962, four shots were exploded about $20 \mathrm{~km}$ off the coast of Jutland. The distances between the shots were $7 \mathrm{~km}$. The explosions were recorded on land in Norway and in Jutland and on the sea-bottom in the Skagerrak.

From the results of 1962 it was obvious that a reversed profile was needed. Then in 1964 some explosions were placed in the water between Jutland and Funen. The charges were from $250 \mathrm{~kg}$ to $3000 \mathrm{~kg}$ TNT.

Positions of shotpoints have been determined by use of Decca. By special means it was possible to obtain the necessary accuracy.

The stations from 1962 were occupied again. Besides the old stations several new ones were added. The explosions were recorded as far as Mykland, $356 \mathrm{~km}$ from the shotpoint.

The results of 1962 were unreversed velocities of $5.45,6.70$, and $8.23 \mathrm{~km} / \mathrm{sec}$. The first plot of the 1964 measurements was presented. A complete report will be published.
\end{abstract}




\title{
PATTERNS OF MAXIMUM AMPLITUDES OF $\boldsymbol{P}_{n}$ AND $P$-WAVES OVER REGIONAL AND CONTINENTAL AREAS
}

\author{
J. N. Jordan, R. A. Black and Ch. C. Bates
}

(Washington, USA)

\section{Introduction}

Seismic body wave amplitudes from explosions and earthquakes have been of interest since the turn of the century. They are pertinent to many problems of seismology such as magnitude-energy relationships, travel times, earth models, etc. From an earth model it is theoretically possible to predict the amplitude patterns from a causative mechanism. Amplitudes, of course, may be used to verify models based independently on travel times.

Since the earlier work of Wiechert (1910), many have worked on elements of the problem both from theoretical and experimental view points. Gutenberg (1926) used amplitudes as an integral part of his argument for a low velocity layer at a depth of about $80 \mathrm{~km}$. This was manifested in low amplitudes from $5^{\circ}$ to $15^{\circ}$.

Richter (1935) and Gutenberg-Richter (1935-1942, 1956) as well as many others have since developed the concept of "seismic magnitude" and published a number of curves showing the attenuation of various seismic phases with distance. These data were taken from earthquakes of undetermined focal mechanism by instruments of varying recording spectrae and calibration precision.

In order to be certain that linear effects associated with earthquake-type source were not affecting the propagation patterns, starting with the Helgoland explosion in 1946, various authors have also reported on seismic amplitude relations with distance from underground explosions. Romney's 1959 paper, for example, is a classical treatise on $P$-wave amplitude attenuation along several radial lines extending from the Logan and Blanca underground nuclear tests in Nevada during 1958. In all cases to date, however, emphasis has tended to be placed on obtaining a standardized attenuation curve with distance and on development of an omnidirectional "station correction" for signal reception. However, with the advent of numerous standardized seismic stations during the early 1960's within the United States, in Canada, and abroad, it is becoming possible to contour the maximum amplitudes of various seismic phases over large areas just as one would draw a weather map. It is the purpose of this paper to present such contoured maps for maximum amplitudes of $P_{n}$ and $P$-waves for a number of well-recorded seismic events in the unified magnitude range of four and five. Because this contouring is experimental, our explanations of the observed patterns of signal strength are to be considered tentative at this time. 


\section{Sources of Data}

The major portion of the data are from the LRSM and WWNSS seismograph programs. Data from existing institutional observatories are incorporated when applicable.

\section{Method of Signal Analysis}

In all cases, the maximum $P$ or $P_{n}$ amplitude as recorded on the short period vertical component was used. This is commensurate with the procedure called for in the determination of " $m_{b}$ " body wave magnitudes. This presents no problem for typical signals, but in the case of asymmetry or at certain distances where travel time "cusps" or manifestations of duplication of phases appear, special care had to be used to arrive at representative values.

For the purpose of this paper, the ratio, amplitude in millimicrons divided by apparent period in seconds, i.e. $\mathrm{A} / \mathrm{T}$, is used in accordance with the custom for computing unified magnitude values. This also tends to equalize disparities in instrumental response.

\section{Signal Amplitude Versus Distance Relationships}

Perhaps at the outset, it would be helpful to review several of the representative amplitude-distance relationships that have been offered to date. Figure 1 shows the results of these studies. Curve (1) in this figure is De Bremaecker's (1955) and is based on amplitudes measured from earthquakes in the western U.S. Curve (2) is the Gutenberg-Richter curve (1956) composed of earthquake data from various regions of the world and may be taken as representing a world average. In practice, this is the smoothed " $Q$ " curve of body wave magnitude determination for " $P$ ". Curve (3) is Romney's (1959) summarizing results from the Logan and Blanca underground nuclear events in the Nevada Test Site. Several things should be noted. Close-in data, i.e. that out to about $1000 \mathrm{~km}$ or so, has roughly a common origin geologically speaking in that all the authors used data from seismic sources occurring in or near belts of geologically active orogenics. In the near regional zone starting at about $150 \mathrm{~km}$, the curves have approximately the same slope but do show some offset. Other differences occur at the beginning of the shadow zone at about $1100 \mathrm{~km}$. De Bremaecker's curve shows a build up in signal level beyond the shadow zone at about $1650 \mathrm{~km}$ whereas the Gutenberg-Richter curve shows the equivalent build-up to occur at about $2100 \mathrm{~km}$. This difference of $450 \mathrm{~km}$ is probably due to the nature of the data sample. The prominent high in signal amplitude at $4400 \mathrm{~km}$ should also be noted, as well as the less well developed "highs" at about $5700 \mathrm{~km}$ and $8800 \mathrm{~km}$, the later being rather broad in scope. Intervening regions, on a world average basis, appear as 
"lows" in signal amplitude. The principal data reported on in this paper will be at continental distances, i.e. from 0 to $4500 \mathrm{~km}$ and at intercontinental distances, i.e. 6000 to $10000 \mathrm{~km}$.

Station corrections adjusting locally observed body wave magnitudes to overall average values have also been noted by Gutenberg as being necessary. Normally,

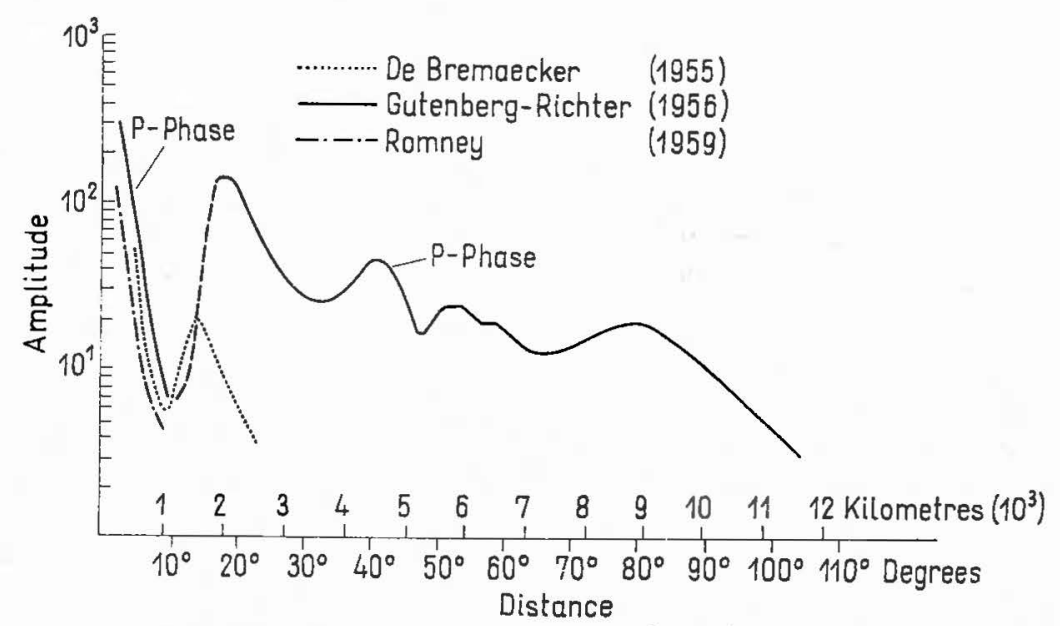

FIG. 1. Selected amplitude-attenuation data of maximum $\boldsymbol{P}_{n}$ and $P$ waves

these local corrections were considered not to require an adjustment exceeding 2.0 (doubling or halving) of the A/T ratio. Subsequent to Gutenberg's and other's formulation of these generalized concepts, Pasechnik (1962) has noted that over the Asian land mass, there is an observed decrease of about 20 percent in $P$-wave amplitudes $\left(m_{b}\right.$ of -0.1$)$ below the average for stations along the USSR's Pacific coastal region and excess of as much as 250 percent in signal amplitude ( $m_{b}$ of $+0.4)$ in the sedimentary regions of Central Asia such as Samarkand. The eleven teleseismic $P$-wave sources used by Pasechnik were megaton explosions in the Marshall Islands region at distances of 4000 to $10000 \mathrm{~km}$. The observed systematic deviations were said to be due to differences in the seismological structure of the districts of the observational stations.

Using a somewhat similar type of analysis and a suite of 130 earthquakes Guyton (1963) has found that the range of average amplitude deviation for 27 standardized seismic stations throughout the United States is about 500 percent ( $m_{b}$ range of 0.72). Amplitudes below the norm were found at stations in the Basin and Range Province and along the Pacific border, while amplitudes above the norm were found at stations on the Colorado Plateau and east of the Rocky Mountain front. Guyton concluded that the signal level was possibly being infiuenced by both a local factor, related to geologic foundation, and by a regional factor, related to regional geologic setting. 
In the instances cited to date, suites of data were averaged to obtain mean values. This practice is acceptable if the phenomenona being studied are essentially reproducible in all azimuths. To check this assumption, let us now turn to determining whether contouring of maximum $P_{n}-P$-wave amplitudes from specific seismic events supports or denies this hypothesis of seismic amplitude distribution.

\section{Seismic Amplitude Patterns from Underground Explosions at the Nevada Test Site}

Figure 2 shows the maximum $P_{n}$ and $P$-wave amplitudes for the Aardvark explosion detonated in alluvium on 12 May 1962. The average magnitude $\left(m_{b}\right)$ o $f$

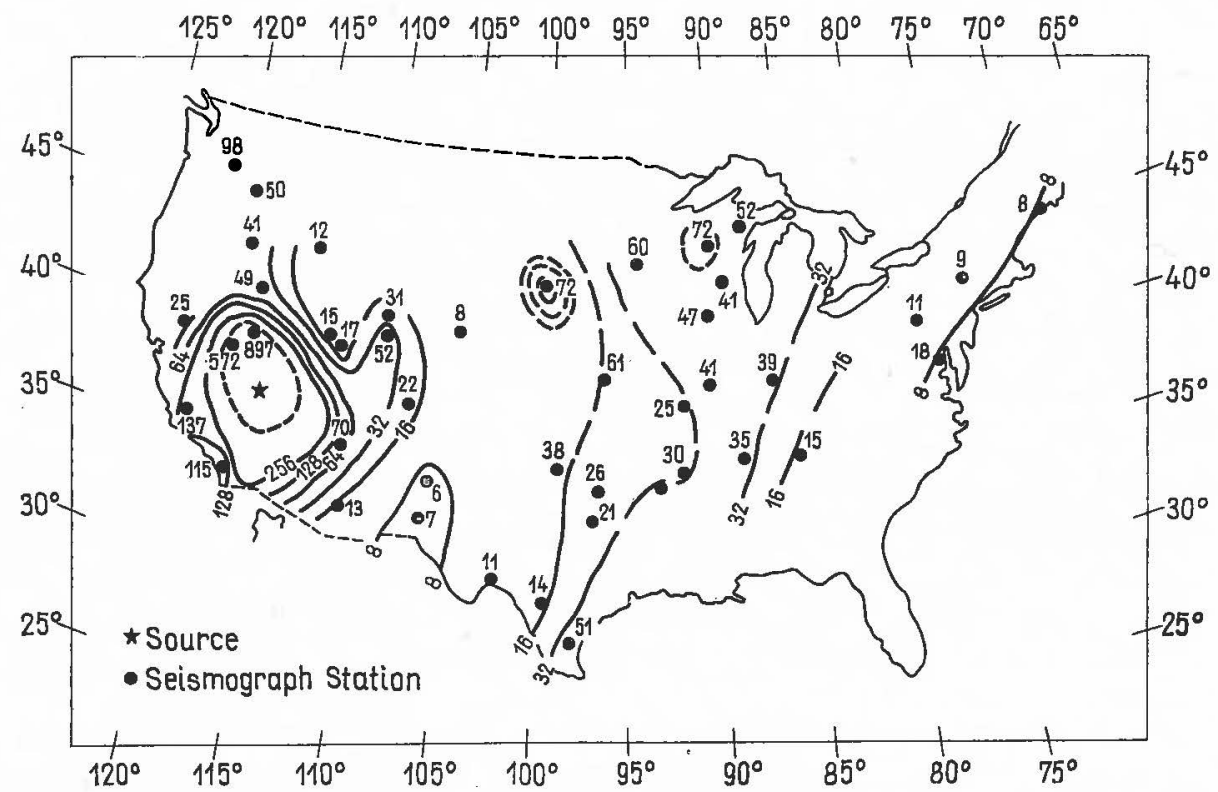

Fig. 2. Generalized contours of $P_{n}$ and $P$ maximum amplitudes $(\mathrm{m} \mu / \mathrm{sec})$ of Aardvark event

this event has been computed as 4.9. The contour interval used on this and subsequent maps is based on a geometric progression, i.e. $1,2,4,8,16 \ldots \mathrm{m} \mu / \mathrm{sec}$, because of the large extremes of signal levels measured close to and at great distances from the source. Although data were not available from large portions of the United States, the contours do show several items worth mentioning. Within a radius of $500 \mathrm{~km}$, the amplitude pattern is generally symmetrical. Beyond that distance, several anomalies appear. They are as follows:

(a) A nose of relatively high seismic amplitude extends northeastward to the Wyoming border from the otherwise symmetrical closed $128 \mathrm{~m} \mu$ contour surrounding the source. 
(b) The Gutenberg "shadow zone" with signal levels normally below $8 \mathrm{~m} \mu$ is well developed about $1000 \mathrm{~km}$ to the southeast, east, and northeast of the source. In contrast, this zone does not appear to exist northnorthwest from the source.

(c) Two single-station, high amplitude anomalies, one at Winner, South Dakota and one at Cornell, Wisconsin ( $72 \mathrm{~m} \mu$ in both cases) at teleseismic distances dominate a closed high in the North Central United States 1500 to $2500 \mathrm{~km}$ from the source.

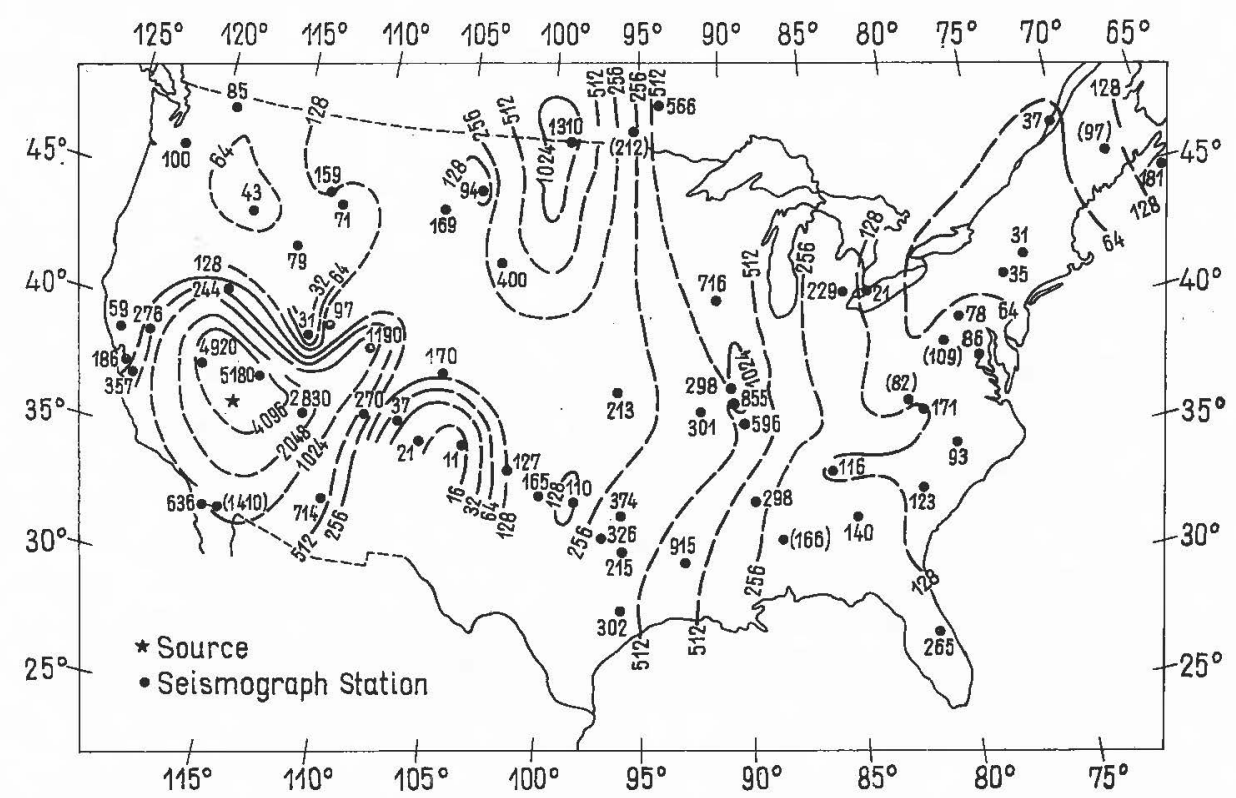

FIG. 3. Generalized contours of $P_{n}$ and $P$ maximum amplitudes $(\mathrm{m} \mu / \mathrm{sec})$ of Bilby event

(d) A second zone of low signal amplitudes with values entirely below $16 \mathrm{~m} \mu$ and sometimes below $8 \mathrm{~m} \mu$ exists along the entire Appalachian region at distances of $3000 \mathrm{~km}$ or greater. This corresponds to the predicted decay of $\boldsymbol{P}$ for this distance according to Gutenberg-Richter.

Figure 3 contours the maximum $P_{n}$ and $P$-wave amplitudes for Bilby, an underground detonation in tuff on 13 September 1963. With the average magnitude for this event being 5.8, the signal level for this event was about eight times greater than that observed for Aardvark and Haymaker, thereby making it possible to contour seismically the noisy eastern United States in much better detail than previously possible. Locations of a number of the Vela mobile seismic observatories had been changed, thereby providing additional new data for contouring regions previously having inadequate data control.

A study of Fig. 3 indicates that the general symmetry observed in the earlier events to a distance of $500 \mathrm{~km}$ also applies to Bilby, as does the nose of high 
seismic amplitudes projecting to the northeast towards Wyoming. The Gutenberg "shadow zone" is large and broad, is centered at about $1000 \mathrm{~km}$ to the south and east of the source, and contains amplitudes as low as $11 \mathrm{~m} \mu$. A smaller low amplitude closure exists to the north of the source, being separated from the "shadow zone" to the east and south by the previously mentioned northeasttrending noise of high signal levels. Prior to Bilby, the mobile observatory at the Winner, South Dakota location was moved and other points substituted in the general region. As a consequence, rather than a single point anomaly at Winner, there now appears a large zone of high seismic amplitude extending north of Winner to at least the Canadian border and centered about $1700 \mathrm{~km}$ from the source. Peak values in this zone exceed $1300 \mathrm{~m} \mu$. A second zone, though narrower, of generally high seismic amplitudes exceeding $512 \mathrm{~m} \mu$ also extended from the Gulf of Mexico north to southern Wisconsin at a distance of about $2200 \mathrm{~km}$ from the $\mathrm{Ne}$ vada Test Site. Beyond that distance, there is a repeat of the poor signal reception area so typical of the Appalachian region, with signal levels going as low as 31 $\mathrm{m} \mu$, before increasing again beyond $4000 \mathrm{~km}$ to a value of $181 \mathrm{~m} \mu$ at Halifax, Nova Scotia, a distance of $4300 \mathrm{~km}$ from the source. From what has been said so far about the repetitive amplitude patterns of $P_{n}$ and $P$-waves about the Nevada Test Site, it appears that these data support the generally held concepts regarding rapid signal attenuation within the first $500 \mathrm{~km}$ and the presence of a pronounced shadow zone centered somewhere between 700 and $1000 \mathrm{~km}$ from the source. Beyond that distance, other factors appear to be coming into play that are capable of causing marked differences in signal level at the same distances but in different azimuths. Several other events such as Haymaker, Mississippi, etc. give essentially the same picture. Let us now turn to seismic events in other locations throughout the United States to see whether these concepts continue to hold.

\section{Oceanic Event Off Southern California}

Figure 4 portrays the amplitude pattern recorded over the United States for a seismic event of unified magnitude 5.1 located approximately $700 \mathrm{~km}$ westsouthwest of San Diego, California. The typical symmetrical decrease in maximum signal amplitude is evident to a distance of nearly $800 \mathrm{~km}$. A strongly developed Gutenberg "shadow zone" is located to the east and northeast at about $1100 \mathrm{~km}$ from the source. Minimal signal values in the 3 to 5 millimicron range occur in this shadow zone, even though values as high as $870 \mathrm{~m} \mu$ were recorded only $330 \mathrm{~km}$ closer to the event, indicating a signal attenuation. Also of interest is the fact that even though highly developed in the eastern sector, the shadow zone does not exist to any demonstrable degree north of the epicenter. Where the shadow zone can be mapped, it begins to end about $1400 \mathrm{~km}$ from the source, and a 200 to $500 \mathrm{~km}$ wide plateau of signal levels exceeding $128 \mathrm{~m} \mu$ trends north-northwestward from Texas to Montana. This plateau does not appear to be symmetrical about the epicenter, for this plateau begins in south Texas about $2500 \mathrm{~km}$ from 
the epicenter, but in Wyoming, only $1750 \mathrm{~km}$ from the epicenter or for a difference in latitude of about $7^{\circ}$. Further east at about 2750 kilometers a second zone of weak reception parallels the just-mentioned band of high signal level, although in this zone, only one station (Niagara, Wisconsin) reports a signal as low as

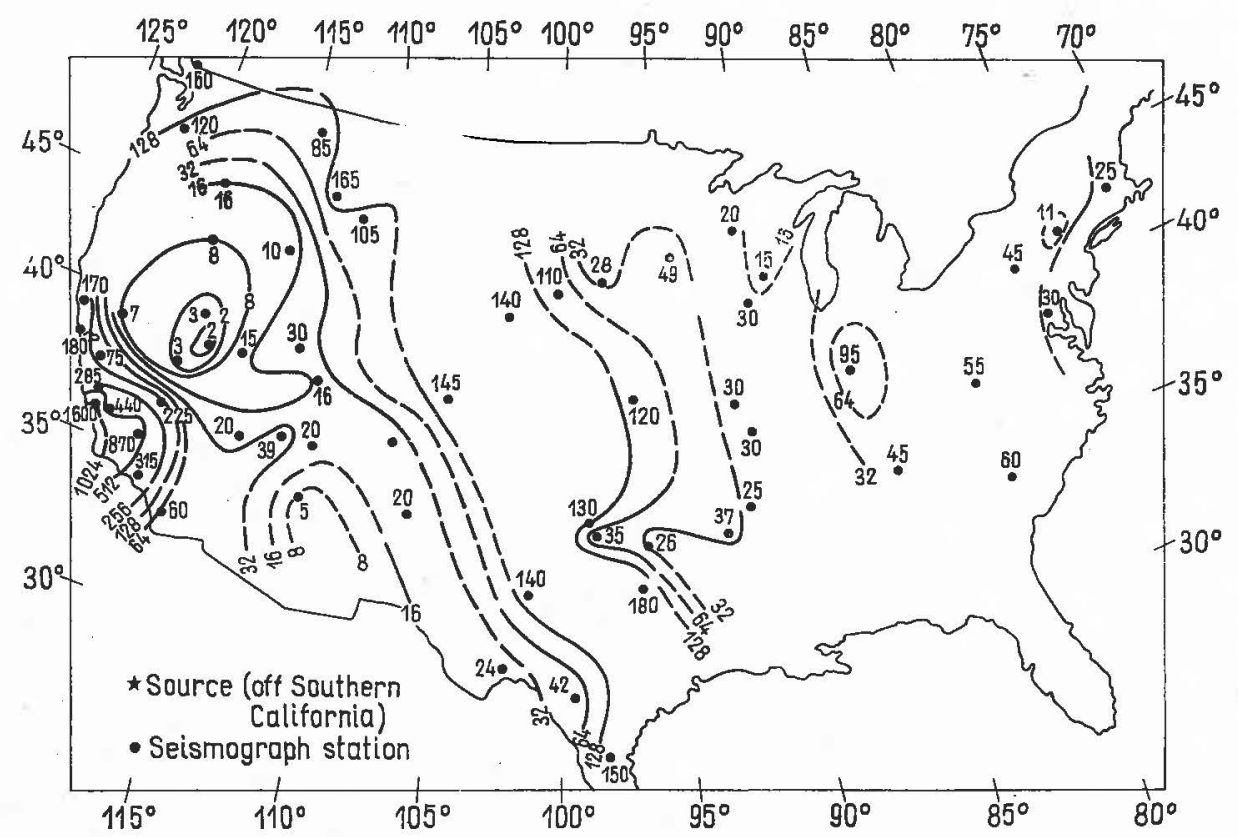

FIG. 4. Generalized contours of $P_{n}$ and $P$ maximum amplitudes $(\mathrm{m} / \mathrm{sec})$ for oceanic event off southern California

$10 \mathrm{~m} \mu$ and most values fall in the 20 to $30 \mathrm{~m} \mu$ range. Here again it might be noted that the Wichita-Ouachita Mountain trend is an area of poorer signal reception than usual, and represents a westward extension of this band of relatively weak signals at a range of about $3400 \mathrm{~km}$ and just to the west of the Appalachian, another build-up by a tapering off to about 15 to $30 \mathrm{~m} \mu$ along the Atlantic coast 3800 to $5000 \mathrm{~km}$ from the epicenter. It may be noted that these values are comparable to the $25 \mathrm{~m} \mu$ maximum signal levels reported from Mould Bay and Resolute Island, Canada $4100 \mathrm{~km}$ from the event. The Shoal event and a Fallon eartquake of similar magnitude showed about the same patterns as the NTS events.

\section{Gnome, the Nuclear Event in New Mexico}

Gnome, the underground nuclear event near Carlsbad, New Mexico, on 10 December 1961 with an average of 4.9 is of particular interest since the event 
is one of the best yet recorded in the United States. Contours of longitudinal body wave amplitudes for this event have already been published by Herrin (1962) and Romney et al. (1962). These plots demonstrated that while the "shadow zone" was highly developed $1200 \mathrm{~km}$ to the west and northwest, signal propagation

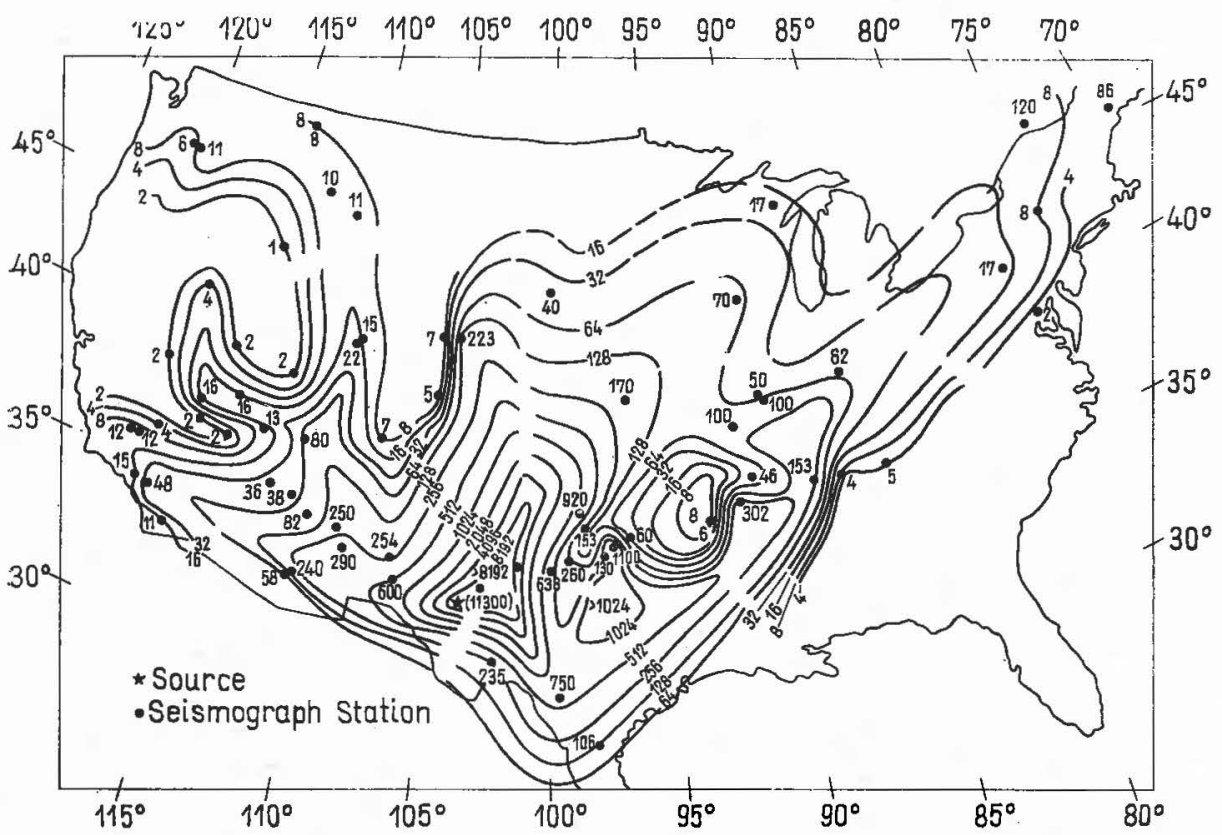

FIG. 5. Generalized contours of $P_{n}$ and $P$ maximum amplitudes $(\mathrm{m} \mu / \mathrm{sec})$ for the Gnome event

was better by a factor of as much as 150 at the same distance to the northeast. In past publications on Gnome, however, little mention has been made of the fact that a shadow effect exists about $1300 \mathrm{~km}$ to the east-northeast of Gnome roughly paralleling the western side of the Appalachian region. Thus, it would appear that it is only in the northeast quadrant of Gnome that the maximum amplitude pattern indicates very little shadow effect to at least as far as $1700 \mathrm{~km}$ from the source. For continuity's sake, we have re-contoured Gnome (see Fig. 5) and also find local evidence of some extremely tight gradients in signal level, thereby suggesting local effects complicating the regional picture. For example, in the Wichita-Ouachita complex that has been mentioned before as being an area of reduced signal strength no matter what the distance is from the source, Mountain Pine, Arkansas reports a level of $6 \mathrm{~m} \mu 1029 \mathrm{~km}$ from the source, while Conway, Arkansas reports a level of $302 \mathrm{~m} \mu$ at $1146 \mathrm{~km}$. The azimuth is essentially identical in both cases. 


\section{- Seismic Amplitude Patterns from Earthquakes in the Central United States}

So far in this paper our attention has been devoted to signals from seismic sources that occurred either surrounded by or within several hundred kilometres of major mountain orogenies of relatively recent age, geologically speaking. It

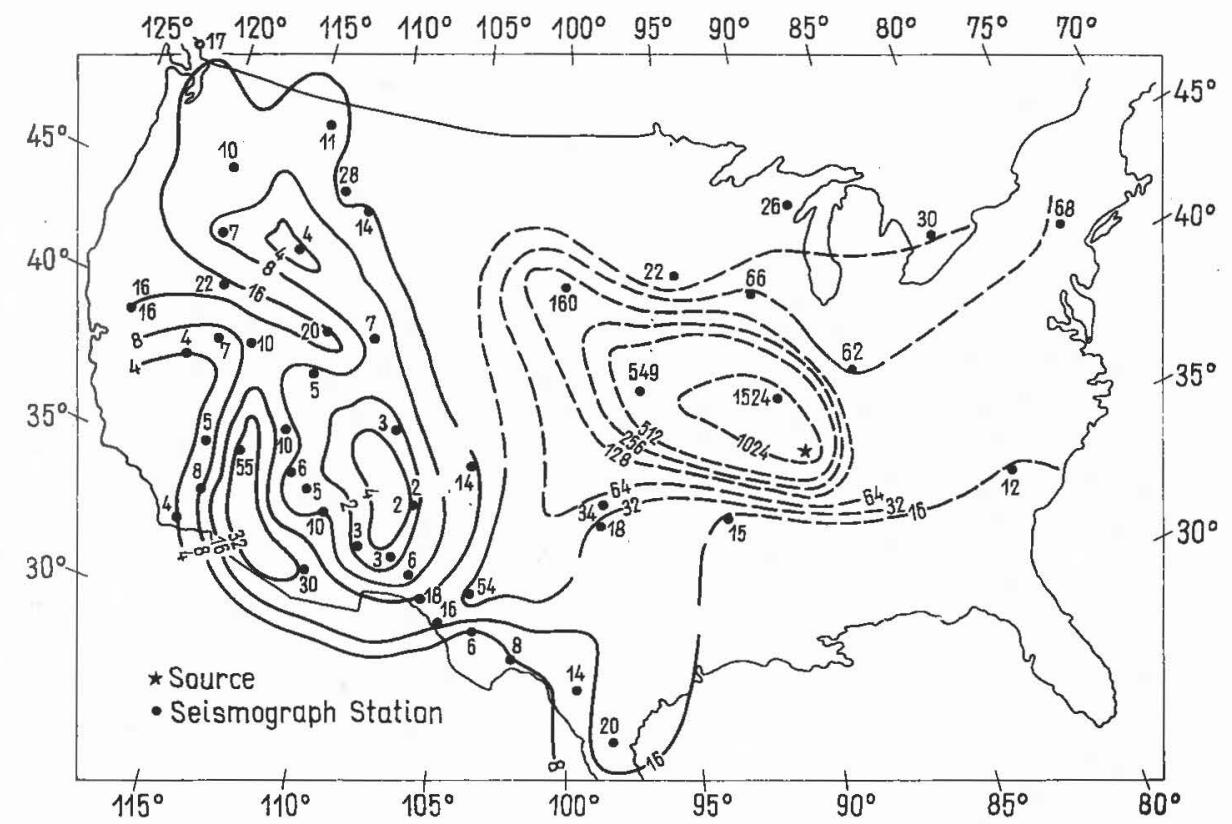

FIG. 6. Generalized contours of $P_{n}$ and $P$ maximum amplitudes $(\mathrm{m} \mu / \mathrm{sec})$ for the New Madrid L. event

is now timely to contour the maximum $P_{n}$ and $P$-wave amplitudes from seismic events occurring nearer the more stable center of the North American Continent. Figure 6 portrays the amplitude data from one such event, the New Madrid, Missouri event of February 2, 1962, which had an average body wave magnitude $\left(m_{b}\right)$ of 4.3, i.e. essentially that of the afore-mentioned Fallon, Nevada earthquake. Although there is a deficiency of data to the east of New Madrid, coverage to the west was relatively complete and the contour pattern shown is considered to be relatively reliable. The immediate zone of high amplitudes about the source appears to be oriented southeast-northwest, with rapid attenuation in the southern quadrant and lesser attenuation to the northeast and to the northwest. For example, Mountain Pine, Arkansas reported a $15 \mathrm{~m} \mu$ maxima at $400 \mathrm{~km}$ from the epicenter, while Delhi, New York at $1400 \mathrm{~km}$ and Winner, South Dakota at $1200 \mathrm{~km}$ reported 68 and $160 \mathrm{~m} \mu$, respectively. The Gutenberg shadow zone in the traditional sense does not appear to be well developed until $1650 \mathrm{~km}$ to 
the west-southwest of the source, e.g. $2 \mathrm{~m} \mu$ at Albuquerque, New Mexico, while to the northwest, the minimal signal level $(4 \mathrm{~m} \mu)$ is not reached until $2200 \mathrm{~km}$ away at Hailey, Idaho, or $600 \mathrm{~km}$ inside the traditional teleseismic zone of improved reception. Also in sharp contrast to the pattern established for $P$-wave propagation out of the Western United States is that beyond the shadow zone only one zone of markedly improved signal reception occurs at distances beyond $2000 \mathrm{~km}$ and that is a narrow zone enclosed by the $32 \mathrm{~m} \mu$ contour between Tucson, Arizona and Lake Mead, Nevada $2200 \mathrm{~km}$ from the source. Implications of these deviations from the assumed norm will be discussed later. However, it should be noted that this particular low is far more circularly oriented about the Nevada Test Site at distance of about $800 \mathrm{~km}$ than it is about the actual epicenter over $1600 \mathrm{~km}$ away in Missouri. Similarly, while the anomalous high signal level in South Dakota could possibly be related to the orientation of the fault plane, the fact remains that this South Dakota station routinely gave anomalous high values for propagation out of the Nevada Test Site.

Another shock, near Poplar Bluff, Missouri on March 3, 1963, of magnitude 4.5 , or 0.2 higher than the former, strengthened the impressions of propagation from this region. The overall pattern is about the same as for the New Madrid shock.

Other events were studied, with origins in Semipalatinsk and Algeria, for regional effects at great teleseismic distances. Brevity does not permit treatment here but it appeared that the regional affects noted herein had manifestations from these events.

\section{Summary and Conclusions}

Having described in some detail the amplitude patterns of A/T for $P_{n}$ and $P$-waves from several seismic events, it is now timely to summarize the findings to date and offer possible explanations for the phenomena observed. With respect to the Nevada Test Site events contoured earlier, all showed major low amplitude seismic anomalies to exist 700 to $1000 \mathrm{~km}$ north and southeast of the source. The shapes and exact radial distances of these shadow zones from the Nevada Test Site varied considerably from map to map. These apparent variations can, in large part, be explained by changes in number and location of recording stations between events, with consequent changes in contouring controls, and by the prevalence of extremely small amplitudes in the 1 to $8 \mathrm{~m} \mu$ range, thereby causing noise levels and seismometer plant coupling to become major disturbing factors at these low signal levels. Superimposed on this roughly concentric zone of low emplitudes about the source, however, there was a repetitive, northeastern-trending nose of high amplitudes distorting the zone and, in the case of Bilby, actually separating the zone into two discrete segments. Since this band of very low amplitude exists at essentially the correct distance from the source, postulated by Gutenberg for a "shadow zone", created by a low-velocity layer in the upper mantle, this phenomenon could easily be considered to be a fixed distance from source phenomena. 
Turning to the contour maps for the two Missouri earthquakes studied, one finds the same two regions to the north and southeast of the Nevada Test Site still showing anomalously low seismic amplitudes. Only now the distance of these two zones from their epicenters is about $1600 \mathrm{~km}$ for the region southeast of the test site, and $2300 \mathrm{~km}$ for the region to the north of the test site. Such distances are obviously quite inconsistent with the $700-1000 \mathrm{~km}$ distance of these features from Nevada Test Site sources if we are to assume the anomalies to be still expressions of the conventional Gutenberg "shadow zone". Also of note is the fact that the two well-developed low-amplitude anomalies to the west of the New Madrid earthquake are very similar in shape and exact location to those shown in the very same region for the Nevada Bilby event, even to the extent that the New Madrid anomalies are separated by a smaller, high amplitude anomaly that approximates the location of the northeasttrending nose of high signal level from the Nevada events. In our opinion, this evidence indicates the distinct possibility that two major regions exist in the western United States where extremely low signal levels cannot wholly be explained by a simple distance relationship from the source but are due at least in part to a superposition of regional effects as well. This contention is further supported by the amplitude pattern observed from the oceanic event off southern California, where very broad, well-developed anomalies of low signals $1100 \mathrm{~km}$ to the east and northeast of the source enclose the same previously mentioned low-amplitude regions.

The case for the low amplitude anomaly occupying the western half of New Mexico being at least partially due to a fixed regional effect can be strengthened by comparing contoured amplitudes observed within the region from seismic events within the United States with contoured amplitudes from seismic events occurring at great teleseismic distances, for in this case the approaching wave front must cross any low-velocity layer essentially vertically when both entering and leaving the upper mantle, rather than being refracted by it. In the case of the Semipalatinsk events where the distance between source and receiver approaches $10000 \mathrm{~km}$, there is still a low amplitude anomaly trending north from the Mexican border to Albuquerque, New Mexico that coincides nicely with the low-amplitude zone from the Nevada Test Site events, from the oceanic event off California, and from the two earthquakes in Missouri. Similarly, the southern Algerian seismic event, again nearly $10000 \mathrm{~km}$ away but in a different quadrant, confirms this particular area as being one of regional low amplitude anomaly. Geologically speaking, this anomaly occupies the Rio Grande Rift Belt, a major tectonic feature of the region. This same region is also one of Tertiary and even Quaternary volcanic rocks and therefore likely to be an area of greater than normal heat flow with a consequent reduction in upper mantle velocity. In fact, U. S. Geological Survey studies (Pakiser, informal communication, 1964) have indicated that seismic measurements do indeed demonstrate this to be a region of abnormally low $P_{n}$ velocities i.e., less than $8.0 \mathrm{~km}$ second in the upper mantle. 
ON DETERMINING THE STRUCTURE OF THE EARTH'S

CRUST OF THE LESSER CAUCASUS BY SEISMIC DATA

N. K. KARAPETIAN

(Leninakan, USSR)

The study of the structure of the earth's crust of the Lesser Caucasus is based on observations of near as well as remote stations.

A teleseismic method has been used for twelve determinations of the thickness of the earth's crust at various points of the Lesser Caucasus and its neighbouring areas. The circles in Fig. 1 indicate the sites where the thickness of the earth's crust has been determined. The numbers within the circles correspond to the value of those thicknesses. The thickness of the earth's crust of the Lesser Caucasus proved to equal $50-54 \mathrm{~km}$. The thickness of the earth's crust under the Caspian Sea, that attains $35 \mathrm{~km}$, has turned out less than on the continent. The thickness of the earth's crust on the eastern shore of the Caspian is $43-46 \mathrm{~km}$, i.e. less than that of the Caucasus.

The thicknesses of the granite and basalt layers of the Lesser Caucasus are determined by the difference in the arrival time of the direct and diffracted waves for earthquakes occurring in neighbouring areas as recorded by the stations of Tsikhis-Jevari, Tbilisi, Akhalkalaki, Nakhichevan, Kirovabad, Borzhomi, Goris and Yerevan. These determinations have averaged the thickness of the granite layer $37 \mathrm{~km}$, while that of the basalt $-15 \mathrm{~km}$.

To estimate the thickness of the earth's crust of the Lesser Caucasus the use of the existing relation between the earthquake focus energy and its dimensions is also suggested. It is assumed, in this connection, that the greatest energy of mechanical stress that could be accumulated before the outbreak of the earthquake in a certain volume of the earth's crust (the so-called volume of hypocentric area) is equal to the energy of the greatest possible earthquake in the given area. The following is the equation between energy $E$ of the most powerful earthquake in the given area and the volume $V$ of the hypocentric area:

$$
E=\frac{1}{2} e x^{2} V
$$

where $e$ is the unified modulus of elasticity of the substance of the earth's crust, $x$ is the limit of deformation of the substance of the earth's crust.

The volume of the hypocentric area can be expressed by the thickness of the earth's crust $d$. Tsuboi's [1] equation $V=9 d^{3}$ can be taken as the first approxima- 
tion. Therefore it is necessary to calculate the energy of the most powerful earthquake of the given area to determine the thickness of the earth's crust under the assumed values $e$ and $x$. Taking into account the frequency spectrum this energy is determined in the following way: the amplitude spectrum of the displacement of the ground [2] is determined by means of electronic computers. Relying on the

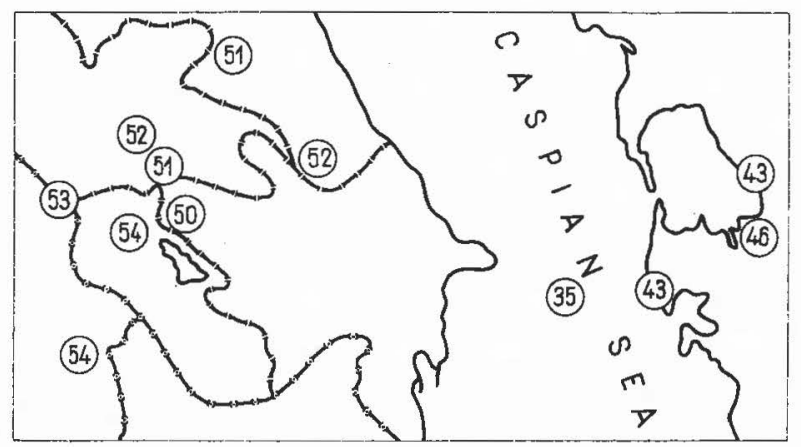

FIG, 1

amplitude spectrum of displacements of vibrations of the ground $\phi(\omega)$ the velocity spectrum is calculated, multiplying the displacement spectrum by the frequency curve $\omega$. Applying Rayleigh's theorem and making use of the obtained magnitude of the modulus of velocity spectrum, the magnitude $I$ is determined, proportional to the density of the energy current (for brevity, let us name this magnitude simply current density) by computing the integral:

$$
I=\frac{1}{\pi} \int_{0}^{\omega} \omega^{2} \phi^{2}(\omega) d \omega
$$

The value of the energy density is determined for particular components of vibrations either analytically (by means of computers) or graphically. The total density of energy is determined along all components in incident waves taking into account the influence of the earth's crust. Next the total energy of the seismic waves is computed. At the same time the energy spectrum may also be obtained; namely, the curve of the distribution of the density spectrum of energy as to frequency [3]. Let us take the determination of the energy density in low blasts as an example. Thirty-three recordings of ground displacement are analysed. They result from nine explosions in rocks, conducted with varying amounts of explosives. The recording of the displacement of the ground under the impact of seismoexplosive vibrations has been made by the seismographs of VEGIK with an approximately constant increase of the order of 1000 for periods over the range of up to $T=1 \mathrm{sec}$. The observations have been made at four points at 
a depth of 2.6-5.9 metres on fundamental basalts (the Roman numerals in Fig. 2). The depth of the instruments varied in consequence of the sharp inclination of the area under investigation. The blasts were set off in the southern and the eastern part of the investigated area (the Arabic numerals in Fig. 2). The observation points of the area were located almost along the lines S-N (I and IV) and E-W (II, I and III).

Amplitude and phase spectra, as well as energy spectra, are drawn for all the investigated recordings, i.e. curves of dependence of the density spectrum of

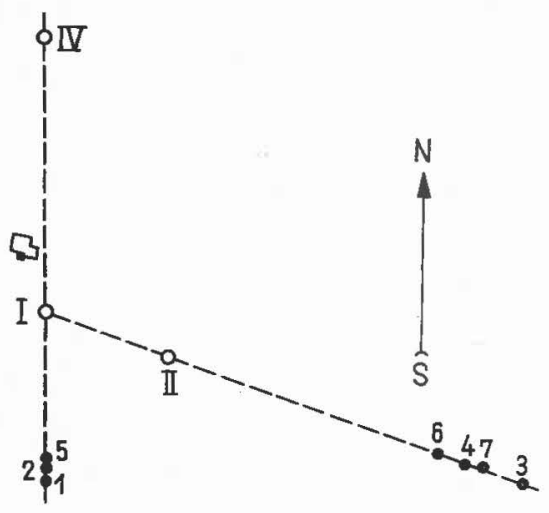

FIG. 2

energy on the frequency. The maximum values of the density spectra of the amplitude and the energy and the corresponding values of the periods are determined. The maximum values of the density spectra of the amplitude and the energy proved to correspond to the short periods. The energy density (to be more precise, the proportional density of energy) was calculated graphically. Numerically it equals the area involved in the curve of distribution of the density spectrum of energy, the axis of the abscissae and the ordinates, corresponding to $\omega=0.83 \cdot 2 \pi$ and $\omega=100 \cdot 2 \pi$. Some correlation is to be found between the energy density and the amount of the charge and also between the epicentric distance and the direction of the explosion. However, we shall not dwell on this correlation since the results have no direct bearing on the subject under discussion.

To determine the thickness of the earth's crust of the Lesser Caucasus, the calculation of the energy of elastic waves of the most powerful earthquakes in the given area is made by the seismogrammes of the stations in Yerevan, Goris and Kirovabad. The energy is calculated taking into account the frequency spectrum (with the division of the waves). No such division has been made while determining the energy of low blasts in view of the short epicentric distances.

Having at our disposal the energy of the most powerful eartquakes of the Lesser Caucasus and introducing some coefficients that take into account the intensity 
of the earthquake, the energy of nonelastic deformations and so on, the mean value of the thickness of the earth's crust of the Lesser Caucasus has been calculated by the formula (1).

\section{REFERENCES}

[1] Tsubor, C.: Journal of Physics of the Earth, 632 (1956)

[2] Karapetian, N. K.: Dokl. AN Armyanskoy SSR, 342 (1962)

[3] Karapetian, N. K.: Dokl. AN Armyanskoy SSR, 371 (1963) 
UNTERSUCHUNG DER DURCH SPRENGUNG ERZEUGTEN OBERFLÄCHENWELLEN KURZER PERIODE

Z. KIss

(Budapest, Ungarn)

\section{Einleitung}

Ein wenig bekanntes Periodengebiet der Oberflächenwellen liegt im Intervall $0,3-0,2 \mathrm{sec}$, welches dem zwischen den Perioden des sog. "ground roll« der Seismik und den allgemein registrierten Oberflächenwellen liegenden Frequenzbereich entspricht. Zur Untersuchung dieses Frequenzbereiches haben wir Messungen durchgeführt, wobei die Oberflächenwellen durch Sprengung erzeugt wurden. In dieser Arbeit werden zuerst die dispergierenden Rayleighwellen behandelt. Die ausführliche Analyse der übrigen beobachteten Wellentypen soll einer späteren Abhandlung vorbehalten bleiben.

\section{Die angewendete Meßmethode und die Apparate}

Einem $18 \mathrm{~km}$ langen Profil entlang wurden in vier Punkten Sprengungen mit verschiedenen Ladungen vollzogen und die erzeugten Wellen an einem Endpunkt des Profils beobachtet. Der Lageplan ist in Abb. 1 dargestellt.

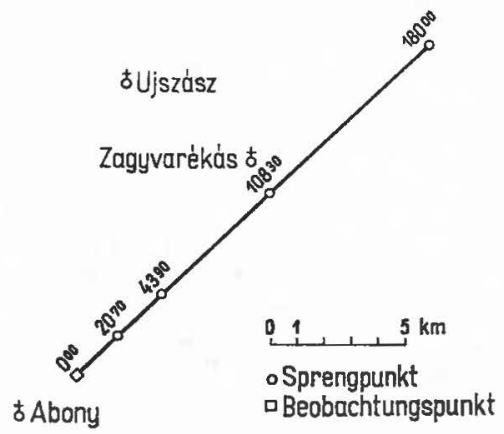

AвB. 1. Lageplan

Die Angaben der einzelnen Sprengungen sind in der später folgenden:̧ Tabelle 1 zusammengestellt.

Die Beobachtungen wurden einem elektrodynamischen 3-KomponentenSeismographensystem vom Typ VEGIK ausgeführt. Die Schwingungsrichtung der Horizontalpendel fiel in der Richtung des Beobachtungsprofils .(Komponent $H_{11}$ ) bzw. senkrecht dazu (Komponent $H_{\perp}$ ) ab. Die vertikale Komponente wird 
mit $\mathbf{Z}$ bezeichnet. Bei dieser Seismographenanordnung erscheinen die Rayleighwellen prinzipiell in der horizontalen Komponente $H_{11}$ und in der vertikalen Komponente $(Z)$, die Lovewellen dagegen in der horizontalen Komponente $H_{\perp}$.

Die Konstanten der Apparate sind die folgenden:

$$
\begin{aligned}
T_{1} & \sim 1,5 \mathrm{sec} \\
T_{2} & \sim 0,08 \mathrm{sec} \\
D_{1} & \sim 0,45 \\
D_{2} & \sim 5 \\
V & \sim 5000
\end{aligned}
$$

Index 1 bezieht sich auf das Pendel, Index 2 auf das Galvanometer. $T$ bedeutet die Eigenperiode, $D$ die Dämpfung, $V$ die Vergrößerung des Apparates. (Bei der entferntesten Sprengung benutzten wir eine Vergrößerung $V \sim 10000$.)

Für die Apparate haben wir die Kurven der Amplitudencharakteristik ( $\bar{U})$ und Phasenverschiebung ( $\tau$ ) berechnet (Abb. 2a und 2b). Die Geschwindigkeit der Registriereinrichtung betrug $20 \mathrm{~mm} / \mathrm{sec}$.*

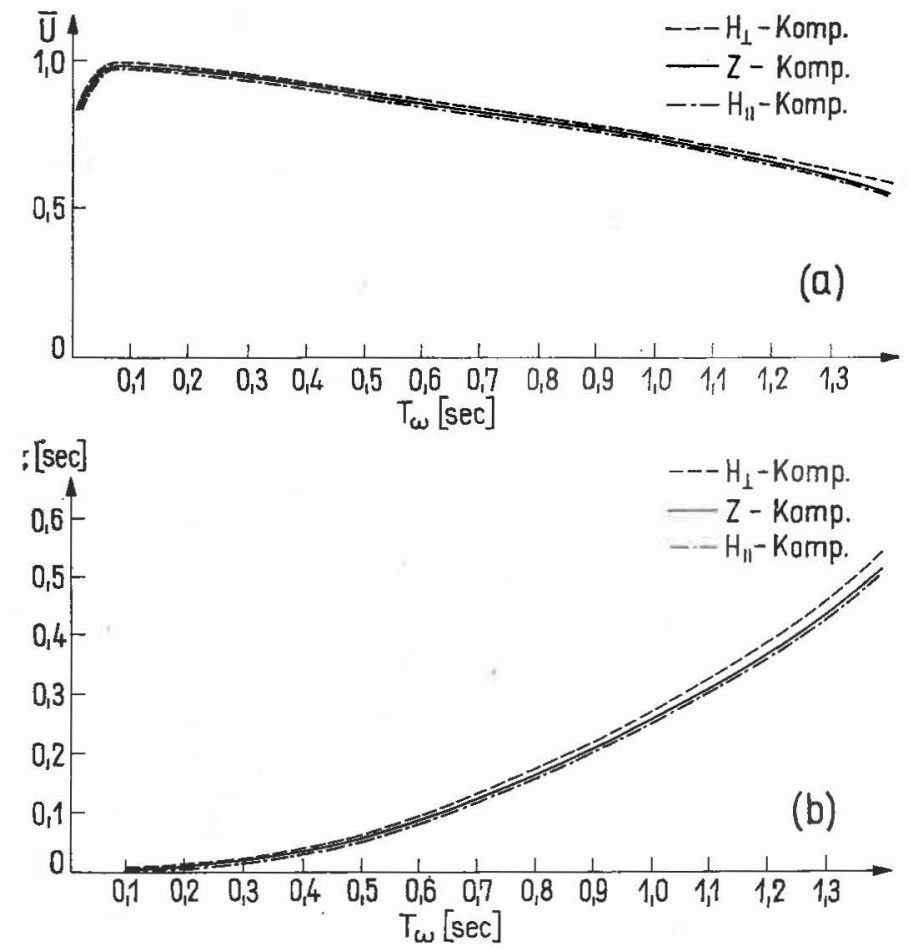

Aвв. 2. (a) Kurven der Amplitudencharakteristik (U). (b) Kurven der Phasenverschiebung $(\tau) . T_{\omega}=$ Periode der Bodenbewegung

* Die Zeitsignal-Einrichtung wurde von Dr. Pál Szemerédy konstruiert. 


\section{Auswertung der Beobachtungen}

Die Seismogramme der zwei ersten Sprengungen (siehe Tabelle 1) sind in Abb. 3 und 4 dargestellt. Die mit $x_{1}$ bezeichneten ersten Einsätze sind die an den verschiedenen Schichten refraktierten longitudinalen Wellen. Die Phase $x_{2}$ ohne

TABelle 1

\begin{tabular}{c|c|c|c}
\hline $\begin{array}{c}\text { Nummer } \\
\text { der } \\
\text { Sprengung }\end{array}$ & $\begin{array}{c}\text { Beobachtungs- } \\
\text { entfernung } \\
\Delta(\mathrm{m})\end{array}$ & $\begin{array}{c}\text { Ladungs- } \\
\text { gewicht } \\
(\mathrm{kg})\end{array}$ & $\begin{array}{c}\text { Spreng- } \\
\text { tiefe } \\
(\mathrm{m})\end{array}$ \\
\hline & 2070 & 5 & 5 \\
2 & 4390 & 20 & 8 \\
3 & 10830 & 70 & 20 \\
4 & 18000 & $4 \times 50$ & 16
\end{tabular}

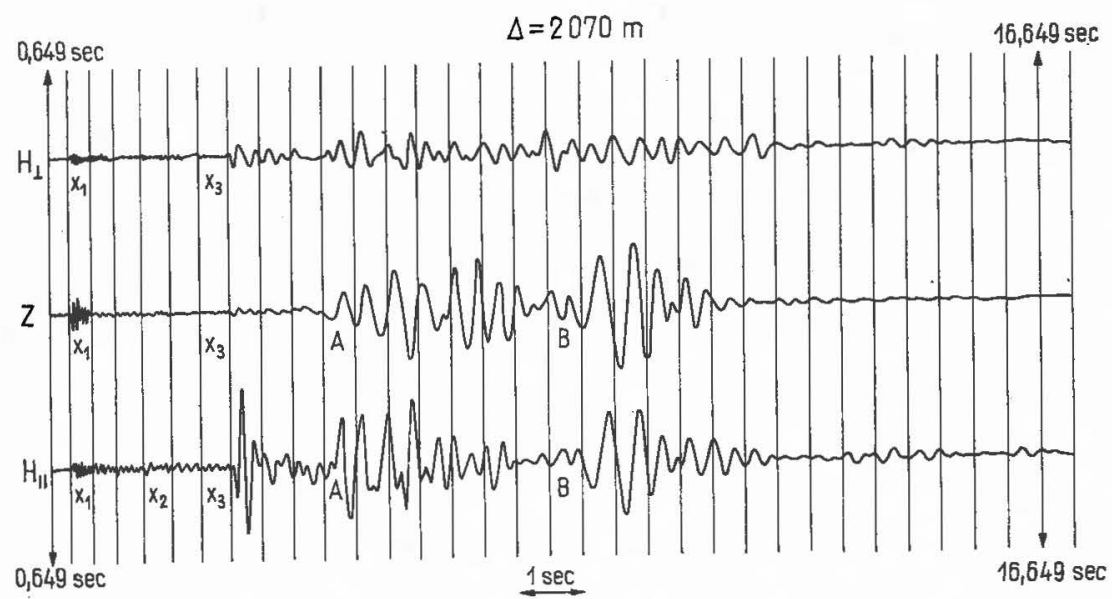

AвB. 3

vertikale Komponente erscheint als eine in der horizontalen Ebene schwingende Transversalwelle, deren scheinbare Geschwindigkeit ungefähr $780 \mathrm{~m} / \mathrm{sec}$ beträgt. Die folgende Phase $x_{3}$ hat eine ebenso kleine vertikale Komponente. Thre scheinbare Ausbreitungsgeschwindigkeit beträgt durchschnittlich $500 \mathrm{~m} / \mathrm{sec}$. Diese Phasen erscheinen in den beiden entfernten Aufnahmen unklar oder überhaupt nicht. In beiden Phasen können zwischen den einzelnen Komponenten Phasenverschiebungen wahrgenommen werden.

Die Oberflächenwellen können bezüglich der Komponenten $H_{\|}$und $Z$ in zwei gut trennbare Gruppen zerlegt werden (auf den Seismogrammen mit den Buchstaben $A$ und $B$ bezeichnet). Früheren Erfahrungen entsprechend sind - bei der 
gleichen Seismographenanordnung und Energiequelle - die Oberflächenwellen der Komponente $H_{\perp}$ schwach. Bei der normalen Sprengungsmethode der Seismik (wie auch in unserem Fall) ist die Scherbeanspruchung gering, und wir konnten deshalb in der Komponente $H_{\perp}$ keine Lovewellen beobachten. Auf diese Wellengruppe, die in den letzteren Komponenten und mit verhältnismäßig größerer Amplitude ungefähr gleichzeitig mit dem Wellenzuge $B$ eintritt, kommen wir später noch zurück.

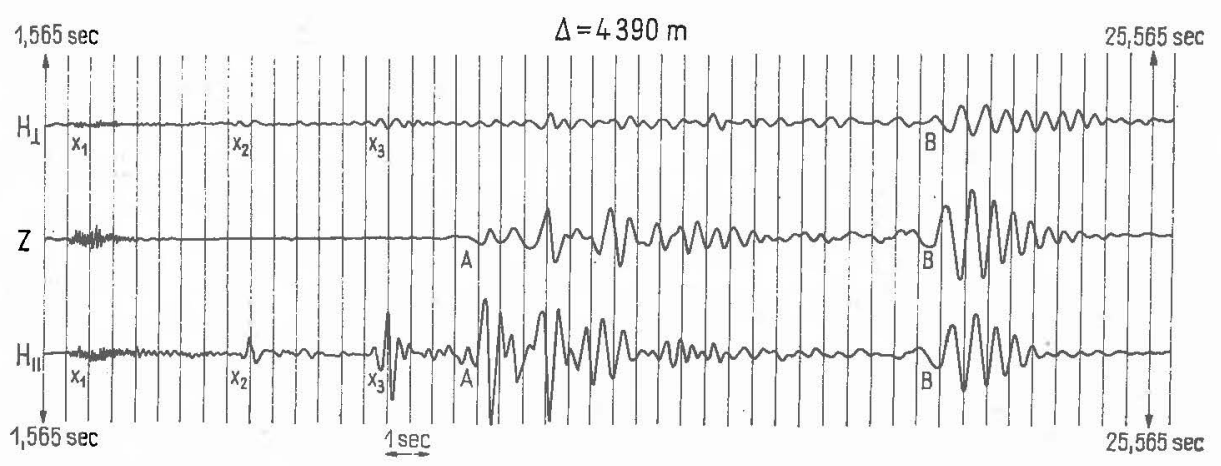

Aвв. 4

Die mit $A$ bezeichnete Oberflächenwellengruppe kann auf den Seismogrammen 1, 2, und 4 gut verfolgt werden. (Auf Seismogramm 3 konnte diese Gruppe infolge eines Registrationsfehlers leider nicht ausgewertet werden.) Die Ausbreitungsgeschwindigkeit $V_{A}$ und die Periode $T_{A}$ der ersten Einsätze sind in Tabelle 2 angegeben.

TABELle 2

\begin{tabular}{|c|c|c|c|c|}
\hline \multirow{2}{*}{$\begin{array}{l}\text { Nummer } \\
\text { der } \\
\text { Sprengung }\end{array}$} & \multirow{2}{*}{$\Delta(\mathrm{m})$} & \multirow{2}{*}{ Komp. } & $V_{A}$ & $T_{A}$ \\
\hline & & & $\mathrm{m} \mathrm{sec}^{-1}$ & $(\mathrm{sec})$ \\
\hline \multirow[t]{2}{*}{1} & 2070 & $Z$ & 352,7 (e) & 0,355 \\
\hline & & $H_{11}$ & 354,3 (i) & 0,319 \\
\hline \multirow[t]{2}{*}{2} & 4390 & $Z$ & 401,2 (i) & 0,547 \\
\hline & & $H_{\text {II }}$ & 393,9 (e) & 0,398 \\
\hline \multirow[t]{2}{*}{3} & 18000 & $Z$ & 412,7 (i) & 0,807 \\
\hline & & $H_{1 \mathrm{I}}$ & 413,5 (e) & 0,654 \\
\hline
\end{tabular}

$\mathrm{i}=$ scharfer Einsatz; e = unbestimmter Anfang

Zwischen den einzelnen Komponenten bestehen auch hier Phasenverschiebungen. Die Gestalt der Bodenbewegung, berechnet für die Anfangsstrecke der Wellengruppe $A$ in der $H_{11}-Z$-Ebene, ist in Abb. 5 dargestellt.*

* Die Berechnung der Teilchenbewegung wurde von Dr. E. Bisztricsány, Z. Kiss und K. Molnár gemeinsam ausgeführt. 
Die Teilchenbewegung wird durch eine elliptische Bahn charakterisiert, deren große Achse horizontal ist. Der Drehungssinn ist nach vorne gerichtet. Später wird die Teilchenbewegung in der Wellengruppe $A$ kompliziert. Es treten Ände-

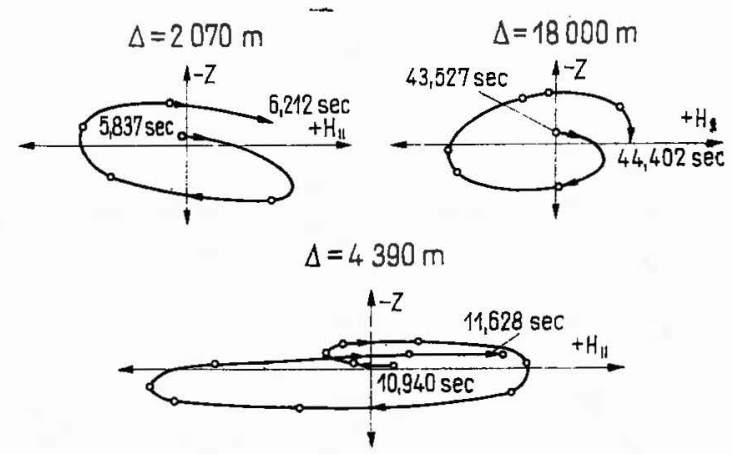

Aвв. 5. Teilchenbewegung des Anfangsabschnitts der Oberflächenwellengruppe $A$

rungen in der Drehrichtung und in den Achsenverhältnissen ein. Innerhalb der Wellengruppen erscheinen an einigen Strecken sehr schwache Dispersionen. Die Wellengruppe $A$ besteht also aus mehreren Wellentypen: dies kann aus den Seismogrammen auch unmittelbar festgestellt werden. Die erste Strecke der Gruppe $A$ ist wahrscheinlich ein sogenannter hydrodynamischer Wellentyp $(H)$ [1]. Diese Wellengruppen und die früher erwähnten Phasen $x_{2}$ und $x_{3}$ sollen in einer späteren Mitteilung behandelt werden.

In unseren Aufnahmen besteht die Oberflächenwellengruppe $B$ aus einem normalen Dispersionswellenzug. Diese Wellen erscheinen hauptsächlich in den Komponenten $H_{1 \mid}$ und $Z$ (Abb. 6). Die berechnete Bahn der Teilchenbewegung ist elliptisch, wobei das Achsenverhältnis $Z / H_{1:}$ annähernd $3 / 2$ beträgt und die Drehrichtung retrograd ist (Abb. 7 und 8).

Auf Grund dieser Tatsachen kann die Wellengruppe $B$ mit einer Rayleighwelle identifiziert werden. Es sei noch erwähnt, daß auch in den Komponenten $H_{\perp}$ Spuren von Rayleighwellen zu beobachten sind, doch können diese den durch die Inhomogenität des durchgelaufenen Mediums hervorgerufenen Wellenfrontänderungen zugeschrieben werden.

Es wurden bezüglich der Rayleighwellen Gruppengeschwindigkeitskurven für die verschiedenen Entfernungen berechnet. Die einzelnen Perioden $(T)$ und die Einsatzzeiten $(t)$ wurden in der üblichen Weise bestimmt [2]. Die Gruppengeschwindigkeit für eine Periode $T$ wurde mit Hilfe der Formel

$$
U=\frac{\Delta}{t+\tau(T)}
$$

erhalten. Hier bedeutet $\Delta$ die Beobachtungsentfernung, $\tau$ die Phasenverschiebung.

Damit wurde stillschweigend vorausgesetzt, daß das Entstehen der Rayleighwellen in der Nähe der Energiequelle erfolgt. Natürlich wäre es vorteilhafter, 


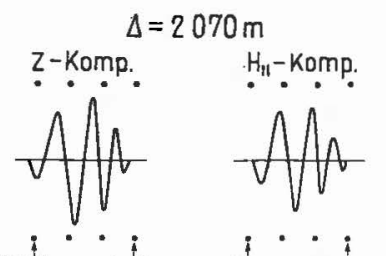

$9,649 \sec 11,149 \sec \quad 9,649 \sec 11,149 \mathrm{sec}$

z-Komp. $\Delta=4390 \mathrm{~m}$

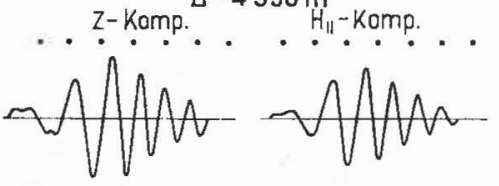

$\underset{20,565 \mathrm{sec}}{\dot{i}} \cdot \underset{23,565 \mathrm{sec}}{i} 20,565 \mathrm{sec} \cdot \dot{23,565 \mathrm{sec}}$
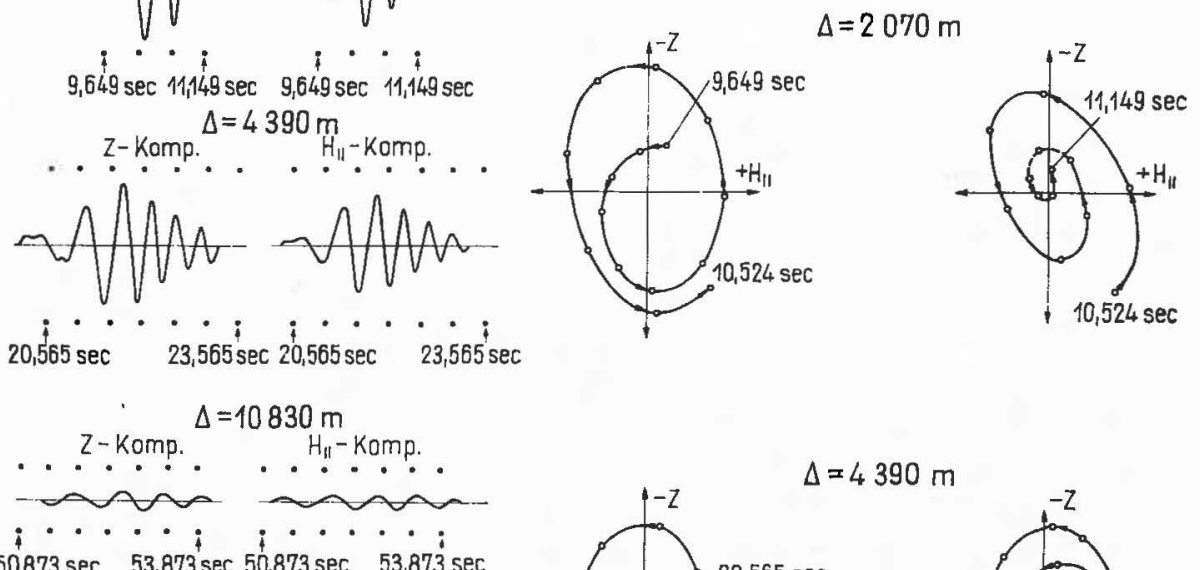

$50,873 \mathrm{sec} \quad 53,873 \mathrm{sec} 50,873 \mathrm{sec} \quad 53,873 \mathrm{sec}$
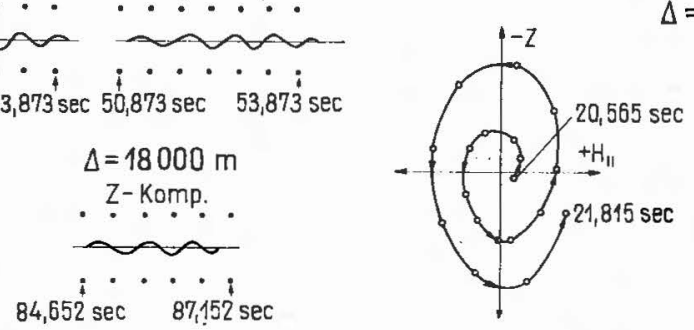

$\Delta=4390 \mathrm{~m}$

Авв. 6. Beobachtete Rayleighwellen mit Dispersion (Wellengruppe $B$ )

AвB. 7. Teilchenbewegung der Wellengruppe $B$ (Rayleighwelle) $\Delta=2070 \mathrm{~m}$ und $\Delta=4390 \mathrm{~m}$
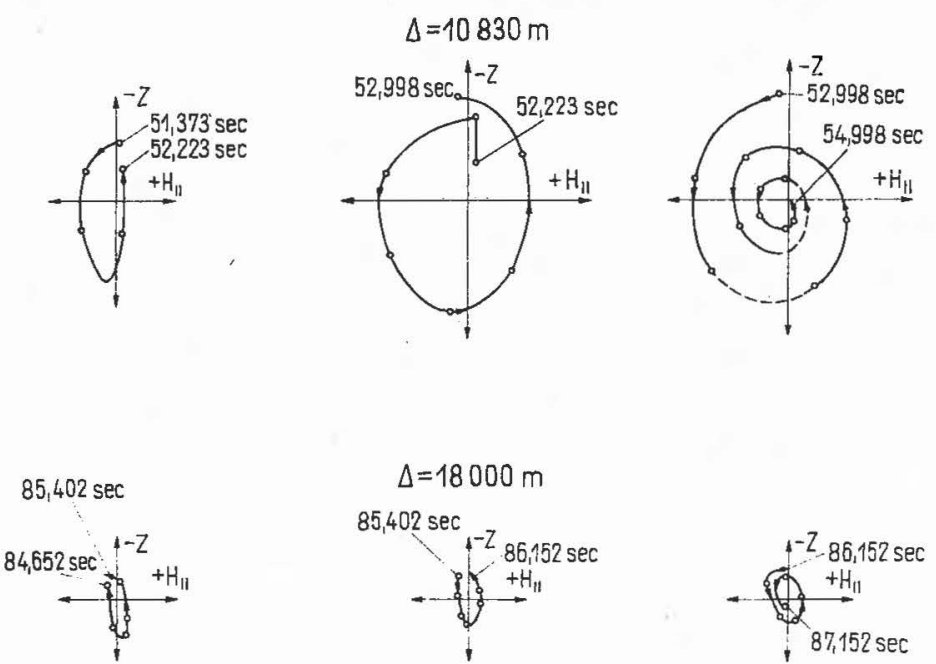

Aвв. 8. Teilchenbewegung der Wellengruppe $B$ (Rayleighwelle) $\Lambda=10830 \mathrm{~m}$ und $\Lambda=18000 \mathrm{~m}$ 
wenn die Angaben einer gemeinsamen Sprengung für alle vorkommenden Entfernungen benützt werden könnten.

Die 4 empirischen Kurven der Gruppengeschwindigkeit sind in Abb. 9 durch Punktreihen dargestellt.
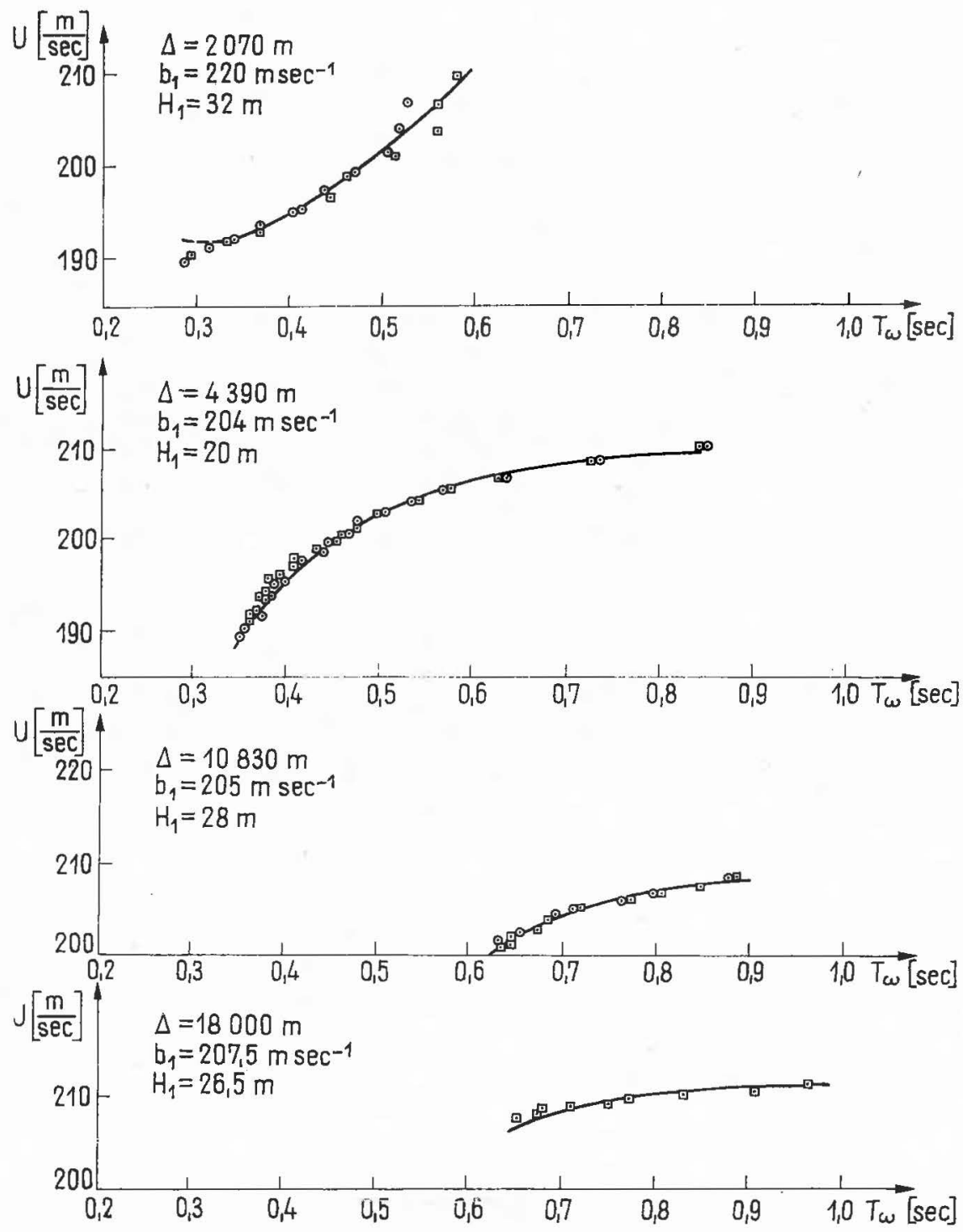

Aвв. 9. Gruppengeschwindigkeitskurven

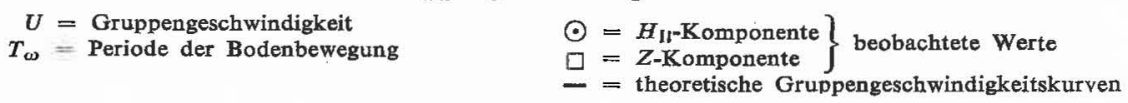


Weiterhin haben wir versucht, die beobachteten Gruppengeschwindigkeiten mit den von verschiedenen Autoren angegebenen theoretischen Dispersionskurven für Rayleighwellen zu identifizieren. Dieser Versuch blieb erfolglos, da die zur Verfügung stehenden theoretischen Kurven im Vergleich zu den empirischen einen zu steilen Anstieg zeigten.

Deshalb haben wir eine neue theoretische Dispersionskurve unter der Voraussetzung berechnet, daß über einem halb unendlichen homogenen Medium eine durch parallele ebene Flächen begrenzte Oberflächenschicht liegt (Abb. 10).

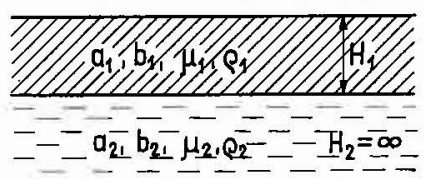

Авв. 10. Vorausgesetztes Strukturmodell

In diesem Fall kann die Periodengleichung für die Grundmodifikation der Rayleighwelle in folgender Form geschrieben werden [3]:

$$
\begin{aligned}
& A-B \operatorname{chf} \alpha_{1} H_{1} \cdot \operatorname{chVf\beta _{1}} H_{1}+ \\
& +C \operatorname{chf} \alpha_{1} H_{1} \cdot \operatorname{shf} \beta_{1} H_{1}+
\end{aligned}
$$

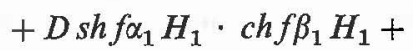

$$
\begin{aligned}
& +E \operatorname{sh} f \alpha_{1} H_{1} \cdot \operatorname{sh} f \beta_{1} H_{1}=0 \text {, }
\end{aligned}
$$

wobei $A, B, C, D, E$ Größen bedeuten, die von der Geschwindigkeit der longitudinalen und transversalen Wellen der beiden Medien, von den Scherkonstanten $\left(\mu_{1}, \mu_{2}\right)$ und von der Phasengeschwindigkeit der Rayleighwellen abhängen.

$$
\begin{array}{cc}
\alpha_{1}=\sqrt{1-\frac{V^{2}}{a_{1}^{2}}} & \beta_{1}=\sqrt{1-\frac{V^{2}}{b_{1}^{2}}} \\
V=\frac{L}{T} & f=\frac{2 \pi}{L}
\end{array}
$$

$L=$ Wellenlänge

$T=$ Periode der Welle

$H_{1}=$ Schichtenmächtigkeit

$a_{1}, a_{2}=$ Geschwindigkeit der longitudinalen Wellen

$b_{1}, b_{2}=$ Geschwindigkeit der transversalen Wellen

$\mu_{1}, \mu_{2}=$ Scherkonstanten

Index 1 bezieht sich auf die obere Schicht, Index 2 auf das unendliche Medium.

Die Gleichung (2) gilt für den Fall:

$$
V<b_{1} \quad \text { und } \quad V<b_{2}
$$


In dem Fall $a_{1}>V>b_{1}$ und $V<b_{2}$ ändert sich die Gleichung (2) formell. Für die Geschwindigkeitsverhältnisse haben wir folgende Werte angenommen:

$$
\begin{gathered}
\frac{a_{2}}{a_{1}}=\frac{b_{2}}{b_{1}}=1,1547, \\
\frac{a_{1}}{b_{1}}=\frac{a_{2}}{b_{2}}=1,7320, \\
\frac{\mu_{2}}{\mu_{1}}=1,4 .
\end{gathered}
$$

Aus den obigen Angaben ergibt sich die Poissonsche Zahl $\sigma_{1}=\sigma_{2}=0,25$ und das Dichteverhältnis $\rho_{2} / \rho_{1}=1,05$.

Auf Grund der Gleichung (2) bzw. deren modifizierten Form und der Werte (3) haben wir die theoretische Phasengeschwindigkeitskurve erhalten (Abb. 11a).
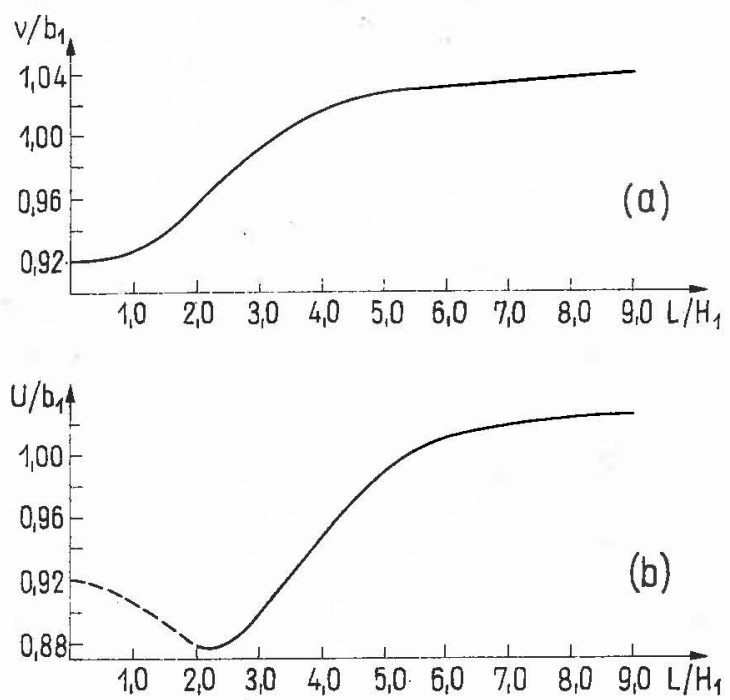

Aвв. 11(a) Theoretische Phasengeschwindigkeitskurve.

(b) Theoretische Gruppengeschwindigkeitskurve

$$
\begin{aligned}
\frac{a_{2}}{a_{1}}=\frac{b_{2}}{b_{1}}=1,1547 ; & \frac{a_{2}}{b_{2}}=\frac{a_{1}}{b_{1}}=1,7320 \\
\sigma_{1}=\sigma_{2}=0,25 ; & \frac{\mu_{2}}{\mu_{1}}=1,4
\end{aligned}
$$

Durch graphische Differenzierung der so erhaltenen Kurve und unter Benutzung der Beziehung 


$$
U=V-L \frac{d V}{d L}
$$

wurde die theoretische Gruppengeschwindigkeitskurve Abb. 11b bestimmt.

Diese theoretische Kurve konnte - durch entsprechende Veränderung der Werte $b_{1}$ und $H_{1}$ - mit den empirischen Gruppengeschwindigkeitskurven verhältnismäßig gut in Einklang gebracht werden. Die zu den mit vollausgezogenen Linien gezeichneten theoretischen Kurven (Abb. 9.) gehörenden Parameter sind in Tabelle 3 zusanimengestellt.

TABeLle 3

\begin{tabular}{c|c|c|c|c|c|c}
\hline \multirow{2}{*}{$\begin{array}{c}\text { Num- } \\
\text { mer } \\
\text { der: }\end{array}$} & \multirow{2}{*}{$\begin{array}{c}\text { Spren- } \\
\text { gung }\end{array}$} & \multicolumn{3}{|c|}{ Parameter der oberen Schicht } & \multicolumn{2}{c}{ Parameter der unteren Schicht } \\
\cline { 2 - 7 } & & $a_{1}$ & $b_{1}$ & $H_{1}$ & $a_{2}$ & $b_{\mathrm{g}}$ \\
\hline & $\mathrm{m} / \mathrm{sec}$ & $\mathrm{m} / \mathrm{sec}$ & $\mathrm{m}$ & $\mathrm{m} / \mathrm{sec}$ & $\mathrm{m} / \mathrm{sec}$ \\
\hline 1 & 2070 & 381,0 & 220 & 32 & 439,9 & 254,0 \\
2 & 4390 & 353,3 & 204 & 20 & 408,0 & 235,6 \\
3 & 10830 & 355,1 & 205 & 28 & 410,0 & 236,7 \\
4 & 18000 & 359,4 & 207,5 & 26,5 & 415,0 & 239,6 \\
\hline & & \multicolumn{3}{|c}{$\sigma_{1}=0,25$} & & \multicolumn{2}{c}{$\sigma_{2}=0,25$} \\
\end{tabular}

Die obigen Ergebnisse zeigen, daß die Rayleighwellen, die in allen vier Entfernungen eine Dispersion zeigen, an die oberen, etwa 20-30 m dicken lockeren Schichten gebundene Wellenzüge sind.

Es wäre wünschenswert, auch den Zusammenhang zwischen den Angaben der Rayleighwellen (Tabelle 3) und den vorliegenden geologischen Verhältnissen zu deuten. Es wurde die Beobachtungslinie eines bekannten Refraktionsprofils der Tiefebene festgesetzt. Das Refraktionsprofil war aber in erster Linie zur Erforschung der Tiefenstruktur des Gebietes geeignet. Nach den Bohrungsangaben von $20-30 \mathrm{~m}$ Tiefe besteht die lockere Schicht entlang dem Profil aus tonigen Sedimenten, selten mit Einkeilungen von Sand und tonigem Sand. Diese informativen Angaben können aber nicht als Grundlage zur Deutung des obigen Zusammenhanges dienen.

Die am besten entwickelten dispersiven Rayleighwellenzüge wurden aus einer Entfernung $\Delta=4390 \mathrm{~m}$ erhalten. Die daraus berechnete Gruppengeschwindigkeitskurve bezieht sich auf ein breites Periodengebiet. Deshalb scheint es zweckmäßig, die daraus abgeleiteten Parameter, wenigstens angenähert mit den die tatsächliche Struktur charakterisierenden Angaben zu vergleichen.

Es wurden in einer Entfernung von $\Delta=4390 \mathrm{~m}$, auf einer Strecke von $3 \times 300 \mathrm{~m}$, Kleinrefraktions-Kontrollmessungen ausgeführt (mit Geophon-Abständen von $6 \mathrm{~m})$. Die Tiefe der Schichtengrenzen sowie die für Longitudinalwellen erhaltenen Geschwindigkeitswerte können aus Abb. 12 abgelesen werden.*

* Die Kleinrefraktionsmessung wurde von Dr. P. Márton ausgewertet. 
Es sei noch erwähnt, daß die Einsätze der direkten Wellen in der Nähe des Sprengpunktes etwas unsicher waren, deshalb haben wir die Durchschnittsgeschwindigkeit $\bar{V}=1000 \mathrm{~m} \mathrm{sec}^{-1}$ aus den Ergebnissen einer früheren Messung übernommen.
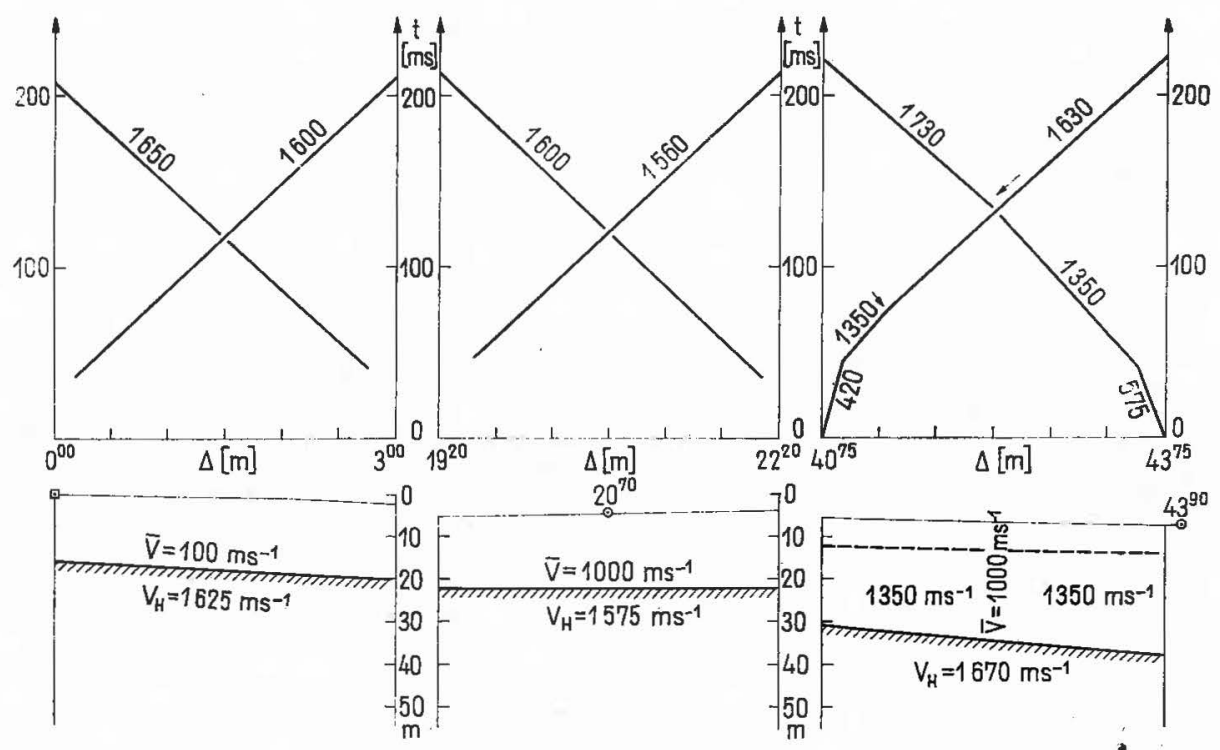

ABB. 12. Laufzeitkurven der Kleinrefraktionsmessung und die konstruierten Schichtengrenzen

\footnotetext{
$V=$ durchschnittliche Geschwindigkeit

$V_{H}=$ Grenzgeschwindigkeit
}

Es wird angenommen, daß die registrierten Rayleighwellen an die in Abb. 12 mit kontinuierlicher Linie eingezeichneten Schichtgrenzen gebunden sind. Die Durchschnittswerte der Tiefe und der Geschwindigkeit der Schicht betragen $H=20,7 \mathrm{~m} V_{H}=1625 \mathrm{~m} \mathrm{sec}^{-1}$.

Die vergleichbaren Werte der zwei Meßmethoden sind in der folgenden Tabelle zusammengestellt:

TABELLE 4

\begin{tabular}{l|c|c}
\hline & $\begin{array}{c}\text { Rayleighwellen } \\
=4390 \mathrm{~m}\end{array}$ & Kleinrefraktion \\
\hline$H_{1}$ & $20 \mathrm{~m}$ & $20,8 \mathrm{~m}$ \\
$a_{1}$ & $353,3 \mathrm{~m} / \mathrm{sec}$ & $1000 \mathrm{~m} / \mathrm{sec}$ \\
$a_{2}$ & $408,0 \mathrm{~m} / \mathrm{sec}$ & $1625 \mathrm{~m} / \mathrm{sec}$ \\
$a_{2} / a_{1}$ & 1,1547 & 1,625
\end{tabular}


Nach den Literaturangaben ist für feuchten Ton bei $a=1000 \mathrm{~m} \mathrm{sec}^{-1}, \sigma \sim 0,49$, (in der Zone von kleiner Geschwindigkeit) für feuchten Ton bei $a=1650 \mathrm{~m} \mathrm{sec}^{-1}$ $\sigma \sim 0,48$.

Wie bereits erwähnt, haben wir bei der theoretischen Phasengeschwindigkeitskurve der Rayleighwellen, als Poissonsche Zahl den Wert $\sigma=0,25$ genommen.

Obwohl die durch die beiden Methoden bestimmten $H_{1}$-Werte sehr schön übereinstimmen, weist der große Unterschied in den Ausbreitungsgeschwindigkeiten (Tabelle 4) auf die Mehrdeutigkeit der Dispersionskurven der Rayleighwellen hin.

\section{Konklusionen und geplante weitere Untersuchungen}

1. Die in das Periodenintervall von ungefähr $0,3-1,0 \mathrm{sec}$ fallenden Oberflächenwellen können gut entwickelte Rayleighwellen mit Dispersion enthalten, die an die lockere Schicht gebunden sind. Es wäre wünschenswert, dieses Periodengebiet bis zu $2 \mathrm{sec}$ auszudehnen.

2. Der Vergleich der aus den Rayleighwellen sowie aus Kleinrefraktionsmessungen abgeleiteten Gesteinsparameter weist auf die Mehrdeutigkeit der Dispersionskurven hin. Es wird vermutet, daß die gemeinsame Deutung der gleichzeitig registrierten Oberflächenwellen verschiedener Typen zu einer engeren Abgrenzung der Mehrdeutigkeit der Dispersionskurven führt. Deshalb sollen die Wellengruppen $A$ (aber auch die Phasen $x_{2}$ und $x_{3}$ ) noch weiter ausgedehnt werden.

3. Weitere Ziele sind die Untersuchung der Existenzbedingungen der Rayleighwellen mit Dispersion sowie die eingehendere Erforschung der Ein- und Mehrschichtenprobleme theoretischer Dispersionskurven $\left(\mu_{2} / \mu_{1}<2\right.$ und $\left.\sigma>0,25\right)$.

Die vorliegende Messung bildet den ersten Teil einer geplanten Forschungsreihe zur Untersuchung der Oberflächenwellen.

Abschließend sei mir erlaubt, meinen Kollegen Dr. E. Bisztricsány, Dr. P. Márton, K. Molnár und Dr. P. Szemerédy, die mir mit wertvollen Ratschlägen geholfen haben, zu danken.

Die technische Hilfe bei den Messungen leistete der Seismische Betrieb des Staatstrusts für die Erdöl- und Gasindustrie, wofür ich auch an dieser Stelle meinen aufrichtigsten Dank ausspreche.

\section{Zusammenfassung}

Diese Abhandlung befaßt sich mit den durch Sprengungen erzeugten Oberflächenwellen, die in einer Entfernung $\Delta=2070-18000 \mathrm{~m}$ registriert wurden und ungefähr in das Periodengebiet 0,3-1,3 sec fallen. In unseren Aufnahmen können die Oberflächenwellen in zwei Hauptgruppen geteilt werden. Die erste (A) besteht aus mehreren Wellentypen, deren erster Teil wahrscheinlich eine sogenannte hydrodynamische Welle ist. Die zweite Wellengruppe ist eine Rayleighwelle mit normaler Dispersion. 
Es wurden Phasengeschwindigkeits- bzw. Gruppengeschwindigkeits-Kurven für die Grundmodifikation der Rayleighwellen bezüglich des Einschichtenproblems berechnet $\left(\mu_{2} / \mu_{1}=1,4, \sigma=0,25\right)$. Auf Grund der Identifizierung der beobachteten und theoretischen Gruppengeschwindigkeitskurven läßt sich feststellen, daß die beobachtete Dispersion der Rayleighwellen an die obere lockere Schicht gebunden ist.

Die Ergebnisse einer Kontroll-Kleinrefraktionsmessung wurden mit den aus der Rayleighwelle abgeleiteten Werten verglichen.

\section{LITERATUR}

[1] Don Leet, L.: Earth waves. Harvard Univ. Press 1950

[2] SAVARENSKy, E. F.: On the determination of group and phase velocities from observations. Izv. Geophys. Ser. 1150-1559 (1959)

[3] КЕйлиС-БороК, В. И.: О поверхностных волнах в слое, лежащем на упругом полупространстве. Изд. АН. СССР. Сер. Геофиз. 2 18-38 (1951) 


\title{
THE INTERNATIONAL UPPER MANTLE PROJECT
}

\author{
L. KNOPOFF
}

(Los Angeles, USA)

It would be presumptuous of me to attempt to describe the entire program of the International Upper Mantle Project, as it is now envisaged, in the short space allotted to me for this contribution. A recent publication describing research programs and proposed research programs, both on a national and an international basis, has been distributed to national committees. ${ }^{1}$

In May 1964, the International Upper Mantle Committee met in Moscow. A number of programs were approved at that meeting for recommendation to national committees. A report on this meeting will be forthcoming shortly. ${ }^{2}$

At least three actions of the International Upper Mantle Committee are of interest to the seismologists present at this assembly. I shall describe them briefly.

\section{A Study of Continental Margins and Island Arcs}

This effort is aimed at determining how two major structural units of the earth's crust, the continents and the oceans, interact at their boundary. The Upper Mantle Committee has laid emphasis upon a study which combines continental margins and island arcs into a single research program. However, the transition from continents to oceans is proposed as an important subject for study in itself. European seismologists have obtained a considerable body of information concerning the detailed variation of parts of the European continent with regard to seismic properties. From observations in the deep sea we know that the crust is considerably thinner than on the continents. What we do not know is the nature of the transition from continents to oceans.

\section{Study of the World Rift System}

A study of rift systems all over the world has been adopted as one of the major programs of the Upper Mantle Project. Although these studies will not be confined to any region or regions, it is clear that geophysicists in any given area of the world have ample opportunity to study rift structures locally. The locations of the major rift systems of the world are well known: the mid-Atlantic ridge the East African rift, the Gulf of California and the San Andreas Fault zone, the Lake Baikal region, etc. Of great interest to the European Seismological community will be, of course, the Rhine and Mjosen grabens. The evidence 
from the Red Sea and the southern part of the Gulf of California is that the crust is thin in these regions. What similarities are present among the world's rifts? Are the oceanic rifts similar or different from the continental rifts? We urge that a cooperative international effort be undertaken to study the European graben systems as part of this major program.

\section{Long Geophysical Profiles}

The Upper Mantle Committee has urged that long profiles be made in many regions of the earth utilizing many modern techniques of geophysics along each profile. Of the many countries active in geophysics today, perhaps only the USSR and the U.S. have sufficient geographical extent so that studies can be made of long profiles which will cross a number of geological provinces. Most of the studies to date have involved regional studies of relatively short extent which have been "spotty" in nature. There has been no general coordination of geophysical studies to allow the programs executed in one area to be linked up with those carried on in another area. In the U.S., where international boundaries do not interfere, the Transcontinental Geophysical Survey is being conducted as part of the U.S. Upper Mantle Program; in this case a three-dimensional structural picture of the crust and upper mantle of the U.S. will be compiled in a band $4^{\circ}$ wide; many geophysical techniques, including seismological, aeromagnetic, geological, etc..., will be applied with great intensity to this band. In addition, a number of other techniques such as heat-flow measurements and deep drilling will be applied where feasible. In Europe, such long profiles would cross many international boundaries, and hence would be a natural project for international cooperation. The Upper Mantle Committee has recommended that at least two long lines be surveyed by a number of geophysical methods in Europe. These lines generally should trend north-south and east-west, although the specific location of the profiles and the areas to be studied must, of course, be left up to the discretion of the investigators involved in the program. This resolution has been referred to the European Seismological Commission because 1. the seismologists will be expected to play a prominent role in excuting this program of research, and 2. the European Seismological Commission represents a highly organized group of geophysicists in Europe, and therefore a body capable of undertaking a program demanding a significant amount of coordination. The resolution of the Upper Mantle Committee has been presented to this meeting at an earlier session by the President of the European Seismological Commission, Professor Zátopek. (The text of the resolutions and reports on the special UMP programs discussed above appears in UMP Report No. 1, pages 11, 12, 21, 23 and 29.)

\section{REFERENCES}

[1] International Upper Mantle Project: Programs and International Recommendations, 1960-63, Los Angeles, January 1964

[2] International Upper Mantle Project: Report No 1, Los Angeles, September 1964 


\title{
SOME RESULTS OF INVESTIGATION OF REGIONAL PECULIARITIES OF TRAVEL-TIMES OF SEISMIC WAVES BY OBSERVATION OF SEISMIC STATIONS OF THE USSR
}

\author{
N. V. Kondorskaya and L. B. Slavina
}

(Moscow, USSR)

The problem of travel-times of seismic waves from an origin to a station is very interesting for studying the structure of the earth's crust and that of its mantle.

In connection with the use of digital computers of great velocity when determining hypocenters on the ground of first arrival times it has become possible to obtain data with accuracy restricted only by the uniformity of arrival times at separate seismic stations and by the accuracy of travel-time tables used. The application of EC gives possibility to obtain, besides information of hypocentre coordinates, a greater number of stational residuals - systematic discrepancies of travel-times of first arrivals of longitudinal waves in comparison with standard averaged travel-time tables of Jeffreys-Bullen - that can be utilized for statistical analysis.

The aim of the present paper is a statistical analysis of stational discrepancies for different stations of the USSR obtained by EC in determining hypocenters disposed all over the globe.

\section{Description of the Program}

The problem consists in finding an origin time $t$ and hypocenter coordinates $\lambda, \varphi, h$ of an earthquake or in finding $t, \lambda, \varphi$ with a fixed focal depth $h[1]$.

If $t_{k}$ and travel-time table $g(\Delta, h)$ were known precisely the problem would have a sole solution satisfying the system of equations

$$
f_{k} \equiv t+\psi_{k}=0
$$

where

$$
\psi_{k}=g\left(\Delta_{k}, h\right)-t_{k},
$$

$\Delta_{k}=$ is an arc epicentral distance to $k$-th station,

$f_{k}=$ is a discrepancy between observed and calculated arrival times to $k$-th station,

$\psi_{k}=$ is an origin-time corresponding to the observed one.

The method of decision is based on determination of a mean-square discrepancy $\psi$ of an origin time. 


$$
\bar{\psi}=\left\{\frac{1}{m} \sum_{i=1}^{m} f_{k_{i}}\right\}^{1 / 2}
$$

It is being sought a totality of $t, h, \lambda, p$ with which $\bar{\psi}$ is minimal. A number of iterations, a mean-square discrepancy of an origin time $\bar{\psi}$, a decision of the problem $(t, h, \lambda, \varphi)$, a number of stations $m^{\prime}$ used in final decision, discrepancies of arrival times of P-waves $f_{k}$, azimuths $\alpha^{\circ}$, and an epicentral distance to each station are being printed as a result of computation.

\section{Stational Data}

We consider here data of the following stations equipped with instruments of the same type: Apatity, Vyborg, Uzhgorod, Kirovabad, Talgar, Frunze, Sverdlovsk, Semipalatinsk, Eltsovka, Yakutsk, Tiksi, Petropavlovsk, YuzhnoSakhalinsk.

Times of first arrivals are being input into EC. Data of longitude and geocentric latitude have been input as a constant parameter.

As a result of the computation, one obtains epicentral distances according to formula $\cos \Delta=a A+b B+c C$, where $A, B, C, a, b, c$ are directing cosines of an epicenter and an observing station connected with their coordinates and allowing for geocentricity. Besides the computer gives an uncorrected residual

$$
\psi_{k}=g_{k}-t_{k}^{p} ; \quad t_{k}^{p}=t_{0}+t\left(\Delta_{i}\right) .
$$

The ellipticity corrections are being input according to Bullen [2]. Then we get a corrected value of discrepancy $f_{k}$ which is a sum of the following quantities

$$
f_{k}=F_{1}(e p)+F_{2}(s t)+F(e p, s t)+C
$$

$F_{1}(e p)$ is a discrepancy connected with deviation from Jeffreys' travel-time tables through a medium composition in the origin location; $F_{2}(s t)$ connects with composition in a station location; $F(e p, s t)$ is a difference obtained at the expense of a path from the epicentre to a station; $C$ is a chance error connected with inaccuracy of processing; etc. $C$ is being eliminated by statistical processing of a great number of epicentres.

\section{The Utilized Material and its Analysis}

We have carried out a statistical analysis of discrepancies for shallow earthquakes occured in different seismic belts in the period of 1961-1963.

The statistical analysis was being carried out by means of construction of standardized averaged curves of discrepancy distribution for any separate station independently of an earthquake origin region. 
Mean values of discrepancy and confidence intervals were being computed according to Student, the following formulas being used. There are discrepancies for $k$ earthquakes $f_{1} \ldots f_{k}$; a mean value of a discrepancy is

$$
\bar{f}=\frac{1}{n} \sum_{i=1}^{n} f_{i}
$$

with $n$ being a number of cases.

According to Student,

$$
\begin{gathered}
S_{f}^{2}=\frac{1}{n}\left[\sum_{i=1}^{n} f_{i}^{2}-n\left(\bar{f}_{i}\right)^{2}\right] \\
P\left\{-t_{n-1}^{\alpha}<\frac{-\bar{f}+m}{S_{f}} \cdot \sqrt{n-1}<t_{n-1}^{\alpha}\right\}=1-2 \alpha
\end{gathered}
$$

where $m$ is a veritable mean value. When $\alpha=5 \%, t_{n-1}^{\alpha}$ was taken equal to 1.645 for given $n>100$.

A confidence interval with $90 \%$ level of confidence $(2 \alpha)$ is

$$
\frac{-S_{f} \cdot t_{n-1}^{\alpha}}{\sqrt{n-1}}+\bar{f}<m<\frac{S_{f} \cdot t_{n-1}^{\alpha}}{\sqrt{n-1}}+\bar{f} .
$$

\section{Results}

There are given standardized graphs of discrepancies constructed for the abovementioned Soviet stations and shown mean statistical quantities of discrepancy values $f_{k}$ on Fig. 1a, b. A negative value of a discrepancy points out at wave delay in comparison with Jeffreys' travel-time table, a positive one points out at outstripping.

First of all it should be noted that individual curves of discrepancy distribution for separate stations differ from the mean statistical curve obtained on the ground of utilized observations of all the Soviet seismic stations and adduced in the paper of N. V. Kondorskaya, T. M. Zhelankina, S. S. Mebel, L. Y. Vartanova [3].

The obtained results of mean discrepancies with respect to each other are in dependence corresponding to S. D. Kogan's results [4]. Indeed, according to Kogan, arrival times to stations Talgar and Frunze are by $1.5 \mathrm{sec}$ later than to Semipalatinsk and Sverdlovsk. According to our data, arrival times to Talgar and Frunze are by $1.15 \mathrm{sec}$ later than to Sverdlovsk and Semipalatinsk. Unfortunately, we have been unable to compare the remaining data because of the difference of taken stations.

It is evident that there exist constant deviations from Jeffreys' travel-time tables for each station. As for being at average, these deviations are being averaged when using observations of different stations of different regions. Comparison 


\section{MIDDLE ASIA}

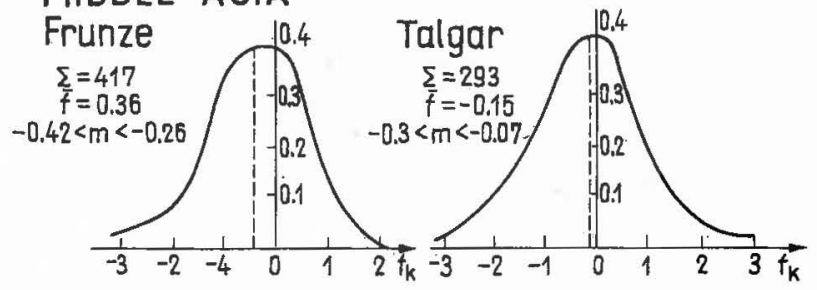

VICINITY OF URAL

Semipalatinsk
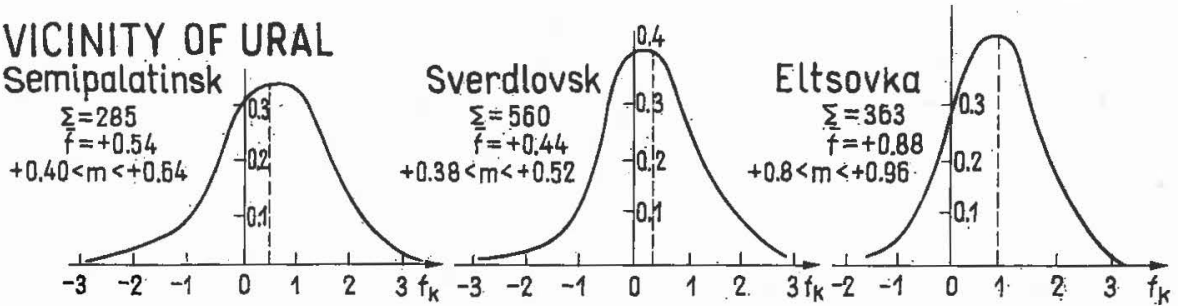

\section{N-W}

Vyborg
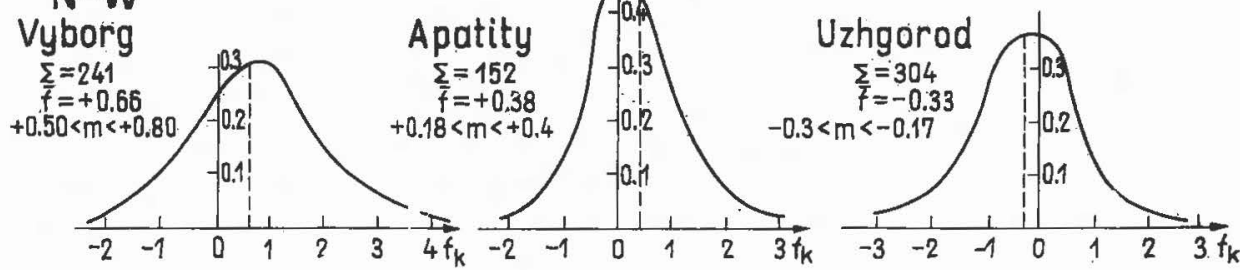

Bakuriani
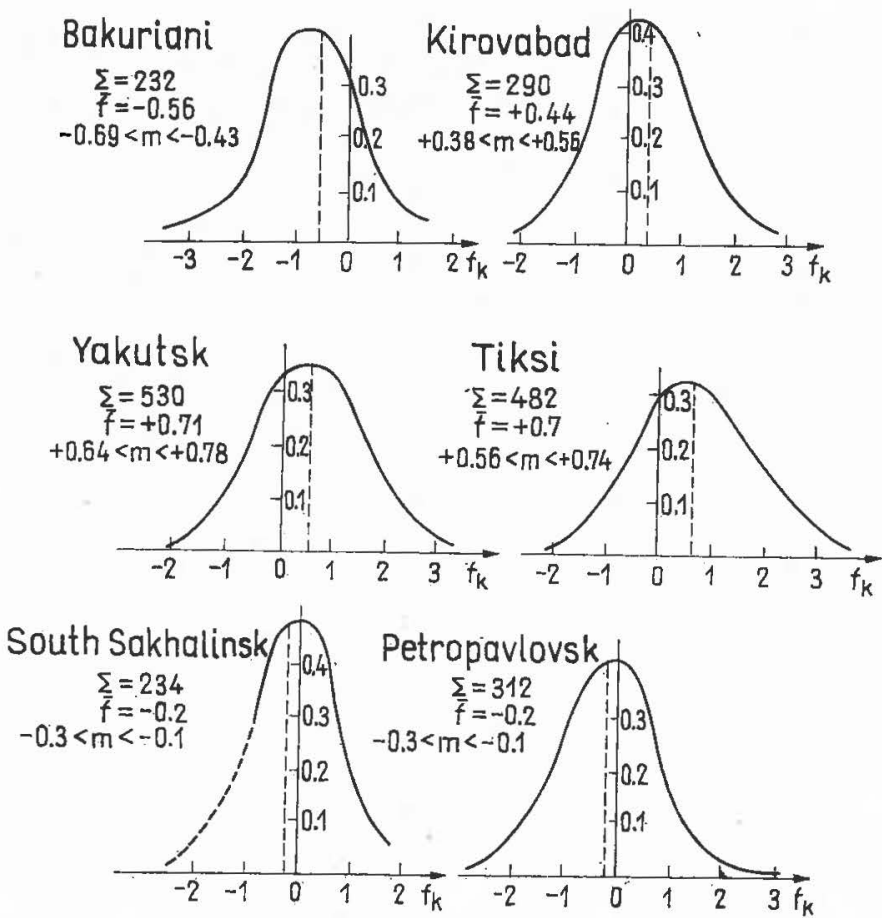

Fig. 1a, b. Standardized curves of discrepancy distribution by separate stations 
of results of discrepancies for separate stations shows that stations disposed at comparatively short distance one from another and in similar geological conditions have, as a rule, similar signs of deviations. Thus all the examined stations can be devided into the following groups:

1. Middle Asia - Talgar, Frunze;

2. the Ural's region - Sverdlovsk, Semipalatinsk, Eltsovka;

3. Northwestern part of USSR - Vyborg, Apatity;

4. Eastern Siberia - Yakutsk, Tiksi;

5. Carpatians - Uzhgorod;

6. Kamchatka - Petropavlovsk;

7. South-Sakhalinsk;

8. the Caucasus - Kirovabad, Bakuriani.

Stations of the Caucasus - Kirovabad and Bakuriani - should be noted particularly for they give quantities of mean discrepancy values with different signs though being disposed at comparatively short distances from each other.

It is natural that such a behaviour of discrepancies may be connected first of all with the structure of the earth's crust. In connection with this we have carried out a comparison of discrepancies for separate stations with tectonic and geologic conditions of their locations, using data of works of the Deep Seismic Sounding (DSS) on the territory of the USSR [7] and other tectonic data. Sections of DSS and data of velocities and M. Discontinuity depths are given in Fig. 2. One can see out of comparison that in the Ural's region there are increases of velocity at $\mathrm{M}$. discontinuity and correspondingly earlier arrival times at its stations as it had been previously noted in Kondorskaya's paper [9] connected with construction of travel-time curves. Similar behaviour of discrepancies at Vyborg and Apatity can be explained by location of these stations. in regions of the ancient Baltic shield with absence of a sedimental thickness and a thinned granite layer (data of DSS).

It is difficult to give any explanation for other regions of station location proceeding from available data about the earth's crust structure. Nevertheless, regularities of discrepancy values for separate stations noted in statistical averaging observations of earthquakes in different regions (here, in the section $\mathbf{I}$, $F_{1}(\mathrm{ep})=0, F(\mathrm{ep}, \mathrm{st})=0, C=0$, and $\left.f_{k}=F_{2}(\mathrm{st})\right)$ point out the possibility to use the obtained results for detailed study of the crust peculiarities in the region of a station. Thus, one can suppose that the crust structure in the region of Tiksi and Yakutsk is similar to that in the region of Sverdlovsk and Semipalatinsk.

With the purpose of using discrepancies for refining epicentral coordinates an influence of terms (1) and (3) in the section I should be investigated.

The following questions were considered:

1. Dependence of $f_{k}$ upon an epicentral distance.

2. Dependence of $f_{k}$ upon an origin location, that is upon azimuth $\left(\alpha^{\circ}\right)$. 


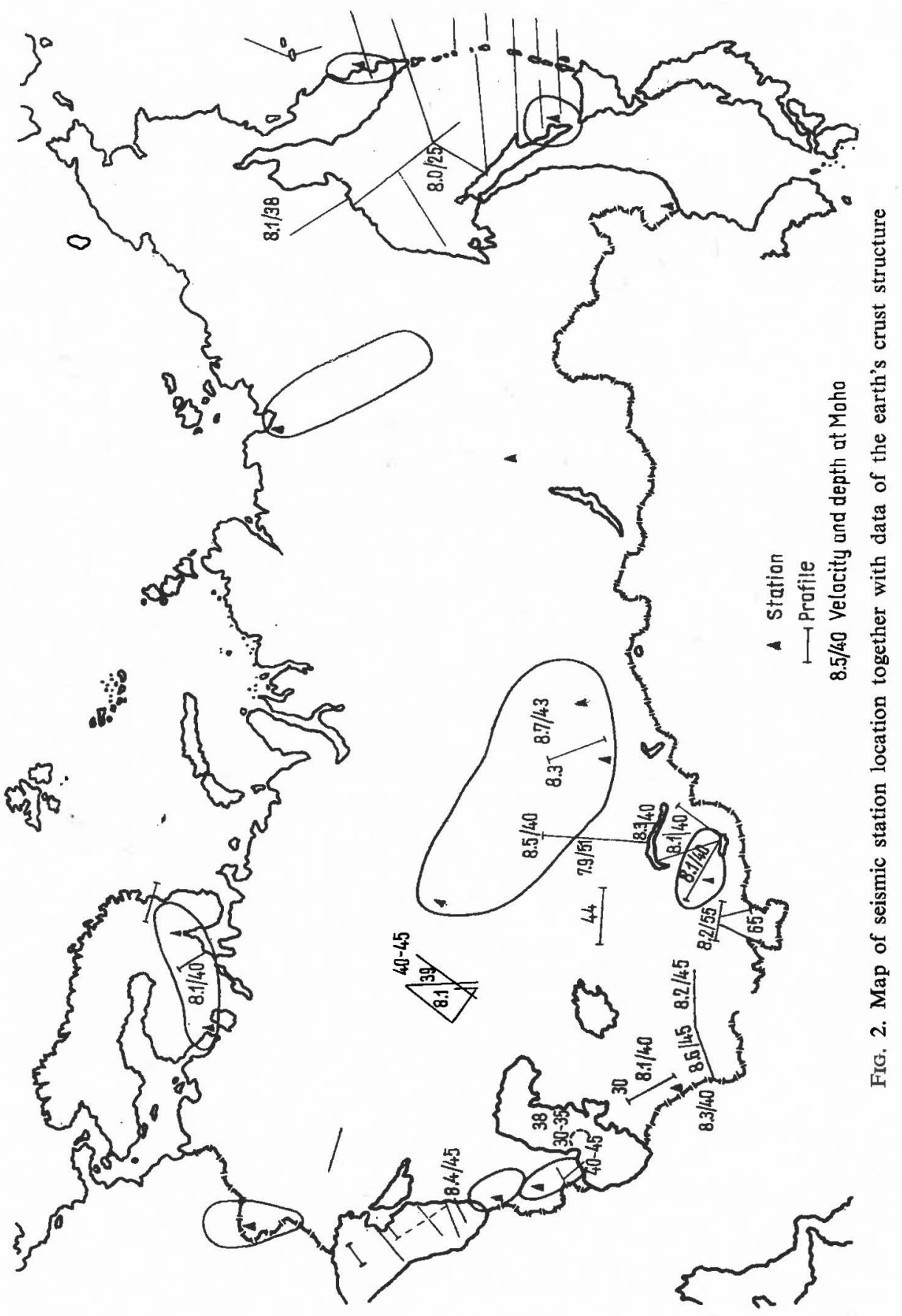


1. Dependence $f_{k}\left(4^{\circ}\right)$

One can see out of Fig. 3, where an example of such a graph is given for Tiksi, that no dependence of $f_{k}$ as a function of $\Delta^{\circ}$ exists, which shows to some extent the mantle homogeneity.

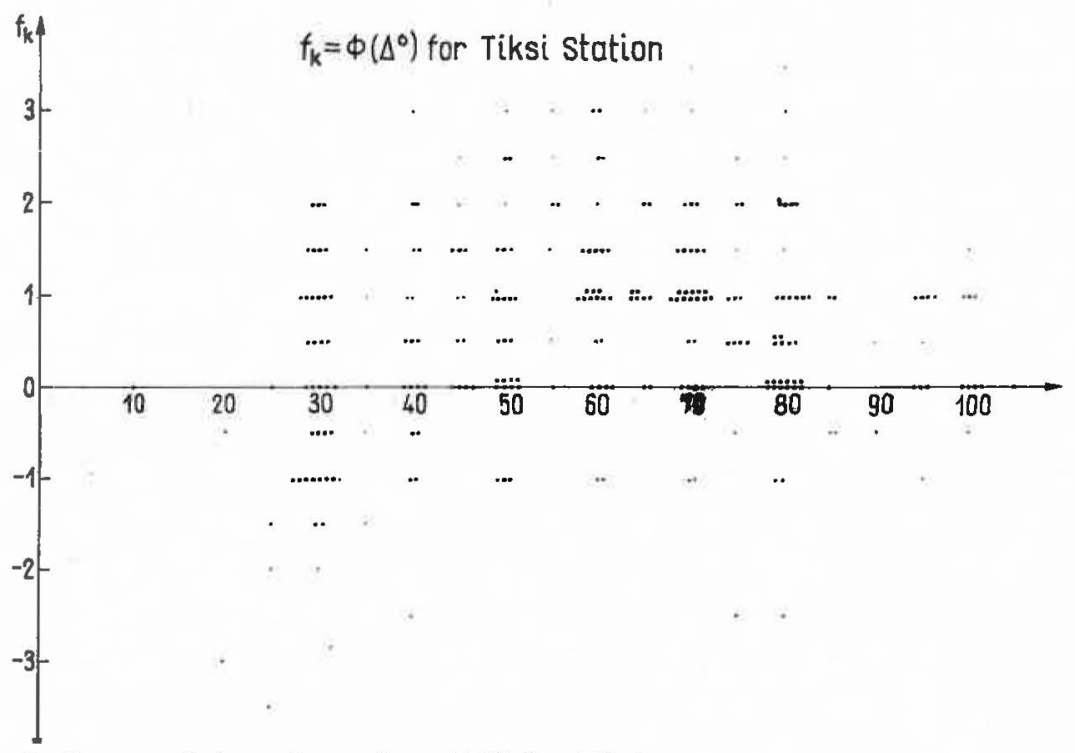

Fig. 3. Curves of dependence $f_{k}=\phi\left(\Lambda^{\circ}\right)$ for Tiksi

\section{Dependence $f_{k}\left(\alpha^{\circ}\right)$}

In their papers on observations of nuclear explosions, S. D. Kogan [4] and H. Jeffreys [5] considered dependence of discrepancy values upon azimuth from an epicentre to a station.

Our aim has been to find a dependence of discrepancy $f_{k}$ on a region for any examined station. For this purpose we have done the following. The globe was devided into 17 regions limited by certain coordinates. Azimuth of each given region was supposed to be constant for convenience of averaging. Then standardized graphs of discrepancy distribution of each station were constructed for separate regions. Mean quantities of discrepancy values are given in Table 1. As one can see, there is some little change of discrepancy value in case of earthquakes in different regions. This change for a number of stations (Frunze, Talgar, and others) is within $1 \mathrm{sec}$, for other ones (Kirovabad, Vyborg) it is within $1.5 \mathrm{sec}$.

It is difficult to trace some regularity in discrepancy values for separate regions, one can note only the fact of existence of difference among quantities of mean 
TABLE 1

\begin{tabular}{l|c|c|c|c|c|c}
\hline \multicolumn{1}{c|}{ Region stations } & $\begin{array}{c}\text { Aleutians } \\
\text { Alaska }\end{array}$ & $\begin{array}{c}\text { Japan } \\
\text { far east }\end{array}$ & $\begin{array}{c}\text { Philip- } \\
\text { pines }\end{array}$ & Indonesia & $\begin{array}{c}\text { Middle } \\
\text { Asia }\end{array}$ & $\begin{array}{c}\text { India } \\
\text { and } \\
\text { Ocean. }\end{array}$ \\
\hline Frunze & -0.99 & -0.26 & +0.20 & +0.14 & +0.17 & -0.75 \\
Talgar & -0.17 & -0.24 & -0.47 & +0.33 & +0.13 & 0.00 \\
Semipalatinsk & +0.40 & - & +0.88 & +1.00 & +0.25 & - \\
Sverdlovsk & -0.43 & +0.57 & +1.30 & +0.73 & +0.42 & +0.70 \\
Eltsovka & +0.10 & +0.85 & +1.36 & +1.08 & +0.79 & +0.58 \\
Vyborg & +0.08 & - & +1.50 & +1.51 & - & - \\
Apatity & +0.07 & - & +1.17 & - & - & +0.98 \\
Bakuriani & - & -0.63 & +0.23 & +0.22 & - & - \\
Kirovabad & -0.20 & +0.07 & +1.37 & +1.23 & - & - \\
Yakutsk & +0.20 & +0.26 & +0.48 & +0.02 & - & +1.15 \\
Tiksi & -0.32 & +0.90 & +0.87 & +0.36 & +0.60 & +1.15 \\
Uzhgorod & -0.60 & - & +0.23 & +0.60 & - & +0.20 \\
S-Sakhalinsk & -0.37 & - & +0.10 & +0.23 & - & - \\
Petropavlovsk & -0.12 & - & -0.23 & -0.34 & - & - \\
& & & & & &
\end{tabular}

values of discrepancies. The obtained data are not sufficient for introduction of refinements into corresponding averaged travel-time tables. The statistical analysis of a more extensive experimental material should be carried out, comparison of stational discrepancies for regions with different thickness of the crust being expedient. Thus, the following regions can be distinguished on the ground of [7]:

1. Europe, thickness is $22-20 \mathrm{~km}$;

2. Greece, $58-60 \mathrm{~km}$;

3. Asia, $35-40 \mathrm{~km}$ and to $60 \mathrm{~km}$ in mountain regions;

4. Japan, $25-30 \mathrm{~km}$;

5. Australia, $35 \mathrm{~km}$;

6. Africa, $35 \mathrm{~km}$;

7. North America, $40-50 \mathrm{~km}$ with thinning to $32 \mathrm{~km}$ towards the coast;

8. South America, 50-60 km;

9. Oceans, 6-7 km;

10. Zone of islands and island arches, $12-16 \mathrm{~km}$.

Finding a dependence between discrepancy value and the crust thickness will allow to obtain additional information about the earth's crust structure in different regions.

\section{REFERENCES}

[1] Piatetskiy-Shapiro, I. I., Keylis-Borok, V. V. and others: Determination of Epicentre Coordinates on EC. DAN AN SSSR, 2 (1963)

[2] Bullen, K. E.: The Ellipticity Coordinates to Travel-Times of P and S Earth. Waves. Monthly Not. of the Roy. Ast. Soc., 42 (1937) 
[3] Kondorskaya, N. V., Zhelankina, T. M. and others: Some Results of Use of Electronic Computers in the Soviet Seismic Service. "Analysis of Seismic Observations on Electronic Computers". Tr. IFZ. Collection (in press)

[4] Kogan, S. D.: "Travel-Times of Longitudinal and Transversal Waves Calculated by Data of Nuclear Explosions Made in the Vicinity of Marshall Islands". Izv. AN SSSR, Ser. Geoph. 3 (1960)

[5] Jeffreys, H.: "Travel Times for Pacific Explosions." Geoph. Journ. of the Roy. Astr. Soc., 72 (1962)

[6] Deep Seismic Sounding of the Earth's Crust in USSR. (Collection of Reports.) (1962)

[7] Gugariy, G. Z., Solovieva, I. A.: The Earth's Crust Structure on Geophysical Data. M. (1963)

[8] Jeffreys, H. and Bullen, K. E.: Seismological Tables. Brit. Ass. for the Adv. of Sciences. 1940

[9] Kondorskaya, N. V.: About Regional Peculiarities of Travel-Times of Seismic Waves. Izv. AN SSSR, Ser. Geoph. 7 (1957) 


\section{LitVINENKo}

(Moscow, USSR)

Seismic soundings (GSS) made upon the Baltic shield [1, 2] are the first detailed investigations of the ancient crystalline shields - districts of early stabilization and deep natural section of the earth's crust. GSS were carried out by the Western Geophysical Trust, the Office of Special Geophysics, Scientific Research Geological Institute of the Soviet Union and the Mining Institute in Leningrad. S. Ankudinov, I. Dekhnich, I. Lenina, I. Litvinenko, K. Nekrasova, L. Platonenkova, A. Sosnovskaya, Z. Telyakova and others took part in these investigations.

Regional seismic profile in Northern Karelia to the west from the town of Kem at first passes along well-exposed ancient structures of gneisses of the Belomor massif and than crosses the younger formations of careliades. Another profile to the north-west from the Kolsky peninsula (enters) the Barentz sea. On the continent it comes along the Pechenga river across the Lovno lake up to the Pados-Tundra and from the north to the south it crosses: the ancient Kolsky gneisses, effusive-sedimentary series of Pechenga, tundra series and gabbro-diorite complex (granulitic formation).

During the investigations together with examination of refracted waves it was possible to use the method of reflected waves. As the result of this it was possible to increase the accuracy of the illumination of the structure of the upper part of the crystallitic crust sections. Close to the day surface it was found a block structure of rate section of the crust. It was caused by the increase of velocity at the depth and on the other, the abrupt changes resulted by the difference of minerologo-petrographical composition of immensely dislocated crystal rocks. The new data about the nature of the groups of waves registrated during the remote explosions were received.

As a result of seismic sounding, according to elastic properties, the lamination of the earth's crust was observed.

This foliation is characterized by the following peculiarites:

1. Conformable occurrence of seismic boundaries from the low depths (4-5 $\mathrm{km}$ ) along the whole section of the crust. The shallow occurrence of the first seismic boundaries allows more close and detailed examination of them by means of a combination of geological and geophysical methods, including drilling.

2. In the crust sections there are sharper seismic boundaries: surface $M$, at the depth of about $40 \mathrm{~km}$; moreover a boundary is characterized by the velocity 
of the elastic waves about $6.6 \mathrm{~km}$ per second at the depth of about $10-15 \mathrm{~km}$ in northern Karelia and a boundary with the alternative values $(\mathrm{V}=6.4-6.9$ $\mathrm{km} / \mathrm{sec}$ ) - in the north-western part of the Kolsky peninsula. In these boundaries the elastic characteristics of the mountain rocks are changed with jumps, which perhaps not only resulted by the pressure and temperature but also owing to the change of minerologo-petrographical structure of the rock.

Other seismic boundaries are distinguished $(\mathrm{V}=6.7-6.9 ; 7.0$ and others), but they are not studied in details yet.

3. Along the upper boundaries of the crust the values of $\mathrm{V}$ are changed. Along the markable boundary (at the depth of $4-8 \mathrm{~km}$ in Pechenga district) $\mathrm{V}$ is changed within high range from 6.4 to $6.9 \mathrm{~km} / \mathrm{sec}$. Similar changes but within smaller range $(\mathrm{V}=6.2-6.45 \mathrm{~km} / \mathrm{sec})$ are traced in North Karelia.

4. There is a connection between the values of $\mathrm{V}$ along the upper seismic boundary and the characteristic according to elastic properties of the blocks of the crust. This connection is especially clear and easy to trace in the NorthWest Kolsky peninsula where more detailed investigations are taking place.

The sections of profiles formed in gneisses and granites at the day surface are characterized by not more than $5.5 \mathrm{~km} / \mathrm{sec}$ and along the first boundary not more than $6.5 \mathrm{~km} / \mathrm{sec}$. Under the effusive-sedimentary series of Pechenga and under gabbro-diorite block the elevated values of $V$ are found to 6.3-6.5 $\mathrm{km} / \mathrm{sec}$ - at the day surface - and to $6.9 \mathrm{~km} / \mathrm{sec}$ along the boundary at the depth of $4-6 \mathrm{~km}$.

5. The topography of the deep seismic boundaries is complicated and characterized by the angle of inclination to $10-15^{\circ}$ and frequent disturbances. According to the universal method of GSS it is possible to trace only the average position of the seismic boundaries. The complex relief of seismic boundaries can be noticed only by means of the reflected waves and in close proximity from the points of explosion. This circumstance, like the change of the velocity, demands further control and may play an important role in determination of the nature of waves, traced at the depth seismic explorations.

6. During the combined exploration of seismic and gravitative data it was found that the essential differences in the values of density for different rock complexes are preserved only to the depth of occurrence of the upper seismic boundaries.

7. The total thickness of the earth's crust along both profiles and possibly for the whole shield is comparatively constant (of about $+40 \mathrm{~km}$ ), but its composition in different structural - facial zones is not similar. Seismic boundaries inside the crust are changed along the profiles and the changes are different.

8. In northern Karelia an elevation similar to East-Karelian synclinorium, characterized by the appearance of late basic magmatism is typical for all the boundaries of the crust.

In the north-west of the Kolsky peninsula a great elevation is marked along the depth boundaries at the joint of granulitic complex and ancient granites and gneisses of the district of the Guirvas lake. Here it is possible to trace ser- 
pentinous belt as a result of regional fracture. It is also possible to observe the elevation of the surface of the layer at about $6.6 \mathrm{~km} / \mathrm{sec}$ velocity which happens under younger diabases of effusive-sedimentary series of Pechenga.

9. The lamination according to elastic characteristics is typical not only for the earth's crust but also for the upper mantle. At the removal more than $300 \mathrm{~km}$ from the shop point a new group of waves was observed perhaps connected with seismic boundary occurring at the depth of $60-70 \mathrm{~km}$ and characterized by the velocity of $9.0 \mathrm{~km} / \mathrm{sec}$.

Besides the described lamination of the earth's crust according to elastic properties it is possible to observe zones of deep crush according to the deviations in tracing the main boundaries of separation and other data. The more typical crush zone is established at the joint of Belomor and Karelian structures. Other zones of deep crushes usually may be observed at the joints of different structuralfacial zones and often are characterized by appearing of the last steps of magmatic activity.

The combination of conformable occurrence of seismic boundaries of separation and steeply dipping zones of disturbances causes a block structure of the crust and therefore a very complicated model of mean, which it is necessary to take into account during the evaluation of the seismic data. Every block of the crust has its own history of development that forms the character of geological section near the day surface.

In the whole the Baltic shield in its crustal structure differs from the crustal structure of the Barents sea. The complex of continental blocks of the shield is considerably elevated in comparison with the sea block. The more interesting blocks corresponding to effusive-sedimentary series of Pechenga and Lovninsky gabbro-diorite block are characterized by comparatively shallow occurrence of the first seismic boundary with a velocity of about $6.6 \mathrm{~km} / \mathrm{sec}(6.4-6.9 \mathrm{~km} / \mathrm{sec})$ which is accepted to name the surface of the so-called basaltic layer. The block crustal structure and shallow occurrence of this layer is also typical, perhaps, for other ancient shields.

Taking into consideration the above mentioned new data, it should be necessary to suppose that the upper seismic boundaries of separation of the shield's crust are the application of samples. The chance in minerologo-petrographic composition owing to the intrusion of the volcanic rocks should be taken into account, but this factor is typical mostly for the separated blocks (the structure of Pechenga and others).

The accepted data demonstrate the restriction and inaccuracy of the determination "basaltic" layer. The composition of at least its upper parts is changed.

The surface of "basaltine" has a compound structure. As it seems in various sections the basaltic layer is formed by different metamorphic and sometimes by volcanic rocks. Its superficial occurrence on the Baltic shield gives the real possibility for the more detailed examination of it under different geological conditions. The solution of this answer will help to find the nature of the lamination of the earth's crust and the upper mantle according to elastic properties. 


\section{REFERENCES}

[1] Litvinenko, I. and Nekrasova, K.: Bull. "Deep Seismic Sounding". Moscow (1962)

[2] Litvinenko, I.: New Seismic Data about Crustal Structure of the Baltic Shield. Reports, $A N$ SSSR, 1496 (1963) 


\title{
THE STRUCTURE OF THE EARTH MANTLE'S UPPER
}

\section{PART ACCORDING TO THE OBSERVATIONS OF THE EARTHQUAKES WITH INTERMEDIATE FOCAL DEPTH}

\author{
A. A. Luck and I. L. Nersesov
}

(Moscow, USSR)

The structure of the upper earth mantle was investigated by the earthquake data at the Pamir-Hindukush epicentral area. The specific property of the latter is local space distribution and the presence of the strong earthquakes frequently generated at a focal depth from 70 to $270 \mathrm{~km}$. To increase the accuracy of the focal geometric coordinate determinations, in the epicentral area, five temporary seismic stations were installed. Elastic waves recorded on the profile of stations, crossing Central Asia, Eastern Kazakhstan, the Altai and the Sayans up to the Lena. An average interval between the stations was $70-100 \mathrm{~km}$. The total length of the profile was about $3500 \mathrm{~km}$. All points of observations were equipped with identical apparature with seismic detectors SKIIIM type and galvanometers GB-4. The seismic channels had flat amplitude curves over the frequency range from 10 to $0.7 \mathrm{cps}$ with an average magnification of approximately 50000 .

Earthquake epicenters were determined by K-epicentrals method (Fig. 1), independent of velocity cross-section of environment medium; focal depths were defined by altered method of theoretical time distance curves.

Velocity cross-section was studied in two stages. First, at a distance of about $1000 \mathrm{~km}$ the subcrustal structure of the upper mantle was estimated to depths of $240-250 \mathrm{~km}$. The interpretation was carried out by a complex technique, main principles of which were taken from well developed methods of seismic prospecting and was based on the 240 earthquake data.

The starting points of this technique are the method of time depth curve (Fig. 2), non-longitudinal travel time curve method, the time field method, "the field" of horizontal travel time curves and the method of vector travel time curve subtractions.

For the greater depths, up to $1400 \mathrm{~km}$ the earthquakes of different focal depths, recorded by all seismic stations throughout the profile, were used and in addition to this the records, obtained by the Soviet stationary seismic stations, were employed. Velocity cross-section of the mantle was achieved by means of longitudinal waves according to the Herglotz-Wiechert method. Experimental results allowed to separate the waves reflected from the main boundaries in the upper mantle. The reflected wave data were associated with the results of other methods. In addition to kinematic data individual dynamic properties of separated waves were used. The combination of kinematic and dynamic features of seismic 
records in comparing with published works provided a considerable increase of the reliability of the resulting velocity profile.

In Fig. 3 velocity cross-section of the upper mantle up to $1400 \mathrm{~km}$ is sketched. Consideration of this figure shows that in the subcrustal layer below the crustal foot at the absolute depth of $85 \mathrm{~km}$ a boundary surface can be distinguished. This

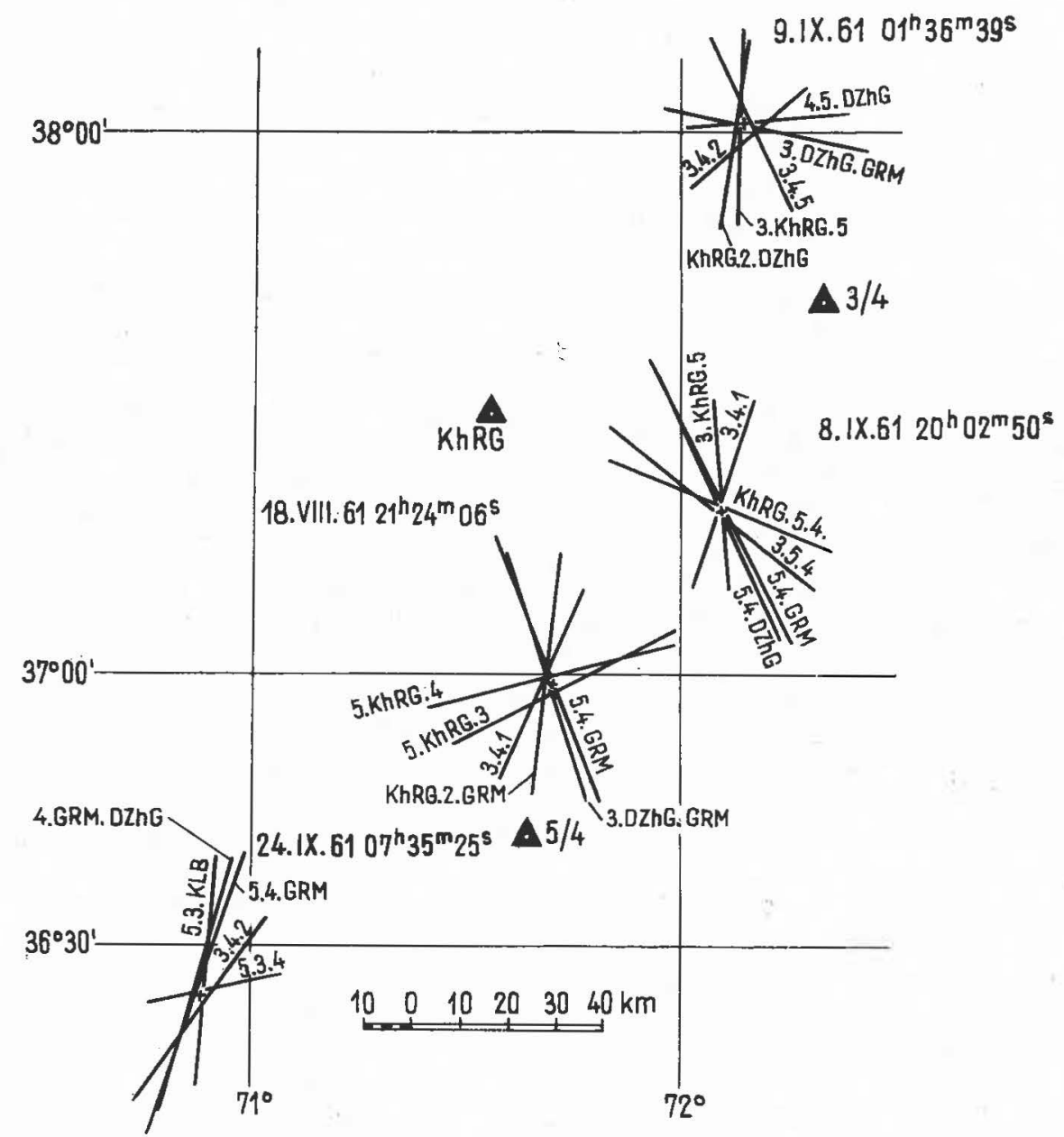

Fig. 1. An example of the K-epicentral determinations

surface can be characterized by increasing velocity. Below, the velocity remains practically constant or has very slight positive gradient. Over the depth range from 100 to $150 \mathrm{~km}$ the channel of lower velocity values of body waves is observed. The reliability of channel separation is due to the methods of non-longitudinal time depth curves and vector travel time curve subtraction. 
Below the depth range from 150 to $200 \mathrm{~km}$ gradual slight velocity increasing of body waves appeared and at a depth of $200 \mathrm{~km}$ a very distinct boundary surface of velocity increasing is seen. The depth range from $200 \mathrm{~km}$ to $400 \mathrm{~km}$ is characterized by various velocity cross-sections of longitudinal and transverse waves. For longitudinal waves over the whole depth range a slight velocity rise

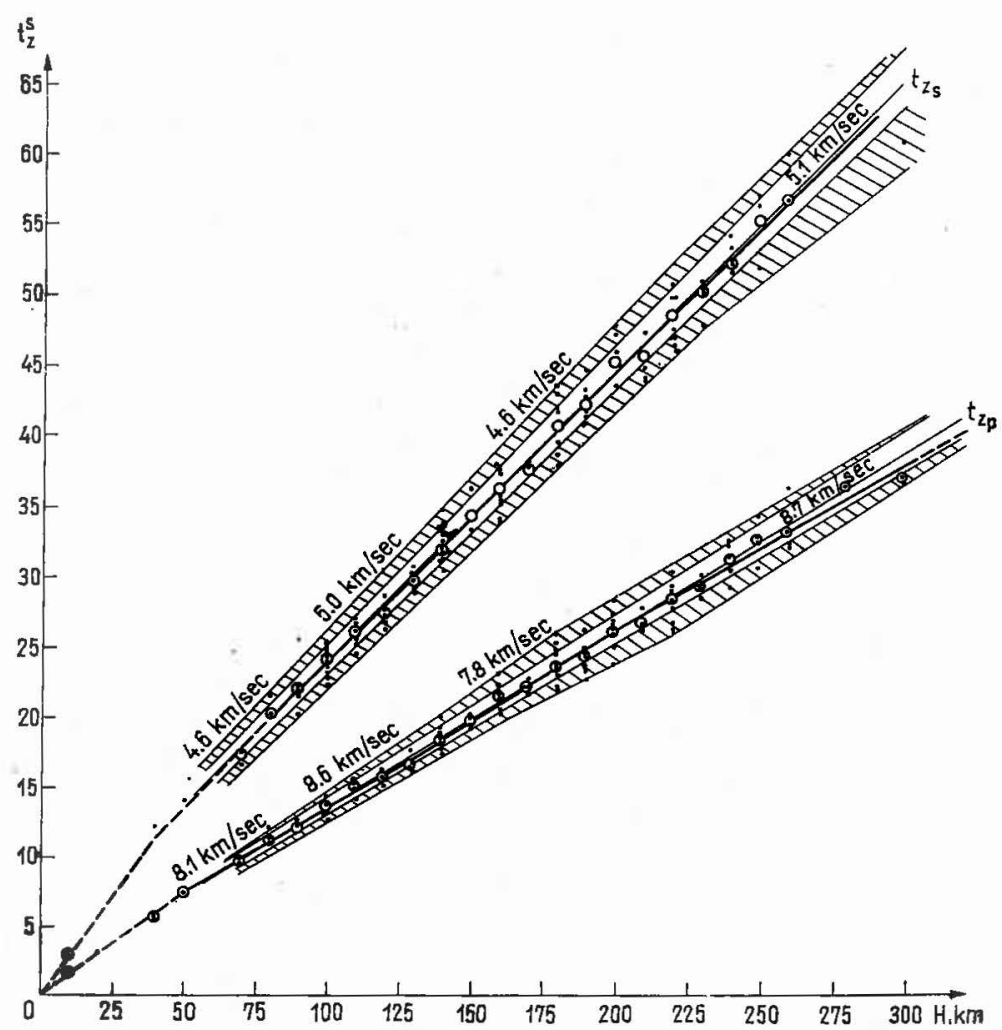

FIG. 2. The time depth curve determined on the Pamir-Hindukush earthquake

from $8.6 \mathrm{~km} / \mathrm{sec}$ to $9.0 \mathrm{~km} / \mathrm{sec}$ takes place. For transverse waves over the depth range from 240 to $400 \mathrm{~km}$ the second channel of the lower velocity values is observed. In the interval of depth range from 400 to $700 \mathrm{~km}$ of the body wave velocity regularly increases. Over the range of $700 \mathrm{~km}$ the velocity gradient rises abruptly up to depths of $780 \mathrm{~km}$. At this depth the boundary surface is characterized by the reducing of the gradient. At the depth of $900 \mathrm{~km}$ the boundary surface observed (although very uncertainly), is characterized by a slight increasing of the gradient of the longitudinal waves. As far as transverse waves are concerned, this boundary is not traced.

Let us briefly consider the cross-section received. At a depth of $85 \mathrm{~km}$ the 
boundary is distinctly șeen for reflected, head and exchange sP-waves and also for the abrupt change of seismic record of the earthquakes located over and directly below the boundary.

As an example of the first waveguide existence theoretical and experimental isochrones built on the basis of the observed cross-section to a depth of $200 \mathrm{~km}$

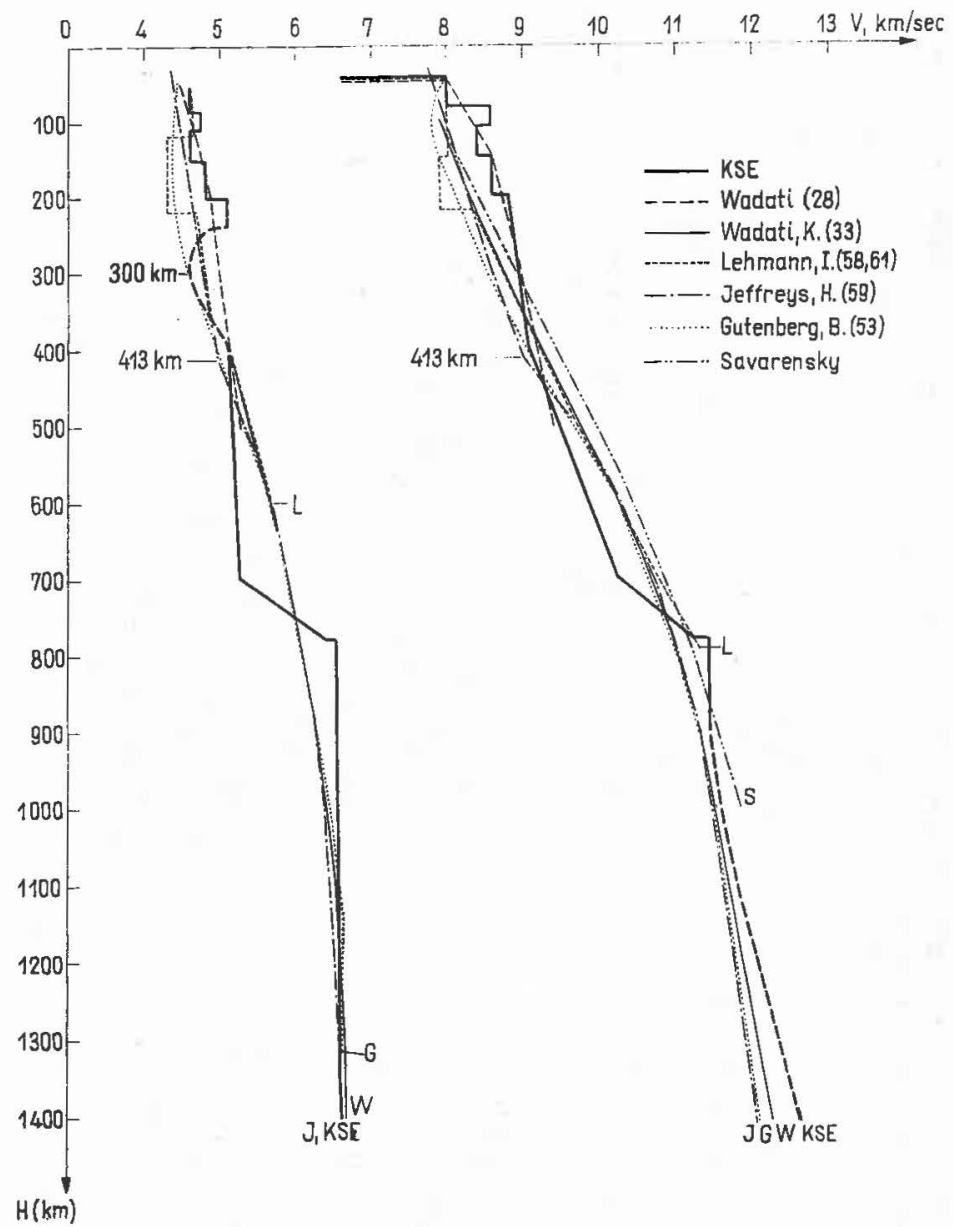

FIg. 3. Velocity cross-section of the earth's upper mantle for the data obtained by different authors

are shown in Fig. 4. The reducing of the longitudinal wave velocity in the waveguide by $0.2 \mathrm{~km} / \mathrm{sec}$ appeared to be insufficient for agreement of computed and experimental results. Here the overfall is likely to be somewhat larger and attains the values of $0.4 \mathrm{~km} / \mathrm{sec}$. It should be noted that the maximum discrepancy of 
theoretical and experimental time field does not exceed $0.8 \mathrm{sec}$ at a distance of $1000 \mathrm{~km}$; this indicates a reliability scheme of the upper mantle cross-section.

Figures 5, 6 and 7 illustrate kinematic time curves of the main wave groups for the focal depth of $200 \mathrm{~km}$. Kinematic data show the following main properties of the wave pattern. The time curve is the first arrivals of the longitudinal

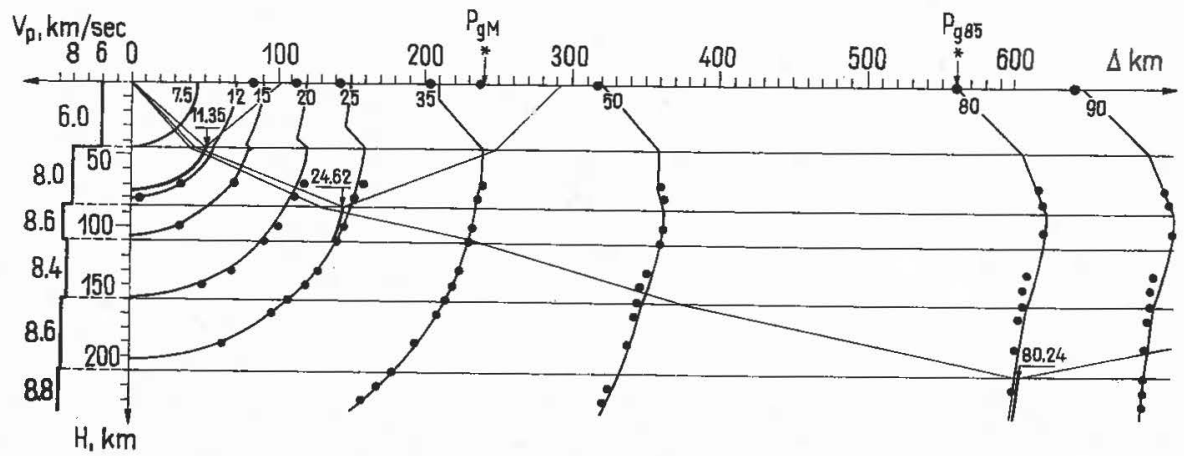

FIG. 4. Comparison of theoretical (thin block line) and experimental isochrones (black points) for the subcrustal layer profile. On the left is the velocity profile curve

waves as dependent on the focal depths, is practically rectilinear over the distance range from $300-500 \mathrm{~km}$ to $1600-1800 \mathrm{~km}$. At a distance of $1800-2200 \mathrm{~km}$ the time curve is characterized by two break points. Two time curve loops distinctly followed by the waves amplitude responses correspond to these points. The first loop is observed by the second arrivals over the distance range from 1200 to $2900 \mathrm{~km}$; the second - from 2000 to $3000 \mathrm{~km}$. The first of the loops is associated with the 400-kilometers boundary; the second - with the 700 kilometers range.

Kinematics of longitudinal waves strongly differ from transverse ones, this indicates the difference of mantle velocity cross-section for two main types of body waves. The time curve of transverse wave breaks abruptly at a distance of $1000 \mathrm{~km}$ and the transverse waves are observed during the times with the lag of 20-35 sec. Such a time curve denotes the uneven velocity reducing at the particular depth. Figure 7 shows the transverse waves reflected from the depth of 240,300 and $390 \mathrm{~km}$, which corresponds approximately to the second waveguide roof, axis and foot. From the longitudinal and transverse waves reflections we can readily determine the boundary surface of $700 \mathrm{~km}$ depth.

In the case of time curve discontinuity the Herglotz-Wiechert method is unavailable for the velocity cross-section determinations. Therefore the transverse waves velocity cross-section has been obtained from the longitudinal waves by rereading the curve of the layer velocities, obtained by an independent method.

Generally the upper part of the cross-section - up to $240 \mathrm{~km}$ - was plotted more precisely than the deeper one. The accuracy of the boundary surface 


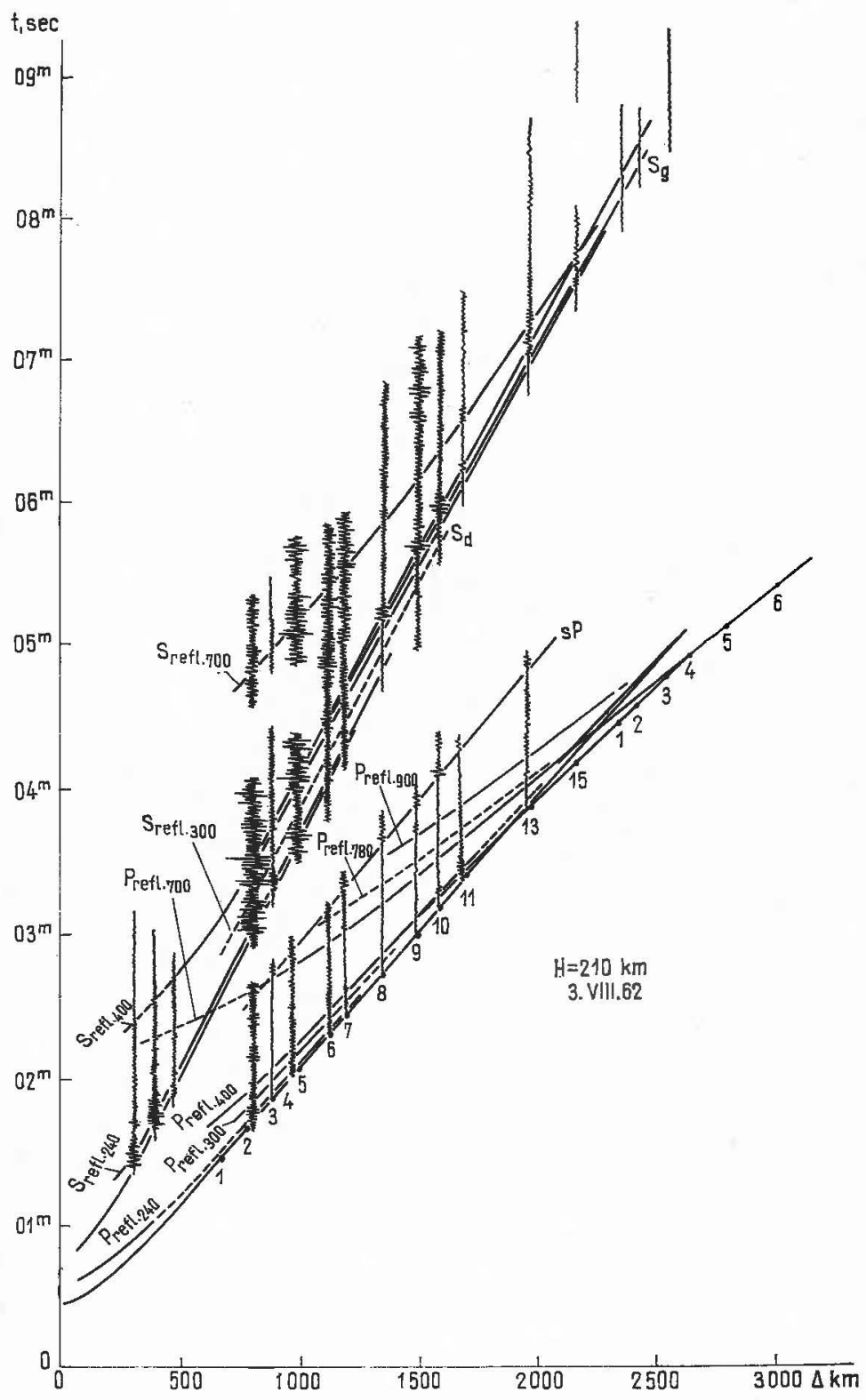

Frg. 5. The travel time curve installation of longitudinal and transverse waves for the earthquake of 3. VIII. $62(H=210 \mathrm{~km})$ 


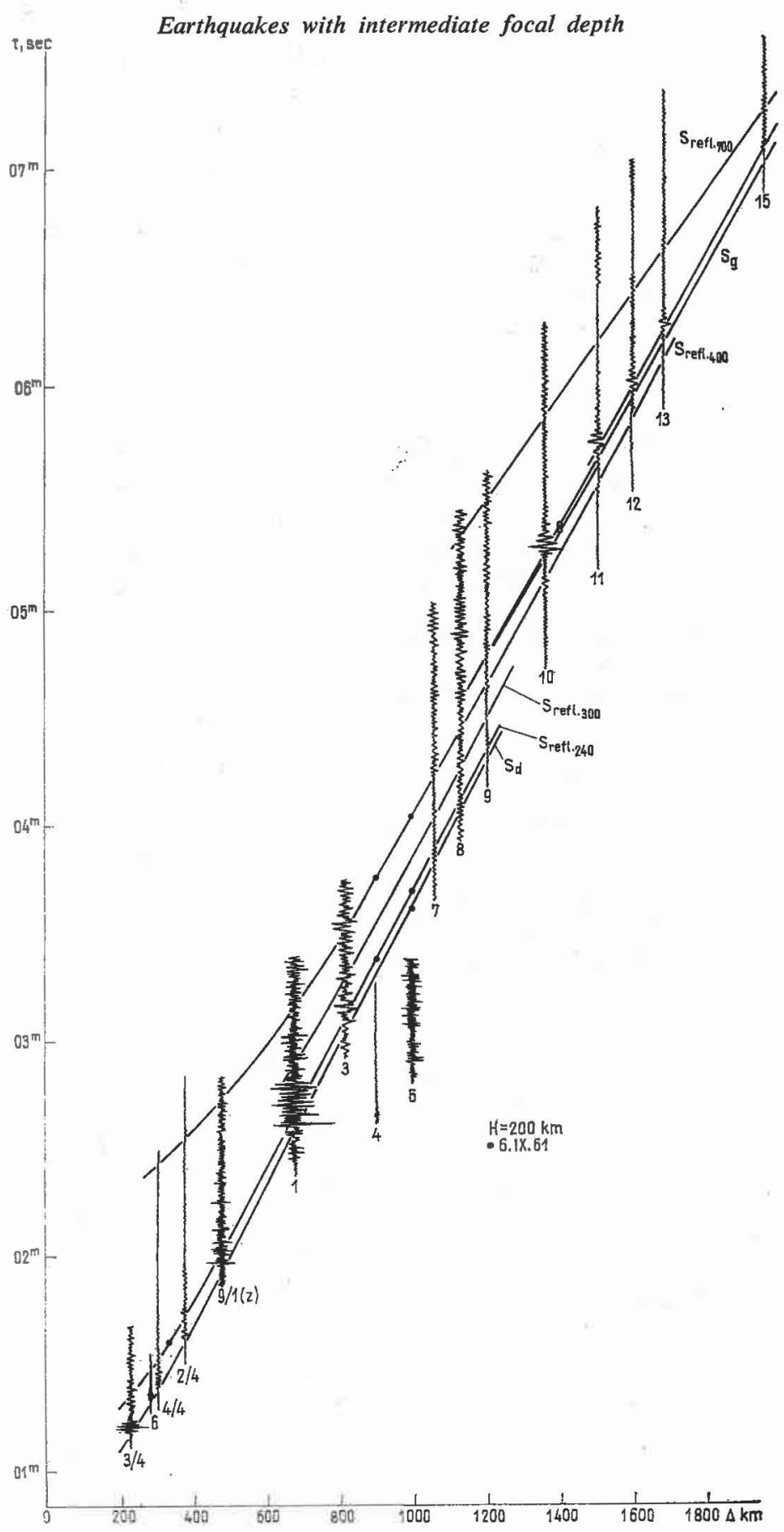

165 


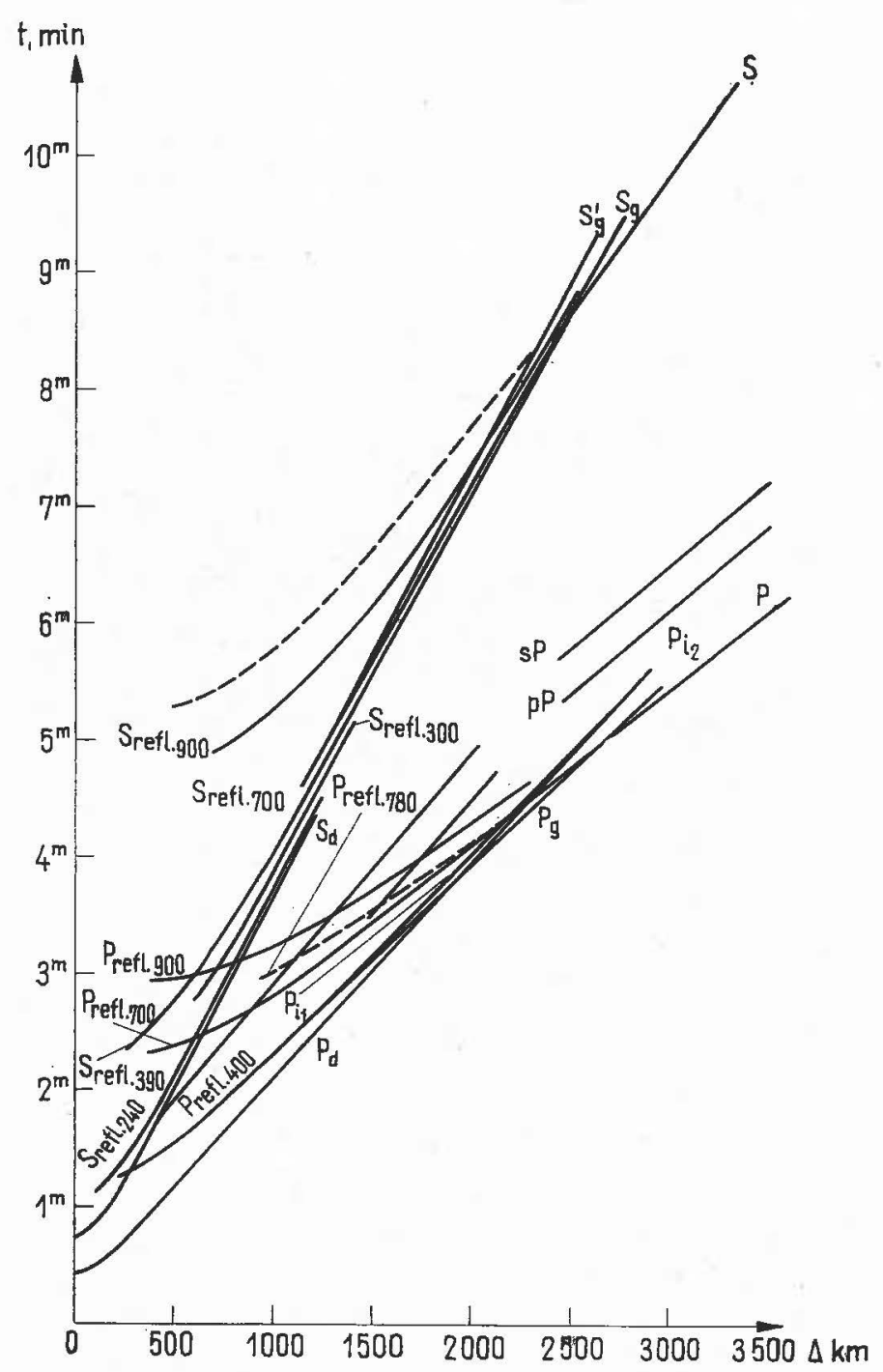

Fig. 7. Summary at travel time curve for focal depth equal to $200 \mathrm{~km}$

determinations in the mantle for the uppermost cross-section can be estimated by $\pm 5 \mathrm{~km}$ and the velocity values - by $\pm 0.1-0.15 \mathrm{~km} / \mathrm{sec}$. At the bottom of the profile the accuracy of the boundary surface determinations varies from \pm 10 to $25 \mathrm{~km}$ and the velocity values - from \pm 0.15 to $0.25 \mathrm{~km} / \mathrm{sec}$. It should be noted that the nature of the transition boundary surfaces and the shape of channel of the lower velocities are presented schematically as the available material does not allow to estimate the profile characteristics more briefly. 
The system for observation did not give any opportunity to separate the waves, reflected from the core of the earth although they should have been distinctly separated beginning from $3000 \mathrm{~km}$. Now the cause of the absence of such reflections remains unknown to us.

Thus, the main features of the mantle cross-section on the area in question, the lower velocity layer is located at a depth of $110-150 \mathrm{~km}$ for the longitudinal and transverse waves, the second waveguide - for the transverse waves only at a depth of $240-400 \mathrm{~km}$ and higher gradient layer - at a depth of 700 $780 \mathrm{~km}$. The obtained mantle cross-section refers to the area investigated and does not pretend to be universal. The cross-section is likely to be somewhat different for various areas throughout the world thus, one of the most significant aims of seismology should be to consider the necessity of study of the structural differences due to the development of geological processes of the earth's upper mantle. 


\title{
DEEP SEISMIC SOUNDING IN HUNGARY
}

\author{
E. Mituch and K. Posgay
}

(Budapest, Hungary)

The work on deep seismic sounding commenced in 1954 with reflection measurements. Valuable deep reflections were obtained at about 10 points with hole-shooting [1, 2].

In 1958 the deep sounding was carried out for the first time by means of the refraction method and this work continued also in the following years [3]. This work was realized by the use of hole-shooting; a diminution of the energy could be observed on greater distances, whereas in several instances no arrivals were obtained at all. This was due to the intensity of seismic ground disturbances, hindering the complete utilization of the sensitivity of our instruments. In order to obtain better results it was necessary to carry out further experiments.

These measurements were directed in 1962 to observations in the proximity of the so called "critical distance". In the proximity of this distance we have observed the expected increase of reflection amplitudes (Fig. 1). It was found that both reflected and refracted waves could be most readily observed in the proximity of the critical distance.

According to this, our experimental measurements were continued in 1963 by a complex method [4]. Our work was based on the use of wide-angle reflections. In this method several observations were arranged near to the shot-points for deep reflections, while others were made at a greater distance for obtaining first arrivals from the Mohorovičić discontinuity (Fig. 2). Since the greatest amplitudes of wide angle reflections appeared at a distance of $50-60 \mathrm{~km}$, our shot-points were arranged with a spacing of about $60 \mathrm{~km}$. In the proximity of the shot-points the shots from the neighbouring shot-points were observed. These observations were carried out over these parts of the profiles in an interval of $22 \mathrm{~km}$ (the spacing of geophones being $200 \mathrm{~m}$ ). For the first refraction events the observations were realized in a distance of 100 and $120 \mathrm{~km}$ from the shotpoint. The observation of deep reflections covered several kilometers in the proximity of the shot-points. Accordingly, this can be regarded as a correlation system observed at intervals.

The following can be reported briefly on the results obtained in 1963 [5].

1. At the "critical distance" generally wide angle reflections were recorded, shown also by the hyperbolic curvature of the time-distance diagram.

2. The amplitudes of wide angle reflections showed is generally a significant energy maximum in the proximity of the critical distance (Fig. 3). 
3. The arrivals from the Mohorovičić discontinuity came in two groups with a time interval of $200-300 \mathrm{msec}$ (Fig. 4). The second group of waves showed much greater energy. This group could be followed over the whole length of the profile (our first profile of this kind was $300 \mathrm{~km}$ long).

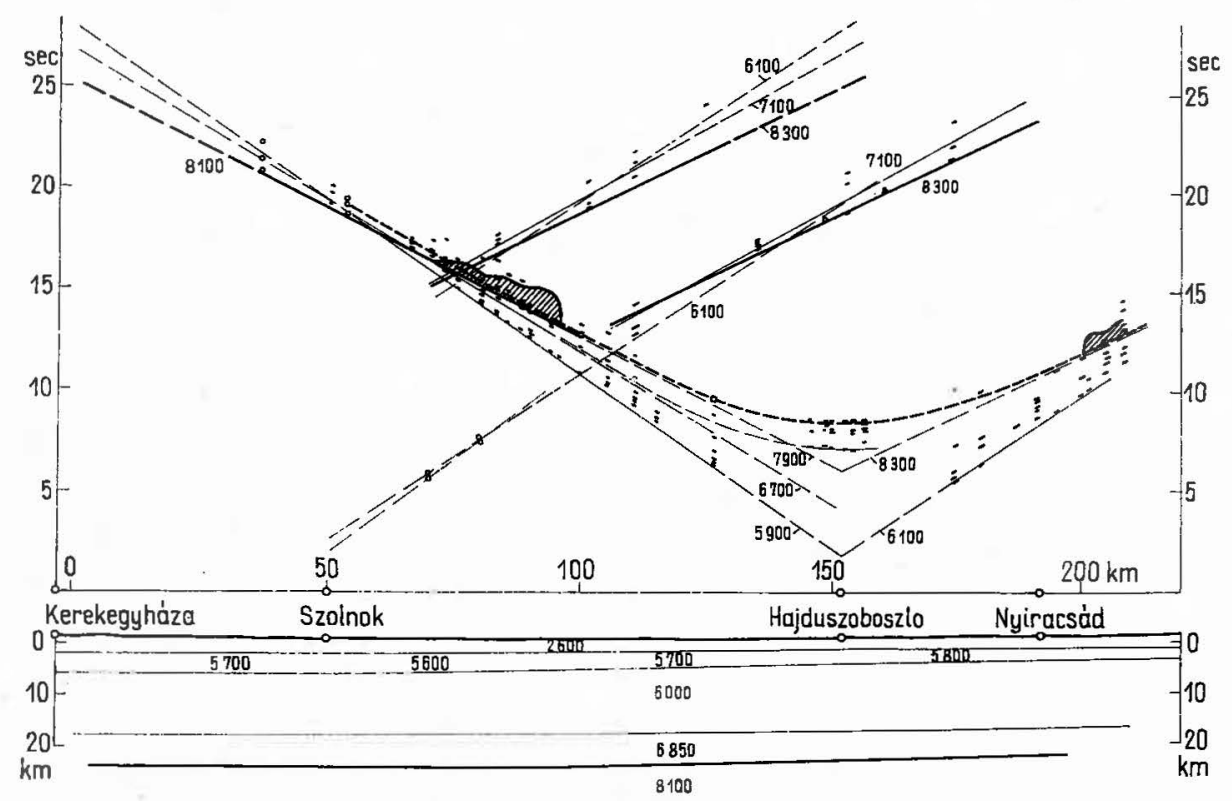

FIG. 1. Time-distance diagram and section on the experimental profile for the investigation of the crust, measured in 1962

4. The Mohorovičić discontinuity appeared with a boundary velocity of 8100 $\mathrm{m} / \mathrm{sec}$.

5. The depth of the Mohorovičić discontinuity varies along our first profile within the range of 24.4 and $27 \mathrm{~km}$ (Fig. 2).

6. Another discontinuity could be traced at a smaller depth. Its boundary velocity was determined as $6100 \mathrm{~m} / \mathrm{sec}$. Its depth varied from 3 to $9 \mathrm{~km}$.

7. Between these boundaries, sometimes another horizon coinciding with the boundary interpreted earlier as the Conrad discontinuity appeared. Its velocity can be set to abt. $6850 \mathrm{~m} / \mathrm{sec}$. Its depth in the eastern part of our profile was $19 \mathrm{~km}$.

After these successful experiments the deep sounding work was planned in 1964 in a strict correlation system. In accordance with this the measurements are conducted along two parallel profiles located at a distance of about $60 \mathrm{~km}$ from each other (Fig. 5). The observations are carried out on the whole length of both profiles. The shots are fired in each case on the other profile against the corresponding observation intervals. The shot-points are arranged between the 


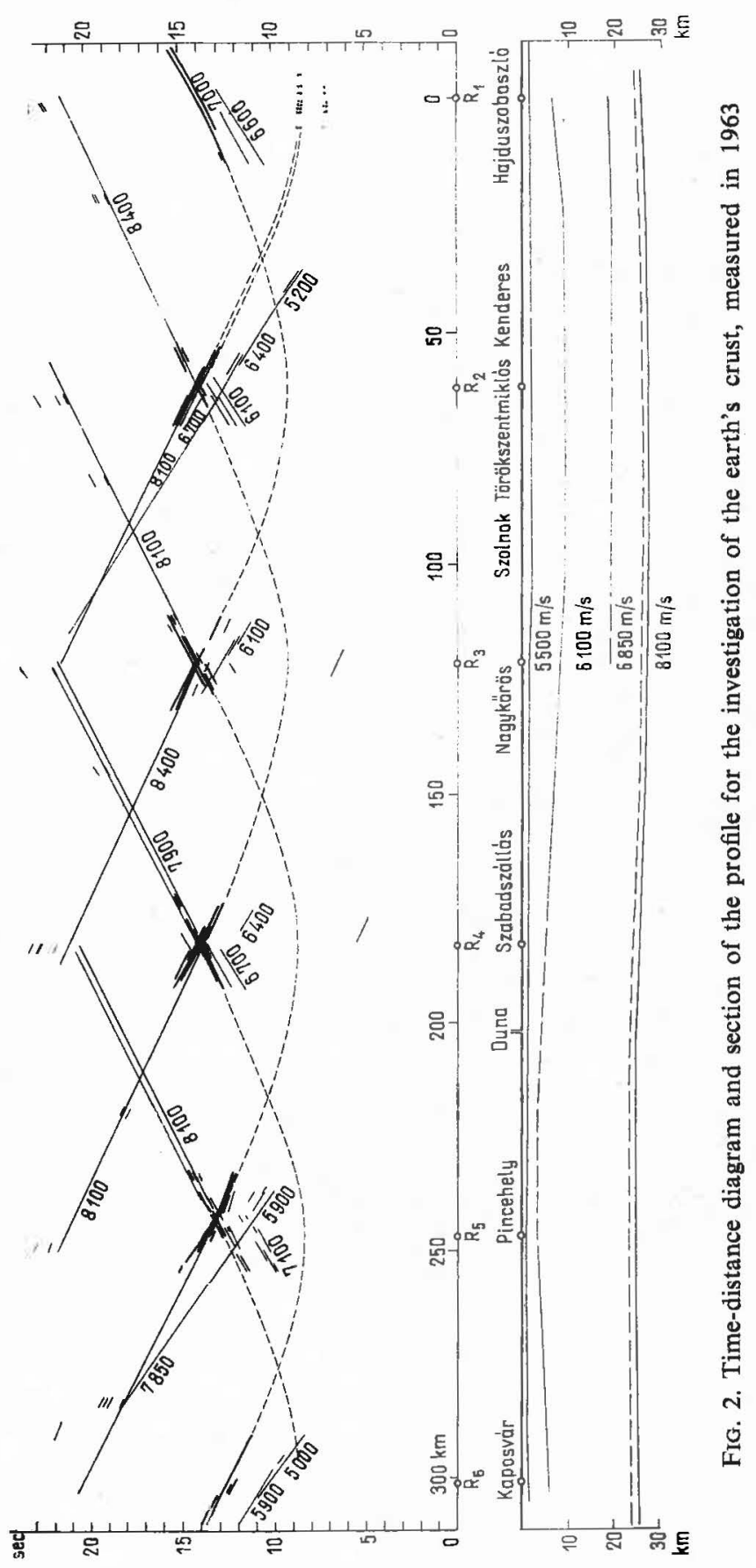




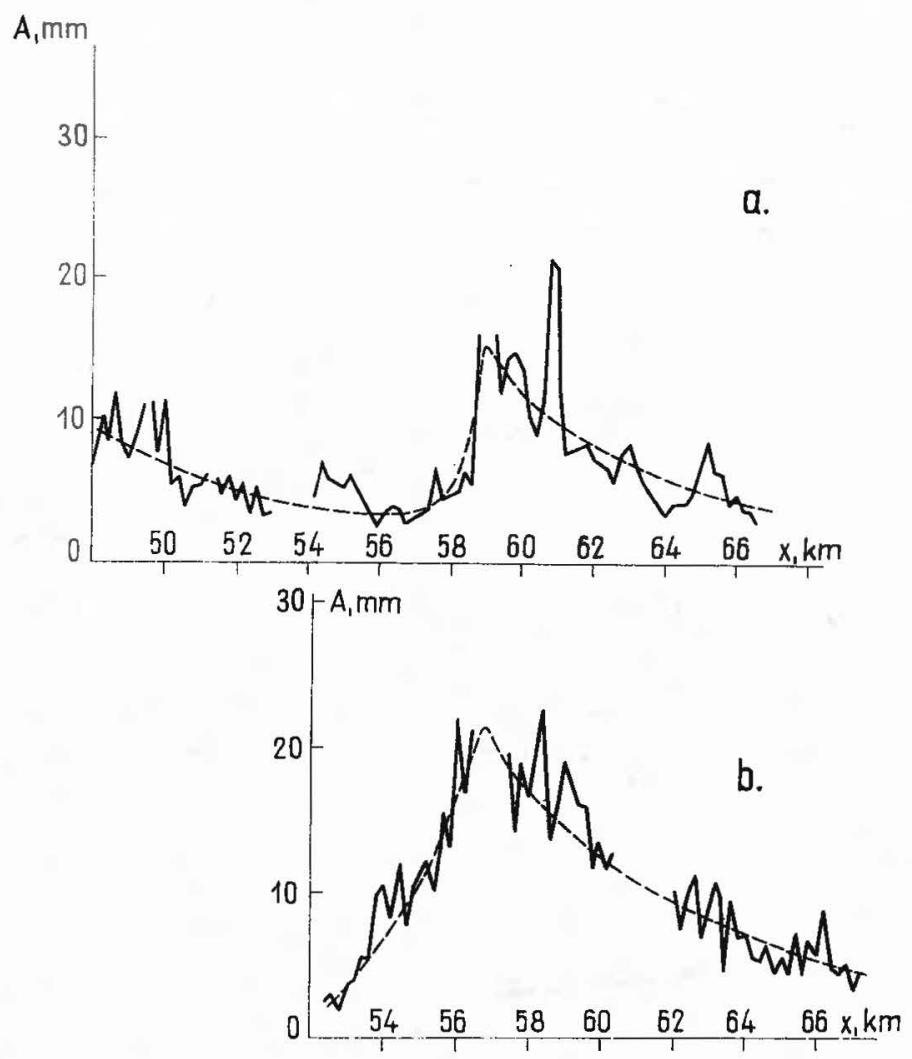

Frg. 3. Amplitude curves. The continuous line indicates the observed data, the dashed line the average value

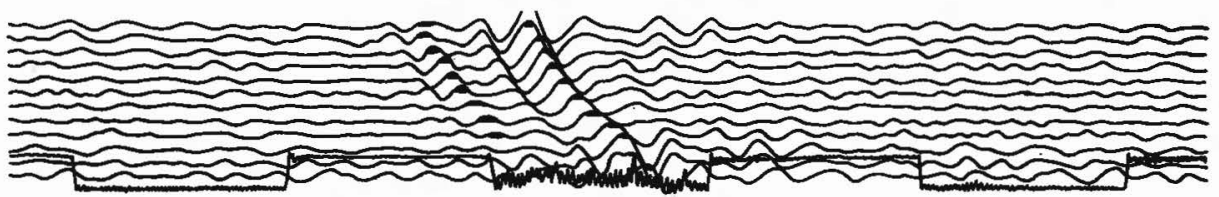

$\overleftrightarrow{0,1 \mathrm{sec}}$

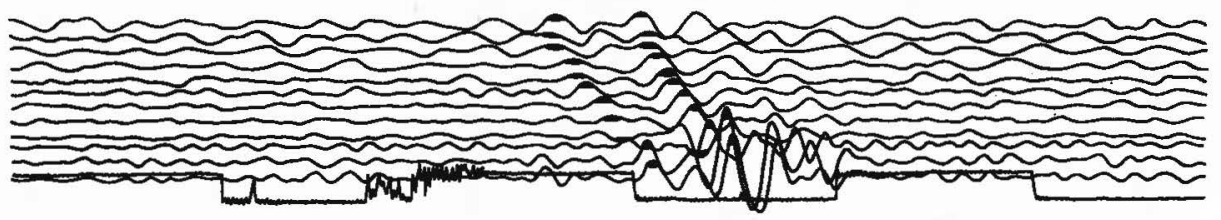

$$
\stackrel{\longmapsto}{0,1 \mathrm{sec}}
$$

FIG. 4. Wide angle reflection from the Mohorovičić discontinuity appearing with double group of waves 


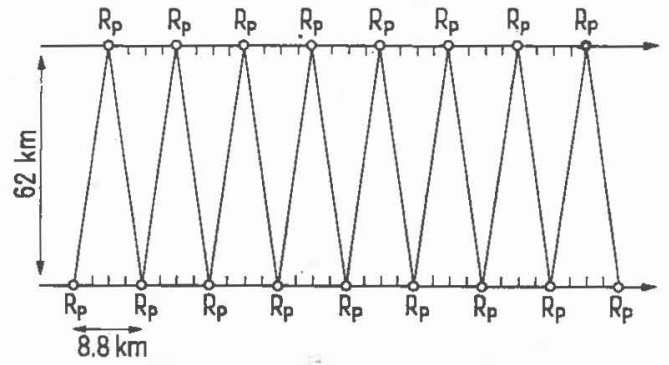

FrG. 5. Scheme of the cross-shooting systems on the profile for the investigation of the crust, measured in 1964

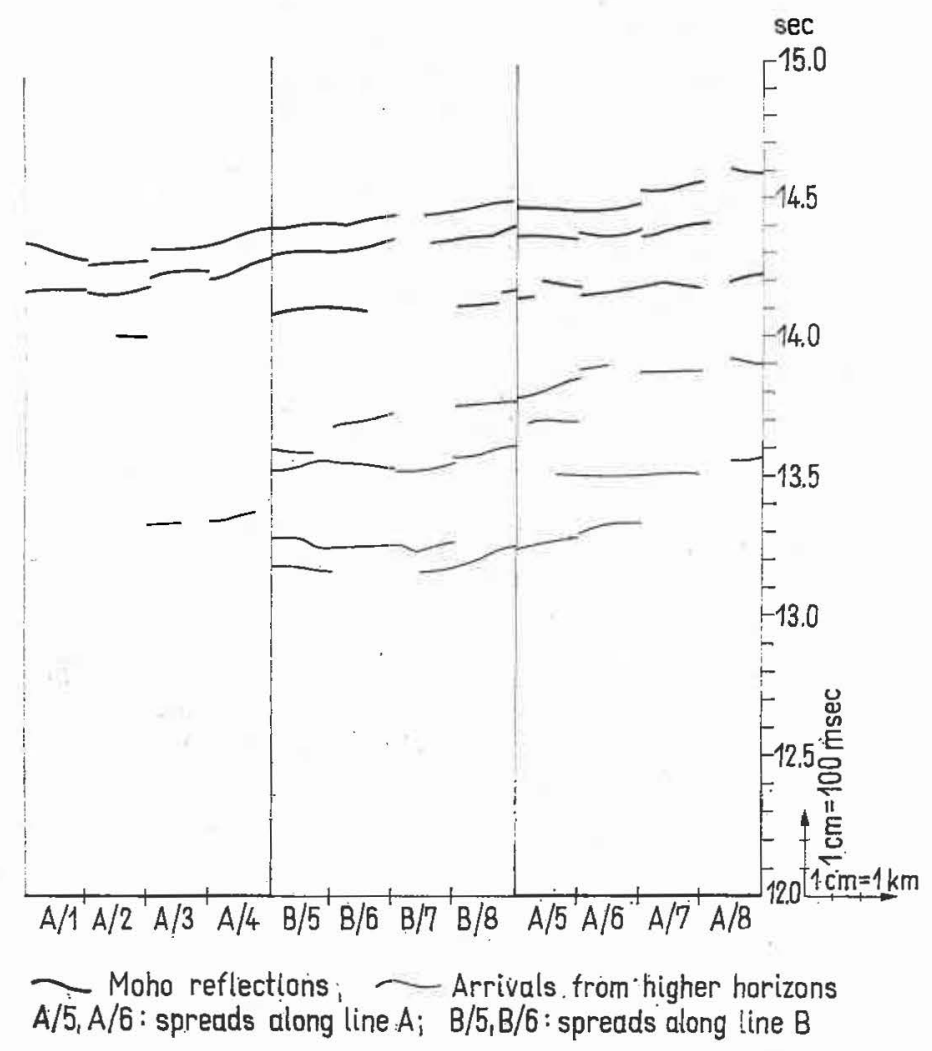

FIG. 6. Section of the time-distance diagram of the profile for the investigation of the crust, measured in 1964 
observation intervals, from which the shots to be observed on the opposite profile are fired.

In this manner it becomes possible to correlate the travel times over the whole length of the system, while the observations are made always at the distance of optimal energy conditions. Figure 6 represents a part of the time-distance diagram on a profile, shot in such an arrangement. The thick lines indicate the arrivals from the Mohorovičic discontinuity. They appeared on the whole profile with smaller interruptions, had a significant energy and could be correlated.

By means of this observation system we have succeeded in realizing the deep seismic sounding as a fast normal routine work. The system has another advantage: the work is done relatively near to the shot point, therefore the resolution factor is fairly great.

This work of deep sounding has been carried out by the seismic department of the Loránd Eötvös Geophysical Institute.

\section{REFERENCES}

[1] GÁlfi, J. and Stegena, L.: Nagymélységü reflexiók Hajdúszoboszló környékén (Deep reflections in the region of Hajdúszoboszló). Geof. Közl. 42 (1955)

[2] GÁLfi, J. and Stegena, L.: Szeizmikus reflexiós méréssel meghatározott néhány adat a földkéreg magyarországi részéről (Some data obtained with seismic reflexion measurements concerning the Hungarian part of the earth's crust). Geof. Közl. 4 1-2 (1957)

[3] Gálfi, J. and Pálos, M.: Refrakciós kéregkutató szelvény a Magyar Medencében (Refraction profile for crustal research in the Hungarian Basin). Geof. Közl. 84 (1960)

[4] Mrtuch, E., Posgay, K. and SÉdy, L.: Szélesszögü reflexiók alkalmazása a kéregkutatásban (The use of wide angle reflexions for the investigation of the earth's crust). Geof. Közl. 132 (1964)

[5] MrтUch, E.: A hazai szeizmikus kéregkutatás újabb eredményei (Recent results of the investigation of the earth's crust by seismic method in Hungary). Geof. Közl. 133 (1964) 


\author{
THE STUDY OF DYNAMICS AND KINEMATICS \\ OF SEISMIC WAVES AT THE PROFILE PAMIR-BAIKAL
}

I. L. Nersesov and T. G. Rautian

(Moscow, USSR)

The nature of kinematic and dynamic characteristics of seismic waves is one of the main problems in the study of the earth's structure.

We aimed to study the wave field by means of observations at the station profile passing through Middle Asia, East Kazakhstan, Altai and Baikal. There were about 50 stations at distances of 20 to $100 \mathrm{~km}$. The length of the profile was $3500 \mathrm{~km}$. SK-M-III seismographs and GB-IV galvanometers with $0.6-10$ cps frequency band and magnification of about 50000 were used.

Both the epicenters along the profile or its extension and the epicenters of transversal direction (India, China, Mongolia) were taken in account. The epicentral distances observed ranged from 20-100 to $3000-3500 \mathrm{~km}$. We studied $\bar{P}, P_{n}, P_{g}$ and $P$-waves and the corresponding transversal waves.

It was found that each of these waves consists of several interfering waves and the dynamics of the whole wave group depends on the type of interfering waves. Each group of waves has different dynamic characteristics in different geological regions intersected by the station profile.

The $\bar{P}$-wave is a straight wave only at distances less than $100-120 \mathrm{~km}$. At distances of $120-200 \mathrm{~km}$ the $\bar{P}$ is replaced by a refracted wave in the earth's crust or the wave reflected by the Conrad boundary. This wave group is quickly attenuated and becomes undetectable at distances of $200 \mathrm{~km}$. At greater distances (from 200 to $500-1200 \mathrm{~km}$ ) the $P_{g}$-waves are detected. The $P_{g}$ consists of a series of multiple waves reflected from the $M$-boundary and of multiple refracted waves in the earth's crust. The velocity of propagation of the $P_{g}$-group is about $6.0 \mathrm{kmps}$.

The group of waves recorded at the distances over $200 \mathrm{~km}$ near the first motions divides into several waves $P_{n}, P_{n}^{1}, P_{n}^{2}$ and $P_{n}^{3}$ reflected from the $M$-boundary and boundaries of the upper mantle. Each of these waves has a maximum amplitude at the moment of its appearance. The velocity of the first motion of the $P_{n}$-group slowly increases from 8.0 to 9.0 , when the distance increases from 200 to $2000 \mathrm{~km}$ corresponding to the velocity of waves replacing each other. The intensity of the whole group is of a pulsing nature (Figs 1-3).

The $P$-wave appears at a distance of about $1500 \mathrm{~km}$ and passes into the first motion at about $2200-2500 \mathrm{~km}$, the velocity of the first motion increasing sharply from 9.0 to $12.0 \mathrm{kmps}$. 


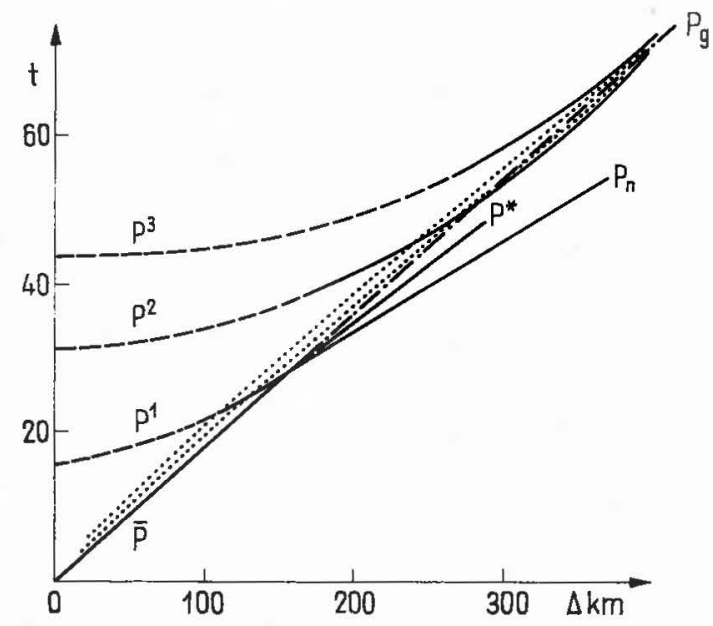

FIG. 1. Travel-time curve illustrated mechanism for $P_{\boldsymbol{z}}$-waves generations

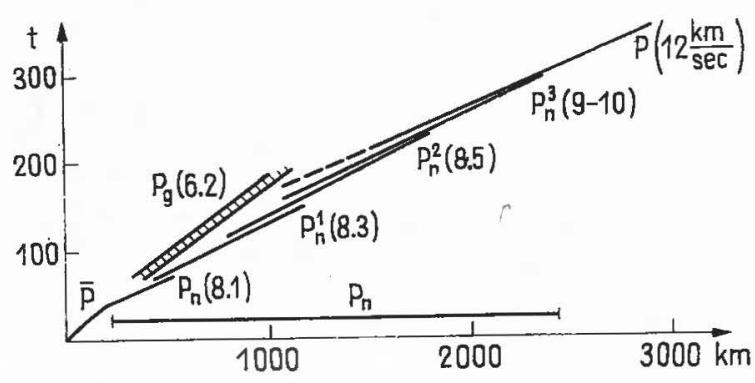

FIG. 2. Schematic travel-time curve for principal phase of longitudinal waves

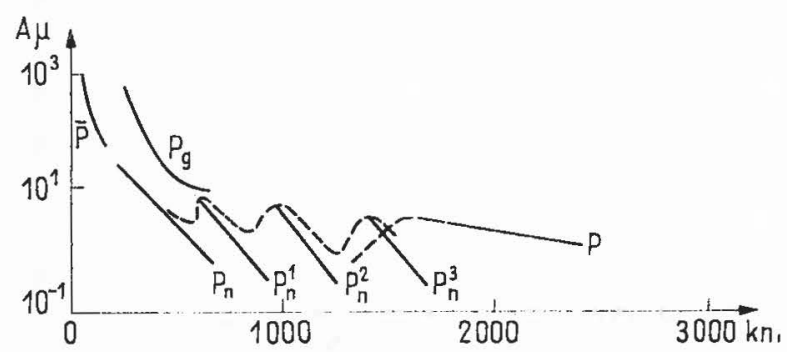

FIG. 3. Amplitude versus distance for principal phases of longitudinal waves 
The corresponding transversal $\bar{S}, S_{n}, L_{g}$ and $S$-waves also exist, while $S_{n}$ waves often are too weak to be observed. The $L_{g}$-wave, on the contrary, can be observed at longer distances than the $P_{g}$-waves because of better reflecting conditions for $S H$-waves than for $P$-waves.

$L_{g}$-waves have interesting regional features. When the epicenter is in the Baikal, East China or Mongolia regions, the dominant periods of $L_{g}$ are about 2-3 sec, whereas for epicenters in other regions the periods are about 5-8 sec and the short period component of $L_{g}(T=2.0 \mathrm{sec})$ is by $10-15$ times weaker than the long period component.

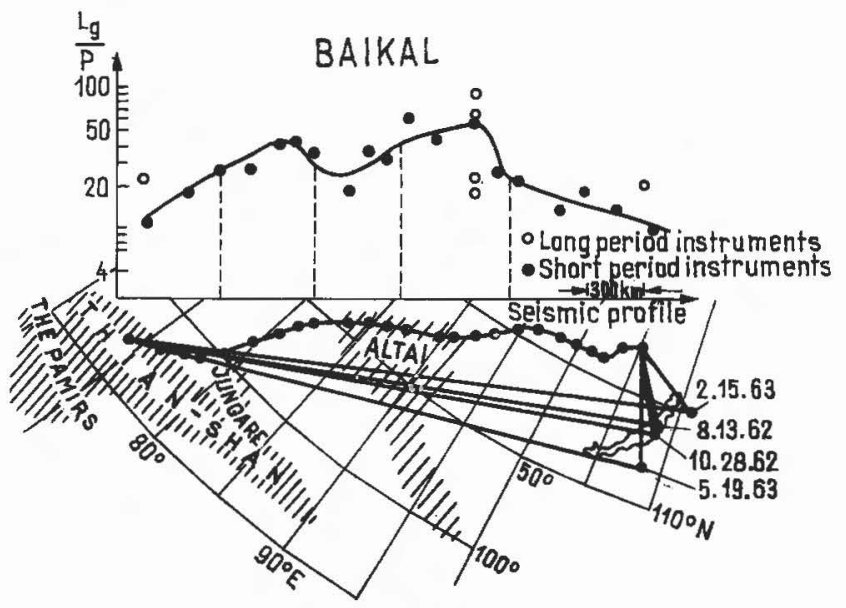

FIGs 4-8. Relative amplitudes of $L_{\mathrm{g}}$-waves along the seismic profile for different epicentral regions. At the foot of each graph is the index map showing epicenters, mountain chains (shaded) and seismic profile

It has been mentioned elsewhere that the $L_{g}$-waves are attenuated after crossing mountain systems. We have recieved numerical data about such an attenuation. The relative intensity of $L_{g}$-waves is described by the ratio $A_{L_{g}} / A_{f}, A_{f}$ being the mean amplitude of oscillations in the interval between the $P$ and $S$-waves. This value permits to eliminate the earthquake magnitude and the influence of the epicentral distance. To study the attenuation of the relative intensity of $L_{g}$, epicenters of transversal direction were used. The station profile was used as a screen on which the mountain systems threw the shadow. It was found that the relative intensity of short period $L_{g}$-waves attenuated by 3-7 times, when passing the Altai, 10 times passing Tibet and 6-8 times passing the Himalaya (Figs 4-8). The assumption of interferentional nature of $L_{g}$-waves permits us to explain their attenuation by the effect of the relief of the reflecting boundaries i.e. $M$-boundary or the earth's surface.

The complex analysis of the dynamic characteristics of $P_{n}, P_{g}, L_{g}$-waves permits of revealing the regional differencies of earth's crust and upper mantle structure. 
The regions of Siberia and the Baikal Lake are characterised by a sharp $M$-boundary. In this situation the head-wave $P_{n}$ is very weak, the value $A_{P_{g}} / A_{P_{n}}$ is about 10-20. The sharpness of the M-boundary promotes strong reflections of shortperiod oscillations of $P$ and $S$ waves. As a result there are intensive

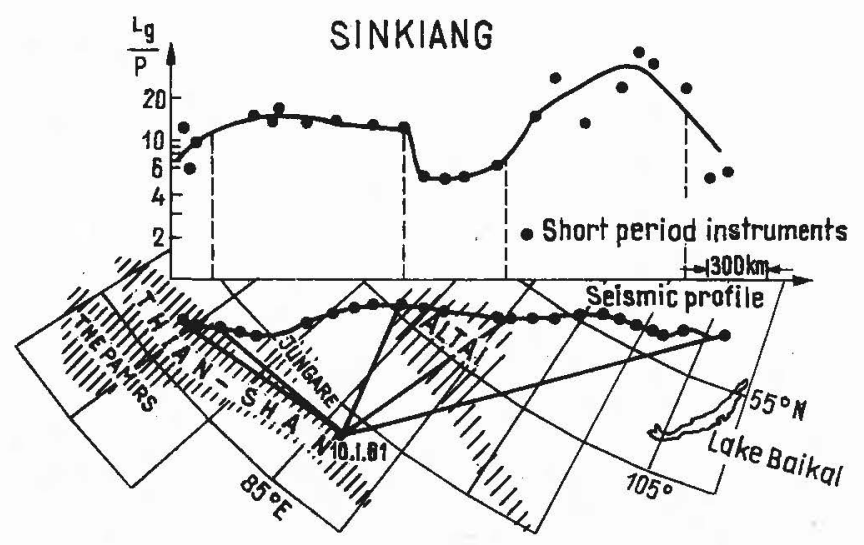

FIG. 5

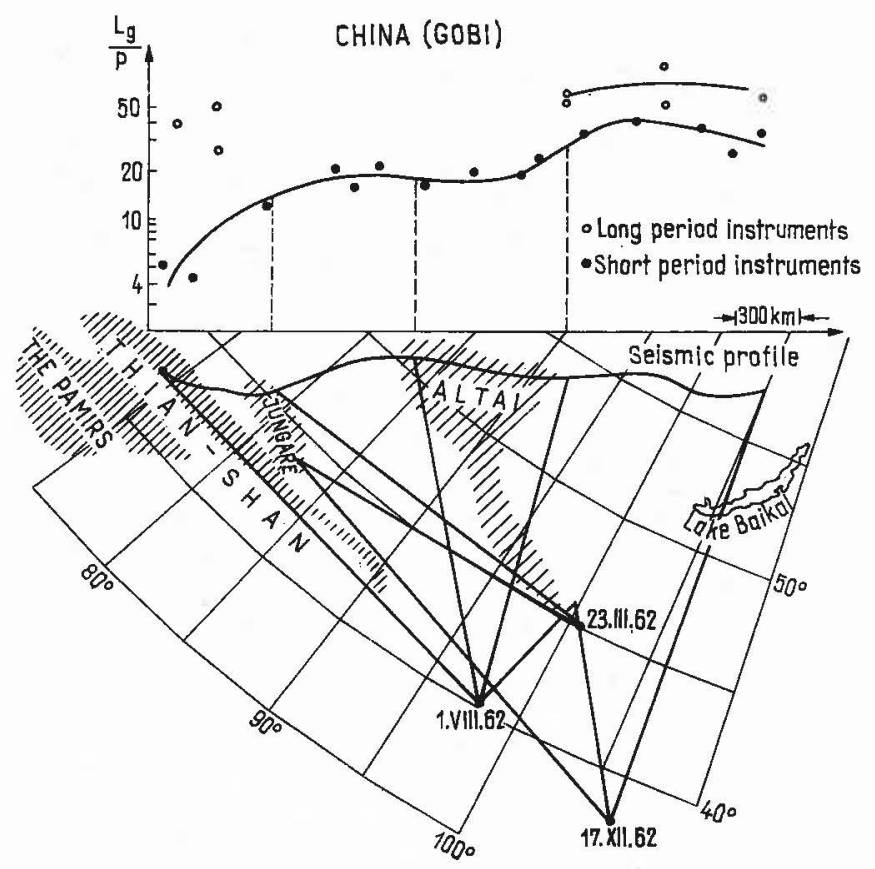

FIG. 6 
high-frequency $P_{g}$ asd $L_{g}$-waves in the Baikal Lake and Siberia regions. The passing $P$ - and $S$-waves lose a considerable part of their energy when crossing the $M$-boundary. That is why $P$ - and $S$-waves of all teleseisms are anomalously weak when recorded by Baikal region sesmic stations. Reversely, Baikal region earthquakes have an anomalously small amplitude of $P$ - and $S$-waves when recorded by all teleseismic stations (Fig. 9).

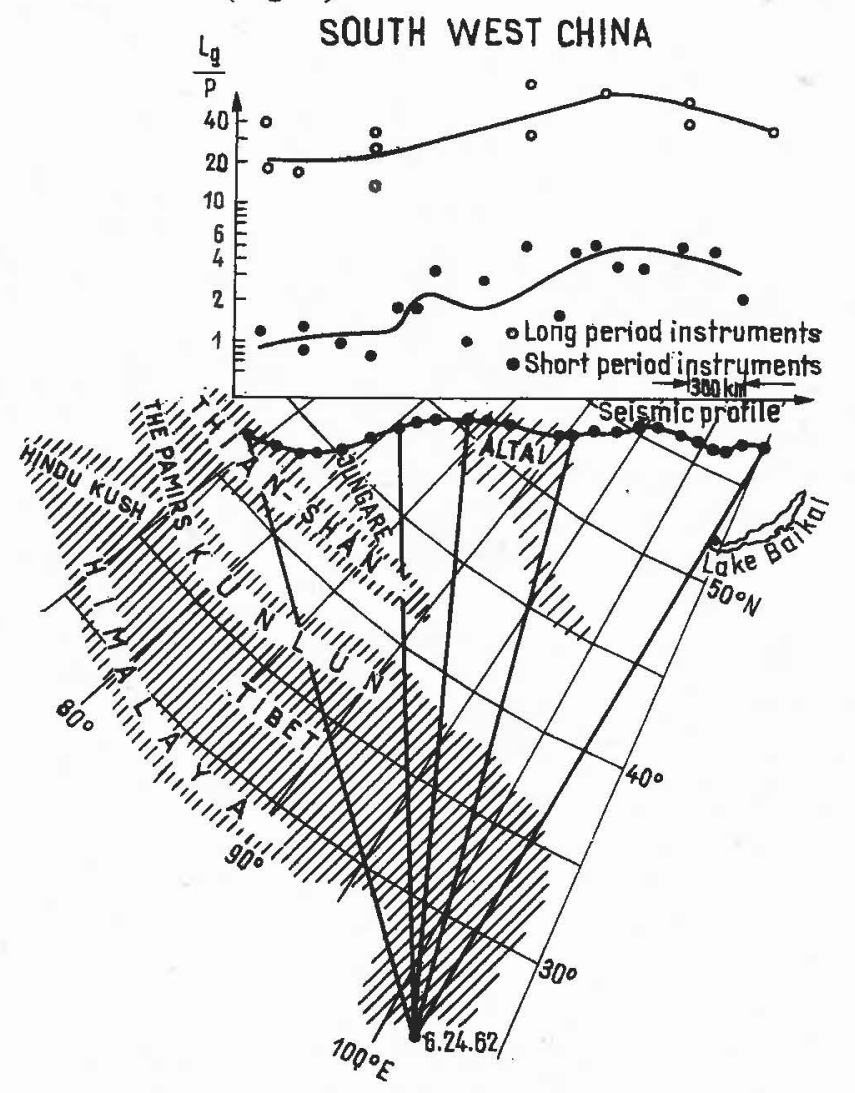

FIG. 7

In the regions of Middle Asia and East Kazakhstan the $M$-boundary being a transitional layer is not as sharp. Owing to these relatively weak reflected waves and relatively strong head $P_{n}$ waves are observed here, so that the ratio $A_{P_{g}} / A_{P_{n}}$ is $2-3$. The value of this ratio changes with the energy of the earthquake. This, in turn, points to the diffused character of the $M$-boundary. For long oscillations periods inherent to strong earthquakes, the $M$-boundary seems to be sharper than for weak earthquakes the typical shortperiod ones (Fig. 10).

In the intermediate zone of the Altai can be observed anomalies in kinematics and dynamics of $P_{n}$. The travel-time curves of the first motion vary depending on 


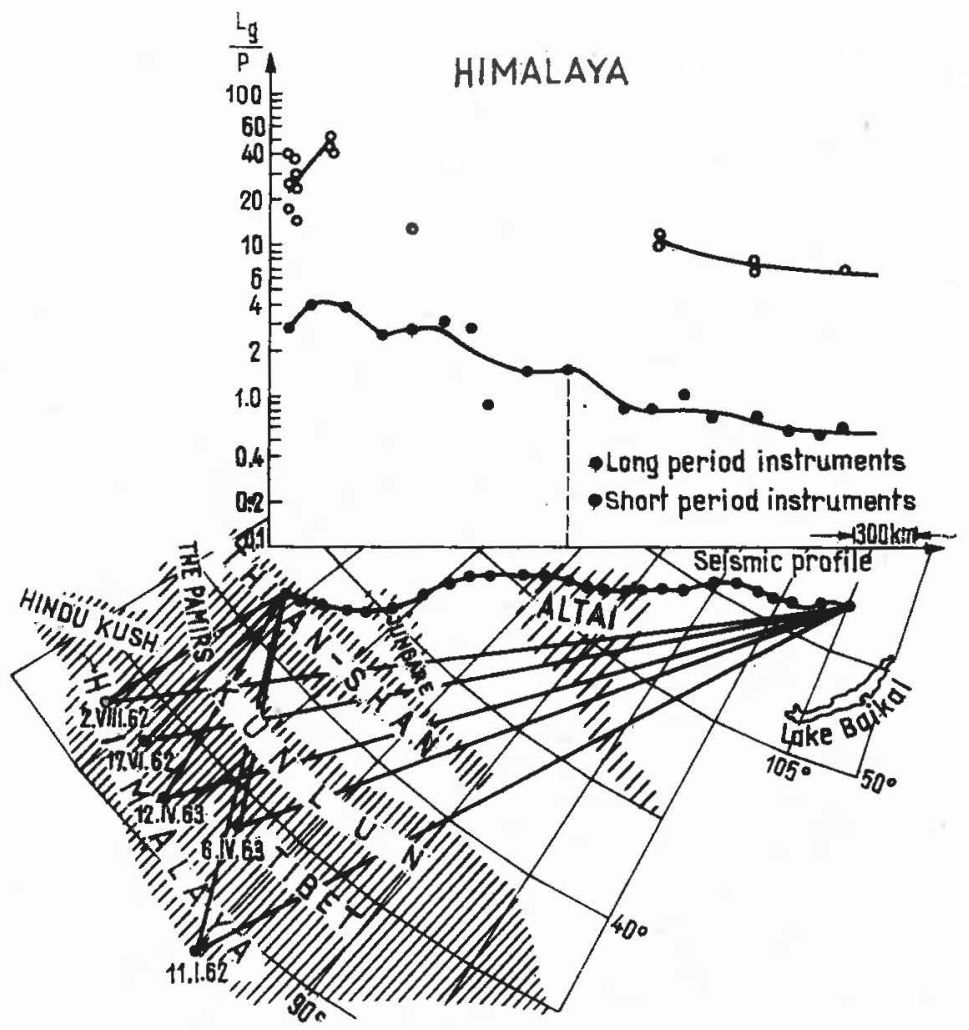

FIG. 8

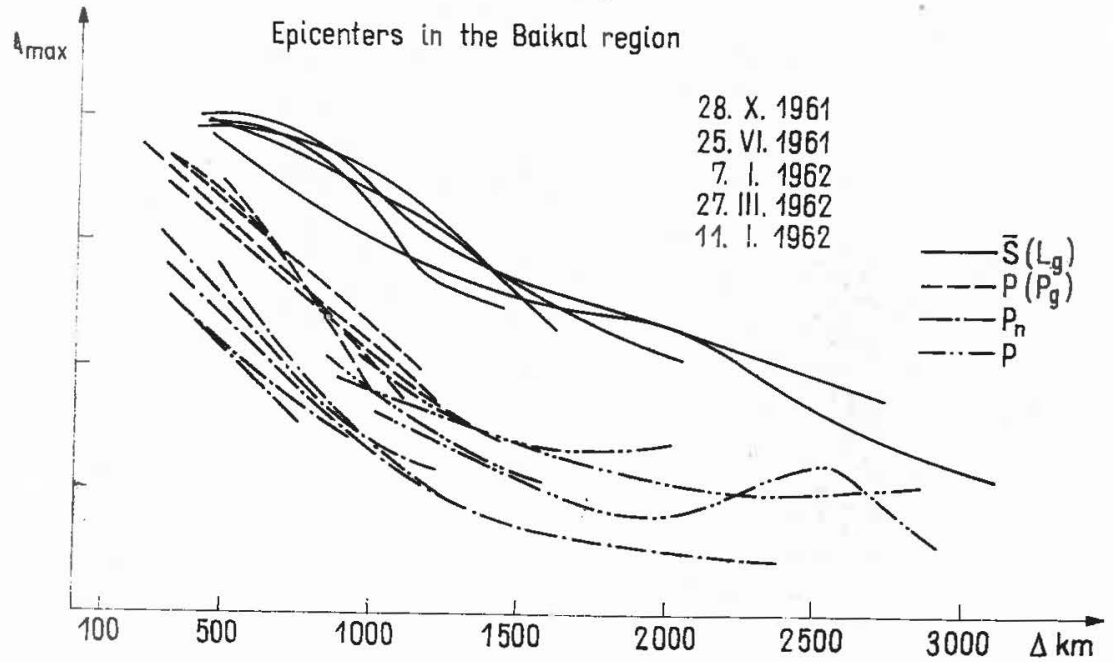

FIGs 9-12. Maximum amplitudes of principal phases versus epicentral distances for three epicentral regions - Baikal (Fig. 9), Middle Asia (Fig. 10) and Altai (Figs 11-12) 


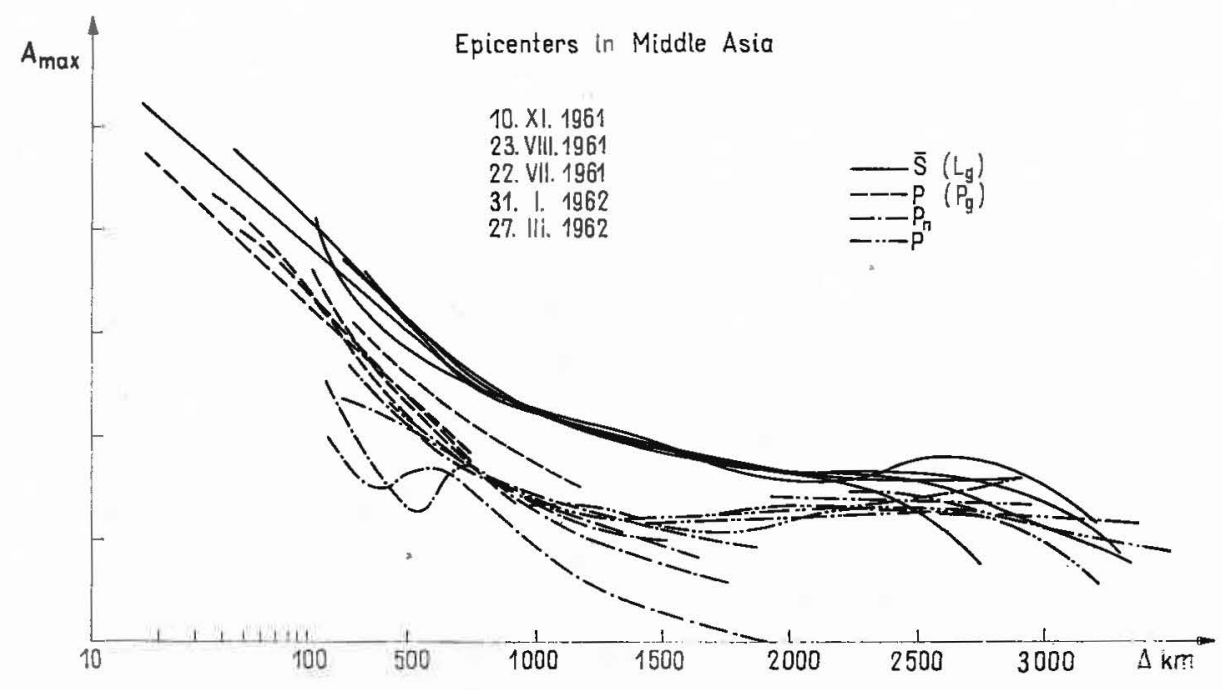

FIG. 10

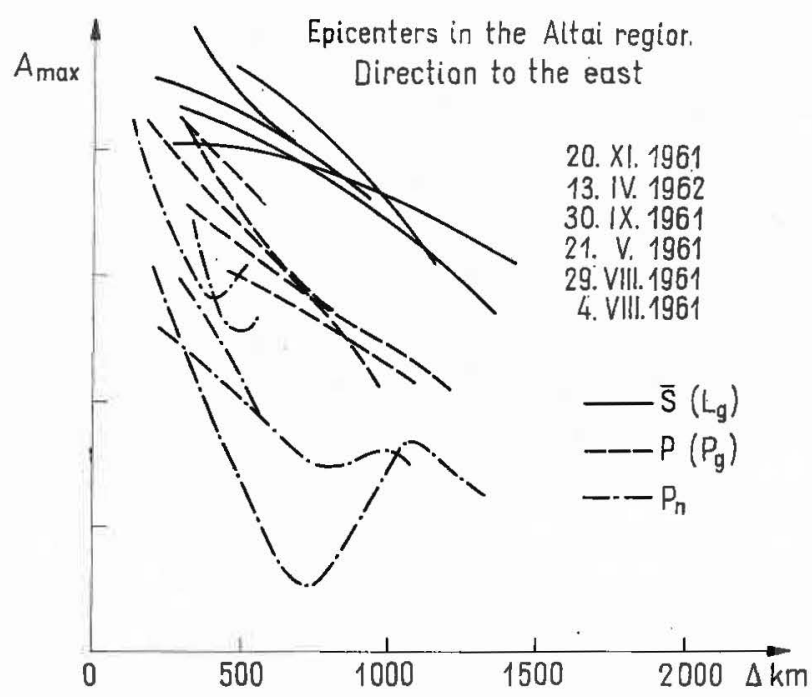

FIG. 11 
the direction of wave propagation and locality of the epicenter. Epicentral distances corresponding to the appearance of a separate wave of the $P_{n}$-group as well as its relative intensity are extremely unstable. This points to the transition from

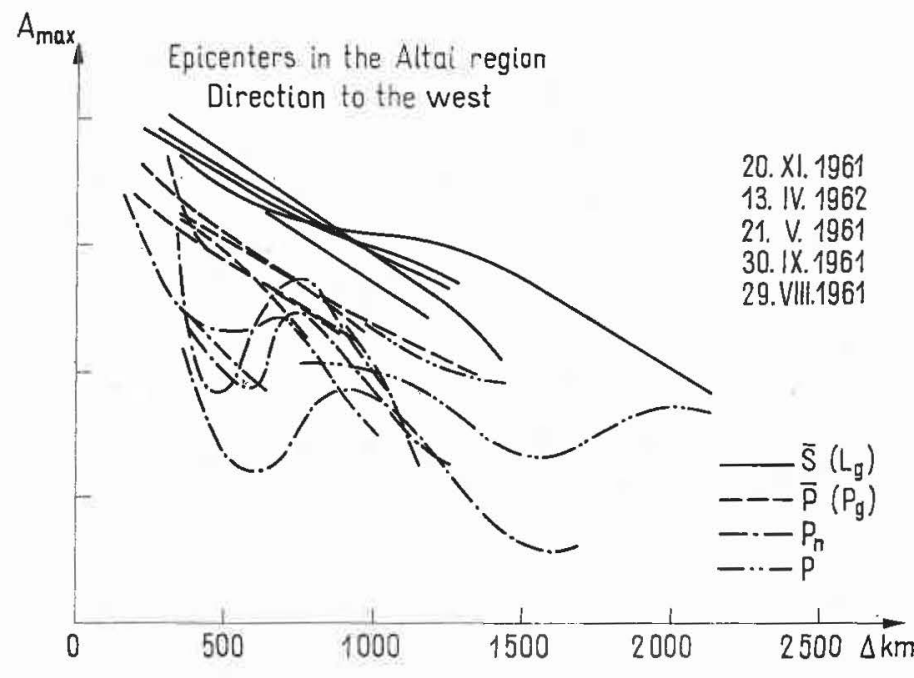

FrG. 12

the geological structures of Siberia to those of Middle Asia existing in the Altai zone. The reconstruction of the structures involves the deep layers reaching the boundaries of the upper mantle (Figs 11-12).

The received data show that the study of seismic wave dynamics should not be considered as an auxiliary research method useful in the interpretation of the nature of the waves. The dynamics of waves contribute fundamentally new information for earth's structure, concerning mainly the qualitative description of the boundaries. 


\author{
FAULT-PLANE SOLUTIONS FOR THE AGADIR, \\ MOROCCO, EARTHQUAKE OF FEBRUARY 29, 1960 \\ AND THE SKOPLJE, YUGOSLAVIA, EARTHQUAKE \\ OF JULY 26, 1963 \\ N. ÖCAL \\ (Cengelkoy-Istanbul, Turkey)
}

The fault-plane method being used at the Dominion Observatory, Ottawa, Canada has been applied for the Agadir, Morocco, earthquake on February 29, 1960 and Skoplje, Yugoslavia, earthquake on July 26, 1963.

For Agadir, a total of 44 data were used, 15 of them has been taken from the Bulletin of B.C.I.S. and 29 from the copies of seismograms which was sent by several stations to the author. For the Skoplje earthquake, a total of 61 data were used, 25 of them has been taken from the copies of seismograms and 36 has been read from the films of SP seismographs of standard network stations of U.S.C.G.S.

In the case of the Agadir earthquake the parameters for plane (a) are, strike $\mathrm{N} 44^{\circ} \mathrm{E}, \operatorname{dip} 87^{\circ}, 5 \mathrm{~N} \mathrm{~W}$, for plane (b), the strike $\mathrm{N} 45^{\circ} \mathrm{W}$, the $\operatorname{dip} 61^{\circ} \mathrm{N} \mathrm{E}$; and in the case of the Skoplje earthquake for plane (a), the strike $\mathrm{N} 35^{\circ} \mathrm{E}$, the dip $86^{\circ} \mathrm{S} \mathrm{E}$, for plane (b), the strike $\mathrm{N} 55^{\circ} \mathrm{W}$, the dip $69^{\circ}, 5 \mathrm{~N}$ E. For both earthquakes the strikes of the planes (a) are in very good agreement with the directions of the fault traces observed in the field.

\title{
CRUSTAL STRUCTURE IN ANATOLIA
}

N. ÖCAL

(Cengelkoy-Istanbul, Turkey)

The crustal structure in Anatolia has been studied by taking into consideration the dispersion of Love waves of seven earthquakes which were recorded by the long-period Galitzin seismographs at the Istanbul-Kandilli Observatory. These seven earthquakes occurred in Iran, in Afghanistan and in Pakistan. The most favourable parameters for the same region of the earth's crust have also been computed and herewith found for the velocity of shear wave in the crust $\beta^{\prime}=3.40 \mathrm{~km} / \mathrm{sec}$, the ratio of shear wave velocities in the crust and in the mantle $\beta^{\prime} / \beta=0.75$, the ratio of rigidities $\mu^{\prime} / \mu=2.20$ and for average crustal thickness $H=37 \mathrm{~km}$. 


\title{
ON DETERMINING FREQUENCY DEPENDENCE OF ABSORPTION COEFFICIENT OF LONGITUDINAL SEISMIC WAVES PROPAGATING IN THE EARTH'S MANTLE
}

\author{
I. P. Passechnik
}

(Moscow, USSR)

Up to the present time the absorption coefficients of longitudinal seismic waves $P_{n}$ and $P$ propagating in the earth's mantle were estimated on the basis of the analysis of the amplitude variation in the indicated maximum waves taken as a function of epicentral distance $[2,6]$. This method in its present form does not permit to investigate with sufficient accuracy the frequency dependence of the absorption coefficient. In this paper an attempt is made to estimate the values of the amplitude absorption coefficients and their frequency dependence by means of a method based on the study of the variation character of the amplitude spectra of $P_{n}$ and $P$ waves recorded from the underground nuclear explosions Logan and Blanca $[2,3,8]$ taken as a function of the epicentral distance. The solution of these problems is important from the point of view of general and applied geophysics, in particular for the estimation of the possibility of recording of the short period components of $P_{n}$ and $P$ waves.

The following assumptions are made in this paper: 1 . The attenuation of $P_{n}$ and $P$ waves due to the absorption. The phenomenon is expressed by the factor $e^{-\alpha L}$, where $\alpha$ is the absorption coefficient depending on frequency $f$, and $L$ is the distance along the seismic ray. 2. The characteristics of the seismographground installations and the earth's crust structure in all observation points at different $\Delta$ are assumed to be similar, while the amplitude variations in $P_{n}$ and $P$ waves due to the irregularities of the upper mantle and crust structure to be frequency independent. 3. The absorption in the lower mantle may be described by some average approximate value of the absorption coefficient $\alpha$ independent of depth. In fact $\alpha$ seems to be a function of depth, however the lack of experimental data does not permit to take into consideration this dependence in the present paper.

Under these assumptions the absorption coefficient of waves and its frequency dependence can be determined from the character of the wave spectral variations with the epicentral distance by means of the method described in paper [1]. The same method can be applied for the determination of the boundary absorption coefficient and its frequency dependence in $P_{n}$ waves from the observations of one profile, the Mohorovičic discontinuity being horizontal.

The data used. Seismic records obtained by the USA and the USSR from the underground nuclear explosions Blanca and Logan made by the USA in Nevada in $1958[2,3,8]$ were used for the computation of the amplitude spectra of $P_{n}$ 
and $P$ waves. Observations in the USA were made by similar instruments, installed every $100 \mathrm{~km}$ along a broken profile, extending to the east of the epicentres up to the distance of $\Delta=2000 \mathrm{~km}$, and then turning to north-east and reaching the distance $\Delta=4021 \mathrm{~km}$. Records of $P_{n}$ and $P$ waves were used that were obtained only by vertical instruments. Records of $P_{n}$ waves were obtained only from a single profile. Therefore in this paper the Mohorovičić discontinuity is assumed to be horizontal along the observation line. In the concrete case under

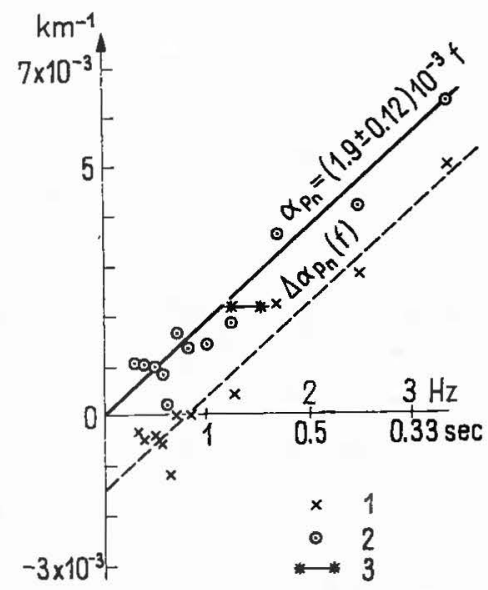

Fig. 1. Frequency dependence of the absorption coefficient difference $\Delta x_{p_{n}}$ and the values of the absorption coefficients proper $\alpha_{P n}$ of $P$ waves recorded in the $L$ range from 203 to $736 \mathrm{~km}$

1 - experimental values of $\Delta \alpha_{P_{n}}, 2$ - experimental values of $\alpha_{P_{n}}, 3$ - boundaries of values $\alpha_{P n}$ determined from amplitude curves in $[2,3]$

consideration this assumption is quite reasonable since as it is shown in special papers [5] the observed lowering of the Mohorovičić discontinuity to the east along the profile in the $\Delta$ range from 0 to $435 \mathrm{~km}$ does not exceed $2.5^{\circ}$, while in the distance range from 435 to $645 \mathrm{~km}$ the Mohorovičić discontinuity is horizontal altogether.

The parameters of the instruments used, their magnification as well as the examples of $P_{n}$ and $P$ records are given in $[3,8]$. The predominant recorded periods of $P$ were in the range of 1.0 to $1.2 \mathrm{sec}$, the train usually consisted of three to four clear oscillations with the duration of 3 to $5 \mathrm{sec}$. The periods of $P_{n}$ waves were 0.5 to $0.8 \mathrm{sec}$, the train consisted of 4 to 5 oscillations.

The spectra of $P_{n}$ and $P$ waves were calculated on electronic computers by the method of parabolic interpolation in the frequency range from hundredths of a cycle to $20-30$ cycles per second. However only the spectral intervals from 0.33 to 3.3 cycles were used for the absorption coefficient computation because of the small instrumental magnification at frequencies less than 0.3 cycles and the errors introduced in the process of digitization for frequencies over 3 to 4 cycles. 
The $P$ waves spectra obtained in the USSR by means of the instruments differs from those used in the USA. Therefore they were preliminarily reduced to the form of the spectrum obtained by the above mentioned instruments by multiplying them by the corresponding magnification ratios of the seismographs.

Determination of $\alpha_{P_{n}}$ and $\alpha_{P}$. The amplitude spectra of $P_{n}$ waves observed in the $L$ range from 203 to $736 \mathrm{~km}$ from the Logan and Blanca explosions (made

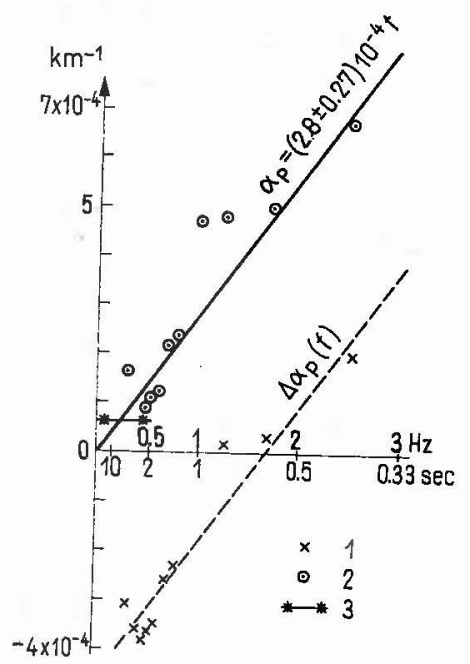

FIG. 2. Frequency dependence of the absorption coefficients $\Delta \alpha_{P}$ and the values of the coefficients $\alpha_{P}$ of $P$ waves recorded in the range from 1968 to $9170 \mathrm{~km}$

1 - experimental values of $\lambda \alpha_{p}, 2$ - values of $\alpha_{P}, 3$ - boundaries of values $\alpha_{P}=6 \cdot 10^{-5}$ $\mathrm{km}^{-1}$ for the waves with periods from 2 to $12 \mathrm{sec}$ according to $[4,6]$

in similar conditions) and the spectra of $P$ waves observed in the $L$ range from 1968 to $9175 \mathrm{~km}$ from Blanca were used for the determination of the amplitude ratios values of the spectral components $\frac{A\left(f_{k}\right)}{A\left(f_{i}\right)}$. The value of $f_{k}$ was taken to be equal to 1 cycle per sec, while the values of $f_{i}$ are taken as: $3.33,2.5,1.67$, $1.25,0.83,0.71,0.62,0.55,0.50,0.40,0.33$ cycles per second. From the ratios $l_{n} \frac{A\left(f_{k}\right)}{A\left(f_{i}\right)}: L$ for the above mentioned ranges of $L$ the angular coefficients of the straight lines approximating these experimental values of the ratios were determined by the method of least squares. The absorption coefficients $\Delta \alpha_{P n}$ and $\Delta \alpha_{P}$ differ from each other and their values are shown in Figs 1 and 2 by crosses. The values of $\Delta \alpha_{P_{n}}$ and $\Delta \alpha_{P}$ were in their turn approximated by straight lines by means of the least square method (dashed line in Figs 1 and 2); the angular coefficients of these straight lines characterize the dependence of the absorption coefficient difference $\Delta \alpha(f)$ on frequency. Through the parallel transference of the straight lines $\Delta \alpha(f)$ to the origin of the coordinates (shown by solid lines in 
Figs 1 and 2) the frequency dependences of the absorption coefficients $\alpha_{P n}(f)$ and $\alpha_{P}(f)$ properly are obtained.

The equations of the approximating straight lines are as follows: for $P_{n}$ waves $\alpha_{P n}=(1.9 \pm 0.12) \cdot 10^{-3} f \mathrm{~km}^{-1}$, for $P$ waves $\alpha_{P}=(2.8 \pm 0.027) \cdot 10^{-4} f \mathrm{~km}^{-1}$.

Here the values with the sign \pm show the mean square deviations of the experimental values from the average values.

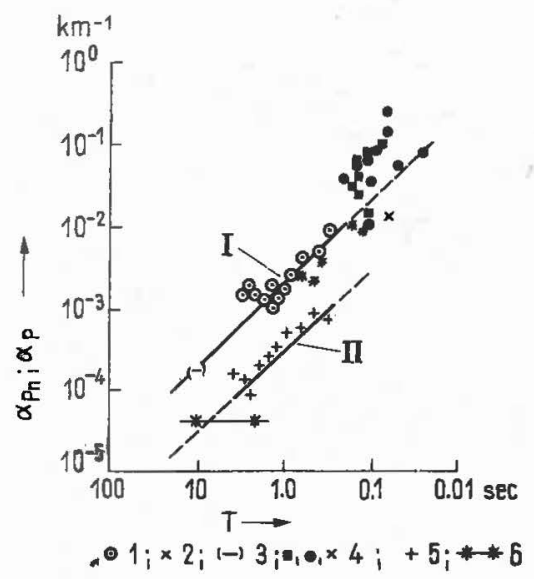

Fig. 3. Summary data concerning the frequency dependence of the absorption coefficient of longitudinal waves propagating 1 - in the earth's crust and the upper mantle, II - in the lower mantle. Experimental data for the upper mantle 1 - from the data of the present paper, 2 - from papers $[2,3,5] ; 3-$ from papers $[9,10] ; 4-$ in crustal rocks: granites, gneisses, marbles, quartzites, crystal schists, basalts according to $[1,11]$ for the lower mantle, 5 - according to the data of the present paper, 6 - according to $[4,6]$

Discussion of results. Figure 3 represents the absorption coefficient values $\alpha_{P n}, \alpha_{P}$ obtained in this paper by spectral methods. In this same figure the values of the absorption coefficients of longitudinal waves for various crystal and metamorphic rocks of the earth's crust determined by amplitude methods for the frequency range from 1 to $50-60$ cycles per sec are plotted using the data of paper [1]. It is evident from Fig. 3 that straight line $I$ for the wide frequency range from 0.1 to $50.60 \mathrm{cps}$ approximates sufficiently well the corresponding experimental values of the absorption coefficients in the crustal and upper mantle rocks. Straight line II is parallel to I, but approximately one order lower, which is connected with smaller values of the absorption coefficients in the lower mantle.

Thus the frequency dependences of the experimental values $\alpha_{P_{n}}$ and $\alpha_{P}$ obtained both through the study of the variation character of the spectral component ratios in $P_{n}$ and $P$ waves spectra depending on the epicentral distance and by means of amplitude methods can be roughly approximated by means of the above mentioned linear dependences for a certain period range, i.e. one must assume that the absorption coefficients correspondingly in the upper and lower mantle linearly depend on frequency. 
The following Table (column 4) contains the values of the $\vartheta$ decrement obtained in this paper by spectral methods and the values of $\vartheta$ (column 6 ) determined from the character of the amplitude decrease with $L$ according to the data of $[2-6,9,12,13]$, in papers $[2,3]$. For $\vartheta$ the same records were used as in the present paper; columns 5 and 7 contain the corresponding values of $Q=\frac{\pi}{\vartheta}$.

TABLE 1

\begin{tabular}{|c|c|c|c|c|c|c|}
\hline \multirow{2}{*}{$\begin{array}{l}\text { Regions } \\
\text { of the earth }\end{array}$} & \multirow{2}{*}{$\begin{array}{l}\text { Wave } \\
\text { type }\end{array}$} & \multirow{2}{*}{$\begin{array}{l}\text { Period } \\
\text { sec }\end{array}$} & \multicolumn{2}{|c|}{$\begin{array}{l}\text { Values obtained } \\
\text { in this paper }\end{array}$} & \multicolumn{2}{|c|}{$\begin{array}{l}\text { From other } \\
\text { determinations }\end{array}$} \\
\hline & & & $\begin{array}{l}\text { Decre- } \\
\text { ment } \vartheta\end{array}$ & $Q$ & Decrement $\vartheta$ & $Q$ \\
\hline .... 1 & 2 & 3 & 4 & 5 & 6 & 7 \\
\hline Earth's crust & $\begin{array}{l}P \\
P \\
P^{*} \\
S^{*}\end{array}$ & $\begin{array}{c}0.3 \\
0.1-1.0 \\
0.5-0.8 \\
1.5-1.8\end{array}$ & $\begin{array}{l}- \\
- \\
-\end{array}$ & $\begin{array}{l}- \\
- \\
-\end{array}$ & $\begin{array}{c}0.016 \\
0.017 \\
0.011 \\
0.012-0.014\end{array}$ & $\begin{array}{c}200^{5} \\
185^{1 z} \\
286^{3} \\
260-224^{3}\end{array}$ \\
\hline $\begin{array}{l}\text { Upper mantle } \\
\text { (near the } \mathbf{M} \\
\text { boundary) }\end{array}$ & $\begin{array}{l}P_{n} \\
P_{n} \\
P_{n}\end{array}$ & $\begin{array}{c}0.3 \\
0.6-0.8 \\
0.3-3.0\end{array}$ & $\begin{array}{l}0.015 \\
0.015 \\
0.015\end{array}$ & $\begin{array}{l}210 \\
210 \\
210\end{array}$ & $\begin{array}{l}0.0015 \\
0.012 \\
-\end{array}$ & $\begin{array}{c}570^{5} \\
260^{2 \cdot 3} \\
-\end{array}$ \\
\hline $\begin{array}{l}\text { Upper mantle to } \\
\text { the depths of the } \\
\text { order of } 600 \mathrm{~km}\end{array}$ & $\begin{array}{l}s_{c} S \\
P_{a}\end{array}$ & ${ }^{25}-12$ & $\overline{-}$ & $\begin{array}{l}- \\
-\end{array}$ & $\begin{array}{c}0.017-0.02 \\
0.012\end{array}$ & $\begin{array}{c}185-151^{13} \\
260^{9 \cdot 10}\end{array}$ \\
\hline $\begin{array}{l}\text { Lower mantle to } \\
\text { the depth of the } \\
\text { order of } 2700 \mathrm{~km}\end{array}$ & $\begin{array}{l}P \\
P \\
P \\
P\end{array}$ & $\begin{array}{r}1.0 \\
2.0 \\
4.0 \\
12.0\end{array}$ & $\begin{array}{l}0.0029 \\
0.0029 \\
0.0029 \\
0.0029\end{array}$ & $\begin{array}{l}1100 \\
1100 \\
1100 \\
1100\end{array}$ & $\begin{array}{l}0.0013 \\
0.0023 \\
0.0080\end{array}$ & $\begin{array}{r}2600^{4} \\
1300^{4} \\
400^{4}\end{array}$ \\
\hline $\begin{array}{l}\text { To the depth of } \\
2900 \mathrm{~km}\end{array}$ & $S c S$ & 25 & - & - & 0.0022 & $1430^{1.3}$ \\
\hline
\end{tabular}

The comparison of the $\vartheta$ values for $P_{n}$ waves determined by different methods from records obtained by similar instruments shows that these values are close; correspondingly close are the values of $Q$. In paper [5] smaller values of $\vartheta$ and correspondingly greater values of $Q$ for $P_{n}$ waves are obtained for the higher frequency instruments.

It should be stressed that the values of $\vartheta$ and $Q$ obtained from $P_{n}$ waves characterize the absorbing properties of a comparatively thin layer of the mantle near its upper boundary.

Discussing the absorption coefficient values and the decrements $\vartheta$ of $P$ waves propagating in the mantle we must take into account the following fact. In connection with the velocity variation in the mantle, with depth the $P$ wave, with the increase of $L$ successively passes through its ever deeper parts. Thus, for instance, the observations of the $P$ waves at $20^{\circ}$ show that the wave reaches the 
depth of the order of $350 \mathrm{~km}$, i.e. the wave path is entirely in the upper mantle where the average velocity of the $P$ waves is about $8.5 \mathrm{~km} / \mathrm{sec}$. At $\Delta=90^{\circ} P$ penetrates to the depth of the order of $2700 \mathrm{~km}$ and its average velocity is of the order of $10.5 \mathrm{~km} / \mathrm{sec}$. The absorbing properties in the mantle seem to decrease with the increase of the depth, i.e. with the increase of the velocity, which is in particular indicated by various values of the coefficients and decrements of the absorption for $P_{n}, P_{a}$ and $P$ waves. Therefore the values of the absorption coefficient for $P$ waves given in this paper must be considered as a certain approximately averaged value.

The decrement value somewhat increases with depth, which is connected with the velocity growth. This leads to a certain decrease of $Q$ in the deeper parts of the mantle. However taking into account the above statements about the probable dependence of $\alpha_{P}$ on depth we must consider the values of the absorption decrement given in the table as approximate and characterizing rather the tendency to the decrement growth with depth than its absolute values. The estimation of the values of absorption coefficients in certain depth intervals of the mantle requires a joint application of the spectra of several wave types $P, P P, P c P, P K P$ and others.

In this paper we used the records of $P_{n}$ and $P$ waves generated by shallow sources of the type of spherical symmetry sources wherein the amplitudes spectra of the spectral components at lower frequencies 0.3 c.p.s. were relatively small. Hence for the more accurate estimate of the $\alpha_{p}$ depends on the frequency in the frequency range from 0.2 to 0.05 c.p.s. Records of strong earthquakes should be used whose maximum of the amplitude spectra is in the frequency range from $0.2-0.1$ c.p.s., and the amplitudes of the spectral components in the above mentioned frequency range have a significant value.

Using the spectral methods we can determine the values of the absorption coefficients of longitudinal and transverse waves and can establish their frequency dependence by means of the techniques described above.

\section{REFERENCES}

[1] Berzon, I. S., Yepinatyeva, A. M., Paryiskaya, G. N. and Starodubrovskaya, S. P.: Dynamic characteristics of seismic waves in real media. Izd. AN SSSR, M. (1962)

[2] Passechnik, I. P.: Izv. AN SSSR, ser. geophys., 12 (1960)

[3] Kogan, S. D., Passechnik, I. P., Sultanov, D. D. and Tzybulsky, V. I.: Trudy IFZ AN SSSR, 15.

[4] Gutenberg, B.: Physics of Earth's Interior, IL. M. (1963)

[5] Ryall Allen and Stuart David, J.: J. Geophys. Res., 68 20, pp. 5821-5835 (1963)

[6] Gutenberg, B.: Bull. Seism. Soc. Am., 48, pp. 269-282 (1958)

[7] Knopoff, L. and MacDonald, G. F.: Revs. Modern Phys. 30, pp. 1178-1192 (1958)

[8] Romney, C. F.: J. Geophys. Res., 6410 (1959)

[9] Khorosheva, V. V.: Izv. AN SSSR, ser. geophys., 11 (1960)

[10] Magnitzky, V. A. and KhorosheVA, V. V.: DAN SSSR, 1352 (1960)

[11] Fedotov, S. A.: Izv. AN SSSR, ser. geophys., 16 (1963)

[12] Khalturin, V. X. and URusov, N. B.: Trudy IFZ AN SSSR, 25 (1962)

[13] Don Anderson, L. and Kovach, R. L.: Proceedings of the National Academy of Sciences, USA, 51 2, pp. 168-173 (1964) 


\title{
LA STRUCTURE DE L'ÉCORCE TERRESTRE EN TCHÉCOSLOVAQUIE A LA BASE DE LA DISPERSION DES ONDES SUPERFICIELLES
}

\author{
K. Peč et O. NovotnÝ \\ (Prague, Tchécoslovaquie)
}

On a procédé à l'étude de deux tremblements de terre; de celui de Panama du 26 VII. 1962 à une magnitude de 7,5 et de celui des Iles Kouriles du 16 III. 1963 à une magnitude égale. Le premier de ces tremblements est caractérisé par un groupe dispersif des ondes de Rayleigh bien développés et l'autre par celles de Love. Pour atteindre les vitesses de phase on a employé les enregistrements des stations suivantes: Budapest, Bratislava, Cheb, Halle an d. Saale, Hurbanovo, Prague, Průhonice, Skalnaté Pleso et Vienne. La plupart de ces stations est munie des séismographes mécaniques du type Wiechert; l'exception font les stations Průhonice, Halle et Budapest, où les enregistrements se font par des appareils éléctromagnétiques.

On a mesuré les temps d'arrivée des sommets et des vallées des parties dispersives des enregistrements de chaque station à part et on y a ajouté les déplacements de phase exprimés en secondes. Les sommets et les vallées particulières ont été mis en correlation par l'emploi des courbes de vitesse des groupes. Les positions des stations ont été projetées au plan tangentiel, touchant le Globe dans le lieu de la station de Prague. De cette manière on a atteint les coordonnées rectangulaires des stations. On a calculé les vitesses de phase et les azimuts, en se servant de la formule suivante:

$$
t_{k}-t_{0}=\left(x_{k}-x_{0}\right) \cos \Theta / c+\left(y_{k}-y_{0}\right) \sin \Theta / c .
$$

Les $t_{k}$ représentent le temps d'arrivée du sommet ou de la vallé de l'onde; $x_{k}, y_{k}$ les coordonnées de la $k^{c}$ station. Les grandeurs indiquées par l'index zéro se rapportent à la station de référence, $c$ est la vitesse de phase et $\Theta$ est l'azimut mesuré de l'axe $x$ orienté à l'est.

Les stations en question sont situées sur deux formations géologiques différentes; elles sont voisines ou appartiennent au Massif Bohême d'une part, ou au système des Carpathes d'autre part. Pour cette raison on a réparti les stations dans les régions situées dans l'une ou l'autre formation géologique. On a également examiné la combinaison des stations posées sur les différents systèmes géologiques. La région 1 comporte les stations: Cheb, Prague, Průhonice, Jena, Vienne; la région 2: Bratislava, Hurbanovo, Vienne; la région 3: Bratislava, Hurbanovo, Skalnaté Pleso, Vienne; la région 4: Cheb, Prague, Průhonice, Jena, Skalnaté Pleso, Vienne. 
Les vitesses de phase, représentatives pour les régions respectives, ont été calculées d'après la formule mentionnée et en tant que le nombre des stations a dépassé 3 , on a utilisé pour compensation la méthode des moindres carrés. La station de Prague a été choisie comme station de référence pour les régions 1 et 4 et la station Vienne pour les régions 2 et 3.

On a employé pour l'interprétation des résultats les courbes de Press et, en partie, les courbes de Glasko, Savarenski et Sečkov. La figure 1 com-

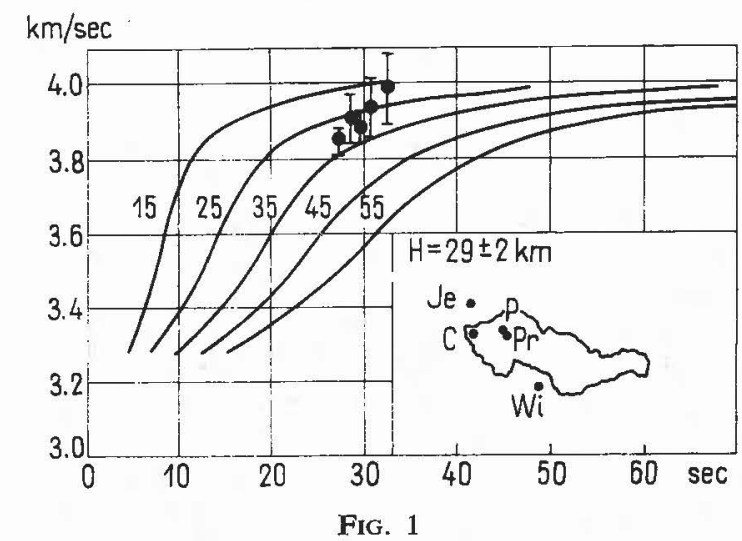

porte les résultats de la région 1 . En attribuant aux vitesses de phase les poids inverses proportionnels à la dispersion des valeurs $\cos \Theta / c, \sin \Theta / c$, nous recevrons sur la base des courbes de Press la profondeur de la discontinuité de Mohorovičić de $29 \pm 2 \mathrm{~km}$; ce résultat répond bien à la valeur de Kárník, dont l'étude des explosions industrielles en Bohême donne la profondeur de $31 \mathrm{~km}$. Il est important que la valeur de $29 \mathrm{~km}$ reste stable, même si nous supprimons n'importe quelle station de la région 1 . Pour cette raison, on peut se fier à cette valeur de profondeur de la discontinuité de Mohorovičić. La région 4 se compose des stations de la région 1 et de celle de Skalnaté Pleso. Ici l'adjonction de cette station agrandit fortement la profondeur de Moho. Cela peut être provoqué par deux faits: ou la déclivité de la surface Moho dans la région de la Haute Tatra est très grande de telle sorte qu'elle n'est point admissible de s'en servir pour l'interprétation d'un modèle à une couche planparallèle, ou bien une grosse faute se produit, soit dans la correction du temps, soit dans les constantes d'appareil de la station. L'influence de la station montagneuse Skalnaté Pleso se manifeste de la même manière dans la région 3 , où par adjonction de cette station d'après les courbes de Press, l'épaisseur de l'écorce augmente de 17 à $24 \mathrm{~km}$.

Il est évident que, pour la région $3, c$ à $d$ dans l'espace Vienne, Bratislava, Hurbanovo, les courbes de Press ne peuvent pas être employées, car elles donnent une petite valeur de la profondeur de Moho. Les résultats seront bien meilleurs par l'emploi des courbes des vitesses de phase des ondes de Rayleigh pour un modèle à deux couches de Glasko, Savarenski et Šečkov. Il faut 
donc choisir la vitesse des ondes transversales de $3.45 \mathrm{~km} / \mathrm{sec}$ et la profondeur de la couche granitique de $10 \mathrm{~km}$. Les courbes donnent la profondeur de Moho dans le terrain-bas slovaque de $24 \mathrm{~km}$, ce qui répond aux résultats de Gálfi et Stegena.

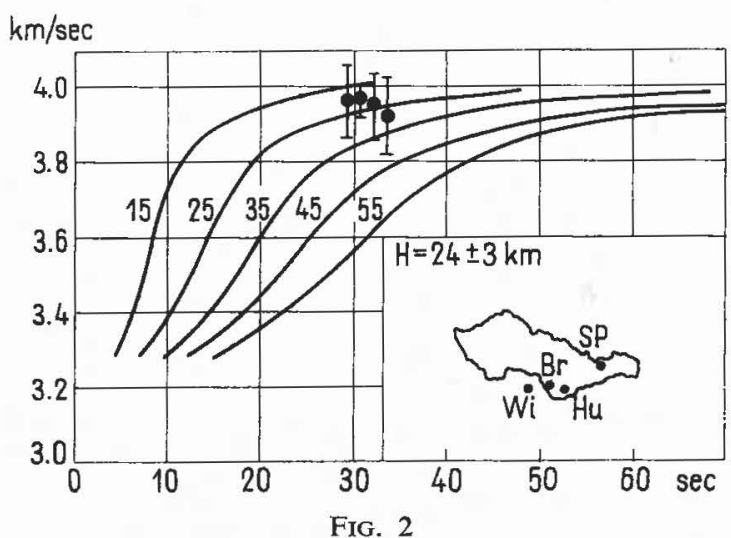

Les courbes de Aki ne peuvent pas être employées pour l'interprétation des vitesses de phase des ondes de Rayleigh, car elles donnent de trop petites valeurs de l'épaisseur de l'écorce.

Les observation de vitesses de phase des ondes de Love ne contredisent pas aux conclusions précedentes; elles sont pourtant - en raison de la grande dispersion des valeurs mesurées - bien moins sûres.

Pour conclure je me fais un agréable devoir de remercier les chefs des stations Budapest, Jena, Halle et Vienne qui par leurs aimables envois des matériaux, ont rendu possible ce travail.

\section{RÉFÉRENCES}

[1] Press, F.: Determination of crustal structure from phase velocity of Rayleigh waves, Part I: Southern California, Bull. Geol. Soc. Amer., 67 (1956)

[2] Glasko, V. B., Savarenski, E. F., Shechkov, B. N.: Dannye o fazovykh i gruppovykh skorostyakh poverkhnostnykh seismicheskikh voln, Izv. AN SSSR, ser. geofiz., 10 (1963)

[3] AKI, A.: Crustal structure in Japan from the phase velocity of Rayleigh waves, Part 1, Bull. Earthq. Res. Inst., 39 (1961)

[4] GÁlfi, J. et Stegena, L.: A generalized method for the determination of crustal thickness by means of $P_{p}$ and $P_{h}$ waves, Geofizikai Közlemények, 12 (1963) 


\title{
STRUCTURE OF THE EARTH'S CRUST AND UPPER MANTLE IN THE SOUTH-EASTERN PART OF THE RUSSIAN PLATFORM
}

\author{
I. V. Pomerantseva, A. N. Moszhenko, I. A. Sokolova, G. V. Egorkina \\ and E. D. TAGHAX \\ (Moscow, USSR)
}

The report contains considerations about the structure of the crust and, partly, the upper mantle of the south-eastern part of the Russian platform resulting from the investigations made by correlation refraction method (CRM), deep seismic sounding (DSS) as well as by observations using seismograph systems of "the Earth" type.

CRM and DSS investigations were made by "VNII Geofizika" in the southeastern part of the Russian platform from 1956 to 1960 under the direction of Yu. N. Godin (Fig. 1).

Commercial CRM and DSS equipments were used in the field [5], which record seismic events in the frequency range of $3-5$ to $30 \mathrm{cps}$.

However, due to the seismometer the frequency pass-band was narrowed to $8-10 \mathrm{cps}$.

The total extent of CRM and DSS profiles run in those years amounts to about 2500 kilometres. Continuous profiling technique was used with the distance of 100 kilometres between the seismometers.

The observation system by profiles permitted to obtain well correlated $t-d$ curves of the events corresponding to the surfaces of "granite" (Precambrian basement), "basalt" and "subcrustal" layers, and also to internal interfaces within them. Maximum length of the $t-d$ curves amounted to 320 kilometres. The intervals between shot points for CRM ranged from 15 to 25 kilometres and those for DSS - from 40 to 70 kilometres.

The investigations resulted in extensive and reliable data which helped to give a new interpretation of some problems concerning the nature of the waves which are recorded by CRM and DSS and concerning the structure of the crystalline crust within the platform $[7,8,9,10]$.

In 1962-1963, experiments were made on a number of DSS profiles of the Russian platform, using seismological systems of "the Earth" type designed by A. N. Moszhenko, "VNII Geofizika" [6, 12].

Observations made with "the Earth" units supplemented, and in a number of areas changed significantly, the idea of the crustal structure in the southeastern portion of the Russian platform. They also helped to develop a technique of studying the structure of the crust and upper mantle of the earth using longitudinal, shear and converted waves recorded by "the Earth" systems from natural earthquakes and artificial explosions. 


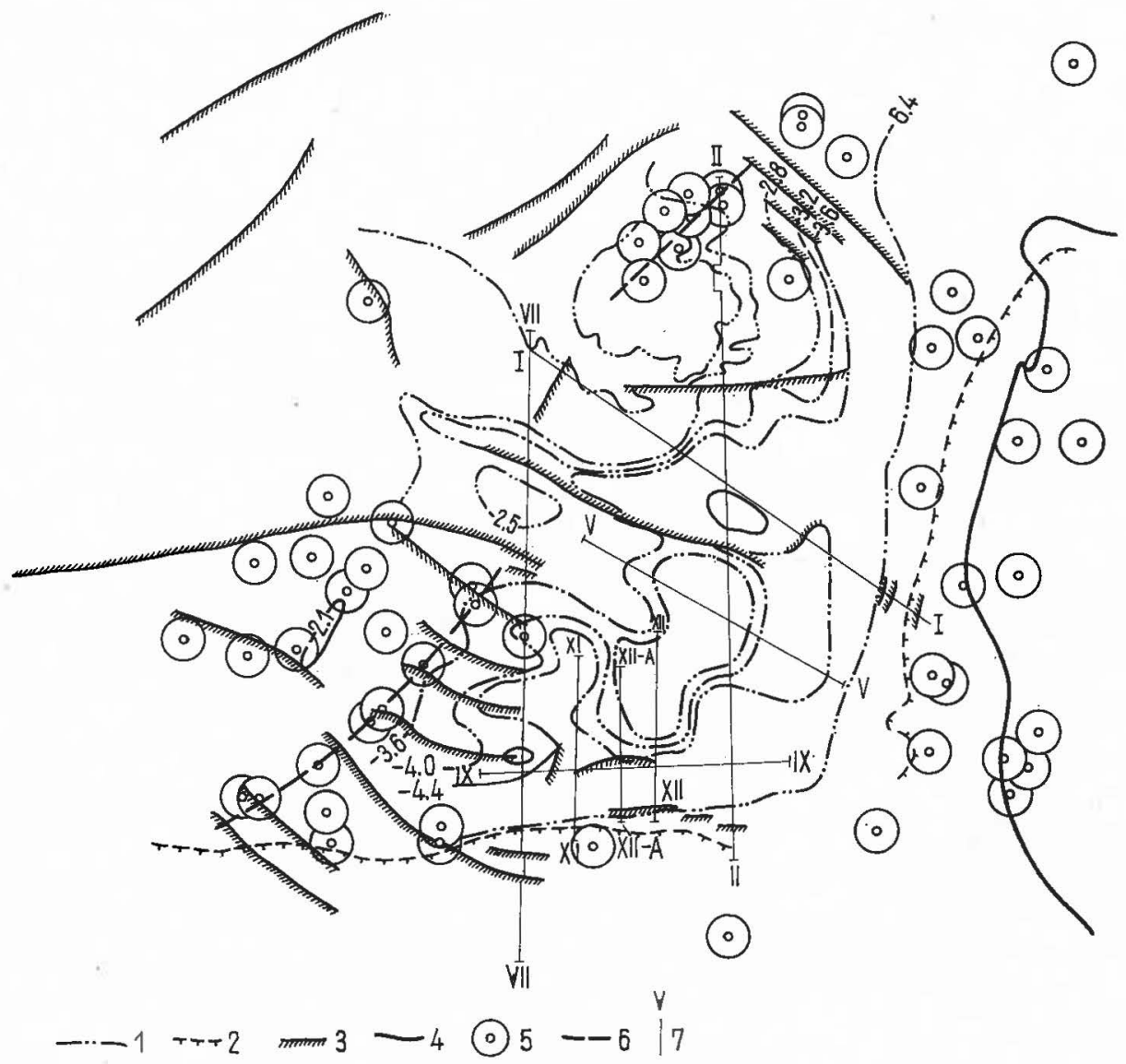

FIG. 1. Structural map of the basement surface in the south-eastern part of the Russian platform and locality map of local earthquake epicentres:

1. Contour lines of the basement surface, 2. Major regional faults separating the Russian platform from the North-Caspian depression (in the south) and from the Urals (in the east), 3. Faults on the basement surface, 4. The Urals folded area, 5. Epicentres of local earthquakes, 6. Faults located by "the Earth" system data

The use of "the Earth" systems in CRM and DSS profiling resulted in a rapid estimation of potentialities inherent in the technique developed.

The design of "the Earth" system shows the following principal differences from the commercial seismological systems:

1. magnetic tape recording with further repeated playback in any pass-band from 0.5 to $20 \mathrm{cps}$; i.e. with frequency analysis of all recorded waves;

2. record playback at 5-to-50-times-greater speeds resulting in frequency transformation and permitting, by means of various filters, to identify events differing by fractions of a cycle; 
3. high amplification up to 500000 and 1000000 (for different filtrations);

4. expanded time marks of a record permitting to mark time with the accuracy of up to $0.010 \mathrm{sec}$.

The complete equipment of "the Earth" system consists of 5 recording units and 1 playback unit.

One field recording unit contains:

1. magnetic recorders with 4 record amplifiers;

2. time synchronizer with count devices;

3. radio receiver for reception of accurate time signals;

4. four VEGIK seismometers.

All the above units of the record system are placed in two cases having an approximate size of $1 \mathrm{~m} \times 0.5 \mathrm{~m} \times 0.3 \mathrm{~m}$.

The playback unit consists of:

1. tape transport;

2. playback amplifier set with 5 (in new model with 36) amplifiers;

3. bifilar oscillograph;

4. cathode oscillograph of ENO-1 type.

Technical characteristics of "the Earth" system:

1. duration of recording -24 hours;

2. speed of tape in recording $-3 \mathrm{~mm} / \mathrm{sec}$;

3. speed of tape in playback -30 to $150 \mathrm{~mm} / \mathrm{sec}$;

4. dynamic range - not less than $30 \mathrm{db}$;

5. Selection of frequency responses and speeds permitting to cover (in playback) a frequency range of 0.5 to $10 \mathrm{cps}$ with right cut-off slope of 18 to 36 $\mathrm{db} /$ octave with interval of 0.1 to $0.3 \mathrm{cps}$;

6. Maximum amplification of the system -5000000 to 1000000 (for different filtration);

7. Record pass-bands -0.5 to $20 \mathrm{cps}$.

Four to nine systems were used for the observations from earthquakes and explosions carried out in the south-eastern part of the Russian platform. The systems were located along interperpendicular profiles.

The distance between the stations varied from 5 to 20 kilometres for the main profile, which was supposed to give a continuous section of the crust, and from 80 to 100 kilometres for the profiles cutting the main one.

Seismic events were recorded on magnetic tape at every system from three seismometers, one of which recorded vertical components of the waves and the remaining two - horizontal components ( $x$ and $y$ ).

Time marks (seconds) from time synchronizer and signals of exact time from Moscow were also recorded on magnetic tape.

Investigations carried out by the above technique resulted in recording longitudinal $P$, shear $S$ and converted $P S, S P$ etc. waves from close earthquakes and explosions ( $\Delta=20$ to 700 kilometres) (Fig. 2) and from distant earthquakes ( $\Delta \approx 2000$ to 14000 kilometres). 


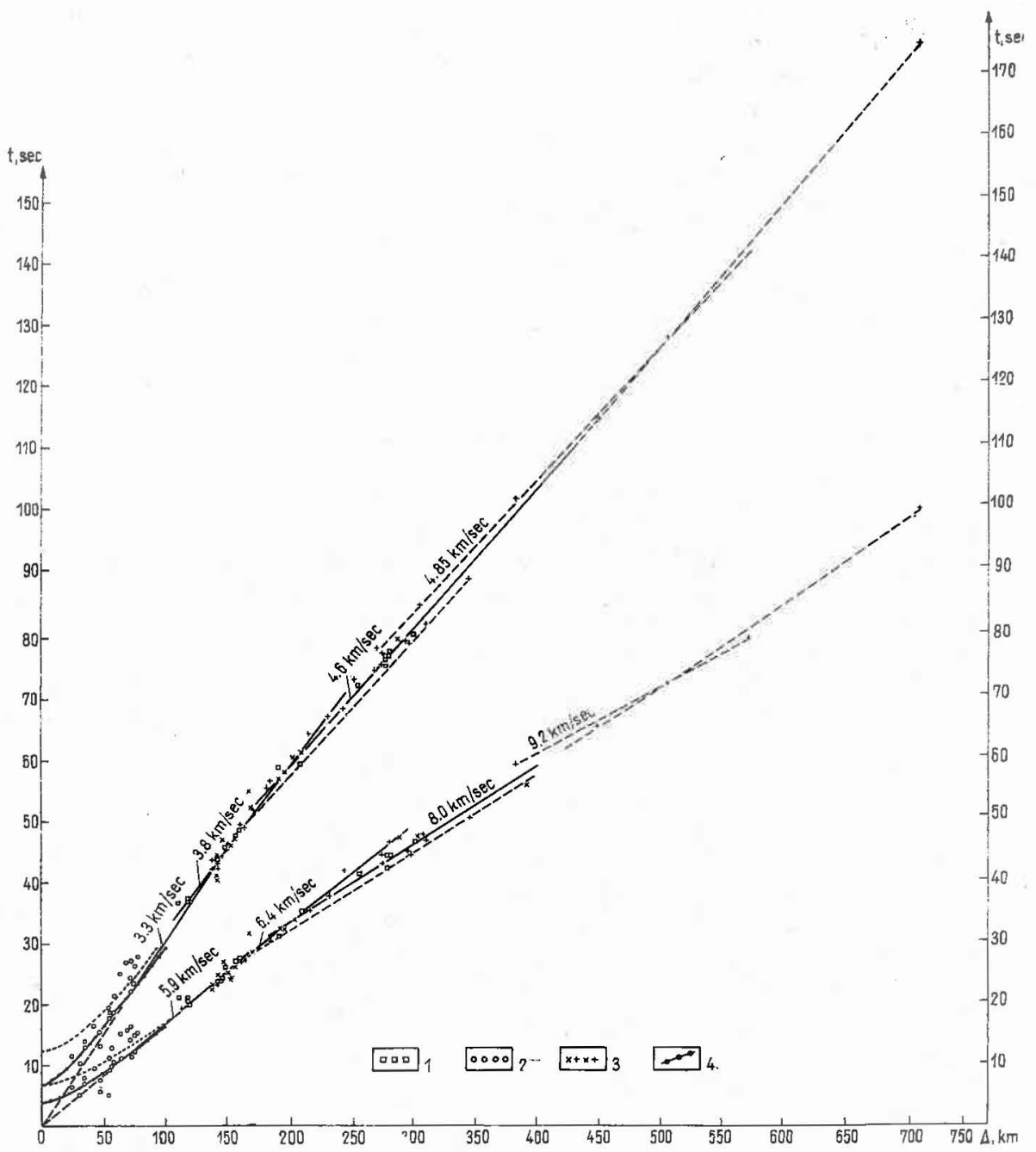

FIG. 2. Time-distance curve of $P$ and $S$ first arrivals from earthquake and explosion recording:

1. Arrival times of the waves recorded from explosions, 2. Arrival times of the waves recorded from earthquakes with hypocentres of 20 to $50 \mathrm{~km}$ deep, 3. Arrival times of the waves recorded from earthquakes with indefinite depth of hypocentres, 4. Theoretical time-distance curves of direct waves with the depth of the foci of 23 and $45 \mathrm{~km}$

Interpretation of records from local earthquakes $[1,13]$ resulted in a picture of the present tectonic activity of the territory studied and in the location of main regional deep faults crosscutting the region.

A section of the crust and upper mantle along one of the base regional CRM and DSS profiles was made from the records of converted waves $[2,3,4]$ of $P S_{n}$ type (Figs 1,3). The section provided much supplementary data for a better 
knowledge of the crustal structure (new and unknown fault zones were located and new interfaces were drawn at depths of 13 to 17 kilometres, etc.).

The results obtained from CRM, DSS and seismological investigations with "the Earth" units are as follows.

The thickness of the crust within the south-eastern portion of the Russian platform amounts to $36-40 \mathrm{~km}$. The crust was found to become gradually thicker towards the North-Caspian depression and the Urals foredeep - 42-43 km mainly due to thicker sediments and slight lowering of the major crustal interfaces (Figs 3, 4).

The thickness of the crystalline crust is 33 to $36 \mathrm{~km}$ and that of the sediments -2 to $5 \mathrm{~km}$.

In the zone of transition to the North-Caspian depression and Urals foredeep, as well as within these regions, the thickness of the crystalline crust is sharply reduced to $26 \mathrm{~km}$, while the sediments become thicker.

"Horizontal" and "vertical" interfaces were found within the crust. "Horizontal" interfaces are such as the basement surface, Mohorovičić discontinuity and internal interfaces within the crust and upper mantle. "Vertical" are fault zones along which individual crustal blocks are moving.

The block structure of the crust is clearly shown by mapping the surface of the Precambrian basement (surface of the "granite" layer) (Figs 1,4).

The depth of the top of the crystalline crust was found to range from $2 \mathrm{~km}$ for the Tatar arch to $5 \mathrm{~km}$ for the zone of transition to the North-Caspian depression and Urals foreddep. Within the latter regions this surface is 13 to 16 $\mathrm{km}$ deep.

Faults at the crystalline surface having magnitudes of displacement of not more than 1 to $2 \mathrm{~km}$ coincide with fault zones at the Mohorovičić surface having shifts amounting to $8 \mathrm{~km}$ (Fig. 4). The largest crustal faults are present in the zones of transition to the North-Caspian depression and Urals foredeep.

Seismological investigations have also located crustal faults within the Tatar arch, along the eastern side of the Zhigulevsko-Pugachevsky arch and Kinelskie dislocations.

All crustal faults are associated with centres of near earthquakes that are evidences of permanent tectonic activity within the eastern part of the Russian platform (Fig. 1). Most of the centres are found inside the crust, within the western and southern margins of the platform.

The behaviour of the Mohorovičić surface and crustal interfaces outside of fault zones is smooth, close to horizontal with dips of not more than $1^{\circ}$ or $2^{\circ}$ (Figs 3, 4, 5).

The zone of transition from the crust to the mantle contains not one, but several ( 2 or 3 ) Mohorovičić surfaces separated by intervals of 5 to $10 \mathrm{kmn}$ (Fig. 3). The depths of these surfaces are 32 to 36,40 to 45,45 to $50 \mathrm{~km}$, respectively.

Below this transitional zone there are several more interfaces. The most distinct of these lie at depths of 55 to 60 and 90 to $110 \mathrm{~km}$.

Thus the zone of transition from the crust to the mantle is not monolithic but 


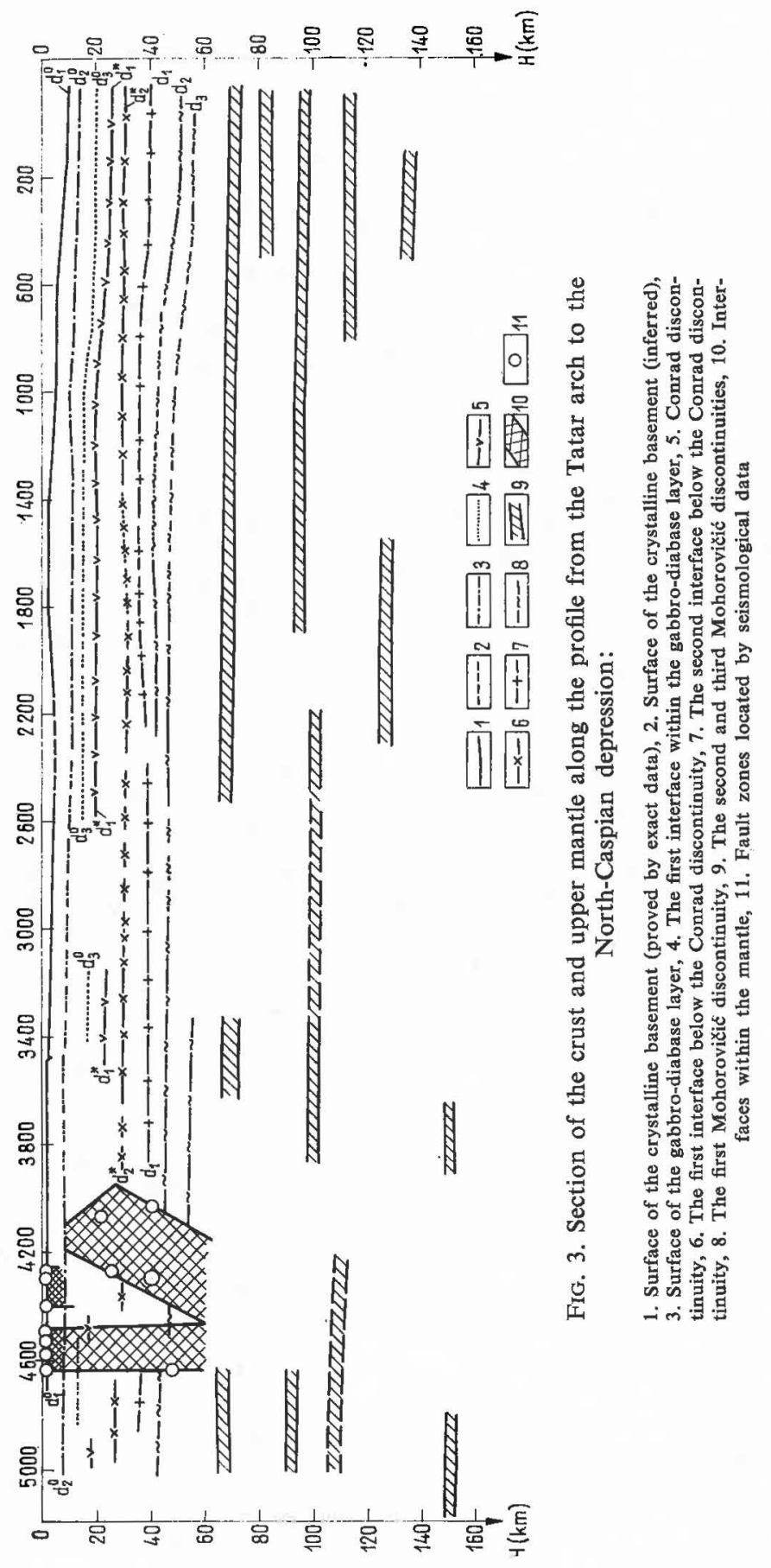




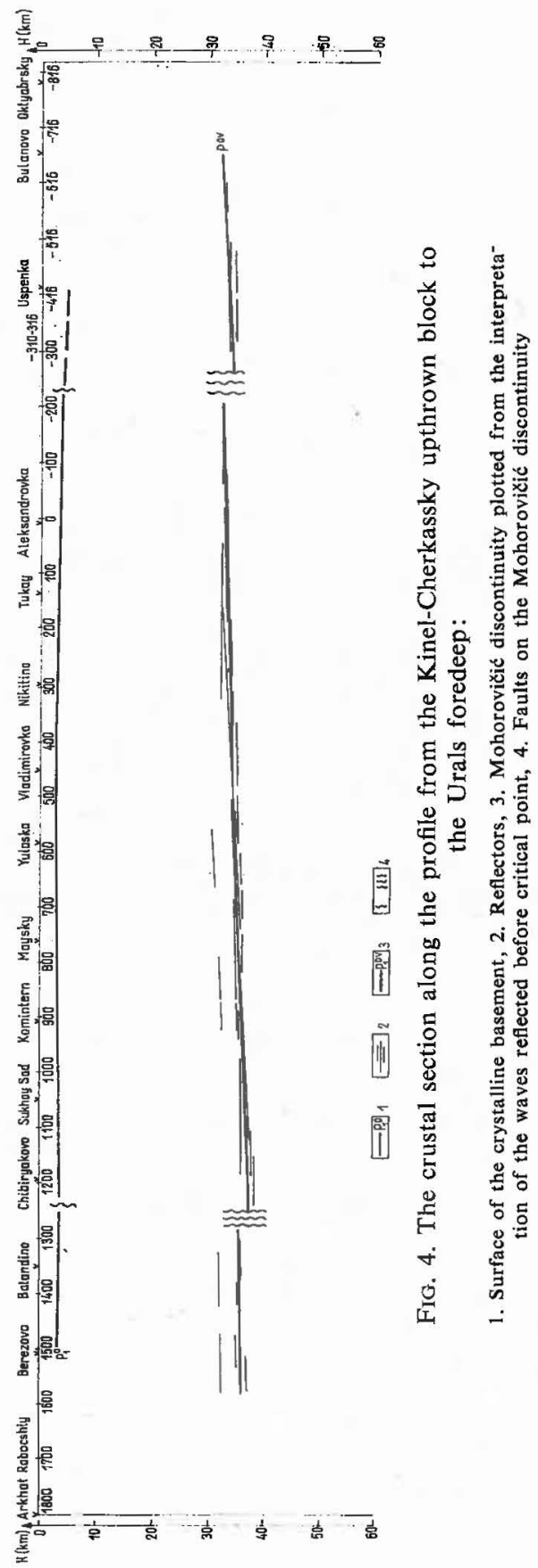


stratified like the crust itself. The difference between the crustal structure and that of the mantle lies in the fact that the crustal layers are 5 to $7 \mathrm{~km}$ thick, while those of the mantle -15 to $25 \mathrm{~km}$ (Fig. 3).

The information obtained from DSS and seismological investigations suggests that the most probable model of the crustal structure is a non-uniform, stratified medium with interfaces of first-order, i.e. with sharp velocity changes at discontinuities (Fig. 5).

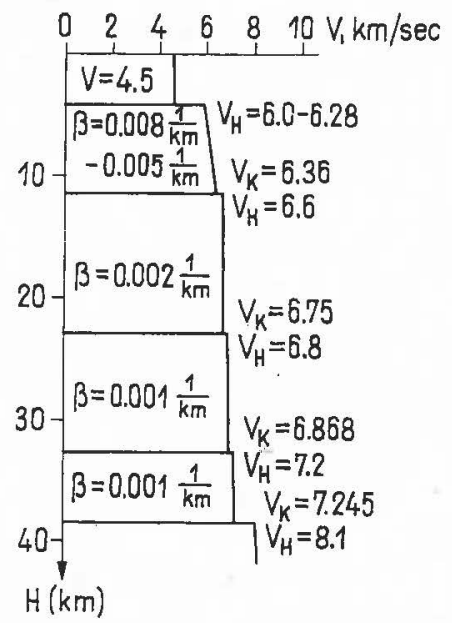

Fig. 5. The crustal structure from DSS and "the Earth" data $\beta$ - "gradient" of velocity increase with depth, $V_{t}$ - velocity at the top of the layer, $V_{b}$ - velocity at the bottom of the layer

The crust of the south-eastern part of the Russian platform is characterized by the following ranges of the main velocity parameters.

1. "Granite" layer. Average thickness equals to $7.5 \mathrm{~km}$. Separate blocks composing the "granite" layer have different thicknesses. The thickness of the layer increases to 9 to $10 \mathrm{~km}$ under the arches (Tatarsky and Orenburgsky). Under the depressions it is reduced. The "granite" layer is a gradient medium, with gradient values, original velocities at the top and rate of velocities increase with depth differing from block to block.

In uplifted areas, where the basement surface is not more than $3 \mathrm{~km}$ deep, velocity increases with depth according to a parabolic law. The order of velocity. increase for lower blocks is near to linear. Values of "gradients" of velocity increase $\beta \approx 0.03-0.041 / \mathrm{km}$ for uplifts and $\beta \approx 0.005-0.0021 / \mathrm{km}$ for the rest of the territory. Original velocities $V_{0}$ are 5.7 and $6.15 \mathrm{~km} / \mathrm{sec}$, respectively. $V_{s}=3.3 \mathrm{~km} / \mathrm{sec}$ on the average.

2. The surface of the "basalt" layer lies at a depth of 8 to $11.5 \mathrm{~km}$, and not 20 to $23 \mathrm{~km}$ as it was believed [11]. The "basalt" layer is also a lowgradient medium $\left(V_{0 p}=6.6 \mathrm{~km} / \mathrm{sec}, V_{s}=3.6 \mathrm{~km} / \mathrm{sec}\right)$.

Velocity at the bottom of the "basalt" layer (with $\beta=0.0021 / \mathrm{km}$ ), at the 
depth of 20 to $23 \mathrm{~km}$ appears to attain $6.75 \mathrm{~km} / \mathrm{sec}$. A possible first-order interface may be present within the layer.

3. The Conrad surface lies at a depth of 20 to $23 \mathrm{~km}$. It is characterized by a small velocity change of about $0.1 \mathrm{~km} / \mathrm{sec}$. The starting velocity at the top of the layer is $6.8 \mathrm{~km} / \mathrm{sec}$ with $\beta=0.0011 / \mathrm{km}$.

4. An abrupt discontinuity was found at a depth of 28 to $33 \mathrm{~km}$ with the velocity changing from 6.85 to $7.2-7.25 \mathrm{~km} / \mathrm{sec}$. Gradient below this discontinuity equals to $0.001-0.00051 / \mathrm{km}$.

5. The first Mohorovičić surface was found to be 32 to $40 \mathrm{~km}$ deep. This discontinuity has a velocity change from 7.2 to $8.0-8.1 \mathrm{~km} / \mathrm{sec}, V_{s}=4.6 \mathrm{~km} / \mathrm{sec}$.

Thus in the south-eastern part of the Russian platform the maximum velocity of seismic waves propagating in the crust does not exceed 7.1 or $7.2 \mathrm{~km} / \mathrm{sec}$.

Values of $V_{H}$ (boundary velocities from head waves) of the order of 7.8-7.7 $\mathrm{km} / \mathrm{sec}$ obtained from the waves reflected from the interface at a depth of 28 to $33 \mathrm{~km}$ are not correct since, from this interface down to $150 \mathrm{~km}$, pre-critical reflections rather than head waves are observed from the shotpoint (I. V. Pomerantseva, in print), from which the determination of $V_{H}$ gives higher velocities of elastic waves propagating in the crust.

\section{REFERENCES}

[1] ANDreyev, S. S.: Interpretation technique for near earthquakes. Trudi Instituta Fiziki Zemli 25 (1962)

[2] ANDREYEV, S. S.: Study of deep crustal structure from converted $P$ waves recorded from earthquakes. Izv. A. N. USSR, ser. geofiz., 1 (1957)

[3] Boulnn, N. K. and Trufelkina, E. I.: Application of converted SP waves recorded from near earthquakes for deep crustal studies. Izv. A. N. USSR, ser. geofiz. 11 (1960)

[4] Boulin, N. K.: Determination of the folded basement depth using converted passing waves of $P S$ type recorded from earthquakes. Izv. A. N. USSR, ser. geofiz., 6 (1960)

[5] Moszhenko, A. N.: Amplifiers for CRM and DSS. Razvedochnaya $i$ promislovaya geofizika, 18 (1957)

[6] Moszhenko, A. N.: Magnetic tape instruments for recording earthquakes and remote explosions. Izv. A. N. Turkmenian SSR, ser. fiz.-techn., chim. i geolog, nauk, $I$ (1961)

[7] Pomerantseva, I. V. and Margotieva, M. V.: On the nature of the waves recorded by DSS. Izd. VNII Geofizika (1959)

[8] Pomerantseva, I. V. and Margotieva, M. V.: Some considerations on the nature of the waves recorded from DSS. In: Deep seismic sounding of the crust in the USSR. Gostoptekhizdat, Moscow (1962)

[9] Pomerantseva, I. V.: Nature of the waves referred to the surface of the basement. Prikladnaya Geofizika, 34 (1964)

[10] Pomerantseva, I. V.: Results of crustal investigations in some areas of the southeastern part of the Russian platforme. Prikladnaya geofizika, 31 (1961)

[11] Pomerantseva, I. V.: Some considerations on the structure and possible composition of the solid crust. DAN USSR, 3145 (1962)

[12] Pomerantseva, I. V., Moszhenko, A. N., Sokolova, I. A. and Egorkina, G. V.: Results of regional investigations with seismological system of "the Earth" type and future prospects of this work. In: Regional geological and geophysical investigations in the marginal zone of the North-Caspian depression. Trudi Nizhne-Volzhskogo NII, Geologia i geofizika, 2 Saratov (1964)

[13] Savarensky, E. F. and KIRnos, D. P.: Elements of seismology and seismometry. Gostoptekhizdat, Second edition (1955) 


\title{
GROUP VELOCITY DISPERSION OF RAYLEIGH WAVES ACCORDING TO SIMFEROPOL OBSERVATIONS
}

\author{
I. I. Popov and S. A. Kapitanova \\ (Moscow, USSR)
}

\begin{abstract}
The seismic station "Simferopol" of the Earth Physics Institute of the USSR Academy of Sciences is systematically carrying out the observations of surface waves with the aim of the study of their velocity dispersion.
\end{abstract}

In this paper some results of observations at the group velocity dispersion of Rayleigh waves are presented, obtained in 1958-1963 by a standard seismograph of general type (SVK) and a special long-period seismograph (SVKD). The curves of seismograph magnifications are presented in Fig. 1.

In all, with indicated aim, 37 earthquakes have been selected which were followed with propagation of rather intensive surface seismic waves. As a rule the foci of the considered earthquakes were in the earth crust, magnitudes varied in the ranges of $51 / 4-71 / 2$, epicentral distances from 3500 to $16800 \mathrm{~km}$, dispersive waves periods - from 12 to 105 seconds, and the group velocities from 2.7 to $4.0 \mathrm{~km} / \mathrm{sec}$.

The lines of waves propagation are shown in Fig. 2, from which it is seen that their majority occupied the Eurasian continent in the ranges of azimuths from observations point from 0 to $90^{\circ}$. In some cases the lines partially passed through the Pacific or Atlantic Ocean.

The group velocities have been determined by existing methods [4, 5]. As a result the experimental curves of group velocities were constructed, the whole complex of which is given in Fig. 3.

The azimuthal dependence of the character of Rayleigh waves according to all the periods observed is well illustrated by the diagram in Fig. 4. The diagram shows the sensitivity of dispersion method to the variations of the earth crust structure by means of surface waves formation and propagation.

The analysis of the obtained data according to the Simferopol observations made it possible to show the following pecularities:

1. The dispersion curves are divided into two groups (Fig. 3); one with relatively small group velocities relates to continental lines of Eurasia, while another with great average values of velocities - to continental oceanic lines.

The longest lines of the waves (Nos 18, 19, 20,21, 22 and 23), passing to the Crimea from the south-western part of the Pacific Ocean (Kermadec Islands, New Hebrides, the Solomon Islands) through the south-western part of Eurasia, as well as the line from the north Atlantic ridge through the Atlantic Ocean and the north-western part of Africa (No. 32) and others (Nos 33, 34, 35 and 37), 
passing through the Atlantic Ocean differ by the highest average values of group velocities, which corresponds to the influence of the properties of the thinnest mainly oceanic, earth crust.

2. The lines of the waves 17 and 18 , and also 23 and 24 are lying in pairs, almost on the same arcs of large circles of the earth, but differ by their length. While

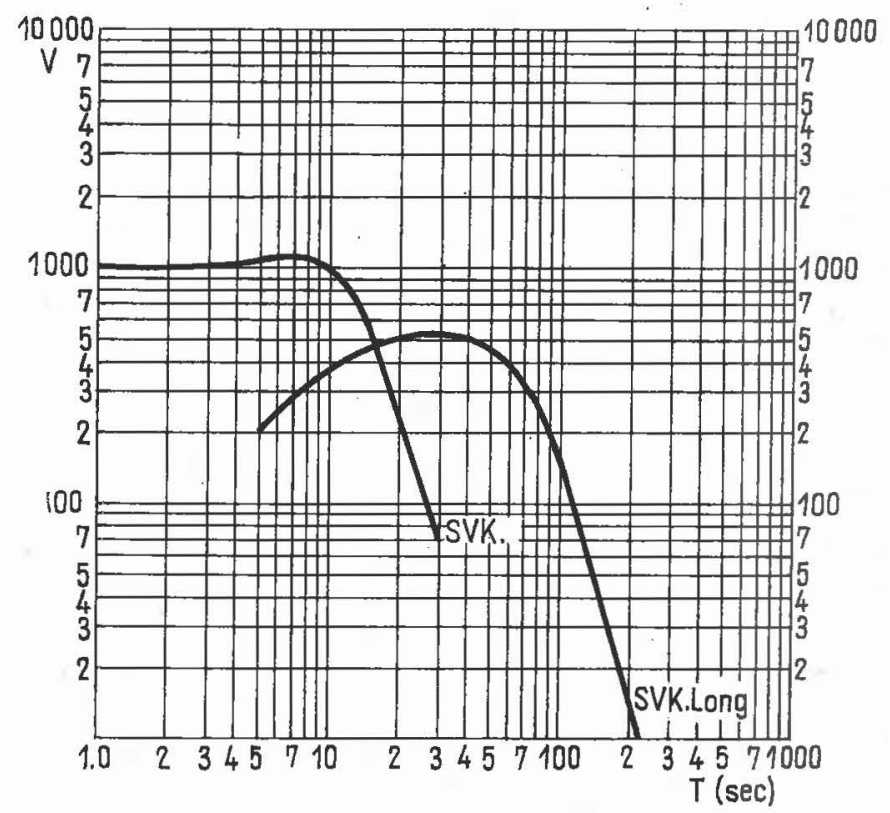

FIG. 1. Curves of seismographs magnification (Simferopol)

the longest arcs correspond to the mixed ways through the ocean and Eurasia, the shortest are continental ones. The difference in dispersion character marks the differentiation of the crustal structure (Fig. 5).

3. The experimental dispersive curves Nos 19,21, 22, 23 and 24 coincide well enough or in concordance continue one another. The line, belonging to these curves where the azimuths are most likely, crosses the Pacific Ocean.

4. The dispersion curves Nos $25,26,27$ and 28 correspond everywhere to continental ways through Eurasia and though their lines are alike by their azimuths and almost coincide with lines Nos 20,21 and 22, they differ by the lowest average group velocities of Rayleigh waves, due to the influence of the thickest continental earth crust (Fig. 6).

5. The wave lines Nos 29, 30 and 31 are passing through Celebes and South China Sea and further through the Eurasian continent. The wave periods in dispersing trains vary from 39 to 90 seconds and in this case the lowest values of average group velocities: $3.2-3.7 \mathrm{~km} / \mathrm{sec}$ correspond to them. The influence of predominant blocks of considerable thicknesses of the crust is evident. It is 


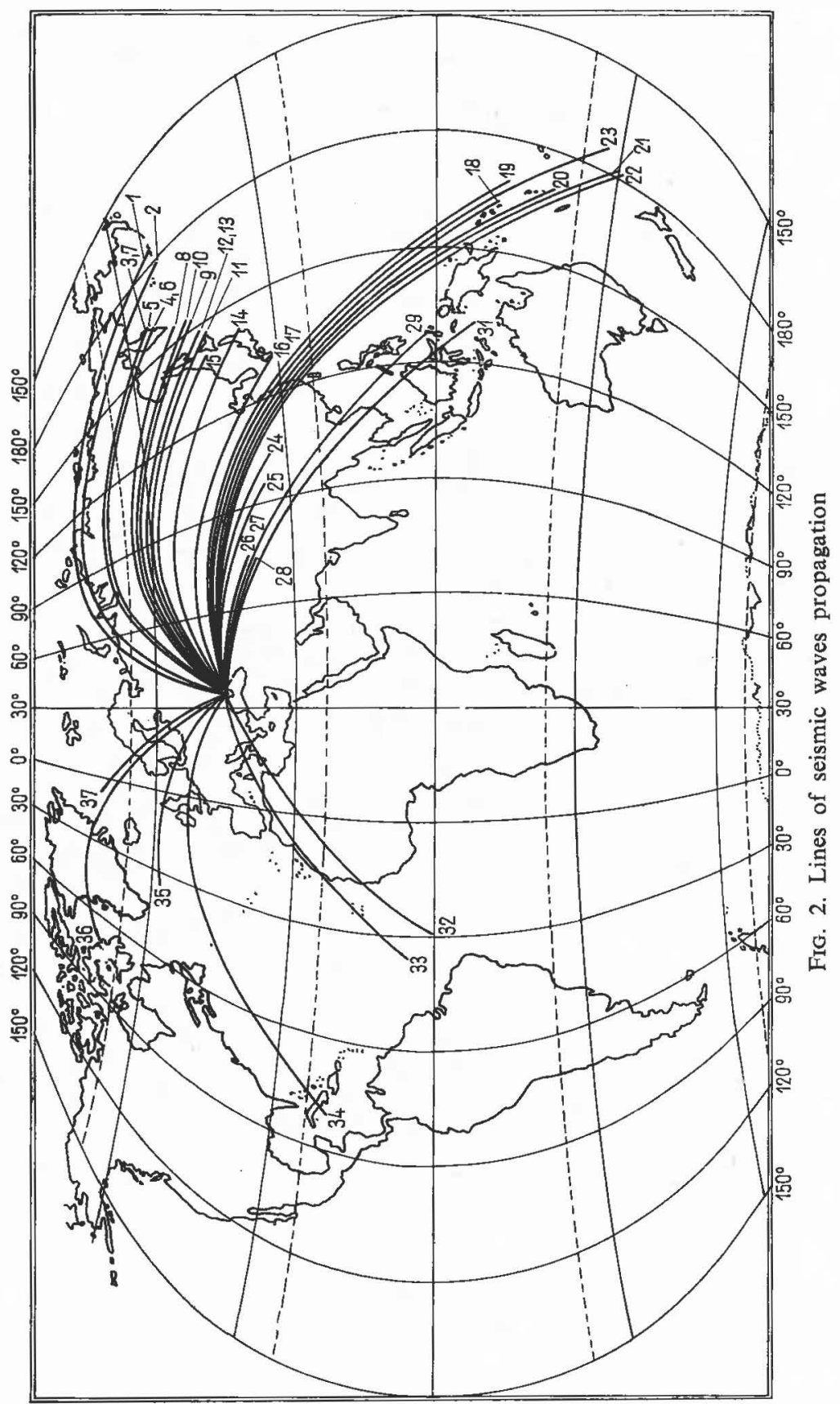




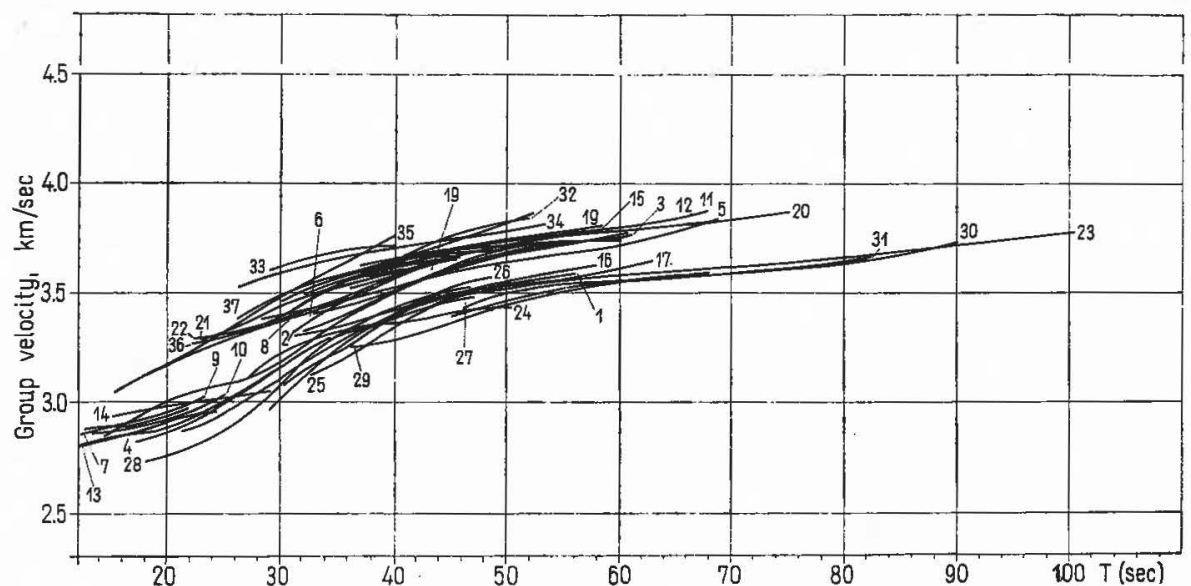

FIG. 3. Experimental curves complex of observed dispersion of Rayleigh waves group velocity

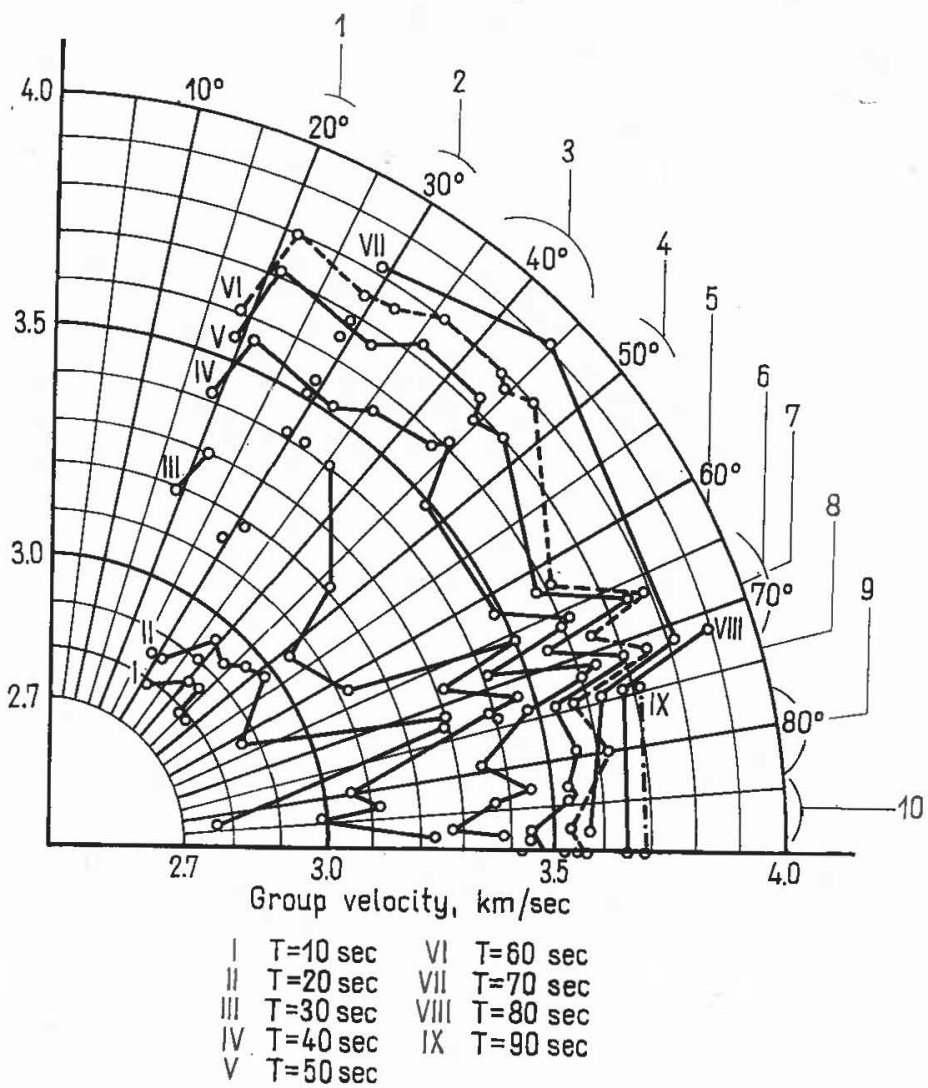

Fig. 4. Azimuthal distribution diagram of group velocities of different waves of period 1. East European Plain, Arctic Ocean, Behring Sea, Aleutian Islands; 2. Ural, Middle Siberian Plateau, Verhoyanks, Akhotskoy Sea, Kamchatka; 3. Stanovo, Akhotskoy Sea, Kuril Islands; 4. Sayan Mountain, Japan Sea, Japan; 5. Altai, Gobi desert, Yellow Sea, Japan; 6. Gobi desert, Pacific Ocean, Solomon Islands, New Hebrides; 7. Tien Shan, Gobi desert, East Chinese Sea, Toward South from Japan; 8. Tien Shan, Pacific Ocean, Melanesia, Kermadec trough; 9. Tien Shan, North-West China; 10. Pamir, Tibet, Himalaya, South Chinese Sea 
possible to explain the sharp decrease of group velocity values almost for all periods with azimuths $62^{\circ}$ and $68^{\circ}$ by the effect of thick blocks of the Altai and Tien-Shan ridges.

6. The fact deserves attention that line 36, passing from Baffin Bay through Greenland, Scandinavia and Europe, greatly differs from all others not only by azimuth, but by the dispersion character of Rayleigh waves.

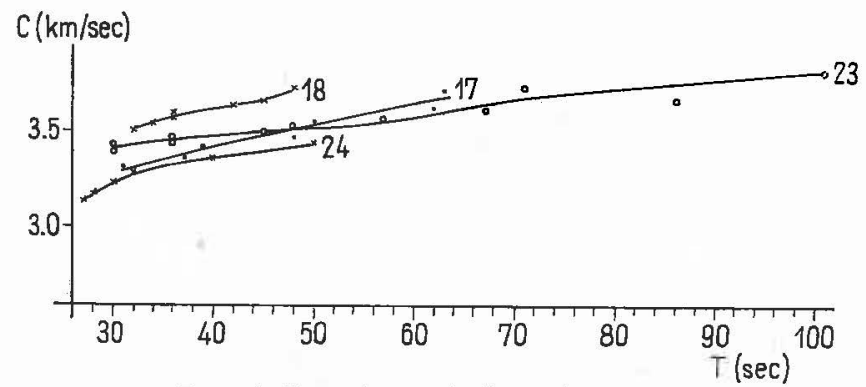

Fig. 5. Experimental dispersive curves

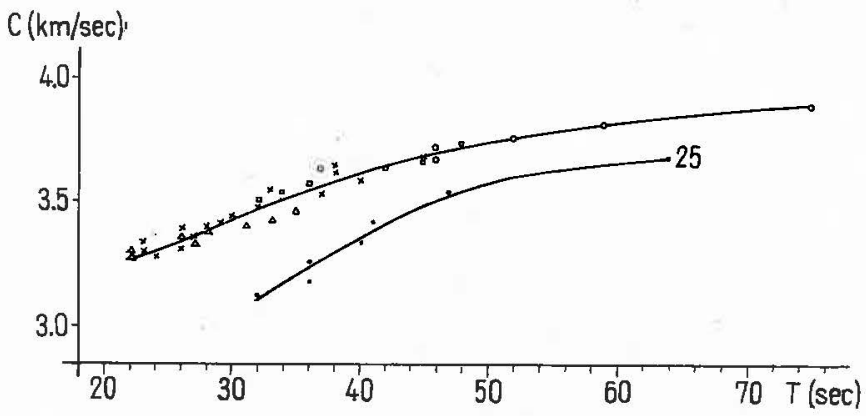

FIG. 6. Experimental dispersive curves

$\square$ - No 18 (The Solomon Islands - the Crimea)

O - No 20 (The New Hebrides - the Crimea)

$\triangle$ - No 23) (Kermadec-Crimea Basin)

$x-$ No 24$\}$

- No 32 (the North Atlantic Ridge-the Crimea)

The observations and their qualitative results show the sensitivity and unquestionable value of the dispersion method for the study of the differences of the interior and properties of the earth crust up to the upper mantle. While the direct task of dispersion calculation comes from idealized imaginations about parameters constancy and properties of layered-homogeneous medium along the way of waves propagation and, therefore, practically may be proved only along short real lines, a reverse task may be solved by the construction of experimental dispersive curves. Though for long lines average results are obtained, they express the properties of real media. The comparison of some points of observation will make it possible to use differential methods and to detail our ideas about dislocation and properties of some great blocks, determining real heterogeneities of the internal structure of the earth's crust. 


\section{REFERENCES}

[1] Popov, I. I.: On Love long-period waves dispersion in continental and oceanic crust on Indonesia-Crimea line, Izv. AN SSSR, ser. geofiz. 10 (1960)

[2] Arhangelsky, V. T., Kirnos, D. P., Popov, I. I. and Soloviev, V. N.: Observations experiment of long-period seismic waves on Simferopol Station, Izv. AN SSSR, ser. geofiz. 5 (1961)

[3] Savarensky, E. F., Popov, I. I. and Lasareva, A. P.: Long-period waves observations of Chile earthquake of 1960, Izv. AN SSSR, ser. geofiz. 8 (1961)

[4] Savarensky, E. F.: On determination of group and phase velocity from observations. Izv. AN SSSR, ser. geofiz. 11 (1959)

[5] "Surface Seismic Waves" - (instruction), under the editorship of E. F. Savarensky, Soviet po seismologii AN SSSR, Moscow (1963) 


\section{RESULTS OF SEISMIC REFRACTION MEASUREMENTS IN SOUTHERN BAVARIA \\ AND ALONG A PROFILE THROUGH THE EASTERN ALPS \\ C. Prodehl}

(Munich, GFR)

From the quarry Eschenlohe near Garmisch at the northern margin of the Alps a seismic profile was observed in $\mathrm{N} \rightarrow \mathrm{S}$ direction. The profile extends across Lago Lagorai, a small lake in the Dolomites, from which in 1961 and 62 the same profile could be observed in $\mathrm{S} \rightarrow \mathrm{N}$ direction. Further on several seismic refraction profiles starting from Eschenlohe were observed towards the Bavarian Molasse Basin. One of these profiles could also be observed in reverse direction starting from Böhmischbruck, a quarry in the crystalline region of the Böhmische Masse.

Along these profiles in the Bavarian Molasse Basin travel-time curves with almost identical velocities were observed. The following mean velocities (longitudinal waves) were found: for the upper crust $5.9 \mathrm{~km} / \mathrm{s}$, for the lower crust $6.5 \mathrm{~km} / \mathrm{s}$ and for the uppermost mantle $8.2-8.4 \mathrm{~km} / \mathrm{s}$.

Contour maps for the Conrad- and Mohorovičić-discontinuity were presented. The Conrad-discontinuity is found below the Bavarian Molasse Basin at a depth of about $19-22 \mathrm{~km}$. It was not possible to follow up the Conrad-discontinuity from the Molasse Basin towards the Alps. The Mohorovičić-discontinuity however could be followed up as a continuous boundary plane from the Bavarian Molasse Basin towards the Alps: Below the northern edge of the Molasse Basin the Mohorovičić-discontinuity is found at a depth of $30 \mathrm{~km}$ and below the Central Alps at a depth of $55 \mathrm{~km}$.

Below the Alps interfaces were found at depths of 10 and $20 \mathrm{~km}$, which separate layers with velocities (longitudinal waves) of $5.9,6.1$ and $6.5 \mathrm{~km} / \mathrm{s}$. Further on a layer - approximately $10 \mathrm{~km}$ thick - was found at a depth of $40-45 \mathrm{~km}$ below the Central Alps having a velocity of $7.2-7.4 \mathrm{~km} / \mathrm{s}$. Depending on the evaluation of the onsets in the seismograms, which allow the setting up of two groups of travel-time curves, for the region of the Southern Alps two models can be constructed: A) The layer having the velocity of 7.2-7.4 km/s cannot be found below the Southern Alps. The Mohorovičić-discontinuity however can be constructed as far as the northern edge of the Po Plain, in which case a maximum depth of $60 \mathrm{~km}$ is obtained below the Quarzporphyr South of Bozen. B) The layer with the velocity of $7.2-7.4 \mathrm{~km} / \mathrm{s}$ can be followed up towards South as far as the edge of the Po Plain rising from $40 \mathrm{~km}$ below the Central Alps up to $25-30 \mathrm{~km}$ below the southern margin of the Alps, while the Mohorovičićdiscontinuity can be constructed only as far as the Quarzporphyr having here 
a depth of $53 \mathrm{~km}$. For the figure the Model B1 was chosen, because it is in general accordance with results by Müller $\&$ Talwani (in preparation) and seems therefore to be the most probable one.

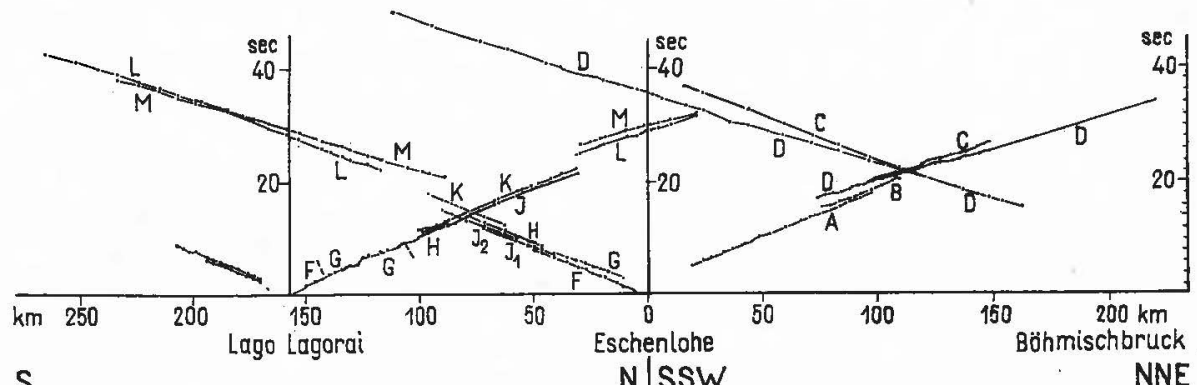

S

N|SSW

NNE

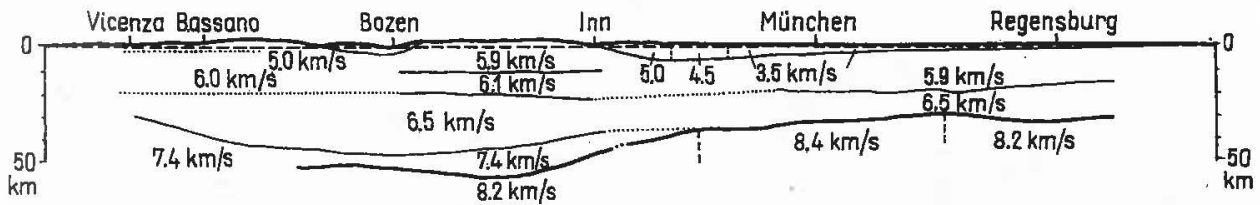

Protile L3/E8

Profile $E 2 / B 2$

Model B1

FIG. 1The travel-time curves in the upper part of the figure correspond to velocities given in the lower part as follows: right side: A $-5.9 \mathrm{~km} / \mathrm{s}, B$ and $C-6.5 \mathrm{~km} / \mathrm{s}, D-8.2-8.4 \mathrm{~km} / \mathrm{s}$; left side: $F-5.0 \mathrm{~km} / \mathrm{s}, G-5.9$ $\mathrm{km} / \mathrm{s}, H-5.8 \mathrm{~km} / \mathrm{s}, J-6.1 \mathrm{~km} / \mathrm{s}, \mathrm{K}-6.5 \mathrm{~km} / \mathrm{s}, \mathrm{L}-7.2-7.4 \mathrm{~km} / \mathrm{s}, \mathrm{M}-8.2 \mathrm{~km} / \mathrm{s}$.

The resulting interfaces are shown in the lower part of the figure. Only the full lines correspond with constructed interfaces. The dashed lines indicate, at which depths changes of velocity were used for the construction of the lower discontinuities, because in this region no corresponding interface could be found from the travel time curves 


\section{THE GROUP CHARACTER OF OBSERVING SURFACE SEISMIC WAVES IN A LAYERED CRUST}

Sh. S. Ragimov

(Moscow, USSR)

In the papers $[1,2]$ on the example of Rayleigh waves propagation in the earth crust it was shown that $n$ layered model of the crust causes the $n$ groups of surface waves. The body waves have been reflected totally at the bottom of the first layer and interfering constructively they form a group of oscillations, which characterizes the first layer properties. In the same way if they are reflected from the second layer, the bottom waves form a group of oscillations, characterizing the properties of both the first and second layers, from the third layer, the bottom waves form the group, characterizing the properties of all three layers and so on. This was illustrated by distant earthquakes records $[1,2]$, where all three groups are in presence. The first group is due to the crust as a whole, the second one - so-called granitic and sedimentary layers, but the third group - only sedimentary layer. The photocopies of the seismograms of the earthquake which occured on March 22, 1957, are shown in Fig. 1.

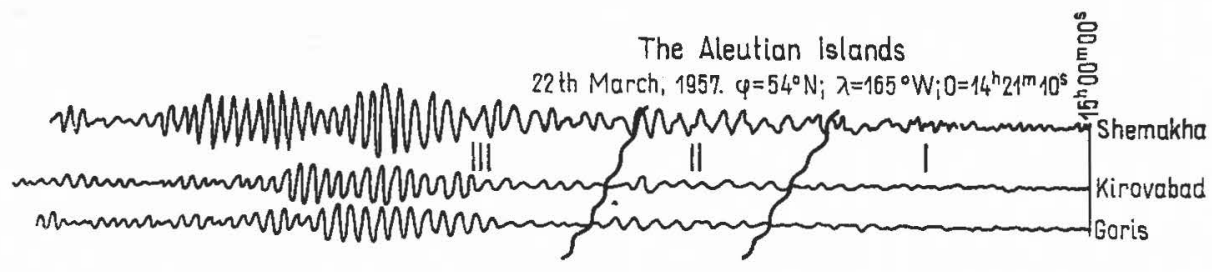

FIG. 1

$$
O=14^{h} 21^{m} 10^{s} ; \quad \phi=54^{\circ} \mathrm{N} ; \quad \lambda=165^{\circ} \mathrm{W} ; \quad M=7 \frac{1}{4}
$$

It illustrates the group character of the surface waves propagation. The same groups of oscillations is possible to see for earthquakes with epicentres in the different parts of the world, relatively the Central Caucasus (Fig. 2). The dispersion data are given in Fig. 3 for these earthquakes. The data are systematized for three fans (I, II, III, in Fig. 2), the nearness of lines along azimuths corresponds to a successive increase of distances inside each of them. On the seismograms of the vertical seismographs Rayleigh surface waves were evaluated. 


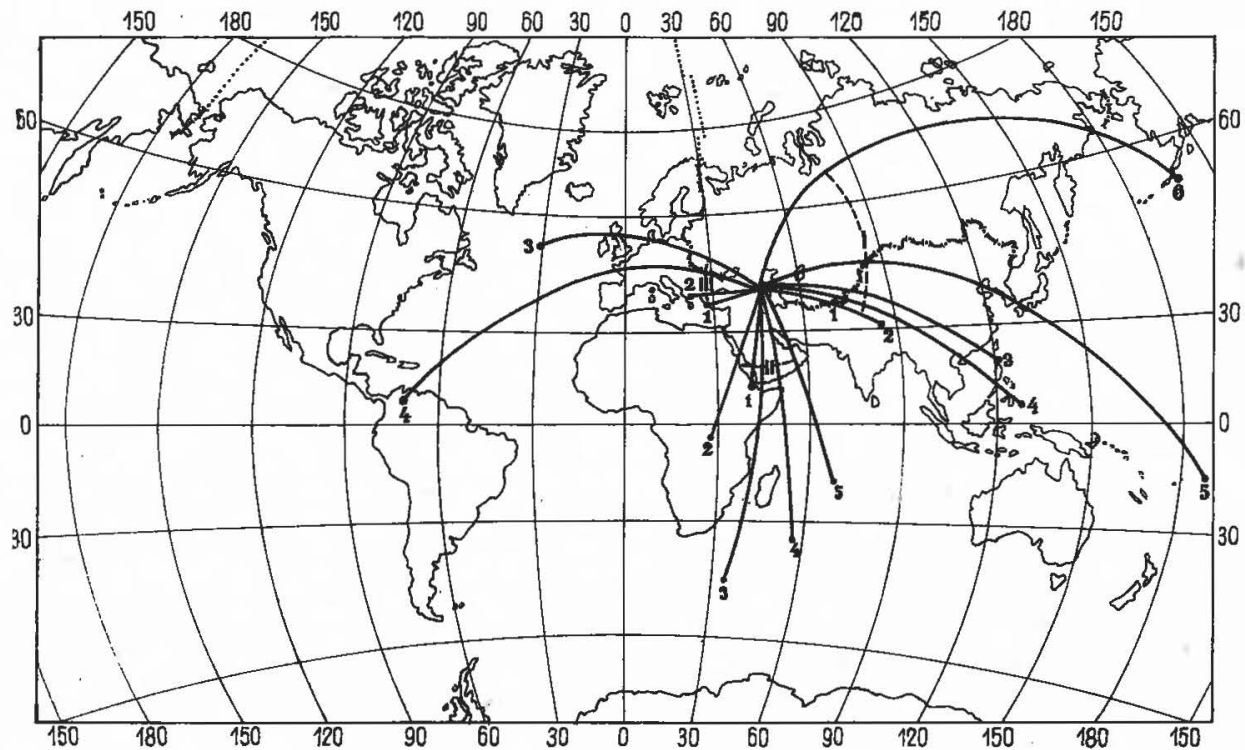

FIG. 2

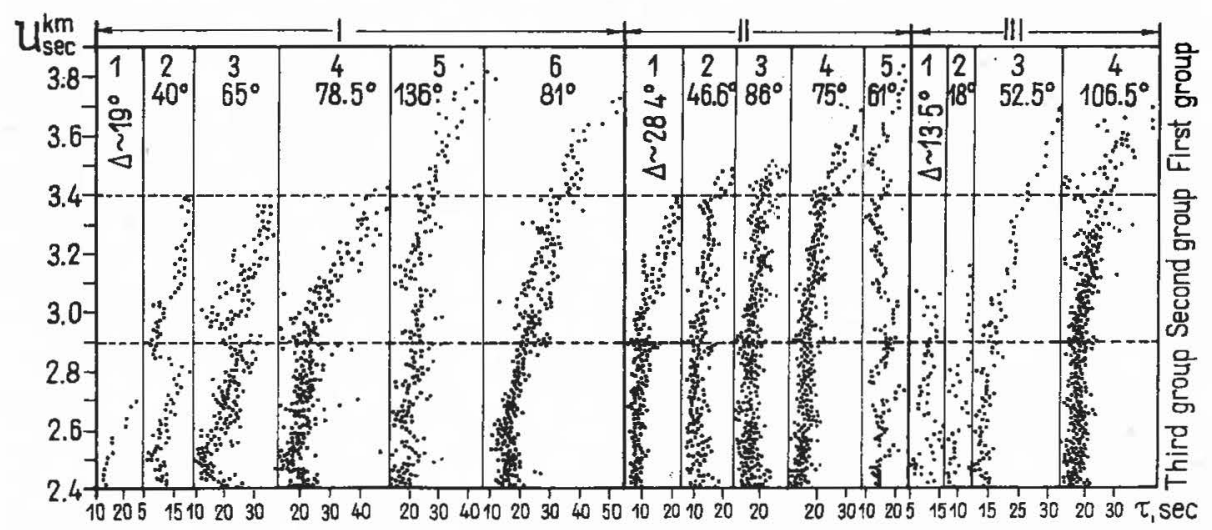

Fig. 3. I fan: 1 The Middle Asia Zone, 4. IV. 1957, $O: 11^{h} 36^{m} 22^{s}, \varphi=36.1^{\circ} \mathrm{N}, \lambda=69.9^{\circ} \mathrm{E}$, $M=5, A z=95^{\circ}, A=19^{\circ} .2$ China, 23. 11. 1954. $O: 06^{h} 40^{m} 31^{s}, \varphi=27.0^{\circ} \mathrm{N}, \lambda=92.0^{\circ} \mathrm{E}$, $A z=95^{\circ}, \Delta=40^{\circ} .3$ The Philippine Islands, 11. VI. 1957, $O: 18^{h} 49^{m} 35^{s}, \varphi=19^{\circ} \mathrm{N}$, $\lambda=120^{\circ} \mathrm{E}, M=61 / 2, A z=85^{\circ}, \Delta \sim 65^{\circ} .4$ Mindanao Island, 24. IX. 1957, $O: 08^{h} 21^{m} 15^{s}$, $\varphi=6^{\circ} \mathrm{N}, \lambda=127^{\circ} \mathrm{E}, M=71 / 4, A z=91^{\circ}, A=78.5^{\circ} .5$ Samoa Islands, 14. IV. 1957, $O: 19^{h} 17^{m} 57^{s}, \varphi=151 / 2^{\circ} \mathrm{S}, \lambda=173^{\circ} \mathrm{W}, M=71 / 4, A z=66^{\circ}, \Delta \sim 136^{\circ}$. 6 Aleutian Islands, 22. III. 1957, $O: 14^{h} 21^{m} 10^{s}, \varphi=54^{\circ} \mathrm{N}, \lambda=165^{\circ} \mathrm{W}, M=71 / 4, A z=17^{\circ} 30^{\prime}$, $\Delta \sim 81^{\circ}$

II fan: 1 Ethiopia, 14. III. 1957, $O: 00^{h} 11^{m} 19^{s}, \varphi=12.5^{\circ} \mathrm{N}, \lambda=41^{\circ} \mathrm{E}, M=41 / 2, A z=190^{\circ}$, $A \sim 28.4^{\circ}$. 2 Belgian Congo, 3. VII. 1954, $O: 00^{h} 32^{m} 54^{s}, \varphi=4.0^{\circ} \mathrm{S}, \lambda=28.5^{\circ} \mathrm{E}, A z=$ $=205^{\circ}, A=46.6^{\circ} .3$ The region of Prince Edward, 4. VIII. 1957, $O: 21^{h} 08^{m} 51^{s}, \varphi=$ $=45^{\circ} \mathrm{S}, \lambda=35^{\circ} \mathrm{E}, M=53 / 4, A z=188^{\circ}, \Delta=86^{\circ}$. 4 The Indian Ocean, 15. VI. 1957, 
At it is seen from Figs $3 a, 3 b$ and $3 c$ separately for each fan with increasing epicentral distances firstly the third group arrives, caused by a sedimentary layer (an approximate interval of velocities is $V=2.4-2.9 \mathrm{~km} / \mathrm{sec}$ ), then the second group, caused by both granitic and sedimentary complex $(V=3.0-3.4$ $\mathrm{km} / \mathrm{sec})$ and at last the first group, caused by the crust as a whole $(V=3.5-4.0$ $\mathrm{km} / \mathrm{sec}){ }^{*}$ All of them also show the group character oscillations of surface waves in a layered crust. This phenomenon must be taken into consideration when the earth crust structure is investigated by the surface waves dispersion.

\section{REFERENCES}

[1] Raglmov, SH. S.: To the question of crustal structure study on group and phase velocities of surface waves, Abstract of Papers, International Association of Seismology and Physics of the Earth's Interior, XIII. General Assembly, 3 International Union of Geodesy and Geophysics, Berkeley, California (1963)

[2] РАгимоб, Ш. С.: Вторая группа волн Релея ДАН Изд. ССР, 199 (1963)

* The first group is not always expressed accurately. Apparently the reason is that the crust structure is not three-layered everywhere.

$O: 00^{h} 44^{m} 15^{s}, \varphi=34^{\circ} \mathrm{S}, \lambda=56^{\circ} \mathrm{E}, M=71 / 4, A z=180^{\circ}, \Delta=75^{\circ} .5$ The Indian Ocean 6. IV. $1955, O: 12^{h} 50^{m} 59^{s}, \phi=17.5^{\circ} \mathrm{S}, \lambda=66.5^{\circ} \mathrm{E}, \quad M=51 / 2, A z=157^{\circ}, \Delta \sim 61^{\circ}$., III fan: 1 Turkey, 22. VII. 1953, $O: 15^{h} 09^{m} 35^{s}, \varphi=39.2^{\circ} \mathrm{N}, \lambda=28.5^{\circ} \mathrm{E}, A z=269^{\circ}, \Delta=$ $=13.5^{\circ} .2$ Greece, 25. V. 1954, $O: 22^{h} 0.3^{m} 35^{s}, \varphi=39.5^{\circ} \mathrm{N}, \lambda=22.0^{\circ} \mathrm{E}, A z=274^{\circ}, \Delta \sim$ $\sim 18^{\circ} .3$ The Atlantic Ocean, 11. XII. 1954, $O: 12^{h} 57^{m} 08^{s}, \varphi=52^{\circ} \mathrm{N}, \lambda=32^{\circ} \mathrm{W}, A z=$ $=310.5^{\circ}, \Delta=52.5^{\circ} .4$ The boundary of Columbia and Venezuela, 21. IV. 1957, $O: 21^{h} 12^{m}$ $26^{\circ}, \varphi=7^{\circ} \mathrm{N}, \lambda=72^{\circ} \mathrm{W}, M=61 / 2, A z=295^{\circ}, \Lambda \sim 106.5^{\circ}$ 


\title{
THE PHASE VELOCITIES DETERMINATION METHOD AND THE EARTH'S CRUST DEPTH ESTIMATION IN AZERBAIJAN BY RAYLEIGH WAVES
}

\author{
Sh. S. Ragimov \\ (Moscow, USSR)
}

It is known that to study the earth's crust by the phase velocities dispersion method of surface waves it is necessary to use the records of those earthquakes, where an epicentre and the seismic stations are in the same profile [1]. On this occasion the earth's crust structure estimation on the Soviet part of Azerbaijan territory by the Rayleigh phase velocities dispersion method we used the earthquakes in Table 1.

TABLE 1

\begin{tabular}{|c|c|c|c|c|c|c|c|c|c|}
\hline \multirow{2}{*}{ No. } & \multirow{2}{*}{ Regions } & \multirow{2}{*}{$\begin{array}{l}\text { Date } \\
1957\end{array}$} & \multicolumn{3}{|c|}{ Time in focus } & \multicolumn{2}{|c|}{ Coordinates } & \multirow{2}{*}{$\mathbf{M}$} & \multirow{2}{*}{$\begin{array}{l}\text { Azimuth } \\
\text { on epi- } \\
\text { centre } \\
\text { from } \\
\text { Azerbaijar }\end{array}$} \\
\hline & & & hour & $\min$ & sec & latitude & longitude & & \\
\hline 1 & Samoa & 14. IV & 19 & 17 & 57 & $15 \mathrm{I} / 2 \mathrm{~S}$ & $173 W$ & $71 / 4$ & $66^{\circ}$ \\
\hline 2 & Philippine Islands & 11. VI & 18 & 49 & 35 & $19 \mathrm{~N}$ & $180 \mathrm{E}$ & $61 / 2$ & $85^{\circ} 30^{\prime}$ \\
\hline 3 & Mindanao Islands & 24. IX & 08 & 21 & 15 & $6 \mathrm{~N}$ & $127 \mathrm{E}$ & $71 / 4$ & $91^{\circ}$ \\
\hline 4 & The Indian Ocean & 15. VI & 00 & 44 & 15 & $34 \mathrm{~s}$ & $56 \mathrm{E}$ & $71 / 4$ & $180^{\circ}$ \\
\hline \multirow[t]{2}{*}{5} & Prince Edward & $4 \mathrm{VHI}$ & 21 & 08 & 51 & $45 \mathrm{~S}$ & $35 \mathrm{~F}$ & 536 & $188^{\circ}$ \\
\hline & ISIands & 4. VIII & 21 & 08 & 31 & $43 \mathrm{~S}$ & 3OE & $3 \% / 4$ & 1800 \\
\hline
\end{tabular}

The epicentres in the table were selected because they situate on the same profile with seismic stations "Shemakha", "Kirovabad" and "Goris"* and because the stations' seismographs during this period were well identifiable and the differences of phase oscillations due to the different dynamical parameters of the instruments were within the permissible error limits [2].

For marking out Rayleigh waves the records of only vertical seismographs "CVK" were evaluated. The results for these earthquakes are presented in Fig. 1. For the earth's crust estimation along the distances together with experimental data the theoretical dispersion curves of main mode of Rayleigh surface waves

* No. 1 earthquake epicentre is approximately in the profile with the stations "Goris" "Shemakha" (the inclination from the profile $\delta_{\alpha}=8^{\circ}$ ), Nos 2 and $3-$ with the stations "Shemakha" - "Kirovabad" $\left(\delta_{\alpha}=4^{\circ} 30^{\prime} ; 1^{\circ}\right)$, Nos 4 and 5 - with the stations "Kirovabad""Goris" ( $\delta_{\alpha}=0^{\circ} ; 8^{\circ}$, see and compare data given in Table 1 and Fig. 1). 
are given. They are calculated for a two-layered model of the earth's crust [3] with parameters

$$
b_{1}=3.4 \mathrm{~km} / \mathrm{sec} ; \quad \rho_{1}=2.5 \mathrm{~g} / \mathrm{cm}^{3} ; \frac{b_{2}}{b_{1}}=1.1268 ; \quad \frac{b_{3}}{b_{1}}=1.3239 ;
$$

$$
\frac{\rho_{2}}{\rho_{1}}=1.0948 ; \quad \frac{\rho_{3}}{\rho_{1}}=1.2044 ; \quad \frac{h_{1}}{H}=0.4 ;
$$

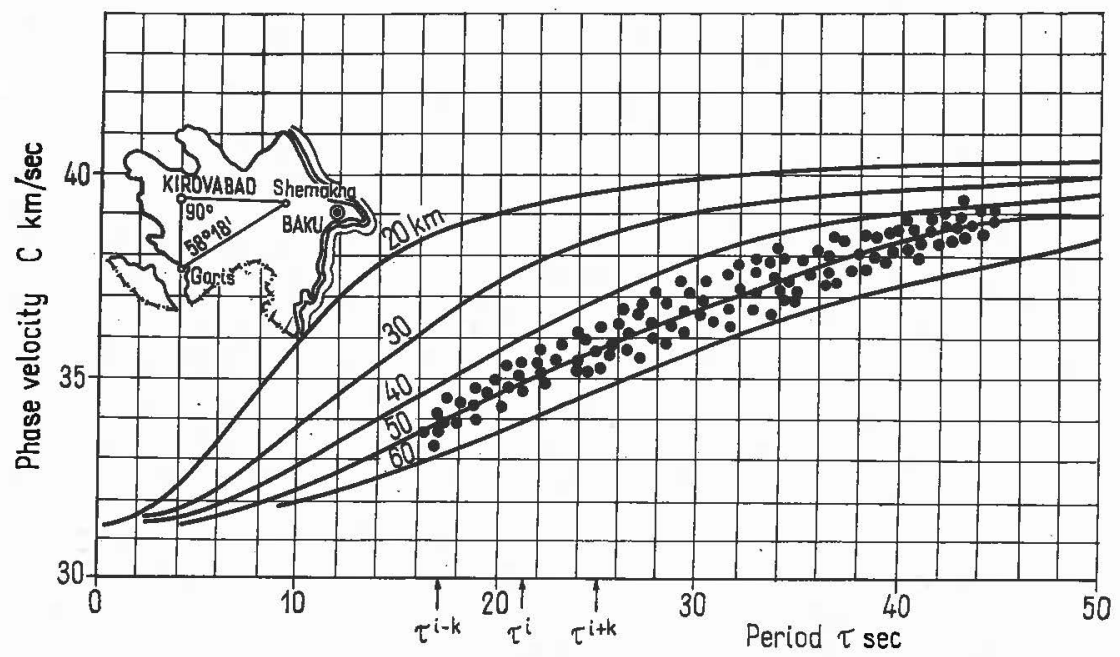

FIG. 1

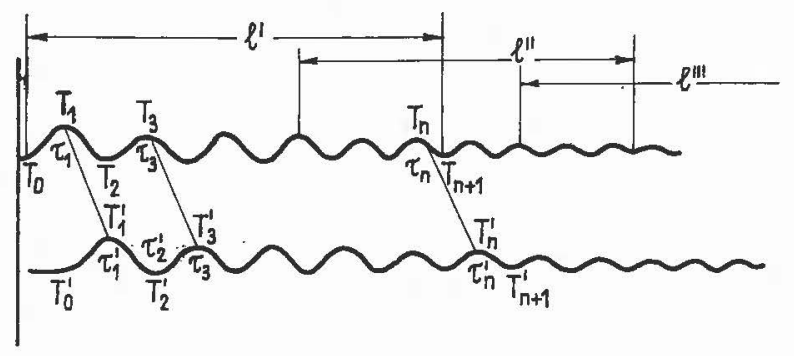

FIG. 2

where $b_{1}, b_{2}, b_{3}, \rho_{1}, \rho_{2}, \rho_{3}, h_{1}, h_{2}$ and $H=h_{1}+h_{2}$ are the designations for the transverse waves velocity, density and thickness layers, respectively.

It is seen from the theoretical curves and experimental data (Fig. 1) that the earth's crust of Azerbaijan is within $45-55 \mathrm{~km}$. This structure is the average for the earth's crust structure of the Soviet Azerbaijan, obtained by the other methods $[4,5]$. It is necessary to remark that at the evaluation of not so clear individual 
parts of oscillation trains on seismograms for control accuracy (the distances $\delta \Delta$ within stations are relatively small ones) we used the following method. According to the integral theorem of averaging (Fig. 2):

$$
C\left(\tau^{i}\right)=\frac{1}{\tau^{i+k}-\tau^{i-k}} \int_{\tau^{i-k}}^{\tau^{i+k}} C(\tau) d \tau=C\left(\frac{\tau^{i+k}+\tau^{i-k}}{2}\right)
$$

we supposed that for interval $\tau^{i-k}-\tau^{i+k}$ the phase velocity curve is rectilinear one and (Fig. 2) $i=I$

$$
\begin{gathered}
C\left(\tau^{\mathrm{I}}\right)=\frac{\delta \Delta}{\delta T^{\mathrm{I}}} ; \\
\tau^{\mathrm{I}}=\frac{\left(\tau_{1}+\tau_{1}^{\prime}\right)+\left(\tau_{2}+\tau_{2}^{\prime}\right)+\ldots+\left(\tau_{n}+\tau_{n}^{\prime}\right)}{2 n}= \\
=\frac{\left(T_{n}+T_{n}^{\prime}+T_{n+1}+T_{n+1}^{\prime}\right)-\left(T_{0}+T_{0}^{\prime}+T_{2}+T_{2}^{\prime}\right)}{2 n} ; \\
\delta T^{\mathrm{I}}=\frac{\left(T_{1}^{\prime}-T_{1}\right)+\left(T_{2}^{\prime}-T_{2}\right)+\ldots+\left(T_{n}^{\prime}-T_{n}\right)}{n} ;
\end{gathered}
$$

$n$ - oscillations number in the interval $l^{I}$.

If one successively calculated in the same way the values $C\left(\tau^{i}\right)$ it would be possible to form all the experimental dispersive curves. The method described here may produce the best result for distances between the stations (less than $100 \mathrm{~km}$ ), while the usual used method, which is based on the times differences from one phase to the next one, is not so suitable for such distances between stations $[6,7]$.

\section{REFERENCES}

[1] Ragimov, Sh. S.: To the question of crustal structure study on group and phase velocities of surface waves, Abstracts of Papers, International Association of Seismological Physics of the Earth's Interior, XIII General Assembly, 3, International Union of Geodesy and Geophysics, Berkeley, California, August (1963)

[2] Savarensky, E. F. and Ragimov, Sh. S. - Саваренский, Е. Ф. и Рагимов ШІ. C.: Определение скорости волн Релея и направления на эпицентр по трем близким станщиям. Изв. $А Н$ СССР, серия геофиз. 12 (1958)

[3] Glasko, V. A., Savarensky, E. F. and Scheckhov, B. N. - Гласко, В. A., Саваренский, Е. Ф. и Печков, Б. Н.: Данные о фазовых и групповых скоростях поверхностных сейсмических волн Изв. АН CCCP, серия геофизическая 10 (1963)

[4] Bagdasarova, A. M., Islamov, K. Sh., Koridalin, E. A. et. al: - Багдасарова, А. М., Исламов К. ІІІ, Коридалин, Е. А. и др.: Сейсмичность восточной части южных отрогов Главного Кавказского хребта и некоторые методические вопросы 
изучения сейсмичности отдельных районов. Сообщение 3, Изв. $A H$ Азербайджанской ССР, сер. геолого-географ. найк б нефти 4 (1961)

[5] Balavadze, В. K. and Shengelaya, G. Sh. - Балавадзе, Б. К. и Шенгелая, Г. Ш.: Основные черты структуры земной коры Большого Кавказа по гравитационным данным. ДАН СССР, 136, 6 (1961)

[6] Press, F,: Determination of crustal structure phase velocity of Rayleigh waves, 1, Bull. Geol. Soc. Amer., 67, 1647-1658 (1956); II: San Francisco Bay region, Bull. Seis. Soc. Amer., 47, 87-88 (1957)

[7] Gutenberg, В. - Гутенберг, Б.: Физика земных недр. Изд. Иностр. литературы. Москва, (1963) 


\author{
V. Z. Ryaboy
}

(Moscow, USSR)

In 1962-1963 along the Kopetdag-Aral Sea profile with a length of $625 \mathrm{~km}$ the earth's crust and upper mantle structure was studied by the DSS method (Gamburtzev 1952).* Field observations were made by means of the continuous longitudinal profile techniques in the process of which meeting and overlapping travel-time curves of deep waves were obtained. The maximum distance at which records of seismic waves were obtained amounted to $615 \mathrm{~km}$.

During field observations generation conditions were monitored and the absolute frequency response of the seismic recording channel was determined, which permitted to measure vertical components** of the real ground displacements within the passband of the channel ( 8 to $18 \mathrm{cps}$ ) and to reduce them to the constant weight of the charges equivalent to 1 ton TNT.

The seismograms yielded over 10 groups of waves corresponding to the interface boundaries in the earth's crust and upper mantle. The apparent velocity values $\left(\mathrm{V}^{*}\right)$ of the recorded waves except the undercritical reflexions vary on the average from 2.0 to $10.0 \mathrm{~km} / \mathrm{sec}$; the values of the effective absorption coefficients $\left(\alpha_{\text {ef. }}\right)$ characterizing the attenuation of waves' amplitudes with distance due to the joint effect of absorption and dispersion vary approximately by 2 orders $\left(\alpha_{\text {ef. }}=\right.$ $=10^{\circ}-10^{-2} \mathrm{~km}^{-1}$ ), the values of the vertical components of ground displacements reduced to the constant weight of the charges equivalent to 1 ton TNT vary by more than 3 orders (from tenths to hundreds of the Angström). The identified group of waves includes head, refracted and reflected waves (both undercritical and overcritical).

Schematic travel-time curves of the principal wave groups recorded along the profile are given in Fig. 1. These curves correspond to the seismo-geological conditions of the central Turkmenian regions. According to the predominant values of the apparent velocities all the identified waves can be divided into 3 great groups corresponding to sediments, the crystal block of the crust and the upper mantle.

* The work was carried out by the "Spetzgeofisica" office of the USSR State Geophysical Committee together with the Board of Geology and Earth's Interior Protection attached to the Turkmenian Council of Ministers and the Uzbek Geophysical Trust.

** For field observations vertical seismographs of the type of HC-3 with the natural frequency of 2 to $4 \mathrm{cps}$ were used. 
The waves originating in sediments are observed at the distance of $8-10 \mathrm{~km}$, the apparent velocity values vary from $2.0-2.5 \mathrm{~km} / \mathrm{sec}$ to $4.0-5.0 \mathrm{~km} / \mathrm{sec}$. These waves will not be dealt with here.
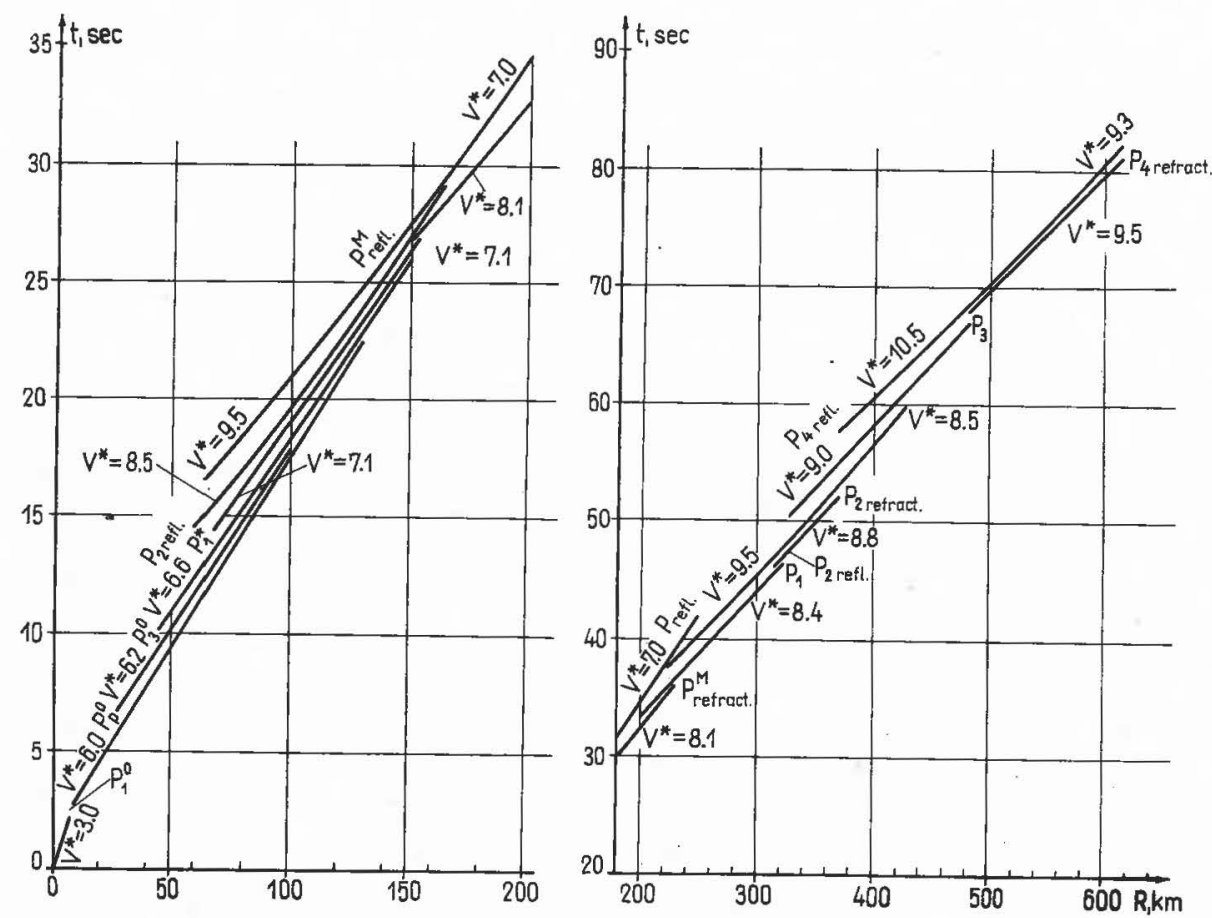

FIG. 1. Schematic travel-time curves of the recorded waves $1,2-$ for the distances $0-200$ $\mathrm{km}$ and 180-615 km, respectively; 3-indexes accepted for the designation of different wave groups; 4 - apparent velocities of waves in $\mathrm{km} / \mathrm{sec}$

The crystal block of the crust gives 5 groups characterizing its surface $\left(\mathrm{P}_{1}^{0}\right)$ and the intermediate interface boundaries in the crust $\left(\mathrm{P}_{2}^{0}, \mathrm{P}_{3}^{0}, \mathrm{P}_{1}^{*}, \mathrm{P}_{2}^{*}\right.$ reff.). Their apparent velocities are 5.8 to $7.3 \mathrm{~km} / \mathrm{sec}$, in some cases they increase to 8.0 to $8.3 \mathrm{~km} / \mathrm{sec}$. These waves are observed at a distance of $160-170 \mathrm{~km}$, beyond which they attenuate. $\mathrm{P}_{2}^{*}$ refl. reflected from the boundary at a depth of 26-28 $\mathrm{km}$ was identified on the records in the distance range from 80 to $170 \mathrm{~km}$ from the shot points. Its apparent velocity decreases with distances from 8.0-8.3 $\mathrm{km} / \mathrm{sec}$ to $7.0-7.3 \mathrm{~km} / \mathrm{sec}$, the refracted waves from the same boundary were not recorded.

Beginning from $160-170 \mathrm{~km}$ to $210-230 \mathrm{~km}$ from the shot points the first arrivals are refracted waves $\left(\mathrm{P}^{\mathrm{M}}\right.$ refract.) corresponding to the Mohorovičić discontinuity. Its apparent velocity is 8.0 to $8.2 \mathrm{~km} / \mathrm{sec}$. In the distance range 


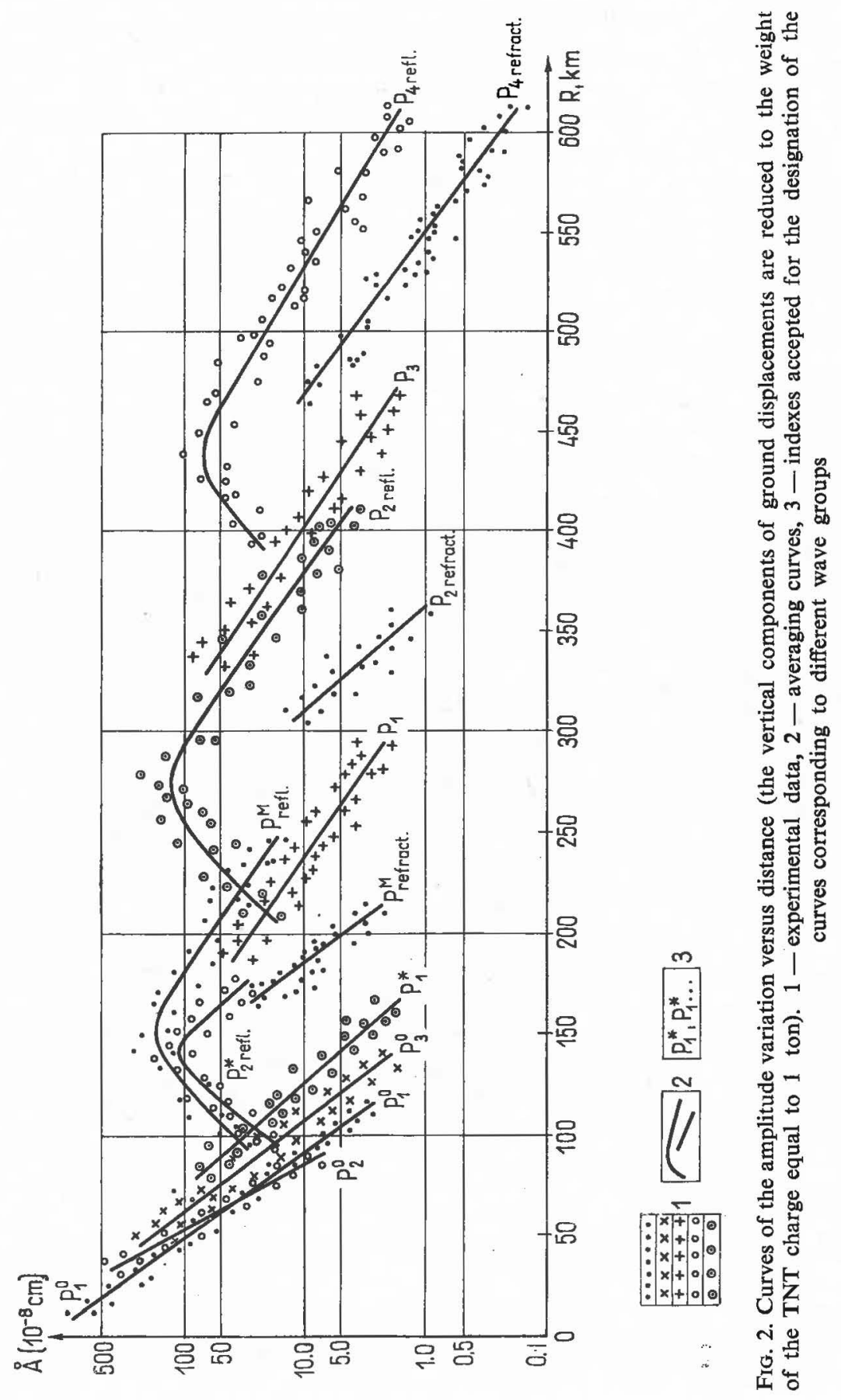


from $80-100 \mathrm{~km}$ to $250-270 \mathrm{~km}$ a reflected wave ( $\mathrm{P}^{\mathrm{M}}$ refl.) was recorded from the same boundary, its apparent velocity decreases with the increase of distance from the shot points from $8.5-9.0 \mathrm{~km} / \mathrm{sec}$ to $6.8-7.2 \mathrm{~km} / \mathrm{sec}$.

In the distance range from $180-200 \mathrm{~km}$ to $615 \mathrm{~km} 6$ more wave groups were recorded $\left(P_{1}, P_{2 \text { refl., }} P_{2 \text { refract. }}, P_{3}, P_{4 \text { ref1. }}\right.$ and $\left.\mathbf{P}_{4 \text { refract. }}\right)$ corresponding to the boundaries in the upper mantle. From the two boundaries located at the depth of the order of $75-80 \mathrm{~km}$ to $115-120 \mathrm{~km}$ both refracted and reflected waves were

1

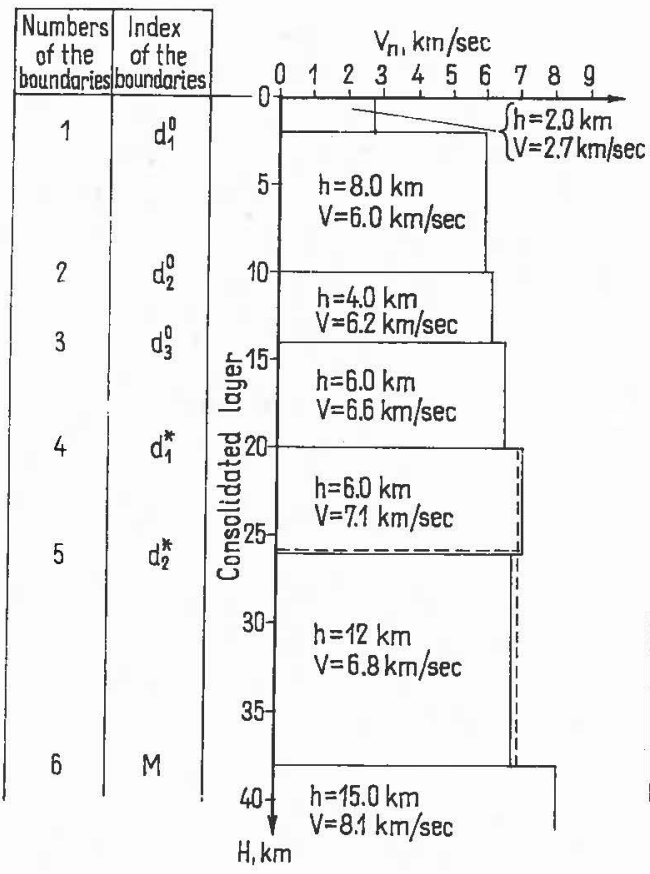

2

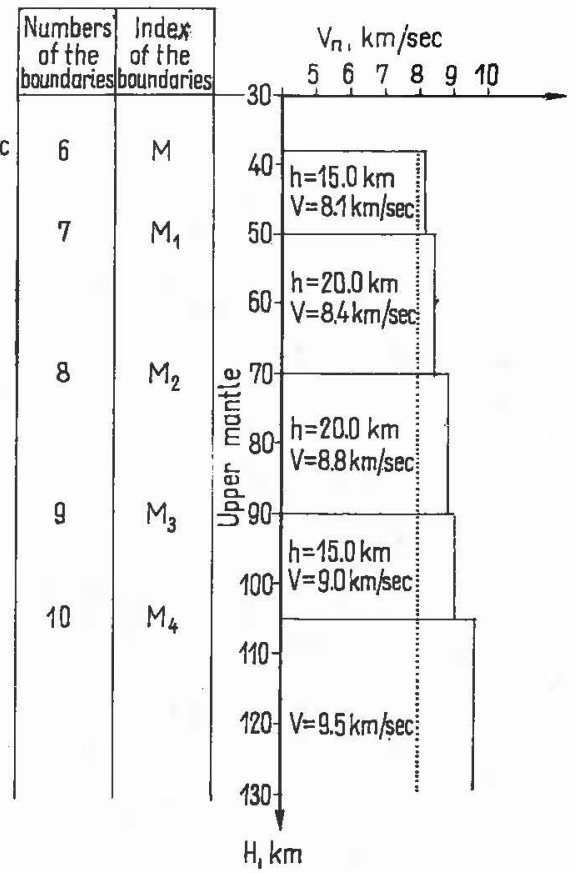

FIG. 3. Velocity cross-section of the crust (1) and upper mantle (2). 3, 4-possible variants of the velocity cross-section

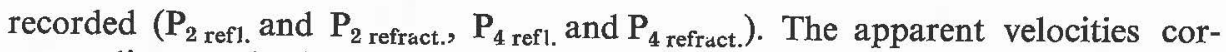
responding to the boundaries in the upper mantle vary from $8.3-8.5 \mathrm{~km} / \mathrm{sec}$ to $9.5-10.0 \mathrm{~km} / \mathrm{sec}$.

Figure 2 shows the curves of the amplitude variation with distance $\left(l_{n} A=\right.$ $=A(R))$. With the increase of the distance from the shot point a decrease of the average level of all recorded waves from the values of the order of 500 Ångströms* to the tenths of Ångström could be stated. The recorded waves differ as to their amplitude level, the attenuation with distance (the values of the effective absorption coefficient) and the character of the amplitude curves (the curves $l_{n} A=$ 
$A(R)$ for some wave groups are well approximated by straight lines of various slope $\left(\mathrm{P}_{1}^{0}, \mathrm{P}_{\Sigma}^{0}, \mathrm{P}_{3}^{0}, \mathrm{P}_{1}^{*}, \mathrm{P}_{\text {refract. }}^{\mathrm{M}}, \mathrm{P}_{2 \text { refract. }}, \mathrm{P}_{1}, \mathrm{P}_{3}, \mathrm{P}_{4 \text { refract. }}\right)$ and for the others they have the shape of curves with sharp maximum $\left(\mathrm{P}_{2 \text { refl., }}^{*} \mathrm{P}_{\text {refl., }}^{\mathrm{M}}, \mathrm{P}_{2 \text { refl., }}, \mathrm{P}_{4 \text { ref1.) }}\right.$ ).

The curves of the recorded waves served to determine the depth of the interface boundaries as well as the values of the interface and layer velocities. Figure 3 represents the velocity cross-section of the crust and upper mantle characterizing the variation of the layer velocities with depth. In cases when the experimental data did not permit a reliable determination of the layer velocity, the values of the interface velocities were taken equal to those of the layers. This is true mainly for the upper part of the consolidated crust. The accuracy of the layer velocity determination is approximately $\pm 0.15-0.20 \mathrm{~km} / \mathrm{sec}$. The depth of the $\mathrm{M}$ boundary in the central Turkmenian regions is 36 to $38 \mathrm{~km}$. The greatest variation occurs at interfaces associated with the roof and foot of the consolidated crust. At these interface boundaries the velocities increase by more than $1.0 \mathrm{~km} / \mathrm{sec}$.

The average velocity of the wave propagation in sediments is $=3.0 \mathrm{~km} / \mathrm{sec}$.

The crystal block of the crust is divided into 5 layers, where the wave velocity varies from $5.7-6.0 \mathrm{~km} / \mathrm{sec}$ to $7.0-7.2 \mathrm{~km} / \mathrm{sec}$. As to the velocity values these layers differ by not more than $0.2-0.5 \mathrm{~km} / \mathrm{sec}$. A wave guide channel with the velocity of $6.8-6.9 \mathrm{~km} / \mathrm{sec}$ is not unlikely to exist in the lower crust. However this result is unreliable since the variation of the layer velocities at the boundary $\mathrm{d}_{2}^{*}$ is comparable with the accuracy of their determination. Except for separate areas of the profiles the layers mentioned above are consistent in their thickness. The layer thicknesses in the crystal block of the crust are on the average from 4 to $10 \mathrm{~km}$.

In the upper mantle for the range of the investigated depths (down to 115$120 \mathrm{~km}) 5$ layers are distinguished where the velocity increases with depth from $8.0-8.2 \mathrm{~km} / \mathrm{sec}$ to $9.0-9.5 \mathrm{~km} / \mathrm{sec}$, while the thicknesses are 15 to $25 \mathrm{~km}$. We have not obtained the data indicating the possible existence of the wave guide channel in the upper mantle within the investigated range of depths. In Central Turkmenia the low velocity layer in the mantle probably exists at greater depths.

As a result of investigations it is established that the crystal block of the crust and the upper mantle (to the depth of the order of 115-120 km) are of layered structure. The upper mantle being a medium layered and little differentiated, as to velocity, it does not differ from the consolidated crust as to its structure. Sediments, consolidated crust and the upper mantle rather strongly differ as to the average gradients of the velocity increase with depth, whose values for the sediments, consolidated crust and upper mantle are as follows: 400 to 600 $\mathrm{m} / \mathrm{sec} / \mathrm{km}, 40 \mathrm{~m} / \mathrm{sec} / \mathrm{km}$ and $20 \mathrm{~m} / \mathrm{sec} / \mathrm{km}$, respectively.

In seismology the velocities of seismic waves equal to $9.0-9.5 \mathrm{~km} / \mathrm{sec}$ are usually associated with the depths of the order of 300 to $400 \mathrm{~km}$ (Gutenberg 1963). This is neither confirmed by our results nor by some other investigations based on the records of strong distant explosions (Butovskaya and Ulomov 1962, Mintrop 1953). The reasons of such a discrepancy must be specially investigated. 


\section{REFERENCES}

[1] Butovskaya, E. M. and Ulomov, V. I.: Travel-time curves of seismic waves and some peculiarities of crustal structure in Central Asia according to data of strong explosions. Deep Seismic Sounding of Earth's Crust in USSR. Collected reports (1962)

[2] Gamburtzev, G. A.: Deep seismic sounding of earth's crust. Reports of the USSR Academy of Sciences, 876 (1952)

[3] Gutenberg, B.: Physics of Earth's Interior, M., I. L. (1963)

[4] Mintrop, L.: Layers of the earth's crust from records of large explosions and earthquakes. Problems of Seismic Prospecting (collected papers), M. I. L. (1953) 


\section{RAYLEIGH AND LOVE WAVES DISPERSION CURVES FOR TWO-AND THREE-LAYERED EARTH'S CRUST \\ E. F. Savarensky, V. B. Glasko and Ya. Sh. Granit}

(Moscow, USSR)

The analysis of theoretical dispersion curves and their dependences on media parameters make it possible to investigate some important characteristics. These characteristics improve considerably the interpretation of observing dispersion and the determination of the structure of the earth's crust [1]. At our investigation as far as possible the parameters of the earth's crust and mantle of the USSR middle European part have been used [2, 3, 4, 5]. The data on transverse waves are taken from the work [6], and the information about densities from [7].

The problem of the number of layers, taken into account, is a complex one. In consequence of the accuracy of observations (1-2\%) and the ranges of observing periods a two, and sometimes a three-layered earth's crust is enough. It had been shown that for periods more than 15 seconds there is no difference between a two and a one-layered crust [8]. The experiments have shown that in our case it was possible to neglect the velocity gradient.

The dispersion of velocities for axial symmetry is the same as for the plane waves. Therefore only a plane wave is considered.

Let us imagine vertical and horizontal components of an elastic displacement vector in the form:

$$
\begin{aligned}
& U=x e^{i \omega}\left(t-\frac{x}{C}\right)+i \frac{\pi}{4} \\
& W=y e^{i \omega}\left(t-\frac{x}{C}\right)-i \frac{\pi}{4}
\end{aligned}
$$

After substituting these expression in equations of oscillations of elastic medium and taking into account the boundary conditions we have in turn the task of own values of parameter $\xi=\frac{1}{C}$. This task in every point $(\xi, \omega)$ is solved by a numerical method. It gives birth to a simple transcendent equation:

$$
f(\xi, \omega)=0
$$

The value of the left part is known in every point $\xi$ at fixed $\omega$, that makes it possible to solve this equation by a numerical method with the help of the standard sub-program for high-speed computers. 
The problem here investigated are the parameters of medium which are piececonstant functions (multilayered model). Such case is included in the previous work [9].

The method has been widely used before us in some electroprospecting tasks [10] This method has advantage in comparison with the matrix one, that can be directly used for the medium with continuously changing parameters.

In this work only a multilayered model is considered. The investigation of Rayleigh and Love plane surface waves dispersion was carried out in two and three-layered elastic crusts, lying on an elastic halfspace.

On the base of given method the program has been formulated for the determination of phase and group velocities, depending on the periods of main tone (fundamental mode) and also the first and second overtones for models with layers number $n$, where $0 \leq n \leq 500$.

The solution of transcendental equation (2) is the program basis at every fixed value of period $T$ or frequency $\omega$ relatively $\xi$ where $\xi=\frac{b_{n+1}}{c}, b_{n+1}$, the transverse wave velocity in halfspace, $C$, phase velocity of surface wave. For the group velocity calculation at some meaning of frequency $\omega$ we take a disturbed value of the root $\xi$, corresponding to the value $\omega+g \omega$.

Then the group velocity is calculated by the formula:

$$
\frac{V}{b_{n+1}}=\frac{1}{\xi+\frac{\Delta}{g}}, \text { where } \Delta=\widetilde{\xi}-\xi
$$

The accuracy of the group velocity calculation has the order $3 \cdot 10^{-3}$, phase velocity $10^{-\infty}$.

On the great frequencies the left part of the dispersion equation is presented asymptotically, that is reached by the decrease of the layers number by program way, as soon as the value $\exp \left[\frac{\omega h_{k}^{*}}{H}\right]$ reaches some critical value, where $h_{k}^{*}=\sum_{i=1}^{k} h_{i}$.

This provides a continuous reliable calculation of dispersion curve in all the given range of periods. The correctness and accuracy is controlled as in the work [11], by repeated calculation of velocities for the previous value of the period by a decreased number of layers.

The described calculations program was prepared in the Moscow University.

We have carried out the calculations for the different theoretical models of the earth's crust, having two or three layers above the halfspace. These models differ from each other by the ratio of layers thickness, or by relative velocities of shear and compressional waves in them.

In Table 1 the calculated data are shown. Calculated dispersion curves represent the dependence of the ratio of phase $(C)$ and group $(u)$ velocities to the trans- 


\begin{tabular}{|c|c|}
\hline$\stackrel{\circ}{-}$ & 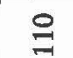 \\
\hline ò & gे \\
\hline$\stackrel{\infty}{0}$ & $\stackrel{\circ}{\circ}$ \\
\hline r & $\stackrel{5}{\sigma}$ \\
\hline$\ddot{0}$ & 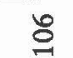 \\
\hline$\stackrel{n}{0}$ & 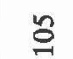 \\
\hline 范 & ت \\
\hline$\stackrel{m}{0}$ & 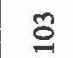 \\
\hline ֻุ & ऽั \\
\hline$\overline{0}$ & $\overline{\mathrm{O}}$ \\
\hline 0 & 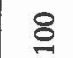 \\
\hline जा & $z \frac{\frac{\infty}{g}}{g}$ \\
\hline
\end{tabular}

ङ

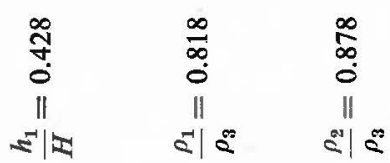

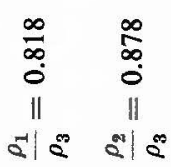

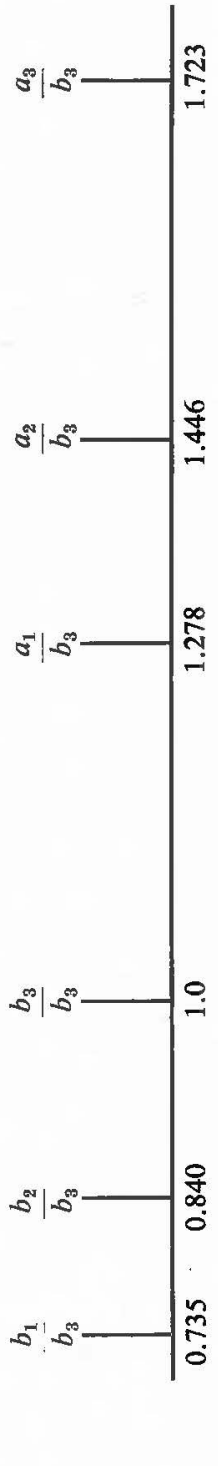

80

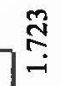<smiles></smiles>

*

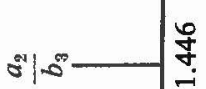

$\approx$

年

*

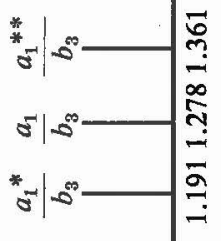

5

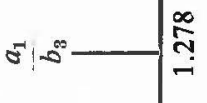

50

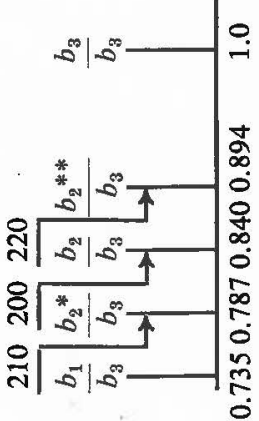

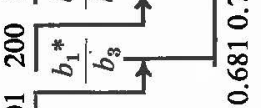

휘

อ 


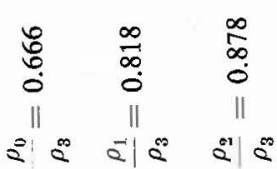

\begin{tabular}{|c|c|c|}
\hline$\stackrel{\text {-j+ }}{\varrho}$ & ֻี กิ & \\
\hline$\stackrel{-10}{\varrho}$ & $\overrightarrow{0}$ & $\stackrel{\circ}{-}$ \\
\hline$\stackrel{\varrho}{\varrho}$ & $\stackrel{0}{0}$ & \\
\hline 桨 & ָ̃ & \\
\hline 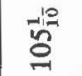 & $\overline{0}$ & $\stackrel{n}{0}$ \\
\hline$\stackrel{\varrho}{\varrho}$ & $\stackrel{\circ}{\circ}$ & \\
\hline$\overbrace{0}^{-1+}$ & $\stackrel{\text { ஸे }}{0}$ & స̃ \\
\hline 망 & $\ddot{0}$ & "̈) \\
\hline$\stackrel{8}{ }$ & $\stackrel{\circ}{0}$ & $\stackrel{0}{0}$ \\
\hline 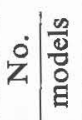 & $=1 \mathbb{Z}$ & $\begin{array}{l}5 \\
+ \\
5\end{array}$ \\
\hline
\end{tabular}

อ

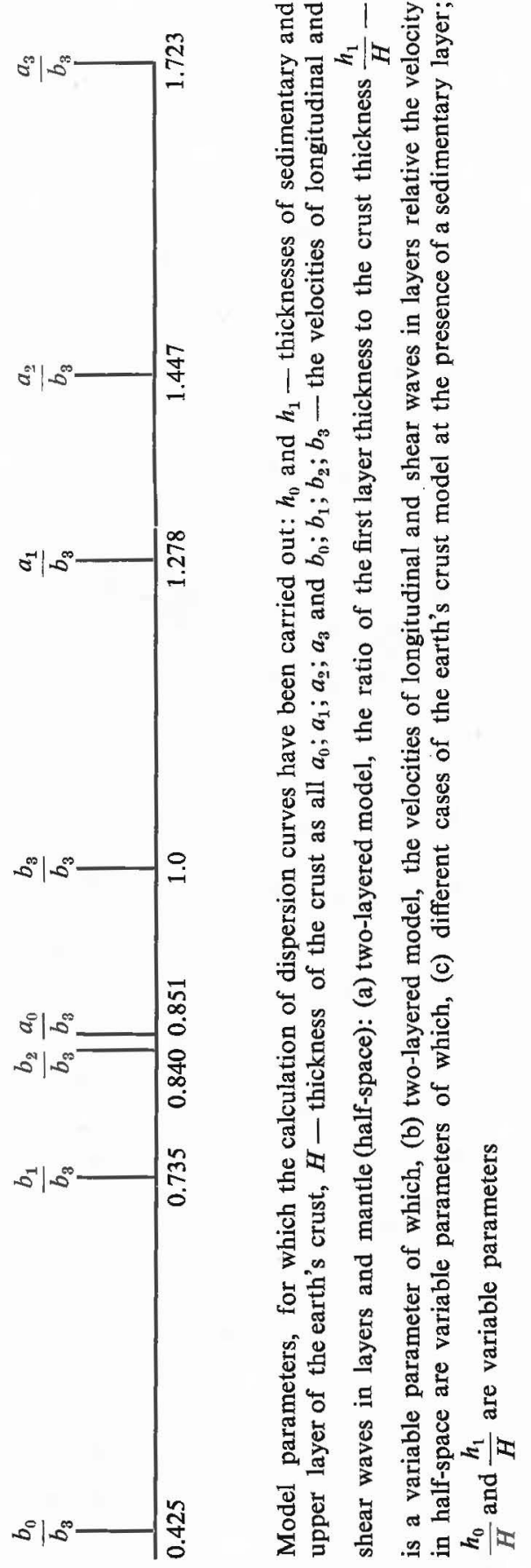


verse wave velocity in the halfspace $\left(b_{3}\right)$ on nonsized period $\rho=\frac{T b_{3}}{H}$, where $H$ is the earth's crust thickness, $T$ the period in seconds.

Thus, the models having different absolute parameters are equivalent, if their relative parameters $\frac{a_{i}}{b_{n+1}}, \frac{b_{i}}{b_{n+1}} \frac{\rho_{i}}{\rho_{n+1}}, \frac{h_{i}}{H}$ coincide $(i=0,1,2)$.

Table 1 shows $\frac{b_{i}}{b_{3}}$ and $\frac{a_{i}}{b_{3}}$ - the values of ratio of transverse and longitudinal waves to $b_{3}$ - velocity in halfspace.

In Table 1a the ratios of the upper layer thickness to the all crust thickness are given and key numbers of them are indicated.

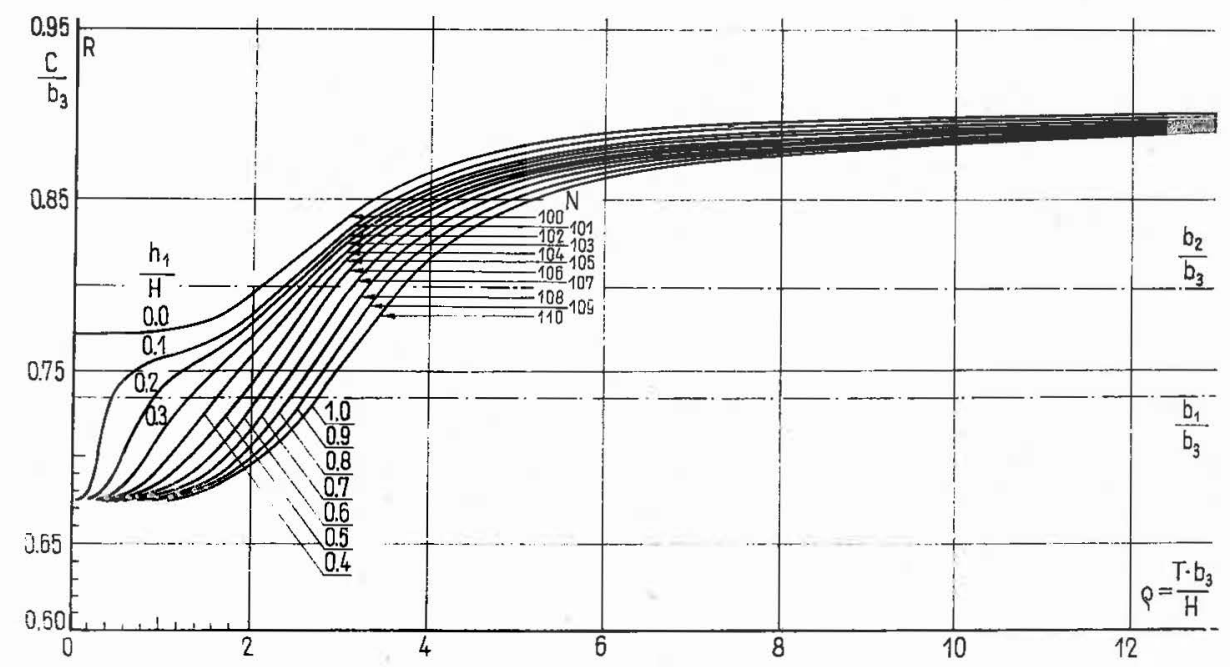

FIG. 1. Dispersion curves of phase velocity $\frac{C}{b_{3}}$ of the main tone first mode of Rayleigh waves for two-layered model. $\frac{h_{1}}{H}-$ is parameter

In Table $1 \mathrm{~b}$ the values of velocities ratios given at their modifying for the first and second layers. The layers thickness $\frac{h_{1}}{H}=0.428$.

In Table 1c the values of initial parameters are given for a three-layered crust.

In Figs 1 and 2 the set of dispersion curves of phase and group velocities of main tone of Rayleigh waves are presented for two-layered models (numbers 100 - 110), having the same velocities but different ratios of layers thickness from 0 to 1 through 0.1 . 
At $\frac{h_{1}}{H}=0$ the phase velocity $C / b_{3}$ asymptotically approximates 0.9194 $\frac{b_{2}}{b_{3}}$ in the vicinity of $\rho=0$.

In the presence of the first layer and on some conditions the group velocity curve has two minimums and one maximum. It is caused by the separate influence

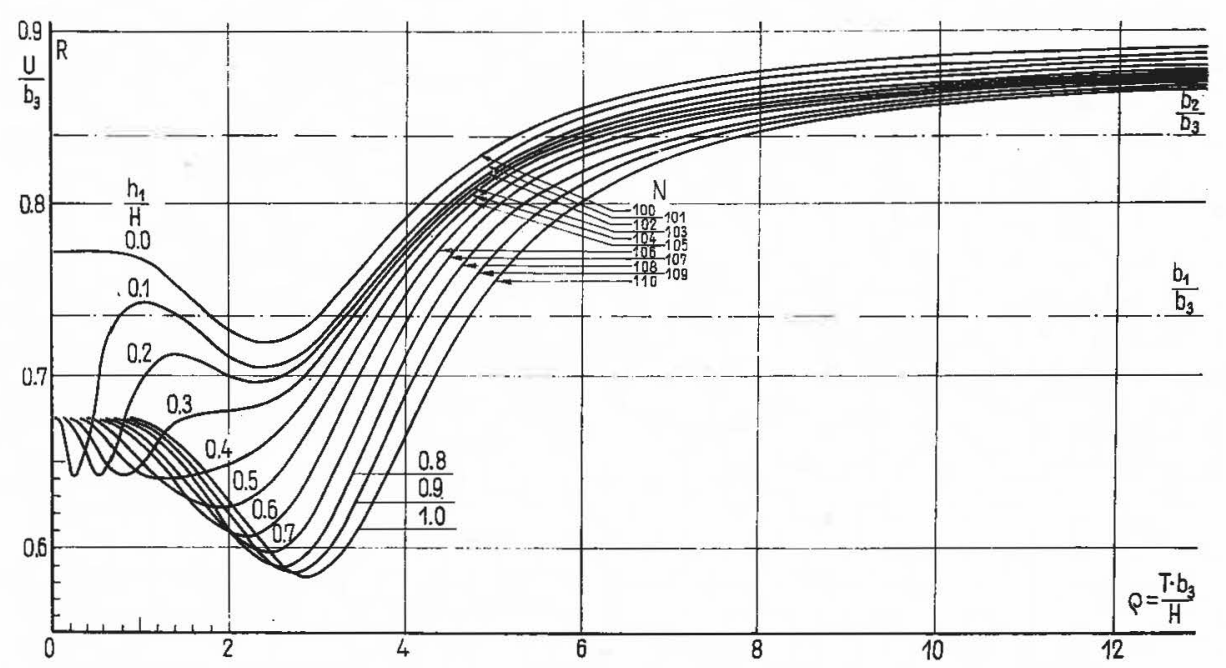

FIG. 2. Dispersion curves of group velocity $\frac{U}{b_{3}}$ of the main tone (first mode) of Rayleigh waves for two-layered model. $\frac{h_{1}}{H} h_{1}$ - is parameter

of the first layer only, and also the first and second layers together at $\frac{h_{1}}{H} \quad$ less than 0.3. With increasing first layer thickness at $\frac{h_{1}}{H}$ about 0.3 maximum and minimum coincide into plateau (bend). At greater $\frac{h_{1}}{H}$ a combined minimum is formed.

In Figs $3 \mathrm{a}$ and $3 \mathrm{~b}$ the families of phase and group velocity of the first overtone $\left(M_{2}\right)$ are presented for the same models. The dependence on $\frac{h_{1}}{H}$ is more sharp. The above mentioned "plateau" takes place at the great relative thickness of the first layer.

Figures 4, 5, 6 are analogues to 1, 2, 3 but for Love wave. 


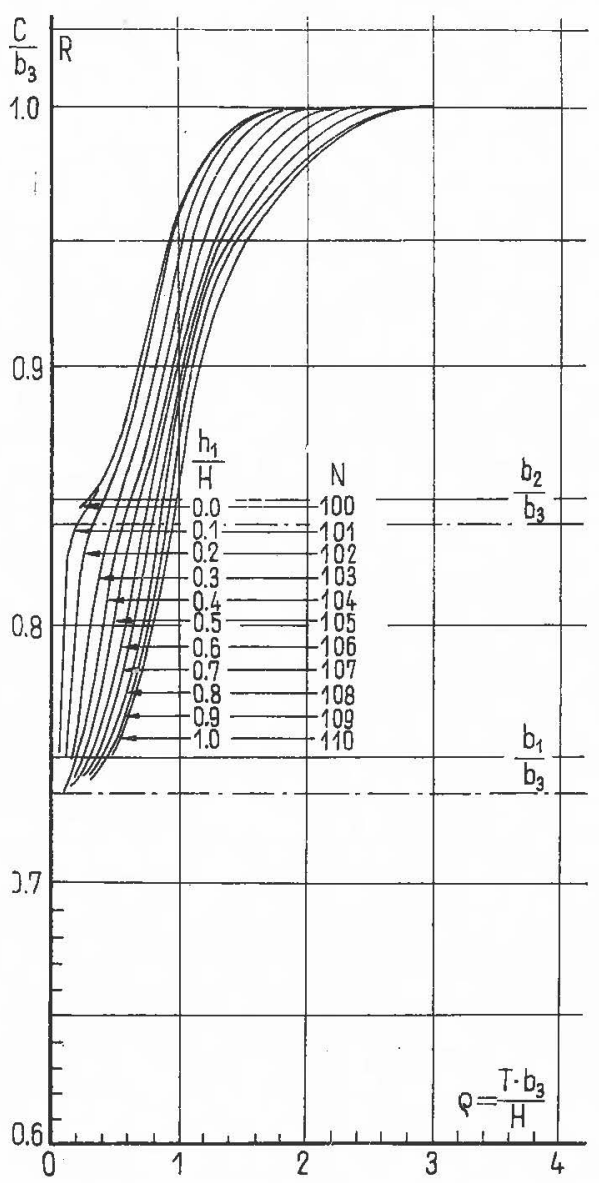

(a)

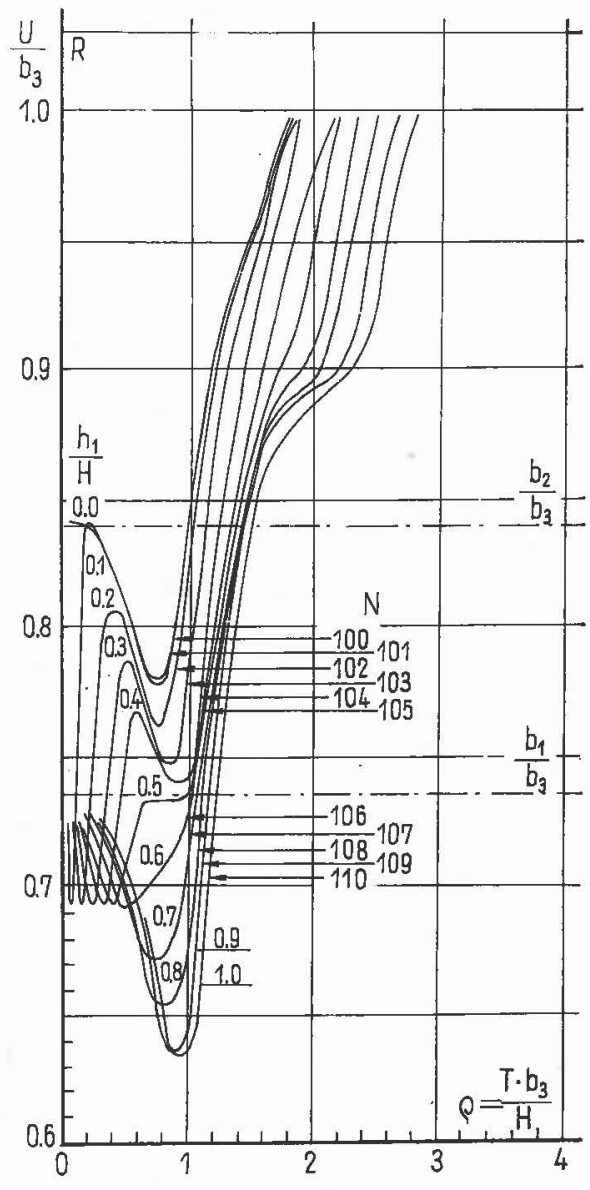

(b)

FIG. 3. Dispersion curves of the first overtone $\left(M_{2}\right)$ (second mode) of Rayleigh waves fo two-layered model. (a) phase velocity $\frac{C}{b_{3}}$, (b) group velocity $\frac{U}{b_{3}}$

In Fig. 7 the velocities and periods in Airy phase in dependence on the layers depths are shown. On the scheme maximums and minimums of the group velocity curves are united.

It is interesting that at the presence of two minimums the value of velocity for Airy phase is sensitive to the variation of the ratio of layers thicknesses for long-period minimums. For short-period minimums the period value is sensitive to the thickness variation, but the velocity is the same for the different models.

For the first overtone of Rayleigh waves $\left(M_{2}\right)$ the velocity in Airy phase is 


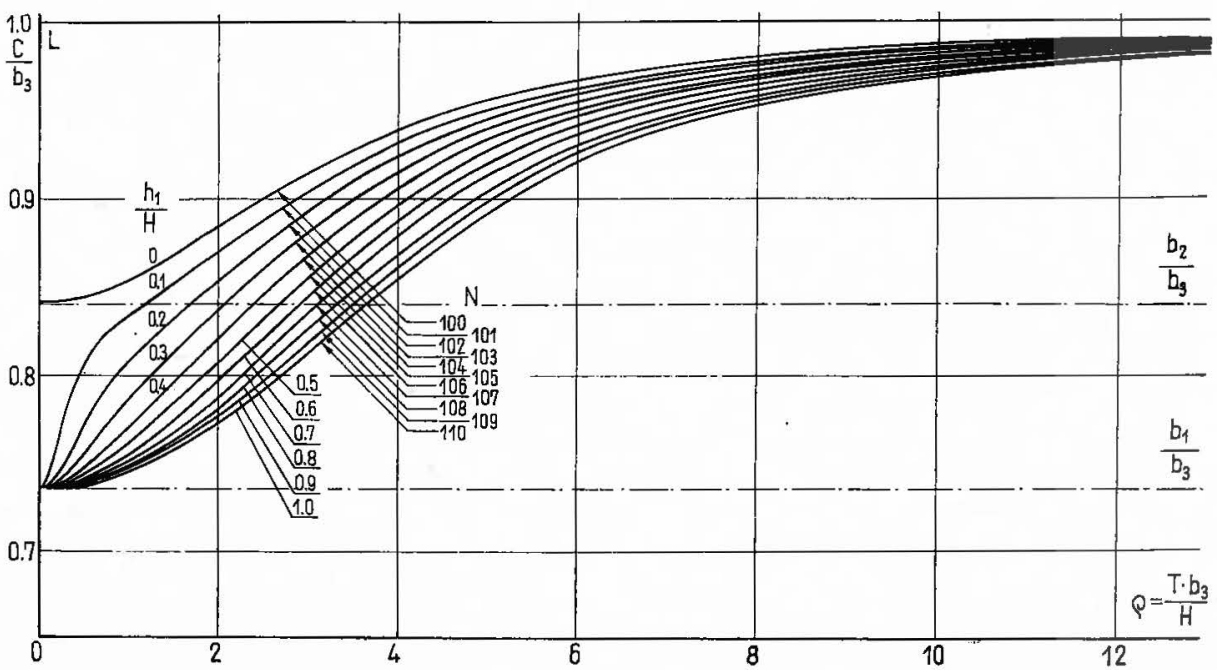

FIG. 4. Dispersion curves of phase velocity $\frac{C}{b_{3}}$ of the main tone (first mode) of Love wave for two-layered model. $\frac{h_{1}}{H}-$ is parameter

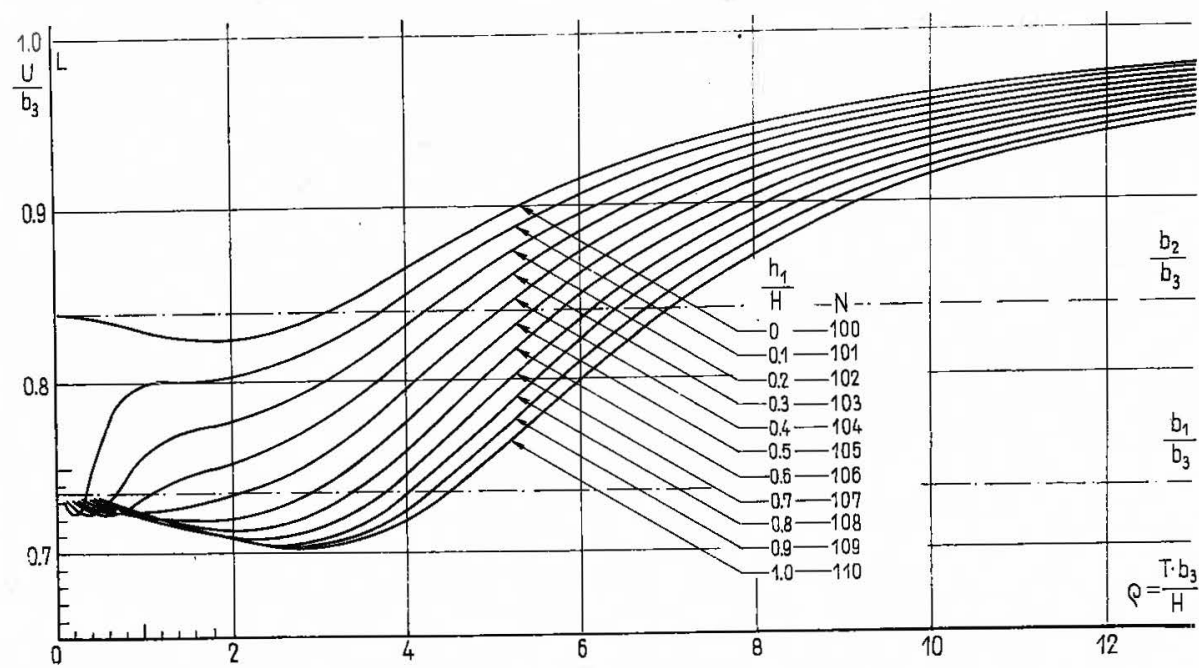

Fig. 5. Dispersion curves of group velocity $\frac{U}{b_{3}}$ of the main tone (first mode) of Love wave for two-layered model. $\frac{h_{1}}{H}-$ is parameter 

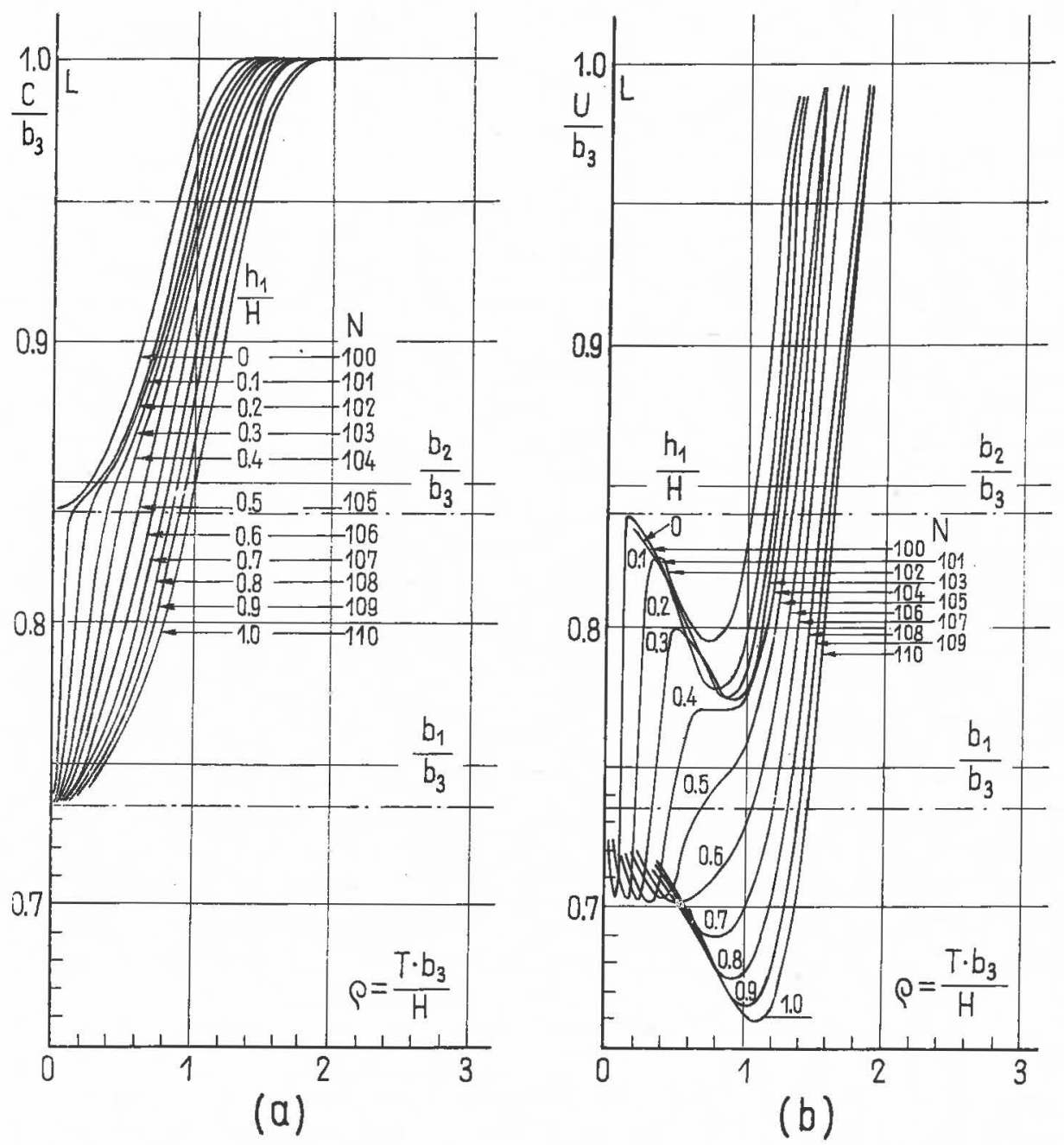

Fig. 6. Dispersion curves of the first overtone of Love waves for two layered model.

(a) phase velocity $\frac{C}{b_{3}}$; (b) group velocity $\frac{U}{b_{3}}$

greater than the corresponding velocity of the main tone, for Love waves, however, it is opposite.

In Fig. 8 the family of the phase and group velocity curves of the main tone of Rayleigh waves is presented for models (No. 200, 201, 202), which differ from each other by velocities in the first layer; in Fig. 9 the same is shown for models $(200,210,220)$, differing by the velocity of compressional waves and shear 


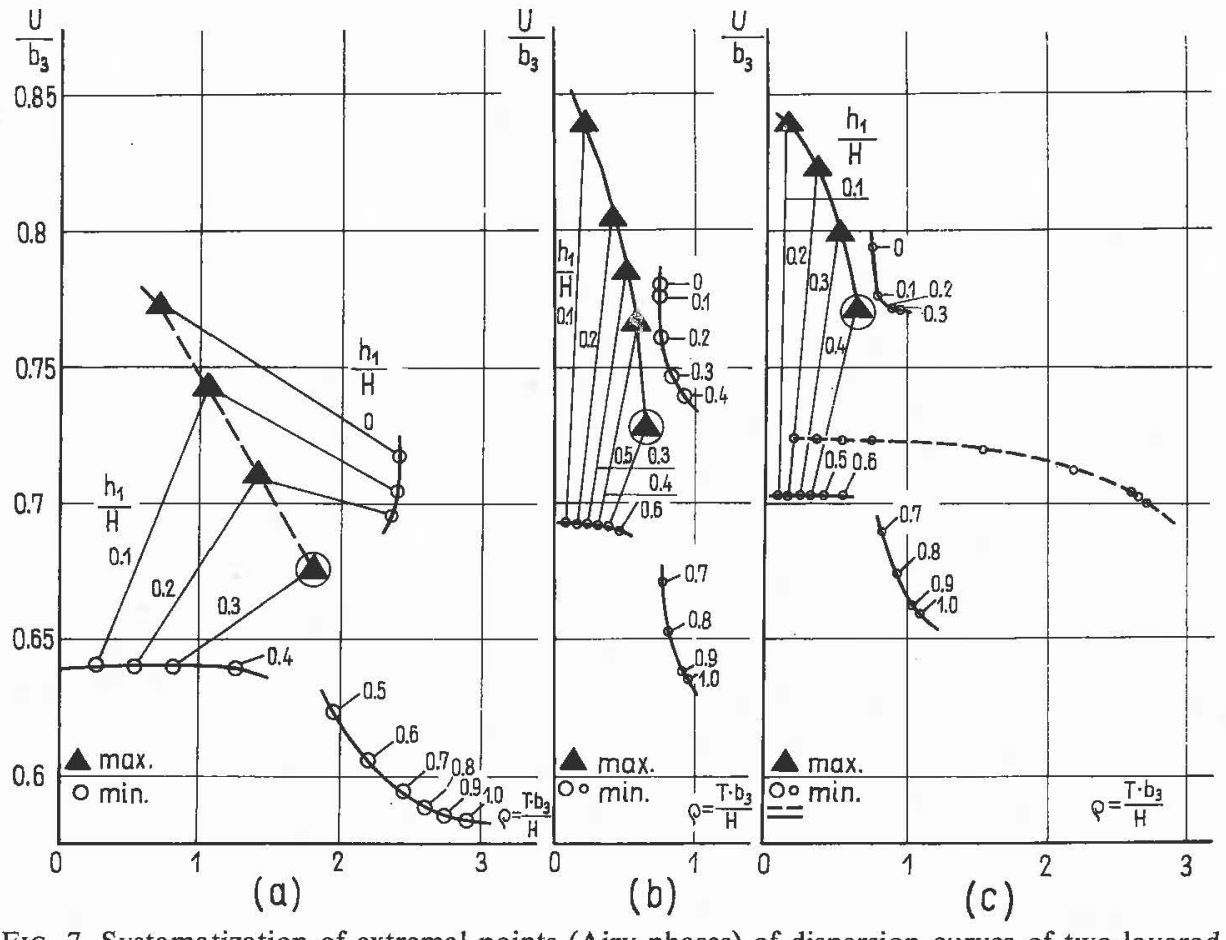

FIG. 7. Systematization of extremal points (Airy phases) of dispersion curves of two-layered model in dependence on $\frac{h_{1}}{H}$ - parameter. (a) main tone of Rayleigh wave; (b) first overtone of Rayleigh waves $\left(M_{2}\right)$; (c) main tone (dotted line) and the first overtone (continuous line) of Love wave

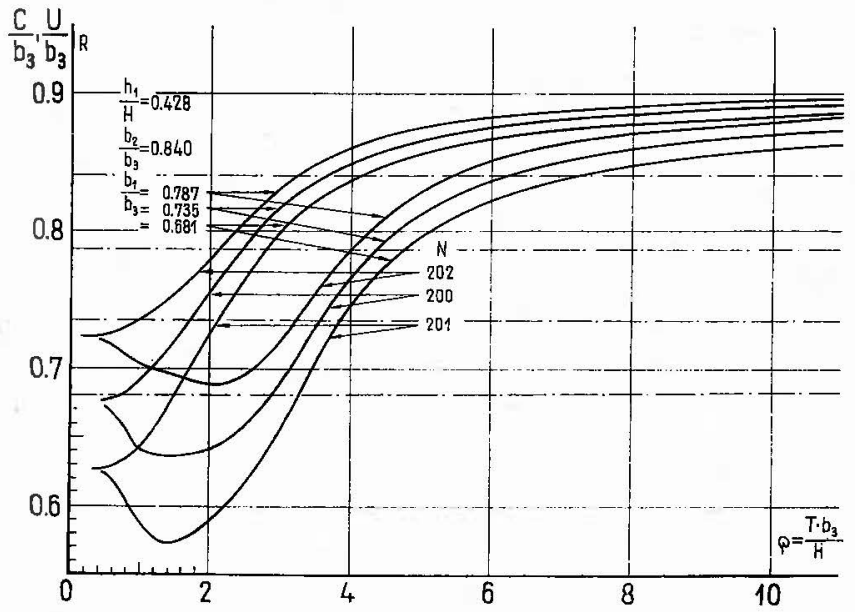

FIG. 8. Dispersion curves of phase $\frac{C}{b_{3}}$ and group $\frac{U}{b_{3}}$ velocities of the main tone of Rayleigh waves depending on parameters: $\frac{a_{1}}{b_{3}}$ and $\frac{b_{1}}{b_{3}}$ 


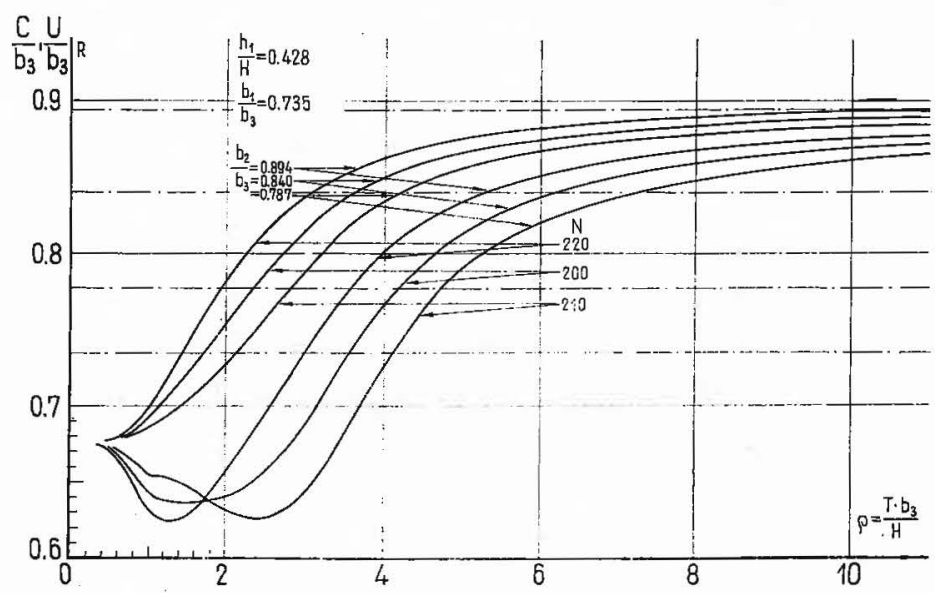

FIG. 9. Dispersion curves of phase $\frac{C}{b_{3}}$ and group $\frac{U}{b_{3}}$ velocities of the main tone of Rayleigh waves depending on parameters: $\frac{a_{2}}{b_{3}}$ and $\frac{b_{2}}{b_{3}}$

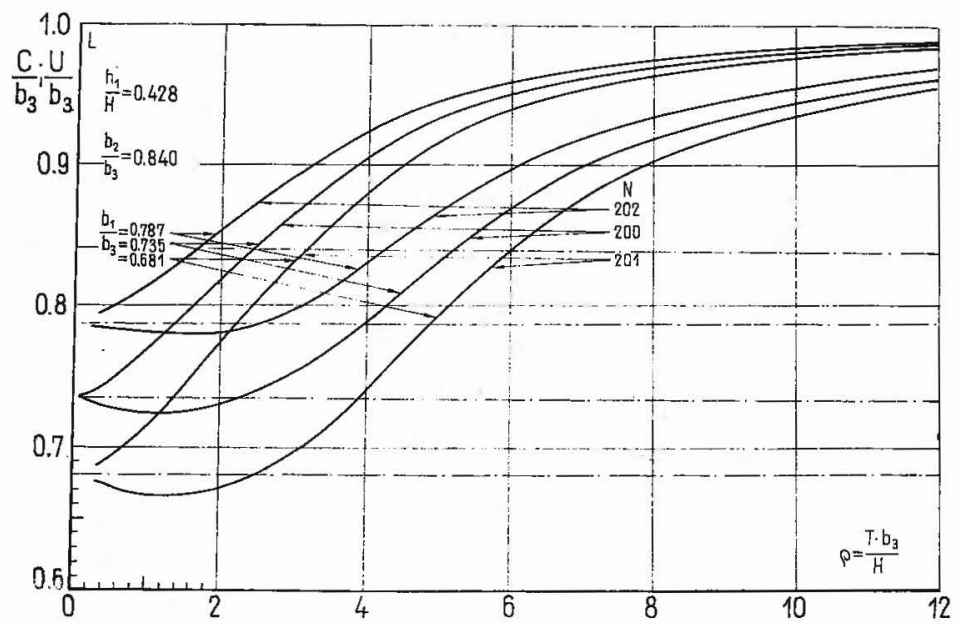

FIG. 10. Dispersion curves of phase $\frac{C}{b_{3}}$ and group $\frac{U}{b_{3}}$ velocities of the main tone of Love waves depending on parameters: $\frac{a_{1}}{b_{3}}$ and $\frac{b_{1}}{b_{3}}$

waves in the second layer. The comparison shows, that at $\rho>3$ the variation of phase velocity is approximately proportional to the variation of relative velocity of shear wave in layer. 


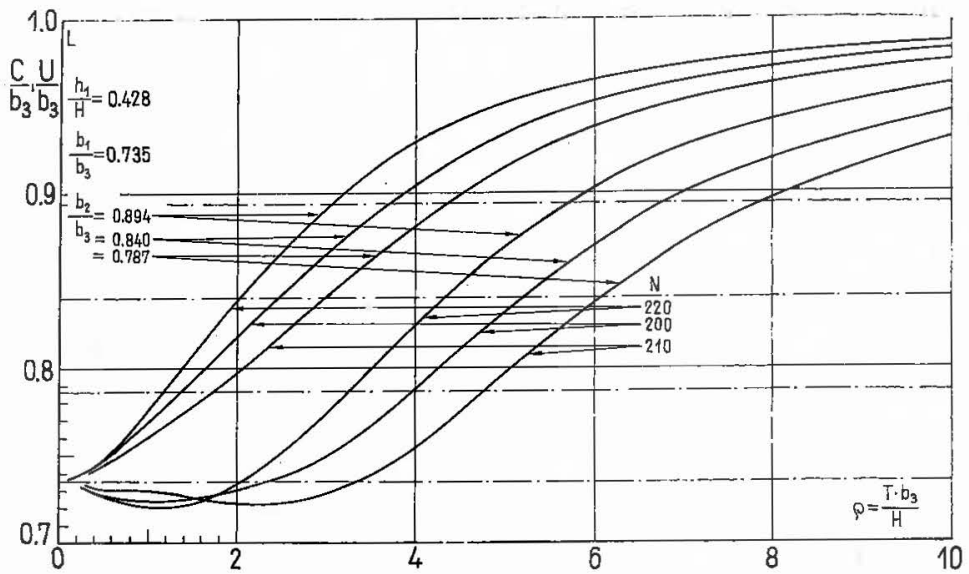

FIG. 11. Dispersion curves of phase $\frac{C}{b_{3}}$ and group $\frac{U}{b_{3}}$ velocities of the main tone of Love waves depending on parameters: $\frac{a_{2}}{b_{3}}$ and $\frac{b_{2}}{b_{3}}$

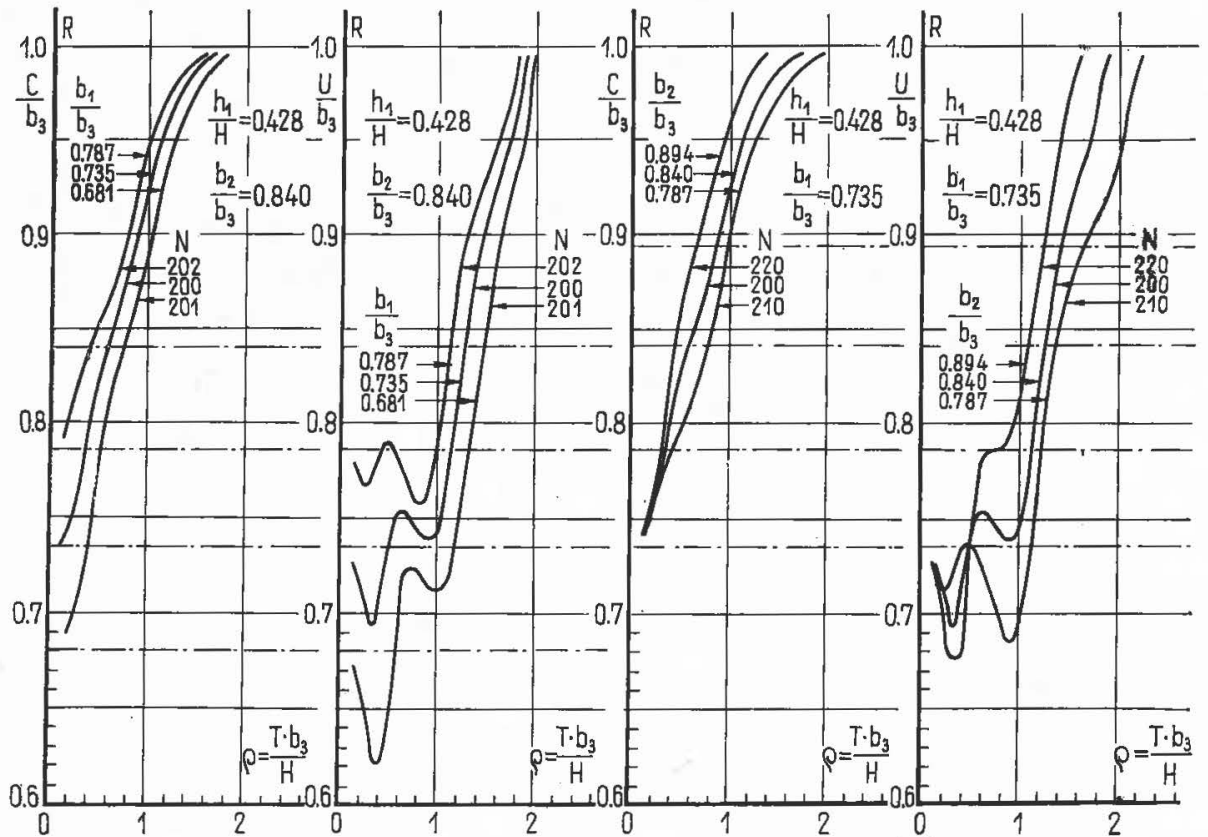

(a)

(b)

FIG. 12. Dispersion curves of phase $\frac{C}{b_{3}}$ and group $\frac{U}{b_{3}}$ velocities of the first overtone of Rayleigh waves: (a) depending on parameters $\frac{a_{1}}{b_{3}}$ and $\frac{b_{1}}{b_{3}}$; (b) depending on parameters $\frac{a_{2}}{b_{3}}$ and $\frac{b_{2}}{b_{3}}$ 
For the group velocities curves the considerable influence of parameter $\frac{b_{1}}{b_{3}}$ on the value of velocity in Airy phase is clearly seen. The displacement period is small. On the other hand, the Airy phase considerably displaces the variation of relative velocity $\frac{b_{2}}{b_{3}}$ in the second layer along the period axis.

Figures 10 and 11 are the same as Figs 8 and 9 but for Love wave.

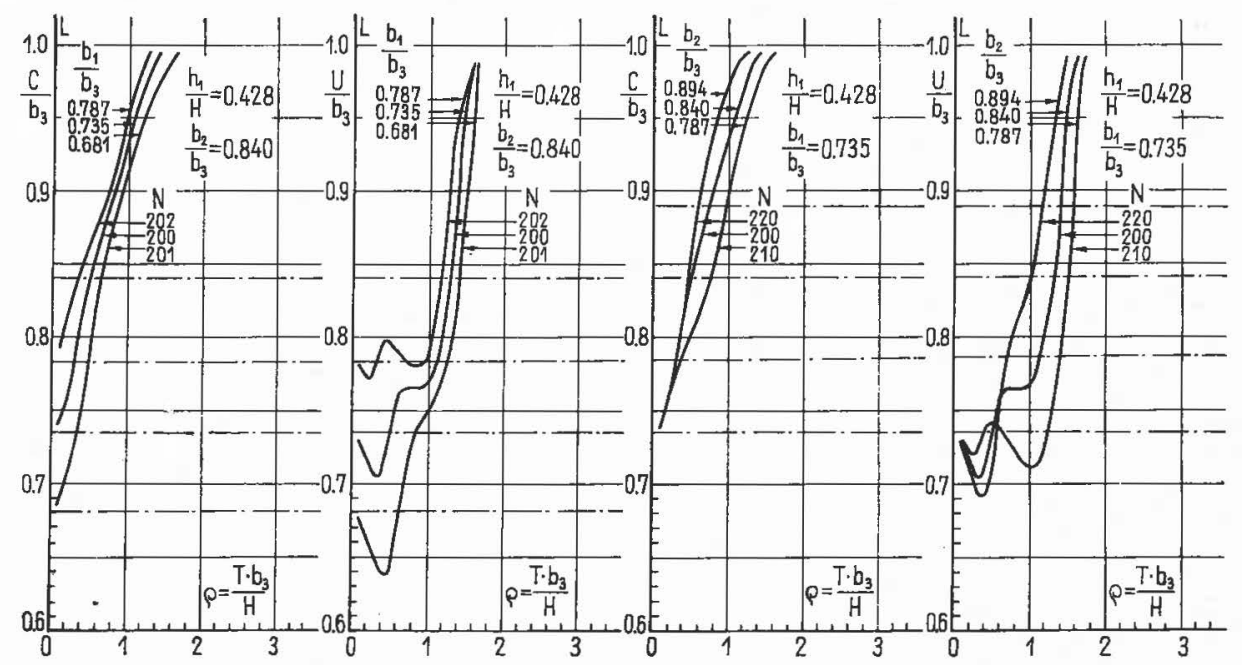

(a)

(b)

FIG. 13. Dispersion curves of phase $\frac{C}{b_{3}}$ and group $\frac{U}{b_{3}}$ velocities of the first overtone of Love waves: (a) depending on parameters $\frac{a_{1}}{b_{3}}$ and $\frac{b_{1}}{b_{3}}$; (b) depending on parameters $\frac{a_{2}}{b_{3}}$ and $\frac{b_{2}}{b_{3}}$

Figures $12 \mathrm{a}$ and $12 \mathrm{~b}$ - curves of phase and group velocities of the first overtone $M_{2}$ of Rayleigh waves at the same parameters, as 8 and 9 the same are seen on Figs $13 \mathrm{a}$ and $13 \mathrm{~b}$ but for Love wave.

Figures 14 and 15 correspond to two-layered earth with the sedimentary layer. Its thickness $\frac{h_{0}}{H}$ is a parameter for Rayleigh and_Love waves.

In Fig. 16 the families of the curves of phase and group velocities of the first overtone of Rayleigh and Love waves are presented.

Figures 14, 15 and 16 represent three models, one of them is two-layered $\left(\frac{h_{0}}{H}=0\right)$, with the ratio of layers $\frac{h_{1}}{H}=0.5$, but two others - three-layered 


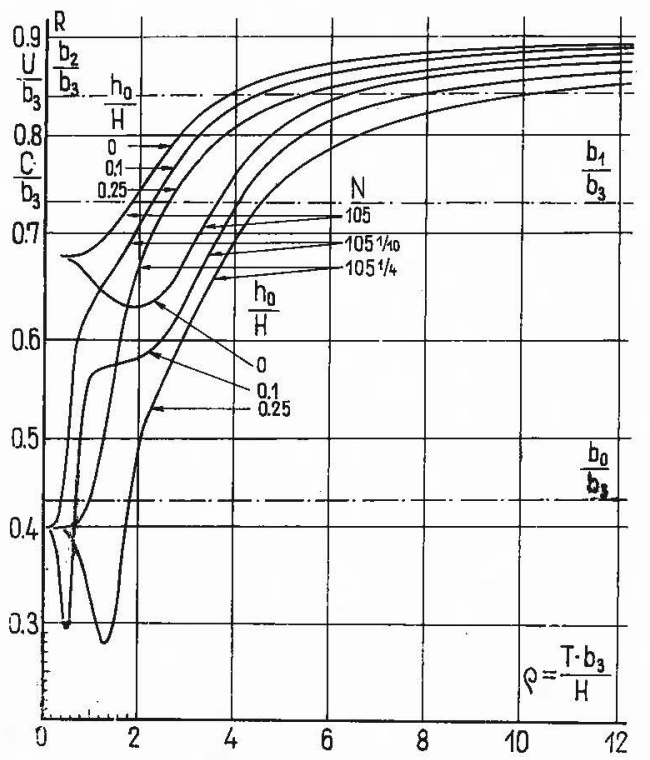

FIG. 14. Dispersion curves of phase $\frac{C}{b_{3}}$ and group $\frac{U}{b_{3}}$ velocities of the main tone of Rayleigh waves for two-layered earth's crust with sedimentary layer. $\frac{h_{0}}{H}-$ is parameter

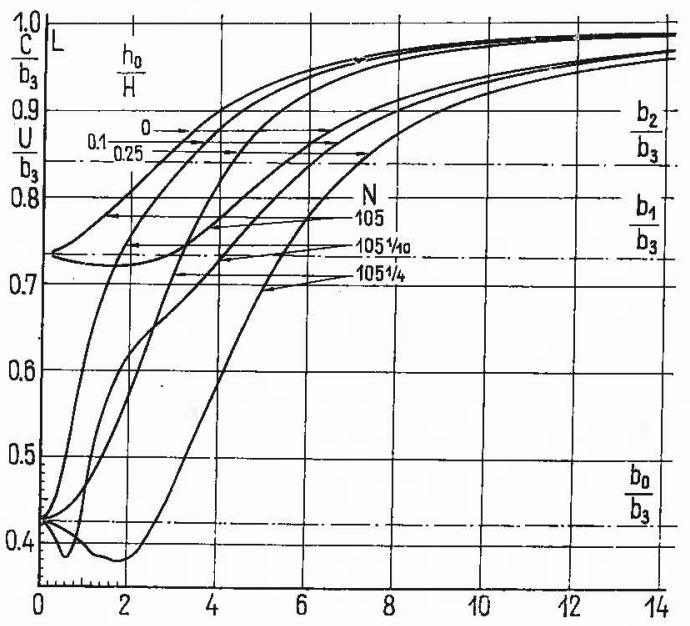

FIG. 15. Dispersion curves of phase $\frac{C}{b_{3}}$ and group $\frac{U}{b_{3}}$ velocities of the main tone of Love waves for two-layered earth's crust with sedimentary layer. $\frac{h_{0}}{H}-$ is parameter 


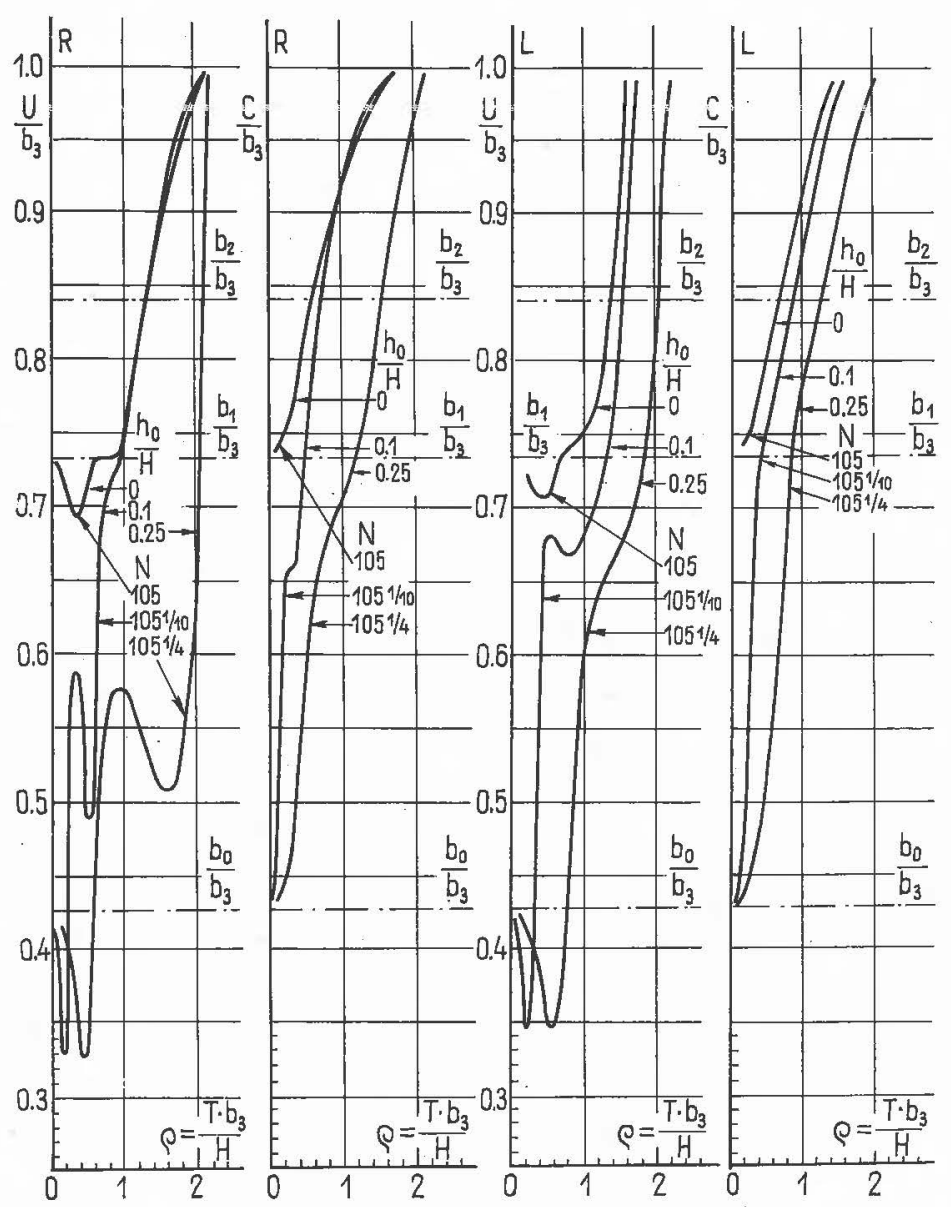

(a)

(b)

Frg. 16. Dispersion curves of phase $\frac{C}{b_{3}}$ and group $\frac{U}{b_{3}}$ velocities of the first overtone for two-layered earth's crust with sedimentary layer. (a) of Rayleigh waves; (b) of Love waves $\frac{h_{0}}{H}-$ is parameter

with sedimentary layers of relative thickness 0.1 and 0.25 correspondingly. The presence of sedimentary layer creates the interval of a sharp gradient of phase velocity at small $\rho$.

In Fig. 17 and 18 analogous curves are given, but for a purely granitic $\left(\frac{h_{0}+h_{1}}{H}=1.0\right)$ or basaltic $\left(\frac{h_{1}}{H}=0\right)$ earth crust and for both Rayleigh and Love fundamental modes. 


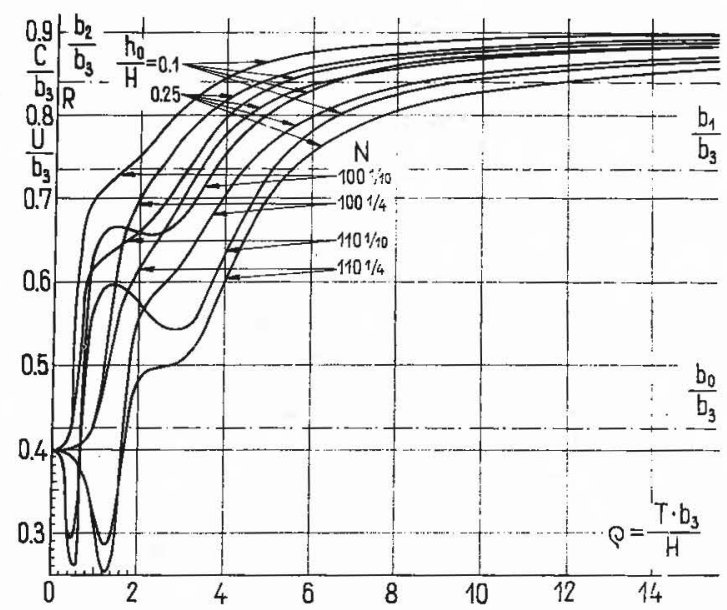

FIG. 17. Dispersion curves of phase $\frac{C}{b_{3}}$ and group $\frac{U}{b_{3}}$ velocities of the main tone of Rayleigh waves at the presence of sedimentary layer in the cases of one-layered basaltic $\left(\frac{h_{1}}{H}=0\right)$ or granitic $\left(\frac{h_{0}+h_{1}}{H}=1.0\right)$ earth's crust. $\frac{h_{0}}{H}$ - thickness of the sedimentary layer is varying

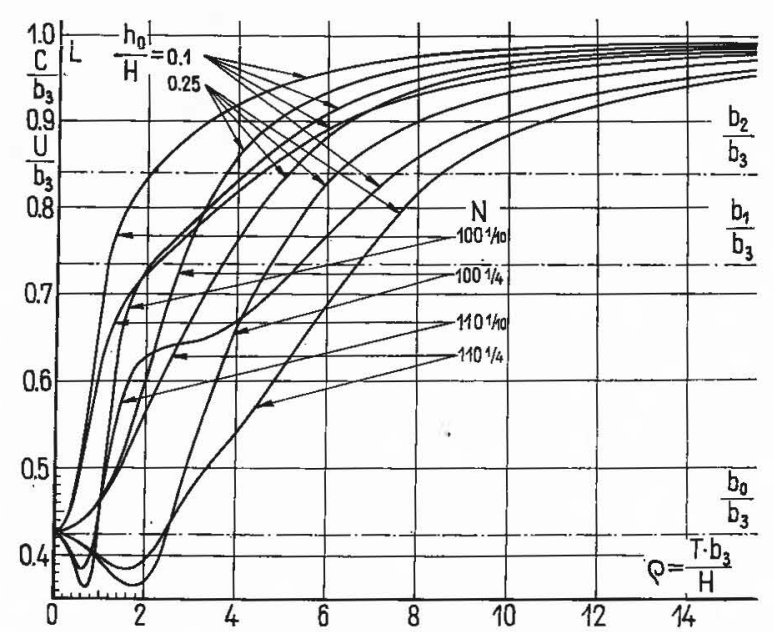

FIG. 18. Dispersion curves of phase $\frac{C}{b_{3}}$ and group $\frac{U}{b_{3}}$ velocities of the main tone of Love waves at the presence of sedimentary layer in the cases of one-layered basaltic $\left(\frac{h_{1}}{H}=0\right)$ or granitic $\left(\frac{h_{0}+h_{1}}{H}=1.0\right)$ earth's crust. $\frac{h_{0}}{H}-$ thickness of the sedimentary layer is varying 
In Fig. 19a the phase and group velocities of the first overtone of Rayleigh waves are given and on Fig. $19 \mathrm{~b}$ - the same for Love waves.

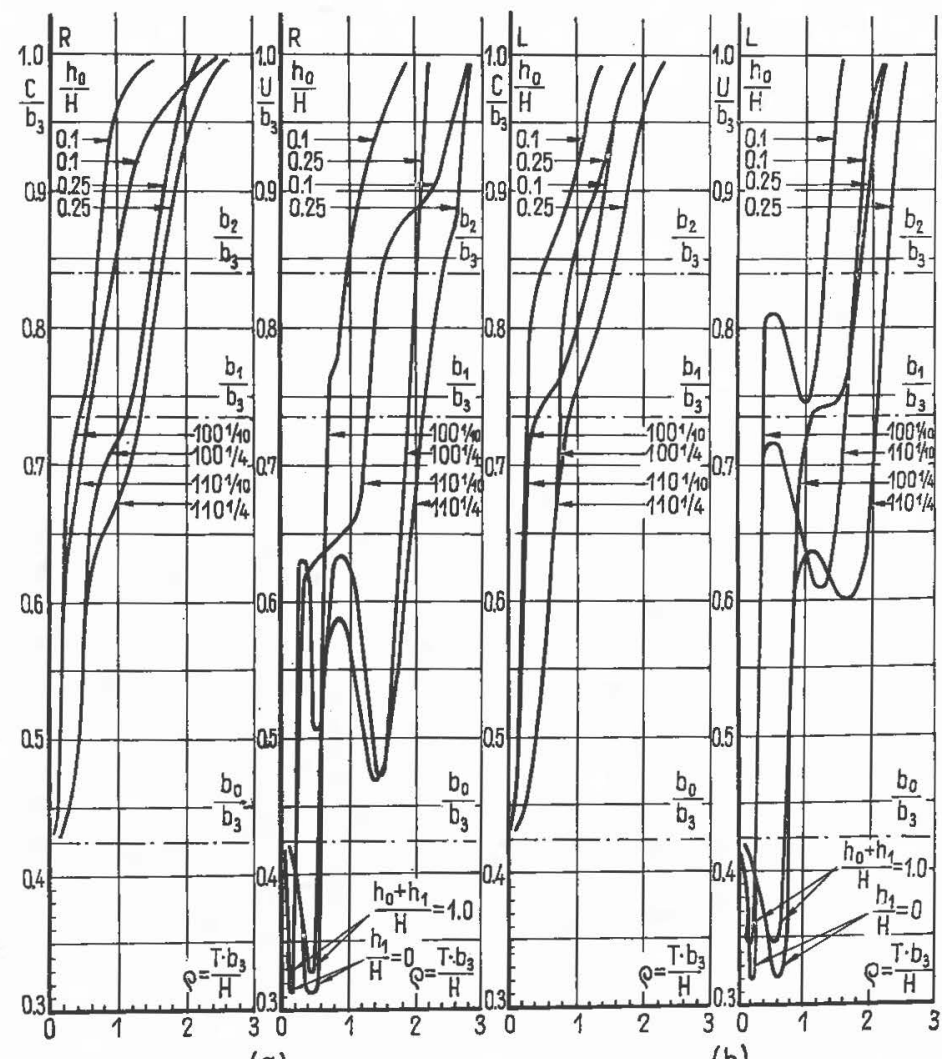

(a)

(b)

FIG. 19. Dispersion curves of phase $\frac{C}{b_{3}}$ and group $\frac{U}{b_{3}}$ velocities of the first overtone at the presence of sedimentary layer in the cases of one-layered basaltic $\left(\frac{h_{1}}{H}=0\right)$ or granitic $\left(\frac{h_{0}+h_{1}}{H}=1.0\right)$ earth's crust. (a) of Rayleigh waves; (b) of Love waves. $\frac{h_{0}}{H}-$ thickness of the sedimentary layer is varying

\section{REFERENCES}

[1] KeIlis-Borok: Interferential surface waves. Trudi Inst. Earth Phys. Acad. Sci. USSR M. (1960)

[2] Litvinenko, I. V. and Nekrasova, K. A.: Peculiarities of the deep seismic sounding on the Baltic shield. "Deep Seismic sounding of the earth crust of the USSR". Sbornik dokladov, p. 187, Gostoptehizdat, L. (1962) 
[3] Egorkin, A. V.: The earth crust structure on south-east of the Russian Platform, see [2] p. 145

[4] Godin, YU. A. and Egorkrn, A. V.: The earth crust structure on south-east of the Russian Platform. Dokl. AN SSSR, 1355 (1960)

[5] Pomeranzeva, I. V.: The results of the works according to investigation of the structure of the earth crust crystalline strata in some regions of south-east of the Russian Platform. Applied geophysics, issue 31 (1961)

[6] Molotova, L. V. and Vasiljev, Yu. I.: On the value of the ratio of the velocities of longitudinal and transverse waves in rock 11. Izv. AN SSSR, ser. geophys. 8 (1960)

[7] Fotiadr, E. E.: Geological structure of the Russian Platform according to the data of the regional geophysical investigations and guide drilling. Gostoptehizdat, issue IV (1958)

[8] JEFFreys, H.: The effect of Love on Heterogenity. MNRAS, geophys. suppl. 22 101$111(1928)$

[9] Glasko, V. B., Savarensky, E. F. and Shechkov, B. N.: Data about phase and group velocities of surface seismic waves. Izv. AN SSSR, ser. geophys., 10 1486-93 (1963)

[10] Tiknonov, A. N.: Method of calculation of electromagnetic fields, disturbed by variable current in layered media. Izv. AN SSSR, ser. geophys., 245-251 (1956)

[11] Haskell, N. A.: The dispersion of surface waves on multilayered media. Bull. Seism. Soc. of Am. 431 17-34 (1953) 


\author{
RAYLEIGH LONG-PERIOD WAVES \\ OF ALASKA EARTHQUAKE ON MARCH 28, 1964 \\ E. F. Sayarensky, O. E. Starovort and S. A. Fedorov
}

(Moscow, USSR)

The dispersion of the group and phase velocities of longperiod-surface waves $(T>75 \mathrm{sec})$ depends on velocity distribution of body waves and density in the earth's mantle. This dependence is determined by the comparison of observed and theoretically calculated dispersion of velocities [1-5].

Long-period surface waves ( $T 100 \mathrm{sec}$ ) occur only at strong earthquakes $(M \geq 8)$, which have large ruptures in the hypocentre. The value dependence of the rupture zone on magnitude is shown, for instance, in [6, 7]. The rupture process of continuity occurs with velocity, like the transversal waves velocity $[1,8]$. With increase of the rupture process the long-period part of the emission spectrum becomes relatively intensive $[8,9]$. This explains long-period oscillations present in the surface waves of strong earthquakes.

During the earthquakes on Alaska* on March 28, 1964 at $M=8.6$ after the main shock and March 30 more than 50 aftershocks had occurred with epicentres in south-south-western direction of the main epicentre (according to USCGS data) (Fig. 1). This also testifies the large dimension of the hypocentral region.

At Alaska earthquake strong Rayleigh long-period waves had been observed.

In the present paper the dispersion of the group velocities of Rayleigh waves is analyzed. These waves have been recorded by the central seismic station "Moskva".

Because of the great intensity of oscillations it was possible to reproduce the complex seismographic record (Fig. 2) of earthquake by successive articulation of seismograph records with different frequency characteristics (Fig. 3). Rayleigh waves are revealed, which repeatedly had run round the Globe. We marked the surface. waves groups from $R_{1}$ to $R_{10}$, where by $R_{1}$ that wave is designated, which came along the shortest way; $R_{3}$ - is $W_{2}$ wave which came from the same side as $R_{1}$; it covered the way of $40000+\Delta_{1} \mathrm{~km}$. It is analogous for other odd waves. The waves with even indexes had run the Globe along the arc of the big circle, but in opposite direction. The wave propagation was through the earth poles.

We have found a sharp difference of amplitudes of the waves, opposite to the direction of the epicentre (Table 1).

${ }^{*} \phi=60.9^{\circ} ; \lambda=147.8^{\circ} ; 28$ March, $1964,03 \mathrm{~h} 36 \mathrm{~m} 14 ; M=8.6$. 
TABLE 1

\begin{tabular}{l|c|c|c}
\hline & \multirow{2}{|c|}{$\begin{array}{c}\text { Amplitude (mm) } \\
\text { Wave }\end{array}$} & $\begin{array}{c}|c| \\
\text { Period: }\end{array}$ \\
& & $T=220 \mathrm{sec}$ & $T=150 \mathrm{sec}$ \\
\hline$R_{2}$ & 32936 & - & 10 \\
$R_{3}$ & 47080 & 2 & 0.5 \\
$R_{4}$ & 72944 & 6 & 1 \\
$R_{5}$ & 87088 & 0.7 & - \\
$R_{6}$ & 112952 & 2 & -
\end{tabular}

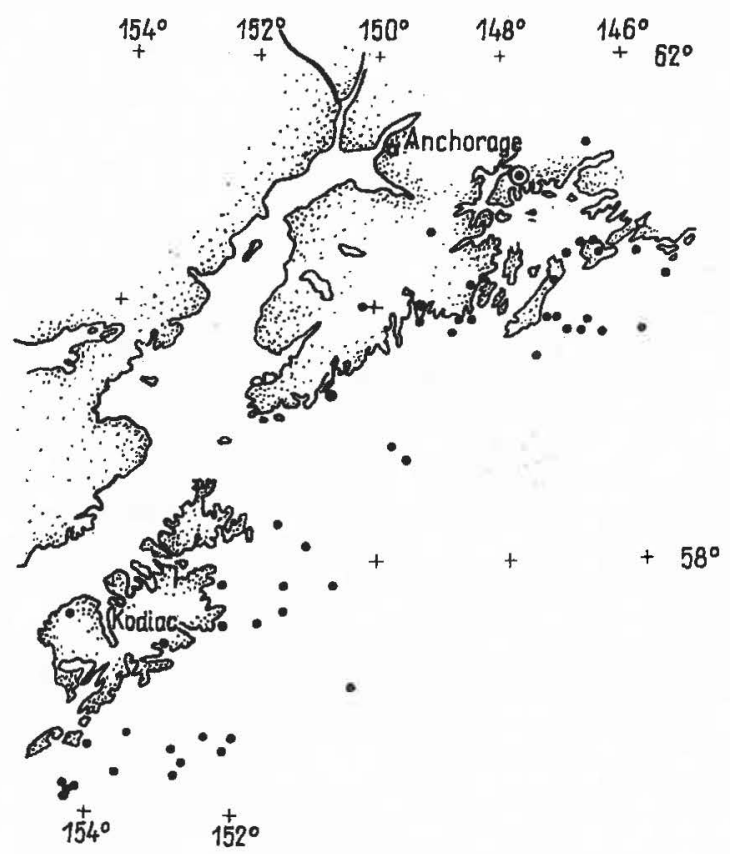

FIG. 1. Aftershock epicentres of Alaska earthquake on March 28, 1964

All the even waves (coming to Moscow from the south) are the most intensive. Such difference in intensity testifies an asymmetrical radiation of energy by a focus. It is connected with orientation of a rupture formation.

It is shown [8], that the amplitudes in the direction of the rupture movement are sometimes greater than in the opposite one. On Alaska the rupture was stretching to the south and it may explain the great intensity of Rayleigh even waves. The position of aftershock epicentres proves also this supposition. Earlier analogous connection had also been marked [10]. 


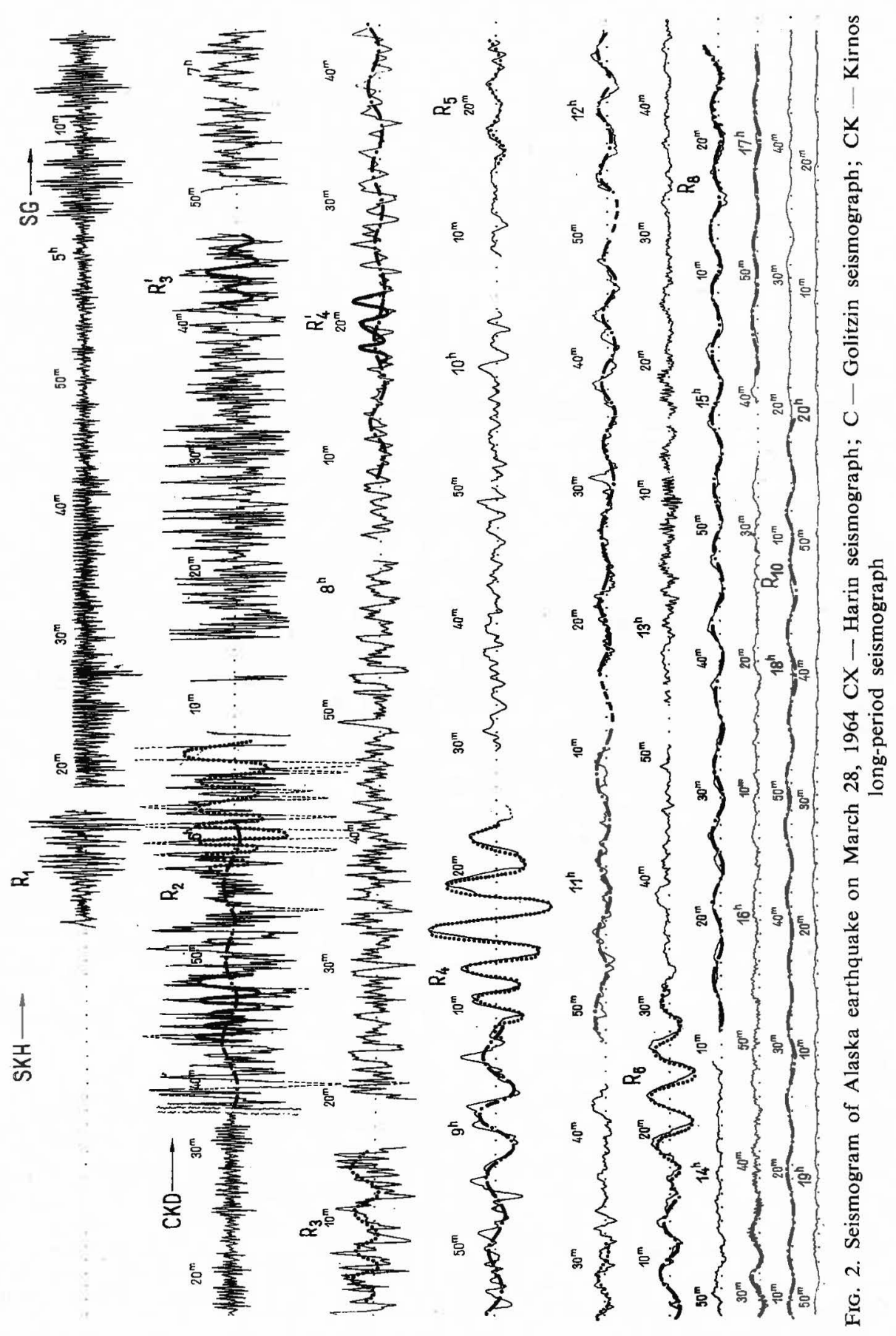


Surface waves group velocity had been determined by us with the help of the standard method.

As the result of seismograms evaluation three branches of dispersion curve of the Rayleigh waves group velocity have been revealed (Fig. 4). The initial branch is characterized by a normal dispersion at the periods from 25 to $32 \mathrm{sec}$ at velocity variation from 3.25 to $3.52 \mathrm{~km} / \mathrm{sec}$ (wave $R_{1}$ ).

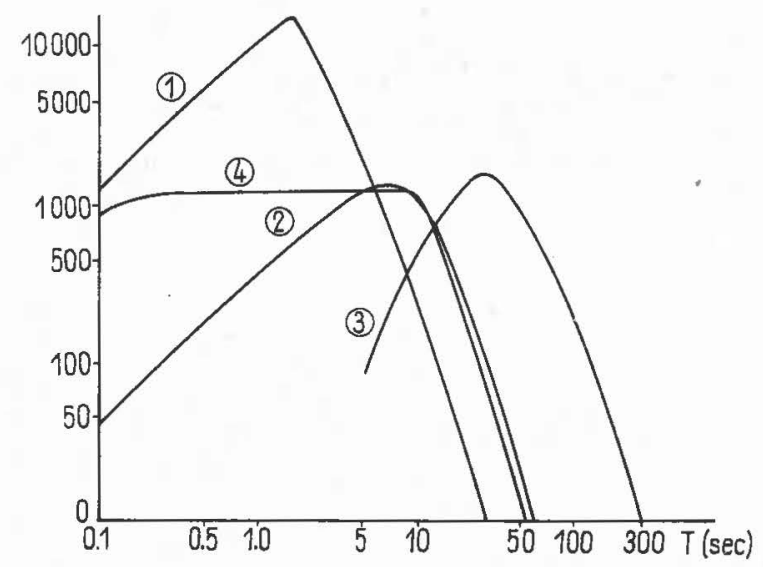

FIG. 3. Frequency characteristics of "Moskva" seismic station seismographs. 1. Harin seismograph $\left(T_{1}=1.5 ; T_{2}=3.8\right), 2$. Golitzin seismograph $\left(T_{1}=9.5 ; T_{2}=9.5\right)$, 3. Kirnos long-period seismograph $\left(T=26, T_{2}=100\right), 4$. Kirnos typical seismograph $\left(T_{1}=12.5\right.$; $\left.T_{2}=1.2\right)$

The second branch of the curve has the anomalous dispersion at periods from 65 to $225 \mathrm{sec}$ and at velocities from 3.90 to $3.55 \mathrm{~km} / \mathrm{sec}$. The last are obtained by the waves $R_{2}, R_{3}, R_{4}, R_{5}, R_{6}$.

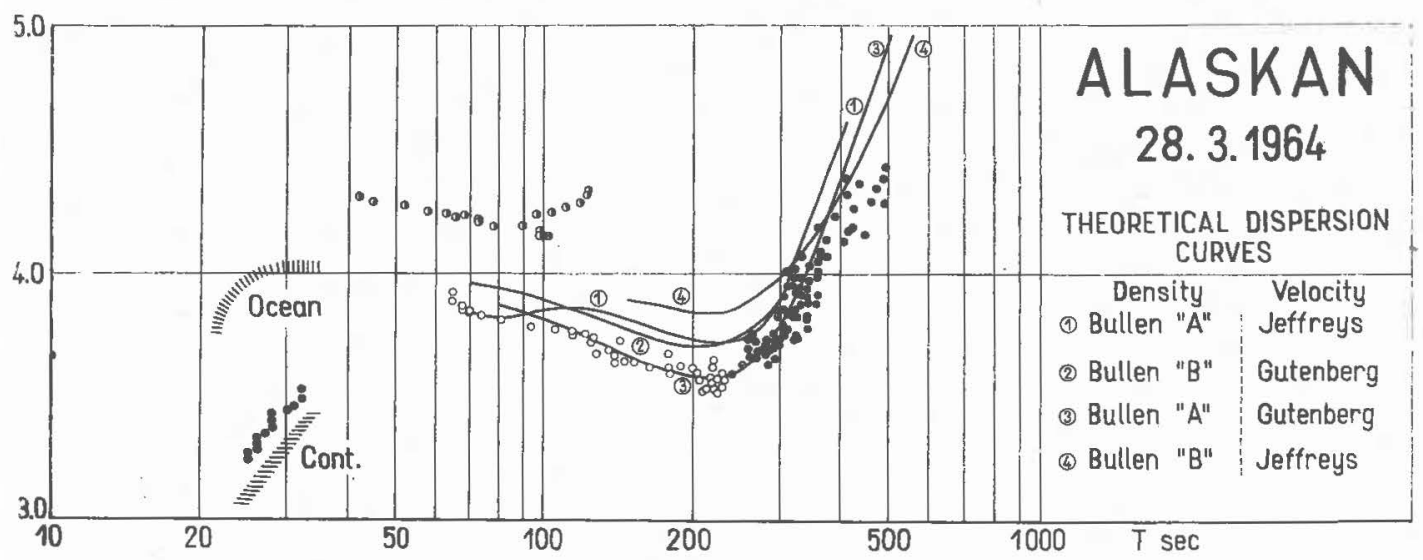

Fr G. 4. Dispersion curves of Rayleigh long-period waves 
The third branch is beginning at the period $T=225 \mathrm{sec}$ and is traced by us to $470 \mathrm{sec}$ at group velocity values from 3.55 to $4.35 \mathrm{~km} / \mathrm{sec}$. At $220-225 \mathrm{sec}$ period a distinct minimum of the group velocity is observed $(3.52-3.59 \mathrm{~km} / \mathrm{sec})$.

Before the groups $R_{1}(04 \mathrm{~h} 06 \mathrm{~m} \mathrm{50s})$ the wave with an apparent period of $60 \mathrm{sec}$ is noticeable. It has a great amplitude and impulsive character.

We have found the surface waves corresponding to the long-period branch of the dispersion curve of the first transversal overtone (Figs 2, 4).

The data obtained by us are compared with theoretical dispersive curves, calculated with regard to the sphericity of the earth and gravity variation [4], (II). $T, 2,3$ curves (Fig. 4) to $T=300 \mathrm{sec}$ are taken from [4]. The curve 4 is constructed as a whole according to the data (II). The data of the velocity variations of the longitudinal and transversal waves and density with depth are given in Table 2.

TABLE 2

\begin{tabular}{c|l|l}
\hline & \multicolumn{2}{|c}{ Distribution of } \\
\hline Curve & velocities & density \\
\hline 1 & Jeffreys & Bullen "A" \\
2 & Gutenberg & Bullen "B" \\
3 & Gutenberg & Bullen "A" \\
4 & Jeffreys & Bullen "B"
\end{tabular}

The velocities according to Jeffreys and Gutenberg have a sharp decrease at the depth of $400 \mathrm{~km}$ (so called " $\mathrm{C}$ " layer of the earth mantle). The main difference between Jeffreys', and Gutenberg's models is that in the last one there is a layer of low velocity at the depth of the order of $150 \mathrm{~km}$ (Fig. 5).

Density variation with a depth in Bullen's " $A$ " model at $400 \mathrm{~km}$ occurs linearly. At great depths the gradient sharply increases. In " $B$ " Bullen model, beginning from $100 \mathrm{~km}$ the density gradient is less, than in model " $\mathrm{A}$ ". Down to a depth of $600 \mathrm{~km}$ the density of " $\mathrm{B}$ " model is greater, than of " $\mathrm{A}$ ".

All these peculiarities of velocity and density distribution influence dispersion curves. The great gradient of velocity at a depth exceeding $400 \mathrm{~km}$ causes at $T=225 \mathrm{sec}$ a minimum of all the dispersion curves. Little densities of the model at $600 \mathrm{~km}$ lead to the decrease of group velocity (see curve 4 at $T=350$ sec on Fig. 4).

The best agreement with observations at $300 \mathrm{sec}$ gives curve 3 (the velocity according to Gutenberg, but the density according to "A" Bullen). At a period greater than $300 \mathrm{sec}$ not one curve gives satisfactory agreement with observations. Therefore the existing ideas about velocity and density section demand the most accurate definition. In particular, it seems to us necessary to decrease the density values at depths more than $600 \mathrm{~km}$ and in some way to modificate velocity distribution and maybe to include the second layer of low velocity at a depth exceeding $500 \mathrm{~km}$. 


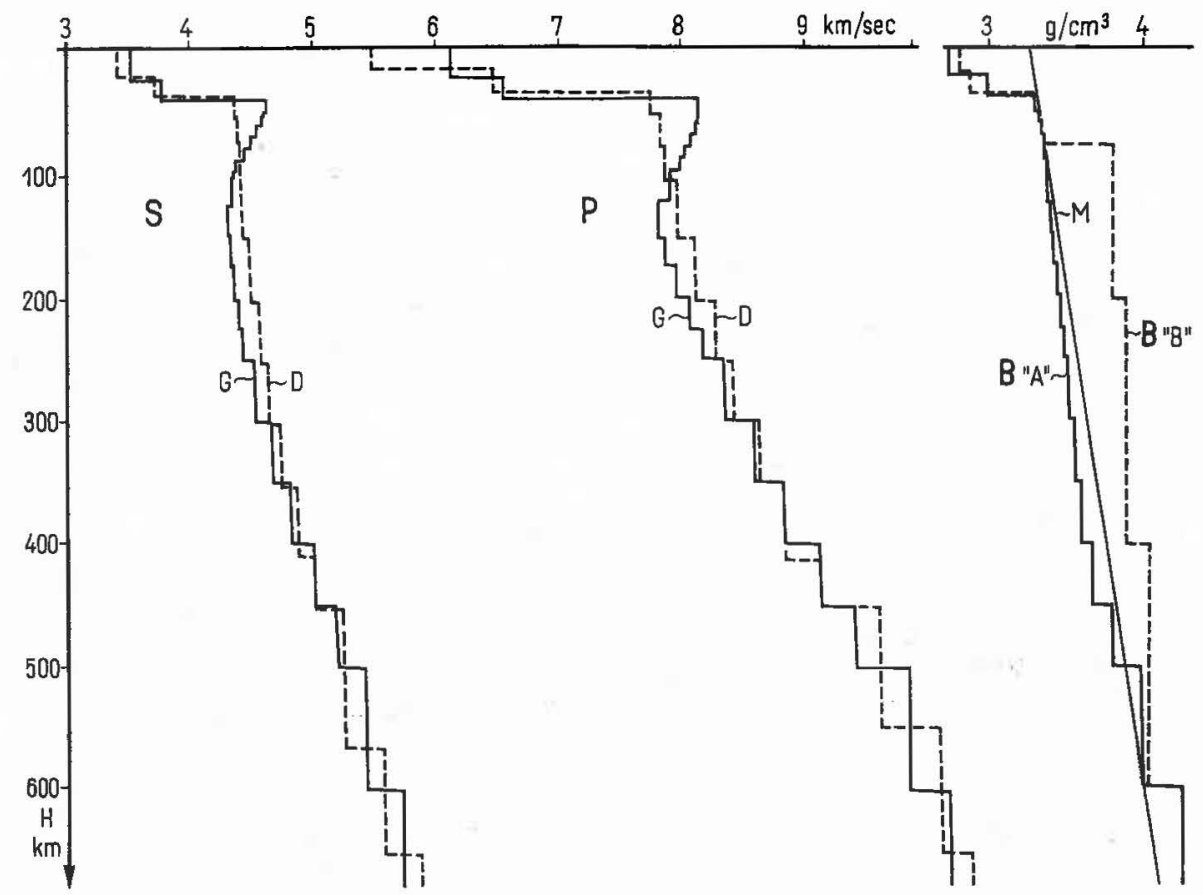

Fig. 5. Curves of velocity and density distribution inside the earth. $S$ - according to Gutenberg, $P$ - according to Jeffreys, " $B_{\mathrm{A}}$ ", " $\boldsymbol{B}_{\mathrm{B}}$ " - according to Bullen, $\boldsymbol{M}$ - according to Molodensky

\section{REFERENCES}

[1] Brune, J., Benioff, H. and Ewing, M.: Long-period surface waves from the Chilean earthquake of May 22,1960, recorded on linear strain seismographs. Journ. Geoph. Res. 669 (1961)

[2] Dorman, J., EwING, M. and Oliver, J.: Study of shear velocity distribution in the upper mantle Rayleigh waves. Bull. Seism. Soc. of Am. 501 (1960)

[3] NAFE, J. and Brune, J.: Observations of phase velocity for Rayleigh waves in the period range 190 to $400 \mathrm{sec}$. Bull. Seism. Soc. of Am. 502 (1960)

[4] BoLt, B. and Dorman, J.: Phase and group velocities of Rayleigh waves in a spherical, gravitating earth. Journ. Geoph. Res. 669 (1961)

[5] Savarensky, E. F., Popov, I. I. and Lazareva, A. P. - Саваренский, Е. Ф., Попов, И. И. и ЛАЗАРЕВА, А. Н.: Наблюдения длиннопериодных воли чилиского землетрясения, 1960. Изв. АН СССР, сер. геоф. 8 (1961)

[6] BercKhemer, H.: Die Ausdehnung der Bruchfläche im Erdbebenherd und ihr Einfluß auf das seismische Wellenspektrum. Gerl. Beitr. zur Geoph. 711 (1962)

[7] KaSaKhaRA, K. - KaCAXAPA, K: О природе сейсмических источников. Сб. "Слебные землетрясеная", М., (1961)

[8] Ben Menahem, A.: Radiation of seismic surface waves from finite moving sources. Bull. Seism. Soc. of Am. 512 (1961) 
[9] Pekeris, C., Alterman, Z. and Abramovichi, E.: Propagation of an SH-torque in a layered solid. Bull. Seism. Soc. of Am. 531 (1963)

[10] Benioff, H.: Mechanism and strain characteristics of the White Wolf fault as indicated by the aftershock sequence. Calif. Dept. Resources, Div. Mines, Bull. 171199 (1955)

[11] Alterman, Z., Jarosch, H. and Pekeris, C.: Propagation of Rayleigh waves in the Earth. Geoph. Journ. Royal Astron. Soc. 4 219-241 (1961) 


\title{
THE INFLUENCE OF THE OBSTACLES ON THE PARAMETERS OF SEISMIC WAVES
}

\author{
A. P. Sinitsyn \\ (Moscow, USSR)
}

\section{Introduction}

The general methods for designing the aseismic high structures was worked out by Savarensky, Medvedev, Nazarov [1, 2, 3] and others. The stability of the masonry dams was considered by the author in $[4,5]$ and there it was obtained that the subgrade reactions under dam foot depend on parameters of the travelling surface wave. The interaction between the surface wave and the structure causes the change of subgrade reactions that is why the stresses in the dam will increase and the safety factor of structure will decrease.

\section{Steady-state Vibrations}

The vibrations of a rigid structure supported by an elastic half space were investigated. Let us assume that the half-space is under the action of the steadystate surface Raleigh wave. If the structure is symmetrical, placed about the top of the wave, it will have only vertical displacements. The subgrade reactions are functions of the time, these forces deform the surface of the half-space and maintain close contact between the structure and the subsoil.

The subgrade surface under the structure must be straight and on the boundary of the foundation the wavy half space can break away from the structure. The close contact between the foot of the foundation and half space will be restored by using a self-balanced forces system. These supplementary reactions smooth out the surface of the half space and a close contact at full lenght of the foundation will be restored. Therefore by the steady-state vibrations at a fixed moment of time the diagram of the reactions will have an increasing value on the boundary of the foundation.

The detailed investigations of this problem made in [6] permitted to make the following statement: if the length of seismic wave is twice the length of the structure foundation, then the sine diagram of the reactions is reduced to zero at the end of the foundation and the supplementary diagram must be of two signes. The values of supplementary diagram decreases as the length of seismic wave increases on account of the small length of the wavy part of the subgrade, which must be smoothed in this case. The vertical component of the inertial forces will give rise to the vertical displacements of the structure which are proportional to the mass and the square of the natural vertical frequency of the structure. The horizontal component of the inertial forces are proportional to the square 
of the natural horizontal frequency of the structure. The concentration of the subgrade reactions on the end of the foundation sometimes exert a small influence on wave propagation and for approximate calculation the sine diagram can be taken for the subgrade reactions.

\section{The Travelling Wave}

When the travelling wave reaches the structure and penetrates under the foundation the investigation is more complicate and must be done by using the step by step method. If the wave penetrates under the foundation on a quarter of its length and the structure is removed from subgrade surface, the system of the reaction forces must be applied on this surface. The surface of the half space

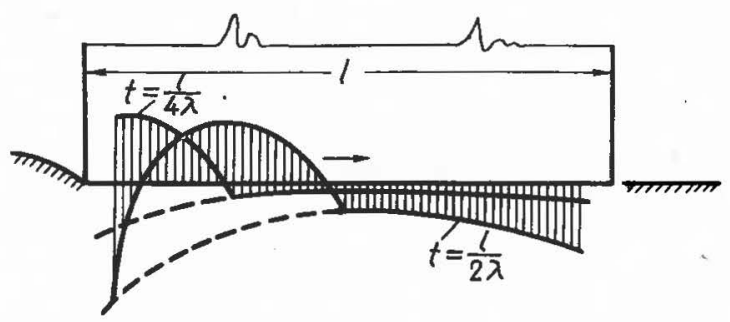

FIG. 1. Diagram of subsoil reactions due to travelling wave

will have the displacements due to this reactions, but the structure is rigid and the surface of the subgrade must be straight that is why in this case also a supplementary system of the subgrade-reactions occurs. The supplementary forces system can be replaced by a main force and a main moment, and the diagram of the reactions acting on half space surface must have a negative part ahead at the wave front, and a concentration of the reactions behind the wave. If the wave penetrated under the foundation on a half of its length the vertical component of the reactions will be as shown in Fig. 1. The main reaction force which is acting between the structure and the subgrade changes its value and the application point, therefore, in this case the travelling load must be taken into account. The suddenly applied travellingload as opposed to the steady state case will give rise not only to six waves $\left(P_{v}, S_{v}, R_{v}, P_{H}, S_{H}, R_{H}\right)$ but still to three further waves $\left(P_{\mu}, S_{\mu}, R_{\mu}\right)$ which arise from the suddenly applied main moment.

\section{Considerations on Plastic Deformations}

The plastic regions in the structures were developed and the changes in the rigidity of the system can arise by action of a strong seismic wave, therefore the subgrade reactions diagram shall be modified. It is interesting to note that the ultimate failure load can be determinated by the limiting values of the subsoil 
strength and the maximum permissible settlement. The appearance of the first plastic area in a structure gives rise to a greatly increased settlement of the rigid structure, whereas there was no such increased settlement of a flexible structure. An elastic stratum whose thickness $h_{0}$ inclined between the structure and the half space reduces the reactions concentration, but the settlements of the structure increase. There is a non linear relationship between the reactions of the subsoil

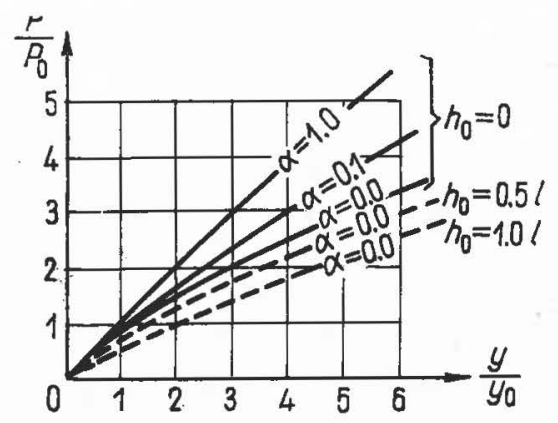

FIG. 2. Relationship between failure load and settlement of structure

and the value of seismic load. This relationship depends on the structure stiffness and also on subgrade stiffness. The value of the limit failure load $P$ for the structure depends on the ultimate value of the subsoil settlement $y$, and this relationship in nondimension form is plotted in Fig. 2. There are $y_{0}$ the subsoil settlement and $\boldsymbol{P}_{\mathbf{0}}$ - limit load is due to the end of the elastic areas. The conclusion is that beyond the elastic limit the reactions and the settlements increase rapidly, but for a rigid structure the increase of the settlements occurs by almost constant limit load. The decrease of the structure rigidity or the increase of subsoil deformation modulus will cause the increase of the ultimate failure load.

\section{The Unsteady Vibrations}

The elastic system which consists of structure and subsoil has the one-sided bonds under the footing of the structure. These bonds will be broken by seismic wave on those parts of the foot where the subgrade reaction due to the dead load of structure is too small. The breaking of bonds cause changes in the rigidity of the system, the centre of stiffness and the centre of mass even symmetrical structures will not lie on a vertical line. The differential equations will be nonlinear or of variable coefficients. Under these conditions the unsteady-state vibrations may occur. They are studied on an easy model of the rigid structure placed on an elastic stratum. The detailed investigations of this problem are carried out in [7]. The dimensions of the unsteady vibrations areas depend on the relation between the natural frequencies of the structure and the parameters of the seismic wave. 
In Fig. 3 these areas are shown, where $C$ is the parameter which describes the dynamic property of the structure, the abscissa $\eta$ - depends on the seismic load and $\xi-$ is the parameter which includes the relationship between dead load and pay load.

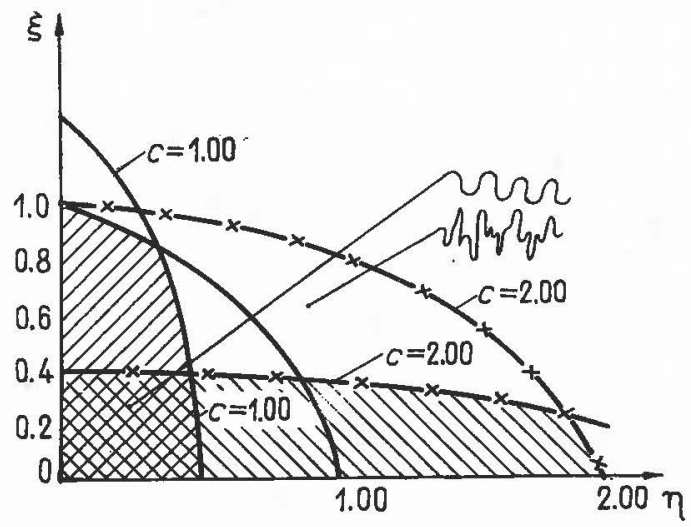

FrG. 3. Areas of unsteady vibrations

\section{Comparison with the Tests}

The vibrations of the structures were experimentally investigated in detail by S. V. Medvedev [2]. The beating by tests of a five story building was investigated.

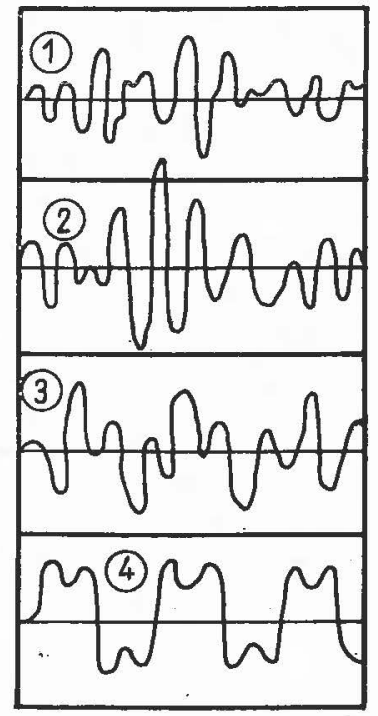

FIG. 4. Diagrams of displacements. 1 - recorded by [2] for five-story building, 2 - recorded by [2] for a masonry building, 3 - recorded by [8] for a system with one degree of freedom, 4 - recorded by [8] for the third subharmonic 
The horizontal displacements diagrams of the different stocks are shown in Fig. 4. These diagrams correspond to the nonlinear vibrations, and the beating there will be observed when the horizontal displacements of the subsoil ceased. The beatings occur by self vibrations of the building. These horizontal vibrations are caused by vertical displacements and rotations of the building. The diagrams recorded by $\mathbf{M}$. Dost and Ch. Atkinson [8] for the trilinear system with one degree of freedom are shown in Fig. 4.

The comparison of the diagrams shown in Fig. 4 confirms the preliminary assumption that the system including structure and subsoil may be a nonlinear system when the bonds on the foot will be partially broken, and the beatings, relative to the nonlinear systems, will occur. The described investigations show the great importance of this problem for the practical purposes. In this paper the difficult problem of the nonlinear vibrations for the system structure subsoil was solved by means of the approximate engineering methods.

\section{REFERENCES}

[1] SAVARENSKY, E. F. and KIRNos, D. P.: The elements of seismology and seismometry. 2nd ed. State Technical Theoretical Publishing Office, Moscow (1955)

[2] Medvedev, S. V.: The engineering seismology. State Constructional and Architectural Publishing Office, Moscow (1962)

[3] Nazarov, A. G.: A method of engineering analysis the seismic forces. The Armenian Academy of Sciences Publishing Office, Yerevan (1959)

[4] Sinitsyn, A. P.: To the problem of calculation the seismic loads to massive structures. The Information Bulletin of the Seismological Council by Academy of Sciences USSR 8 Moscow (1960)

[5] SinITSYN, A. P.: The influence of the travelling seismic wave on the massive structures. The Scientific Work of the Institute of the Physics of the Earth 17 Moscow (1961)

[6] Sinitsyn, A. P.: The beam on two layered half space beyond the elastic limit. Investigations on Structure Theory $X$ Moscow (1961)

[7] Sinitsyn, A. P.: On the causes of beating arises by structure vibrations. The Scientific Work of the Institute of the Physics of the Earth 22 Moscow (1963)

[8] Dost, M. and AtKinson, C. P.: Electronic computer simulation of a system with a trilinear restoring function. Proceedings of the third U.S. Nat. Congress of Applied Mechanics, ASME (1958) 


\title{
DEEP SEISMIC RESEARCH IN UKRAINE
}

\author{
V. B. Sollogub, N. I. Pavlenkova and A. V. Chekunov
}

(Kiev, USSR)

Almost all varieties of the primary geotectonical elements are present in Ukraine and the water area of the Black Sea. They are: Precambrian Shield, young Epihercynian platform and a series of Mesozoic depressions within it, Alpine folded zone with foredeeps and the "sub-oceanic" depression. The above mentioned geostructures form evolution series.

Lately the main features of waves field and deep structure of the elements have been known from the data of deep seismic sounding (DSS) along the regional line Black Sea-Voronezh Massif.

A DSS program on the line has been performed by the Geophysical Institute of the Ukraine Academy of Sciences for the last three years.

All recorded waves were subdivided into five groups according to kinematic and dynamic character and their association with certain layers of the earth's crust:

1. with horizons in the sedimentary series,

2. with basement surface and boundaries near it,

3. with horizons within "the granitic" layer,

4. with "basaltic" layer surface and boundaries within it,

5. with Mohorovičić discontinuity and interfaces in the upper mantle.

The main waves recorded everywhere are as follows:

1. refracted with penetration into layer (rarely refracted) wave $\mathrm{P}^{\circ}$ from the basement surface (surface of the "granitic" layer),

2. refracted wave $P *$ from the surface of the "basaltic" layer,

3. reflected wave Prefl. from the bottom of the earth's crust.

Let us consider the most interesting questions of recent research.

\section{A) Waves from the Interfaces in "Granitic" Layer}

Work has been done concerning the upper part of the earth's crust structure within the Ukraine Shield $[3,5,6]$ where the basement is exposed or overlain by a thin cover. Recorded distinct refracted and sometimes reflected waves from the shallow interfaces within the "granitic" layer $(2.0-10.0 \mathrm{~km})$, usually follow the wave from the basement surface.

Waves associated with the interfaces in the "granitic" layer are complex: reflected ones at the point of arrival and refracted waves from the same boundary.

Reflected waves are not always observed. 
Waves shapes are often distorted due to disturbances and here traces are sharply broken, while short random synphase lines and diffracted waves are recorded. The picture shows a flat-bedded structure mainly because of indistinct velocity differentiation of the medium.

The phenomena take place as a rule on the border-lines of blocks with different geologic division and in places of contacts.

It would be most desirable to know whether or not gentle seismic interfaces occur at shallow depths within vertical-bedded Precambrian crystalline series. For this it may be necessary to revise or specify old points of view on the geologic structure of deep parts of an old folded-metamorphic complex.

\section{B) The Nature of Waves Patterns from Large Depths}

Publications on DSS show [1], that observed waves from interfaces within the earth's crust and Mohorovičić discontinuity usually display a complex pattern of synphase lines with almost the same values of apparent velocity.

Separate phase correlation within the pattern may be performed rarely because of changes in their intensity ratio.

There existed different suggestions as to the nature of the pattern. Some seismologists are favourably inclined to the thought that pattern formation is associated with the existence of the upper part of the earth's crust, especially of the sedimentary cover. Thus, the pattern may be due to generation of multiple waves in the sedimentary cover, composite waves that run one part of their way as compressional wave and another - as shear wave, and to some peculiarity of explosion (aftershocks, "ghosts") and so on.

But seismic studies have demonstrated a pattern when both the shot-point and recording stations are located on the Shield, where seismic waves do not run through the sedimentary series (Fig. 1).

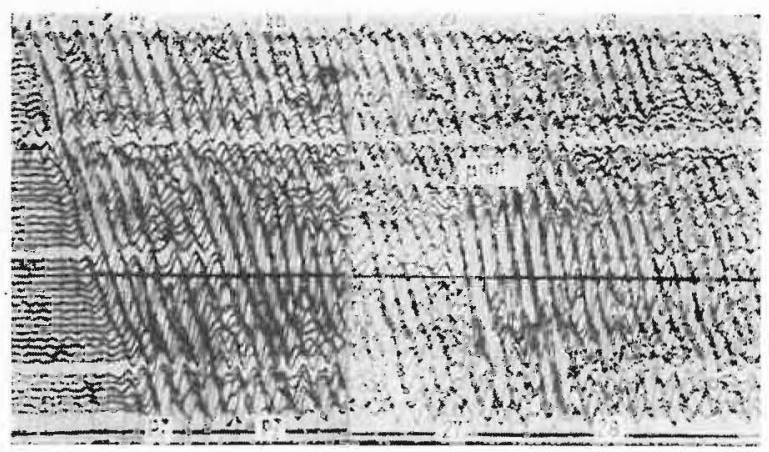

FIG. 1

Seismic line 1; shot point 50; reception interval 195.0-200.0 km; charge weight $450 \mathrm{~kg}$; geophones location interval $100 \mathrm{~m}$; seismic station series SS-30/60 KMPV (designed for correlation method of refracted waves); amplification 5; date 1963; recorded by Geophysical Institute of the Ukraine Academy of Sciences 
Moreover, in the case of a sedimentary cover some deep waves are recorded as simple two or three phase oscillations and some deep waves as a complex pattern (Fig. 2).

The most complex pattern is observed on the record of waves from the Mohorovičić discontinuity within the Black Sea-Voronezh Massif line. The pattern

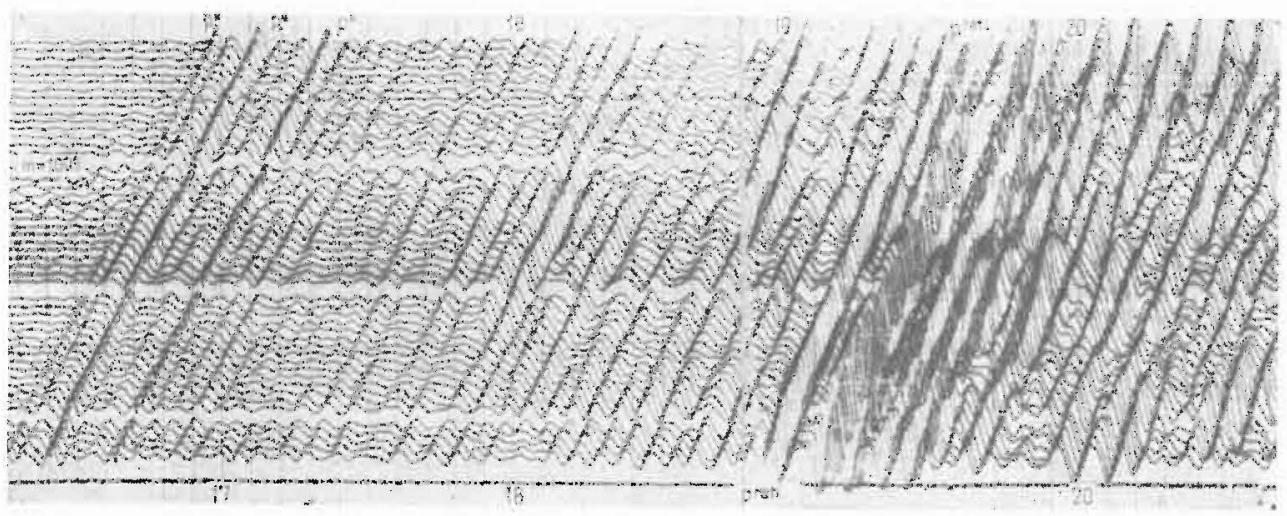

FIG. 2

Seismic line 1; shot point 215; reception interva1 124.5-129.4 km; charge weight $200 \mathrm{~kg}$; geophones location interval $100 \mathrm{~m}$; seismic station series SS-30/60 KMPV (designed for correlation method of refracted waves); filtration 1-mk (small steepness); amplification 5; date 1962; recorded by Geophysical Institute of the Ukraine Academy of Sciences

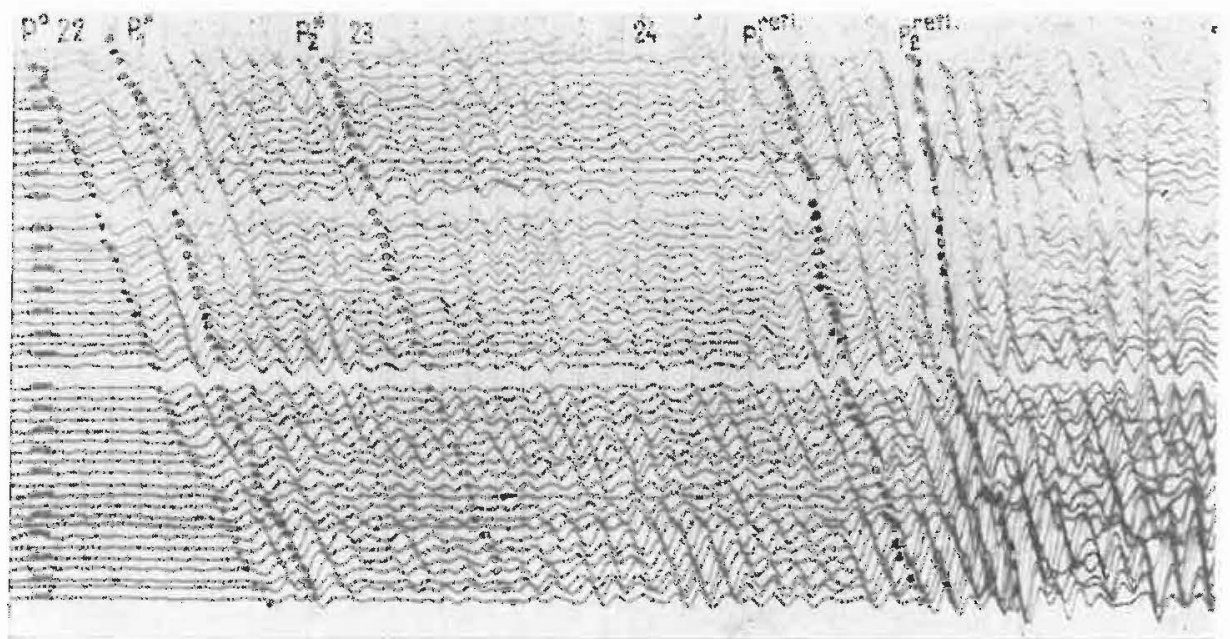

FIG. 3

Seismic line 1; shot point 150; reception interval 35.0-40.0 km; charge weight $750 \mathrm{~kg}$; geophones location interval $100 \mathrm{~m}$; seismic station series SS-30/60 KMPV (designed for correlation method of refracted waves) filtration 1-mk (small steepness); amplification 5; date 1963; recorded by Geophysical Institute of the Ukraine 
seems to be of multiphase (up to 7-8 phase) and of strong intensity (Fig. 2). In the Dnieper-Donets depression we can see the forking of the pattern into two separate parts of complex shape (Fig. 3).

To the north-west from the Dnieper-Donets depression the wave shape becomes simpler and consists of two-three phase oscillations (Fig. 4) within the

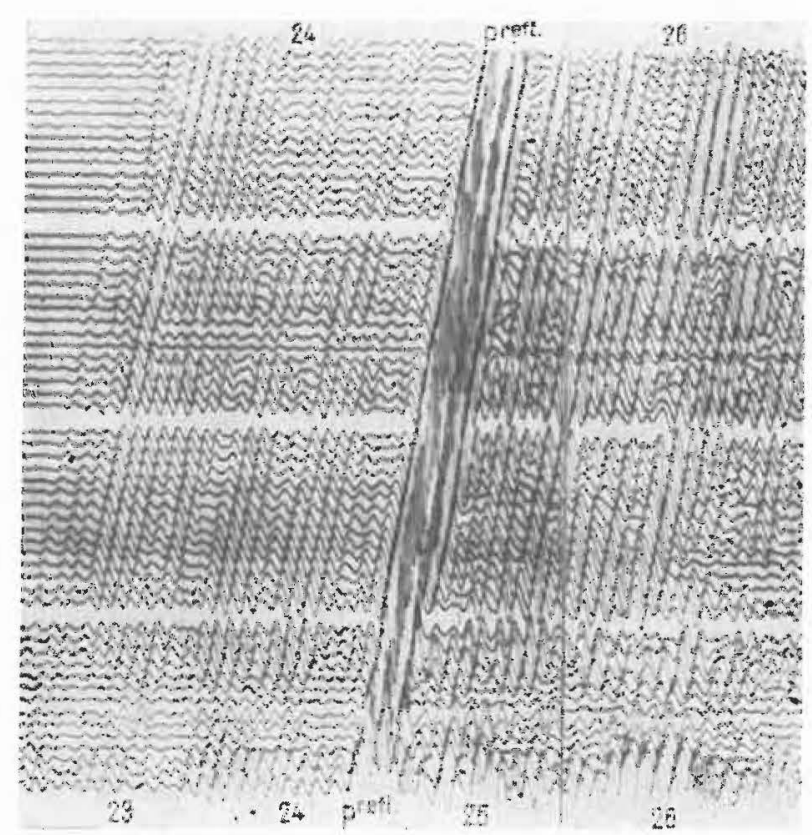

FIG. 4

Seismic line Kanev-Novgorod-Seversk; shot point 121.8 ; reception interval $(-5.9)+(-11.7)$; charge weight $360 \mathrm{~kg}$; geophones location interval $100 \mathrm{~m}$; seismic station series SS-30/60 KMPV (designed for correlation method of refracted waves); filtration 1-mk (small steepness); amplification 5; date 1962; recorded by Trust "Ukrgeophysrazvedka"

Kanev-Novgorod-Seversk line. The oscillations are well correlated at observations on large distances. Wave form from the Mohorovičić discontinuity on the record is like that of a reflected wave from reference horizon in sedimentary cover (for example, reflected wave from anhydrite-gypsum horizon in the Precarpathian foredeep).

The information gives the possibility to suggest that pattern formation is mainly defined by physical properties and deep structure of seismic interfaces.

Thus, Mohorovičić discontinuity within the Kanev-Novgorod-Seversk line, is probably "plain" enough and formed by the sharp change in characteristic impedances. The discontinuity in the region, where the Black Sea-Voronezh Massif line spreads is very complicated. 
Peculiarities of records of deep waves doubtlessly yield certain geological informations as to the structure and properties of interfaces in the earth's crust and mantle.

\section{C) Seismic Characteristics of Deep Faults}

A number of deep faults are discovered along the Black Sea-Voronezh Massif line. Criteria of a deep fault in records are as follows:

(a) break in the correlation of the main waves, not connected with a peculiar structure of the upper part of the geologic section (e.g.: saline deeper fold, disturbances in sedimentary series and others) (Fig. 5),

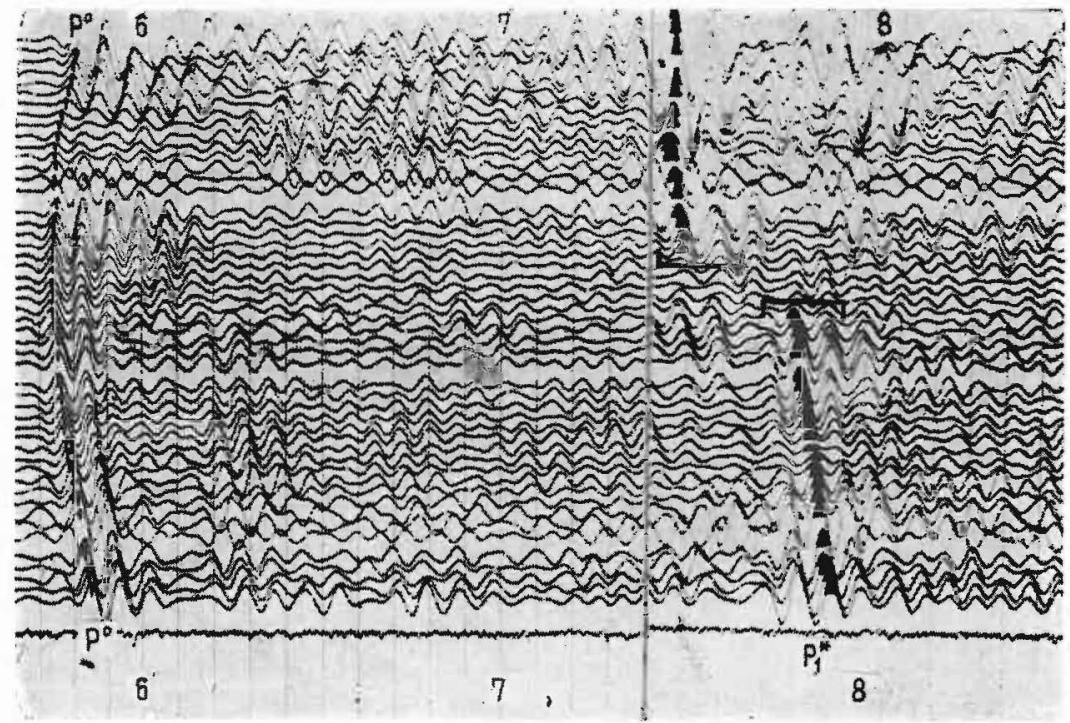

FIG. 5

Seismic line III; off shot point 2; reception interval $48.5-53.4 \mathrm{~km}$ off distance $30 \mathrm{~km}$; charge weight $40 \mathrm{~kg}$; geophones location interval $100 \mathrm{~m}$; seismic station series SS-30/60 KMPV (designed for correlation method of refracted waves); filtration 1-mk (small steepness); amplification 5; date 1962; recorded by Geophysical Institute of the Ukraine Academy of Sciences

(b) appearance of groups of diffracted waves, outlining a narrow disturbed zone (Fig. 6). The waves have great intensity, especially in the part of timedistance curve minimum and help to determine disturbed zone depth,

(c) existence of intensive but short in length synphase lines of "strange" waves (Fig. 7). The nature of the waves is not treated simply, though we can suggest them to be reflected waves from subvertical surfaces in the place of a disturbed zone. Apparent velocities are great enough $(13-7 \mathrm{~km} / \mathrm{sec})$ and the wave character is repeated on the surge system of time-distance curves, 
(d) change in the total character of the wave field, velocities in the sedimentary cover and velocities at the boundaries, wave adsorption or intensity ratio of different wave types.

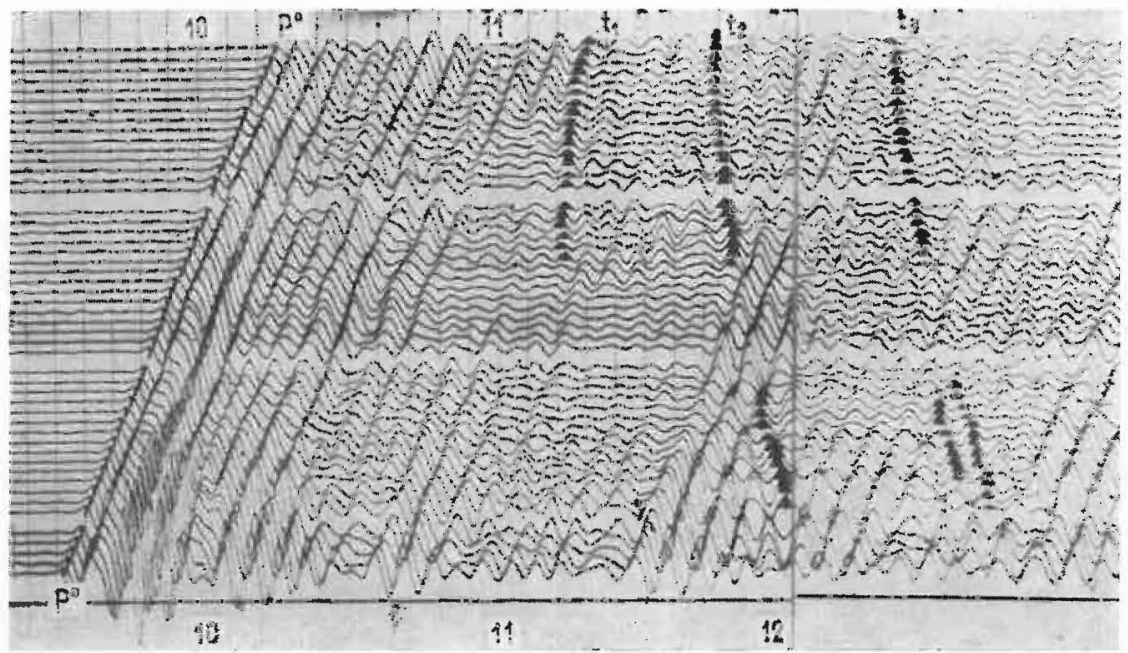

FIG. 6

Seismic line III; shot point 105; reception interval $62.1-67.0 \mathrm{~km}$; charge weight $200 \mathrm{~kg}$; geophones location interval $100 \mathrm{~m}$; seismic station series SS-30/60 KMPV (designed for correlation method of refracted waves); filtration 1-mk (small steepness); amplification 5; date 1962; recorded by Geophysical Institute of the Ukraine Academy of Sciences

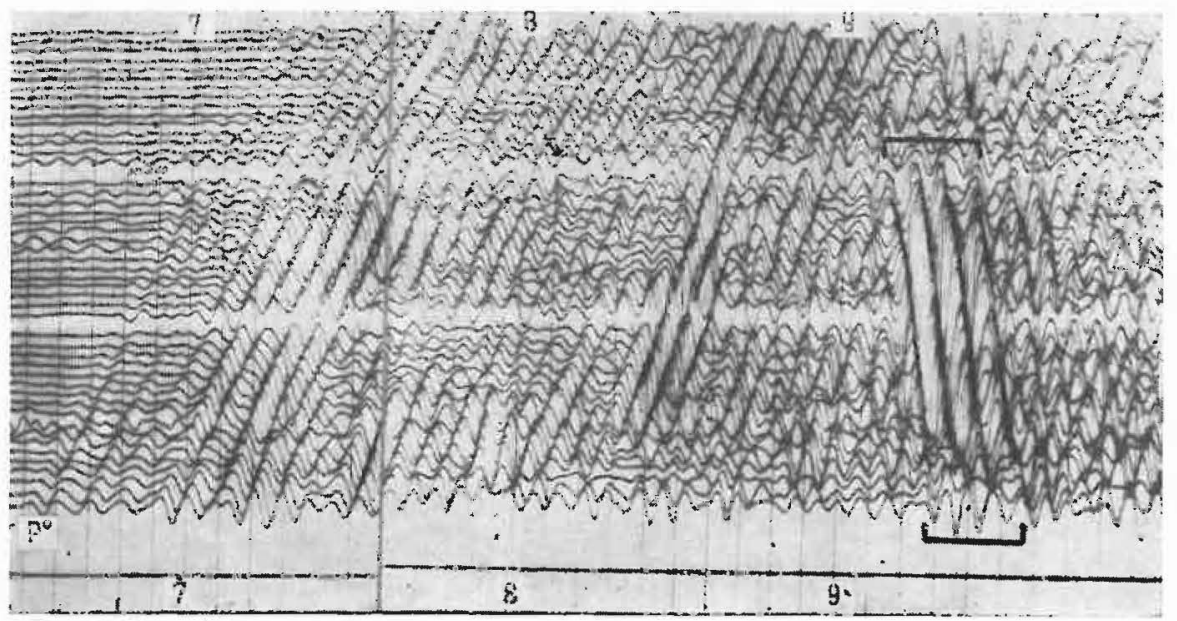

FIG. 7

Seismic line I; shot point 50; reception interval 8.4-13.1 km; charge weight $120 \mathrm{~kg}$; geophones location interval $100 \mathrm{~m}$; seismic station series SS-30/60 KMPV (designed for correlation method of refracted waves); filtration 1-mk (small steepness); amplification 5; date 1963; recorded by Geophysical Institute of the Ukraine Academy of Sciences 


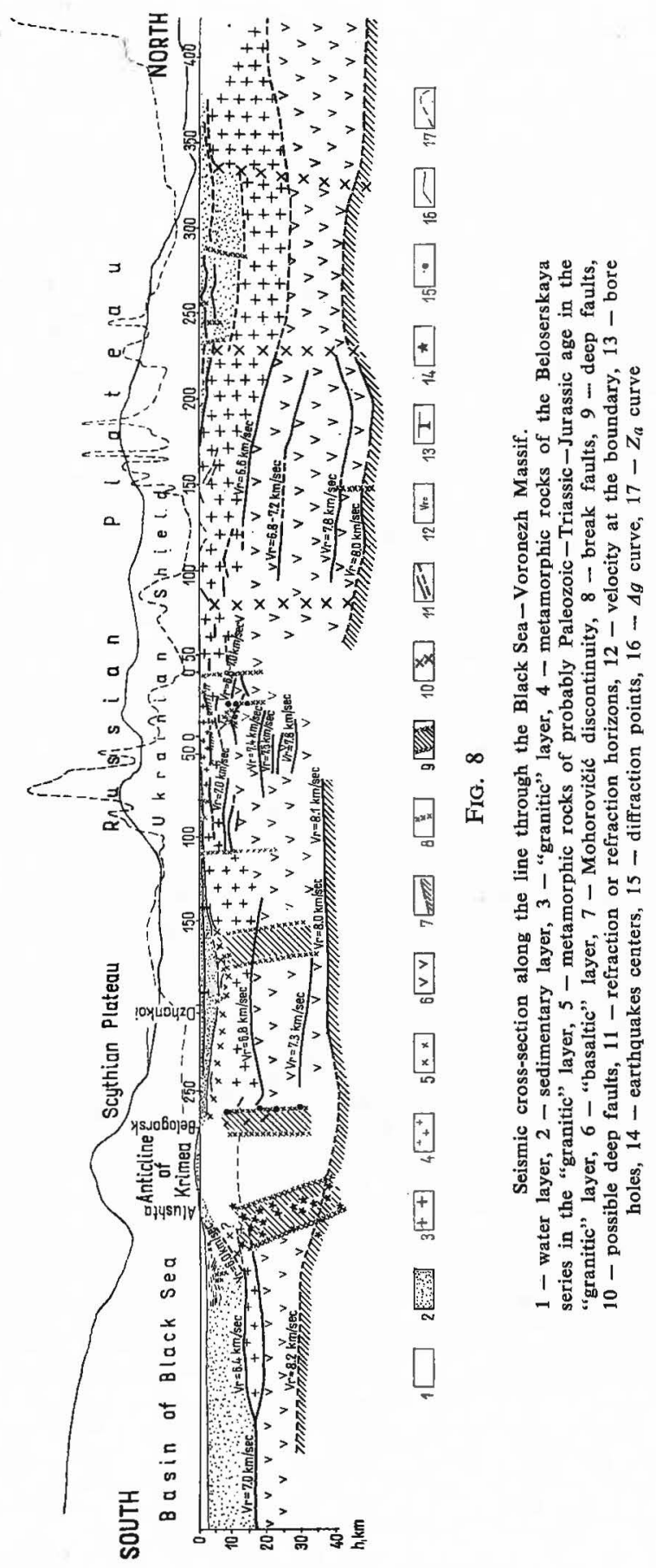




\section{D) The Earth's Crust Structure along the Black Sea-Voronezh Massif Line}

The difference in structure of the earth's crust in different geotectonical elements is clearly seen. From one region to another the thickness of the earth's crust and the layers which form it are varied.

The surface of the "granitic" layer occurs at a depth of $10-11 \mathrm{~km}$ in the Dnieper-Donets depression, rises to $6.0-6.5 \mathrm{~km}$ in the Sovashskaya depression, lies at depths of 5-14 km in the north part of the Black Sea and is exposed in the Ukraine Shield.

A hundred kilometers to the south from the Crimea a "granitic" layer is thinned out [4].

In many investigated regions the "granitic" layer has a clear bedded structure. Thus, on the south slope of the Ukraine Shield in the Belosersk magnetic anomaly region a number of continuous gentle seismic interfaces with large velocities at the boundaries are traced, beginning from the depth of $2-3 \mathrm{~km}$; for example, a seismic horizon at the depth $5-8 \mathrm{~km}$ has a velocity of $7.0-7.1 \mathrm{~km} / \mathrm{sec}$ at the boundary.

From the value of the velocity at the boundary the interface is to be related to the surface of the "basaltic" layer. Therefore, the Belosersk region appears to be an uplifted block, as compared to the adjacent parts of the Ukraine Shield.

The Conrad discontinuity has been traced along the line and shows a complicated elief. It forms a syncline similar to the basement relief in the DnieperDonetsk depression area and an anticline of $5 \mathrm{~km}$ in amplitude in the Sivashskaya depression region.

In the Crimea the "basaltic" layer rises and becomes thicker, the interfaces within the layer being traced along the line.

The Mohorovičić discontinuity forms an anticline and its depth varies in the range of $45-50 \mathrm{~km}$. As it was mentioned above it is identified by a zone and not by a surface. To the south the Mohorovičic discontinuity rises and in the Belosersk region it occurs at the depth of $35 \mathrm{~km}$, in the plain of Crimea and Presivashje it lies at the depth of $35-40 \mathrm{~km}$, in the mountainous Crimea it sinks to $45-50 \mathrm{~km}$ and in the Black Sea trench it rises again to $22-30 \mathrm{~km}$.

The line runs through a number of deep faults, which frame the Crimea megaanticlinorium, separates the Russian and Scythian platforms and makes a border line between the Dnieper-Donets depression, the Voronezh Massif and the Ukraine Shield.

Thus the earth's crust has a complex block structure [7]. Relations of the structure of the upper part of the earth's crust to its deep structure are outlined in the paper.

\section{REFERENCES}

[1] Deep seismic sounding of the earth's crust in the USSR, Sb. dokl., Gostoptechizdat, L. (1962) 
[2] Demmenko, J. B., Manuta, M. Y., Lisenko, V. A. and Spikhina, L. M.: Results of seismic research of deep structure of the Earth's crust in West Ukraine, Geophys. Sb. Institute geophys. AN Ukraine SSR, 57 (1963)

[3] Kaljushnaya, L. T., Sollogub, V. B. and Chekunov, A. V.: Characteristics of the elastic waves from the interface in the crystalline basement in the south of the Belosersky Region and its deep structure. Geophys. sb. Instituta geophys. AN Ukraine SSR 810 (1964)

[4] Neprochnov, J. P.: Deep structure of the Earth's crust under the Black Sea trench from seismic data. Bull. MOIP, otd. geol. v. XXXV 4 (1960)

[5] Sollogub, V. B., Chekunov, A. V., Chilinsky, L. A. and Garkalenko, I. A.: Resuits of experimental seismic survey on the study of the inner structure of crystalline basement of the north part of the Krivoroshsky Basin, Geophys. sb. Instituta geophys. AN Ukraine SSR 13 (1962)

[6] Sollogub, V. B., Chekunov, A. V., Kaluushnaya, L. T., Chilinsky, L. A. and KhaRECHKO, G. E.: Inner structure of the crystalline basement in the south-west of the Korostensky Plateau from seismic data, Geophys. sb. Instituta geophys. AN Ukraine SSR, 57 (1963)

[7] Subbotin, S. I., Sollogub, V. B. and Chekunov, A. V.: The Earth's crust structure of the primary geostructural elements of the Ukraine area, DAN Ukraine SSR, 1532 (1963) 


\title{
AMPLITUDE CURVES OF SEISMIC BODY WAVES
} AT DISTANCES SMALLER THAN $12^{\circ}$

\author{
J. VANĚK and C. RADU \\ (Prague, Czechoslovakia)
}

During the 7th session of the E.S.C. in Jena J. Vanĕk and J. Stelzner [1] reported about the amplitude-distance curves for seismic body waves at distances greater than $12^{\circ}$, which were derived on the basis of observations of a network of Central European stations. Due to the importance of reliable amplitude curves in relation to the structure of the earth's upper mantle the study of amplitude curves at smaller distances seemed to be very desirable.

The aim of the present communication is to report about the investigation of the fine structure of the amplitude curves of $\mathrm{P}$ and $\mathrm{S}$ waves at distances between $5^{\circ}$ and $12^{\circ}$. For this purpose observations of 49 selected shallow earthquakes recorded at the seismic station Bucharest and at the stations of our Central European network [1] were used. Their epicentres were situated in Turkey, Eastern Mediterranean and Greece in distances between $5^{\circ}$ and $14^{\circ}$ from Bucharest and in distances greater than $12^{\circ}$ from the Central European stations.

First, the station corrections $S$ for the station Bucharest relating to Prague were determined by usual methods [2] comparing the magnitudes obtained from the observations of individual wave types at Prague and Bucharest; during this procedure earthquakes with epicentral distances greater than $12^{\circ}$ were used.

The same definition of the amplitude-distance curve $A^{*}(\Delta)$ as in the previous work [1] was accepted, namely

$$
A_{j}^{*}(\Delta)=\log \left(A_{j} / T_{j}\right)-M_{j}
$$

where $A_{j}$ is the maximum ground amplitude in microns and $T_{j}$ the corresponding period in seconds of the individual wave group $j$ and $M_{j}$ is the earthquake magnitude, which has the function of a normalizing quantity in this case. The value $M_{j}$ for every earthquake was estimated as an average of all the observations obtainable at our network of Central European stations. This was possible since the epicentral distances of these stations were greater than $12^{\circ}$ for our selected earthquakes and the existing third approximation of the magnitude calibrating functions for body waves [3] could thus be used. A weight $p$ was coordinated to every magnitude $M_{j}$ according to the number of observations, on the basis of which the average was estimated.

After correcting the Bucharest observations by the appropriate station corrections a set of values $\left(A_{j}{ }^{*}, \Delta\right)$ was obtained. In the distance range considered rapid changes of $A_{j}{ }^{*}$ were observed. The course of the amplitude curves was 
determined by the same method as in previous work [1]. The whole set of observations was divided into natural intervals. The observations in every interval were represented by their centre of gravity, taking into account the weights of individual points of observation. Then a smooth curve was drafted so that it passed through all the representative points allowing for the tendency of detailed distribution of observations. The resulting amplitude curve for the horizontal component of $\mathbf{P}$ waves can be seen in Fig. 1. We see that the shape of the amplitude curve

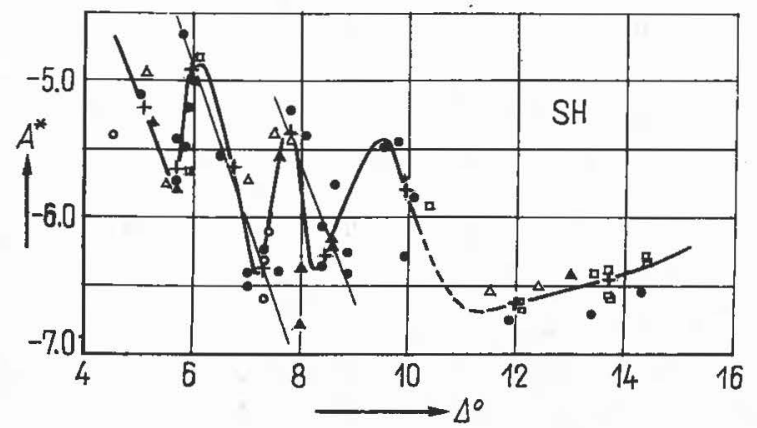

FIG. 1. Amplitude curve $A^{*}(\Delta)$ for PH waves. Observations of Bucharest with different weights are indicated as follows: open circles for weight 1 , open triangles for weight 2 , full circles for weight 3 and 4, full triangles for weight 5 and more. Observations of Central European stations are indicated by squares. Crosses denote representative points of natural intervals

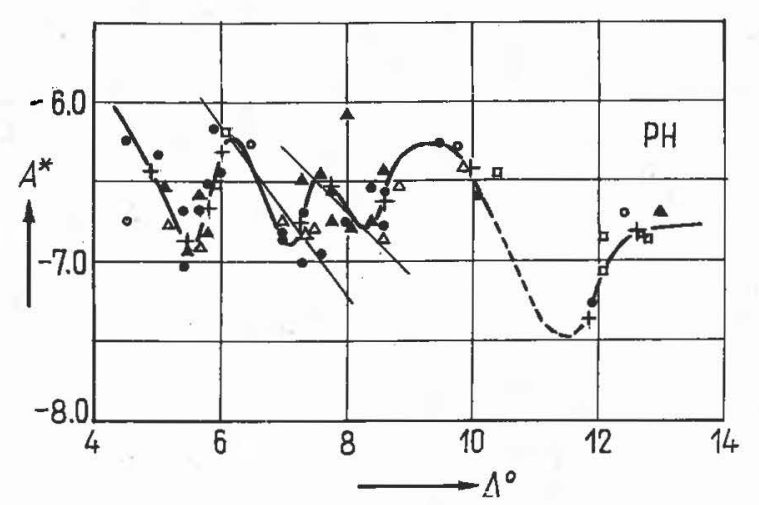

FIG. 2. Amplitude curve $A^{*}(\Delta)$ for $\mathrm{SH}$ waves. Symbols as in Fig. 1

is rather complicated. A similar picture was obtained for SH waves (Fig. 2).

As can be seen from the figures the amplitudes of body waves are very variable in the distance range between $5^{\circ}$ and $10^{\circ}$. If we construct amplitude curves as a smooth line, clear oscillations can be observed in the distance range in question. The magnitude of these oscillations is safely above the errors of observations, the mean quadratic error of a single observation in $A^{*}$ lying between \pm 0.1 and 
\pm 0.2 . It is important that epicentral distances, at which maxima and minima of the oscillations occur, coincide excellently for $\mathrm{P}$ and $\mathrm{S}$ waves. The reliability of oscillations constructed in the figures is not the same for all of them. The oscillations, the maxima of which are around $6^{\circ}$ and $9.5^{\circ}$, are much more certain than the oscillation with the maximum near $7.5^{\circ}$, where the scatter of observations is considerable. The increased scatter can be partially influenced by the earthquake mechanism, because the azimuth difference between Bucharest and Central European stations can attain up to $50^{\circ}$ at some of our selected earthquakes. Nevertheless, it is improbable that the earthquake mechanism could influence substantially the shape of amplitude curves due to random distribution of the azimuth differences with epicentral distance.

What is the cause of such an instability of amplitudes of body waves observed in the distance range considered? This question is nearly connected with the structure of the upper mantle quite beneath the Mohorovičić discontinuity. There are two possible explanations:

1. The construction of amplitude curves as smooth curves with oscillations is correct. Then the oscillations are probably representing the zone of interference of waves reflected from beneath the Mohorovičić discontinuity. In this case a certain velocity gradient must be assumed in these depths.

2. There are several discontinuities of the first or second order between the Mohorovičić discontinuity and the assumed low-velocity channel. In this case the amplitude variability observed can be explained as a mixture of amplitude curves of waves bounded to individual discontinuities.

To decide between the above two possibilities further special investigations are necessary, keeping in mind that a combination of both variants is not exluded.

The course of amplitude curves at distances greater than $10^{\circ}$ shows that in the case of the existence of a low-velocity channel in the upper mantle the shadow zone would begin at $9.5^{\circ}$ reaching the minimum amplitudes near $11.5^{\circ}$ for both $\mathrm{P}$ and $\mathrm{S}$ waves. Then a clear increase of amplitudes occurs having its maximum near $19^{\circ}$, where another region of oscillations can be observed. The lack of sufficient observational data, however, did not allow to derive amplitude curves in the distance range between $10^{\circ}$ and $12^{\circ}$ with much certainty. To complete this a further study is necessary using observations of other suitably situated seismic stations.

It must be emphasized that owing to the possible regional differences in the upper mantle structure the resulting amplitude curves are strictly valid for the southeastern part of the European continent.

\section{REFERENCES}

[1] J. VANĚK and J. STELZNER: Oscillatory character of amplitude curves for seismic body waves. Veröff. Inst. Bodendynamik und Erdbebenforsch. Jena, 77205 (1964)

[2] J. VANĚK and J. STELZNER: Einheitliche Bestimmung von Erdbebenmagnituden für mitteleuropäische Stationen. Travaux Inst. Géophys. Acad. Tchécosl. Sci. 136 (1960)

[3] J. VANĚK and J. STELZnER: The problem of magnitude calibrating functions for body waves. Ann. Geofis. 13393 (1960) 


\title{
THREE-DIMENSIONAL SEISMIC MODELS OF THE EARTH'S UPPER MANTLE
}

\author{
L. Waniek, J. VaněK, Z. Pros and K. Klíma \\ (Prague, Czechoslovakia)
}

Recently, several efforts have been made to complete our knowledge of the structure of the earth's upper mantle by detailed observation of seismic body waves, especially by their amplitude-distance curves [1,2]. During these investigations difficulties appeared in the interpretation of observed results. This is first of all caused by the fact that theoretical investigations, which are mostly limited to the application of the ray theory, do not at present allow for the solution of some basic problems of the propagation of elastic waves in inhomogeneous media $[3,4]$.

For this reason measurements on appropriate seismic models seem desirable. Nevertheless, measurements of this kind meet fundamental difficulties in producing models with continuously variable velocity. The problem has already been attacked by the two-dimensional modelling technique [5-8]. These investigations, however, have met serious technological difficulties, especially in fabricating a model with negligible horizontal variation of the vertical distribution of velocity. Moreover, the possibilities of modelling the high-frequency range of wave spectra of body waves are rather limited. It is questionable, in principle, whether the representation of the wave propagation in three dimensional space by that in a plate is a sufficient approximation of actual conditions.

Therefore, we tried to develop a method of producing three-dimensional models, in which the continuous variation of velocity was realized by a large number of thin horizontal layers with negligible velocity differentiation between the individual layers. In this case the modelling medium must satisfy the following necessary conditions: sufficient range of velocity variation, possibility of producing very thin layers of constant thickness on a relatively large surface, ideal contact between individual layers and between medium and pick-ups of the modelling apparatus, homogeneity of elastic properties in horizontal direction and relatively small attenuation of elastic waves in 1-Mc/s frequency range which must be used to keep models within reasonable dimensions. We have found that the foregoing properties could be obtained by multicomponent gels. In the work recorded here, a three-component system of water, glycerol and photogelatin was applied. In a medium of this type the velocity of longitudinal waves was between 1.5 and $1.9 \mathrm{~km} / \mathrm{sec}$ (Fig. 1). The required velocity can be obtained by different combinations of the three components, which in a certain range permits controlled attenuation. 
For model measurements a special apparatus developed in the Geophysical Institute, Czechosl. Acad. Sci., was used [9]. Only longitudinal waves were studied applying a lead titanate zirconate piezo-ceramic transmitter with free frequency of $1 \mathrm{Mc} / \mathrm{sec}$ and a special splinter receiver of the same material for recording the vertical component of P-waves on the surface of the model. A typical oscillogram obtained on the surface of gel-model is shown in Fig. 2.

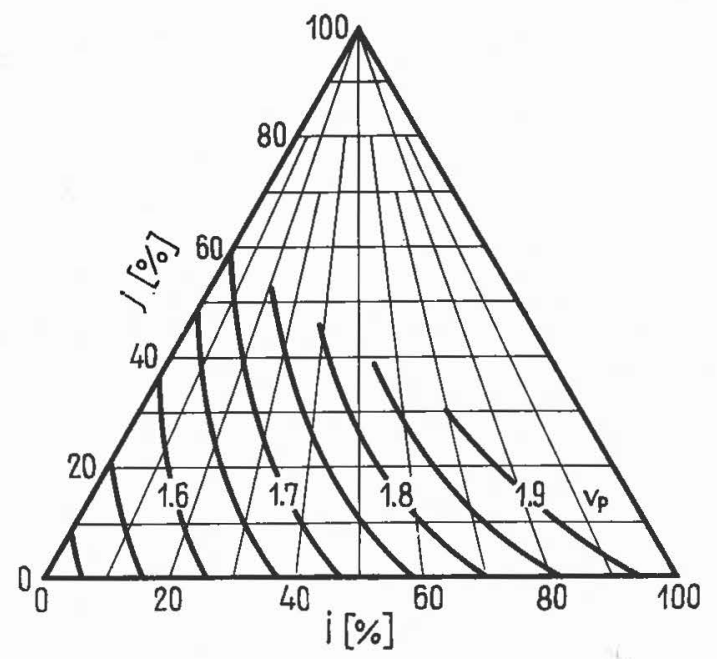

Fig. 1. Velocity dependence for three-component system of water, glycerol and photogelatin. Velocity $v_{P}$ in $\mathrm{km} / \mathrm{sec}$ at $1 \mathrm{Mc} / \mathrm{sec}$, concentration $i$ of glycerol in water and $j$ of gelatin in the glycerol-water mixture are given in weight percentages

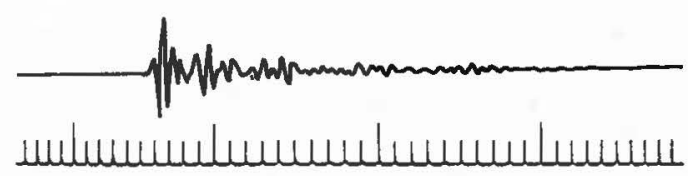

FIG. 2. Typical oscillogram of the wave pattern on the surface of a model. Time marks in $\mu \mathrm{sec}$

To demonstrate the applicability of gel medium for models of the upper mantle we have realized two variants of velocity-depth distribution, which seemed most probable after ray theory calculations from seismic amplitude-distance curves.

The first variant can bee seen in Fig. 3. The velocity distribution is characterized by a channel of low velocity with the minimum at $110 \mathrm{~km}$ and one dis- 
continuity of the 2 nd order. The amplitude curve was obtained for the source in the crust. No corrections for the actual earth's crust and curvature are introduced in the figure. The model in question consisted of 63 layers $1.5 \mathrm{~mm}$ thick characterized by $i$ varying between 0 to $64.8 \%, j$ equalling $10 \%$ and $v_{p}$ varying between 1.51 to $1.84 \mathrm{~km} / \mathrm{sec}$.
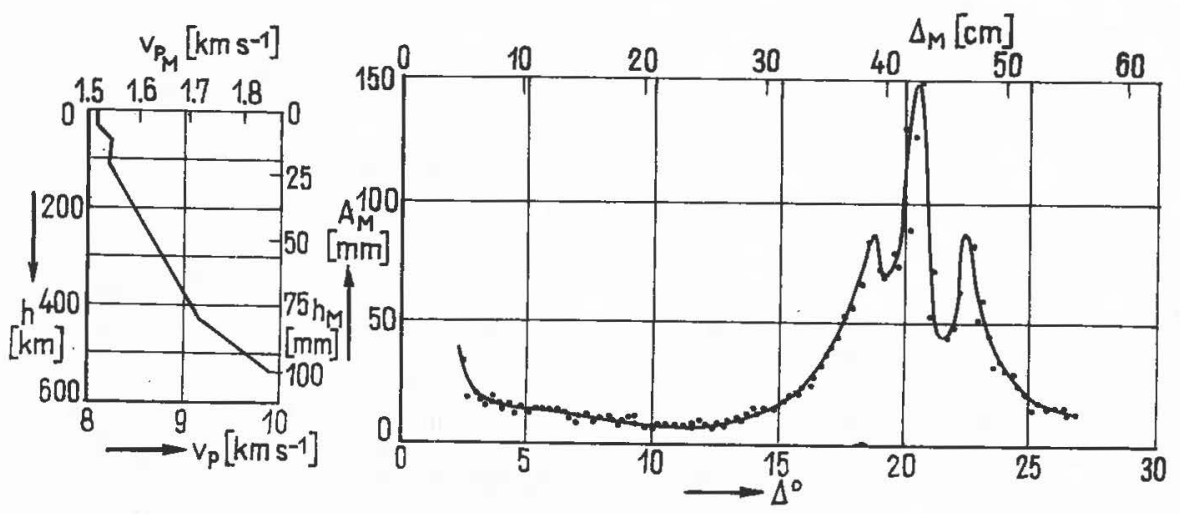

FIG. 3. Amplitude-distance curve of P-waves measured on the first model of the earth's upper mantle. On the left side velocity-depth distribution. Depth $h$ in $\mathrm{mm}$ on the model and in $\mathrm{km}$ in the earth's upper mantle, epicentral distance $\Delta_{M}$ in $\mathrm{cm}$ on the model and $\Delta^{\circ}$ actual epicentral distance in degrees, $A_{M}$ maximum amplitude in P-wave group

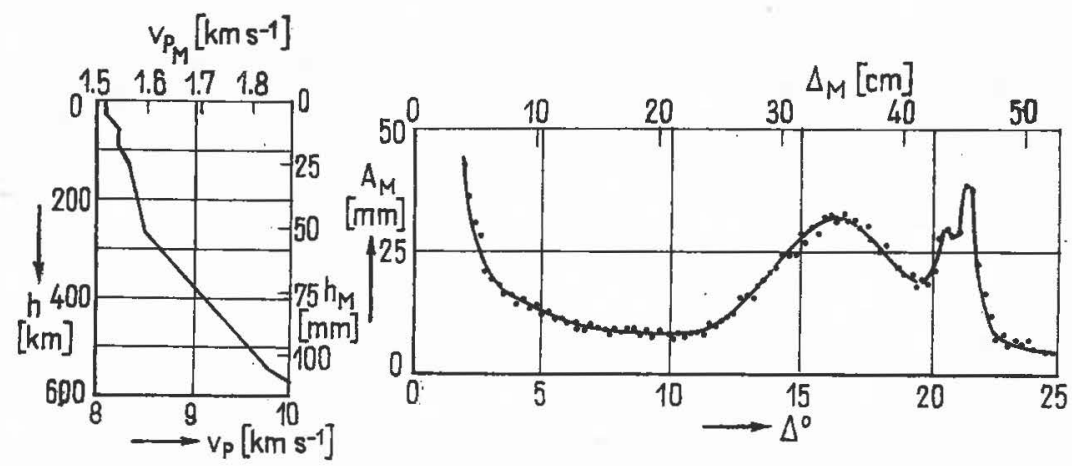

FIG. 4. Amplitude-distance curve of P-waves measured on the second model of the earth's upper mantle. The meaning of symbols as in Fig. 2

The results for the second variant of the velocity-depth distribution are shown in Fig. 4. Here the channel is narrower and there are several discontinuities of the $2 n d$ order. The amplitude-distance curve was measured under the same conditions as in the first case. This model consisted of 69 layers, $i$ varying between 0 to $80 \%, j$ equalling $10 \%$ and $v_{p}$ varying between 1.52 to $1.87 \mathrm{~km} / \mathrm{sec}$. From the last two figures we can see that for different velocity distributions a quite. 
different shape of amplitude curve of P-waves can be observed. This result is very promising for further development of the modelling technique described.

Comparing the amplitude-distance curves for the two velocity distributions investigated with seismological data the first variant seems to be much more probable than the second one. This is evident from Fig. 5. In the upper part

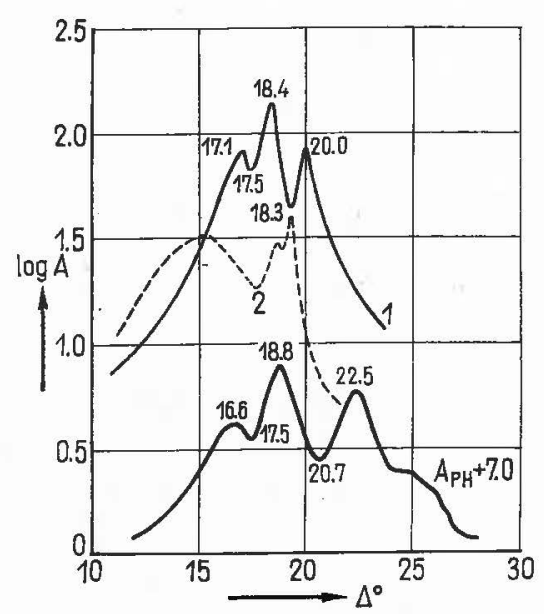

Fig. 5. Amplitude-distance curves on the models (curves 1 and 2) and amplitude-distance curve obtained from seismological data (curve $A_{P H}$ )

of this figure amplitude curves obtained by measuring at the models are given. Here the influence of the earth's curvature was roughly taken into account. The amplitude curve drafted bellow by heavy line represents the amplitude curve of P-waves obtained by Vaněk and Stelzner [1] from seismological data. We see a good agreement between amplitude curve for the first variant of the velocitydepth distribution and seismological data. For comparison the exact values of distances of local maxima and minima are also given.

Further investigations into the models of several possible variants of the upper mantle structure are now being carried out.

\section{REFERENCES}

[1] VANÉx, J. and Stelzner, J.: Nature 187, 491 (1960); Gerlands Beitr. Geophys. 71 p. 105 (1962)

[2] Golikova, G. V., Yanovskaja, T. B. and GelčinskiJ, B. J.: Dokl. AN SSSR 145 p. 315 (1962); Yanovskaja, T. B. and Asbel, I. Y., Geophys. J. 8 p. 313 (1964)

[3] Babič, V. M. and Alekseev, A. S.: Izv. AN SSSR, ser. geofiz., 17 (1958)

[4] Petraš̌n, G. I. et al.: Voprosy teorii rasprostranenija seismičeskich, 5 (Leningrad 1961)

[5] Oliver, J.: Earthquake notes, 2729 (1956)

[6] Healy, J. H. and Press, F.: Geophys. 25987 (1960)

[7] Rizničenko, J. V., Šamina, O. G. and Chanutina, R. V.: Izv. AN SSSR, ser. geofiz. p. 497 (1961)

[8] RizniČenko, J. V. and S̆amina, O. G.: Izv. AN SSSR, ser. geofiz., p. 223 (1963)

[9] Pros, Z.: Travaux Inst. Géophys. Acad. Tchécosl. Sci. 153 (1962) 


\title{
CHARACTERISTICS OF THE CRUSTAL STRUCTURE IN SOME ZONES OF TRANSITION FROM CONTINENT TO OCEAN
}

\author{
P. S. WEIZMAN
}

(Moscow, USSR)

The deep structure in zones of continent to ocean jointing is of great importance for substantiating hypotheses about the direction of the development of the earth and, in particular, of its accessible parts - the crust and upper mantle. There are various types of such zones of jointing or, as they are called, of transition zones. However, clearly distinguished are two types: 1. smooth transition zones (of the Atlantic type) and 2. complex transition zones (of the Pacific type). To the second type belongs, e.g. the zone of transition from the Andean range in South America to the Pacific via Atakama deep trough and the marginal ocean ridge. IGY investigations were carried out just in this region.

Crustal structure was determined from the results obtained by DSS observations carried out in 1957-1958 by the complex geological-geophysical expedition of the Academy of Sciences of the USSR.

The results obtained concerning the entire region are described in the Collected papers [1].

In the Kurile-Kamchatka zone various deep structures are found. This zone contains the jointing strips of the substantially different blocks, composing the crust and mantle. Because of its importance let us consider this zone separately.

Figure 1 shows a system of DSS profiles and sections constructed from them. Such a representation permits to demonstrate clearly the general variety of separate deep structure characteristics, as well as the similarity between some of them.

The region can be roughly divided into zones differing substantially in structure: 1. The Okhotsk Sea (its part adjacent to the island ridge), 2. the zone lying between the island ridge and the deep-oceanic trench and 3. the eastern slope of the deepoceanic trench and the ocean ridge turning into the ocean bed.

The island arc zone has practically not yet been studied. The crustal structure of the Okhotsk Sea will not be particularly discussed here.

To the east of the deep-oceanic trench the crust is of the oceanic type and consists of a $5-7 \mathrm{~km}$ basaltic layer and a thin sedimentary one (about 1-2 km thick). The granitic layer is absent.

To the west of the deep-oceanic trench axis, extending to the island arc, the crustal structure is more complex.

The depth of the $\mathrm{M}$ surface and the presence of all three layer components indicate a continental type of crustal structure in this zone. The velocities in 


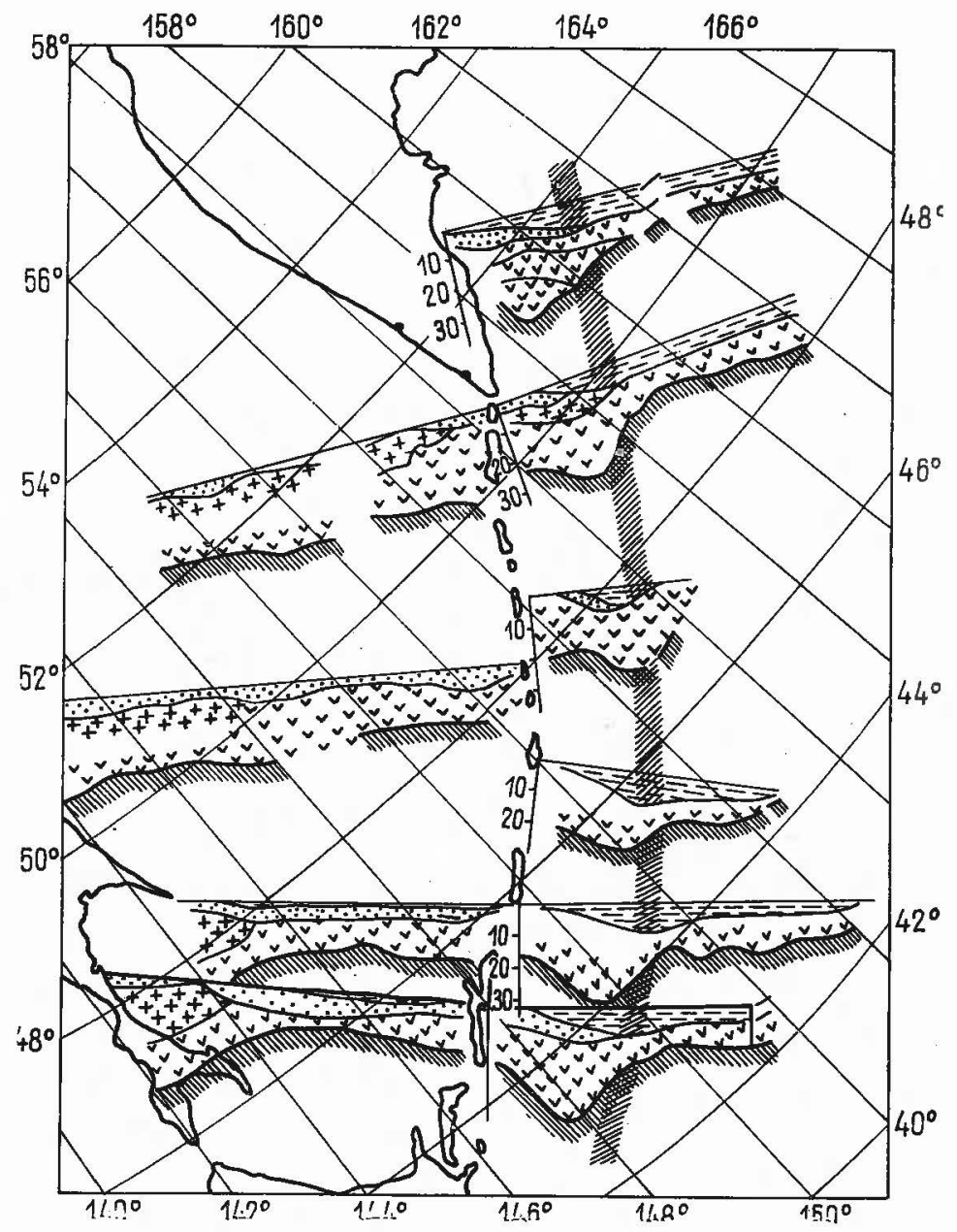

Fig. 1

the sediments and in the granitic layer, which might include young volcanic rocks, are however somewhat lower than usual. The maximum thickness of this peculiar complex continental block is confined to the western slope of the deepoceanic trench. The continental type of the crust is particularly clearly expressed in the south and north of the zone. In its central part the layering is not so clear and the structure is rather of the subcontinental type.

The characteristic features of the continental block structure will be now considered, using profile $9-10$. They are: 1 . the presence of three layers, including the granitic one, which is thinning out at the axis of the trench; 2 . the presence of a trench expressed in two deep layers - the basaltic and the subcrustal. The 
higher trench is expressed, the more it is shifted in eastern direction towards the ocean. In the sections the deep disjunctive dislocations are not clearly revealed, but they probably occur here. This is clearly demonstrated by the equal average velocity V(z) curves plotted by the Kondratjev method [2] for all profiles. Samples of such curves are given on profile 7-0 (Fig. 1). They are obviously discontinued near the axis of the trench.

All these features can be followed over the entire zone, however, less clearly in its central part.

All the islands of the arc lie on the western part of the line of maxima $M$ discontinuity depths the central and southern islands line in a zone of a relatively thin crust, whereas the northern islands belong to a zone of a substantially thicker crust. In some places, near the island arc, small values of velocity $\mathrm{V}$. for the $M$ surface are found, equal to $7.5-7.3 \mathrm{~km} / \mathrm{sec}$.

A qualitative correlation was made between the data concerning the crustal structure on one hand, and characteristics of the geophysical field-, gravitational and, in a lesser degree, magnetic data on recent vertical movements, volcanism and seismicity, on the other. A comparison is made between the laws governing the relation between $\mathrm{H}_{M}$ and water-depth $\mathrm{h}$ obtained from a series of DSS profiles and the global average relation between the position of the M-surface and topography.

Data obtained from aeromagnetic observations (carried out by Gainanov and Soloviev [3]) show that strong magnetic anomalies correspond to places, in which heterogeneous blocks are jointed.

Correlation with data given by Gorjachev [4], confirms the assumption made by geologists that the southern and northern parts of the island arc are zones of maximum rates of recent uplift, whereas the central part presents a zone of relative downwarping. The relatively strongest manifsstation of recent volcanism is confined to the central part of the arc, but a still greater maximum is found in the northern direction towards Kamchatka. Manifestation of seismicity is stronger in the northern and southern part of the zone.

Figure 2 shows the bathimetry and the gravity anomaly curves plotted across the fundamental structures for the most northern and two southern DSS profiles and for the line running parallel to the most southern DSS profile, crossing Hokkaido and coming out via the deep-oceanic trench into the ocean bed. Similarity of the bed relief to the main characteristics of the gravity anomalies (a gravity minimum is everywhere confined to the Western slope of the trench and near the axis of the trench a maximum gradient exists) permits to assume that, qualitatively, the crustal structure along this line little differs from that along the DSS profiles.

The gravity anomaly minimum, confined to the Western slope of the trench is similar to the so called Vening-Meines lines, which he explained by "buckling" of the crust near the axis of trenches: Similar anomalies were explained in other cases by nearness of the axis of the trench of a very thick sedimentary layer in an unthickened crust. It would be probably more correct to assume that these 
gravity anomaly singularities were connected with a crustal block thickening confined to the Western slope of the trench.

A comparison between the M-surface map and bed relief shows that, for the most part of the investigated region, a connection existed between the depth of the M-surface and the large forms of land relief. This relation does not exist
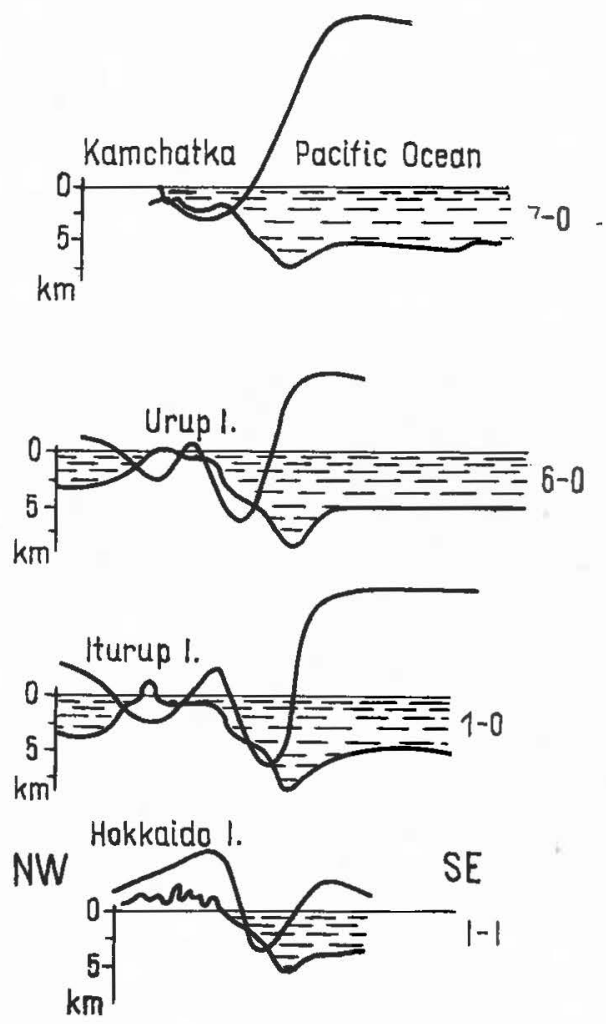

Fig. 2

anymore in the region of the shelf, neither at the western slope of the trench. The curve given in Fig. 3 shows the relation between $\mathrm{H}_{M}$ and water depth $\mathrm{h}$ for the world ocean. This averaged curve was plotted from data collected jointly with Solovieva [5]. The cluster of points defines the scatter band. The ascending branch of the curve determines the state of general isostatic equilibrium and corresponds to depths up to $5 \mathrm{~km}$. For larger depths, i.e. for deep-oceanic trenches, a descending branch of the curve is characteristic, showing unbalance of isostatic equilibrium. All the values obtained for the Okhotsk Sea fall into the scatter band, near the fundamental curve.

The remaining symbols in Fig. 3 relate to DSS profiles in the ocean. For each 
profile its own relation between $\mathrm{H}_{M}$ and water depth $\mathrm{h}$ may be considered, since the entire sequence of depths from 0 up to $8 \mathrm{~km}$ near to the axis of the trench exists. For the eastern slope of the trench and the ocean ridge the sequence of points for each profile falls on the descending branch of the curve. For the shelf region and the western slope of the trench an inverse relation is obtained by the

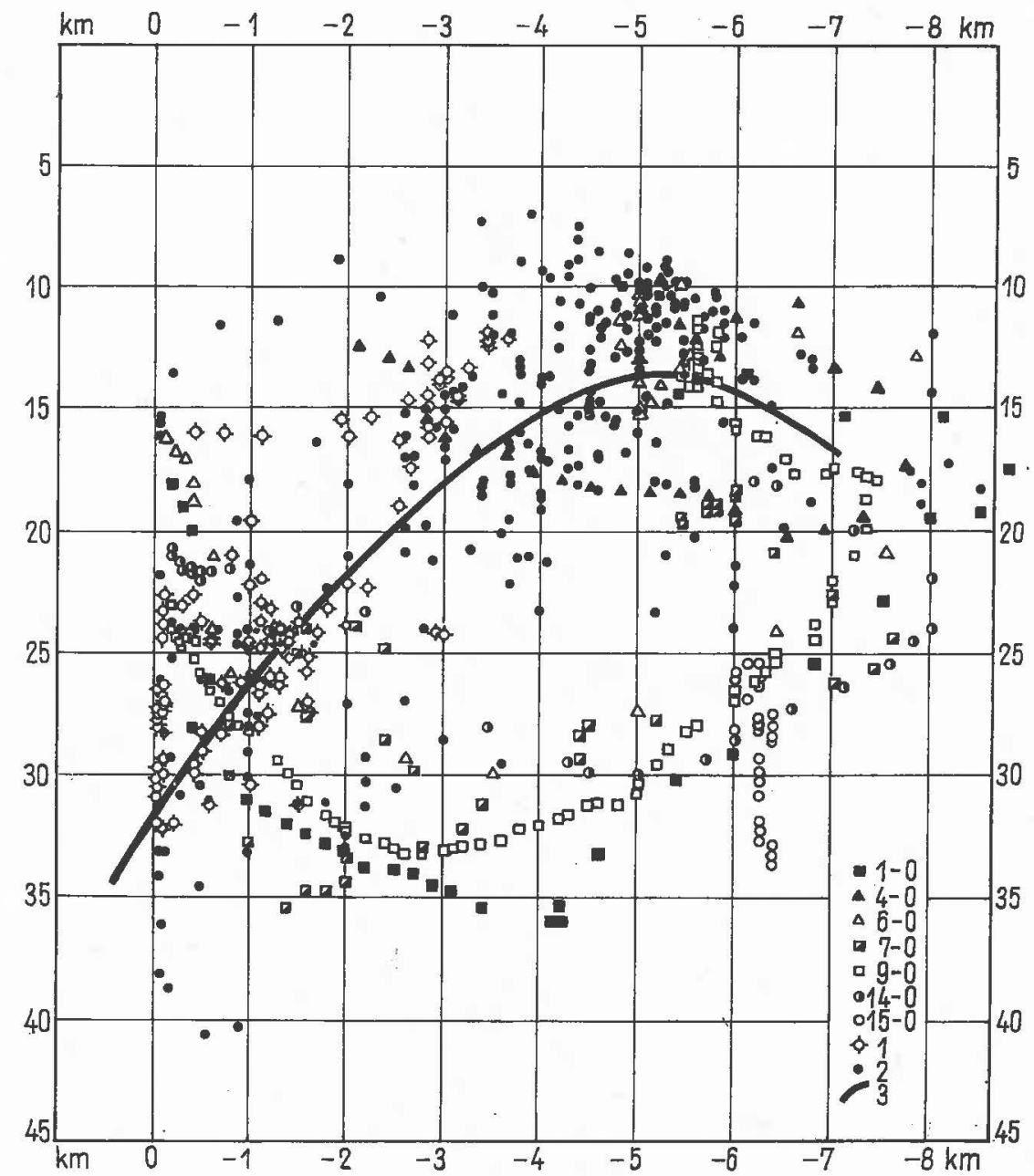

Fig. 3a

averaged curve. For the southern and northern profiles this relation considerably exceeds the boundaries of the scatter band. As a result in the shelf zone and zone of the western slope of the trench, in the region under investigation, unbalance 
of the isostatic equilibrium takes place, which is stronger in the southern and northern part of the zones.

Figure 4 shows crustal sections made along the most southern and most northern profiles. The depths of the earthquake foci, taken from a seismicity Atlas [6] and from data obtained by high-sensitivity instruments (Fedotov, Kuzin and others $[7,8]$ ) are also given there. The group of deepest earthquakes (down to

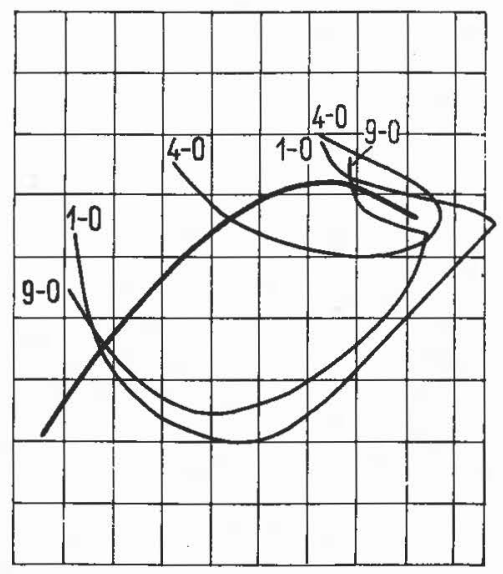

Fig. $3 b$

about $650 \mathrm{~km}$ ) was confined to the Western part of the Okhotsk Sea. This area is separated by an interval of about $100 \mathrm{~km}$ from a region of earthquake focus concentration, steadily extending along the entire zone and occupying all the possible depths down to about $200 \mathrm{~km}$. The main bulk of foci lies in a zone having an inclination close to that of the surface connecting the axis of the deep oceanic trench with the axis of the trough in the M-surface and descending in the western direction below the Okhotsk Sea. The emergence of the zone on the earth's surface is confined between the island arc and the axis of the deep oceanic trench.

There are indications that the eastern slope of the Kurile-Kamchatka trench subside relatively to the western [9]. It was shown by Balakina [10] that the strains in the earthquake foci of the Pacific-belt are mainly compressive.

The zone of earthquake focus concentration, embracing also the earth's crust and upper mantle, is probably similar to that called by Gamburzev and Belousov $[11,12]$ a seismically slackened zone, or a "system of seismic scars".

Similar distribution of foci are given in a series of investigations for other transition zones and systems of island arcs and oceanic trenches (Gutenberg and Richter [13], Rudich [14], Benioff [15], Petrushevsky [16] and others).

As an example a similar picture is given in Fig. 5 for the zone of transition from the Pacific, via the Atakama oceanic trench to the Andean range in South 
America. The sections were constructed from data given by Benioff [15], Raitt [17], and Woollard [18]. Examination of the Pacific seismic belt (Gutenberg [13]) shows that in the west the zone of focus concentration is confined to trenches and island arcs, while in the east, to trenches and coastal ridges everywhere inclined downwards below the continent. Behind the trenches there are few foci in the

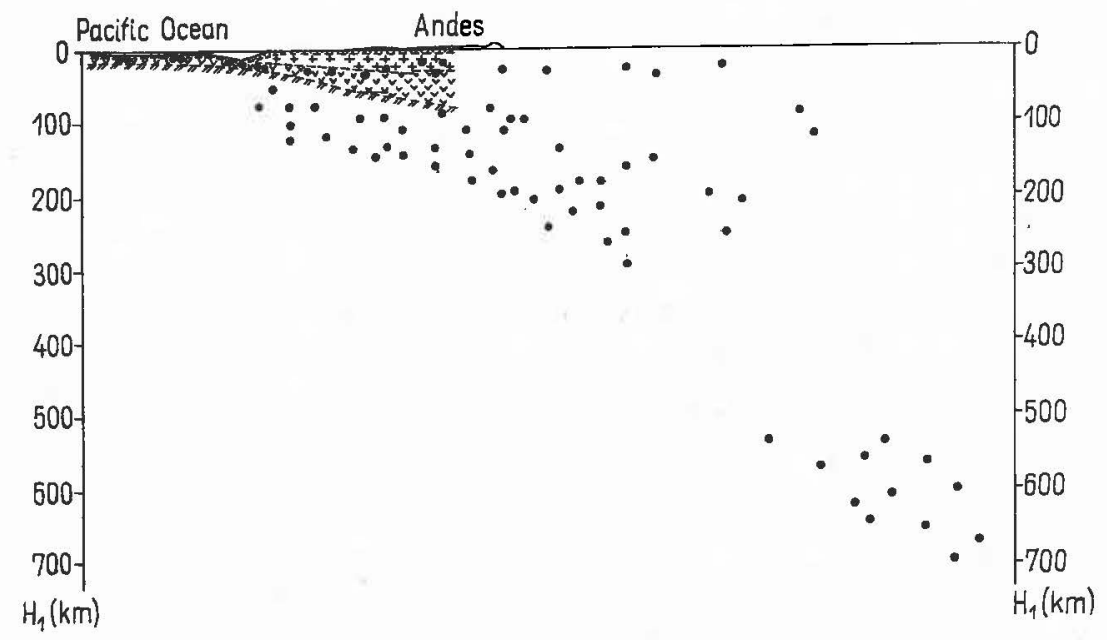

Fig. 4

ocean. The gigantic plate of the oceanic type crust under the Pacific is bordered by two seismically slackened zones inclined downwards below the continent.

It has been recently revealed that in some regions the earth's upper shells consist of a block structure. Blocks of different sizes are found: from the smallest, included in separate layers, to very large ones which are often traversed by seismic boundaries. A paper by Risnichenko and Kosminskaya [19] is devoted to this problem.

Down to depths of about $200 \mathrm{~km}$ the blocks considered in the present paper are composed by the crust and upper mantle. This means that blocks of the first order, corresponding to continents and oceans, are discussed. Zones of focus concentration lying between such blocks will be called shift zones. Within separate blocks of this kind different types of crust can be considered.

For the section given in Fig. 6, data obtained by the author and also taken from publications by others were used. This section extends from the southern part of the Kurile-Kamchatka zone in the Pacific to the Andean range in South America. The Pacific crust-mantle block is clearly outlined, being separated from the continental blocks of Asia in the east and of South America in the west by shift zones, or zones of earthquake focus concentration, extending below the continents. If the shift zones lying in the interior and in deep-oceanic trenches on the surface of the earth were considered to border the Asian continental 
block, then they would include the marginal Okhotsk Sea and the island arc, like the Andean range in South America.

In Fig. 6 the averaged positions (Gutenberg) of the low velocity zones are marked. They occupy depths of $50-200 \mathrm{~km}$ under oceans and $100-200 \mathrm{~km}$ under continents. In the Kurile-Kamchatka shift zone no low velocity layer

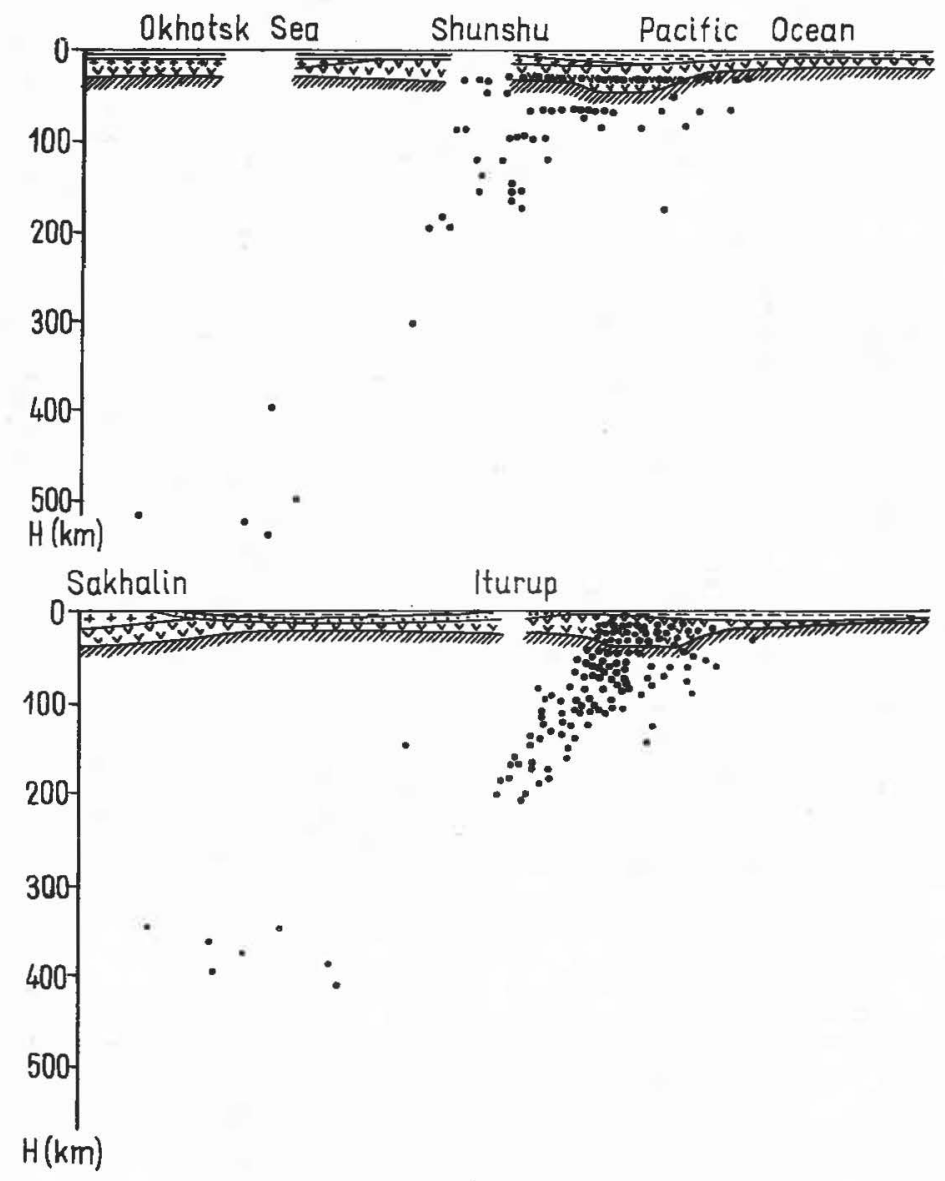

Fig. 5

was found by Fedotov [7, 8]. However, in absolute value the velocities observed there were somwhat lower than at corresponding depths below continents and oceans. In one of his papers Gutenberg pointed out, that in the Kurile-Kamchatka zone low velocities for P and S waves occupy depths from the foot of the crust down to about $200 \mathrm{~km}$. It appears that a shift zone is characterized by velocities lower than those of the ajoining parts of continental and oceanic blocks. 
According to seismological data the properties of the upper mantle below continents and oceans were considered so far to differ in the position of the low velocity zone. There is, however, every reason to consider the position of the low velocity zone within continents (see the works of Lukk and Nersesov in press), as well as within oceans (Woollard [17]) subject to considerable local variations.

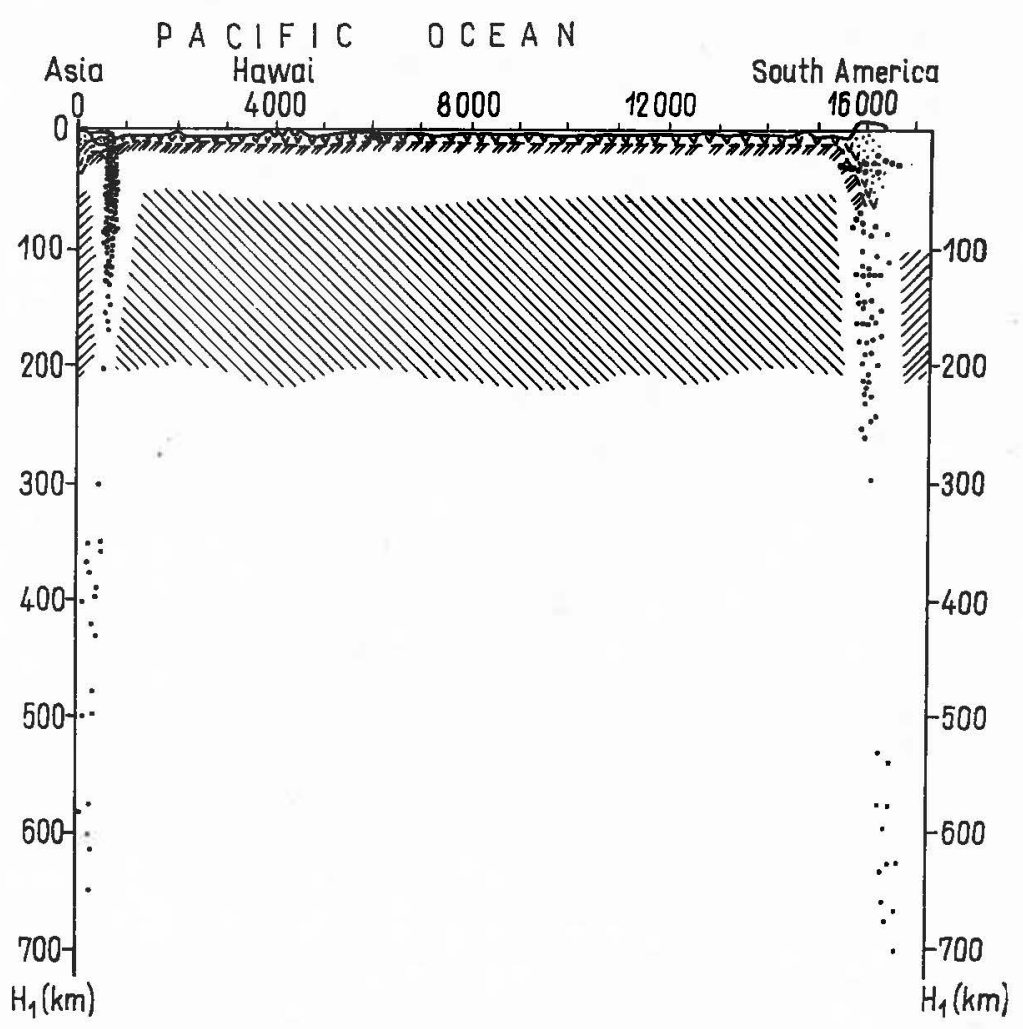

Fig. 6

A picture similar to that represented in Fig. 6 - of shift zones separating seismically more stable blocks - is observed on different scales in higher layers. Thus in the crust zones of focus concentration they separate more stable regions. A great number of weak earthquake foci fall close to known fractures. 


\section{REFERENCES}

[1] Kosminskaya, I. P. and Galperin - Косминская, И. П. и Галперин,: Строение коры в области перехода от азиатского континента к Тихому океану. Результаты МГГ, Изд. АН СССР, Москва (1964)

[2] Kondratev, O. K. and Gamburtsev, A. G. - Кондратьев, О. К. и Гамбурцев, А. Г.: Результаты сейсмических исследований в прибежной части восточной Антарктиды. Труды ИФЗ АН СССР, (1963)

[3] Solovev, O. N. and Gainanov, A. G. - Соловьев, О. Н. и Гайнанов, А. Г.: Особенности глубинного геологического строения переходной зоны от азиатского материка к Тихому океану в районе Курило-Камчатской островной луги. Советская геология 3 (1963)

[4] GoRyachev, A. V. - Горячев, А. В.: Основные закономерности геотектонического режима Курило-Камчатской складчатой зоны. ДАН СССР, 1421 (1962)

[5] Gurary, G. Z. and Soloveva, I. А. - Гурарий, Г. 3. И Соловьева, И. А.: Строение земной коры по геофизическим данным. Изв. АН СССР, М. (1963)

[6] Атлас землетрясений в СССР (1911-1957 г. г.) Изд. АН СССР, М. (1962)

[7] Fedotov, S. A., Kuzin, I. P. and Boвкov, M. F. - Федотов, С. А., Кузин И. П. и Бобков, М. Ф.: Детальные сейсмологические исследования на Камчатке в 19611962. Изв. АН СССР, серия геофизическая (1964)

[8] Fedotov, S. A., Gagdasarova, A. M., Kuzin, I. P. and Tarakanov, R. Z. - Федoтов, С. А., Гагдасарова, А. М., Кузин, И. П. и Тараканов, Р. 3.: О сейсмичности и глубинном строении юга Курильской островной дуги. ДАН СССР, 153 3 (1963)

[9] Savarensky, E. F. and KiRnos, D. P. - Саваренский, Е. Ф. и Кирнос, Д. П.: Элементы сейсмологии и сейсмометрии ГИТТЛ, М.-Л., (стр. 113). (1949)

[10] Balakina, L. M. - Балакина, Л. М.: Общие закономерности в направлениях главных напряжений, действующих в очагах землетрясений Тихоокеанского тектонического пояса. Изв. АН СССР, сер. геофиз., 2 (1962)

[11] Gamburtsev, G. А. - Гамбурцев, Г. А.: Состояние и перспективы прогноза землетрясений. Бюл. Совета Сейсмологии. I (1955)

[12] Gamburtsev, G. A. and Beloyusov, V. V. - Гамбурцев, Г. А. и Белоусов, В. В.: Прогнозы землетрясений. Избр. Труды Г. А. Гамбурцева, Изд. АН СССР, М. (1960)

[13] Gutenberg, B. and Richter, C.: Seismicity of the Earth. N. Y. (1954)

[14] Rudich, E. M. - Рудич, Е. М.: Основные закономерности развития Приморья, Сахалина и Японии, как зоны перехода от континента к океану. Изд. АН ССCР, М. (1962)

[15] Beniof, H.: Seismic Evidence for the Faultorigin of Oceanic Deeps. Bull. Geol. Soc. Am. 60 (1949)

[16] Petrushevsky, В. А. - Петрушевский, Б. А.: Вопросы геологической истории и тектоники Восточной Азив. Изд. Н. М. (1964)

[17] Fisher, R. and RaITt, R.: Topography and Structure of the Peru-Chile Trench. DeepSea Research, 9 (1962)

[18] Woollard, G. and Strange, W.: Gravity Anomalies and Crust of the Earth in the Pacific Basin. The Crust of the Pacific. Geophysical Monograph 6 (1962)

[19] Riznichenko, Yu. V. and Kosminskaya, I. P. - Ризниченко, Ю. В. и Косминская, И. П.: О природе слоистости земной коры и верхней мантии. ДАН $C C C P, 153,2$ (1963) 


\section{PRELIMINARY RESULTS OF DEEP SEISMIC SOUNDING IN POLAND}

B. Wojtczak-Gadomska, A. Guterch and J. Uchman

(Warsaw, Poland)

Research work on the structure of the crust of the earth by means of deep seismic soundings was instituted in recent years by the Institute of Geophysics, P. A. Sces.

Measurements were made in December 1960, September and December 1961 on the profile Radynia-Gdańsk Bay (profile length $485 \mathrm{~km}$ ), and in December

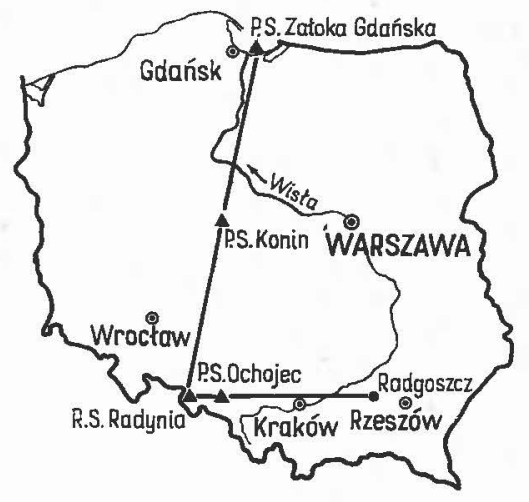

FIG. 1. Location of profiles and shotpoints

1963 on the profile Radynia-Radgoszcz $(242 \mathrm{~km})$. Location of the profiles and shotpoints are shown in the map (Fig. 1). Charges of from 500 to $3000 \mathrm{~kg}$ conventional seismic explosive were used. Recording of seismic waves was accomplished by means of a multi-channel seismic prospecting equipment, lent by Przedsiębiorstwo Geofizyki Przemyslu Naftowego Cracow and Przedsiębiorstwo Poszukiwań Geofizycznych, Warsaw.

Distance between the measuring points was approximately $25 \mathrm{~km}$. For measurement low-frequency $(7-10 \mathrm{~Hz})$ geophones were used, one per channel, and groups of higher-frequency $(25-35 \mathrm{~Hz})$ geophones in series connection.

The number of waves recorded at the particular measuring points was fairly considerable, namely in general over five, sometimes over 10 . Their accurate correlation was unfortunately not possible owing to relatively great distances between measuring points and non-uniform instrumentation. 
Travel Time curves and their interpretation

\section{A) Profile Radynia-Radgoszcz}

Travel-time curves of the interpretated waves for Radynia-Radgoszcz profile are shown in Fig. 2. The waves with apparent velocity value $6200 \mathrm{~km} / \mathrm{sec}$ correspond probably to waves refracted on the granitic layer. Their curves show an intercept time $t_{0}=1.45 \mathrm{sec}$.

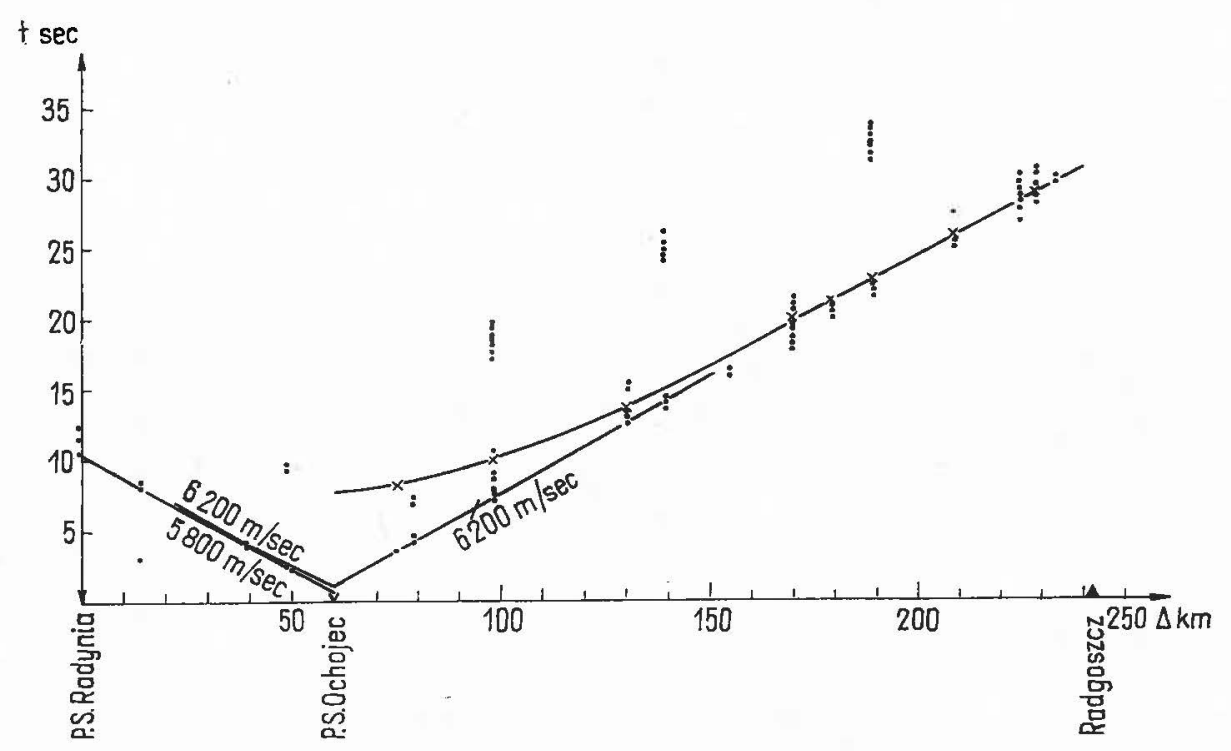

FIG. 2. Travel-time curves for Radynia-Radgoszcz profile

$$
\text { o }-\boldsymbol{P}_{\boldsymbol{g}} \text { waves, } \mathrm{x}-\boldsymbol{P}_{\text {refl }} \text { wave, - }- \text { other waves }
$$

Assuming for the sediment layer a mean velocity of $4300 \mathrm{~m} / \mathrm{sec}$, a depth of $4300 \mathrm{~m}$ to the granitic layer is obtained at the shotpoint Ochojec.

The further interpretation of deep-soundings data went primarily in the direction of segregation of waves reflected $P_{\text {ref } 1}$ from the Moho discontinuity beyond the critical angle. The dominant importance of those waves in the region beyond the starting point of the refraction wave has been demonstrated by many authors in theoretical research on dynamic crust models [1], [4] as well as on the basis of experimental data [1], [4], [6].

The singled-out waves $\left(\mathrm{P}_{\text {ref } 1}\right)$, whose travel-time curves are in Fig. 2, have the following characteristics allowing to consider them as waves reflected from the $M$ boundary.

1. Their apparent velocities, determined from the particular seismograms, show $t^{\text {he }}$ general trend to diminish from very high values (practically tending to $\infty$ )

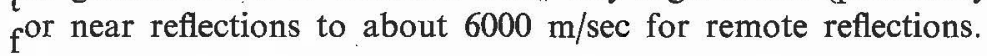


2. The apparent velocity on the straight part of the travel-time curve is approximately $6500 \mathrm{~m} / \mathrm{sec}$.

3. The effective velocity for the whole crust determined from the time-distance curve of the $P_{\text {refl }}$ waves by means of the method of constant differences, is $6200-6300 \mathrm{~m} / \mathrm{sec}$.

4. The ratio of amplitudes of the $P_{\text {ref } 1}$ waves to the $P$ waves recorded in the first arrivals on distances of $120-180 \mathrm{~km}$ lies within the range from about 3 to about 15 .

Crustal thickness below the shotpoint Ochojec, determined from the intercept time $7.73 \mathrm{sec}$ of the time-distance curve of $\mathrm{P}_{\text {ref1 }}$ waves for mean velocity 6200 $\mathrm{m} / \mathrm{sec}$, is $24.0 \mathrm{~km}$.

\section{B) Profile Radynia-Gdansk Bay}

Owing to the relatively more ample measuring material from the $236 \mathrm{~km}$ sector Radynia-Konin, the interpretation concentrated primarily on that sector.

The travel-time curves of the interpreted waves are in Fig. 3. Up to $80 \mathrm{~km}$ distance from the shotpoint Radynia the first arrivals are waves with an apparent velocity of $6100 \mathrm{~m} / \mathrm{sec}$. These are probably waves refracted on the granitic layer surface, their curve indicating an intercept time of $0.97 \mathrm{sec}$. Assuming for the

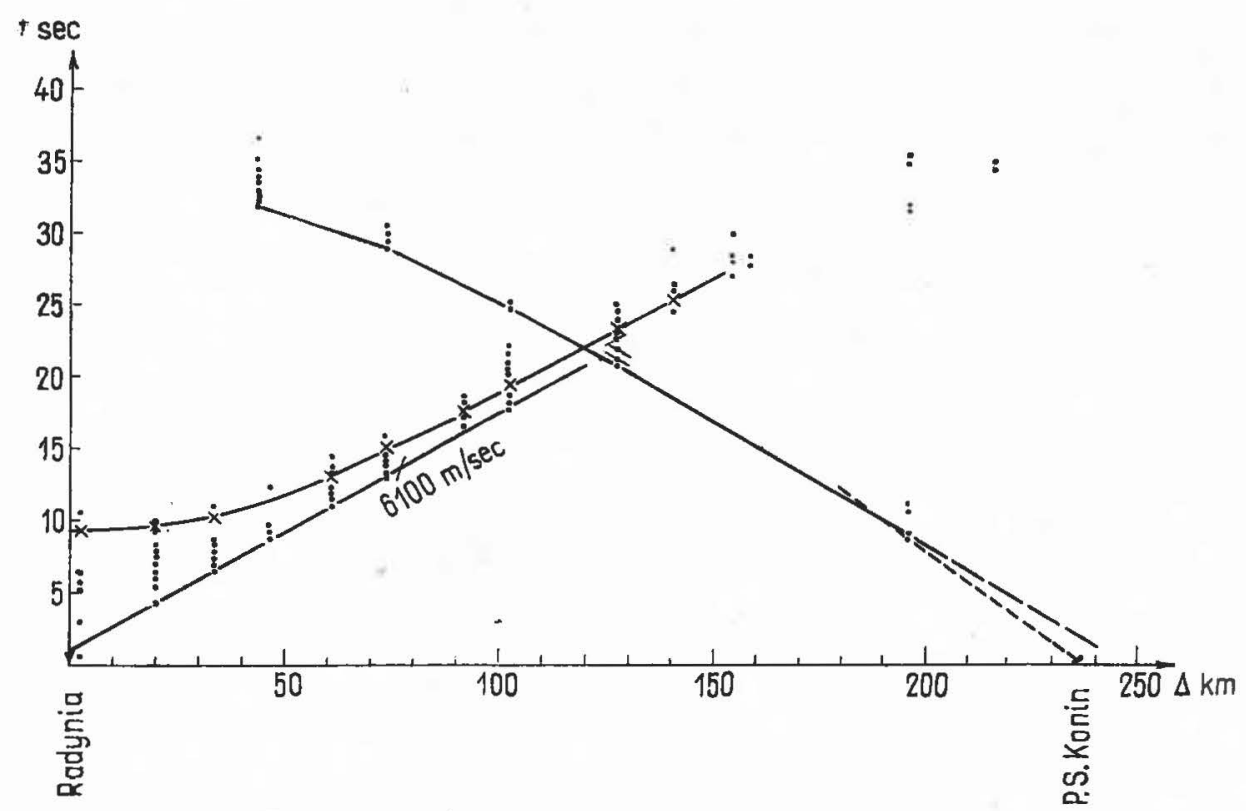

FIG. 3. Travel-time curves for Radynia-Konin profile o $-\boldsymbol{P}_{\boldsymbol{g}}$ waves, $\mathrm{x}-\boldsymbol{P}_{\text {refl }}$ waves, - other waves 
sediment layer a mean velocity of $4300 \mathrm{~m} / \mathrm{sec}$ we obtain for the shotpoint Radynia a depth value to the crystallic bed surface of $2900 \mathrm{~m}$.

Similarly as in profile $A$, the $\mathrm{P}_{\text {refi }}$ waves reflected from the M boundary were separated too. They show the following characteristics:

1. their apparent velocities defined from the particular seismograms diminish from very high values at $2-20 \mathrm{~km}$ distances to $6000-7000 \mathrm{~m} / \mathrm{sec}$ on distances of over $70 \mathrm{~km}$ from the shotpoint,

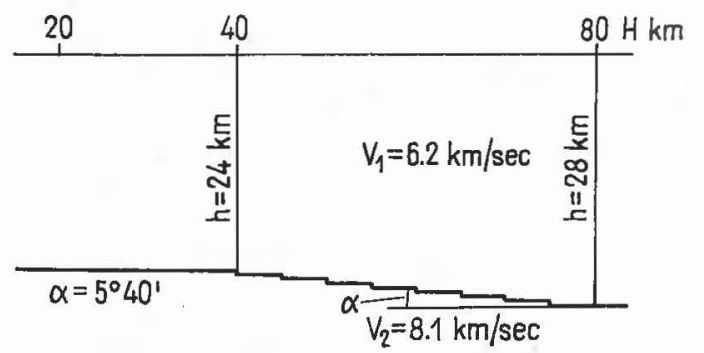

FIG. 4. Crustal model for profile Radynia-Radgoszcz

2. the apparent velocity on the straight part of the $P_{\text {ref1 }}$ wave curve is 6400 $\mathrm{m} / \mathrm{sec}$,

3. their effective velocity computed with the method of constant differences is $6000 \mathrm{~m} / \mathrm{sec}$.

Assuming from the $P_{\text {ref } 1}$ waves curve an intercept time equalling $9.16 \mathrm{sec}$ and a mean velocity of $6200 \mathrm{~m} / \mathrm{sec}$, a value of $28.5 \mathrm{~km}$ was obtained for the crustal thickness below the shotpoint Radynia.

Northwards from shotpoint Konin no first arrivals with velocity of the order of $6000 \mathrm{~m} / \mathrm{sec}$ are obtained up to $95 \mathrm{~km}$ distance. The available material is insufficient for accurate computation of depth to the crystallic bed. According to approximate estimation it is not less than $12 \mathrm{~km}$.

To get a more acsurate conception of the crustal structure on the observed profiles, a number of crust model variants, composed of homogeneous layers, were considered for the purpose of finding the model giving best agreement between the computed travel-time curve and the experimental curve for waves reflected from the $\mathbf{M}$ boundary.

For the profile Radynia-Radgoszcz (sector Ochojec-Radgoszcz) several three-, two- and one-layered models were tested with $M$ surface placed horizontally, as well as inclined at different angles. Computations were made for models with total thickness of crust from 24 to $28 \mathrm{~km}$. Velocities in the whole crust were approximately $6200 \mathrm{~m} / \mathrm{sec}$. In result of consecutive approximations a model with a steplike increase of depth to the $M$ surface was chosen (Fig. 4), whose traveltime curve of reflected waves shows the best agreement with the experimental curve of the waves reflected from the boundary.

This model indicates a crustal thickness of $24 \mathrm{~km}$ at a distance of approximately $40 \mathrm{~km}$ from shotpoint Ochojec, whence the $\mathrm{M}$ boundary begins to sink gradually, 
reaching about $28 \mathrm{~km}$ depth at $80 \mathrm{~km}$ distance (the reflection was recorded at approximately $160 \mathrm{~km}$ ).

A similar interpretation was made for profile Radynia-Konin.

The best agreement with experimental curve shows the crustal models as in Fig. 5, with steplike change of depth to $M$ boundary.

In accordance with this model the depth of $M$ surface diminishes from $28.5 \mathrm{~km}$ at shotpoint Radynia to $26.3 \mathrm{~km}$ at $25 \mathrm{~km}$ distance northwards from Radynia.

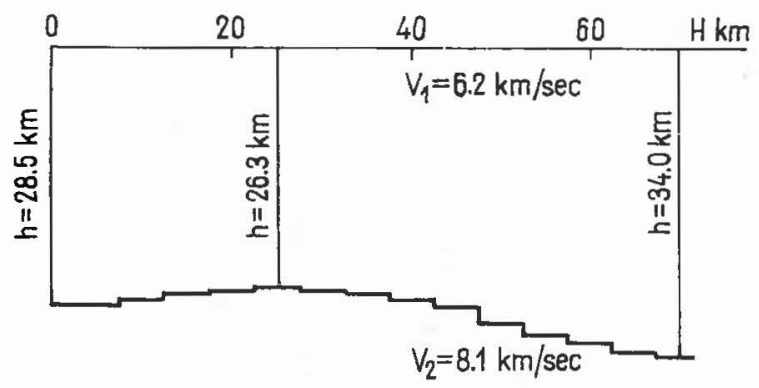

Fig. 5. Crustal model for profile Radynia-Konin

Then the $M$ surface begins to increase gradually, reaching about $34 \mathrm{~km}$ depth at $70 \mathrm{~km}$ distance from the shotpoint (the reflection was recorded at approximately $140 \mathrm{~km}$ ).

Our deep soundings had the character of point soundings. The obtained traveltime curve of the waves reflected from the $M$ boundary is then an average curve and for this reason the model with steplike depth of boundary $-M$ is an admissible representation of the reflecting surface.

A tentative dynamic interpretation was made in respect to the experimental data obtained on the profile Radynia-Radgoszcz (for both shotpoints). It was assumed that the waves recorded as first arrivals $\left(P_{\mathrm{I}}\right)$ at the distances $120-180 \mathrm{~km}$ from the shotpoints Radynia and Ochojec were waves refracted at the M boundary. For kinematic reasons this assumption is legitimate for a crustal model of approximately $25 \mathrm{~km}$ thickness. On this basis a curve was plotted indicating the ratios of amplitudes of $\frac{\mathbf{P}_{\text {ref1 }}}{\mathbf{P}_{\text {refr }}}$ waves to distance.

The experimental curve together with the curve computed for crustal models of $25 \mathrm{~km}$ and $30 \mathrm{~km}$ thickness are shown in Fig. 6. The respective computations were based on the theoretical relationship $\frac{\mathrm{P}_{\text {ref1 }}}{\mathrm{P}_{\text {refr }}}=f(\Delta)$, given by A. M. Epinatieva [2]. In computation of the theoretical curves the refraction at the boundary surface between the sediment layer and the crystallic bed was taken in consideration. The dependance of the modulus of the reflection coefficient on the angle of wave incidence on the M surface was taken from the curve given in paper [5] 
for the ratio of velocities $\frac{V_{1}}{V_{2}}=0.75$ and of densities $\frac{\rho_{1}}{\rho_{2}}=0.85$.

The computation were made for the waves frequency equalling $10 \mathrm{~Hz}$. The maximum of the experimental curve occurring at a distance of $142 \mathrm{~km}$ is derivated by $4 \mathrm{~km}$ in respect to the maxima of the curves $1,2,3$, computed for model $a$ )

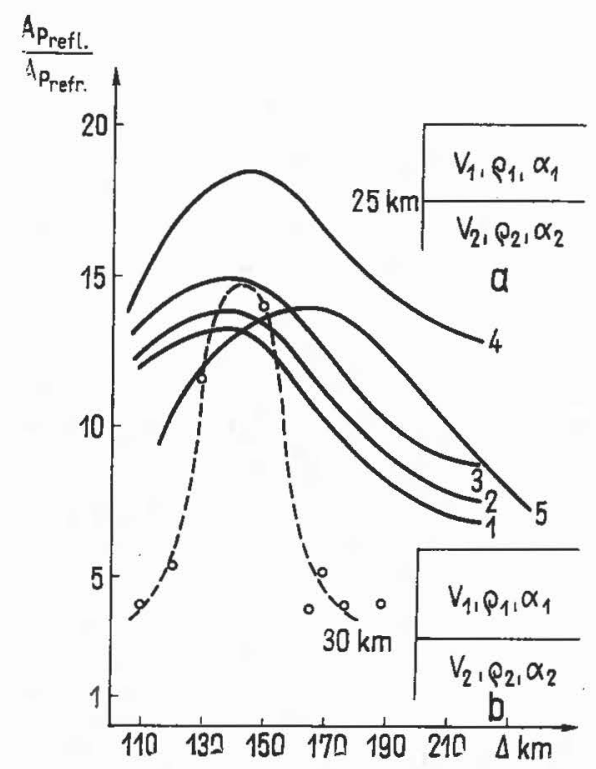

FIG. 6. Curves of relations $\frac{A_{P_{\text {refl }}}}{A_{P_{\text {refr }}}}=f(\Delta)$

experimental curve $\frac{A_{\text {Pref } 1}}{A P_{\text {refr }}}=f(\Delta)$ for profile Radynia-Radgoszcz;

computed curves for crustal model $V_{1}=6.25 \mathrm{~km} / \mathrm{sec}, \rho_{1}=2.88 \mathrm{~g} / \mathrm{cm}^{3}, V_{2}=8.1$

$\mathrm{km} / \mathrm{sec}, \rho_{2}=3.38 \mathrm{~g} / \mathrm{cm}^{2}$;

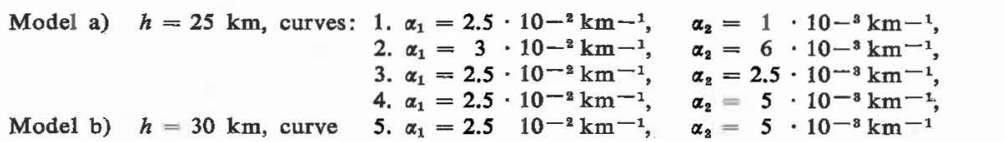

with $25 \mathrm{~km}$ thickness. On the other hand, the maximum of the curve computed for model $b$ ) with $30 \mathrm{~km}$ thickness occurs at a distance of $165 \mathrm{~km}$. Thence results that a model of $26 \mathrm{~km}$ thickness corresponds to the experimental curve. This conclusion supports the kinematic interpretation performed on the profile Radynia-Radgoszcz.

The discrepancy between the experimental curve and the computated curves $1,2,3$ (finding its expression in greater steepness of the experimental compared with the computed curves) is pointed out in numerous studies [2], [4] and has a good reason. Furthermore, the M surface was found to be inclined on the studied 
profile, whereas the corresponding experessions on which the theoretical computations were based, were derived for horizontal discontinuity boundaries.

Comparison of the obtained results with the crustal thickness values derived by Z. Fajklewicz [3] from gravimetric computations indicates that the gravimetric data exceed in both cases the seismic results by the same figure of $5-6 \mathrm{~km}$.

Measurements on both profiles shall be continued in 1964 and the following years.

\section{REFERENCES}

[1] Alekseev, A. S. et al.: Izv. AN SSSR, ser. geofiz., 11 (1963) 1 (1964)

[2] EPINATIEvA, A. M.: Trudy Inst. Fiziki Zemli AN SSSR 14 (1960)

[3] FAJKLEwicz, Z.: Glebokość zalegania nieciaglości Mohorovicicia w Polsce wedīug danych grawimetrycznych [Depth to the Moho discontinuity in Poland according to gravimetric data. Manuscript ready for publication]

[4] Glubinnee sejsmičeskoe zondirowanie zemnoj kory v SSSR [Deep seismic sounding of the Earth Crust in SSSR], Leningrad 1962

[5] McCamy, K., Meyer, R. P. and Smith, T. J.: Bull. Seism. Soc. Am., 4 (1962)

[6] Tatél, H. E. and Tuve, M. A.: Geol. Soc. Am. Spec. Paper 62 35-50 (1955) 
DETERMINATION OF THE THICKNESS OF THE EARTH'S CRUST IN THE CARPATHIANS FROM THE DISPERSION OF THE SURFACE WAVES FROM CHILEAN

\author{
EARTHQUAKES IN 1960 \\ O. I. YuRKeVICH and B. I. VoloseCKY
}

(Kiev, USSR)

In 1960 the network of the seismic stations of the Ukrainian SSR consisted of four stations established in the Carpathians. The stations are situated along both sides of the Carpathians mountains - Lvov and Chernovtsy in the Precarpathians, Uzhgorod and Rakhov - in the Transcarpathians. Thus, the waves of the Chilean earthquakes, when stations are duly chosen, are transsecting the Carpathian range in two locations (Fig. 1).

The Precarpathian stations, Lvov and Chernovtsy are both equipped with the typical instrument SK, while Uzhgorod and Rakhov possess the regional instrument SCH. For registering the phase disfigurements due to imperfections of the instrument, for each station corrections were made after the phase displacement.

For the determination of the eart crusth's thickness in the Carpathian zone the following data of earthquakes were used:

\begin{tabular}{|c|c|c|c|c|c|c|c|}
\hline \multirow{2}{*}{ No. } & \multirow{2}{*}{ Date } & \multicolumn{3}{|c|}{ Origin time } & \multicolumn{2}{|c|}{ Location } & \multirow{2}{*}{$M$} \\
\hline & & $h$ & $m$ & $s$ & $\phi$ & $\lambda$ & \\
\hline 1. & 21. V. 1960 & 12 & 02 & 50 & 37.5 & 73.5 & 7.5 \\
\hline 2. & 22. V. 1960 & 10 & 30 & 39 & 38.0 & 73.5 & 7.4 \\
\hline 3. & 25. V. 1960 & 08 & 34 & 33 & 45.0 & 76.0 & 7.1 \\
\hline 4. & 6. VI. 1960 & 05 & 55 & 44 & 45.5 & 73.5 & 7.5 \\
\hline 5. & 20. VI. 1960 & 02 & 01 & 08 & 38.0 & 73.5 & 7.2 \\
\hline
\end{tabular}

The determination of the earth's crust thickness was performed according to the dispersion of the surface waves by means of the phase velocity-method along two square-crossings of the Carpathian mountains: Uzhgorod-Lvov and Rakhov-Chernovtsy. For all the earthquakes the epicenter azimuths were established on all stations, as well as profile azimuths for Uzhgorod-Lvov and Rakhov-Chernovtsy.

Direction azimuths "epicenter-station" differ in average from the direction azimuth Uzhgorod--Lvov at $20^{\circ}$, while from the direction azimuth RakhovChernovtsy at $8^{\circ}$. The distance between the stations Uzhgorod and Lvov is 182 $\mathrm{km}$, and between Rakhov and Chernovtsy $136 \mathrm{~km}$.

The situation of epicenters relative to the stations (azimuth $70^{\circ}$ ) resulted in the fact, that the transversal Love-waves were well recorded on the component 
$\mathrm{N}-\mathrm{S}$, and very indistinctly on the component E-W. It is to be noted, that it is very difficult to correlate in equity both components: the component E-W is rather disfiguring than correcting the record of the component N-S; thus, when the determinations of the eart crusth's thickness were made according to Lovewaves, only the N-S component was used. As a final result, the thickness of the earth's crust in the Uzhgorod-Lvov direction was established after the entrance

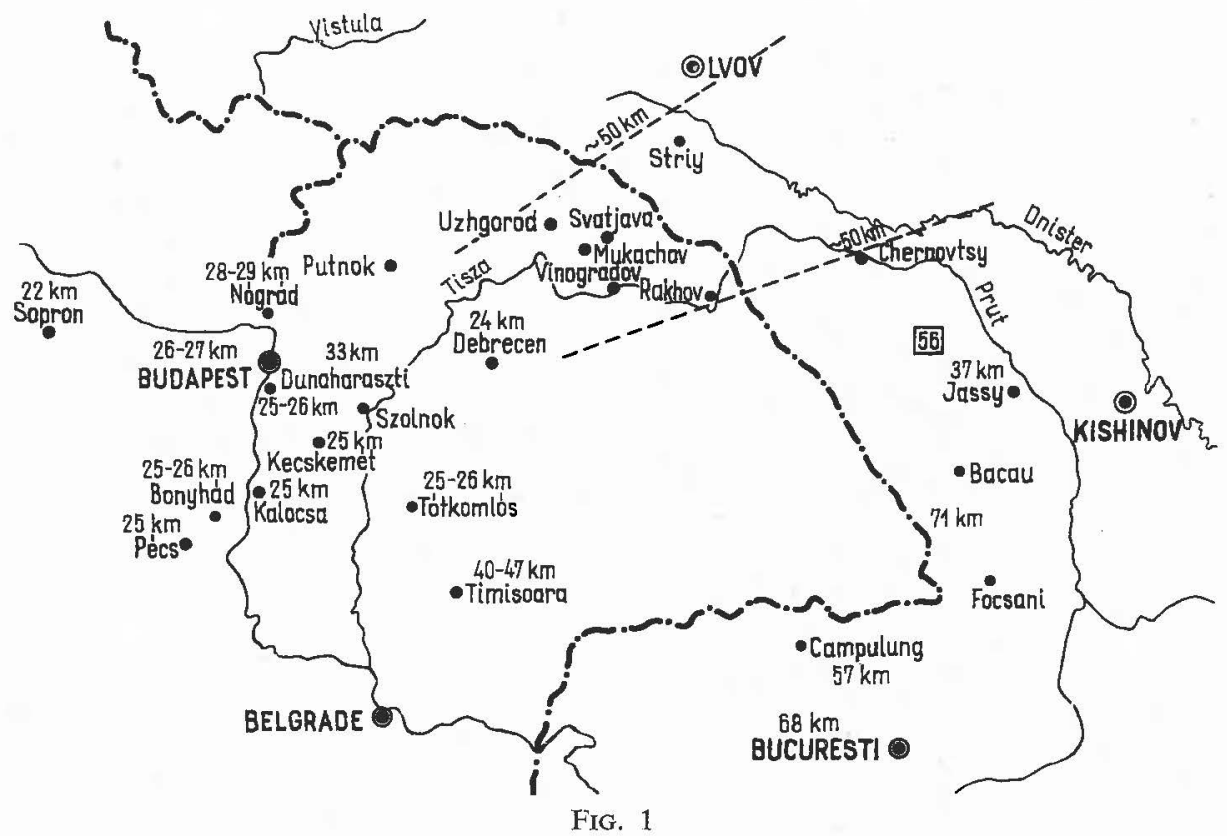

of the transversal Love-waves and the longitudinal Rayleigh-waves while in the Rakhov-Chernovtsy direction, because of very weak entrances of the Lovewaves - only according to Rayleigh-waves.

Dispersion curves were calculated for models by interrelations of the velocities of dispersion of the transversal seismic waves in granitic and basaltic layers and in the subcrustal substratum and relatively to the densities of different-strata are $b_{2} / b_{1}=1.127, b_{3} / b_{1}=1.324, \rho_{2} / \rho_{1}=1.095, \rho_{3} / \rho_{1}=1.204$ and at the most probable values of the velocity of the transversal waves radiating in the Carpathians are $b_{1}=3.3 \mathrm{~km} / \mathrm{sec}$, and $b_{1}=3.4 \mathrm{~km} / \mathrm{sec}$. The interrelation of stratal thickness was chosen as $h_{1} / H=0.3,0.5$ and 0.7 for the general thickness of the general crust $H=35,45,55$ and $65 \mathrm{~km}$.

The clearest picture of Rayleigh-waves is displayed in the direction UzhgorodLvov: at $b_{1}=3.3 \mathrm{~km} / \mathrm{sec}, H=45 \mathrm{~km}$, at $b_{1}=3.4 \mathrm{~km} / \mathrm{sec}, H=55 \mathrm{~km}$.

Along the direction of Rakhov-Chernovtsy the dispersion was recorded in a shorter range of periods and after Rayleigh-waves only. The earth's crust thickness in this direction was established to be $50-60 \mathrm{~km}$. 


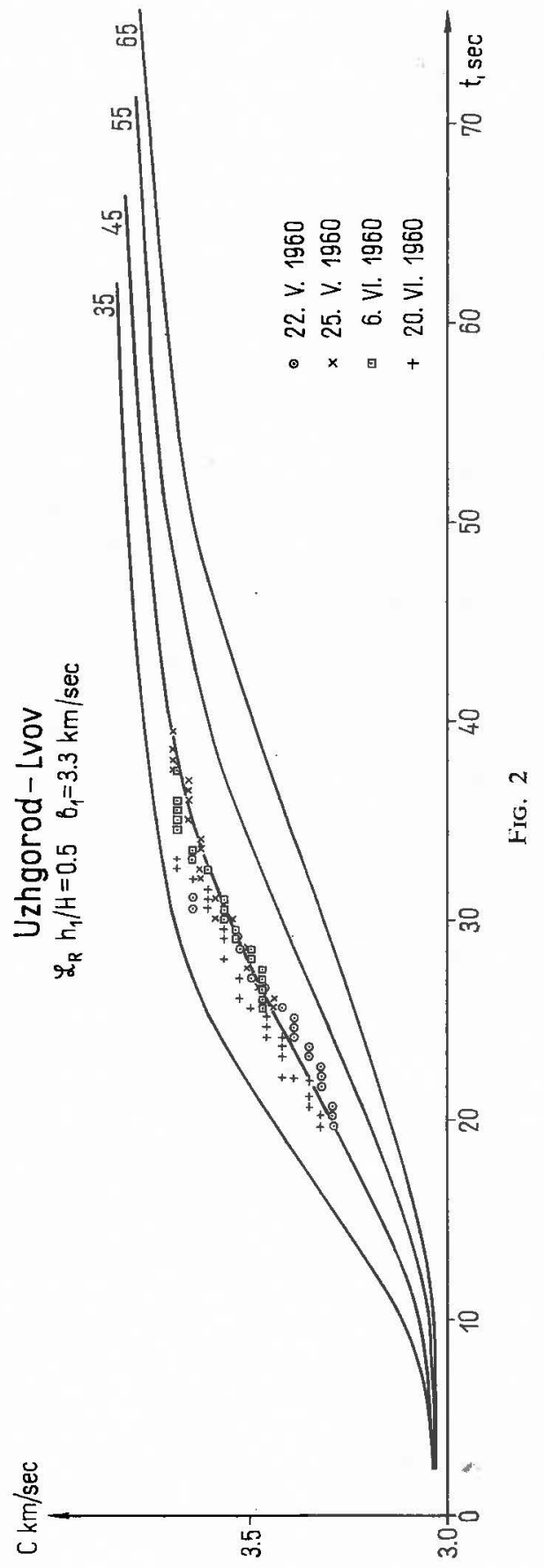




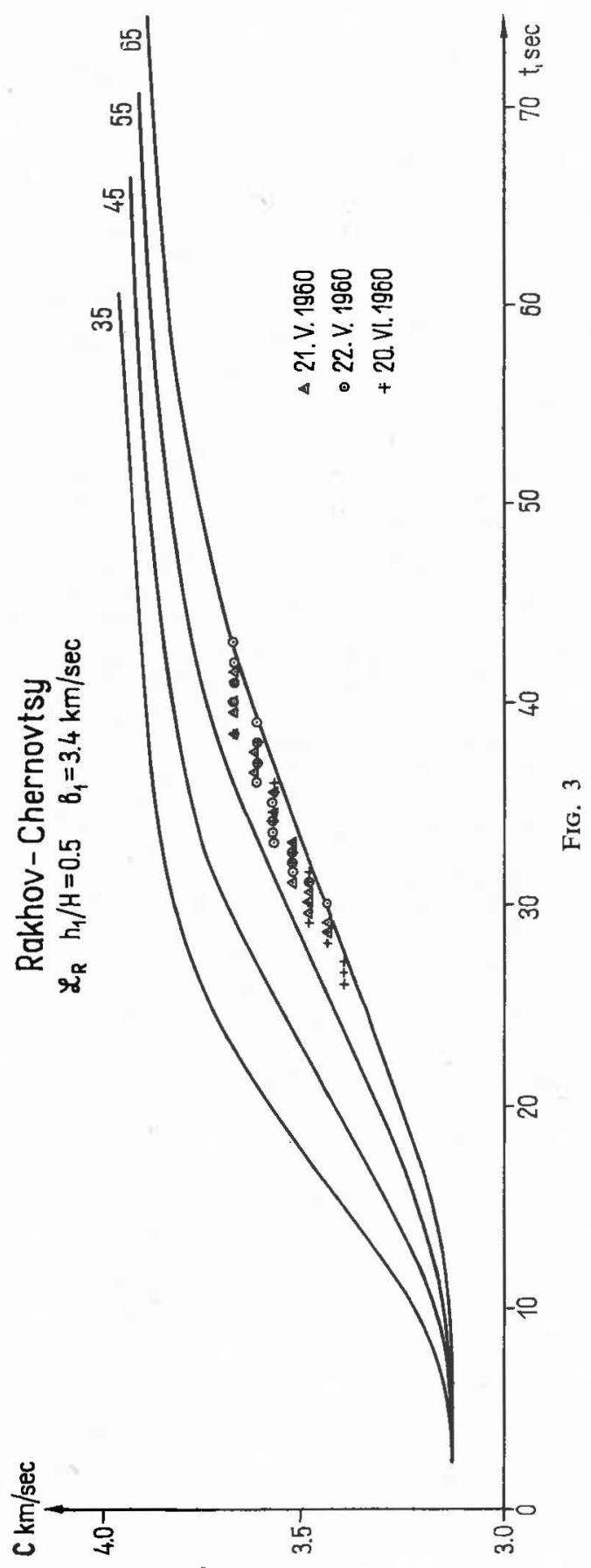




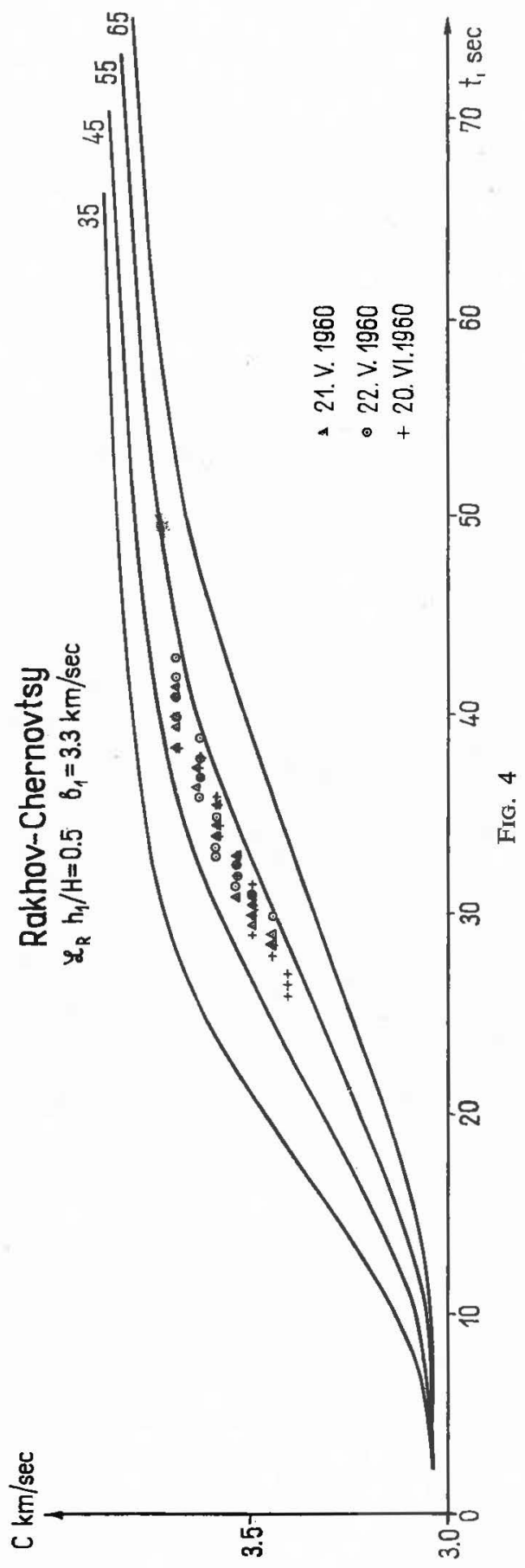




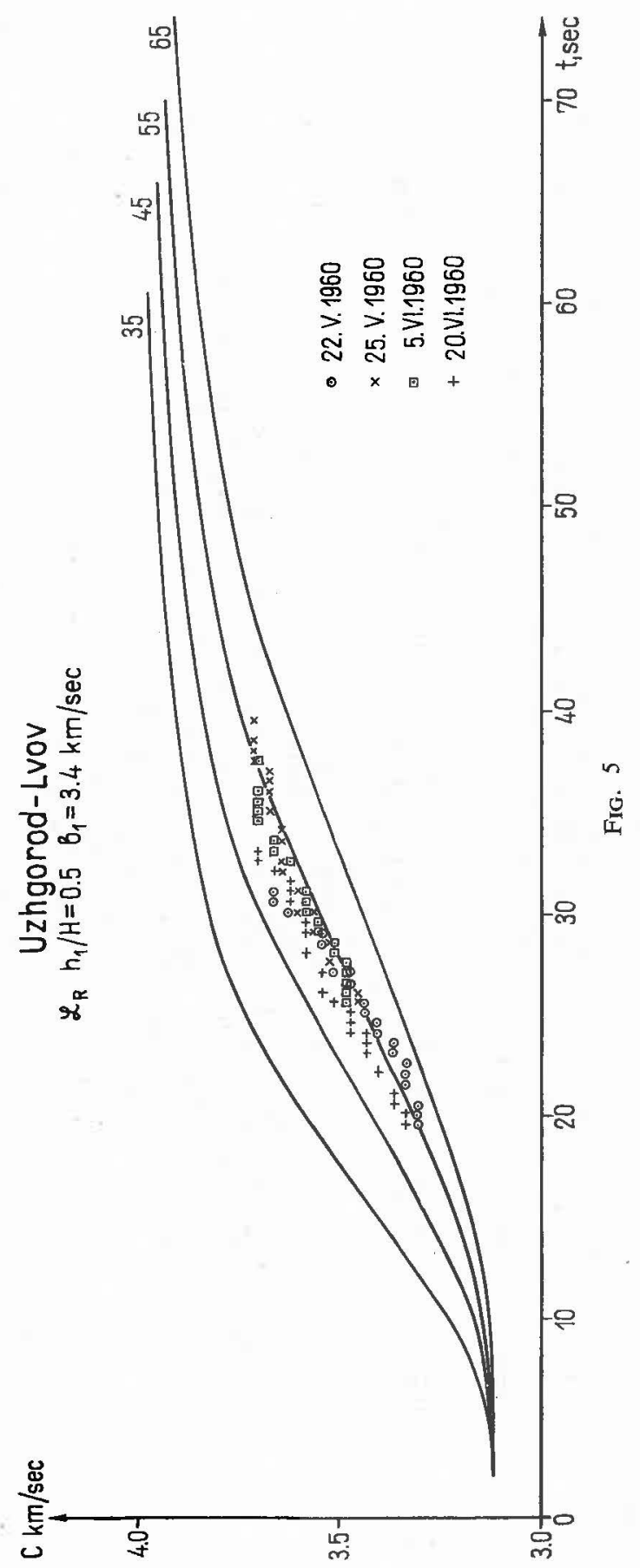


Thus, the following conclusion may be deducted: the thickness of the earth's crust along two almost normal transsections of the Carpathian mountains range is about $50-55 \mathrm{~km}$, at the interrelation of the granitic and the entire thickness of the earth's crust is $h_{1} / H=0.5$.

Until now, the thickness of the earth's crust in the Carpathian region had been established by near earthquakes and in one instance from gravitational and magnetic data. The most complete determinations are referred to the territory Rumanian Popular Republic, and a few, to the south-western part of Transcarpathians and to the Hungarian Plain.

The greatest thickness of the earth's crust in the Carpathians was recorded at the arc of overbending of the Carpathian Mountains - ranging circa $70 \mathrm{~km}$, and from the transitions to the foreland it is gradually decreasing, in Cîmpulung region the crust thickness is $57 \mathrm{~km}$, in Bucharest region is $68 \mathrm{~km}$; out of the two determinations of the earth's crust thickness in the Iasi region the most probable one is $37 \mathrm{~km}$.

On the territory of the Ukrainian SSR, besides the determinations of the earth's crust thickness at the Ukrainian crystalline massiv, according to the dispersion of surface waves, the thickness of the earth's crust has been establisted by means of recording the nearest earthquakes, and by aid of gravitational and magnetic data.

In 1959, the Institute of Earth's Physics under the direction of G. I. Galperin had traced the profiles DSS. The main of these investigations was the study of the optimal methodics and adaptation of the instrument under complicate Carpathian conditions; but simultaneously results were obtained regarding the thickness of the sedimentary layer upon the Ukrainian crystalline massiv (its absence has been established) and on the Carpathian mountains range (the sedimentary complex ranging between $7-8 \mathrm{~km}$ ).

As it is evident from the collected data that the thickness of the earth's crust under the Carpathian mountains, obtained by the dispersion of superficial waves, are $H=50-55 \mathrm{~km}$.

The determinations of the earth's crust thickness according to the dispersion of the surface waves of the Chilean earthquakes may be considered as preliminary.

When more detailed determinations will be performed, the dispersion curves will be compared with theoretically calculated curves for different velocities and different interrelations of thickness as well as granitic and basaltic layers, with regard of the sedimentary ones, likewise of the general thickness of the earth's crust as a whole, adaptable for different regions of the Carpathian mountains and of Precarpathians.

\section{REFERENCES}

[1] Borisov, A. A. and Kruglyakova G. I. - Борисов, А. А. и Круглякова, Г. И.: О глубинном строении земной коры Закарпатья. Известия $A H$ CCCP, cep. геоф. № 11 1962, И-о АН СССР, Москва 
[2 ] Galperin, E. I. Raykher, L. Ts. and Shaffansky, A. V. - Гальперин, E. И., Райxep, Л. Ц. и Шаффанский А. В.: Пересечение Восточных Карпат рекогносцировочным профилем глубинного сейсмического зондирования (ГС 3). Инбормачионный бюллетень МГГ № 4 И-о АН СССР Киев 1961

[3] GÁfi, J. and StegenA, L. - Гальфи, Я. и Штегена, Л.: Будова земной кори в Угорщини. Геологичний жсурнал т. ХХ, вип. 3. В-о АН УРСР, Киев 1960

[4] Gurary, G. Z. and Soloveva, I. А. - Гурарий, Г. З. и Соловьева, И. А.: Строение земной коры по геофизическим данным И-о Геологического Института $А Н$ CССР, Москва 1963

[5] KARnIK, V.: Zemetresni v Novogradskych Horach (Nograd). 20. II. 1951. Práce Geofysikalnih, Ustavu Cs. Ak. Ved. Geofys. sbornik, 2 (1953)

[6] Petrescu, C. and Radu, C.: Structura scoartei terestre in R. P. R. Studii şi cercetari VII. 2 E-ra Académisi RPR. (1962) 
SEISMICITY AND MAGNITUDE 



\title{
SEISMICITY AND QUATERNARY STRUCTURAL ACTIVITY IN THE NORTHERN RHINE DISTRICT
}

\author{
L. AHORNER
}

(Cologne, GFR)

By "Northern Rhine district" we mean NW Germany and the adjoining areas of Belgium and the Netherlands. In this part of Europe the seismicity is comparatively small. Only about three or four times per century larger earthquakes with a maximum intensity of grade VII or VIII of the MM-scale (magnitude 5-6) occur in the area of 170000 square $\mathrm{km}$, which includes the Lower, Middle and parts of the Upper Rhine valey, the Hercynic Rhenish massif (Rheinisches Schiefergebirge, Ardennes) and their surroundings. Nevertheless the above named region is of interest to the seismologist, because the earthquake activity can here be closely related to the well known younger geological tectogenesis, which is relatively simple in its moving style.

To prove the objective relations between the seismicity and the regional structural activity a special study has been undertaken of the earthquake activity of the Northern Rhine district covering the period 1750-1963. For this reason detailed macroseismic and microseismic investigations had to be carried out to determine the foci and other basic earthquake parameters as accurate as possible. Observation material has been taken from existing literature (e.g. from monographs and catalogues of Sieberg 1940, Sponheuer 1952, Charlier 1951, Van Rummelen 1945, Visser 1949, a.o.) and, beginning with 1903, also from station bulletins of nearby seismic stations. Besides this for shocks later than 1903 all available seismograms and the original macroseismic observation material were collected and re-interpreted. Thus for larger earthquakes in 1903-1963 the accuracy in epicenter determination is mostly better than $\pm 5-10 \mathrm{~km}$. In the same order lies the error in depth determination. The focal depths were calculated macroseismically by the method of Sponheuer (1958) or microseismically using the time difference $P_{g}-P_{n}$. Magnitudes are based on macroseismic observations according to formulas given by Sponheuer (1962) or on recorded amplitudes at nearby stations. With the results of the above mentioned investigations three seismic maps were prepared; each with an original scale of $1: 500000$.

The epicenter map (Fig. 1) gives the location of the epicenters classified according to magnitudes and accuracy in determination. For the time interval 1900-1963 the map contains shocks with magnitude $M \geq 2.5$ (full circles), for 1750-1899 those with $M<3.5$ (open circles). The heaviest earthquake in the Northern Rhine district during the last 200 years was that of February 1756 with its epicenter near Düren, west of Köln. Its magnitude is estimated to be near 6 (see also 
Sponheuer 1958). Another heavy shock with $M=5.8$ occurred in June 1938 in the Belgium region southwest of Bruxelles. 8 shocks in the last 200 years were of magnitude 5.0-5.4. The focal depths vary between several and $25 \mathrm{~km}$ as a maximum. Thus all foci lie within the earth's crust. Very superficial shocks (triangles

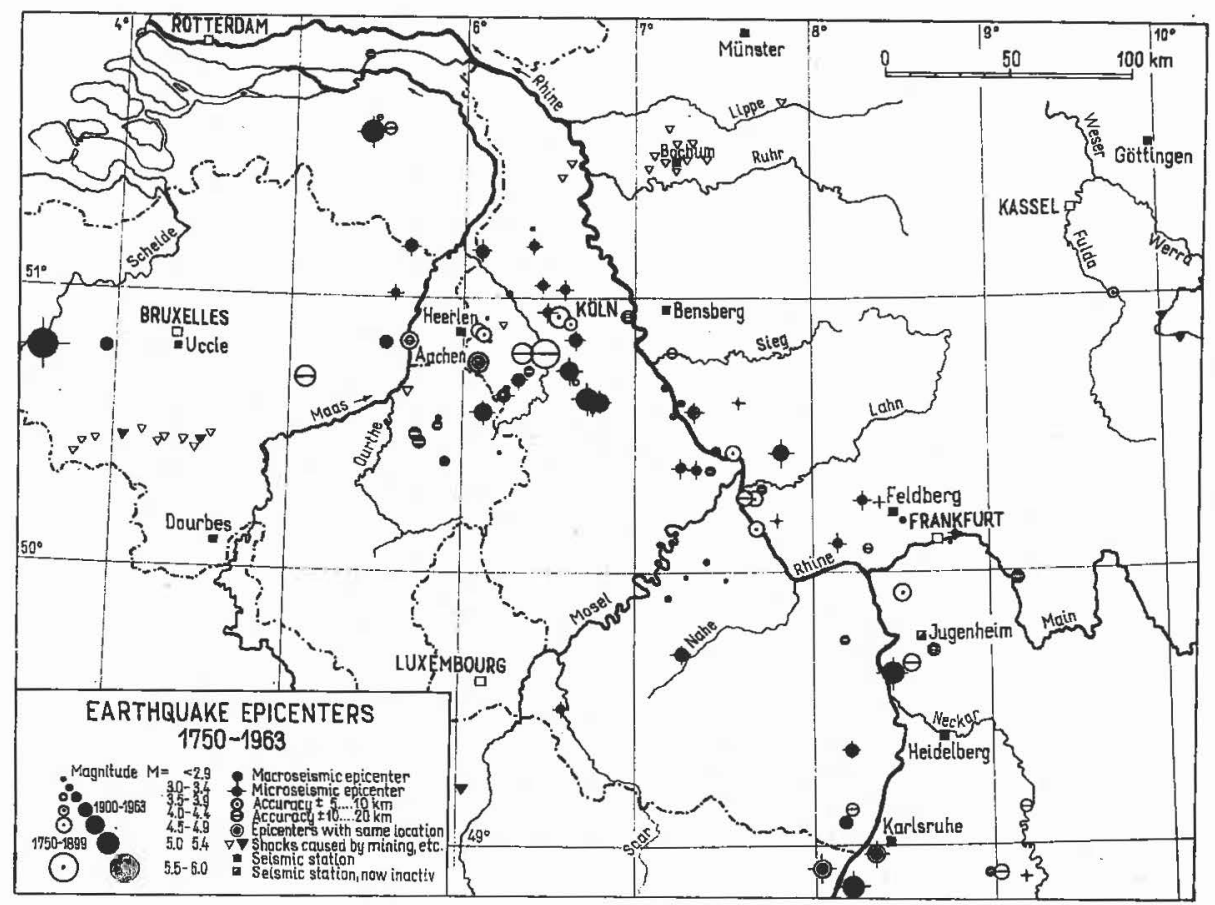

FIG. 1. Epicenter map

in the map) were observed in some mining districts, e.g. Ruhr coal basin, Werra district, Belgium coal basins south of Bruxelles. These shocks are obviously caused by mining work and therefore of no use for seismo-tectonic studies. As our epicenter map shows, nearly all true earthquakes lie within a zone approximately following the river-course of the Rhine.

In order to demonstrate this fact more clearly, a map of released seismic energy (Fig. 2) was compiled. The method used for the preparation of the map is as follows. For each shock the energy $E$ was calculated from the magnitude $M$ with the formula of Gutenberg \& Richter

$$
\log \mathrm{E}=9.4+2.14 \mathrm{M}-0.054 \mathrm{M}^{2} .
$$

The epicenter map was subdivided by a grid of lines measuring $0.2^{\circ}$ in length and width. On each cross-point of the grid lines the cente $r$ of a counting circle, drawn on transparent material, was put. The energies of a ll shocks lying within 


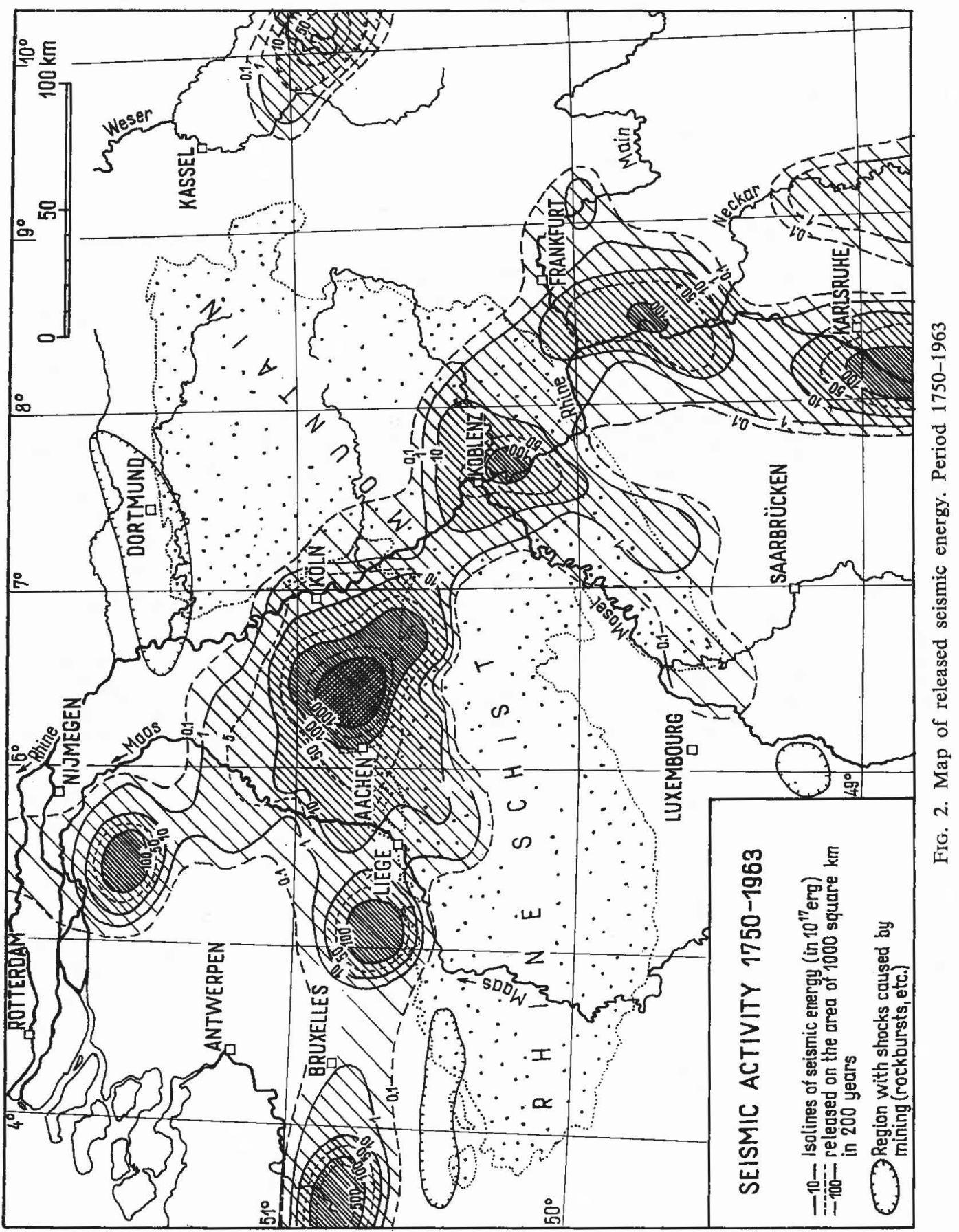


the circle were summed up and the total amount noted on the center-point. From the so obtained values of $\Sigma E$ finally isolines were drawn giving the seismic energy in units of $10^{12} \mathrm{erg}$ per 1000 square $\mathrm{km}$ during the last 200 years. In order to suppress very local shocks the lowest drawn isolines were those of $0.1 \cdot 10^{17} \mathrm{erg}$.

The so prepared map of seismic energy, released in 1750-1963, demonstrates very well the existence and the general course of a - let's say - "Rhenish" seismo-active zone, which goes in nearly the same way as the Rhine valley from

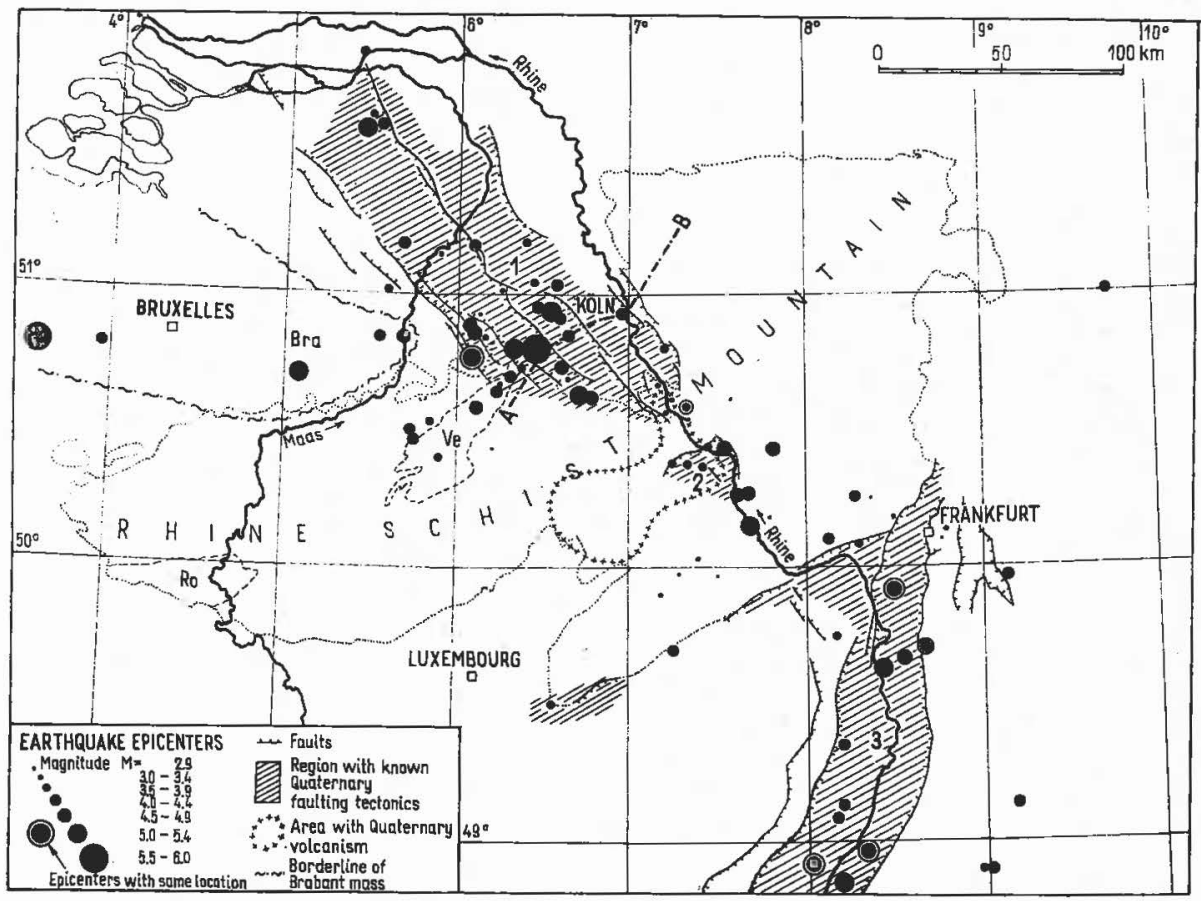

FIG. 3. Seismo-tectonic map of the Northern Rhine district. 1 = Lower Rhenish graben, 2 = Koblenz basin, $3=$ Upper Rhenish graben

Karlsruhe in the South, touching Koblenz and Köln, to the area of NijmegenRotterdam in the North; thus dividing the Hercynic Rhenish massif into a western and an eastern part. The highest amount of seismic energy was released in the Lower Rhenish area between Köln and Aachen, where locally more than 2000 . $\cdot 10^{17}$ erg per 1000 square $\mathrm{km}$ and 200 years can be calculated.

A secondary branch of the seismo-active zone is situated in the northwestern foreland of the Rhenish massif, crossing the Belgium territory from Liège to Bruxelles and Ostende. Its axis goes nearly East-West, that is parallel with the folds of the Cambro-Silurian Brabant mass. The Hunsrïck area in the southwestern border district of the Rhenish massif shows a smaller lateral branch. 
Other seismo-active regions besides the zones above mentioned are of minor importance. Great parts of the Northern Rhine district are even practically aseismic, that means, no shocks with $M \leq 3.3$ have occurred here in the last 200 years.

The knowledge of the marked "Rhenish" seismo-active zone leeds to the question: What relations are given to the geo-tectonical situation? In response we present a seismo-tectonic map of the Northern Rhine district (Fig. 3). Those regions are marked with hatching where stronger Quaternary faulting tectonics are known. They correspond mainly to the large regional fracture zones of the Lower Rhenish graben, the Koblenz basin, and the Upper Rhenish graben, where for longer geological times up to the present epoch vertical block movements are predominant with normal faults and regional flexure zones on the boundaries between regions of relative uplift and subsidence. The distribution of the epicenters (full circles on the map) gives the course of our "Rhenish" seismoactive zone. It is easy to find out, that there is a close coincidence between earthquake activity and Quaternary structural activity. Nearly all areas with very young fault structures in some extent show comparatively high seismicity, whereas fracture zones of pre-Quaternary or even pre-Neogene age, for instance the Hessian grabens between Frankfurt and Kassel, or the "Nord-Süd-Zone" of the Eifel, are largely aseismic. Only in the Belgium part of our seismo-active zone and in some parts within the Rhenish massif very young fault structures are still unknown. However, we have to consider that Quaternary faults are often hardly to prove by geological field work, especially in areas, where qualified sediments from the Quaternary epoch (e.g. river gravels) are absent.

From the analysis of seismicity it becomes clear, that the structural continuation of the Quaternary Upper Rhenish graben does not lie in the region of the Hessian grabens, as we may expect from Stille's idea of the "Mittelmeer-MjösenZone", but in the regions of the Middle and the Lower Rhine valley.

The Quaternary structural activity is especially well known in the Lower Rhenish graben zone by recent studies of Quitzow \& Vahlensieck (1955) and Ahorner (1962). Active faults form here good visible fault scarps in the modern morphology. A cross-section through the Lower Rhenish graben zone is given in Fig. 4. Gravity field, general geological section, Quaternary tectonics, precisions levelling and seismological vertical-section are combined to demonstrate the correlations between the present earthquake activity and crustal block movements in Tertiary, Quaternary and recent times. From Fig. 4 we can see, that a narrow structural zone in the western part of the Lower Rhenish graben, caracterized by relative high seismicity and relative "deep" shocks, shows also the highest velocity gradients of vertical crustal block movements in geological and recent times. For the time interval 1933-1952 an average rate of relative subsidence exceeding $0.5-1 \mathrm{~mm}$ per year can be calculated from precision levelling.

The observed maximum focal depths of $20-25 \mathrm{~km}$ permit the conclusion, that at least some main fault structures of the Lower Rhenish graben are reaching down into the earth's crust below the Conrad discontinuity, probable even in 


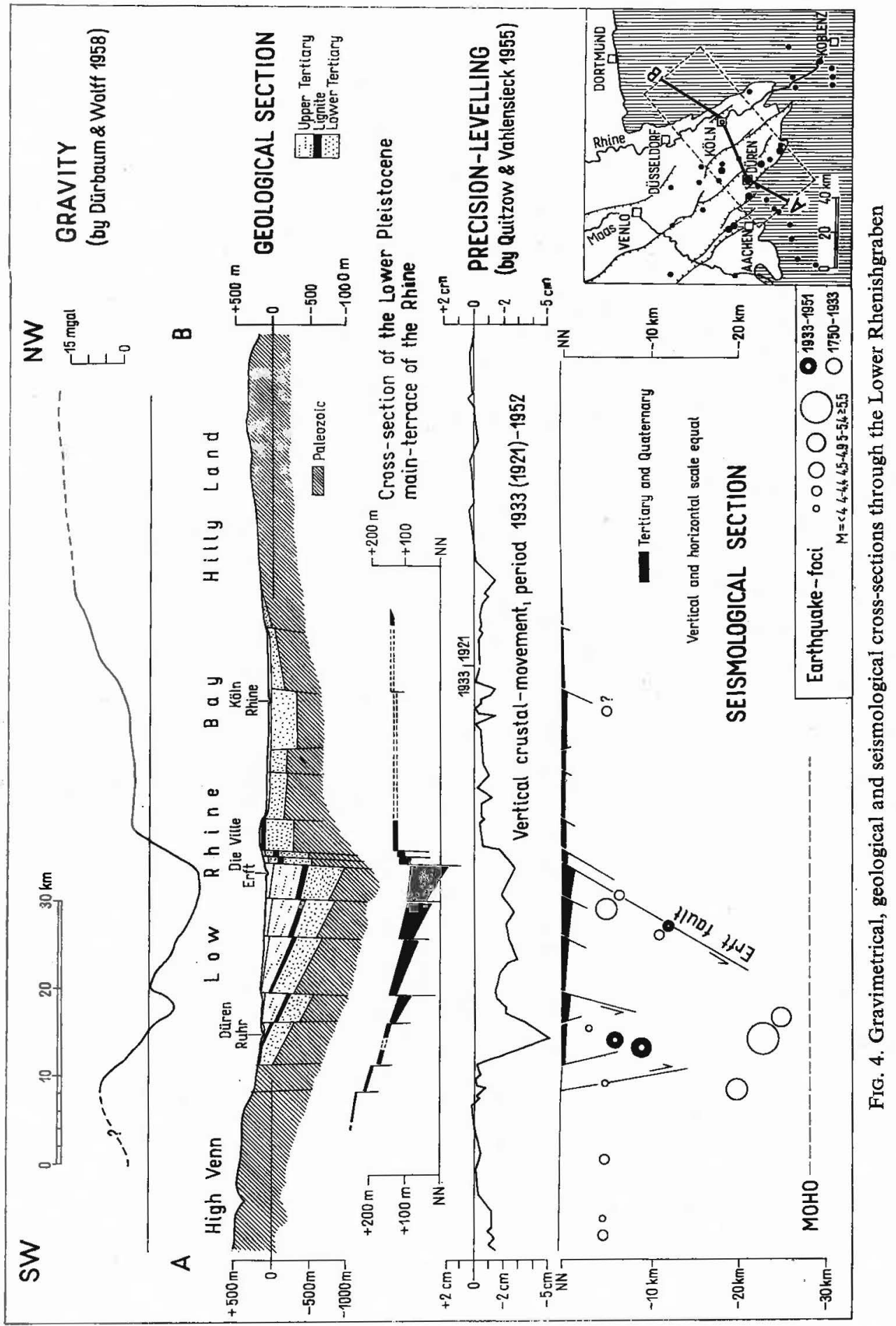


the nearness of the Mohorovičić discontinuity, which we may expect from explosion seismic work in about $30 \mathrm{~km}$ below the surface.

Some of the active fault zones are well exposed in larger excavations. As a typical example the outcrop-sketch of the significant "Erft-Sprung" forming the western border line of the Ville lignite district west of. Köln is given (Fig. 5).

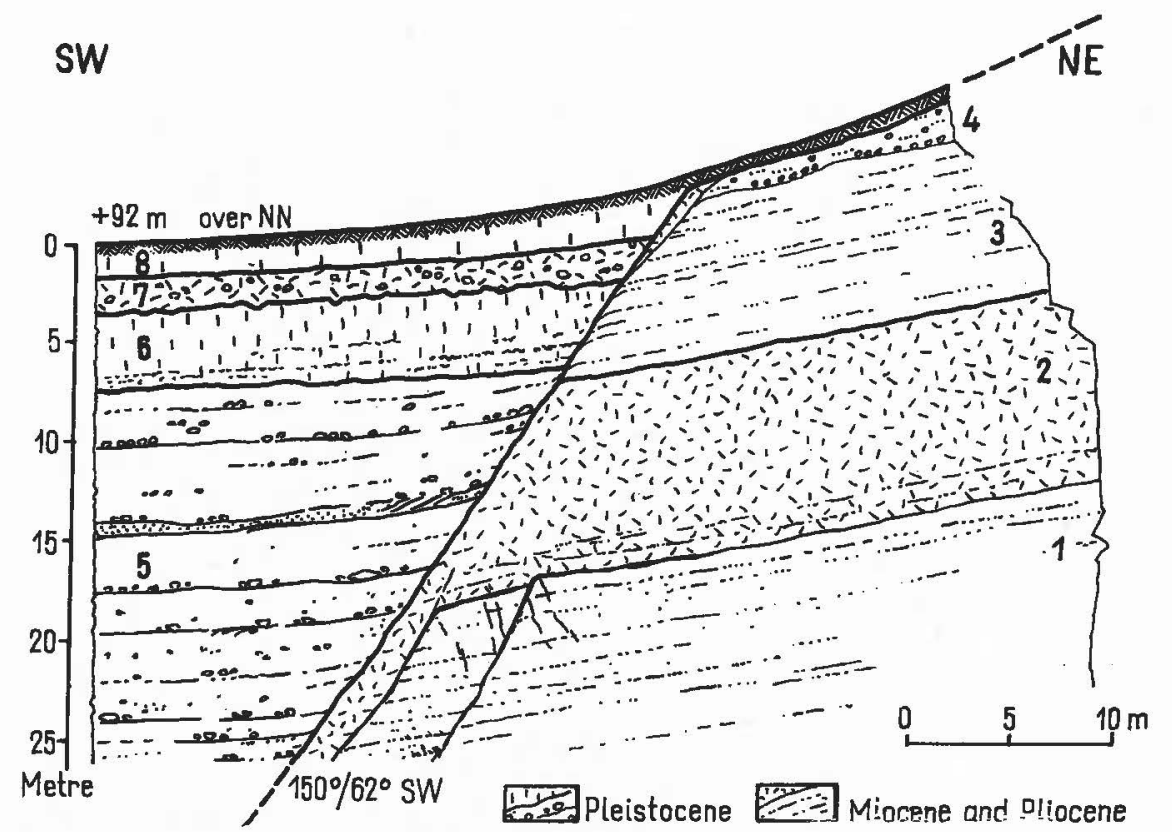

FIG. 5. Outcrop-sketch of an active fault. Erft-Sprung, Zentraltagebau Frechen, west of Köln.

6-8 = Loess, etc.; Younger Pleistocene (Riss, Würm), $5=$ River gravels of the main terrace of the Rhine; Older Pleistocene (Günz), $4=$ Pliocene, $1-3=$ Miocene

Quaternary river gravels of the main terrace of the Rhine (left in the figure on the downthrow side) are dislocated by this fault more than $100 \mathrm{~m}$ in the vertical. Even the very young loess layer is thrown. The total amount of post-Miocene throw exceeds more than $350 \mathrm{~m}$ (see also Fig. 4).

With the known hight differences of corresponding stratigraphic horizons on both sides of the "Erft-Sprung" system diagrams were prepared showing the development of vertical fault displacements with geological time (Fig. 6). The absolute time scale in million years used in the diagrams as ordinate is based mainly on Potassium-Argon dates published by Frechen (1959) and Evernden a.o. (1964). Diagram "a" gives the growth of Neogene-Quaternary fault displacement with absolute time; the total amount of post-Lower-Miocene throw is set $100 \%$. Diagram " $b$ " gives the calculated mean values of dislocation velocity in $\mathrm{mm}$ per year for limited stratigraphic units. Both diagrams indicate that during 
the Quaternary epoch the faulting activity seems to be more intensive than in Tertiary times back to nearly 20 million years. Of course our diagrams are not exact, as we do not know, whether dislocation movements are continuous within longer stratigraphic periods or not. But they show undoubtfully, that the Quaternary structural activity is of great significance for the tectogenesis of the Lower Rhenish

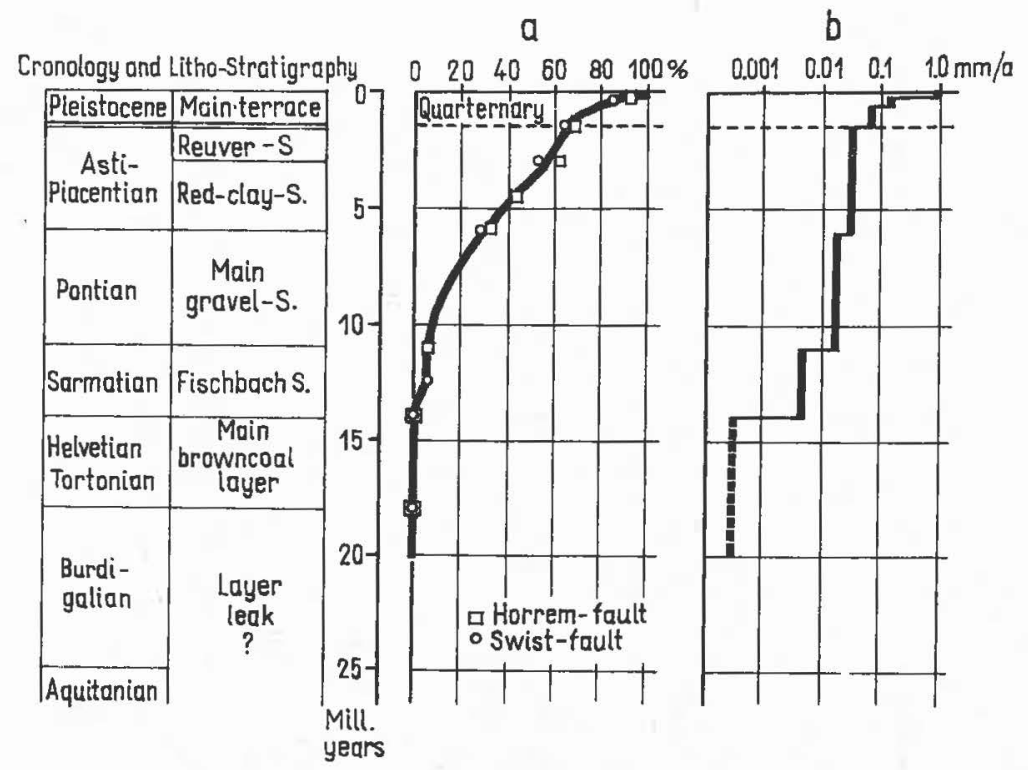

FIG. 6. Development of the vertical fault displacement with geological time, Erft-Sprung system.

Diagram a: growth of the total post-Lower-Miocene throw;

$\mathrm{b}$ : mean values of dislocation velocity in mm per year

graben zone. Geotectonical analyses in the Upper Rhenish graben and in the Koblenz area should lead to quite similar results. In all these regions the Quaternary rates in the development of the Neogene-Quaternary block movements are high and the structural activity is continuing with high efficiency until the present time. Therefore, the above mentioned statements seem to be typical for most regions of high seismicity in the Northern Rhine district.

It should be noted, that the knowledge of the correlation between Quaternary structural activity and seismicity might be of practical interest too, for instance in compiling a map of probable seismic risk.

Finally, the author refers to a paper by Schwarzbach (1963), who recently also points out a close relationship between the seismicity of parts of Central Europe and Quaternary structural activity - not structural activity in general.

Acknowledgments. This investigation has been carried out at the Abteilung für Erdbebengeologie, Geologisches Institut, Köln. The author is indebted to the 
Director, Prof. M. Schwarzbach, for various help and useful discussions; he also thanks the Directors of the neighbouring seismic stations, who have supported him with material. Financial help was given from the Deutsche Forschungsgemeinschaft, Godesberg.

(This is a brief report, a detailed study with the complete observation material will be published later.)

\section{REFERENCES}

[1] AHORNER, L.: Untersuchungen zur quartären Bruchtektonik der Niederrheinischen Bucht. - Eiszeitalter $u$. Gegenwart 13 24-105, Öhringen (1962)

[2] Charlier, Ch.: Etude systématique des tremblements de terre belges récents (19001950). 4e Partie: La Séismicité de la Belgique. - Publ. du Serv. Séism. et Gravim. de l'Obs. Roy. Belg., Ser. S, 10 60, Bruxelles (1951)

[3] Evernden, J. F., Savage, D. E., Curtis, G. H. and James, G. T.: Potassium Argon dates and the Cenozoic Mammalian Chronology of North America: - Am. Jour. Science 262 145-198, New Haven (1964)

[4] Frechen, J.: Die Tuffe des Laacher Vulkangebietes als quartärgeologische Leitgesteine und Zeitmarken. - Fortschr. Geol. Rheinld. u. Westf. 4 363-370, Krefeld (1959)

[5] Quitzow, H. W. and VAhlensiecK, O.: Über pleistozäne Gebirgsbildung und rezente Krustenbewegungen in der Niederrheinischen Bucht. - Geol. Rdsch. 43 56-67, Stuttgart (1955)

[6] SiEbERG, A.: Beiträge zum Erdbebenkatalog Deutschlands und der angrenzenden Gebiete für die Jahre 58 bis 1799. - Mitt. deutsch. Erdbebend. 21-111, Berlin (1940)

[7] Sponheuer, W.: Erdbebenkatalog Deutschlands und der angrenzenden Gebiete für die Jahre 1800 bis 1899. - Mitt. deutsch. Erdbebend. 3 1-195, Berlin (1952)

[8] Sponheuer, W.: Die Tiefen der Erdbebenherde in Deutschland auf Grund makroseismischer Berechnungen. - Ann. di Geofisica 11 157-167, Roma (1958)

[9] SPONHEUER, W.: Untersuchungen zur Seismizität von Deutschland. - Veröff. Inst. Bodendyn. Erdbebenf. Jena 72 23-52, Berlin (1962)

[10] Schwarzbach, M.: Seismizität und Bruchtektonik in Mittel-Europa. - Ann. Mus. Geol. di Bologna, Serie 2, 31 1-5, Bologna (1963)

[11] Van Rummelen, F. H.: Overzicht van de tussen 600 en 1940 in Zuidlimburg en omgeving waargenomen aardbevingen (etc.). - Meded. Jaarversl. Geol. Bureau 19421943, $151-130$, Maastricht (1945)

[12] VISSER, S. W.: Seismologie. - 160 p., Gorinchem 1949 


\title{
SEISMOLOGICAL DATA ON EUROPE SEISMOTECTONIC MAP
}

\author{
V. I. BUNE
}

(Moscow, USSR)

\section{Materials' characteristic and the methods of representation}

The main materials about Europe seismicity for 1901-1955 were collected by V. Karnik and represented by five maps of epicentres [1]. The earthquakes with foci in the earth's crust are represented on four maps, depending on shocks intensity in epicentre: $J_{0}=6^{\circ}(M=4-43 / 4) \quad J_{0}=7^{\circ}(M=4.8-5.4), \quad J_{0}=8^{\circ}(M=$ $=5.5-6.2)$ and $J_{0}=9^{\circ}-11^{\circ}(M=6.3-8.3)$. On the fifth map the deep earthquakes' epicentres are given with a division according to depth into two groups: $60-300 \mathrm{~km}$ and more than $300 \mathrm{~km}$. The deep earthquakes according to magnitude are divided into the same groups as earthquakes with foci in the earth's crust. The Europe seismotectonic map is compiled in a little scale $(1: 2500000)$ and the simple platting of this map of numerous epicentres of earthquakes would make the map very overburdened. In most seismic regions there are so many epicentres, that it is impossible to plat on them even on an outline map of such scale. It was necessary to use a simple and objective method of earthquakes' numerous epicentres presentation with account of their force and depth for drawing these data on the Europe tectonic map. It was necessary also to take into consideration comparatively little accuracy the overwhelming majority not more than $\pm 0.3^{\circ}$ of coordinates for determination of epicentres. For the solution of this problem the epicentres presentation method was selected in the isolines form suggested by Yu.V. Riznichenko for the compilation of seismic activity maps [2,3]. It was considered to make the most efficient use of this method for a comparative characteristic of the occurrence areas of numerous and not very hopefully determined earthquakes with $J_{0}=6^{\circ}-8^{\circ}$. It was not possible to plot on the map great number of epicentres of the strongest earthquakes with foci in the earth's crust $\left(J_{0}=9^{\circ}-11^{\circ}\right)$, in deep and also weak little-active regions. It was possible to use all the information for joint analysis of the data about seismicity and tectonics.

Recurrence isolines are related to the earthquakes with $J_{0}=7^{\circ}$. For the data recalculation of the weakest $\left(J_{0}=6^{\circ}\right)$ and strongest earthquakes $\left(J_{0}=8^{\circ}\right)$ to conditional recurrence $J_{0}=7^{\circ}$ the graph of earthquakes' recurrence is constructed with $J_{0}=6^{\circ}-12^{\circ}$ (Fig. 1). The graph is constructed on the base of V. Karnik maps for 1901-1955 period. From the graph it is seen that the points, corresponding to the logarithms of earthquakes number with $J_{0}=7^{\circ}-11^{\circ}$, are well put on the straight line with the angular coefficient $\gamma=0.48$. Thus, the earthquakes' number $N_{i}$ with intensity in epicentre $7-11^{\circ}$ may be determined from the equation: 


$$
\begin{gathered}
\log N_{i}=\log N_{7}-\gamma(i-7) \\
i=7-11
\end{gathered}
$$

The earthquakes' number with $J_{0}=6^{\circ}$ occurred to be twice less than it was possible to suppose at a linear extrapolation of a graph into the weakest

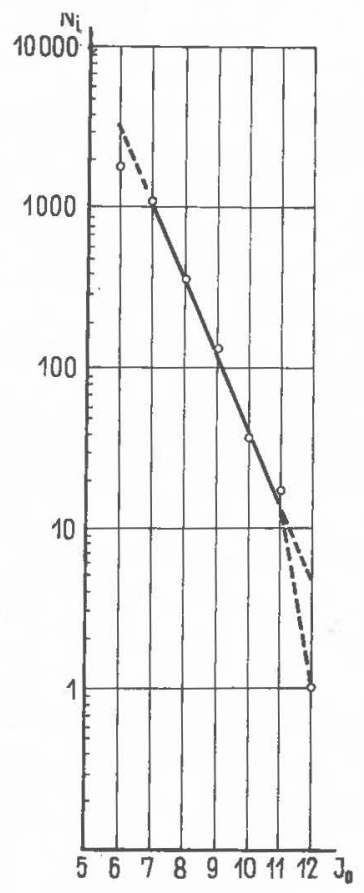

FIG. 1. Earthquakes' number with different intensity in Europe for 1901-1955 according to V. Karnik data

earthquakes' area. Apparently this shows that the great number of such earthquakes was skipped over at catalogues' compilation. On the recurrence graphs' base the formula is suggested:

$$
N_{7}=\frac{1}{3}\left(0.5 N_{6}+N_{7}+3 N_{8}\right)
$$

The coefficients in this formula are determined with the recurrence graph account, from which it is that

$$
\frac{N_{7}}{N_{8}}=\frac{N_{8}}{N_{9}}=\frac{N_{9}}{N_{10}}=\frac{N_{10}}{N_{11}} \sim 3
$$

For earthquakes with $J_{0}=6$ this ratio is not observed and therefore the coefficient is not taken as 0.3 but 0.5 . For convenience of subsequent work the earthquakes 
of all three shown groups $\left(J_{0}=6^{\circ}-8^{\circ}\right)$ are plotted on one map, on the same map the net through $0.2^{\circ}$ is plotted and with every net knot the circle, the square of which was $1000 \mathrm{~km}^{2}$ was combined. The epicentres' number was calculated, which were in circle ranges and by formula (2) $N_{7}$ was determined. Through the points with equal values $N_{7}$ the isolines are drawn. As earthquakes' data have been collected for same 55 years, it occurred to be most conveniently to relate the final figures to the period of 50 years. The figures on isolines show what earthquakes' number with the intensity of $7^{\circ}$ had occurred for 50 years on the area in $1000 \mathrm{~km}^{2}$. In fact it may be three times lesser earthquakes' number with $J_{0}=8^{\circ}$ or 2 times greater earthquakes' number with $J_{0}=6^{\circ}$. The area which is equal to $1000 \mathrm{~km}^{2}$ is great enough, though the decrease of averaging area at the compilation of this map did not seem to be rational because of the little accuracy of epicentres' coordinates determination. The average accuracy of epicentres' coordinates determination does not exceed $\pm 30 \mathrm{~km}$, i.e. epicentres' majority can freely move in a circle ranging with a square of $1000 \mathrm{~km}^{2}$. Therefore as a whole during the map compilation it was impossible to expect great accuracy. In some regions, e.g. in France, Crimea and some others where the accuracy of determination of the coordinates is better it was possible to compile a very accurate map, though, in the first approximation it is convenient to have in all the cases the same data interpretation method. In this case from the analysis of the well studied regions the possibilities of the map improvement at the use of the most accurate materials will be clear. Assuming in all the cases the possibility of earthquakes' epicentre displacement in the indicated area ranges, we obtained possible areas of shocks with intensity $7^{\circ}$ at the earthquakes with foci in the earth's crust. This result is an intermediate one for the map compilation of the seismic zoning and is of a great interest.

\section{The Main Regularities of Seismoactive Zones in Europe}

The zones with activities $N_{7}>1.0$ (Fig. 2) where already the earthquakes with $J_{0} \geq 9^{\circ}$ were observed are situated in the following regions: Portugal, Italy (the Apennine peninsula and Sicily), Yugoslavia, Albania, Greece, Bulgaria, Turkey, USSR (the Caucasus), Algeria. The biggest zone with maximum frequency of earthquakes' recurrence with $J_{0} \geq 9^{\circ}$ is situated on the Balkan peninsula (Yugoslavia, Bulgaria, Albania, Greece). The second zone accordingly includes the west coast of Turkey and the islands in the Aegean Sea, the third one is situated on the Apennine peninsula (Italy). The rest of the zones are of little dimensions. The great number of earthquakes with $J_{0} \geq 9^{\circ}$ had occurred in the zones with recurrence of earthquakes with intensity $7^{\circ} N_{7} \geq 1.0 .165$ earthquakes with $J_{0} \geq 9^{\circ}$ for 1901-1955 are plotted on the map. In the zones with recurrence $0.1<N_{3}<1.028$ earthquakes had occurred. In the zones with $N_{7}<0.14$ such earthquakes had occurred. All the rest of the earthquakes with $J_{0} \geq 9^{\circ}$ had occurred in the zones with $N_{7}>1.0$. The area of this zone is not large in comparison with 
the area where earthquakes in general are observed. It confirms a high concentration of the most seismoactive zones.

The obtained conclusions need a subsequent more precise definition. At first it is necessary to mark that there is no firm belief that all the information about $6^{\circ}, 7^{\circ}$ and it may be $8^{\circ}$ earthquakes, which had taken place in Turkey and Algeria were included in the catalogues and corresponding maps, compiled by V. Karnik. It is possible that this explains that just in Turkey, in the zone with activity less than 1.015 (from 28), earthquakes with $J_{0} \geq 9^{\circ}$ had occurred.

\section{Reviews, Notes and Recommendations Regarding Further Collections and Analysis of Materials}

The seismotectonic map of Europe was discussed at the meetings of the European Seismological Commission in Jena in 1962 and sent to review together with the explanatory note. The critical notes and new additional materials concerning seismic data are obtained from Czechoslovakia (A. Zátopek), Denmark (H. Jensen), Finland (E. Vesanen, E. Pentillä), France (E. Peterschmitt, J. P. Rothé), Great Britain (E. Tillotson), Greece (A. Galanopoulos), Iceland (H. Sigtryggsson), Italy (P. Caloi), Poland (T. Olczok), Sweden (M. Båth), Turkey (K. Ergin, M. Ipek).

It is necessary to consider in details the recommendations according to the materials' generalization method. Apparently the problem of the necessity of the use of full informations about earthquakes which intensity is $J_{0} \geq 9^{\circ}$ for all the historical period is the most important one. Unfortunately the complete materials about such earthquakes are still not gathered and therefore not every strong earthquake which had occurred in Europe to 1900 are plotted on the map. It is difficult to expect that for all the territory it will be possible to gather information about the weakest earthquakes, as even for the 20th century there are the isoseismals' maps only approximately for $1 / 5$ earthquakes the intensity of which is $8^{\circ}$ or more.

Though the collection and publication of isoseismals' maps, especially of strong earthquakes with $J_{0} \geq 8^{\circ}$ are of a great significance, as epicentres' coordinates may be more exactly determined on the base of isoseismals' maps. It is necessary to use the seismic scale, suggested by S. V. Medvedev, V. Karnik and W. Sponheuer.

Besides accurate definition of earthquake epicentres position it is important to specify a real seismic energy in earthquake foci. The maps, on which the force in the focus is characterized by a certain intensity in epicentre, does not seem to be successful enough. On the one hand, in the different countries during the different periods, different scales were used for intensity determination. On the other hand, even at using one and the same scale different investigators in different ways evaluating the destroying effect in many cases have a difference which is equal to $1^{\circ}$ of intensity. At last, the earthquakes' destroying effect does not depend on energy only in the source and the depth of earthquake focus, but also on a construction quality and local ground conditions. At the 
comparison of the seismicity with tectonics all these effects are not significant. For these aims it would be better to use earthquakes' classification on the base of instrumental data by elastic waves' energy or magnitude. At present, it was not successful to carry this out since only a little part of European earthquakes data are collected.

This problem can not be carried out well without participation of all the European countries' seismologists.

\section{LITERATURE}

[1] KARniK, V.: Epicentre maps for Europe, Studia geoph. et geod. 5 (1961)

[2] RiznichenKo, Yu. V.: On quantitative determination and mapping of seismic activity, Annuali di Geofisica, Roma, 122 (1959)

[3] Riznichenko, Yu. V. and Nersessov, I. L.: A detailed study of the seismic regime in the Garm epicentral region, Ann. geofis. 142 (1961) 


\title{
INTERRELATIONS BETWEEN EARTHQUAKES AND GEOLOGICAL STRUCTURES
}

\author{
I. E. GubIN
}

(Moscow, USSR)

This paper is a progressive report on seismic zoning, which deals with the interrelations between earthquakes and seismogenetic geological structures. These quantitive interrelations were determined empirically by juxtaposition of geological structures of the USSR territory with seismic data (especially macroseismic ones). There are several space and quantitative conformities of seismic data to geological structures.

The first conformity. Earthquakes originate on the planes of the faults, that are between differently moving geological structures. This is well known and examples are not necessary.

The second conformity. The greater is the depth of seismogenetic structures or that of the seismogenetic faults, the deeper are the foci of earthquakes in the given territory and vice versa.

For instance, near the coasts of Kamchatka and the Kurile Isles, in contact of two large deeply rooted structures of the Pacific Ocean and the Asiatic continent the earthquakes foci are from a few up to $600 \mathrm{~km}$ deep. The named region is supposed to be modern geosyncline.

In East Siberia, Tadjikistan, Turkmenya, Caucasus, Crimea and in the Carpathian Mts, in Mesozoic and Alpine folded belts the foci were in the earth crust. Only in three regions in contact of large peculiar structures the foci were from a few till $100-200 \mathrm{~km}$ deep: in the middle part of the Pamirs; in the middle part of the Caspian Sea (?) and the Wranch district of Carpathian Mts (Rumania).

In the north part of Central Asia, in the Altai and in the Baikal districts, in the faulted platforms, on contacts of active (moving) block structures the foci occurred only in the earth crust.

There is the same space conformity of foci to the local seismogenetic structures. For instance, in the region of folded mesozoic and tertiary layers, where the folding is disharmonic with respect to the Palaeozoic substratum, the foci were shallow in these folds, as they did occur in a lot of regions of Central Asia and of the Caucasus. In the regions of large block structures, which penetrate deeper into the earth crust, the foci were deeper. Such examples may be continued.

The third conformity. The larger is the new fault, or the greater is the area and the amplitude of displacement of a structure on the surface of an old fault, the larger and stronger is the earthquake.

This conformity is caused by the magnitudes of geological structures. The 
larger and more consolidated a structure is, the larger is the fault originated by the displacement of this structure and the stronger is the earthquake and vice versa. Consequently, each seismogenetic structure, with given parameters, has its own highest level of strength of the earthquakes.

Several seismogenetic geological structures of different types and dimensions with the highest levels of earthquakes' strength pertaining to them were determined empirically on the Tadjikistan territory. They have been recommended as local standards of different seismogenecity (Gubin 1960, 1962). Such standards were also determined, but preliminarily, in a few other territories of the USSR. They are based on the following data.

The strongest earthquakes $(M=7-8.5)$ were registered in the earth crust on contacts of large, active block structures, mostly in the faulted platforms, in the districts of Baikal Lake and Central Asia. The extension for each above mentioned block structure is not less than $40-50 \mathrm{~km}$. Lesser earthquakes occured on contacts of lesser blocks.

Weaker earthquakes $(M=5.5-7)$ were registered on planes of the faults in the regions of folded mesozoic and tertiary layers, which are less consolidated than platforms. They did occur in many districts of the Caucasus and Central Asia.

In the regions of smaller fold structures, of the same type, lesser earthquakes were registered. In the districts of unfaulted structures only very weak earthquakes occured. They were caused by little disruptions of different parts of structures.

The fourth conformity. The greater is the speed of displacement of the structure, the shorter is the period of the time needed for the accumulation of stress and maximum earthquakes, which can be originated from this structure occur more frequently, and vice versa. In a lot of structures, due to their too little speed of displacement, the maximum earthquakes were not registered until now.

The fifth conformity. The ratio of quantities of weak, middle and strong earthquakes in seismogenetic structures of different types and age is unequal (Richter 1959, Gubin 1960, Vvedenskaya 1961, Soloviev 1961).

Standards of the above-mentioned conformities, that were determined empirically in several districts of the USSR, cannot be taken as universal ones. Geological structures internally are too individual and their tectonic interrelations are too unequal. Consequently, these standards require specification for every district by its historical seismic and tectonic data. Only after specifying these standards may they be used for seismic regionalization. An elaborated set of such standards will much facilitate the work.

In this short paper we cannot discuss all the peculiarities and difficulties of such a seismic zoning or regionalization. But we may state, in general outline, that zones of earthquakes' origination may be determined by the position of large active tectonic faults and also by smaller ones. The depth of foci can be predicted by the depth of active structures and faults. One may hazard to guess the magnitude of future earthquakes by the magnitude of the respective seismogenetic structure. It is done by juxtaposition of a given structure with the set of structures 
taken as the standards of local seismogenecity. To a given structure we ascribe the strength of earthquake of an appropriate standard, that has the same size and geological peculiarities.

The determination of frequency of earthquakes must be based on the prediction of speed of displacement of a given structure. But we cannot predict the fluctuation of speed of displacement. Consequently we may predict only an average frequency of maximum earthquakes, with the help of historic seismic data on large tectonic zones.

Accordingly to the above-said, one may and must depict on maps of seismic regionalization (Gubin 1964): zones of earthquakes' origination; magnitude and grade of intensity of future maximum earthquakes in these zones; their average frequency in every zone; the depth of foci of future maximum earthquakes in these zones and probable areas of propagation of earthquakes.

Such a seismic mapping requires the knowledge of geology, historical seismicity and geophysical data of a given territory. Consequently this mapping can be done only in regions where seismogenetic geological structures and their speed of displacement can be measured. Seismotectonic maps of this type have been made for mountain districts of Tadjikistan and Turkmenia (Gubin 1960). The construction of these maps has shown the necessity of progressive elaboration of seismotectonic method of this mapping, including elaboration of set of standards of seismogenecity and a precise and practicable method of measuring the parameters of geological structures.

In cases when such seismotectonic maps of seismic zoning can not be compiled, we may construct usual seismostatistical maps of seismic regionalization (Richter 1959 , Gubin 1964), where only the grades of intensity of future earthquakes in given regions are shown.

\section{REFERENCES}

[1] Savarensky, E. F., Soloviev, S. L. and Harin, D. A. : Atlas of earthquakes of the USSR, Akad. Nauk SSSR, Moscow, 1962 (in Russian)

[2] Vvedenstaya, N. A.: Earthquakes of Central Asia. In: Earthquakes in te USSR, Akad. Nauk SSSR, Moscow, 1961 (in Russian)

[3] Gubin, I. E.: Regularities of seismic phenomena on the territory of Tadjikistan (geology and seismicity). Akad. Nauk SSSR, Moscow, 4641960 (in Russian)

[4] Gubin, I. E.: Seismicity and geological structures of Central Asia. Studia geoph et geod. 6 Praha (1962)

[5] Gubin, I. E.: On earthquakes forecasting. lzwest. Akad. Nauk SSSR, ser. geophys., 8 (1964) (in Russian)

[6] Richter, C. F.: Seismic Regionalization. Bull. Seism. Soc. of Am., 492 (1959)

[7] SolovieV, S. L.: Essay on seismicity of the USSR. In: Earthquakes in the USSR, Akad. Nauk SSSR, Moscow (1961) (in Russian)

[8] Tectonic map of the USSR. Ed. A. A. Bogdanov, Moscow (1961) (in Russian) 


\section{DIE SEISMIZITÄT AUF DEM GEBIET \\ DER DEUTSCHEN DEMOKRATISCHEN REPUBLIK}

W. SPONHeuer

(Jena, DDR)

Das seismisch aktive Gebiet der DDR wird hauptsächlich von der sächsischthüringischen Großscholle gebildet. In geologischer Hinsicht ist dieses Gebiet ein Teil des in Bruchschollen zerfallenen mitteleuropäischen Raumes, dessen heutige geologische Situation größtenteils eine Folge der saxonischen Gebirgsbildung ist. Im Gegensatz zu den großen erdumfassenden Erdbebenzonen, die durch die jungen Kettengebirge und durch gewaltige Brüche gekennzeichnet sind, haben Bruchschollenländer eine verhältnismäßig geringe seismische Aktivität. Schon früher habe ich darauf hingewiesen, daß in Mitteleuropa die seismische Aktivität von Norden nach Süden zunimmt, während im Untergrund von Süd nach Nord eine immer stärker werdende Konsolidierung festzustellen ist.

Die Seismizität des in Frage kommenden Gebietes wird einmal für den Zeitraum 1800 bis 1900, zum anderen von 1900 bis 1960 untersucht. Auf der Grundlage von makroseismischen Erdbebenkatalogen $[4,5]$ sind verschiedenartige Karten der Erdbebentätigkeit zusammengestellt worden. Die Karten der Epizentren mit den Maximalintensitäten vermitteln die Anzahl der Herde, ihre Verteilung und lassen in vielen Fällen Beziehungen zu den tektonischen Störungen erkennen. Selbstverständlich ist das der Karte von 1800 bis 1900 zugrunde liegende Material nicht so vollständig wie das für den Zeitraum von 1900 bis 1960 . Dennoch läßt sich, wie die Abbildungen 1 und 2 ersehen lassen, eine weitgehende Übereinstimmung in der Verteilung der Epizentren erkennen. Ihre Häufung ist besonders groß im sächsischen Raum zwischen Saale und Elbe. Sie verdichten sich noch weiter im Bereich des Vogtlandes. Diese Nord-Süd-Tendenz ist tektonisch begründet, da mindestens zwei Verwerfungszonen dieses Gebiet kennzeichnen. Einmal handelt es sich um die Finnestörung, die südlich des Harzes ausgehend, etwa parallel zum Thüringer Wald verläuft und deren Fortsetzung bis über Gera hinaus nach Süden zu verfolgen ist. Der Herd des bisher energiereichsten Bebens Mitteldeutschlands vom 6. März 1872 bei Ronneburg steht höchstwahrscheinlich mit dieser Störung im Zusammenhang. Die andere Störung, die sogenannte Hallesche Marktplatzverwerfung, verläuft von Halle im Bogen nach Süden, setzt sich in herzynischer Richtung fort und endet kurz vor der erzgebirgisch gerichteten Störung, die in der Zwickauer Gegend beginnt. Im allgemeinen ist durch die Vielzahl der Herde der Überblick über solche Karten der Epizentren schwierig zu gewinnen. Da nicht nur die maximale beobachtete Intensität für jedes Epizentrum, sondern auch die Anzahl und Energie der Beben als maßgebende 


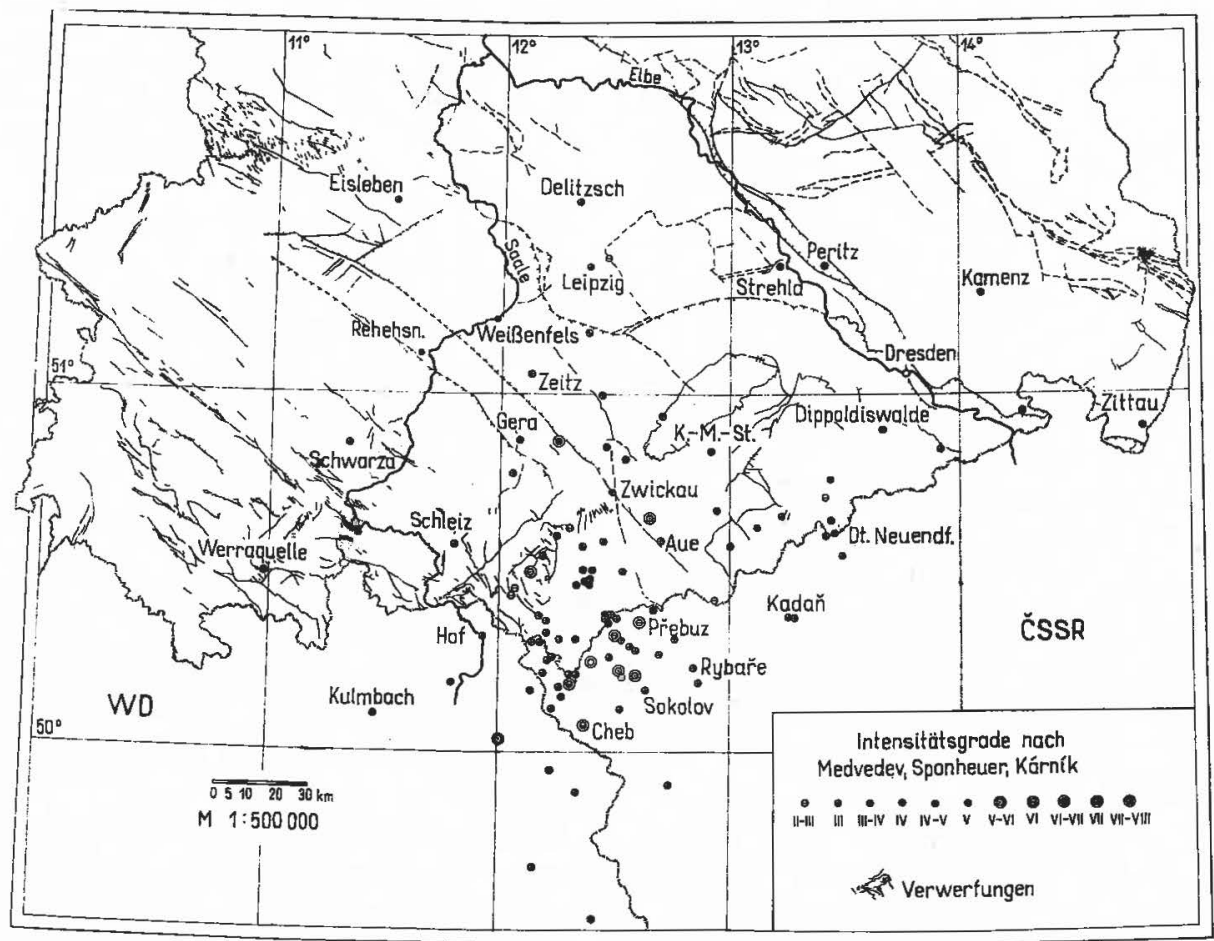

ABB. 1. Epizentren maximaler Intensität innerhalb des Gebiets der sächsisch-thüringischen Großscholle im Zeitraum 1800 bis 1900

Größen auftreten, ist für die Beurteilung der Seismizität eine Epizentrenkarte nicht allein grundlegend. Um dies zu berücksichtigen, wurde in der Karte der Epizentren auch die Summe der Energie und die Anzahl der Beben für jedes Epizentrum eingetragen. Wegen der einfacheren Schreibweise wurde der Logarithmus der Energie, abgerundet auf ganze Zahlen, durch Signatur angegeben und die Anzahl der Beben durch arabische Ziffern bezeichnet.

Für die Berechnung der Magnitude bzw. der Energie der Erdbeben wurden wie schon an anderer Stelle [3] zwei Beziehungen von Gutenberg-Richter benutzt :

und

$$
M=2 / 3 I_{0}+1
$$

$$
\log E=1,6 M+11 \text {. }
$$

Da in Gl. (1) $M$ nur von $I_{0}$ abhängt, ist es möglich, auch die Magnitude schwacher Erdbeben zu bestimmen, von denen lediglich $I_{0}$ bekannt ist. Auf diese Weise konnte auch die Energie der vogtländischen Schwarmbeben abgeschätzt werden. Leider ist das Beobachtungsmaterial besonders an Tagen großer Stoßhäufigkeit 


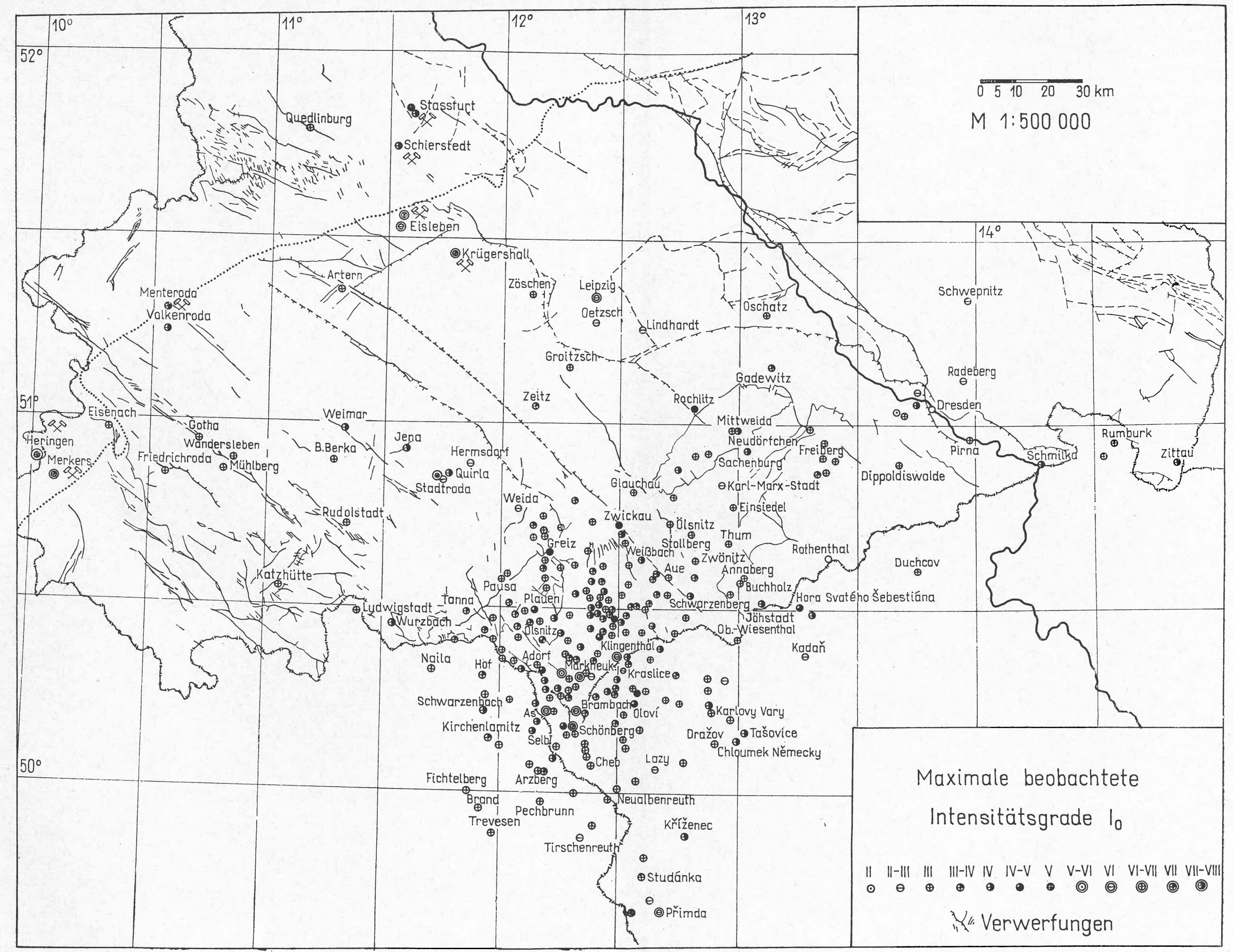

Авв. 2. Epizentren maximaler Intensität innerhalb des Gebiets der Deutschen Demokratischen Republik im Zeitraum 1900 bis 1960 


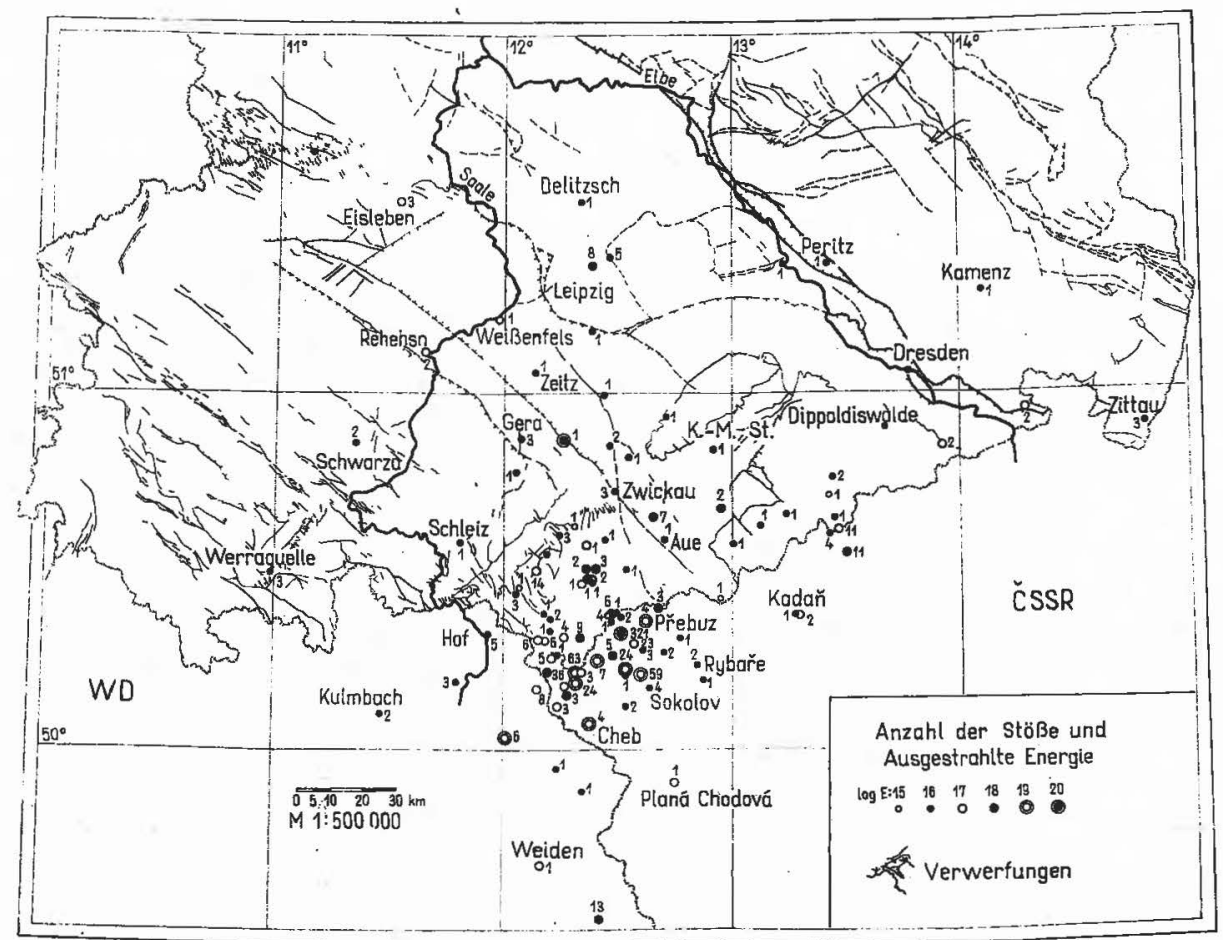

Авв. 3. Epizentren mit Angabe der AnzahI der Erdbeben und des log der Energiesumme innerhalb des Gebiets der sächsisch-thüringischen Großscholle im Zeitraum 1800 bis 1900

so wenig detailliert, daß oftmals die Festlegung von Stoßzahl und Intensität fraglich ist. $\mathrm{Da}$ aber für die Energiebestimmung die stärkeren genauer beobachteten Stöße mehr ins Gewicht fallen, ist eine noch brauchbare Abschätzung der seismischen Aktivität zu erwarten. Die Abbildungen 3 und 4 zeigen auf den ersten Blick die Schwerpunkte der seismischen Aktivität bzw. Energieabgabe. Der Vergleich beider Karten zeigt, daß beispielsweise die Energieverteilung im Vogtland sich nur wenig unterscheidet. Man erkennt in der Karte 1800-1900, von der Brambacher Gegend ausgehend, eine nach Norden gerichtete Zone von Epizentren starker Energieentfaltung, die bei Markneukirchen in die erzgebirgische Richtung abbiegt. Die Karte dieses Jahrhunderts zeigt eine ganz ähnliche Energieverteilung, ebenso wie die Auffächerung im Süden durch das Epizentrum von Asch. Vielleicht ist die Aufteilung der Energie in zwei Richtungsbereiche als Hinweis zu werten, daß an der Entstehung der Schwarmbeben zwei tektonische Systeme beteiligt sind. Gegenüber der Karte der älteren Epoche geht aus der von 1900 bis 1960 hervor, daß infolge des besseren Beobachtungsmaterials mehr Epizentren, z. T. mit größerer Aktivität, auch am Rande des vogtländischen Schüttergebietes auftreten. Dies gilt besonders für die Gegend nördlich von Klin- 


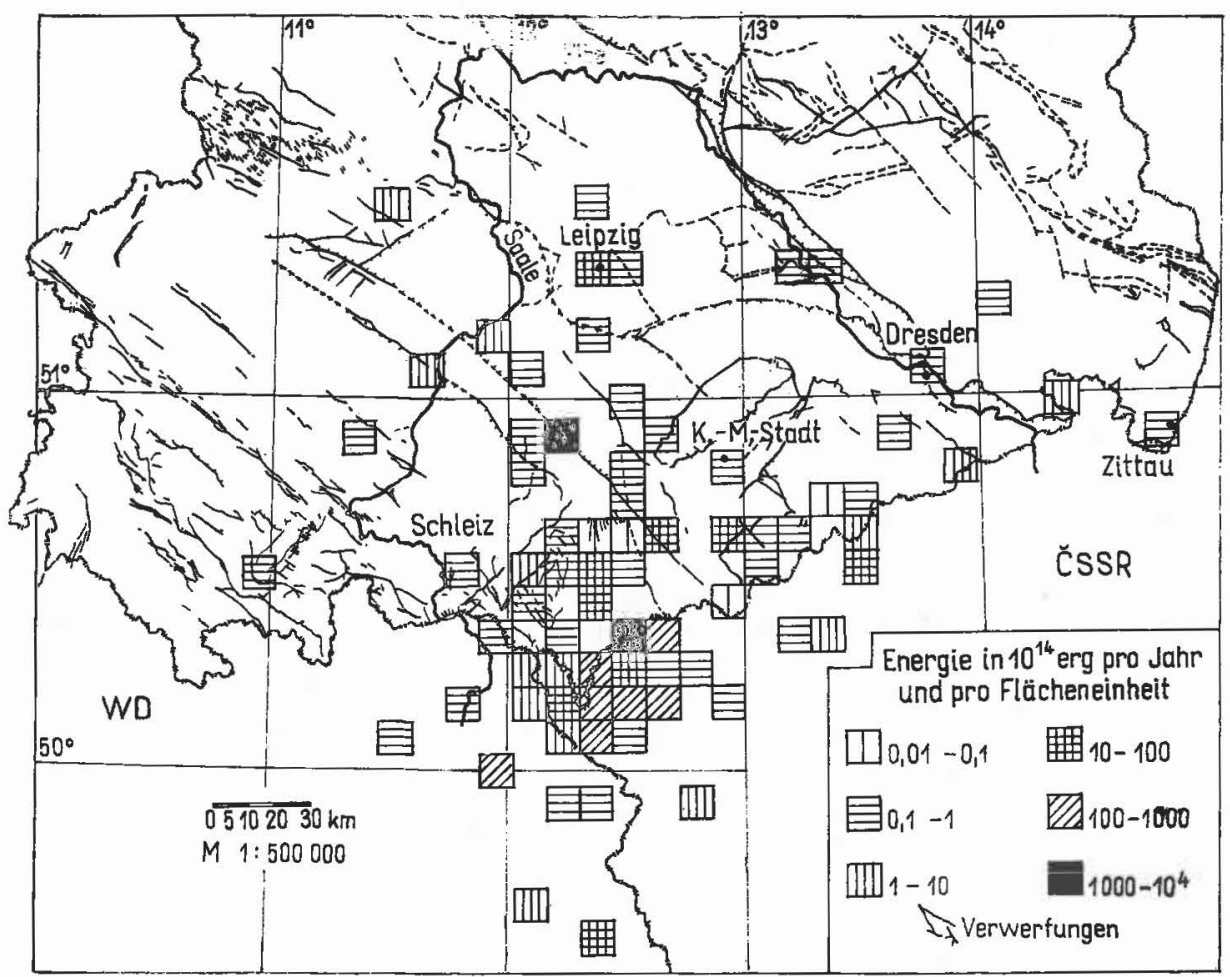

Aвв. 5. Seismische Energie pro Jahr und Flächeneinheit innerhalb des Gebiets der sächsischthüringischen Großscholle im Zeitraum 1800 bis 1900

genthal und das Gebiet von Ölsnitz bis Greiz. Auf beiden Karten treten die Randstörungen des Thüringer Waldes etwa auf der Linie Eisenach - Gehren und die Gotha - Saalfelder Störungszone seismisch nur wenig angedeutet hervor. Auch der gewaltige Erzgebirgsabbruch tritt abgesehen vom Gebiet des Vogtlandes relativ unbedeutend in Erscheinung. Ähnlich verhält es sich mit dem Elbtalgraben. Wie auch in anderen Fällen zeigt sich hier also, daß bedeutende Störungszonen nicht unbedingt seismisch besonders aktiv sein müssen.

Wie Båth [1] und neuerdings auch Galanopoulos [2] gezeigt haben, läßt sich die Seismizität durch die je Flächen- und Zeiteinheit ausgelöste Energie beschreiben. Eine solche Karte entsteht dadurch, daß man über eine Karte der vorher beschriebenen Art ein Netz legt und die Energie innerhalb jedes Rechtecks summiert. Je weitmaschiger das Netz gewählt wird, umso geringer wird das Auflösungsvermögen. Dies hat jedoch den Vorteil, daß die Schwerpunkte der seismischen Aktivität klarer hervortreten. In den Karten der Abbildungen 5, 6 und 7 sind die Energien für je $25 \mathrm{~km}^{2}$ Flächeneinheit für verschiedene Gitterweiten bestimmt worden. Um die Karten vergleichen zu können, wurde die Energie 


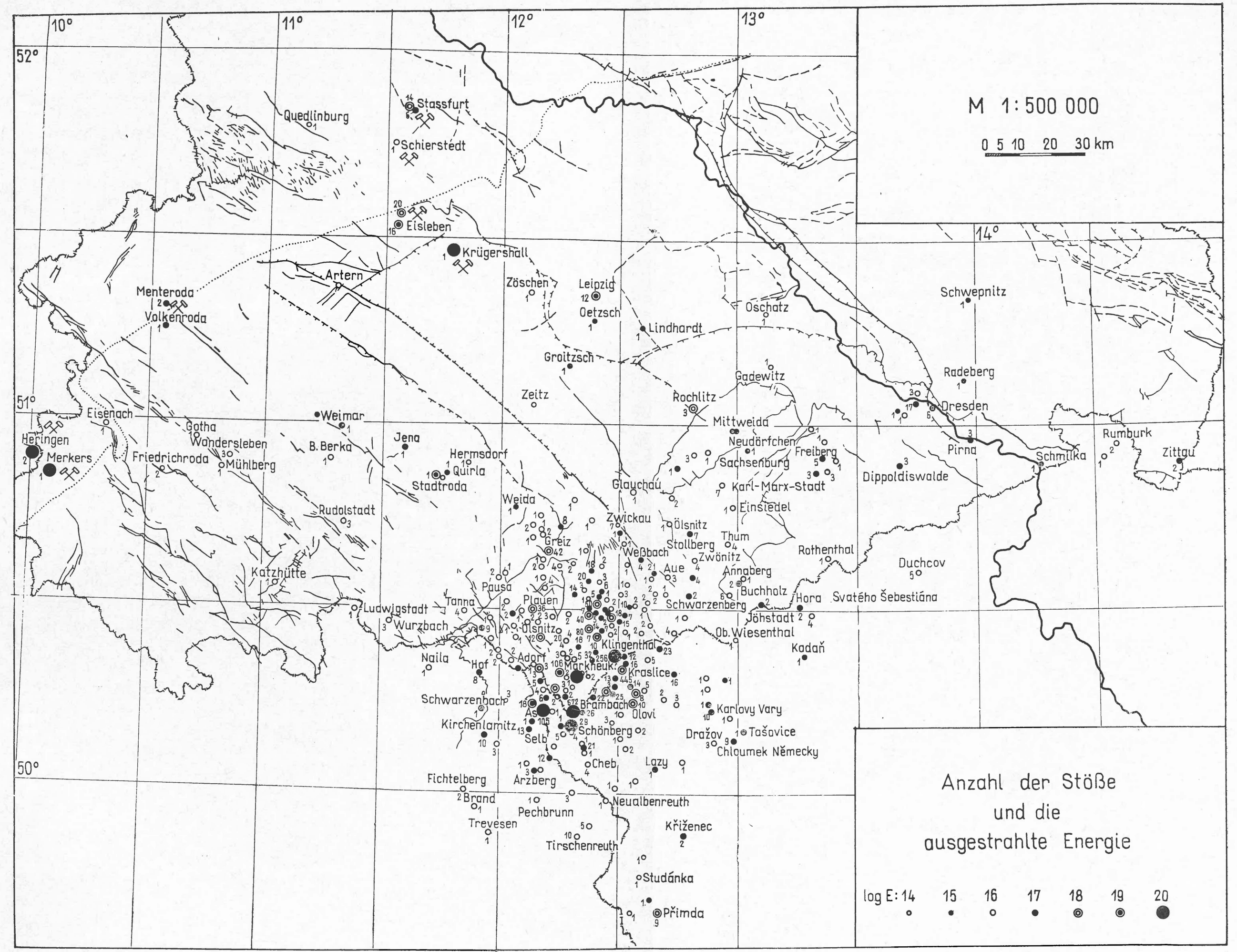

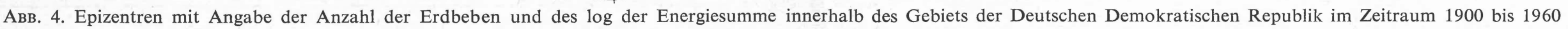




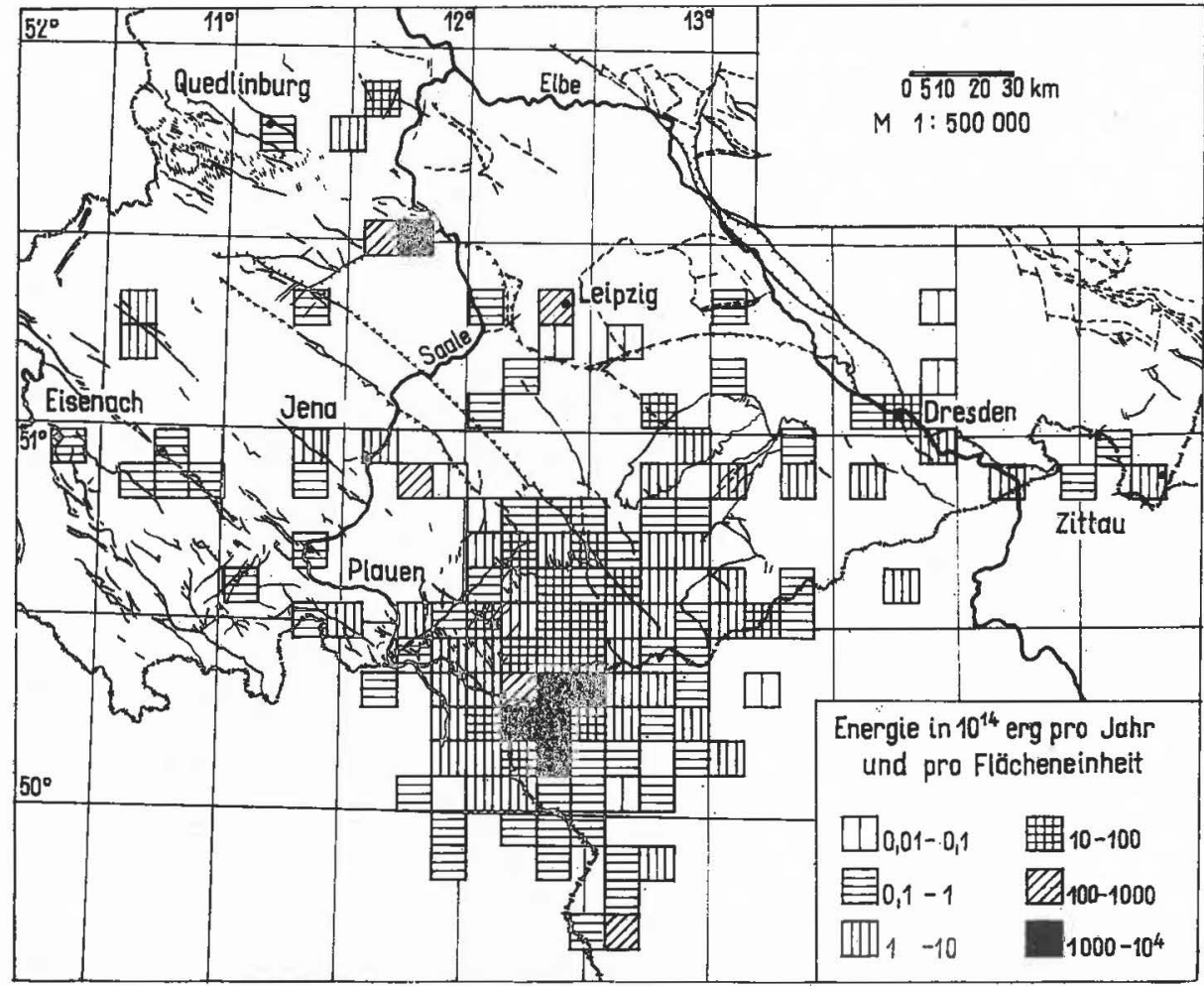

Aв8. 6. Seismische Energie pro Jahr und Flächeneinheit innerhalb des Gebiets der Deutschen Demokratischen Republik im Zeitraum 1900 bis 1960

für jedes Viereck auf die gleiche Flächeneinheit bezogen. Während in der Karte mit dem dichteren Netz $(5 \times 5 \mathrm{~km})$ noch mehrere Schwerpunkte maximaler Energie auftreten, ergibt die Karte mit dem weitmaschigen Netz von $10 \times 10 \mathrm{~km}$ einen einzigen Schwerpunkt, das vogtländische Schwarmbebengebiet. Ein Vergleich mit der Karte des vorigen Jahrhunderts läßt dieses Gebiet ebenfalls als das seismisch aktivste erkennen. Ein zweiter Schwerpunkt weist auf das große mitteldeutsche Erdbeben von 1872 mit dem Herd bei Ronneburg hin. Durch die seismische Aktivität im Vogtland wird geradezu die Frage aufgeworfen, welche tektonischen Ursachen dafür sprechen, daß in dieser Region durch die Schwarmbeben die stärkste Energieabgabe erfolgt. Wie schon gesagt, ist diese Stelle die Kreuzung zweier Gebirgssysteme, nämlich des Erzgebirges mit dem Gebirgszug des Thüringer und Böhmer Waldes. Es läßt sich nachweisen, daß sich solche tektonischen Kreuzungsstellen häufig durch eine gesteigerte seismische Tätigkeit auszeichnen. Auch das zweite große Schwarmbebengebiet im deutschen Raum, das Mainzer Becken mit dem Groß-Gerauer Herdgebiet, liegt beispielsweise auf der Kreuzung des Rheintalgrabens mit dem Saar-Nahe-Graben. Die 


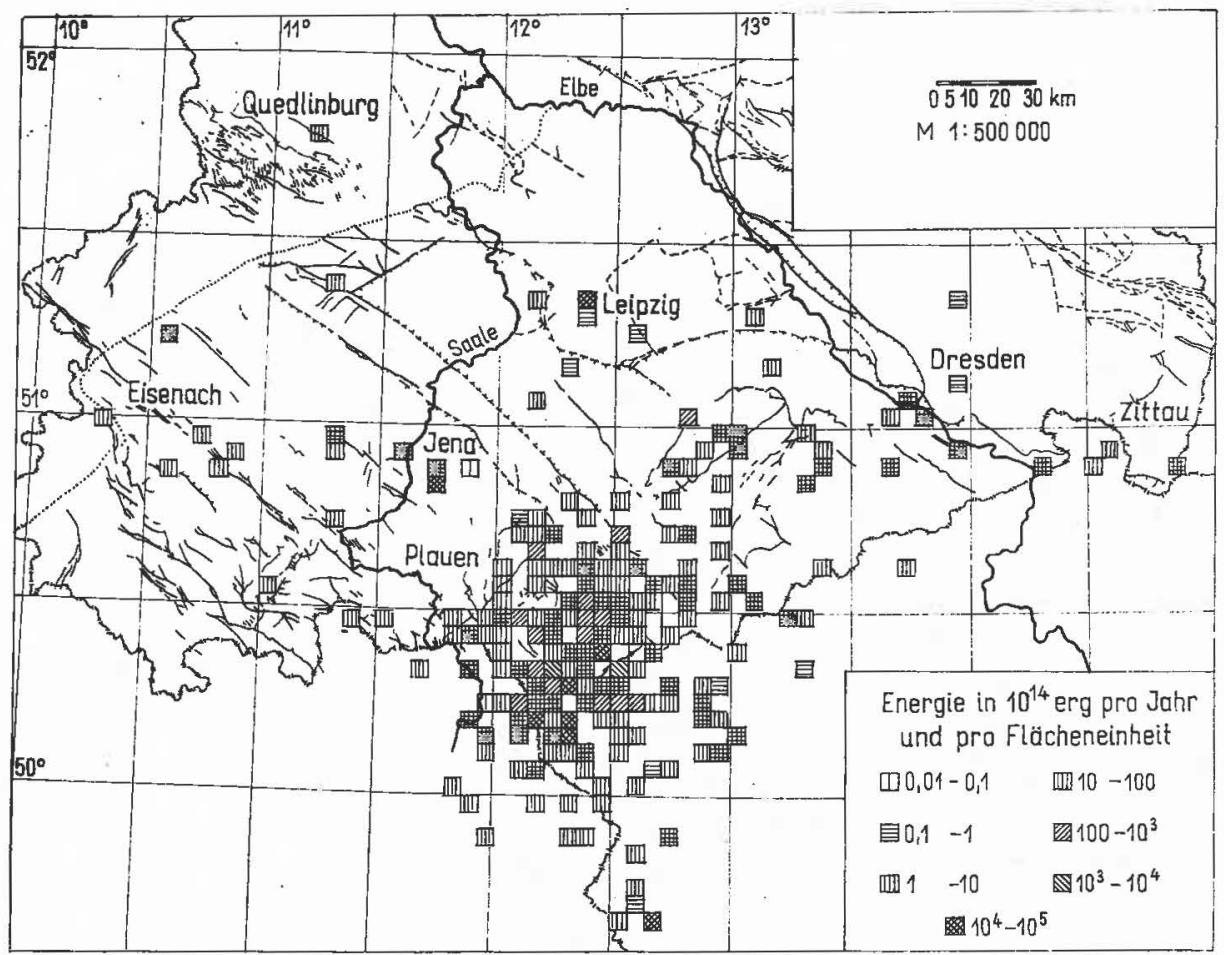

Aвв. 7. Seismische Energie pro Jahr und Flächeneinheit innerhalb des Gebiets der Deutschen Demokratischen Republik im Zeitraum 1900 bis 1960 (bei kleiner Gitterweite)

bisher noch offene Frage, warum in diesen Schwarmbebengebieten die angesammelte seismische Energie nur in vielen kleinen Beträgen freigegeben wird, kann dadurch erklärt werden, daß an den hier tätigen Herdvorgängen immer nur kleine Teile der Verwerfungen beteiligt sind. Auch das bei Schwarmbeben häufig beobachtete sprungweise Wechseln der Herde während einer Schwarmbebenperiode scheint dafür zu sprechen. Eine weitergehende Klärung des Schwarmbebenproblems ist aber sicherlich nur auf Grund mikroseismischer Untersuchungen mit hochempfindlichen Geräten im Bebengebiet möglich.

\section{LITERATUR}

[1] ВА̊TH, M.: Seismicity of Fennoscandia and Related Problems. Gerl. Beitr. z. Geoph. 633 173-208 (1953)

[2] Galanopoulos, A. G.: On Mapping of Seismic Activity in Greece. Annali di Geofisica 16 I 37-100 (1963)

[3] SPONHEUER, W.: Untersuchung zur Seismizität von Deutschland, Veröffentlichungen des Institutes für Bodendynamik und Erdbebenforschung in Jena. 72 23-52 (1962)

[4] Sponheuer, W.: Erdbebenkatalog Deutschlands und der angrenzenden Gebiete für die Jahre 1800 bis 1899. Mitteilungen des Deutschen Erdbebendienstes 3195 (1952)

[5] SPONHEUER, W.: Erdbebenkatalog Deutschlands 1900-1960 (Manuskript) 


\title{
SEISMICITY OF MACEDONIA
}

\author{
A. ZÁTOPEK \\ (Prague, Czechoslovakia)
}

The seismic catastrophe of Skopje, southern Yugoslavia, on July 26, 1963 $\left(42^{\circ} 00^{\prime} N, 21^{\circ} 27^{\prime} E, H=04^{\mathrm{h}} 17^{\mathrm{m}} 11^{\mathrm{s}} \mathrm{GMT}, I_{0}=9^{\circ} M M, h=5 \mathrm{~km} \pm, M=6.0\right)$, initiated an extensive study of the seismicity of the Skopje region and that of the territory of the Popular Republic of Macedonia, of which the city of Skopje is the capital.

The territory of Macedonia is situated in the Mediterranean seismic belt. It was quoted by many authors (Montessus de Ballore, Sieberg, Gutenberg and Richter, etc.) as an area with elevated seismicity. Its seismic activity was studied three decades ago by J. Mihailović and treated in various connections in his papers.

Let us now give a general description and more detailed characteristics of Macedonian seismic areas as a part of results of the author's research performed in the framework of a joint UN TA/UNESCO Mission to Skopje, December 1963-March 1964.

The seismologically active zones of Macedonia are given in Fig. 1.

In the seismic history of Macedonia, the Vardar zone appears as a region, where earthquakes occur frequently, and the Skopje region is considered to be the most mobile part of the Vardar zone. We shall see that it may be true as it concerns the frequency, but not regarding the energy of earthquakes observed.

The documentation about Macedonian earthquakes before 1904 (foundation of the Seismological Institute of Belgrade), especially about the minor shocks, is very poor.

The seismic history of the Skopje region is reduced, before 1904, practically to a rather scarce description of the earthquake catastrophe of Skupi in 518 a. D., and that of Skopje in 1555. The old town of Skupi was situated about 4-5 km northwest from the centre of the present Skopje; this is said to have been partly demolished in 1555 .

During our century, the region of Skopje was affected by a group of damaging earthquakes in the summer of 1921, besides of a number of minor shocks, felt after 1900. The last destructive earthquake of July 26, 1963, was followed by a series of over 500 aftershocks registered as yet by the Mainka pendulums of the Skopje Seismological Station, partly also macroseismically felt.

The region suffered several times from earthquakes originated in more distant sources, e.g. from the Gnjilani region in southern Serbia.

In the Vardar zone of central Macedonia, one finds ruins of the town Stcbi 
the destruction of which is also ascribed to an earthquake (mostly to that of 518 a. D.).

In the part of the Vardar zone passing through southern Macedonia, there is a very active region of Valandovo, where heavy destructions have been caused by two earthquakes occurred on March 7 and 8, 1931. The energy of these two earthquakes represents a maximum known in Macedonia.

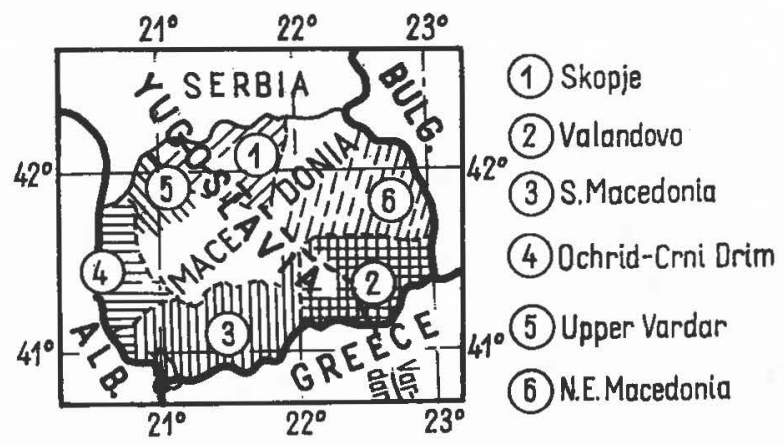

FIG. 1. Seismically active zones of Macedonia

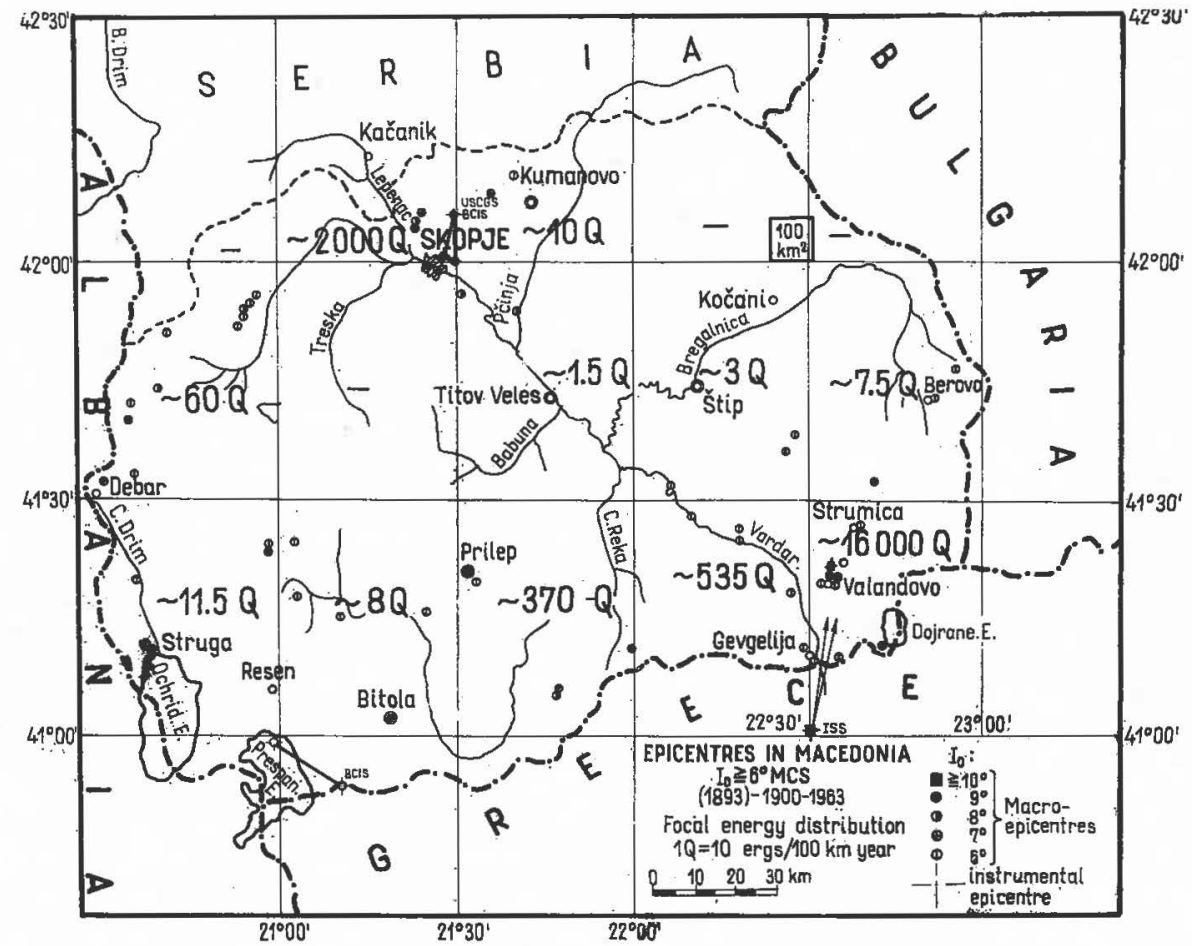

FIG. 2. Epicentres $I_{0} \geqq 6^{\circ}$, (1893)-1900-1963, with the corresponding energy distribution in Macedonia 


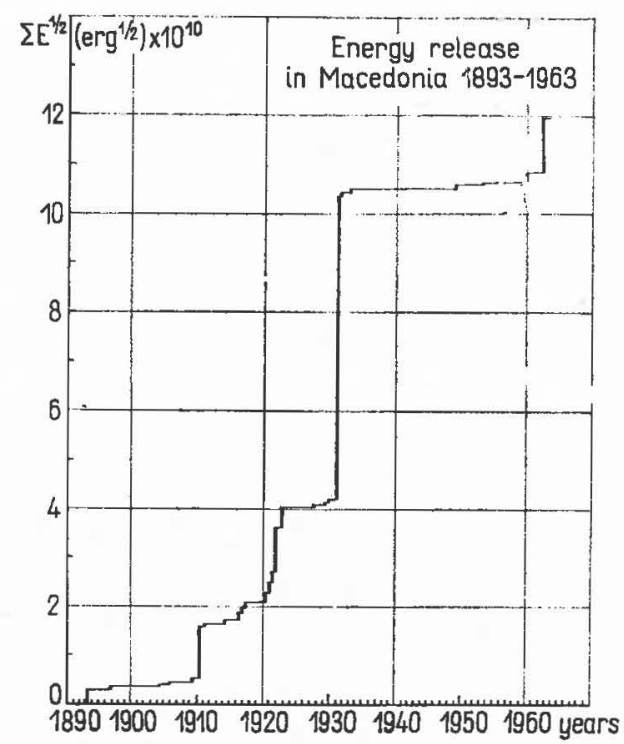

Fig. 3. Energy release and elastic strain rebound in Macedonian foci, 1893-1963

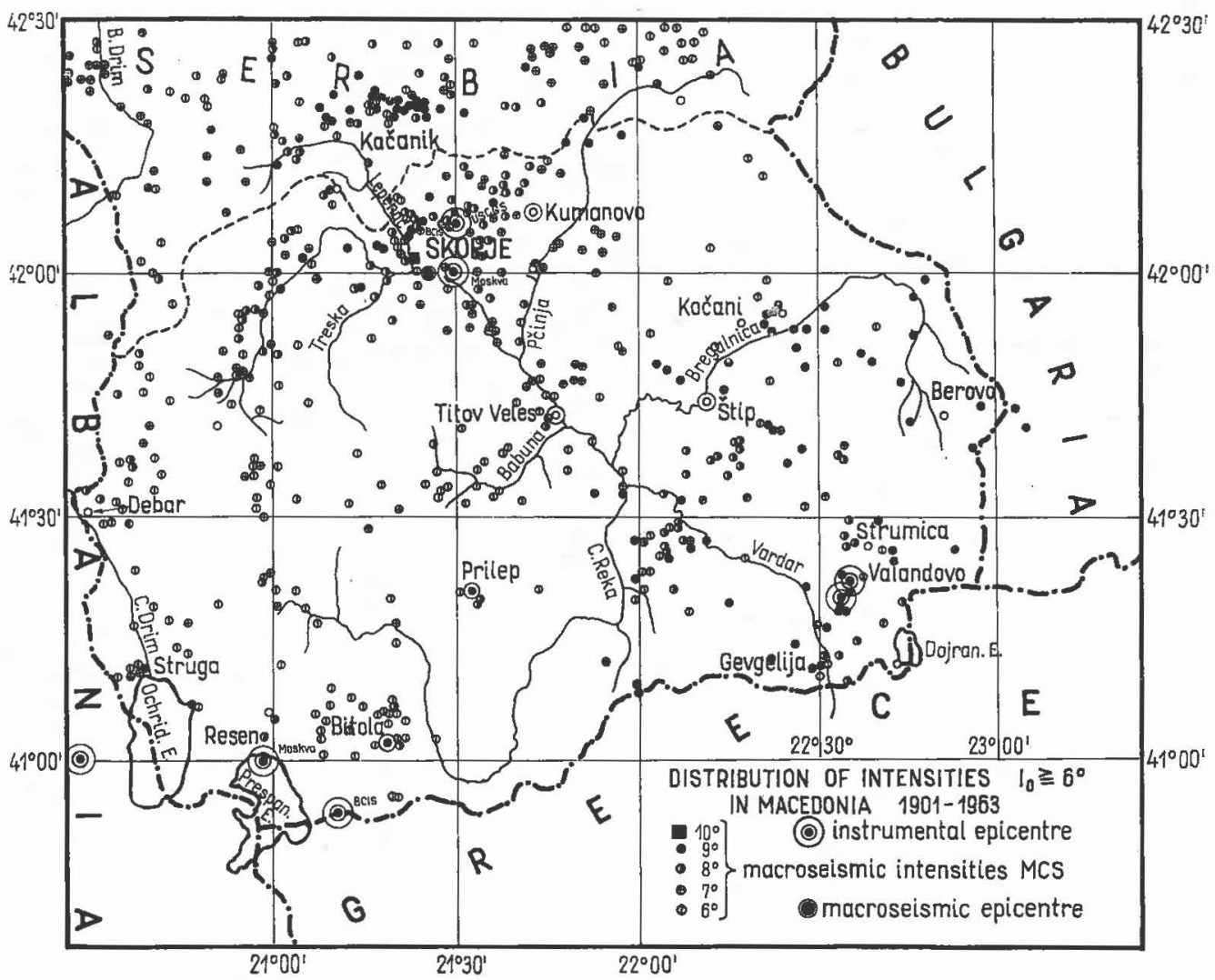

FIG. 4. Distribution of intensities $I_{0} \geqq 6^{\circ}$ in Macedonia, 1901-1963 
The southern and southwestern part of Macedonia is often visited by earthquakes too. Many strong shocks come from the foci situated in Albania and in Greece. Such earthquakes were felt on March 30, 1921, and March 15, 1958, without serious effects, but e.g. in 1858 and 1871 serious damages to structures are reported from Bitola in southern Macedonia. From the Lake of Ochrid, a seismic zone proceeds northward along the Crni Drim, the origin of numerous earthquakes of the western frontier territory of Macedonia.

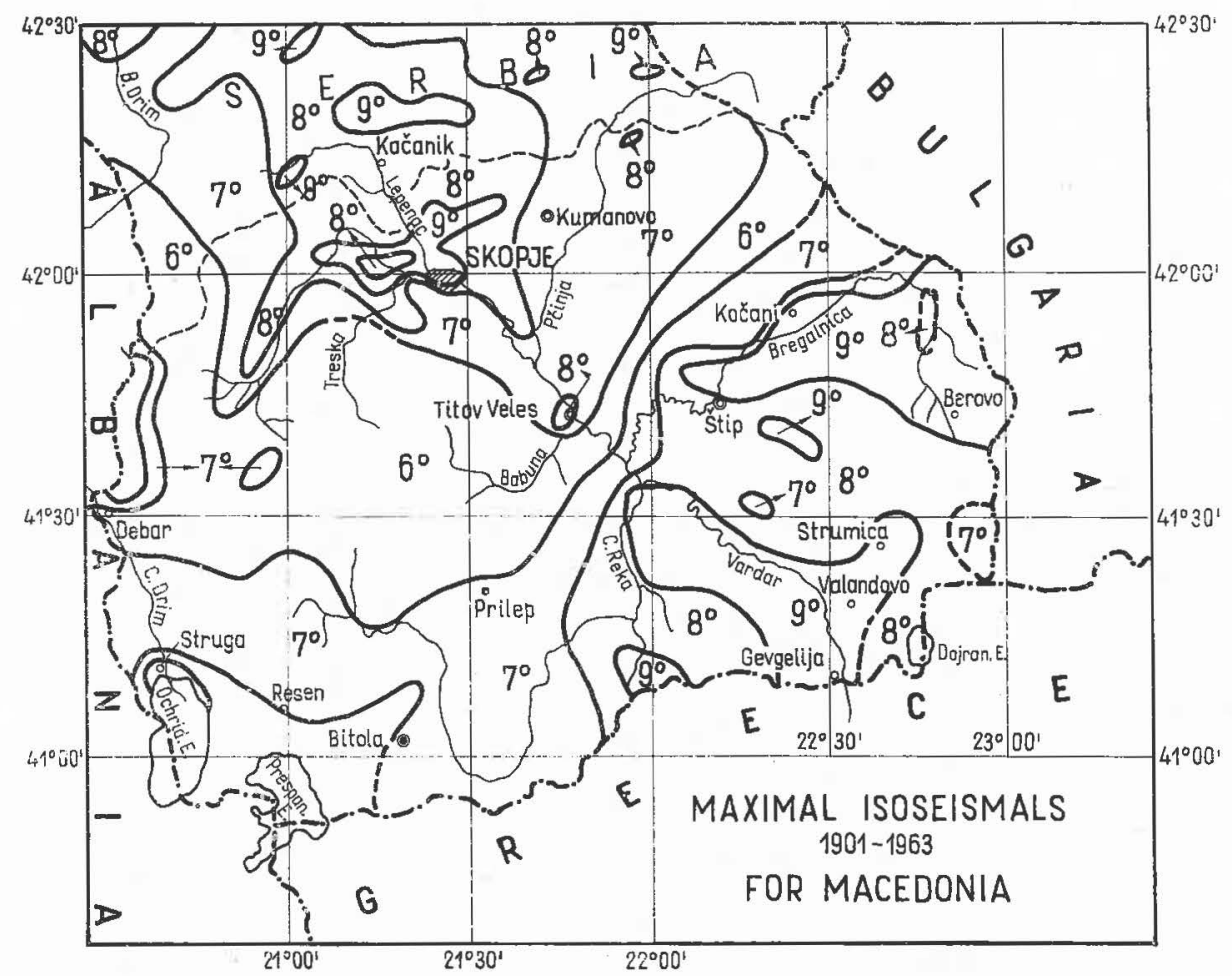

FIG. 5. Maximal isoseismals for Macedonia, 1901-1963

The upper flow of Vardar, in northwestern Macedonia, including the region of Mavrovo, in the zone passing from Gostivar to Tetovo, is distinguished by minor shocks. From time to time a stronger earthquake takes place too, often with curiously distributed intensities as that of Gradec in 1960.

From the effects of external foci those in the northeastern part of Macedonia are to be mentioned. There catastrophic effects have been caused by the earthquake whose epicentre was situated in the region of Simitli in the valley of the Struma river in western Bulgaria. This shock of April 4, 1904, caused in Macedonia serious damages to buildings, and a number of persons were killed.

This is the general picture of the distribution of seismic activity in Macedonia. 
A map of epicentres for $I_{0} \geqq 6^{\circ} M M$, (1893)-1900-1963 is represented in Fig. 2. In this map the energy density distribution of foci situated on the Macedonian territory, expressed in $Q$-units, is also represented $\left(1 Q=10^{15} \mathrm{ergs} / 100 \mathrm{~km}^{2}\right.$ year). According to Fig. 2, the most important autochtone seismic region in Macedonia is that of Valandovo (in consequence of the earthquakes of March 7

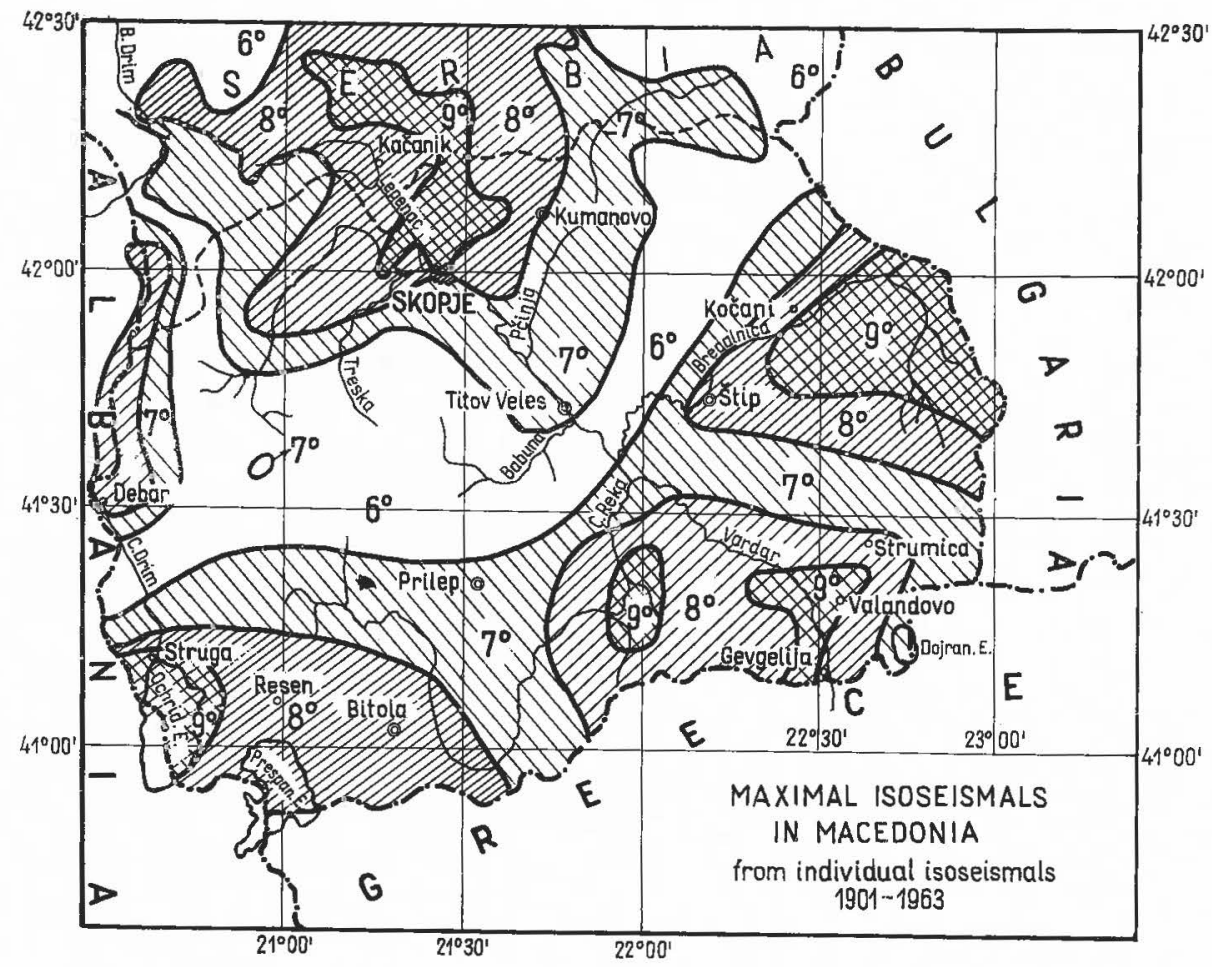

Frg. 6. Maximal isoseismals for Macedonia derived from isoseismal maps of individual earthquakes, 1901-1963

and 8,$1931 ; I_{0} \max \rightarrow 10^{\circ} M M, M=6.7$ and 7.1, respectively). The Skopje region appears to be the second one. Naturally, to receive a whole characteristic of the seismic effect on the Macedonian territory we should have elaborated the neighbouring areas in the same way.

The earthquakes of Macedonia are to be classified as superficial and shallow ones. Their focal depths, during the period considered, did probably not exceed $20 \mathrm{~km}$.

Energy release and elastic strain rebound for Macedonian foci, as a function of time, are given in Fig. 3. Periods of elevated activity may be seen in 1910 in southern Macedonia, 1920-1923 in northern and northwestern Macedonia, 1931 at Valandovo, southeastern Macedonia, and 1963 in the Skopje region. 
The energy liberated by the strongest Macedonian earthquakes 1900-1963 may be estimated as follows: Valandovo, March 7, 1931, $E \sim 10^{21} \mathrm{ergs}$, March 8 , $1931, E \sim 10^{22}$ ergs; Skopje, July 26, 1963, $E \sim 10^{21}$ ergs (according to the instrumental data). The order of energy radiated from external sources may be given by $10^{22}$ ergs for the focus near Uroševac (southern Serbia) on Aug. 10, 1921, and $10^{22}-10^{23}$ ergs for the earthquake of April 4, 1904, mentioned above and located in western Bulgaria.

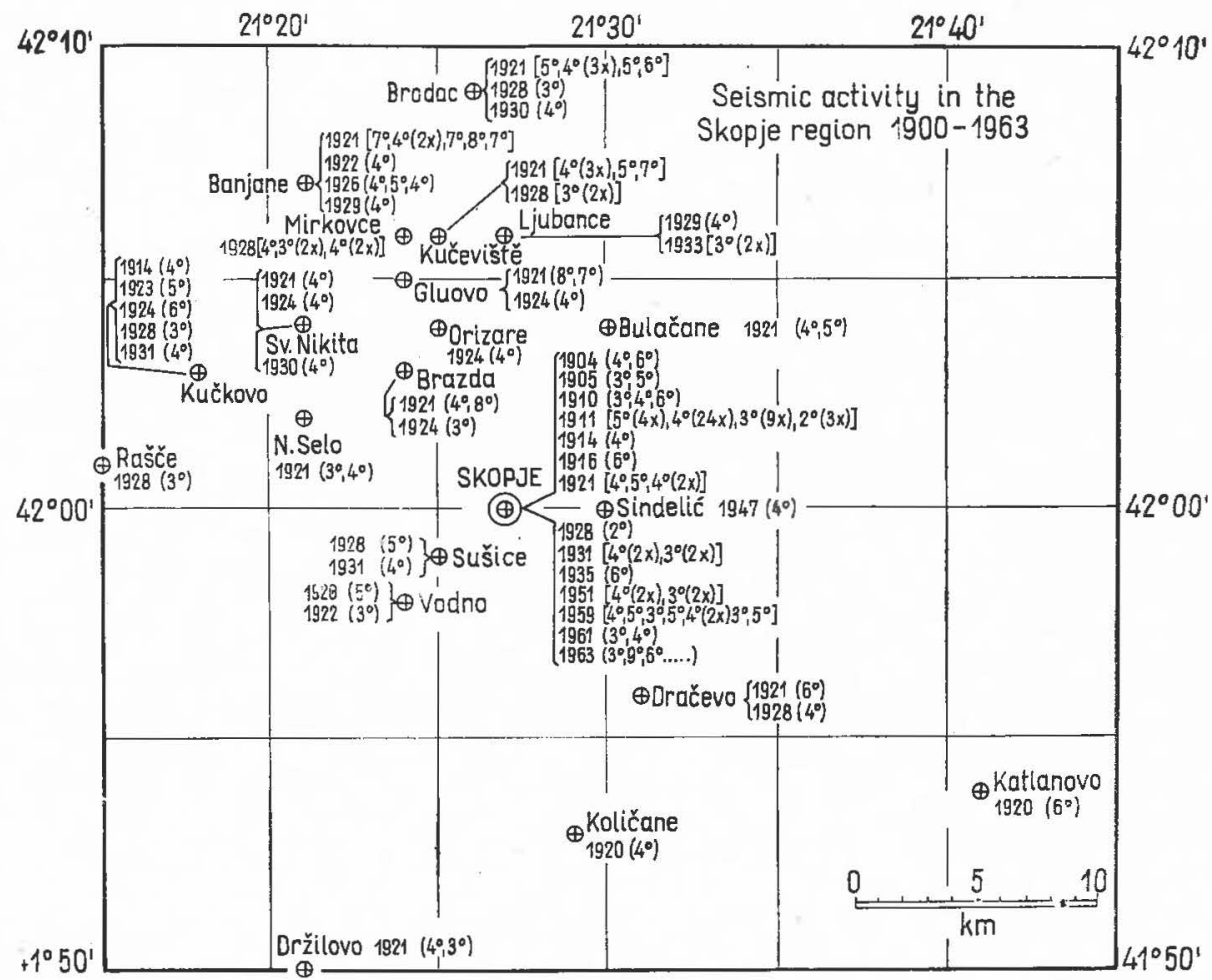

FIG. 7. Seismic activity in the Skopje region, 1901-1963

A map of intensities for $I \geqq 6^{\circ}$ for 1901-1963 is represented in Fig. 4. Here it is possible to see the influence of external sources on the distribution of seismic effects, as observed in Macedonia. Basing on this map a map of maximal intensities was constructed. It is plotted in Fig. 5.

Another version of such a map has been drawn by superposition of the maps of isoseismals of individual shocks possessing $I_{0} \geqq 6^{\circ}$ (Fig. 6). Both of these maps conserve the main features in spite of a higher degree of generalisation of the second map, and of the fact that the basic isoseismal maps were constructed independently by seismologists of the Seismological Institute in Belgrade. The intensity background of $6^{\circ}$ forms a belt crossing the Vardar zone in central 
Macedonia roughly in the WSW-ENE direction. Outside this zone all structures are dangered by earthquakes and must be protected in a measure corresponding to the maximal intensity. Inside the zone, buildings protected against wind effects may be considered automatically protected also against the earthquake up to $6^{\circ}$. Nevertheless, it is not excluded (see ruins of Stobi) that a destructive earthquake might occur in this zone, too. (A swarm of more than 50 weak earthquakes, recorded at the Skopje station on Oct. 19/20, 1963, was located near Gradsko in central Macedonia. That demonstrates that the seismic activity is still living in this region.)

An empirical relation between the epicentral intensity $6^{\circ} \leqq I_{0} \leqq 10^{\circ}$ and magnitude $M$ for mall focal depths was obtained by adjustment: $M=0.58 I_{0}+0.7$. This formula is valid for the territory of Macedonia as a whole.

The number $N$ of earthquakes having an epicentral intensity $I_{0}$ and which occurred in Macedonia during the period considered may be empirically expressed by an adjusted formula $\log N=4.31-0.43 I_{0}$. This means one $10^{\circ}$ earthquake within

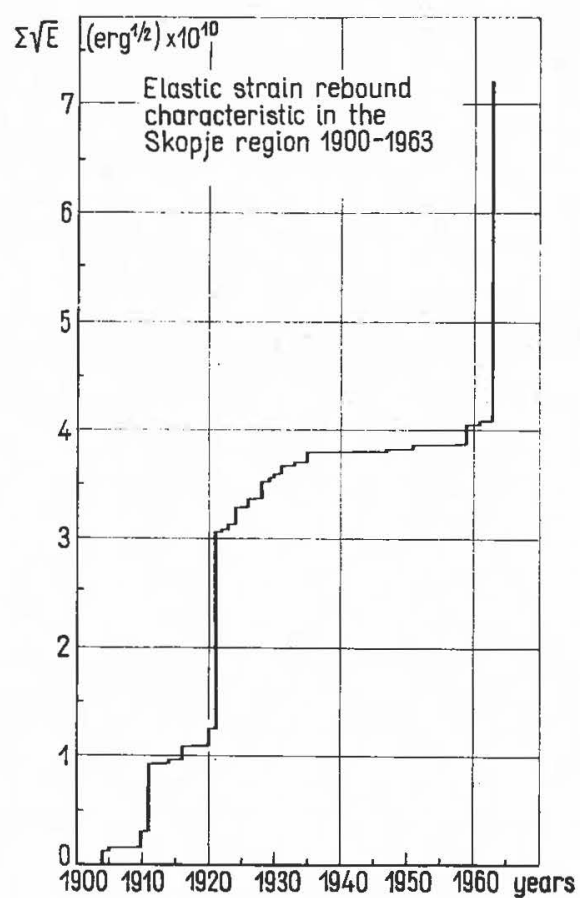

Fig. 8. Energy release and elastic strain rebound in the Skopje region, 1901-1963

about 68 years, $9^{\circ}$ within 25 years, $8^{\circ}$ within 10 years, $7^{\circ}$ within 3.5 years, and $6^{\circ}$ within 1 year, respectively. Reduced to one year interval the formula gives $\log N_{1}=2.47-0.43 I_{0}$. 
Substituting $M$ for $I_{0}$ we get $\log N=4.83-0.74 M$, and $\log N_{1}=2.98-0.74 M$, respectively. This means one earthquake of magnitude 7 within about 158 years, that of magnitude 6 within about 29 years, etc.

As it concerns the seismic activity in the Skopje region, it is represented in Fig. 7, where epicentres, years and intensities are plotted. By means of this map, an elastic strain rebound characteristic as function of time was represented in Fig. 8. It shows the main active periods in 1910, in 1921 (followed by a moderate activity during the period 1921-1935), and in 1963 (preceded by a relatively very long quiet period).

Remark: A detailed study of the seismicity of the Skopje region and of Macedonia was also made by N. N. Ambraseys (1963/64). 


\title{
TÄTIGKEITSBERICHT DER SUBKOMMISSION FÜR DIE SEISMISCHE UNTERSUCHUNG DES KARPATEN- UND BALKANGEBIETS IM ZEITRAUM 1962-1964
}

\author{
A. ZÁTOPEK
}

(Prag, Tschechoslowakei)

Nach der Formulierung des Arbeitsprogramms in Jena wurde es klar, dal3 sich die Aufgaben der seismischen Untersuchung des Karpaten- und Balkangebiets in drei Gruppen gliedern:

1. Untersuchung der Seismizität, die zur Interessensphäre der Subkommision für Seismizität gehört.

2. Entwicklung des Netzes der seismischen Stationen in den Karpaten- und Balkanländern.

3. Seismische Untersuchung der Erdkrustenstruktur, vor allem seismische Tiefensondierungen.

ad 1. In den meisten Ländern hat man die Bearbeitung der Nationalkataloge im Hinblick auf die Vervollständigung der Angaben über ältere und schwächere Erdbeben bereits vollzogen, obwohl die Arbeiten noch bei weitem nicht als abgeschlossen betrachtet werden können. In der Kommission für Seismizität wurde dies eingehend diskutiert und ich habe darüber im Rahmen meines Berichts über die Tätigkeit der ESK zum Teil bereits referiert. Zunächst wurden die Einzelstöße studiert, z. B. die Erdbeben im Gebiet des Kaspischen Meeres in der UdSSR, weiterhin das katastrophale Erdbeben vom 26. Juli 1963 in Skopje sowie dessen Nachstöße, dann das jugoslawische Erdbeben vom 13. April 1964 in Slawonski Brod; die Untersuchung der im Gebiet von Vrancea in Rumänien beobachteten Erdbeben wurde fortgesetzt und die vogtländischen Erdbeben im Grenzgebiet der DDR und der CSSR sowie die in den Ostalpen im Dezember 1959 bzw. im Juli 1964 entstandenen Erschütterungen wurden auf dem Gebiet der ČSSR verfolgt.

Die Generalversammlung der Internationalen Assoziation für Seismologie und Physik des Erdinnern hat die in Jena besprochene modifizierte makroseismische Erdbebenskala diskutiert und eine Kommission für die Vereinheitlichung der makroseismischen Skalen unter der Leitung von Dr. Sponheuer gebildet. Die karpatische Subkommission verwendet diese Skala nach Möglichkeit konsequent, wo immer ihr statistischer Charakter dies zuläßt. Die Arbeit nach dieser Skata erfordert natürlich, die Sammlungsmethoden der Beobachtungen so zu verbessern, da $B$ man mit ihrer Hilfe repräsentative Werte für makroseismische Intensitäten ermitteln könne.

Die Seismizität von Makedonien (Jugoslawien) wurde nach den von der ESK in Alicante und Jena festgesetzten Prinzipien studiert, wobei auch die seismo- 
tektonischen Beziehungen eingehend untersucht wurden. Im Rahmen der UNESCO wurden beim Neuaufbau von Skopje die Ergebnisse mehrerer Missionen praktisch vollkommen ausgenutzt.

In der revidierten Karte der seismischen Rayonierung der Ukrainischen und Moldauischen Republik wurden neue Kriterien (Wiederholungstendenz, Magnitudenklassifikation, Genauigkeitsbestimmung der Ortung von Bebenherden usw.) verwendet und für den demnächst erscheinenden Erdbebenatlas der Sowjetunion vorbereitet.

Die Probleme des Mechanismus der Herdvorgänge wurden mit Anwendungen in der UdSSR (teilweise in Zusammenarbeit mit der ČSSR), in Jugoslawien und in Polen theoretisch untersucht. Das in der Resolution 3 empfohlene systematische Studium wurde jedoch noch nicht in Angriff genommen.

ad 2. Das Erdbeben von Skopje hat gezeigt, daß das Netz der seismischen Stationen auf der Balkanhalbinsel den modernen Anforderungen der Untersuchung dieses aktiven Gebiets noch bei weitem nicht genügt. Die Netzdichte ist gering, die Empfindlichkeit der Instrumente im allgemeinen ungenügend. Auch im Karpatengebiet ist die Qualität des Netzes den Bedürfnissen einer progressiven Forschung noch nicht angemessen. Elektromagnetische Seismometer mit einer in der Resolution 3 empfohlenen geradlinigen, konstanten Fręquenzcharakteristik im Periodenbereich von 0,1 bis $10 \mathrm{sec}$ und mit einer Vergrößerung der Größenordnung $10^{3}$ wurden zwar an einigen Stationen in der Tschechoslowakei, in der Deutschen Demokratischen Republik (die wegen der seismischen Tiefensondierungen den Karpatenländern zugeordnet wird), in Polen, Ungarn und Bulgarien eingeführt. Einige Stationen (UdSSR, ČSSR, Ungarn) wurden auch mit anderen hochempfindlichen Instrumenten versehen. Es besteht jedoch Mangel an langperiodischen Seismometersätzen, an deren Entwicklung in der DDR, in Polen und schon längere Zeit in der UdSSR gearbeitet wird.

Die obenangeführten Instrumentensysteme Kirnosschen Typs (meistens in der UdSSR hergestellt) sind zwar als homogen anzusehen, bilden aber infolge lokaler Abweichungen im Einsatz kein wirklich homogenes Netz.

Fortlaufend registrierende elektromagnetische Seismographen gibt es in der Balkanzone, außer Athen, nur in Sofia und in Ljubljana. Mit Hilfe der Vereinigten Nationen soll nun zunächst in Makedonien, dann in Jugoslawien und schließlich auf der ganzen Balkanhalbinsel ein modernes Netz mit entsprechenden standardisierten Sätzen von hochempfindlichen Kurzperiodenseismographen, mittel- und langperiodischen sowie "strong-motion «-Instrumenten ausgebaut werden. Dies soll für Jugoslawien von einer aus UNESCO-Experten und jugoslawischen Seismologen bestehenden Kommission vorbereitet werden.

Zwecks einheitlicher und möglichst schneller Weiterleitung der seismologischen Angaben an die Interessenten soll in Moskau vom Institut für Physik der Erde alle 10 Tage ein operatives Bulletin ausgegeben werden.

ad 3. In Jena wurde bei der karpato-balkanischen Subkommission eine von Frau Dr. I. P. Kosminskaja geleitete Koordinationsgruppe geschaffen, die eine ökomonische Durchführung der in Osteuropa vorgesehenen seismischen Tiefen- 
sondierungen vorschlagen sollte. Nach einem Briefwechsel gelang es zunächst auf mehreren Teilkonferenzen bilaterale Vereinbarungen zwischen der DDR, der ČSSR, Polen und Ungarn über die Messung von über mehrere der genannten Länder laufende Profile zu erzielen. An diesen Verhandlungen nahm auch der Präsident der ESK teil, der auch auf der Konferenz in Moskau anwesend war, wo im Juni 1964 unter der Leitung von Dr. Kosminskaja ein zeitlicher Plan ausgearbeitet wurde, um in den Jahren 1964 bis 1967 sechs internationale Profile durchzumessen. An der Moskauer Konferenz nahmen Vertreter der UdSSR, der Tschechoslowakei, von Polen, Ungarn und Bulgarien teil. Eine weitere Konferenz über die seismischen Tiefensondierungen soll in der Tschechoslowakei Ende 1964 veranstaltet werden.

Einige der angeführten Profile wurden bereits gemessen. In den polnischen Profilen ergab sich für die Tiefe der Mohorovičić-Diskontinuität in der Polnischen Tiefebene ein Wert von $26 \mathrm{~km}$ und in Westpolen, nördlich der Karpaten ein Wert von $29 \mathrm{~km}$.

Soviel über die Arbeiten, die im Rahmen der karpatisch-balkanischen Subkommission in bezug auf die Resolution 3 ausgeführt wurden.

Die anderen Arbeitsrichtungen und Ergebnisse habe ich in meinem Bericht über die Tätigkeit der ESK dargelegt.

Aus dem Gesagten ergeben sich folgende Empfehlungen:

1. Es wird empfohlen, das Netz der seismischen Stationen im Karpaten- und Balkangebiet durch Aufstellung von homogenen und standardisierten Seismographen an den Hauptstationen entsprechend den Vorschlägen der Internationalen Assoziation für Seismologie und Physik des Erdinnern zu vervollkommnen und durch die notwendige Anzahl von geeigneten Neben- oder Hilfsstationen, vor allem in den seismisch aktiven Gebieten zu verdichten;

2. die Katalogisierung der älteren und schwächeren historischen Erdbeben fortzusetzen und das Standardisieren auch auf die Auswertungsmethoden $\mathrm{zu}$ erweitern;

3. alle makroseismisch beobachteten Erdbeben sorgfältig zu studieren und die Dokumentation in den einzelnen Ländern zu zentralisieren;

4. die Sammlungsmethoden der makroseismischen Beobachtungen und deren Auswertung nach der modifizierten Skala Medwedew-Sponheuer-Kárnik ständig $\mathrm{zu}$ verbessern;

5. die Ergebnisse der seismischen Tiefensondierungen möglichst bald zu interpretieren;

6. bei der Auswertung und Bearbeitung der seismologischen Beobachtungen die zeitgemäße Technik, unter maximaler Heranziehung der Mechanisations- und Automatisations-Methoden, systematisch anzuwenden. 

INSTRUMENTS AND DEVICES 



\title{
NEW NETWORK OF SEISMOLOGICAL STATIONS IN HUNGARY
}

\author{
E. Bisztricsány, D. Csomor, L. Egyed and Z. Kiss
}

(Budapest, Hungary)

A few years ago the network of seismological stations was still in a very neglected state. Altogether 5 horizontal seismographs of mechanical registration have been working at 5 stations. Urban traffic hindered the development of the stations in question. The organization of a new network of stations became urgent.

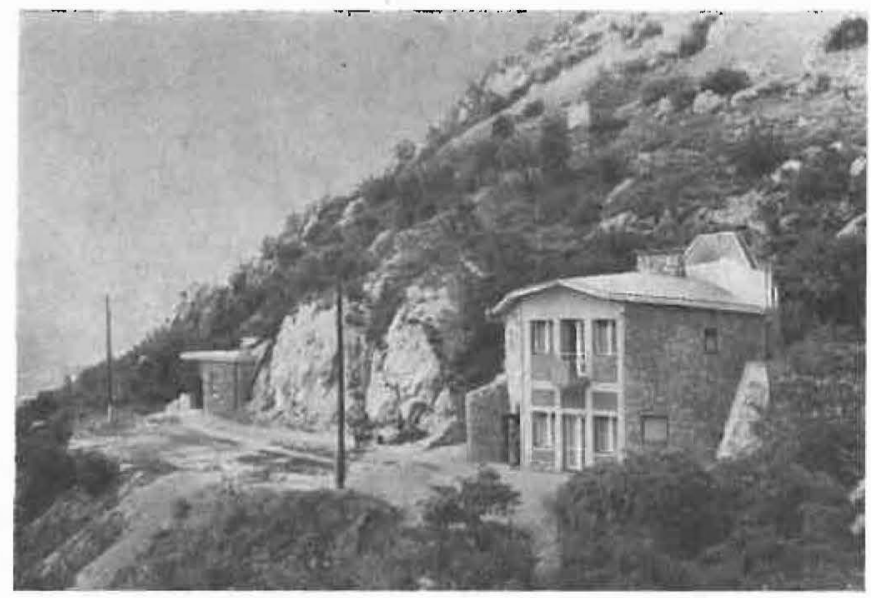

Fig. 1. Working rooms built in front of both entrances

The first up-to-date station has been set up in Budapest. The expenses were relatively low. A former ammunition-magazine of the second World War situated in the interior of the Sashegy (Mountain) was made use of. The level hight of the station amounts to $190 \mathrm{~m}$ (Fig. 1). The Sashegy is made up by Triassic dolomite, Fig. 2 shows the plan of the station. The rooms where the instruments are installed are situated deep in the interior of the hill, their temperature is as high as $11^{\circ} \mathrm{C}$ both in summer and winter. A $20 \mathrm{~m}$ thick dolomite layer overlies the rooms of the station. Refrigerators of $2.5 \mathrm{~kW}$, situated at the 2 entrances assure the relative humidity of 70 percent of the station. The concrete blocks of the instruments are built directly on the dolomite bed. In the room marked by No. 1 in Fig. 2 is a horizontal pendulum of Wiechert type (Fig. 3) magnification 200, natural period $10 \mathrm{sec}$. The velocity of the smoked paper is $22 \mathrm{~mm} / \mathrm{min}$. 


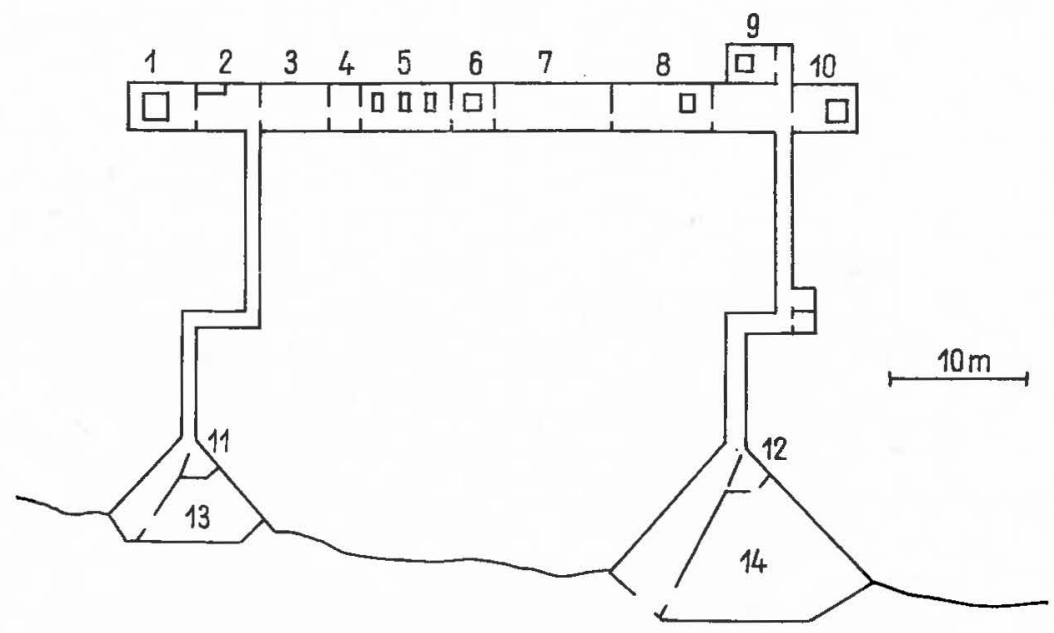

Frg. 2. Plan of Station. Application of rooms: 1. Wiechert pendulum, 2. Accumulator charger and paper smoker, 3. room for direct registration, 4. time control equipment, 5. electrodynamic pendulum, 6. Anderson-Wood pendulum, 7. recorders, 8-10. disused rooms, 11-12. refrigerators, 13-14. working rooms

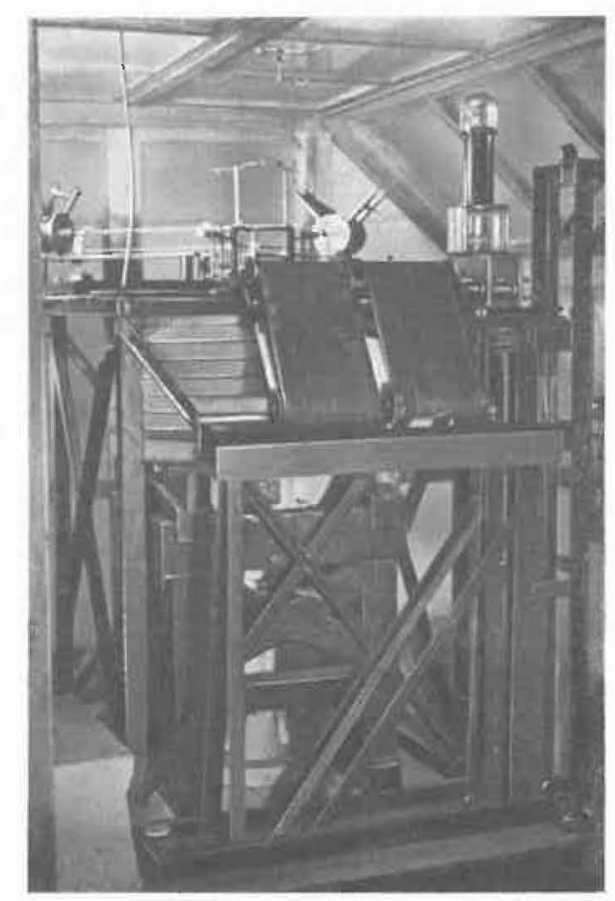

FIG. 3. Wiechert pendulum 
In a neighbouring room a battery charger is installed. In an interior room a time-marker is to be found while in another room a horizontal seismograph of Anderson-Wood type of 1000 magnification, $T=0.8 \mathrm{sec}$, the velocity of the

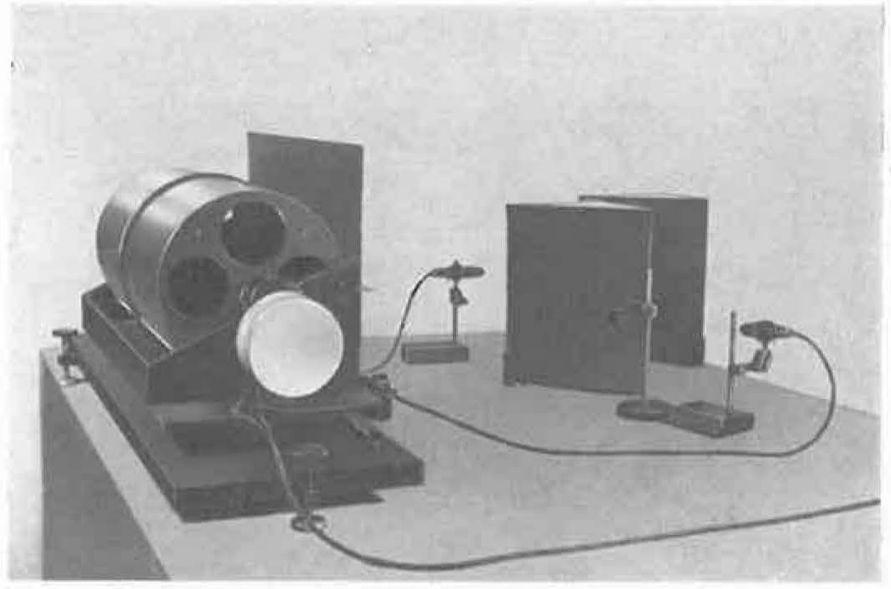

FIG. 4. Anderson-Wood pendulum

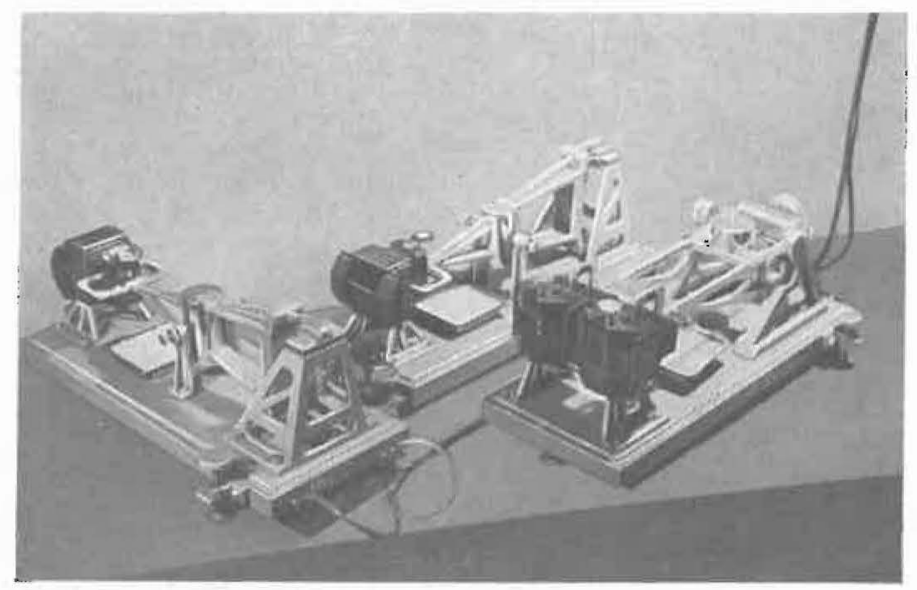

FIG. 5. Kirnos pendulum

paper amounts to $60 \mathrm{~mm} / \mathrm{min}$ (Fig. 4). Most of the equipment will be arranged in a room with 3 blocks. At present a Kirnos system of 3 components is operating, the natural period of the horizontal pendulums amounts to $12.5 \mathrm{sec}$, that of the galvanometers to $2 \mathrm{sec}$, their magnification is 1000 , the magnification of the vertical pendulum is 650 the velocity of the paper is $30 \mathrm{~mm} / \mathrm{min}$ (Fig. 5). Moreover, a Vegik pendulum with three components is operating, its magnification is 
17000 , natural period $1 \mathrm{sec}$, that of the galvanometer $0.08 \mathrm{sec}$. The velocity of the paper is $80 \mathrm{~mm} / \mathrm{min}$ (Fig. 6).

With the exception of the Wiechert pendulum the registration takes places on photographic paper (Fig. 7). At the front of the entrances there are working rooms.

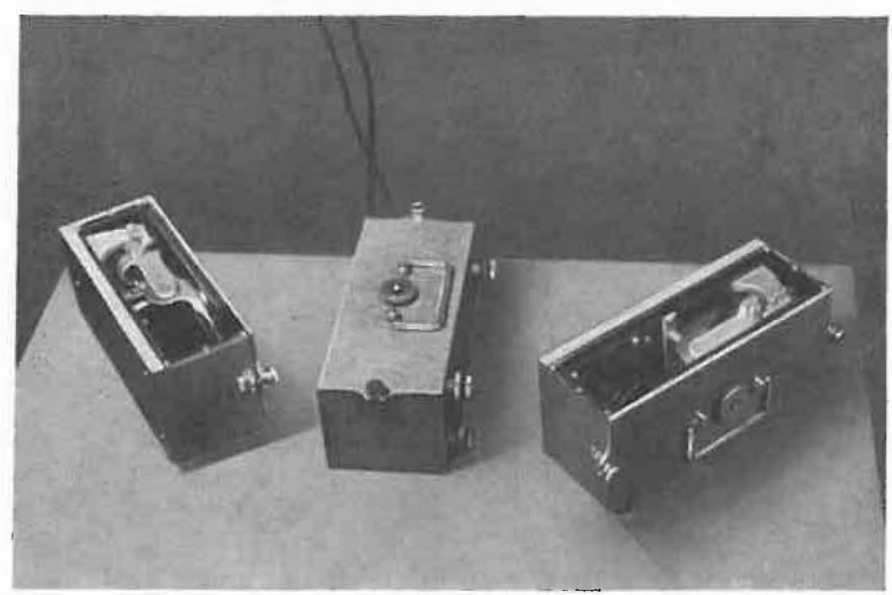

Frg. 6. Vegik pendulum

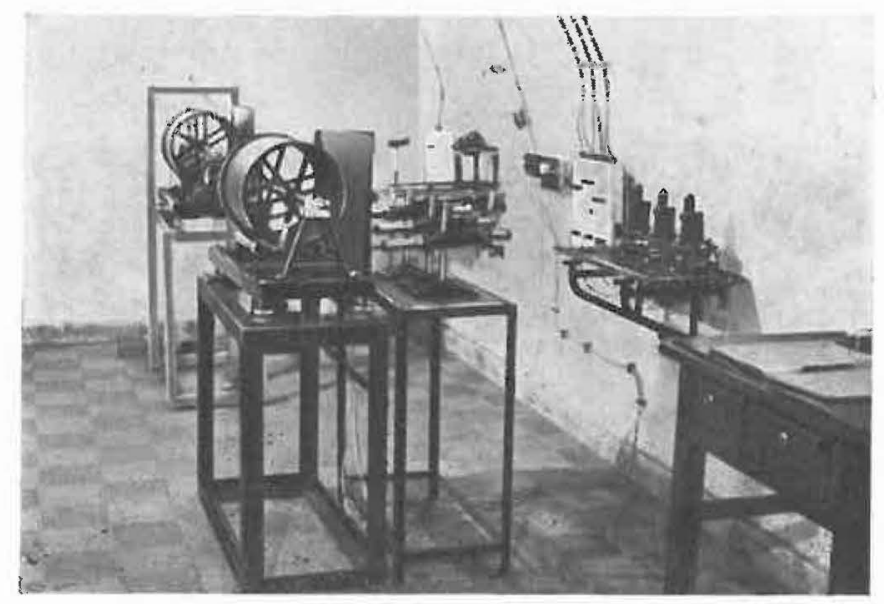

FIG. 7. Room of the recorders

Our second new seismological station has worked continuously since 1963 in Mátra Mountains. It is located $20 \mathrm{~km}$ from the nearest town Gyöngyös, on the top of a $940 \mathrm{~m}$ high mountain (Fig. 8). 
At this station a vertical pendulum of Kirnos type is operated, $T_{p}=12.5$, $V=700$, the velocity of the photographic paper is $30 \mathrm{~mm}$. There has also been a Vegik Seismograph for eight months $T_{p}=1 \mathrm{sec}, T_{g}=0.08 \mathrm{sec}$, magnification of

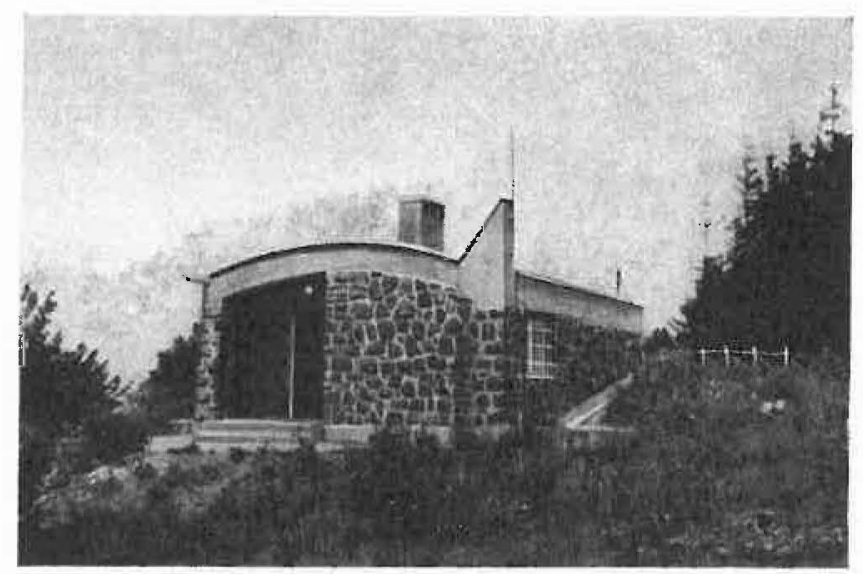

FIG. 8. Seismological station, Piszkéstetö

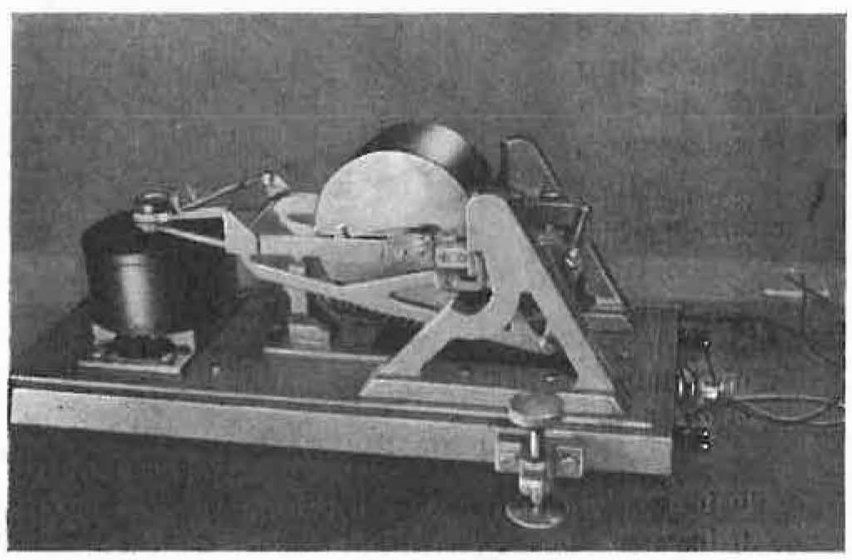

FIG. 9. Modified Kirnos vertical pendulum constructed in Budapest

40000 and drum speed is $60 \mathrm{~mm} / \mathrm{min}$. Although the region is covered by woods and there is a mine working in a distance of a few kilometers, the ground disturbance is very low. Hence, the starting up of a home made modified Kirnos vertical pendulum (Fig. 9) of great magnification became possible; $T_{p}=1.2 \mathrm{sec}$, $T_{g}=0.08 \mathrm{sec}, V=250000$. A relative humidity of 65 percent is fixed by an air conditioning equipment. 


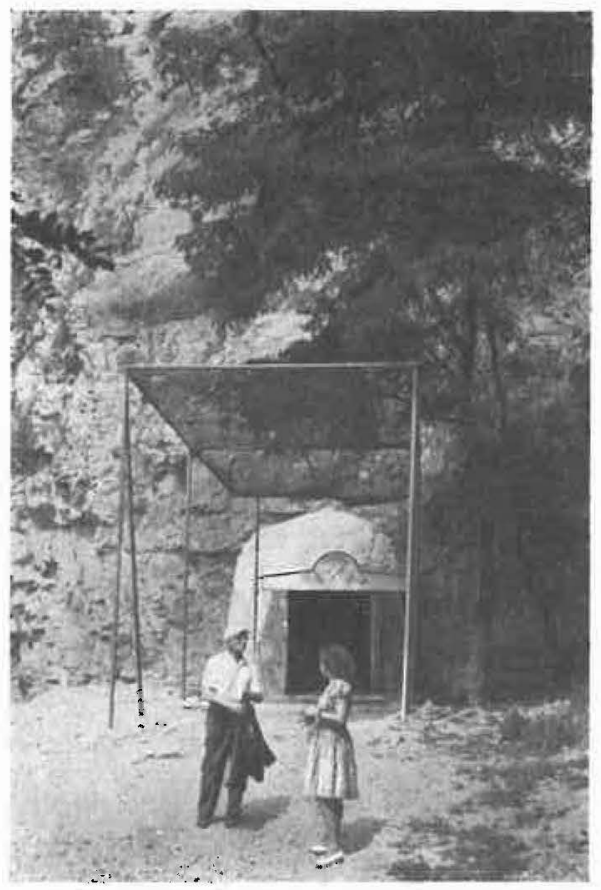

FIG. 10. Entrance of the Sopron station

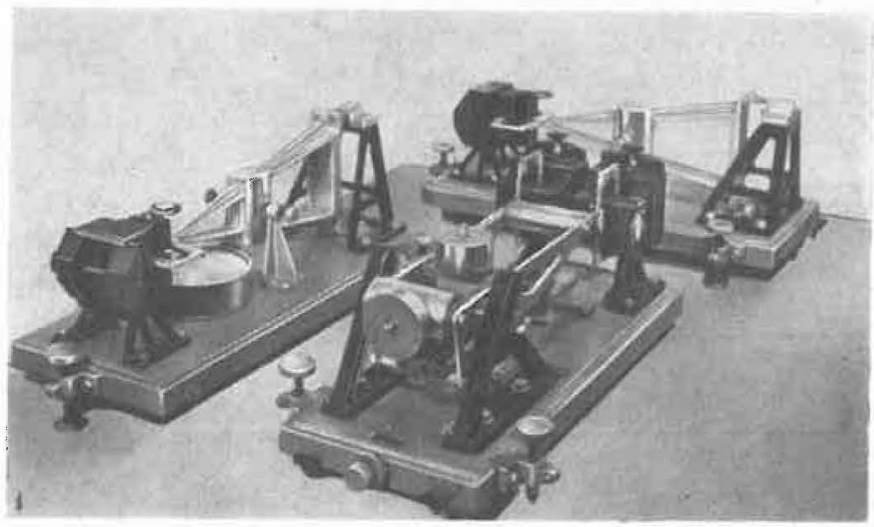

FIG. 11. Kirnos pendulum

The third new station is situated in a distance of a few kilometres from the town Sopron (Fig. 10). The blocks of the instruments are built on crystalline schist (Fig. 11). A Kirnos seismograph system of 3 components is operating there.

The relative humidity of 65 percent is fixed by an air conditioning equipment similar to that of the Mátra station. 


\title{
PHASE VELOCITIES OF RAYLEIGH WAVES \\ ON THE RUSSIAN PLATFORM
}

\author{
G. N. Bozhko and O. E. Starovort
}

(Moscow, USSR)

For some years past there has been a great development in the application of the method of the surface waves' phase velocities for the investigation of local geological regions [1-3]. This method consists of the determination of phase velocities by means of (not less than two) stations, situated on a section of a great circle in a line with the earthquake epicentre, as well as of the comparison of experimental data with theoretical dispersion curves for an estimate of the crust structure on the section between the stations.

Here we give some results of the determination of the phase velocities of Rayleigh surface waves of the mine tone on the Russian platform (Fig. 1) as well as a preliminary estimate of the mantle thickness.

The choice of the Russian platform is explained by the fact, that it is a considerably large geological region with undisturbed tectonics. The surface waves on the section between the stations travel through a practically similar section.

For instance, the Moscow-Pulkovo route is situated on the Moscow cynclinal, which consists of the ancient foundation mostly covered with a uniform horizontal sedimentary layer.

These conditions are most satisfactory for the application of the phase velocity method. An analysis of surface Rayleigh waves' records has been carried out, the recording seismic stations being those of "Moscow", "Pulkovo", "Lvov".

TABLE 1

The earthquakes considered

\begin{tabular}{|c|c|c|c|c|c|c|c|c|c|}
\hline \multirow{2}{*}{ No. } & \multirow{2}{*}{ Data } & \multirow{2}{*}{\multicolumn{3}{|c|}{ Origin time }} & \multicolumn{2}{|c|}{ Coordinates } & \multirow{2}{*}{$M$} & \multirow{2}{*}{ Region } & \multirow{2}{*}{$\begin{array}{c}h \\
\mathrm{~km}\end{array}$} \\
\hline & & & & & $\phi^{2}$ & $\lambda^{\circ}$ & & & \\
\hline 1 & 18. 8.1959 & 06 & 37 & 18 & $44.5 \mathrm{~N}$ & $111 \mathrm{~W}$ & $71 / 2$ & $\begin{array}{c}\text { The Rocky } \\
\text { Mountains }\end{array}$ & - \\
\hline 2 & 18. 8. 1959 & 15 & 26 & 10 & $44.5 \mathrm{~N}$ & $111 \mathrm{~W}$ & $61 / 4$ & $\begin{array}{c}\text { The Rocky } \\
\text { Mountains }\end{array}$ & - \\
\hline 3 & 6. 11.1960 & 04 & 38 & 16 & $52.2 \mathrm{~N}$ & $159.8 \mathrm{~W}$ & $61 / 2$ & Kamchatka & 32 \\
\hline 4 & 11. 5.1962 & 14 & 11 & 54 & $17.2 \mathrm{~N}$ & $99.7 \mathrm{~W}$ & 7 & Mexico & normal \\
\hline 5 & 19. 5. 1962 & 14 & 58 & 13 & $17.1 \mathrm{~N}$ & $99.4 \mathrm{~W}$ & 7 & Mexico & normal \\
\hline 6 & 28. 3. 1963 & 00 & 15 & 50 & $66.5 \mathrm{~N}$ & $19.5 \mathrm{~W}$ & 5 & Greenland Sea & 15 \\
\hline 7 & 4. 9.1963 & 13 & 32 & 09 & $71.4 \mathrm{~N}$ & $73.4 \mathrm{~W}$ & 6 & Baffin-Island & - \\
\hline 8 & $15.10,1963$ & 09 & 59 & 26 & $62.2 \mathrm{~N}$ & $18.5 \mathrm{~W}$ & 5 & Iceland & 33 \\
\hline
\end{tabular}


Figure 2 shows the records. The technical characteristics of the seismograph are given in [4].

The choice of earthquakes was such a one, that pairs of stations and epicentres were situated on one section of the great circle. The rest of the earthquakes are such, that the angle between the lines stations-epicentre and station-station is about $5-7^{\circ}$.

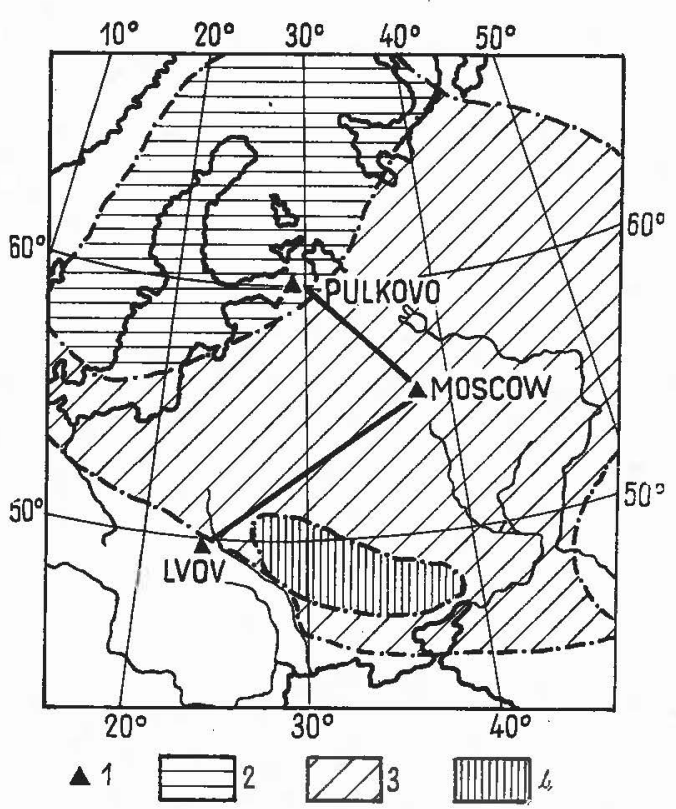

FIG. 1. The locations of the seismic stations and the homogeneity of geological spaces. 1 - seismic stations, 2-Baltien shield, 3-Russian Platform, 4-Ukrainian crystalline rock mass

This condition is only satisfied by earthquakes $3,4,5,6,8$. The calculation of the phase velocity was made on Moscow-Pulkovo and Moscow-Lvov routes (Fig. 1) on the base of method [5].

During the determination of velocities in connection with the application of different types of seismographs corrections were made in phase change, i.e. real determination of the earth displacement. Figure 2 shows the plotted record after estimation of phase disfigurations of the seismograph.

The records of the earthquake, on the stations Moscow and Pulkovo that occurred in September 4, 1963, have been digitized with $\Delta t=2 \mathrm{sec}$.

Spectral analysis was made by $\mathbf{M}-20$ electronic computer. The comparison (Fig. 3) of phase velocities, calculated directly on the base of seismogramms and phase spectors show satisfactory results. The average difference between velocities does not amount to more than $0.03 \mathrm{~km} / \mathrm{sec}$ (of order $1 \%$ ). 


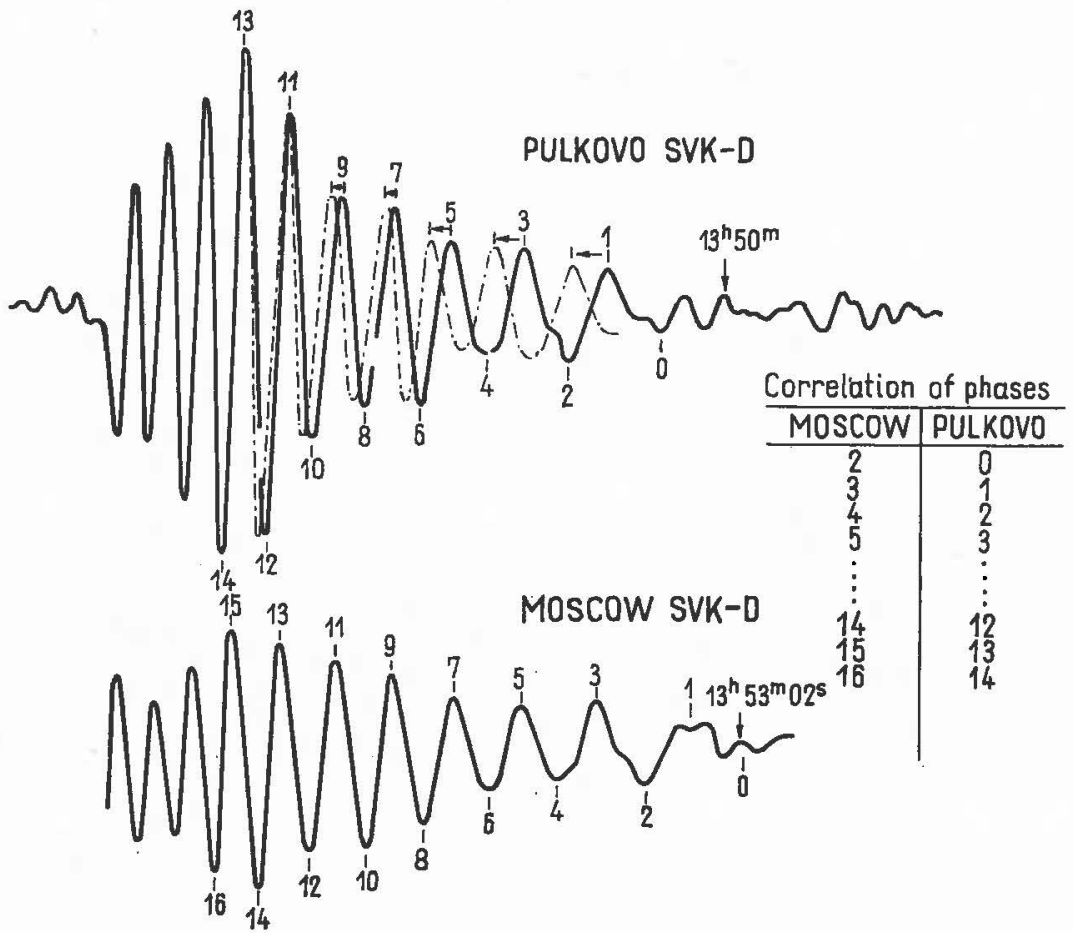

Fig. 2. The record of the earthquake September 4, 1963 on Moscow and Pulkovo stations and correlation of the phase

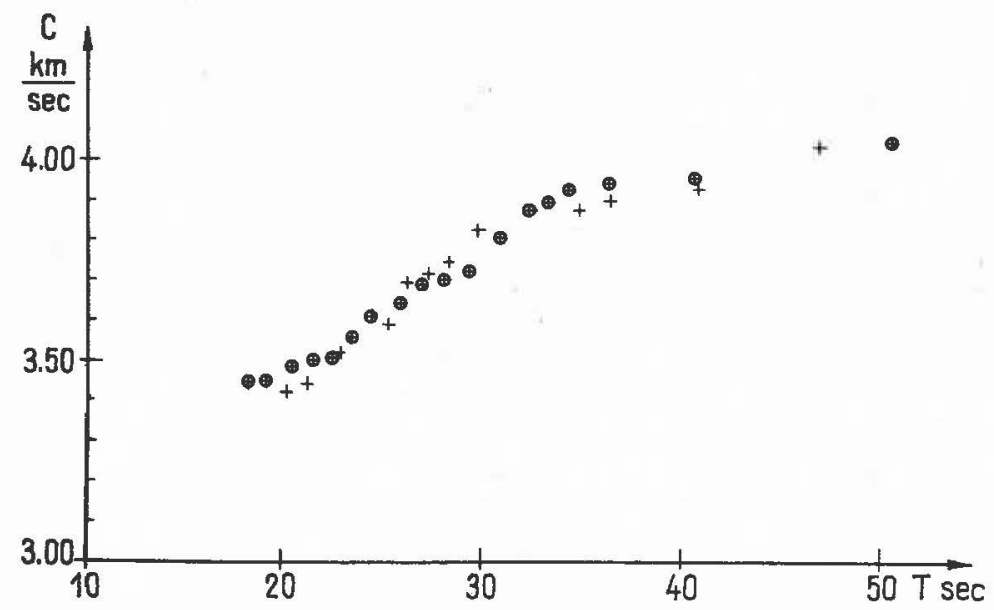

Fig. 3. Comparison computed of the phase velocity by means of "hand method" (cross) and Fourier analysis method (cross in circle) 
On the Moscow-Pulkovo route $(\Delta=622 \mathrm{~km})$ phase velocities are determined for the waves with periods from $15 \mathrm{sec}$ at the relative change of the velocities from $3.27 \mathrm{~km} / \mathrm{sec}$ to $4.08 \mathrm{~km} / \mathrm{sec}$ (see Fig. 4). The difference between velocity values for the period is not more than $0.1 \mathrm{~km} / \mathrm{sec}$.

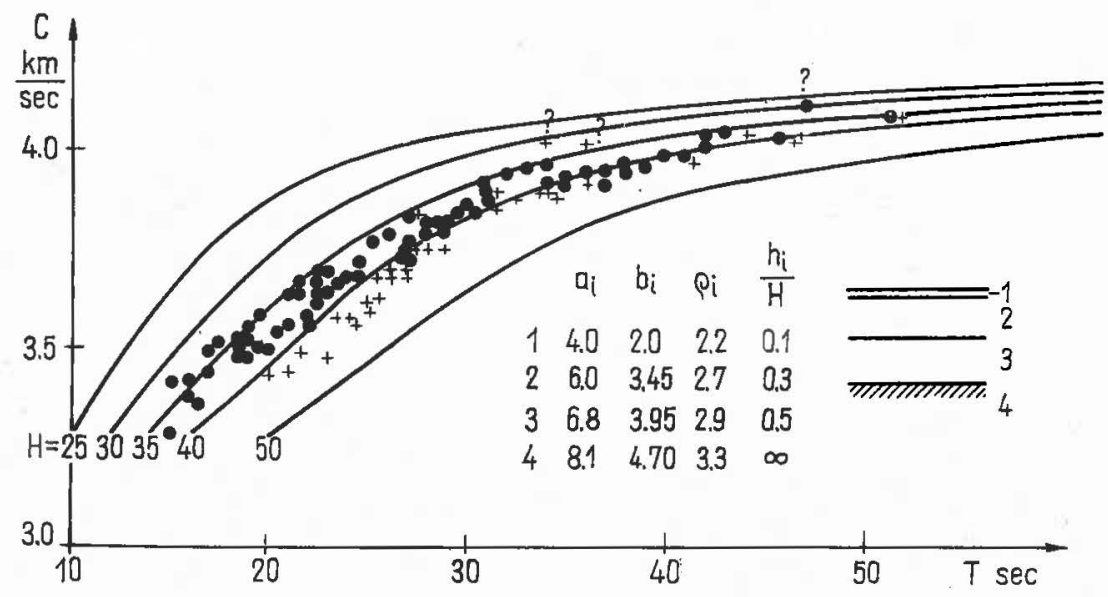

Fig. 4. Dispersion curves of the phase velocity for the trace Moscow-Pulkovo. The earthquake epicentres are located in range (solid circle) and not in range (cross) with stations. Theoretical curves for three-layers model. Parameters of the layers: compressional $(\alpha)$ and shear $(\beta)$ waves velocity in $\mathrm{km} / \mathrm{sec}$; density in $\mathrm{gm} / \mathrm{cm}^{3}$ and $h_{1,2,3}$-thickness of the layers in $\mathrm{km} ; H=h_{1}+h_{2}+h_{3}$

The difference between the values obtained from earthquakes lying in the line station-station and those obtained from earthquakes not satisfying, this condition can be clearly discerned. The former are 4, 5, 6, 8 in Table 1 and the latter are $1,2,7$.

The latter give reduced values of the phase velocity (of the order $0.1 \mathrm{~km} / \mathrm{sec}$ ) for periods from $20 \mathrm{sec}$ to $28 \mathrm{sec}$. This fact can be explained by different refractions of surface waves travelling from the epicentre to the station by various routes.

On the Moscow-Lvov route the phase velocities are calculated for one earthquake (see earthquake 3, Table 1) for waves' periods from $18 \mathrm{sec}$ to $20 \mathrm{sec}$ (phase velocities $3.25-3.75 \mathrm{~km} / \mathrm{sec}$ ).

Obtained dispersion curves of the phase velocities of Rayleigh surface waves on the Moscow-Pulkovo and Moscow-Lvov routes were compared with theoretical curves (Figs 4, 5).

Theoretical dispersion curves have been computed [6] for the layer parameters characteristic of the Russian Platform.

The phase velocities on the Moscow-Pulkovo route are satisfactorily in keeping with the theoretical çurve (Fig. 4). The total thickness of the crust and the sedimentary layer on this route is accordingly $38-40 \mathrm{~km}$ and $3.5-3.7 \mathrm{~km}$. 


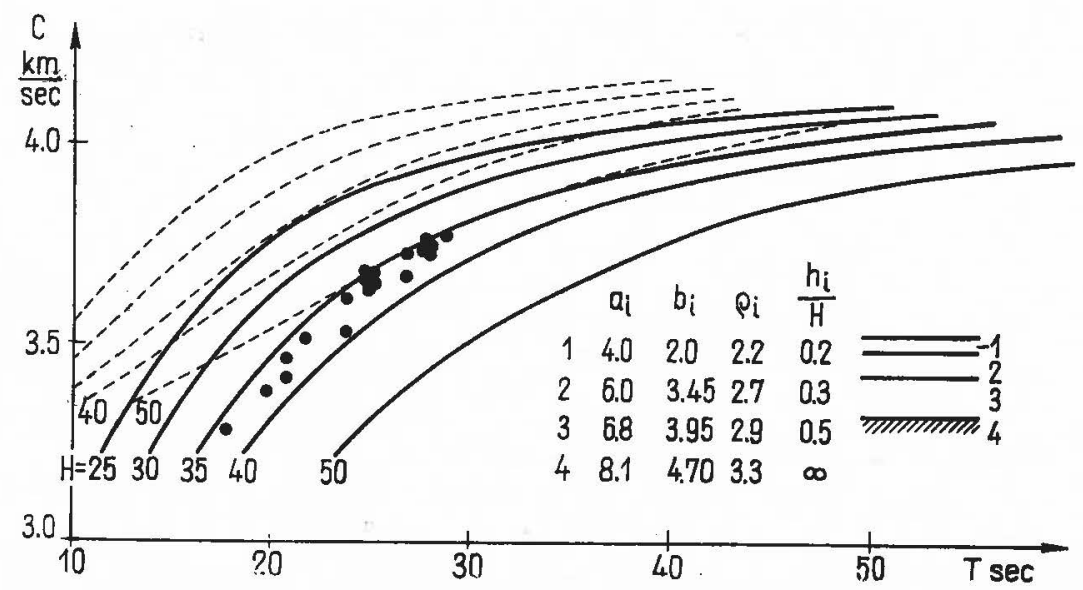

Fig. 5. Dispersion curves of the phase velocity for the trace Moscow-Lvov. The observed (circle) data and the theoretical curves for two-layer (dashed lines) and three-layer (heavy lines) model

But the absence of agreement between experimental and theoretical data in the range of periods from $15 \mathrm{sec}$ to $25 \mathrm{sec}$ shows, that the thickness of the sediments have been somewhat overestimated. The dispersion curves on the MoscowLvov route compared with the theoretical curves give the crust thickness about 36-38 km (Fig. 5).

\section{REFERENCES}

[1] Press, F.: Determination of crustal structure from phase velocity of Rayleigh waves. Bull. Seism. Soc. of Am. 471 (1957)

[2] Brune, J. and Dorman, J.: Seismic waves and earth structure in the Canadian shield. Bull. Seism. Soc. of Am. 531 (1963)

[3] Tryggvason, E.: Crustal thickness in Fennoscandia from phase velocities of Rayleigh waves. Annali di Geofisica 143 (1961)

[4] Параметры и частотно-амплитудные характеристики приборов сейсмических станций СССР, 1963. АН СССР, ИФ3, Москва, (1963)

[5] Руководство по обработке наблюдений над поверхностными волнами под ред. Е. Ф. Саваренского. Созет по сейсмологии АН СССР, М. (1964)

[6] Гласко, В. Б., Саваренский, Е. Ф. и Шечков, Б. Н.: Данные о фазовых и групповых скоростях поверхностых волн. Изв. АН СССР, сер. геоф. № 10 (1963) 


\section{SUR L'EXISTENCE DE LA COUCHE DE FAIBLE VITESSE DANS LA PARTIE SUPÉRIEURE DU MANTEAU EN ROUMANIE}

T. IosiF

(Bucharest, Roumanie)

Les informations sur la structure de l'intérieur de la Terre sont basées spécialement sur l'étude de la propagation des ondes séismiques à l'intérieur de la Terre.

Sur la base de la distribution de la vitesse des ondes séismiques en fonction de la profondeur, la Terre peut être divisée en quelques zones internes qui correspondent aux variations accentuées des propriétés élastiques du milieu.

Jusqu'à présent il n'existe pas d'opinion unique sur la structure du manteau, et en particulier sur sa partie supérieure. Il reste comme un problème controversé l'existence ou l'inexistence dans la partie supérieure du manteau de la soi-disante limite de $20^{\circ}$ et du canal de l'asténosphère avec la décroissance des valeurs des vitesses de propagation des ondes séismiques de volume.

L'objet de notre travail est constitué par l'essai d'expliquer le caractère de la variation de la vitesse de propagation des ondes séismiques dans le manteau avec la profondeur; pour cela nous avons accordé une attention spéciale à la structure de la partie supérieure du manteau en Europe et au problème de l'existence ou de l'inexistence de la couche de faible vitesse.

La méthode de base pour la détermination de la dépendence des vitesses des ondes élastiques en fonction de la profondeur à l'intérieur de la Terre est la méthode Wiechert-Herglotz.

La vitesse croît avec la profondeur aussi bien pour les ondes $P$ que pour les ondes $S$. Mais, si à partir d'une certaine profondeur, la vitesse décroît avec la profondeur, avec une telle rapidité que l'inégalité

$$
\frac{d v}{d r}-\frac{v}{r}>0
$$

soit satisfaite, alors la méthode Wiechert-Herglotz ne permet pas de déterminer la distribution des vitesses aux profondeurs, de constater où a lieu cette inégalité. Dans ce cas, on observe à la surface de la Terre seulement de faibles ondes diffractées.

Ceci montre que, si la couche de faible vitesse existe et si à son intérieur l'inégalité est valable [1], alors les informations directes sur la variation de la vitesse dans cette couche, sur la base des observations des durées de parcours des ondes des tremblements ne peuvent pas être obtenues.

C'est pour cela que pour obtenir la distribution des vitesses en fonction de la profondeur dans la partie supérieure du manteau, il est nécessaire d'avoir des 
observations aux temps de propagation pour les séismes dont les foyers sont situés à l'intérieur de la couche de faible vitesse.

Les foyers des séismes de Vrancea sont situés justement aux profondeurs où la vitesse atteint une valeur minima $(80-160 \mathrm{~km})$.

Si l'on construit et réduit l'hodographe des ondes séismiques $P$ et $S$ à la surface, qui correspond à la profondeur du foyer, nous nous trouvons dans les conditions d'application de la méthode de Wiechert-Herglotz, et à des profondeurs plus grandes, cette méthode est appliquable.

De tous les tremblements de terre profonds de la magnitu de $M>5$, qui ons eu lieu au cours des dernières trente années dans la région de Vrancea, nout

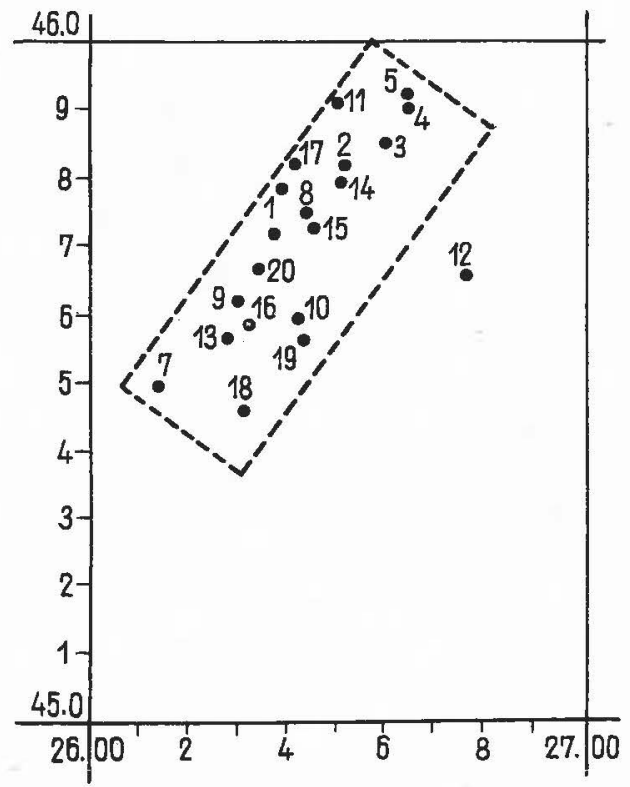

Fig. 1. La distribution des épicentres des tremblements profonds de Vrancea

avons choisi 20, et redéterminé les coordonnées et le temps dans le foyer. Pour tous ces séismes on a établi des hodographes des ondes longitudinales et transversales. Notons que cette source originale des séismes dans la région de Vrancea a une telle particularité, que tous les séismes profonds qui y ont lieu, sont situés dans la limite d'une petite zone, qui s'étend dans la direction SW-NE (Fig. 1).

C'est à sept séismes que nous avons réduit les bodocrons des ondes séismiques longitudinales et transversales à la surface qui correspond à la profondeur du foyer.

Les séismes ont des profondeurs entre 80 et $160 \mathrm{~km}$ et sont donnés dans le tableau 1. 


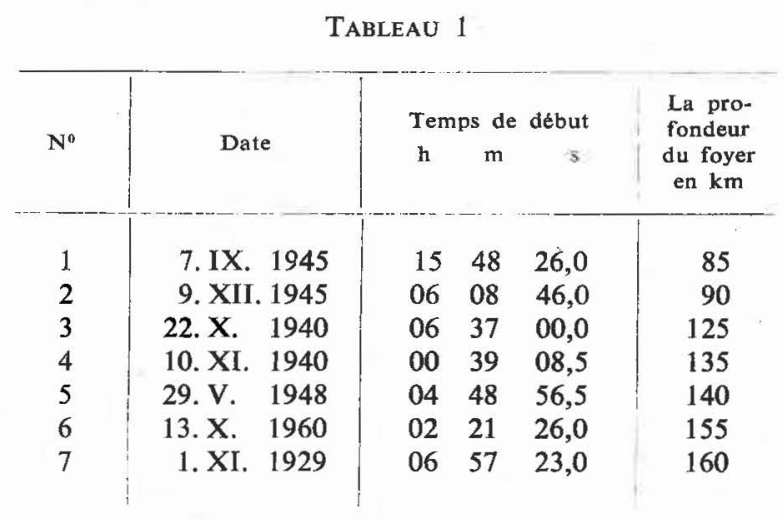

La réduction a été fait sur la base du choix des grandeurs des dérivées des hodocrones ayant les mêmes valeurs.

Pour l'approximation de l'hodographe nous avons employé la parabole du $3^{\mathrm{e}}$ degré, appliquée par intervalles de distances épicentrales:

$$
t_{i}=f(\Delta)=a_{0}+a_{1} \Delta_{i}^{2}+a_{2} \Delta_{i}+a_{3} \Delta_{i}^{3}
$$

où $t_{i}$ est le temps de propagation des ondes séismiques jusqu'à la station $i$ et $\Delta_{i}$ la distance épicentrale de la station correspondante.

Le début du temps peut être pris à volonté puisque y entre un membre permanent $a_{0}$.

Le contrôle des calculs est basé sur le fait qu'il intègre le dérivé du hodographe que nous devons obtenir par l'hodographe initial.

C'est un problème qui donne naissance à beaucoup de difficultés.

Les figures 2 (2a pour les ondes longitudinales, $2 b$ pour les ondes transversales) présentent les valeurs de vitesse des ondes séismiques longitudinales, $P$, et transversales, $S$, dans le manteau, obtenues sur la base des hodographes de ces sept tremblements.

Comme on peut remarquer sur la représentation graphique, les vitesses des ondes séismiques obtenues sur la base des tremblements individuels sont suffisamment en bon accord entre elles.

Les vitesses des ondes longitudinales et transversales accroissent avec la profondeur dans le manteau, au-dessous du foyer.

A partir de la profondeur de $730 \mathrm{~km}$ on observe une atténuation accentuée de la croissance des vitesses avec la profondeur. C'est justement à ces profondeurs que sont situés les foyers des plus grands séismes profonds. C'est cette profondeur que nous proposons comme limite de séparation entre les couches $B$ et $C$.

Nous avons obtenu les courbes des vitesses moyennes comme moyenne arithmétique des valeurs individuelles des vitesses à la profondeur donnée. La partie 


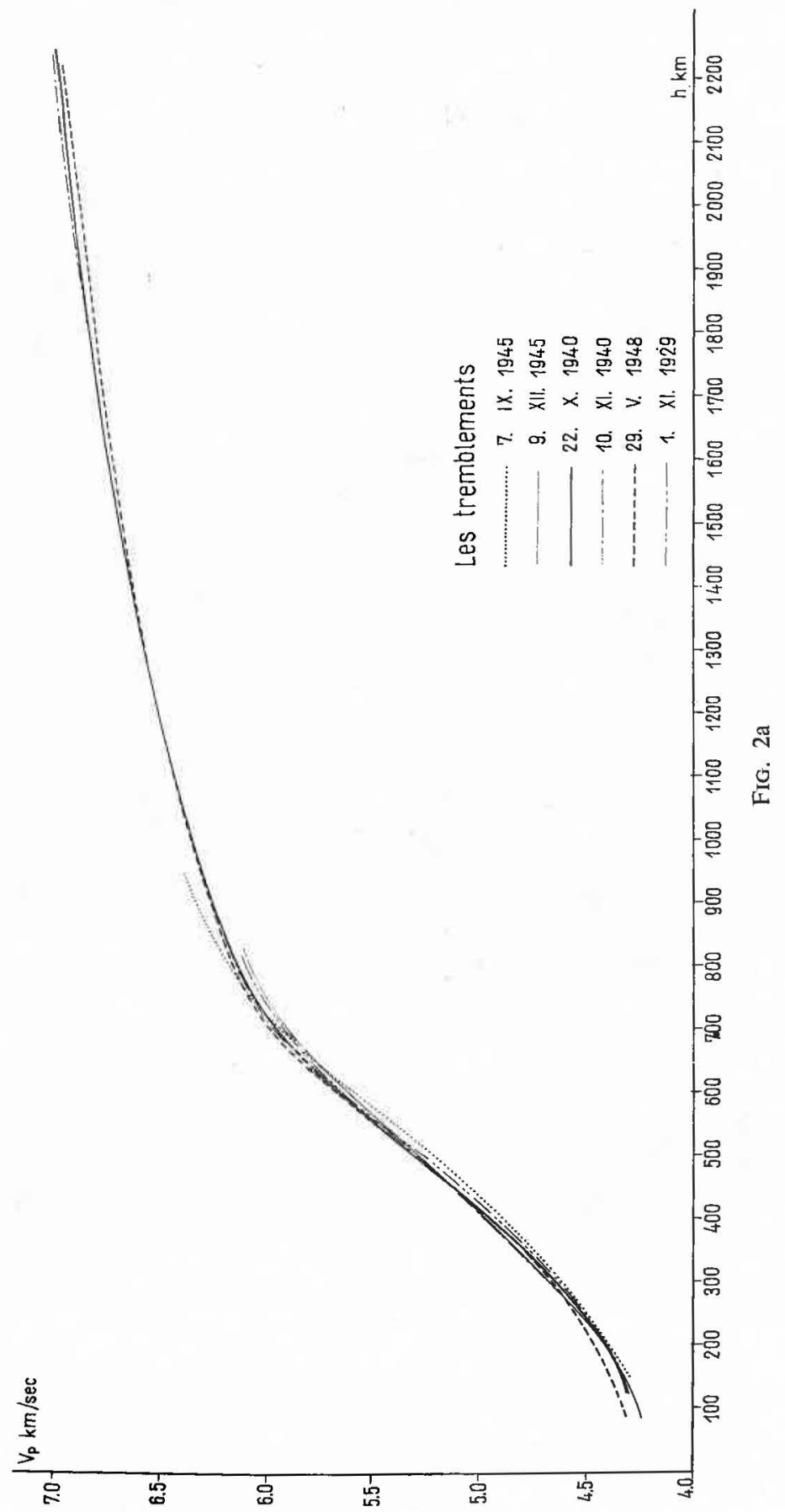




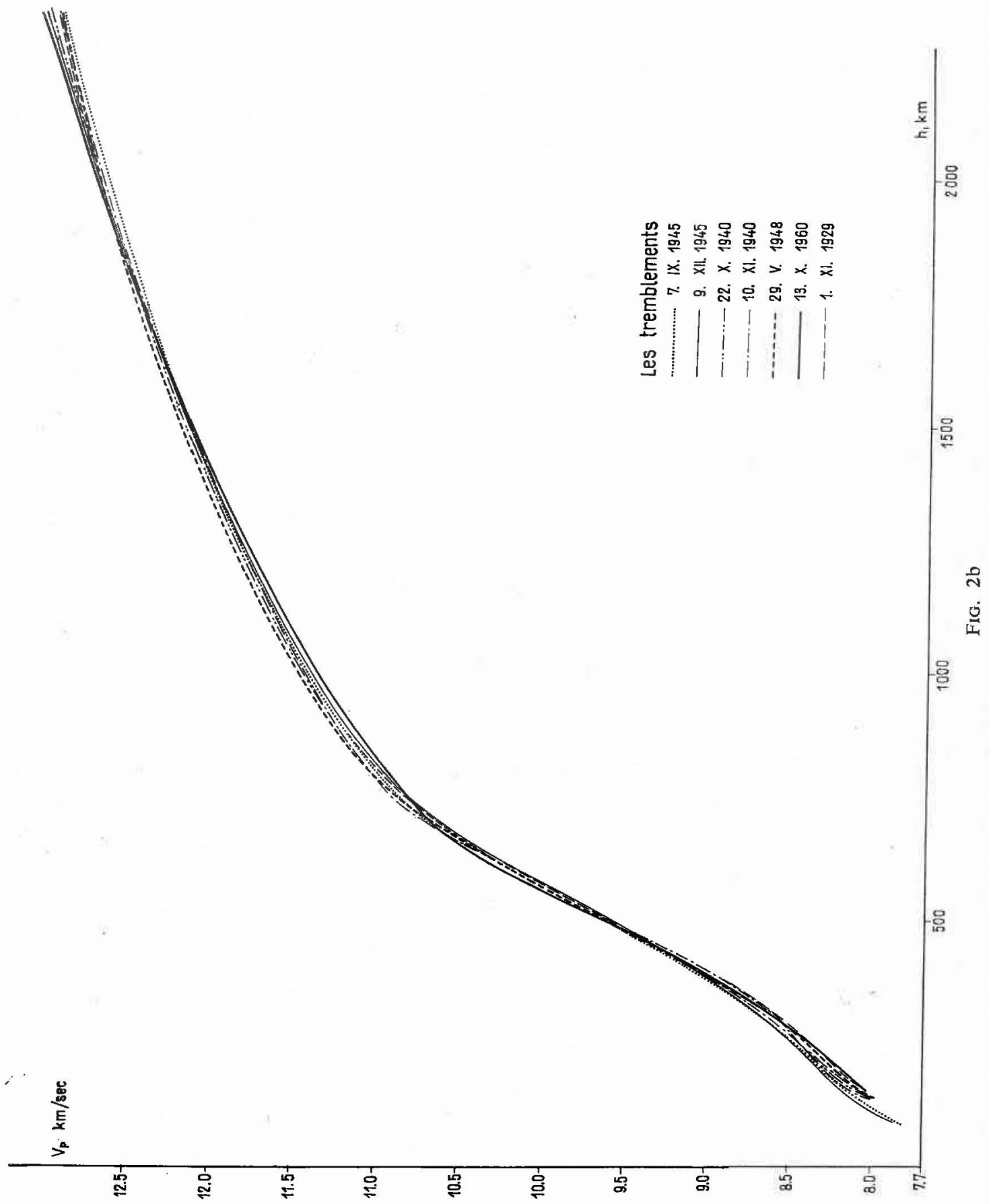


de la courbe de l'onde $P$ présente une exception se rapportant aux profondeurs jusqu'à $200 \mathrm{~km}$. Sur cette portion, la courbe a été tracée par les valeurs d'autour $\mathrm{du}$ foyer, puisque les valeurs trouvées aux niveaux des profondeurs des foyers nous paraissent les plus probables.

TABLEAU 2

Les valeurs moyennes des vitesses

\begin{tabular}{|c|c|c|c|c|c|}
\hline$\underset{(\mathrm{km})}{\mathrm{h}}$ & $\underset{\mathrm{km} / \mathrm{sec}}{V P}$ & $\underset{\mathrm{km} / \mathrm{sec}}{V_{\mathrm{s}}}$ & $\underset{(\mathrm{km})}{\mathrm{h}}$ & $\begin{array}{c}V_{P} \\
\mathrm{~km} / \mathrm{sec}\end{array}$ & $\begin{array}{c}V_{S} \\
\mathrm{~km} / \mathrm{sec}\end{array}$ \\
\hline 100 & 7,885 & 4,300 & 1100 & 11,595 & 6,413 \\
\hline 133 & 7,970 & 4,312 & 1133 & 11,649 & 6,440 \\
\hline 166 & 8,070 & 4,345 & 1166 & 11,700 & 6,463 \\
\hline 200 & 8,200 & 4,403 & 1200 & 11,750 & 6,488 \\
\hline 233 & 8,330 & 4,472 & 1233 & 11,803 & 6,513 \\
\hline 266 & 8,460 & 4,550 & 1266 & 11,850 & 6,534 \\
\hline 300 & 8,597 & 4,639 & 1300 & 11,888 & 6,553 \\
\hline 333 & 8,737 & 4,732 & 1333 & 11,955 & 6,572 \\
\hline 366 & 8,893 & 4,830 & 1366 & 11,985 & 6,592 \\
\hline 400 & 9,059 & 4,852 & 1400 & 12,031 & 6,608 \\
\hline 433 & 9,236 & 5,027 & 1433 & 12,074 & 6,628 \\
\hline 466 & 9,421 & 5,147 & 1466 & 12,114 & 6,646 \\
\hline 500 & 9,612 & 5,256 & 1500 & 12,153 & 6,666 \\
\hline 533 & 9,802 & 5,375 & 1533 & 12,198 & 6,680 \\
\hline 566 & 9,998 & 5,487 & 1566 & 12,233 & 6,698 \\
\hline 600 & 10,183 & 5,605 & 1600 & 12,276 & 6,715 \\
\hline 633 & 10,369 & 5,713 & 1633 & 12,312 & 6,733 \\
\hline 666 & 10,539 & 5,818 & 1666 & 12,353 & 6,745 \\
\hline 700 & 10,684 & 5,915 & 1700 & 12,388 & 6,762 \\
\hline 733 & 10,810 & 5,995 & 1733 & 12,423 & 6,778 \\
\hline 766 & 10,907 & 6,060 & 1766 & 12,458 & 6,790 \\
\hline 800 & 10,997 & 6,115 & 1800 & 12,490 & 6,807 \\
\hline 833 & 11,081 & 6,160 & 1833 & 12,523 & 6,815 \\
\hline 866 & 11,155 & 6,202 & 1866 & 12,557 & 6,832 \\
\hline 900 & 11,227 & 6,235 & 1900 & 12,589 & 6,847 \\
\hline 933 & 11,293 & 6,272 & 1933 & 12,620 & 6,860 \\
\hline 966 & 11,358 & 6,303 & 1966 & 12,700 & 6,868 \\
\hline 1000 & 11,421 & 6,333 & 2000 & 12,681 & 6,885 \\
\hline & 11,480 & 6,356 & 2033 & 12,712 & 6,895 \\
\hline \multirow{2}{*}{1066} & 11,540 & 6,390 & 2066 & 12,741 & 6,907 \\
\hline & & & 2100 & 12,771 & 6,915 \\
\hline
\end{tabular}

Le tableau 2 et la figure 3 présentent les valeurs des vitesses moyennes des ondes séismiques longitudinales $\bar{V}_{P}$ (Fig. 3a) et transversales $\bar{V}_{S}$ (Fig. 3b).

Ces figures montrent également les vitesses des ondes longitudinales, $P$, et transversales, $S$, d'après Jeffreys aux profondeurs $33-2100 \mathrm{~km}$, et d'après Gutenberg jusqu’à $600 \mathrm{~km}$. 


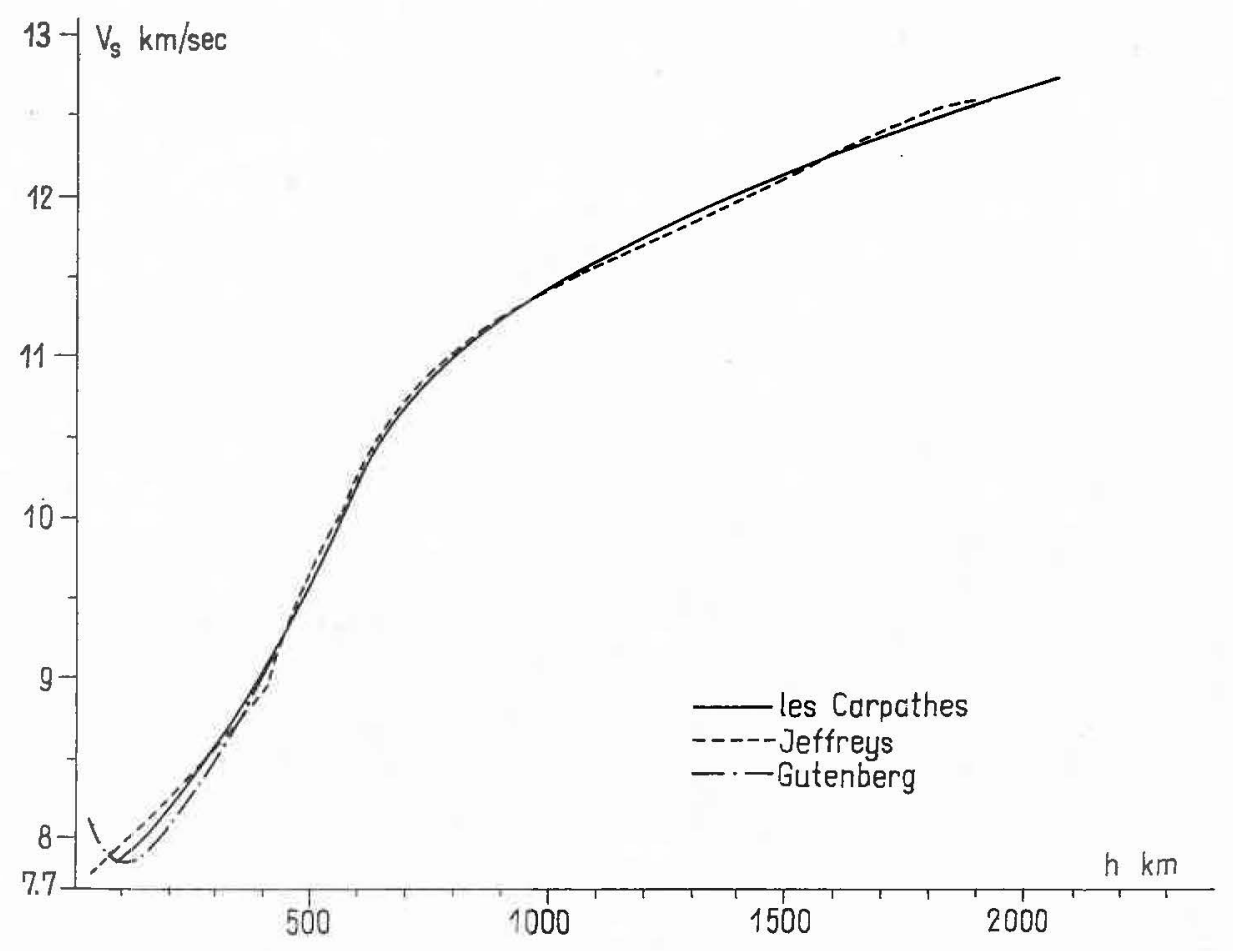

FIG. 3a

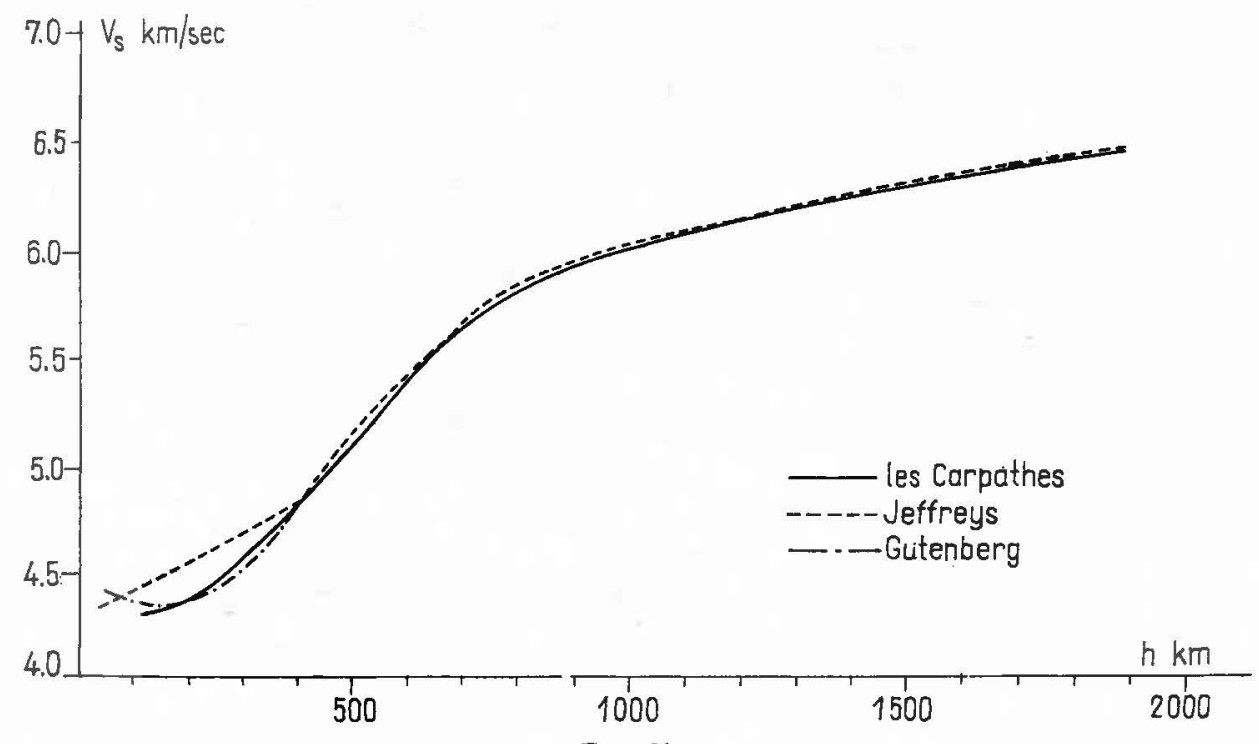

FIG. 3b 
La comparaison de la distribution des vitesses en fonction de la profondeur, distribution obtenue par nous sur la base des données des séismes profonds de Vrancea, avec les fonctions de Jeffreys et Gutenberg, montre qu'elles sont en bon accord, à partir de la profondeur de $430 \mathrm{~km}$.

Il n'y a pas d'indication sur l'existence d'une limite nette de séparation, ou bien sur la limite de premier ordre dans la partie supérieure du manteau, à une profondeur dépassant $100-150 \mathrm{~km}$.

D'après nos données à la profondeur de $100-200 \mathrm{~km}$ une décroissance de la vitesse des ondes $P$ et $S$ a eu lieu.

Pour les ondes $P$, la décroissance est irisignifiante, tandis que pour les ondes $S$ elle est bien marquée, atteignant une valeur de $0,2 \mathrm{~km} / \mathrm{sec}$.

Si l'on compare nos courbes avec celles de Jeffreys, il semble que la couche de faible vitesse contient des intervalles différentes pour les ondes $P$ et $S$. Pour les ondes $P$, la couche de faible vitesse s'étend jusqu'à $250 \mathrm{~km}$, pour les ondes $S$, jusqu'à $400 \mathrm{~km}$.

Cette conclusion sur l'existence d'une couche de faible vitesse pour les ondes $P$ et $S$ est basée sur les données concernant les vitesses de ces ondes sur la surface de Mohorovičić $(4,5 \mathrm{~km} / \mathrm{sec}$ pour les ondes $S$, et $8,1-8,2 \mathrm{~km} / \mathrm{sec}$ pour les ondes $P$ ).

Les hodographes de Jeffreys et Bullen, qui étaient comparés à nos résultats obtenus, sont des hodographes moyens pour les tremblements de terre du globe entier, puisqu'ils sont établis en utilisant les données de plusieurs séismes qui ont eu lieu dans de diverses régions de la Terre.

Les courbes de la dépendance de la vitesse des ondes séismiques en fonction de la profondeur de Gutenberg ont été également établies suivant les données des séismes parvenus de différentés régions.

Les valeurs des vitesses obtenues dans cet ouvrage sont «individuelles»: elles ont été obtenues sur la base des données des séismes profonds de Vrancea (80$160 \mathrm{~km}$ ) et dont les épicentres sont situés dans une région épicentrale relativement petite.

En sortant de la méthode appliquée de calcul des vitesses, il faut rapporter les valeurs obtenues à la zone des épicentres des Carpathes ainsi qu'à la zone de semi-distances entre les épicentres et les stations d'observation.

\section{RÉFÉRENCES}

[1] Wiechert, E. et Geiger, L.: Bestimmung des Weges der Erdbebenwellen im Erdinnern, Physik Z. 11 (1910)

[2] IosIf, T.: Considerații asupra cutremurelor din Vrancea, Studii și cercetări de Astron. si seism. 1 (1957)

[3] JefFreys, H.: The times of the core waves, Mon. Not. Roy. Astron. Soc. Geophys. Suppl. 4 (1939)

[4] Gutenberg, B.: Wave velocities at depths between 50 and 600 kilometres, Bull. Seism. Soc. Amer. 43 (1953) 


\author{
THEORETISCHE UNTERSUCHUNGEN \\ ÜBER EINEN EINGEBETTETEN SEISMOGRAPHEN \\ R. MAAZ \\ (Jena, DDR)
}

Auf der Tagung der Europäischen Seismologischen Kommission in Jena hat der Autor der folgenden Mitteilung einen Vortrag »Zur Wechselwirkung zwischen einem mechanischen Empfänger und einer longitudinalen Planwelle« gehalten. Darin wurde ein kugelförmiges Gestell eines Seismographen in einem homogenen isotrop elastischen Vollraum betrachtet, wobei das darin angebrachte Gehänge linear translatorische Bewegungen in Richtung der Wellennormale einer gegebenen longitudinalen harmonischen Planwelle ausführt. Die Ergebnisse sind streng deduktiv gewonnen und graphisch dargestellt worden [1,2].

Um auch den Einschwingvorgang zu erfassen, wird im folgenden eine longitudinale Planwelle mit weitgehend beliebiger Wellenform zugrundegelegt. Da die Annahme einer festen Verbindung zwischen dem. Gestell und dem Medium nicht immer gerechtfertigt ist, wird auch der Fall betrachtet, da $\beta$ das Medium am Gestell gleitet. Bei der experimentellen Untersuchung des aus dem Empfänger und dem umgebenden Medium gebildeten schwingungsfähigen Systems, pflegt man die Empfängerbewegung nicht durch eine elastische Welle sondern durch direkt eingeprägte Kräfte zu erzeugen. Im folgenden werden die beiden Möglichkeiten der Schwingungserregung aufeinander bezogen.

Hinsichtlich der mathematischen Entwicklung der folgenden Ergebnisse und Formeln sei auf den Artikel »Theoretische Untersuchung eines im elastischen Medium eingebetteten mechanischen Empfängers eines Seismographen in einer longitudinalen Planwelle" verwiesen [3]. - Nach einer teilweisen Aufspaltung der beim vorliegenden Problem auftretenden Wellengleichungen nach der Zeit $t$ und den Kugelkoordinaten führt die Laplace-Transformation zu einer Lösung der Aufgabe. Hierzu wurden die natürlichen Anfangsbedingungen für den Empfänger zugrundegelegt.

Die Gestellbewegung $p(t)$ ergibt sich zwanglos als Faltungsintegral

$$
p(t)=\int_{0}^{t} f\left(t^{\prime}\right) g\left(t-t^{\prime}\right) d t^{\prime}
$$

über die Wellenform und die inverse Laplace-Transformierte $g(t)$ einer rationalen Funktion $g(s)$ der komplexen Variablen $s$. Für den Fall, daß das Gehänge verschwindend klein $(\varepsilon=0)$ und die Masse des Empfängers gleich der verdrängten Mediummasse ist $(\gamma=1)$, lautet $g(t)^{`}$ sehr einfach: 


$$
g(t)=2 \sqrt{3} \frac{a}{R} \exp \left(-\frac{3 a}{2 R} t\right) \sin \left(\frac{1}{2} \sqrt{3} \frac{a}{R} t\right) .
$$

$R$ bedeutet den Radius der Gestellkugel, $a$ die Ausbreitungsgeschwindigkeit von rotationsfreien elastischen Wellen.

Die umgekehrte Aufgabe verlangt, die Wellenform $f(t)$ aus einem vorliegenden Seismogramm oder der daher bekannten Gestellbewegung $p(t)$ zu berechnen. Hierzu dient die Formel

$$
f(t)=p(t)+\frac{R}{a} \dot{p}(t)+\frac{1}{3} \gamma(1-\varepsilon)\left(\frac{R}{a}\right)^{2} \ddot{p}(t)+A+B+C,
$$

worin $A, B$ und $C$ Faltungsintegrale über $\ddot{p}(t)$ und andere gegebene Funktionen sind. Für $\varepsilon=0$ verschwinden $B$ und $C$. Gilt noch $\gamma=1$, so verschwindet auch $A$, so $\mathrm{da} \beta$ dann die Wellenform äußerst einfach zu berechnen ist.

Die Formel für $f(t)$ bietet auch im allgemeinen Fall $(\varepsilon \neq 0 ; \gamma \neq 1)$ die Möglichkeit, ein Kriterium für die hinreichend gute Wiedergabe der Wellenform durch die Gestellbewegung $p(t)$ herzuleiten.

Das bisher Gesagte trifft gleichermaßen für festen wie gleitenden Kontakt zwischen dem Medium und dem Gestell zu. Die Ergebnisse unterscheiden sich in den Funktionen $g(t), A(t), B(t)$ und $C(t)$, abgesehen vom Fall $\varepsilon=0, \gamma=1$. In diesem Spezialfalle sind also die Gestellbewegung und die Berechnungsformel für die Wellenform von der speziellen Art des Kontaktes unabhängig.

Die Gestellbewegung infolge einer Kraft, die mit der Größe $K(t)$ am Gestell angreift, läßt sich ebenfalls als Faltung

$$
p(t)=\int_{0}^{t} K\left(t^{\prime}\right) h\left(t-t^{\prime}\right) d t
$$

darstellen, wobei $h(t)$ ähnlich wie $g(t)$ gebaut ist. Wiederum ist der Fall $\varepsilon=0^{\text {? }}$ $\gamma=1$ mathematisch sehr einfach und physikalisch dadurch ausgezeichnet, daß sich die jeweilige Art des Kontaktes zwischen dem Medium und dem Gestell nicht auswirkt.

Beim experimentellen Studium des Verhaltens eines im Medium eingebetteten mechanischen Empfängers unter dem Einfluß einer elastischen Welle bereitet es große technische Schwierigkeiten, eine definierte Wellenform zu erzeugen. Demgegenüber ist es einfacher, dem mechanischen Empfänger eine Kraft mit gut bekanntem zeitlichen Verlauf einzuprägen. Experimente mit derartigen eingeprägten Kräften werden aber erst dann seismologisch interessant, wenn sie zu Experimenten mit seismischen Wellen in Beziehung gesetzt werden. Dies geschieht dadurch, daß der zeitliche Verlauf $K(t)$ der Kraft am Gestell ermittelt wird, welche dieselbe Gestellbewegung $p(t)$ bewirkt wie eine elastische Welle mit der interessierenden Wellenform $f(t)$. Das trifft zu, wenn 


$$
K(t)=4 \pi \rho R a^{2} f(t)-\int_{0}^{t} f\left(t^{\prime}\right) U\left(t-t^{\prime}\right) d t^{\prime}
$$

mit

$$
U(t)=c_{1} \exp \left(-c_{2}^{2} t\right) \sin \left(c_{3} t+c_{4}\right) .
$$

Gibt man sich den Kraftverlauf $K(t)$ vor, so errechnet sich die äquivalente Wellenform gemäß

$$
f(t)=\frac{1}{4 \pi \rho R a^{2}} K(t)+\int_{0}^{t} K\left(t^{\prime}\right) V\left(t-t^{\prime}\right) d t^{\prime},
$$

worin $V(t)$ analog zu $U(t)$ gebildet ist.

Neben der Kraft am Gestell soll nun auch eine dem Gestell parallele Kraft am Gehänge mit dem Verlauf $k(t)$ wirken. Die für eine Kraft am Gestell abgeleiteten Formeln gelten auch in dem allgemeineren Fall, wenn man in ihnen die Funktion $K(t)$ durch eine fiktive Kraftfunktion $\widetilde{K}(t)$ ersetzt,

$$
\widetilde{K}(t) \equiv K(t)-k(t)+\int_{0}^{t} k\left(t^{\prime}\right) W\left(t-t^{\prime}\right) d t^{\prime} \Rightarrow K(t),
$$

worin die Funktion

$$
W(t)=\frac{\omega}{\sqrt{1-\alpha^{2}}} \exp (-d \omega t) \sin \left(\omega t \sqrt{1-\alpha^{2}}+c\right) \text { für } \alpha<1
$$

von der Dämpfungskonstanten $\alpha$ des mechanischen Empfängers und seiner Eigenfrequenz $\omega$ abhängt. Für $\alpha=1$ und $\alpha>1$ gelten entsprechende Ausdrücke für $W$.

Wird der mechanische Empfänger durch elektromagnetische Kräfte zu Schwingungen erregt, so daß er als Sender seismischer Wellen fungiert, so greifen am Gehänge und am Gestell entgegengesetzt gerichtete Kräfte gleichen Betrages an,

$$
K(t)+k(t)=0 .
$$

Dementsprechend lautet die fiktive Kraftfunktion

$$
\widetilde{K}(t)=-2 k(t)+\int_{0}^{t} k\left(t^{\prime}\right) W\left(t-t^{\prime}\right) d t^{\prime} .
$$

Es erhebt sich die Frage, wie die Kraftgröße $k(t)$ zu wählen ist, um eine gewünschte fiktive Funktion $\widetilde{K}(t)$ zu erzeugen, die wiederum zur Realisierung einer interessierenden Wellenform benötigt wird. $\mathrm{Zu} \widetilde{K}(t)$ gehört eine am Gehänge angreifende Kraft gemäß 


$$
-2 k(t)=\widetilde{K}(t)+\int_{0}^{t} \tilde{K}\left(t^{\prime}\right) X\left(t-t^{\prime}\right) d t^{\prime},
$$

wobei $X$ ähnlich wie $W$ gebaut ist.

Für $\widetilde{K}(t)$ hat man $4 \pi \rho R a^{2} f(t)-\int_{0}^{t}\left(t^{\prime}\right) U\left(t-t^{\prime}\right) d t^{\prime}$ einzusetzen, damit dieselbe Gestellbewegung wie infolge einer seismischen Welle mit der Wellenform $f(t)$ entsteht. Die umgekehrte Frage nach der Wellenform $f(t)$, welche hinsichtlich der Gestellbewegung einem vorgegebenen Verlauf $k(t)$ einer Kraft am Gehänge äquivalent ist, läßt sich durch Kombination von bereits erwähnten Formeln beantworten.

\section{LITERATUR}

[1] MAAZ, R.: Theoretische Untersuchung der Wechselwirkung zwischen dem mechanischen Empfänger eines Seismographen und einer longitudinalen harmonischen Planwelle im Vollraum. Veröff. Inst. Bodendynamik Erdbebenforsch. Jena, 73 (1963)

[2] MAAZ, R.: Zur Wechselwirkung zwischen einem mechanischen Empfänger und einer longitudinalen harmonischen Planwelle. 7. Tagung der Europäischen Seismologischen Kommission vom 24. 9. bis 30. 9. 1962 in Jena. Veröff. Inst. Bodendynamik Erdbebenforsch. Jena, 77 251-255 (1964)

[3] MAAZ, R.: Theoretische Untersuchung eines im elastischen Medium eingebetteten mechanischen Empfängers eines Seismographen in einer longitudinalen Planwelle. Pure and Applied Geophysics (1964) 


\title{
DATA ACQUISITION AND PROCESSING EQUIPMENT FOR EDINBURGH ROYAL OBSERVATORY
}

\author{
R. PARKS \\ (Edinburgh, Great Britain)
}

Just over two years ago, a new seismological research group was set up at Edinburgh. From time to time visiting seismologists ask the question: "What is to be the equipment for the base station?"

In the case of Edinburgh, this question has to be considered in the light of the recent developments at Eskdalemuir, some fifty miles to the south, where two new recording stations have been established. The first station records continuously on magnetic tape in multichannel FM form, from a large array of short-period vertical seismometers, while the second produces conventional chart records from a set of seismometers covering three components of long period and three components of short period, an installation of the type which is now standard at a large number of stations around the World. The specially constructed vault for the second station also provides some space for other research workers to install their own instruments.

It is evident that a further station producing conventional records from the rather more noisy site at the Royal Observatory would not represent a useful addition. The contributions from the new group fall under two broad headings: a set of portable recorders for field work, and a data-processing centre which will build up a library of tapes and carry out detailed analysis.

One of the main parameters determining the characteristics of an array is the inter-element spacing, and the freedom to vary this according to the type of wavemotion to be studied is a valuable one. Furthermore, as coverage of the globe from existing array stations is far from complete, there is a need for a selfcontained installation which can be set up in any region at short notice.

For an array of short-period instruments, the requirements for relative timing accuracy are fairly stringent, and it would be desirable to record all channels simultaneously on a single tape deck. However, even with a permanent array the problems of linking all seismometers to a single recorder are formidable, as outlined in some of the previous papers, and if emphasis is placed on mobility a single-recorder system becomes impracticable, especially for work in populated areas.

The equipment which has been developed for field work comprises four portable recording sets, which may be either distributed as independent stations or combined as a single array of any desired configuration. Each set is provided with a crystalcontrolled time encoder, and to permit the relative timing to be established 
accurately a radio receiver in each recording set is automatically switched on for twenty seconds every hour, and its output (after suitable shaping) is superimposed on one signal channel. Broadcast time signals are not available on as regular a schedule as would be desirable for checking absolute timing, but it will be possible to use any burst of speech or music tone for comparing the timing between recording sets.

Each recording set is capable of handling up to six seismometer channels, and provision is made for the alternative use of radio or cable links between seismometers. On a basis of cost, radio compares favourably with cable for distances greater than two miles, but on a basis of convenience radio is preferable even for much shorter runs. Twelve channels in the $150 \mathrm{Mc} / \mathrm{s}$ band have been allocated by the G.P.O. for limited use in this country. At these frequencies propagation is limited to line of sight, which would be a major handicap in mountainous terrain if only a single recorder were used, but with the four independent recorders the limitation is not serious.

A unit containing an amplifier and FM modulator is located adjacent to each seismometer, using a carrier mid-frequency of $960 \mathrm{~Hz}$ which is transmitted to the recorder over either a screened cable or the speech channel of a normal walkie-talkie transmitter/receiver pair. Radio communication up to a range of three miles is obtainable with simple whip aerials, and a useful extension could be achieved by the use of more elaborate aerials. At the recorder the carrier is divided down to $120 \mathrm{~Hz}$ mid-frequency for recording at a tape speed of 0.133 inches/sec. In the present version a level response to ground velocity is obtained for the range 0.1 to $3 \mathrm{sec}$ period, and further development is planned to extend this to the range 0.1 to $100 \mathrm{sec}$. Studies in the longer period range are those involving wide spacings of the array, but fortunately the requirements for timing accuracy can then be relaxed substantially.

The tape ransport is a commercial unit modified for low speed, low power consumption, and operation from 24-volt batteries. The tapes and recorder batteries are changed at two-day intervals; when radio links are used the batteries for each sbsmometer unit are also changed at two-day intervals, but with cable links the atteries will operate for approximately four days between changes. The FM tape standards have been based on those already in use by other groups. With sixteen tracks on 1-inch tape each reel can be used for two passes each taking eight tracks (covering time code and flutter-compensation channels in addition to the six seismometers). A significant additional reason for favouring 1-inch tape is its suitability for future development of digital recording from a direct-digitizing seismometer.

The basic processing technique is the velocity-filtering operation, which involves summation of the signals in two groups, with appropriate weightings and with time-lags corresponding to the time taken for a chosen wave-front to pass across the array, followed by cross-correlation of the outputs of the two groups.

Originally the project had been planned in terms of the development of specialpurpose analogue equipment, as for example a tape-deck with staggered heads 
for introducing time-lags. However, the use of a large general-purpose digital computer is now preferred, because of the freedom to change the techniques of analysis by merely writing a fresh programme. It also simplifies the problem of combining data from several recording stations to form a single array. The main functions of the data laboratory are therefore the building of a library, the preparation of data for computer input, and the playback of computer output, with some facilities for preliminary assessment of data.

In the initial stages, the library will use the computer format for the sake of convenience, but it is envisaged that a more economical format can be developed later, as the computer standards are based on a much higher degree of freedom from drop-outs than is justified with seismic data. However, the digital form (even at high density) is preferred to FM on the grounds of reduced contamination of data with repeated transcriptions, together with ease of detection of such contamination as may occur.

Ideally the library should be collected on a very general basis, with the object of providing a stock of suitable material for a wide range of studies which have not yet been set out in detail. However, the tape cost for such a library would be excessive, and in practice events will have to be chosen more carefully. Obviously all the largest events should be included, but it would also be desirable to collect a series of events which fall below the limit of detection on a conventional record.

One of the major obstacles to the use of a digital computer for large data flows is the requirement that the data should be grouped into blocks separated by gaps. The equipment for buffering data into such blocks makes up a substantial proportion of the cost of a data laboratory, and there is a strong case for proposing that the operation should be handled by the computer. The technical problem of providing back-spacing operation of the computer tape handler are trivial, and this would permit the input of data in continuous digital form with only block markers and no interblock gaps, but no computer with such a facility is available in this country, although at least one has been in operation in the U.S.A. for several years. This is at Rice University, where a direct-digitizing seismograph system has been developed to take advantage of it.

A further difficulty arises in the matter of checking of input. Usually the computer assumes the input to be of very high quality, and check bits are provided for longitudinal or transverse parity (or both). If an error is detected by these checks, the input tape is re-read several times, and if the indication is still unsatisfactory the entire input is rejected. In general the seismic data is of much lower quality, with contamination ranging from seismic noise (e.g. sheep) to system noise (e.g. drop-outs on the analogue tape). Elaborate checking of the input tape transfer is simply not worthwhile. The data could be accepted without check bits, and a programme applied to detect erroneous readings and substitute more acceptable figures such as the mean of adjacent readings. Data containing errors should not be rejected outright, but should only have the faulty portion tagged. However, rather than entirely abandoning input checking, it is worth including a simple check such as transverse parity, to permit detection of 
an equipment fault before a large amount of computer time has been used up.

The equipment for digitization comprises a 24-channel playback deck for FM tapes, a sampling switch, an analogue-to-digital converter, a core store buffer, and a digital tape handler. The maximum data rate used is 20000 samples per second, corresponding to a speed-up ratio of 28 for a 24-channel recording of signals up to $10 \mathrm{~Hz}$. Control is effected by a unit reading the time code, to start and stop the operation at preset time readings. For the sake of simplicity, preaddressed computer tapes are used and the check-sum which is normally written after each block is omitted.

For the playback of digital tapes to give analogue output, the core store is again used as a buffer. The principal output device is a 4-channel pen recorder handling frequencies up to $1000 \mathrm{~Hz}$, and other facilities include variable bandpass filters, analogue multipliers and operational amplifiers. For monitoring purposes an audio amplifier and loudspeaker have proved to be extremely valuable; using speed-up ratios in the region of 30 to 100 , much of the seismic information falls within the audible range, and an experienced operator can readily assess the characteristics of events at various distance ranges, and of background noise.

For the operation of summation without lags, relatively simple equipment is sufficient, but the chief benefit of a spaced array lies in the velocity-filtering effect obtained by the insertion of lags. This requires considerably more equipment, and for field work it may be worth investigating array configurations which yield some improvement without the use of lags. The simple three-component set is particularly rewarding: by summing the two horizontal components in appropriate ratio and sign to derive a radial component, and then correlating this radial component with the vertical component, a clear indication of the type of particle motion is obtained. For the $\mathbf{P}$ arrivals the correlator gives a positive output, for the $\mathrm{S}$ arrivals a negative output, and for the surface-wave arrivals an oscillating output. This opens up such possibilities as the recognition of weak $\mathrm{S}$ arrivals in the presence of a strong $\mathrm{P}$ signal.

The main analysis has been planned on the basis of using the Atlas computer at Manchester, sending the data on magnetic tape and the programme either indirectly by posting punched tape or directly by the use of the land-line link which is already serving Edinburgh University. Even in favourable circumstances this involves some days of delay before the full output is available, and delays of a week or more are by no means uncommon.

Obviously there is a great attraction in having laboratory facilities for processing with lags, even in a limited way. A scheme is now under development to achieve this by the use of the core store in conjunction with special addressing and readout facilities. Given the data in multiplexed digital form on computer tape, the problem is chiefly one of addressing. For any specified set of time lags there is a corresponding set of address spacings, giving a movable frame which can be swept through the data in the store. The capacity of the store is two complete Atlas blocks, so that while one block is being processed the other can be refilled 
with fresh data. A special arithmetic unit derives the spaced addresses from information set in by the operator on a simple plug-board, defining the array configuration and the incremental time-lags required. To reduce the cost and complexity of the equipment, the data as read out from the store are converted to analogue form before the summation and correlation operations.

Although it is not considered that a permanent conventional seismograph station at Edinburgh would provide a worthwhile contribution to the existing world network, an underground vault has been constructed for the development and testing of new types of seismometer system. Particular attention has been paid to minimising the disturbances due to the presence of operators in the vault, by the provision of a suspended floor and controlled heating and ventilation. 


\section{FREQUENZABHÄNGIGE KOPPLUNG \\ BEI ELEKTRODYNAMISCHEN SEISMOGRAPHEN *}

Ch. Teupser

(Jena, DDR)

Ein elektrodynamischer Seismograph besteht aus zwei schwingungsfähigen Systemen, dem mechanischen Empfänger und dem Galvanometer, deren Bewegung meist durch zwei lineare Differentialgleichungen zweiter Ordnung beschrieben werden kann:

$$
\begin{aligned}
& \ddot{\Theta}+2 \alpha_{s} \omega_{s} \dot{\Theta}+\omega_{s}^{2} \Theta=-\frac{\ddot{X}}{l}+\frac{4 \alpha_{s} \omega_{s} \alpha_{g} \omega_{g}}{\kappa} \lambda \dot{\Psi}, \\
& \ddot{\Psi}+2 \alpha_{g} \omega_{g} \dot{\Psi}+\omega_{g}^{2} \Psi=\kappa \dot{\Theta} .
\end{aligned}
$$

$\Theta$ und $\Psi$ sind die Winkelausschläge, $\alpha_{s}$ und $\alpha_{g}$ die Dämpfungen und $\omega_{s}$ und $\omega_{g}$ die Eigenfrequenzen des mechanischen Empfängers bzw. Galvanometers. $l$ ist die reduzierte Pendellänge, $\kappa$ der sog. Übertragungsfaktor. Die elektrodynamische Kopplung hat zur Folge, daß durch die bei einer Bewegung der Galvanometerspule erzeugte Gegen-EMK eine Rückwirkung, gekennzeichnet durch den Faktor $\lambda$, auf den mechanischen Empfänger eintritt. Diese Rückwirkung ist der Geschwindigkeit der Galvanometerbewegung proportional und nicht immer ein vernachlässigbarer Parameter des elektrodynamischen Seismographen. Es ist $0<\lambda<1$.

Durch Einführung von Kapazitäten und Induktivitäten in den Kopplungskreis können die Eigenschaften des Seismographen geändert werden. Im allgemeinen wird dadurch die Ordnung der Differentialgleichungen erhöht. Alle bisher durchgeführten Betrachtungen über die Charakteristiken, die Einschwingvorgänge und die Eichmethodik bei elektrodynamischen Seismographen müßten erweitert werden. Es zeigt sich nun, daß dies nicht notwendig ist, da unter gewissen Bedingungen die Ordnung der beiden Differentialgleichungen erhalten bleibt.

Diese Bedingung hat bei allen untersuchten Schaltungen zur Folge, daß sich die Eigenperiode und die Dämpfung des mechanischen Empfängers nicht ändern und da $B$ die erste Ableitung von $\Theta$ alleinige Störfunktion in der Galvanometergleichung bleibt.

Die Parallelschaltung eines aus Induktivität, Widerstand und Kapazität gebildeten Reihenresonanzkreises (Abb. 1) ist schon in einem Vortrag auf der Regio-

\footnotetext{
* Mitteilung Nr. 5 aus dem Institut für Geodynamik Jena der Forschungsgemeinschaft der Deutschen Akademie der Wissenschaften zu Berlin.
} 
naltagung in Moskau im Juni d. J. erörtert worden [1]. Daher sollen die Ergebnisse dieser Betrachtungen nur kurz referiert werden. Der Reihenresonanzkreis muß die gleiche Eigenfrequenz wie das Galvanometer haben, wenn die Ordnung der Differentialgleichungen erhalten bleiben soll. Die Dämpfung des Galvanometers kann beliebig erhöht werden. Wenn sie größer als bei Kurzschluß vorgeschrieben wird, ändert die Rückwirkung des Galvanometers auf den mechanischen Empfänger ihr Vorzeichen. Es gilt also $-\infty<\lambda<1$. Wenn die Dämpfung

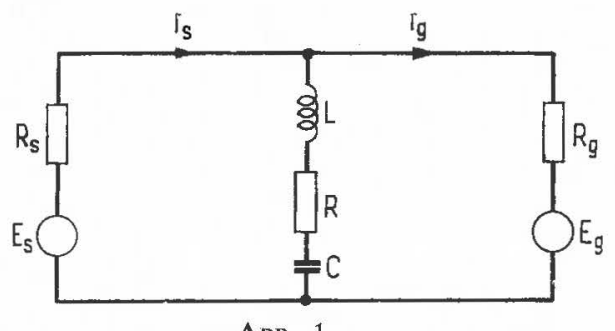

AвB. 1

gleich der bei Kurzschluß gewählt wird, verschwindet die Rückwirkung exakt. Im Fall, daß die geforderte Galvanometerdämpfung kleiner als die Kurzschlußdämpfung ist, kann man einen rückwirkungsfreien Seismographen bauen, indem man die Kurzschlußdämpfung durch einen Widerstand in Reihe mit dem Galvanometer oder durch einen magnetischen Nebenschluß herabsetzt. Es ist auch bei umgekehrter Voraussetzung die Rückwirkung durch zusätzliche Einschaltung eines Parallelresonanzkreises völlig ausschaltbar (Abb. 2).

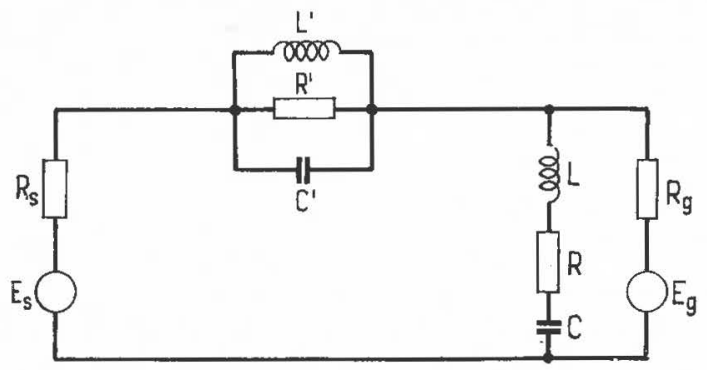

Авв. 2

Wird dem Galvanometer nur Widerstand $R$ und Kondensator $C$ parallelgeschaltet, so wirkt dies wie eine Erhöhung der Eigenperiode des Galvanometers. Dessen Dämpfung ändert sich ebenfalls. Die Bedingung über die Beibehaltung der Ordnung der Differentialgleichungen liefert

$$
\omega_{g} R C+\frac{1}{\omega_{g} R C}=2 \alpha_{g k} .
$$


Es muß also die Kurzschlußdämpfung $\alpha_{g k}>1$ sein, damit man reelle $R$ and $C$ erhält. Die Beziehung zwischen alter und neuer Eigenfrequenz ist gegeben durch

$$
\omega_{g}^{\prime}=\omega_{g}\left[1+\frac{R_{s} R_{g}}{\left(R_{s}+R_{g}\right) R}\right]^{-\frac{1}{2}} .
$$

$R_{s}$ und $R_{g}$ sind die inneren Widerstände der Tauchspulen des mechanischen Empfängers und des Galvanometers. Die erste Gleichung liefert bei gegebener Kapazität zwei Widerstände $R$ und damit auch zwei Eigenperioden. Die Dämpfung ist

$$
\alpha_{g}^{\prime}=\frac{\omega_{g}^{\prime}}{\omega_{g}}\left[\alpha_{g}+\frac{\omega_{g} R_{g} R_{s} C}{2\left(R_{s}+R_{g}\right)}\right] .
$$

Diejenige Lösung, die eine große Erhöhung der Eigenperiode liefert, kann daher eventuell eine Erniedrigung der Dämpfung zur Folge haben. Wenn die Lösung mit der geringen Erhöhung der Eigenperiode gewählt wird, erhöht sich die Dämpfung immer.

Wesentlich neu in der Theorie der elektrodynamischen Seismographen ist das Auftreten einer der Beschleunigung proportionalen Rückwirkung. In Gl. (1) tritt dann auf der rechten Seite zusätzlich der Summand

$$
\frac{2 \alpha_{s} \omega_{s}}{\kappa} \mu \ddot{\Psi}
$$

auf. Der Faktor der beschleunigungsproportionalen Rückwirkung hängt von der Änderung der Eigenperiode $a b$

$$
\mu=-\frac{\left(\alpha_{s}-\alpha_{s 0}\right)}{\alpha_{s}} \frac{\left(T_{g}^{\prime 2}-T_{g}^{2}\right)}{T_{g}^{\prime 2}} \frac{R_{g}}{R_{s}} .
$$

$\alpha_{s o}$ ist die Dämpfung des mechanischen Empfängers bei offener Wandlerspule. Aus dieser Formel geht hervor, daß $\mu<0$.

Wird dem Galvanometer nur Widerstand und Induktivität parallelgeschaltet, so wirkt dies wie eine Erniedrigung der Eigenperiode. Es tritt dann in (1) eine der Verrückung proportionale Rückwirkung

$$
\frac{2 \alpha_{s} \omega_{s} \omega_{g}^{\prime 2}}{\kappa} \nu \Psi
$$

auf. Die Formeln sind ganz analog denen bei der Schaltung mit Kondensatorglied. Es $m u ß \alpha_{g k}>1$ sein und es gilt $v<0$.

Durch Induktivitäts- bzw. Kapazitätsglied in Serienschaltung mit dem Galvanometer erreicht man ebenfalls eine Erhöhung bzw. Erniedrigung der Eigenperiode. Damit die Ordnung der Differentialgleichungen erhalten bleibt, war bei der Parallelschaltung die notwendige Bedingung $\alpha_{g k}>1$ zu erfüllen. Jetzt muß der 
viel schwerer zu erfüllenden Bedingung, offene Galvanometerdämpfung $\alpha_{g 0}>\mathrm{I}$, entsprochen werden. $\mathrm{Da} \alpha_{g 0}>1$ selten der Fall ist, muß man die offene Galvanometerdämpfung erhöhen, indem man dem Galvanometer erst einen Reihenresonanzkreis parallelschaltet. Es treten jetzt wieder der Beschleunigung oder der Verrückung proportionale Rückwirkungen auf. Diese haben aber positive Vorzeichen und sind immer kleiner als 1 .

Wichtig ist zu erwähnen, daß man unter gewissen Bedingungen, die für Parallelund Serienschaltung teilweise sogar entgegengesetzt sind, bei einer vorgegebenen Änderung der Eigenperiode und der Dämpfung des Galvanometers erreichen kann, daß die geschwindigkeitsproportionale Rückwirkung verschwindet.

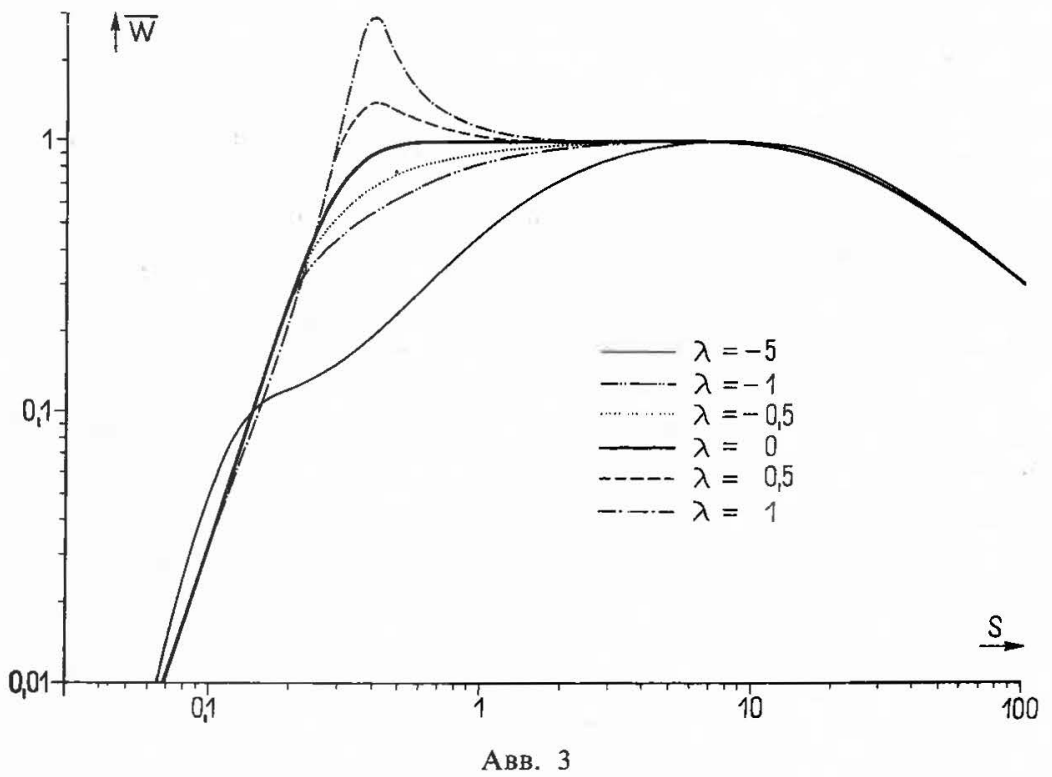

Durch Einschaltung von Blindwiderständen in den Kopplungskreis treten bisher nichtbekannte Rückwirkungen auf. Es ist daher Aufgabe einer verallgemeinerten Theorie, den Einfluß dieser Rückwirkungen auf die Eigenschaften des Seismographen zu untersuchen. Den Betrachtungen ist obiges, durch die beiden zusätzlichen Rückwirkungen verallgemeinertes Differentialgleichungssystem zugrundezulegen. In der verallgemeinerten Theorie gilt nicht mehr der Vertauschungssatz, der besagt, daß die Eigenschaften eines elektrodynamischen Seismographen erhalten bleiben, wenn man die Eigenperioden und die Dämpfungen des mechanischen Empfängers und des Galvanometers vertauscht.

Der Einfluß der verschiedenen Rückwirkungen ist für alle wichtigen Abstimmungen der elektrodynamischen Seismographen untersucht worden. Besonderes Augenmerk ist dem sog. Verrückungsmesser, also demjenigen Seismographen, der in einem breiten Frequenzintervall die Bodenverrückung getreu wiedergibt, 
zugewandt worden. Das Ergebnis der Untersuchungen soll hier nur an Hand der Amplitudencharakteristiken wiedergegeben werden. In der Abb. 3 ist der Einfluß der geschwindigkeitsproportionalen Rückwirkung zu sehen. Für $\lambda>0$ sind die Kurven bereits bekannt. Es sind aber auch für $\lambda<0$ Charakteristiken gezeichnet worden. In der Nähe der Eigenfrequenz des mechanischen Empfängers erreichen die Verzerrungen ihre größten Beträge. Ganz ähnlich liegen die Verhältnisse bei der verrückungsproportionalen Rückwirkung (Abb. 4). Die Diskus-

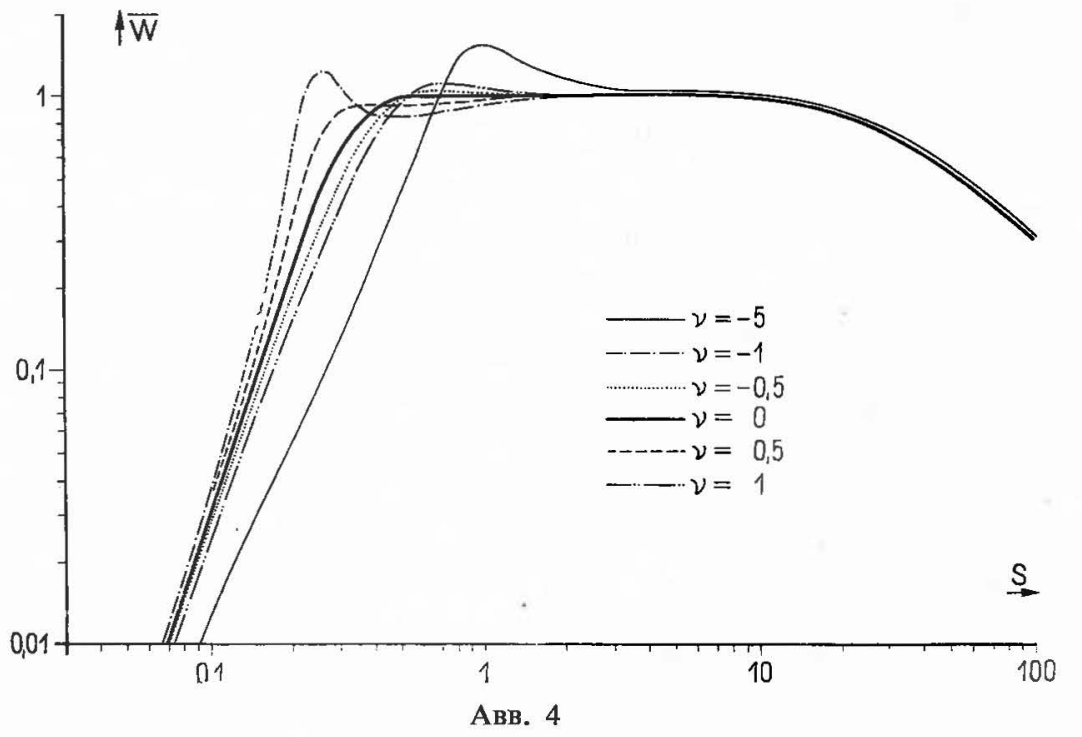

sion der beschleunigungsproportionalen Rückwirkung bei dem Verrückungsmesser führt zu dem überraschenden und auch erfreulichen Ergebnis, daß diese Rückwirkung keinen großen Einfluß auf die Eigenschaften des Seismographen hat. Wenn $|\mu|<1$, ist sie vernachlässigbar. Nur für sehr große (negative) $\mu$ $\mathrm{mu} ß$ sie in Betracht gezogen werden. Es wird definiert

$$
\zeta=-\mu \frac{\omega_{s}^{2}}{\omega_{g}^{2}} .
$$

Beim Verrückungsmesser ist $\omega_{s} \ll \omega_{g}$. Man kann nun zeigen, daß unter der Bedingung $\lambda<0$

$$
0<\zeta<1
$$

ist. Für diese sehr großen Rückwirkungen ist der Einfluß in der $\mathrm{Abb} .5 \mathrm{zu}$ sehen. Man erkennt daraus, da $B$ die Verzerrungen selbst bei der maximal möglichen Rückwirkung $\zeta=1$ nicht sehr groß sind. Die Herabsetzung der Vergrößerung um einen konstanten Faktor im frequenzabhängigen Bereich wollen wir dabei nicht als Verzerrung der Amplitudencharakteristik bezeichnen. 
Die Behauptung, daß kleine beschleunigungsproportionale Rückwirkungen keinen Einfluß auf die Amplitudencharakteristik haben, ist auch experimentell nachgeprüft worden. Zu diesem Zweck wurde die Vergrößerung eines Horizontalseismographen in Abhängigkeit von der Frequenz auf einem Schütteltisch bestimmt. Dem Galvanometer war ein Widerstands-Kondensatorglied parallel-

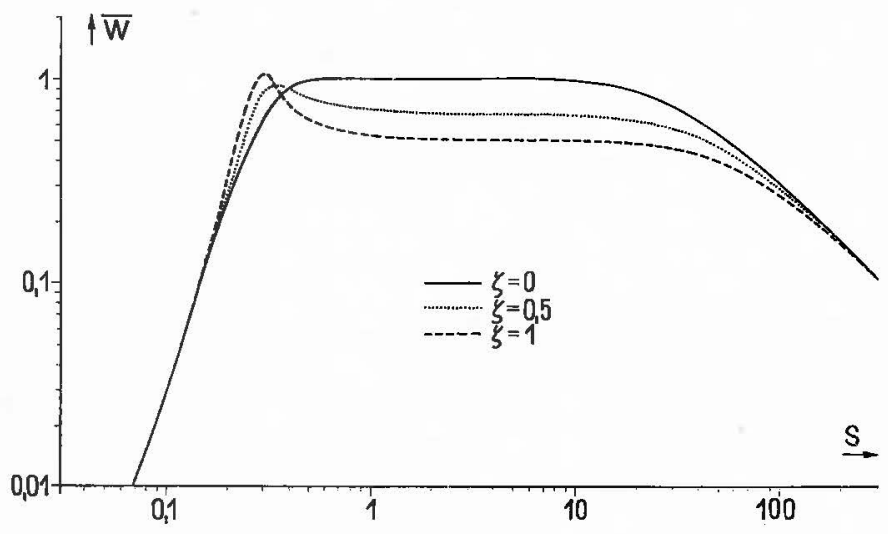

ABB. 5

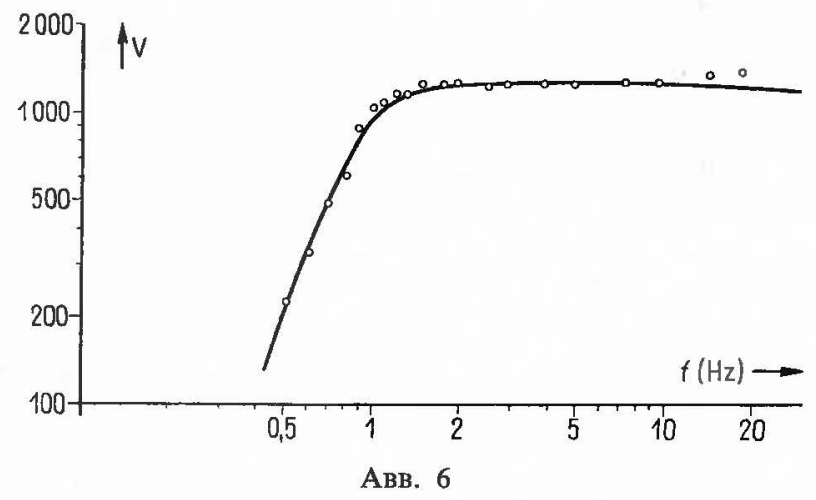

geschaltet. Ohne dieses Glied war $\lambda \approx 1$. Mit ihm ist $\lambda=0, \mu=-0,84$. Da die Änderung der Eigenperiode des Galvanometers nur $6 \%$ beträgt, entspricht die Amplitudencharakteristik fast der in völlig rückwirkungsfreiem Fall. Die ausgezogene Kurve in Abb. 6 ist die theoretische für den rückwirkungsfreien Seismographen, die Kreise sind experimentelle Werte. Man kann also beim Verrückungsmesser schon durch ein $R$ - $C$-Glied die Rückwirkung praktisch ausschalten. Die im allgemeinen Fall notwendige Induktivität kann eingespart wer- 


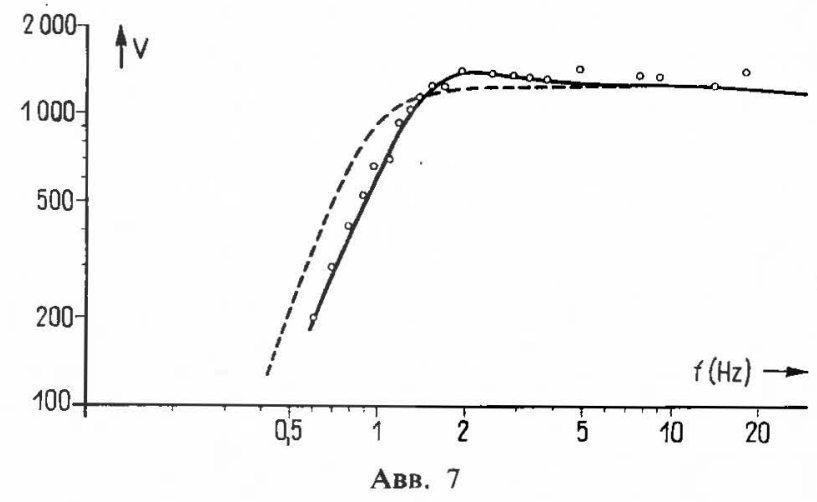

den. Es ist oben schon gesagt worden, daß die verrückungsproportionale Rückwirkung beim Verrückungsmesser einen größeren Einfluß hat. Ein experimentelles Beispiel für den gleichen Betrag der Rückwirkung, nämlich $v=-0,84$, zeigt Abb. 7. Die experimentellen Werte stimmen wieder gut mit der theoretischen Kurve überein. Deutlich ist auch der Unterschied gegenüber der gestrichelten Kurve des rückwirkungsfreien Falles zu erkennen. Eine ausführliche Darstellung der aufgezeigten Ergebnisse erfolgt in [2].

\section{LITERATUR}

[1] Teupser, Ch.: Die Rückwirkung und deren Ausschaltung bei elektrodynamischen Seismographen. Vortrag Regionaltagung Moskau (1964)

[2] Teupser, CH.: Verallgemeinerung der Theorie elektrodynamischer Seismographen durch frequenzabhängige Kopplung. Veröffentlichung des Instituts für Geodynamik Jena, 2 (1965) 


\title{
ELECTROMAGNETIC VELOCITY SEISMOGRAPHS WITH NON-NEGLIGIBLE REACTION OF THE GALVANOMETER
}

\author{
V. TOBYǍš \\ (Prague, Czechoslovakia)
}

Besides the values of displacement and acceleration of the ground movement during passing seismic waves, there is the third important kinematic quantity of movement - velocity. In some cases it is suitable to record in a certain range of periods this quantity with the limited distortion of the amplitudes. Such type of seismograph with the constant velocity sensitivity we shall call velocity seismograph.

For realisation of the electromagnetic velocity seismographs with galvanometric recording (without amplifier), we have two wellknown different combination of their constants.

I. The first type is a seismometer and galvanometer of different periods with equal damping constants of both systems smaller than critical.

II. The second type is a seismometer and galvanometer of contrary equal period and both systems overdamped.

In the first case, where the period $T_{2}$ of one system is much smaller than the period $T_{1}$ of the second one $\left(T_{2}=T_{1} k_{T}, k_{T} \ll 1\right)$, we can derive for the optimum flat response characteristic the dependence of damping constants on the ratio of periods $k_{T}$ in the form $D_{1}=D_{2}=\left(1-k_{T}\right) / \sqrt{2}$. Here limiting conditions for phase response characteristic were not taken into account.

The values of periods $T_{1}, T_{2}$ control the bandpass of seismograph; the band width is for $k_{T} \leqq 0.1$ equal to $T_{1}$ with error less than $2 \%$. The amplitude response curve is symmetrical according to period $T=T_{1} \quad k_{T}$ :

In the second type of velocity seismograph there is the bandpass regulated by both constants - periods and dampings. The band width is equal to $\Delta T=$ $=1.29 T_{1} D_{1}$ and the amplitude response curve is symmetrical according to line $T=T_{1}$.

For the comparison of amplitude response curves of both types there are in Fig. 1, their courses for the same bandpass. The first type $\left(T_{2}=T_{1} k_{T}, D_{1}=\right.$ $\left.=D_{2}=1-k_{T} / \sqrt{2}, k_{T} \ll 1\right)$ is better than the second $\left(T_{1}=T_{2}, D_{1}=D_{2} \gg 1\right)$. It is clear from the method of derivation of constants: in the first case is the velocity sensitivity taken into consideration for the whole response characteristic, in the second case separated for seismometer and galvanometer.

All what has been said about relations among constants holds correct for a negligible reaction of galvanometer, i.e. for zero coupling coefficient $\sigma^{2}$ and therefore for zero magnification. This coupling coefficient lies in the ranges between 
zero and one. To reach great sensitivity of seismograph with small dimensions and weight of seismometer or with robust galvanometer measuring system it is necessary to use a non-negligible value of coupling coefficient. Then the partial constants of seismograph, i.e. the periods $T_{01}, T_{02}$ and damping constants $D_{01}$, $D_{02}$ of galvanometer and seismometer computed with presupposition of zero coupling differ from the actual equivalent constants.

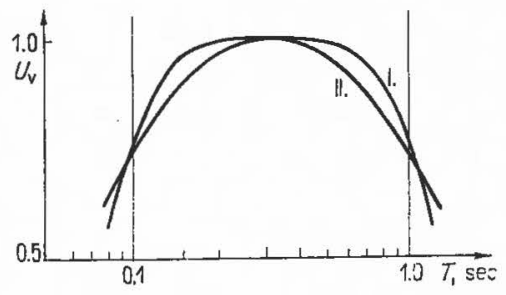

For both types of electromagnetic velocity seismographs formulae for calculation constants were derived for two eases which appear in practice more often:

1. For fixed partial periods of seismometer and galvanometer we enumerate the other partial constants, i.e. dampings so that the equivalent constants fulfil the above mentioned conditions for velocity seismograph (for the first type the relations among constants and period ratio, for the second type the equality of periods and damping constants). This case takes place by controlling the sensitivity of seismograph without regulating both periods.

2. For a fixed bandpass of seismograph (i.e. for fixed equivalent constants) we have to compute all partial constants in dependence on the value $\sigma^{3}$. This case takes place when the same bandpass is regulated in different types of seismographs.

For the first type of seismograph with fixed partial periods the dependence of equivalent periods and partial damping constants on coupling is nearly linear. The greater period decreases, the smaller one increases and the necessary partial damping constants are equal and decrease with coupling. The example given in the Table 1 for period ratio $k_{T_{\theta}}=0.1$ shows, that the maximum difference

TÁBLE 1

\begin{tabular}{c|c|c|c}
\hline$\sigma^{2}$ & $T_{1}(\mathrm{sec})$ & $T_{2}(\mathrm{sec})$ & $D_{01}=D_{02}$ \\
\hline 0.000 & 1.000 & 0.100 & 0.636 \\
0.108 & 0.990 & 0.101 & 0.630 \\
0.221 & 0.980 & 0.102 & 0.623 \\
0.339 & 0.969 & 0.103 & 0.616 \\
0.463 & 0.959 & 0.104 & 0.609 \\
0.592 & 0.949 & 0.105 & 0.602 \\
0.728 & 0.938 & 0.107 & 0.595 \\
0.871 & 0.927 & 0.108 & 0.589 \\
1.000 & 0.918 & 0.109 & 0.582
\end{tabular}


for maximum coupling $\left(\sigma^{2}=1\right)$ does not exceed $10 \%$ according to zero coupling. For period ratio $k_{T_{0}}=0.05$ these changes are only $5 \%$ and with decreasing of the period ratio they are further decreasing.

In the second type of velocity seismograph another relation must be used among partial constants to give necessary equivalent ones. Instead of equivalent periods, there are different partial periods and instead of an overdamped system there is maximum critical damping.

One example of this case is in Table 2 for the same bandpass as for $k_{T}=0.1$ of the first type. The change of partial damping constants is here about $10 \%$. The equivalent periods are fixed and therefore the changes in band width are only due to damping variations.

TABLE 2

\begin{tabular}{c|c|c}
\hline$\sigma^{ \pm}$ & $D_{01}=D_{v 2}$ & $D_{\mathrm{t}} \approx D_{\mathrm{z}}$ \\
\hline 0.000 & 1.000 & 2.410 \\
0.178 & 0.982 & 2.366 \\
0.271 & 0.973 & 2.345 \\
0.366 & 0.964 & 2.323 \\
0.465 & 0.955 & 2.301 \\
0.566 & 0.946 & 2.280 \\
0.669 & 0.937 & 2.258 \\
0.776 & 0.928 & 2.236 \\
0.887 & 0.919 & 2.214 \\
1.000 & 0.910 & 2.193 \\
& & \\
\end{tabular}

The partial damping constants are in both types less than critical and the differences between periods are significant. From this reason the changes of partial constants for fixed bandpass are also small.

In Table 3 we have the boundary values for minimum and maximum coupling coefficient for fixed bandpass and several ratios of periods $k_{T}$. (The period $T_{1}$ of the first type seismograph is here equal to $1 \mathrm{sec}$; the equivalent periods $T_{1}=$ $=T_{2}, D_{1}=D_{2}$ are given in the lower part having the same bandpass for the second type.)

TABLE 3

\begin{tabular}{c|c|c|c|c}
\hline \multicolumn{2}{c|}{$\sigma^{2}=0$} & \multicolumn{3}{|c}{$\sigma^{2}=1$} \\
\hline $1 / k_{T}$ & $D_{01}=D_{02}$ & $D_{01}=D_{02}$ & $T_{01}(\mathrm{sec})$ & $1 / k_{T_{0}}$ \\
\hline 10 & 0.636 & 0.598 & 1.08 & 11.6 \\
20 & 0.672 & 0.646 & 1.04 & 21.8 \\
30 & 0.683 & 0.664 & 1.03 & 31.9 \\
40 & 0.689 & 0.674 & 1.02 & 41.9 \\
50 & 0.693 & 0.680 & 1.02 & 51.9
\end{tabular}




\begin{tabular}{l|c|c|c|c|c|c|c}
\hline \multirow{2}{*}{$1 / k_{T}$} & \multirow{2}{*}{$T_{1}(\mathrm{sec})$} & $D_{1}$ & \multicolumn{2}{|c}{$\sigma^{2}=0$} & \multicolumn{3}{|c}{$\sigma^{2}=1$} \\
\cline { 4 - 8 } & & & $T_{01}(\mathrm{sec})$ & $1 / k_{T_{0}}$ & $D_{01}=D_{02}$ & $T_{01}(\mathrm{sec})$ & $1 / k_{T_{0}}$ \\
\hline 10 & 0.316 & 2.41 & 1.45 & 21.2 & 0.924 & 1.59 & 25.2 \\
20 & 0.224 & 3.46 & 1.51 & 45.9 & 0.962 & 1.58 & 49.7 \\
30 & 0.182 & 4.25 & 1.53 & 70.4 & 0.974 & 1.57 & 74.1 \\
40 & 0.158 & 4.91 & 1.54 & 94.3 & 0.982 & 1.56 & 98.2 \\
50 & 0.141 & 5.49 & 1.54 & 119.0 & 0.980 & 1.56 & 122.0
\end{tabular}

Maximum differences in constants of the first seismograph are less than $10 \%$ for $k_{T}=0.1$ and they decrease with decreasing period ratio. The second type of velocity seismograph shows a similar situation. It has usually greater partial damping constants, but the partial period ratio is more than twice smaller for the same bandpass.

The electromagnetic accelerographs and displacement seismographs have always one overdamped system. Because galvanometers are usually used as overdamped systems, this condition limits the possibility of their choice. The velocity seismograph of the first type has partial damping constants of both systems smaller than critical. The range of their values is $\sim 0.6-0.7$, which can be reached by most galvanometers. In the second type of velocity seismograph the equivalent constants have significant values. The substitution with another partial constant reduces the maximum damping constant to the critical (for zero coupling) and the minimum value is about 0.9 (for $k_{T}=0.1, \sigma^{2}=1$ ), and they are again in the range of common galvanometers.

These types of electromagnetic velocity seismographs, from the point of view of possibility realisation and great coupling in the system seismometer-galvanometer are very suitable, and they have some advantage according to seismographs with constant displacement and acceleration sensitivity.

The full text will be published in the Studia geophysica et geodaetica 9 (1965). 


\title{
CLOSING SPEECH
}

\author{
A. ZÁTOPEK \\ President of the ESC \\ (Prague, Czechoslovakia)
}

\section{Ladies and Gentlemen, Dear Colleagues}

We are now going to close the VIII ${ }^{\text {th }}$ Assembly of the European Seismological Commission. Therefore, we have to summarize its activities in order to draw conclusions how to better its work in the future.

The progress reports presented at the beginning of the Assembly reflected undoubtedly the fact that there was a work which has been done, and that a further progress has been reached in solving the problems resulting from the programme of the ESC and the resolutions adopted in Jena and at the foregoing Assemblies. Simultaneously, we have seen that the structure of our Commission should be adjusted in a way which would be more appropriate to obtain a more productive method of work. It was very good, at least in my opinion, that we succeeded in finding a wider basis for the investigation of the earth crust structure by means of explosions and I should express, on behalf of our Commission, many thanks to Prof. Closs for his initiative and his great and successful effort concerning this difficult task.

I think also that the transformation of the working group for the Upper Mantle Project into a corresponding Sub-Commission was necessary, taking into consideration the variety of problems involved in its research work. It will be desirable to group these problems conveniently and to have some points of concentration in the working programme, but it is only a task of internal organization of work which Vice-President Savarensky, Chairman of this Sub-Commission, certainly will solve in the near future.

Also the creation of the Sub-Commission for microseisms and of the working group for the study of the focal mechanism of European earthquakes, working in the framework of the Sub-Commission for Seismicity, was useful.

The links established for a better coordination between individual Sub-Commissions may bring good fruits as it concerns the exchange of opinions and introduction of progressive ideas or experience into the work in a large scale.

I also think that we did not establish a clumsy and burocratic system when we legalized the position of a Secretary Adjoint in the Bureau of the Commission.

As it concerns the purely scientific parts of our meeting, I think that the system of isolated communications of many speakers should be replaced by a system of several not too numerous reporters, who would be able to present concentrated reports on given subjects, and the individual contributions could be then treated in the discussions. This system may lead to a considerable time economy and bring a deeper analysis of the problems in question, if, naturally, the participants could be made acquainted with the individual topics of their interest in time. 
But that is also a question of the organization of work and a task for the corresponding Presidents or Chairmen.

In any case a thematic concentration and coordination are the first order task in the future work of the ESC and the preparation of contributions for the next meeting, if scientific symposia would be included, will require a sufficiently long time interval, longer than it was the use as yet.

I do not know in what way we establish the contacts with UNESCO and organize the implementation of the UMP resolution, concerning the long profiles. But anyway, we must be very busy in finding out the possibilities.

As it concerns the general scientific level of the meeting, I think it was very good and a number of the contributions presented here may be considered to be of value for a better knowledge of the earth and for the development of seismology. New valuable contacts and a fruitful exchange of ideas and opinions belong also to the positive balance of our meeting.

We were working in a very agreable milieu. This was given to our disposal by the Hungarian Academy of Sciences and we enjoyed the famous and wonderful Hungarian hospitality. We are very grateful for these opportunities. But it meant a lot of work for many people to whom we are greatly indebted. First, it was the Organizing Committee, headed by our colleague and my friend Prof. Egyed, with Dr. Bisztricsány and Dr. Csomor as "spiritus agentes" and the ladies working in the Bureau of the Assembly. This organization was working all the time at our meetings with admirable devotion and extreme ability in the polylingual agenda of our programme. Many, many, thanks also for the thousands of "petits riens" which have been arranged by them for the benefit of our Assembly and its individual participants. In the same sense we have to thank the group of the Ibusz Bureau.

As it concerns our Assembly itself I should like to express my most cordial thanks to our Secretaries Mr. Peterschmitt and Mr. Van Gils who fulfilled their difficult and intricated tasks on a traditionaliy high level; I thank also Prof. Rothé and Prof. Knopoff for their collaboration and for their good general judgement on our Assembly; I further thank our Vice-Presidents Savarensky and Vesanen for their devoted collaboration; beside this we are indebted to Prof. Savarensky for his chairmanship of the Symposium on the Upper Mantle, as well as to all Chairmen of the individual sessions; I thank all speakers who took part in contributions and discussions. Many thanks are addressed to Prof. Meisser for his chairmanship in the Voting Committee, to Prof. Toperczer, and to all those who have assisted in formulating and checking resolutions or contributed in any way to the success of the Assembly.

We must also thank in advance the Hungarian Academy of Sciences for printing the volume on our Assembly, and the editorial Board for taking care of the preparation of this publication.

I thank all of you, wishing you very much success in your life and work for the ESC in your home countries.

Herewith, I close the VIII ${ }^{\text {th }}$ Assembly of the European Seismological Commission. 



\section{LIST OF PARTICIPANTS}

\section{Ahorner}

\section{Cologne, GFR}

G. ANGenheIster Munich, GFR

E. Bederke Göttingen, GFR

Cl. Behnke Hannover, GFR

E. BiszTricsÁNY Budapest, Hungary

M. BerCKHEMER Frankfurt, GFR

I. BRouCEK

Bratislava, Czechoslovakia

V. I. BunE Moscow, USSR

V. CervenÝ Prague, Czechoslovakia

H. Closs Hannover, GFR

L. Constantinescu Bucharest, Romania

D. Csomor Budapest, Hungary

G. DoHR Hannover, GFR

L. EGYED Budapest, Hungary

E. M. Fournier D'Albe Unesco, France

O. Förtsch Munich, GFR

A. G. Galanopoulos Athens, Greece

J. GÁLFY Budapest, Hungary

P. GIESE Munich, GFR

J. M. VAN GILS Uccle, Belgium

M. GRANDJEAN Algeria, Algiers

\section{E. Grigorova}

Sofia, Bulgaria

I. E. GuBin Moscow, USSR

D. GüTH Jena, GDR

J. HJELME Copenhagen, Denmark

L. HiersemanN Halle, GFR.

M. HoPPE Leipzig, GDR

REV. R. INGRAM Dublin, Ireland

H. JENSEN Copenhagen, Denmark

J. N. JoRDAN Washington, USA

N. K. KARAPETIAN Leninakan, USSR

\section{Z. KIss} Budapest, Hungary

L. KNOPOFF Los Angeles, California

Ch. KNothe Freiberg, GDR

S. D. KoGAN Moscow, USSR

H. KorHONEN Oulu, Finland

B. KunZ Vienna, Austria

I. M. Kunnetsova Moscow, USSR

Mme Y. Labrouste Paris, France

U. Luosto Helsinki, Finland

R. MAAZ Jena, GDR

H. MARTIN Jena, GDR 


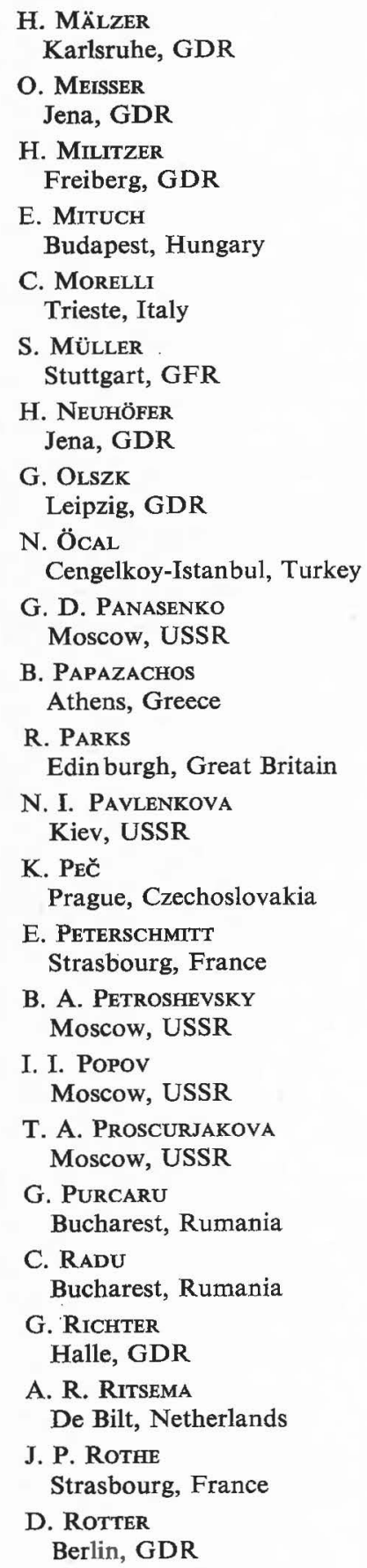

L. RUPRECHTOVÁ Prague, Czechoslovakia

E. F. SAVARENSKY Moscow, USSR

D. SEIDL Stuttgart, GFR

M. A. Sellevoll Bergen, Norway

B. Simon Budapest, Hungary

L. B. Slavina Moscow, USSR

A. A. Sorsky Moscow, USSR

A. Sörnes Bergen, Norway

W. SPONHEUER Jena, GDR

J. STELZNER Jena, GDR

A. TÁrCZI-HoRNOCH Sopron, Hungary

P. TeIKARI Helsinki, Finland

Ch. Teupser Jena, GDR

E. TILLOTSON England

V. TOBYÁš Prague, Czechoslovakia

M. TOPERCZER Vienna, Austria

J. UCHMAN Warsaw, Poland

W. UlLMANN Jena, GDR

J. VANĚK Prague, Czechoslovakia

R. VEES Mainz, GFR

E. Vesanen Helsinki, Finland

L. WANIEK Prague, Czechoslovakia
A. ZÁtopeK Prague, Czechoslovakia 